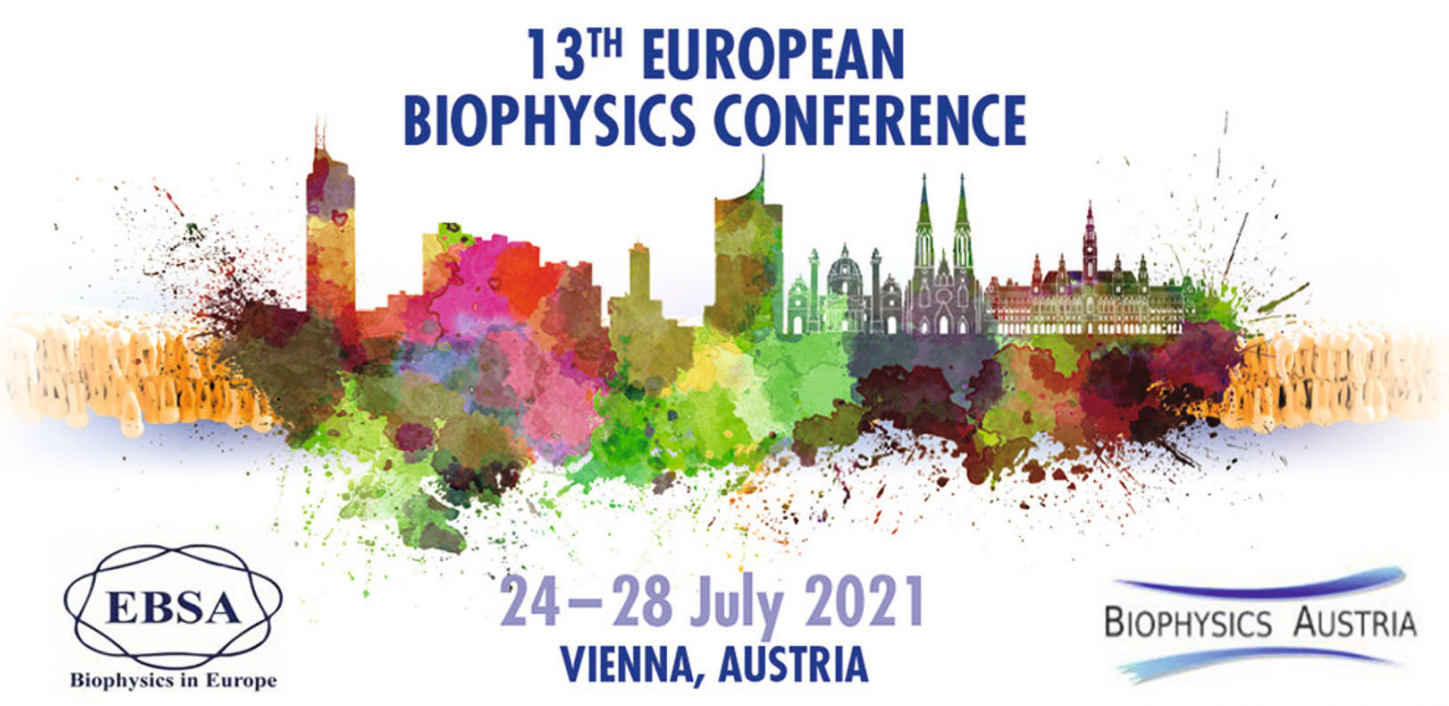

$13^{\text {th }}$ EBS A congress

July 24-28, 2021, Vienna, Austria 


\section{Acknowledgements}

\section{Silver sponsors:}

- Bruker Nano Surfaces Division

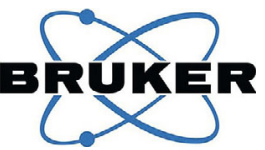

- Beckman Coulter

BECKMAN

COULTER

Life Sciences

Bronze sponsors:

- Boehringer Ingelheim

- Elements

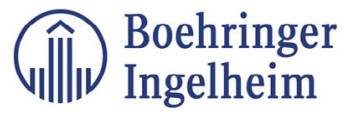

\section{elements}

\section{Exhibitors:}

- Fida Biosystems

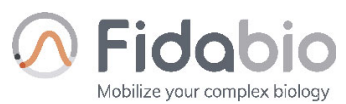

- Olympus - Life Science Solutions

\section{OLMMPUS}

\section{Other Sponsors:}

- Austrian Physical Society

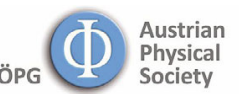

- Biophysical Society

Biophysical Society

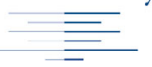

- DFG -- Deutsche Forschungsgemeinschaft

7 G Deutsche

Forschungsgemeinschaft

German Research Foundation
- PicoQuant

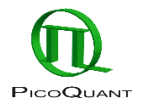

- REDSHIFT Bio

REDSHIFTbỉo
- Teledyne Photometrics 71- TELEDYNE PHOTOMETRICS

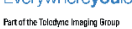




\section{$13^{\text {th }}$ EBSA European Biophysics Congress Vienna (Austria), July 24-28, 2021}

Organized by Biophysics Austria

\section{CONFERENCE CHAIR}

Elena E. POHL

Institute of Physiology, Pathophysiology and Biophysics,

University of Veterinary Medicine Vienna

Vice President of Biophysics Austria

Vice President of EBSA

\section{LOCAL ORGANIZING \& SCIENTIFIC COMMITTEE}

Klaus GROSCHNER, Institute of Biophysics, Medical University Graz

Carl-Philipp HEISENBERG, Institute of Science and Technology Austria, Klosterneuburg

Peter HINTERDORFER, Institute of Biophysics, Johannes Kepler University Linz

Andreas HORNER, Institute of Biophysics, Johannes Kepler University Linz

Thomas A. KLAR, Institute for Applied Physics, Johannes Kepler University Linz

Robert KONRAT, Max F. Perutz Laboratories, University of Vienna

Georg PABST, Insitute of Molecular Biosciences, University of Graz

Birgit PLOCHBERGER, University of Applied Sciences, Linz

Elena E. POHL, Institute of Physiology, Pathophysiology and Biophysics,

University of Veterinary Medicine Vienna

Peter POHL, Institute of Biophysics, Johannes Kepler University Linz

Ruth PRASSL, Institute of Biophysics, Medical University Graz

Erik REIMHULT, Institute for Biologically inspired materials,

University of Natural Resources and Life Sciences, Vienna

Gerhard SCHÜTZ, Institute of Applied Physics, TU Wien

Thomas STOCKNER, Institute of Pharmacology, Medical University of Vienna

José Luis TOCA-HERRERA, Institute of Biophysics,

University of Natural Resources and Life Sciences, Vienna

Bojan ZAGROVIC, Max F. Perutz Laboratories, University of Vienna 


\section{EBSA EXECUTIVE COMMITTEE}

Jacqueline CHERFILS, Centre National de la Recherche Scientifique (CNRS), France Mauro DALLA SERRA, National Research Council of Italy, Italy

Robert GILBERT, University of Oxford, UK

Helmut GRUBMÜLLER, Max-Planck Institute of Biophysical Chemistry, Germany

Sarah KÖSTER, Georg-August-University Göttingen, Germany

André MATAGNE, University of Liège, Belgium

László MÁTYUS, University of Debrecen, Hungary

Pierre-Emmanuel MILHIET, INSERM, CNRS, Montpellier University, France

Jesús PERÉZ-GIL, Universidad Complutense, Spain

Elena E. POHL, University for Veterinary Medicine Vienna, Austria

Nuno C. SANTOS, Universidade de Lisboa, Portugal

John M. SEDDON, Imperial College London, UK

Ilpo VATTULAINEN, University of Helsinki, Finland

Anthony WATTS, University of Oxford, UK

Anthony J. WILKINSON, University of York, UK

Dejan ŽIKIĆ, University of Belgrade, Serbia

\section{INTERNATIONAL SCIENTIFIC COMMITTEE}

Maria T ALONSO, Valladolid, Spain

Oleg BATISHCHEV, Moscow, Russia

Peter JÖNSSON, Lund, Sweden

Sarah KÖSTER, Göttingen, Germany

Adriana MIELE, Lyon, France

Ludo RENAULT, Leiden, Netherlands

Nuno CORREIA SANTOS, Lisbon, Portugal

John SEDDON, London, United Kingdom

Petra SCHWILLE, Martinsried, Germany

Mauro DALLA SARRA, Rome, Italy

Ilpo VATTULAINEN, Helsinki, Finland

Viktorie VLACHOVÁ, Prague, Czech Republic 


\section{Welcome to $13^{\text {th }}$ EBSA Biophysics Congress in Vienna 2021.}

Dear colleagues,

Dear friends,

On behalf of the Austrian Biophysical Society (Biophysics Austria) and the European Biophysical Societies Association (EBSA), I should like to extend a warm welcome to all of you at the $13^{\text {th }}$ EBSA meeting in beautiful Vienna.

EBSA2021 is one of the first scientific meetings to be held on-site after the lengthy period of virtual congresses. During the global COVID-19 pandemic, we all became acutely aware of how much we miss the excitement of catching up with colleagues and friends, of motivating discussions, of arranging new cooperations, of being exposed to new ideas and different perspectives and, not least, of discovering new cultures, cities and countries - everything that the meeting in Vienna offers us.

For the first time in the history of biophysical congresses, the $13^{\text {th }}$ EBSA meeting will take place in hybrid form, both on-site and online, allowing every interested biophysicist worldwide - independent of the travel situation - to join and contribute to this event. This combination will open up a new era in congress organization and we are confident that it will become the main modus for future congresses.

The 2021 meeting is unique in many ways. In 2021, the Austrian Biophysical Society celebrates its $60^{\text {th }}$ birthday. Austria has a long-standing tradition in biophysics. Biophysics Austria was founded in 1961 and is among the oldest biophysical societies in Europe.

In 1971, 50 years ago, with the "collaboration of sister societies and under the co-sponsorship of the International Union for Pure and Applied Biophysics (IUPAB)" Biophysics Austria hosted the first European Biophysics Meeting in Biophysics in Baden near Vienna. The opening lecture was given by Max F. Perutz, who won the 1962 Nobel Prize for his work on the structures of haemoglobin and myoglobin. The diverse program of the first congress included 19 symposia that covered among others, Biophysics of Transport Processes, Regulation of Virus Protein Synthesis, The Cardiac Electric Field, Water in the Function of Biological Systems, Bioenergetics, Radiation, and Environmental Biophysics, and Origin of Life. Many of these topics are still acutely relevant today. 
Unfortunately, 26 years elapsed before the second congress has taken place in Orléans, France (1997). Since 2003 the EBSA Congress is a biennial event and has developed into one of the two most essential biophysicist gatherings in the world.

This years' congress will feature 90 invited speakers, who will share their latest exciting discoveries. There will be more than 90 slots reserved for junior researchers to present their results in short talks. EBSA2021 also features cross-disciplinary plenary lectures to foster discussions at the frontiers of current biophysical research, including single-molecule biophysics; high resolution optical and force microscopy; ion channels and transporters; lipids and membranes; structural biology; theoretical biophysics; cell mechanics and many other subjects. Basic research has paved the way for the successful fight against the current pandemic, specifically for the development of mRNA vaccines (such as those from Biontech Pfizer and Moderna). The battle against Covid-19 will also be a major topic at the EBSA 2021 Congress.

At the time of writing this welcome, participants from 39 countries have submitted 563 abstracts. Session chairs, members of the scientific committee, and the EBSA Executive Committee members have performed excellent work in assembling the scientific program and EBSA member societies have contributed by suggesting speakers.

For the first time, a public lecture open to all interested in science will start the congress. Nobel laureate Ada Yonath will honour the contribution of Erwin Schrödinger to life sciences by outlining her view on the age-old question of "What is life?". Her talk is particularly apt as Schrödinger's book of the same title also addressed the lay reader. In 2021 we celebrate the $60^{\text {th }}$ anniversary of the Austrian physicist and Nobel Prize winner Erwin Schrödinger.

EBSA is continuing the collaboration with the sister societies that started 60 years ago. Austrian Physics Society is sponsoring Ada Yonath's lecture, while the Biophysical Society of America and the IUPAB are supporting the lectures of Karolin Luger and Francisco Bezanilla. The Biophysical Society of America will also present twelve awards of US $\$ 500$.

We are grateful to all plenary and invited speakers who have volunteered to travel to Vienna despite the pandemics-mediated uncertainties caused by the pandemic during the months leading up the meeting. We are looking forward to their brilliant talks and their equally important, encouraging contributions to the discussion of short talks and posters. EBSA is awarding bursaries to facilitate the participation of 60 talented young colleagues and Biophysics Austria is providing prizes for the best three virtual posters.

Many devoted scientists have contributed to the preparation of the event. We have received substantial input from the session chairs, from the editor of the European Biophysics Journal, Rob Gilbert, and from members of EBSA's executive committee. Special thanks 
go to Anthony Watts, Jesús Pérez-Gil, John Seddon, Tony Wilkinson, André Matagne and Éric Quiniou.

We also thank the professional congress organizers from RAM/Columbus, headed by Renate Androsch-Holzer, and acknowledge the continuing support of the Vienna Convention Office and the Austria Center Vienna. EBSA2021 has also profited from multiple industrial sponsors.

The local organizing committee is highly pleased and honoured to host the scientific communities of the European Biophysics Associate Societies. We have gone to great lengths to make the EBSA 2021 Meeting in Vienna as enjoyable and memorable as possible and hope that each and all of you will feel the inspiring charm of our city. We trust you will love your stay in Vienna and look forward to seeing you again at the $14^{\text {th }}$ EBSA Congress in Stockholm, Sweden in 2023.

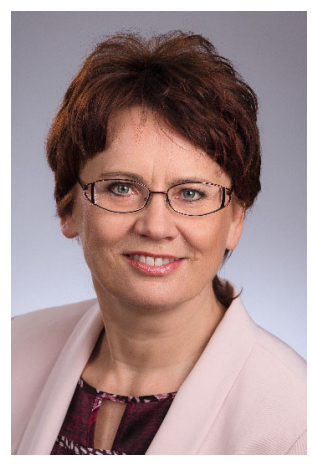

Elena E. Pohl

Chair of EBSA 2021

Vice-President of the EBSA

Organization Committee: Klaus Groschner

Andreas Horner

Georg Pabst

Birgit Plochberger

Peter Pohl

Erik Reimhult

Rainer Schindl

Gerhard Schütz

Thomas Stockner 


\section{The EBSA Executive Council at a committee meeting in Vienna in January 2020.}

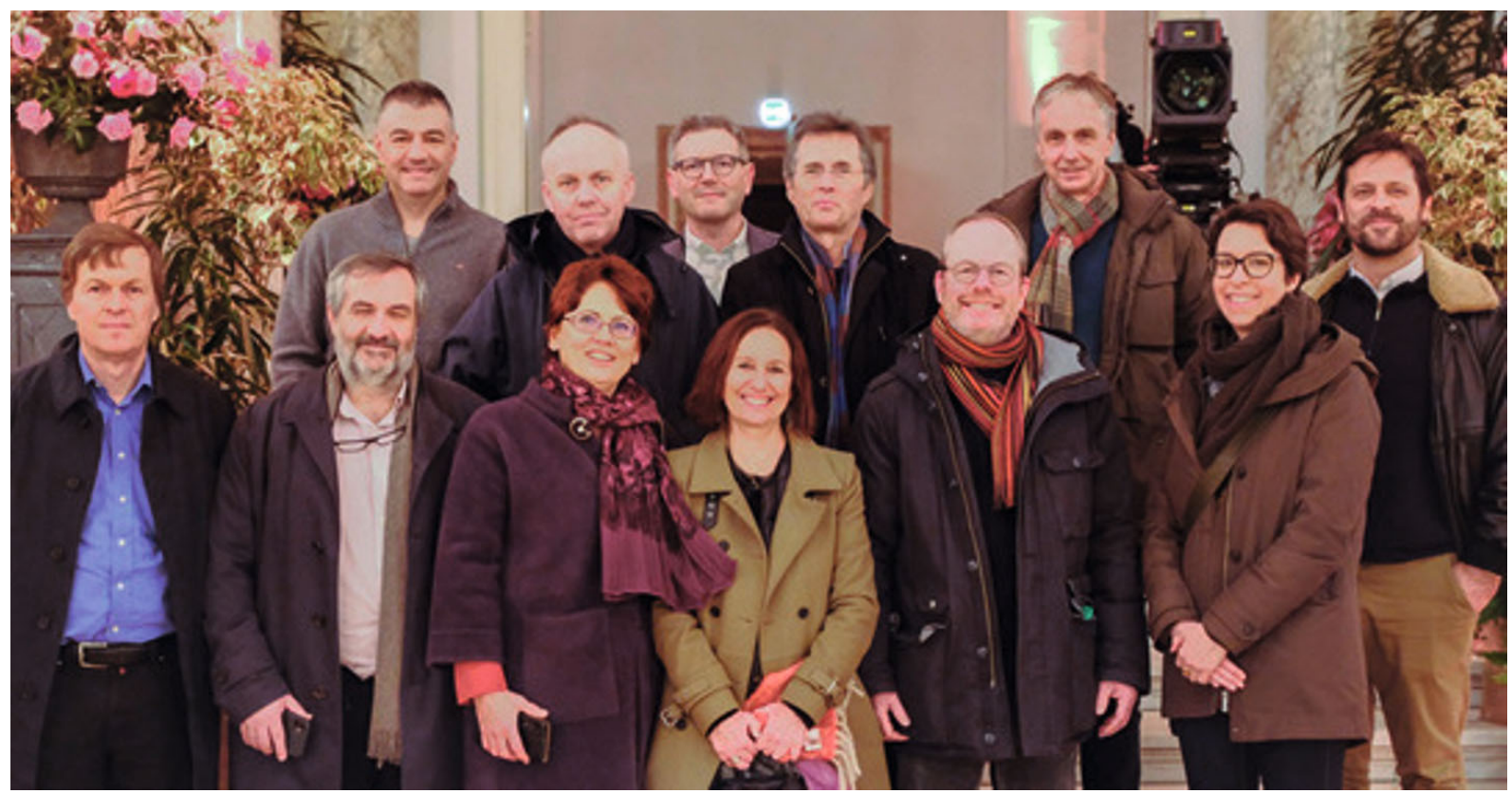

Members are (from left to right): Helmut Grubmüller, Göttingen, Germany; Jesús PérezGil, Madrid, Spain; Dejan Žikić, Belgrade, Serbia; Robert Gilbert, Oxford, England; Elena Pohl, Vienna, Austria; Mauro Dalla Serra, Trento, Italy; Jacqueline Cherfils, Paris, France; John Seddon, London, England; André Matagne, Liege, Belgium; Anthony Wilkinson, York, England; Sarah Köster, Göttingen, Germany; Nuno Santos, Lisbon, Portugal. Not present are: Ilpo Vattulainen, Helsinki, Finland; László Mátyus, Debrecen, Hungary; Pierre-Emmanuel Milhiet, Montpellier, France; Anthony Watts, Oxford, England. 


\section{Welcome from the President of EBSA}

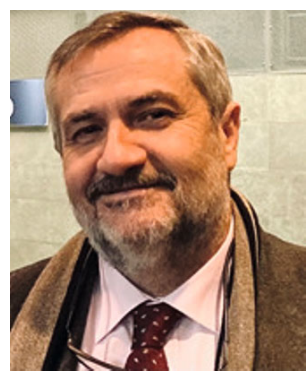

The world is dealing with one of its greatest challenges, and thanks to the amazing contribution of science and the development, in record time, of vaccines against SARS-CoV2 virus, we are starting to envision the light at the end of the tunnel of the Covid-19 pandemic. It is at this time, when our biannual date with European Biophysics come onto the agenda, as a new edition of the European Biophysics Congress will be opening in Vienna 24-28 July 2021 (https : / www . ebsa2021 .org). Biophysics is contributing significantly, in close collaboration with so many other disciplines, to a solution of the Covid-19 problem, and we will have in Vienna a good selection of biophysical studies and concepts related with the most recent research efforts during Covid-19 time.

EBSA is really grateful to our host colleagues from the Austrian Biophysical Society for accepting the challenge of organizing our Congress in Vienna during these extremely difficult times, despite being full of uncertainties. They are making every effort to have an at least partially physically present Congress, where we can start again to meet and restablish the irreplaceable flavor associated with exchanging ideas and collaboration opportunities in Biophysics, face to face. The rapid evolution of the sanitary conditions across the different countries in Europe, including the progressive extension of the vaccination coverage, makes the scenario somehow unpredictable, but we are hopeful of joining a good sample of European biophysics in person in Vienna. EBSA is really indebted to Prof. Elena Pohl, from University of Veterinary Medicine Vienna, and her team, for taking the lead to assemble an attractive scientific programme, in the tradition of the spectacular past European Congresses celebrated in Dresden (2015), Edinburgh (2017) and Madrid (2019), with a very dynamic organization, ready to adapt to any conceivable possible situation.

The congress in Vienna has been organized to combine both physical and on-line activities, facilitating the participation of everybody in the biophysical world, no matter the country or particular sanitary condition at any context. This obligated constraint has added an additional level of complexity to the local Organizing Committee that we all in EBSA have to heartily acknowledge. Thanks Elena, we 
will be always indebted to you and your team for your contribution to sustain Biophysics and bringing Biophysics again to the focus, in such a tough time.

This Vienna Biophysics Congress will thus allow once more the exchange of scientific ideas behind the science that is currently running in the laboratories of the 34 biophysical societies under the umbrella of EBSA in the European area, representing around 5000 scientists that are members of EBSA through their respective national societies. An illustrative number of teams will be presenting their latest achievements in Biophysics in Vienna, and a complementary group of them will be present and equally available through the screens. EBSA is also indebted to Springer-Nature, the publisher of the European Biophysics Journal (EBJ), for its commitment to disseminate Biophysics across Europe and beyond, with its support for our Congress in Vienna, which will encounter in EBJ a unique tool to spread the last novelties brought to Biophysics in Vienna.

In addition, the EBSA Congress in Vienna will permit us once more to establish solid collaborative connections with our fraternal societies extending support for Biophysics across the whole planet. EBSA is associated with the International Union of Pure and Applied Biophysics (IUPAB), which will be present in Vienna through different talks and sessions. EBSA also maintains fruitful collaborative links with the US-based Biophysical Society, which is also supporting our congress with an offer of bursaries and poster prizes, as a way to promote collaborative Biophysics across the continents.

Once more, EBSA is also proud to recognize in 2021, in the context of the Vienna Congress, the achievements of some of our star biophysicists, who have been awarded the two main biannual EBSA Prizes: The EBSA Young Investigator Award, with a cash prize supported directly by EBSA, and the Avanti/EBSA Award for Membrane Biophysics, generously supported by Avanti Polar Lipids Inc. This year the Young Investigator Award has been given to Yaser Hashem, from Bordeaux University, for his outstanding contributions to cryoelectron microscopy and structural biology studies of macromolecular complexes. The Avanti/EBSA Award has gone to Elina Ikonen, from University of Helsinki, for her seminal contribution to the study of structure-function determinants in biological membranes. Both awardees will deliver plenary lectures in the context of the Congress, and have

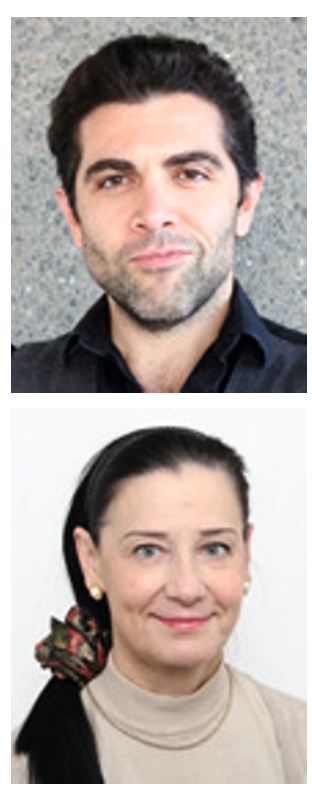


accepted to prepare a contribution that will be published in EBJ to aid the dissemination of the fundamental biophysical concepts to which they have contributed.

EBSA will be delighted to welcome anybody interested in Biophysics to Vienna, and to facilitate exchange of ideas and support to all the national biophysical societies under EBSA umbrella. We will warmly welcome the Presidents of all the societies to the traditional Presidents' Meeting, that will be set in a hybrid (physical/virtual) mode, in the context of the Congress here in Vienna. We also encourage the voting delegates from all the societies to bring (or send) their suggestions and contributions to our EBSA General Assembly, for the benefit of the future development of Biophysics in Europe during the next few years.

We thank you all for your participation and we trust you will have a great experience, whether physical or virtual, benefiting and enjoying the congress, the science and meeting with others.

Jesús Pérez-Gil

President of EBSA (2019-2021) 


\section{Satellite Meetings}

Proton transport and proton-coupled transport

Vienna, 22-24 July 2021

Organiser: Peter Pohl

Johannes Kepler Universität, Linz, Austria

The meeting brings together European Scientists interested in interfacial protons and related transport phenomena. Proton transfer is crucial in numerous biological and chemical processes, e.g., in cellular proton pumps or hydrogen fuel cells. Proton transfers are among the most common biochemical reactions, being involved both in the use of chemical energy in mitochondrial respiration and in light initiated oxygen fixing photosynthetic reactions. The dysfunction of proton transporters was reported to have implications for cancer, obesity, or gastritis - to name a few examples. Many details of the underlying proton transfer mechanisms are still unresolved, and understanding how confined water mediates proton dynamics remains a fundamental challenge in biophysics. Furthermore, in the biotechnical field, the deep understanding of proton transfer will enable biomimetic applications, such as recreation of ATP biosynthesis.

Research-Data Management in Biophysics

Vienna, 24 July 2021, 9:00 to 16:00

Organisers: Carsten Baldauf

Fritz-Haber-Institut der Max-Planck-Gesellschaft, Berlin, Germany

Hans-Günther Döbereiner

Institute of Biophysics, University Bremen, Germany

The aim is to engage part of the audience of the European Biophysics Conference in a discussion about research-data handling in general and about the peculiarities of biophysics data. Experimental data are only meaningful when methods, machinery, sample, measurement conditions, and measured quantities are known. Likewise, computational data is only worth keeping if all aspects of the underlying simulation are recorded in detail as well. To create and provide data according to the FAIR principles (findable, accessible, interoperable, reusable), we need extensive annotation, i.e., a systematic metadata catalogue. Such data structures can only be developed in a community effort. Specific topics of this workshop are:

- Background on research data

- Identifying needs and strategies specific to biophysics regarding

- User and software interfaces of data handling platforms

- Strategies for developing (meta)data standards 


\section{Plenary Lectures}
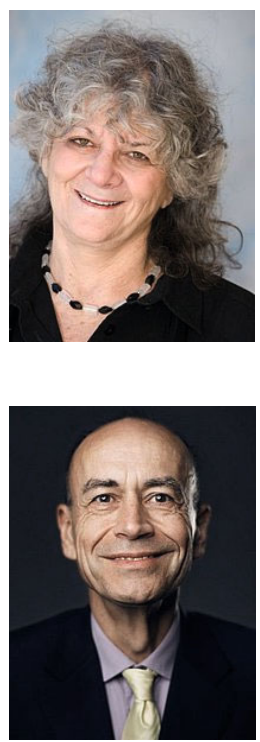

Thomas Südhof

Nobel Prize in Physiology or Medicine 2013

Department of Molecular and Cellular Physiology

Stanford University School of Medicine, USA

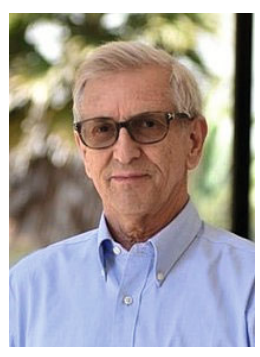

\section{Francisco Bezanilla}

Department of Biochemistry and Molecular Biology

University of Chicago

Illinois

USA

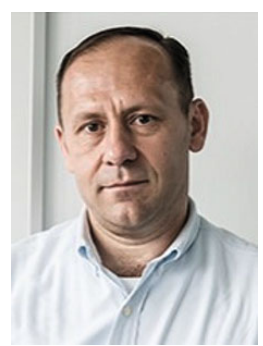

\section{Raimund Dutzler}

Department of Biochemistry

University of Zurich

Zurich

Switzerland
Saturday, July 24, 2021

17:00-18:00

From origin of life to modern medicine
Sunday, July 25, 2021

\section{8:30-9:15}

The molecular logic of synapse formation: From structure to function

Sunday, July 25, 2021

13:15-14:00

Voltage sensors and the opening of ion channels
Monday, July 26, 2021

8:30-9:15

The TMEM16 family of calcium-activated ion channels and lipid scramblases 


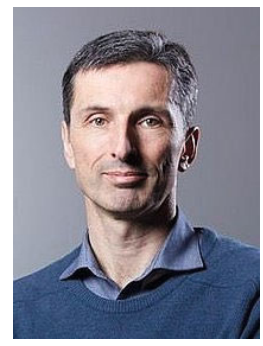

\section{Gerhard Hummer}

Department of Theoretical

Biophysics

Max Planck Institute of Biophysics

Frankfurt am Main

Germany

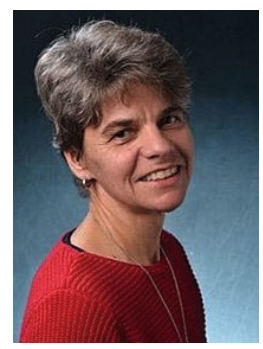

\section{Karolin Luger}

Biochemistry

University of Colorado

Boulder, Colorado

USA

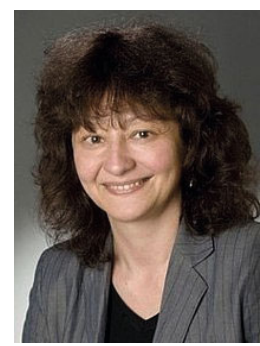

\section{Marina Rodnina}

Physical Biochemistry

Max Planck Institute for

Biophysical Chemistry

Göttingen

Germany

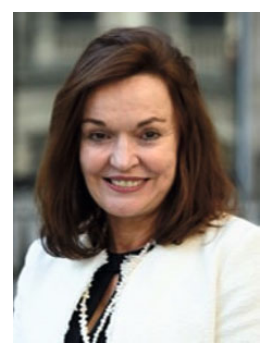

\section{Carol Robinson}

Chemistry Physical and

Theoretical Chemistry Laboratory

University of Oxford

United Kingdom
Monday, July 26, 2021

\section{8:30-9:15}

Molecular simulations of SARS-CoV-2 spike and its interactions with the human host

Monday, July 26, 2021

13:15-14:00

Adventures in the chromatin jungle: Nucleosome

remodelers, archaeal slinkies, and giant viruses

Tuesday, July 27, 2021

13:15-14:00

Protein folding inside the ribosome
Wednesday, July 28, 2021

\section{8:30-9:15}

Protein complexes subjected to tandem mass spectrometry reveal allosteric binding partners 


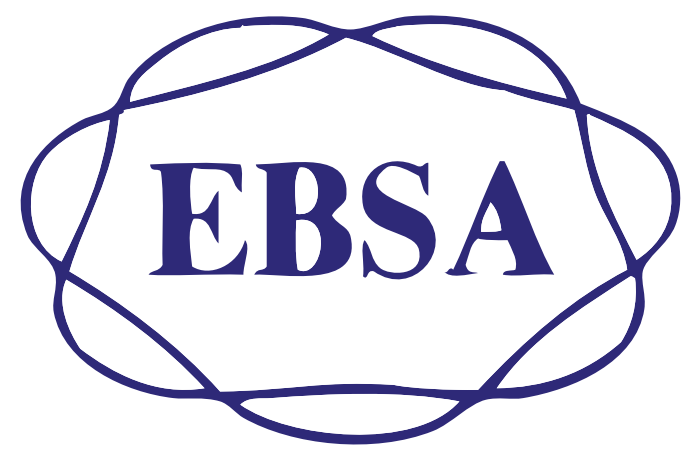

Congresses of the

\section{EUROPEAN BIOPHYSICAL SOCIETIES' ASSOCIATION}

$1^{\text {st }}$ EUROPEAN BIOPHYSICS CONGRESS, 1971, BADEN, AUSTRIA

$2^{\text {nd }}$ CONGRESS, 1997, ORLEANS, FRANCE

$3^{\text {rd }}$ CONGRESS, 2000, MUNICH, GERMANY

$4^{\text {th }}$ CONGRESS, 2003, ALICANTE, SPAIN

$5^{\text {th }}$ EBSA $/ 15^{\text {th }}$ IUPAB / SFB CONGRESS, 2005, MONTPELLIER, FRANCE

$6^{\text {th }}$ CONGRESS, 2007, LONDON, UNITED KINGDOM

$7^{\text {th }}$ CONGRESS, 2009, GENOA, ITALY

$8^{\text {th }}$ CONGRESS, 2011, BUDAPEST, HUNGARY

$9^{\text {th }}$ CONGRESS, 2013, LISBON, PORTUGAL

$10^{\text {th }}$ CONGRESS, 2015, DRESDEN, GERMANY

$11^{\text {th }}$ EBSA $/ 19^{\text {th }}$ IUPAB / CONGRESS, 2017, EDINBURGH, UNITED KINGDOM

$12^{\text {th }}$ CONGRESS, 2019, MADRID, SPAIN

$13^{\text {th }}$ CONGRESS, July 24-28 2021, Vienna, Austria

$14^{\text {th }}$ EBSA CONGRESS in Stockholm, Sweden

(see http://www.ebsa.org/ for details) 


\section{EBSA Bursaries}

Abidine, Yara - Sweden

Affatigato, Luisa - Italy

Aleksanyan, Mina - Germany

Anisimov, Mikhail - Russian Federation

Anthony, Nicholas Stephen - Italy

Baldini, Francesca - Italy

Bano, Fouzia - Sweden

Bednáriková, Zuzana - Slovak Republic

Bera, Krishnendu - Czech Republic

Bernát, Ondrej - Slovak Republic

Borges Araújo, Luís Pedro - Portugal

Cainero, Isotta - Italy

Canepa, Ester - Italy

Casas Ferrer, Laura - France

Cerutti, Elena - Italy

Denieva, Zaret - Russian Federation

Deviri, Dan - Israel

do Rêgo Barros Fernandes Lima,

Maria Augusta - Italy

Dudás, Bálint - France

Džupponová, Veronika - Slovak Republic

Dzurillová, Veronika - Slovak Republic

Hovan, Andrej - Slovak Republic

Hurmach, Vasyl - Ukraine

Iaparov, Bogdan - Slovak Republic

Jacko, Juraj - Slovak Republic

Janaszkiewicz, Angelika - France

Kalutskiy, Maksim - Russian Federation

Kolmogorov, Vasilii - Russian Federation

Krasnobaev, Vladimir - Russian Federation

Lazzeri, Gianmarco - Germany
Leomil, Fernanda - Germany

Lopanskaia, Iuliia - Russian Federation

Madariaga Marcos, Julene - Germany

Mohebi, Ali - Italy

Oravczová, Veronika - Slovak Republic

Perego, Laura - Italy

Petrišič, Nejc - Slovenia

Pierzynska-Mach, Agnieszka Marta - Italy

Pinigin, Konstantin - Russian Federation

Pupkis, Vilmantas - Lithuania

Ridolfi, Andrea - Italy

Robles-Ramos, Miguel ángel - Spain

Ruppelt, Dominik - Germany

Sandu, Nicoleta - Romania

Scodellaro, Riccardo - Italy

Scollo, Federica - Czech Republic

Shekunov, Egor - Russian Federation

Slabý, Cyril - Slovak Republic

Socrier, Larissa - Germany

Spodniaková, Barbora - Slovak Republic

Sudhakar, Swathi - United Kingdom

Svorinic, Andrea - Germany

Timoshenko, Roman - Russian Federation

Tóth, Ágota - France

Trianni, Alberta - Italy

Troiano, Cassandra - Italy

Vaneev, Aleksandr - Russian Federation

Velísková, Martina - Slovak Republic

Volovik, Marta - Russian Federation

Zdorevskyi, Oleksii - Ukraine 


\section{Scientific Program}

(Locations and timings of lectures are in the accompanying Program Schedule)

SATURDAY, JULY 24, 2021

Austria Centre Vienna (ACV)

Bruno-Kreisky-Platz 1

1220 Vienna, Austria

16:30 - 17:00 Welcome Address

17:00 - 18:00 PUBLIC LECTURE

Sponsored by OePG - Austrian Physical Society

Ada E. Yonath, Rehovot, Israel

Laureate of the Nobel Prize in Chemistry 2009

From origin of life to modern medicine

Chair: Elena E Pohl, Vienna, Austria

18:00 - 19:00 Welcome Cocktail

This supplement was not sponsored by outside commercial interests. It was funded entirely by the publisher. 
SUNDAY, JULY 25, 2021

\section{8:30 - 9:15 PLENARY LECTURE \\ Thomas Südhof, Stanford, USA}

Laureate of the Nobel Prize in Physiology or Medicine 2013

The molecular logic of synapse formation: From structure to function

Chair: Claudia Steinem, Göttingen, Germany

\section{9:30 - 12:15 1. INTERFACIAL PROTONS}

Chairs: Mihail Barboiu, Montpellier, France

Stephan Block, Berlin, Germany

Invited speakers ${ }^{*}$

Ana-Nicoleta Bondar, Bucharest, Romania

Dynamic hydrogen-bond networks for proton binding and conformational coupling

Sylvie Roke, Lausane, Switzerland

A water window on membrane biochemistry: Quantifying surface potential, free energy and binding constants.

Tobias Weinert, Villigen, Switzerland

Time resolved structural biology - Bacteriorhodopsin paves the way to a dynamic future

\section{Short talks ${ }^{*}$}

Stefania Brescia, Linz, Austria

The proton channel Hv1 is water-impermeable

Katharina Gloria Hugentobler, Berlin, Germany

Measuring enzyme activity in proteoliposomes on single-enzyme level

Jochen S Hub, Saarbrücken, Germany

Lipid composition and lipid-protein interactions shape the free energy

landscape of pore and stalk formation

\section{9:30-12:15 2. CHANNELS AND $\mathrm{CA}^{2+}$ SIGNALING}

Chairs: Rainer Schindl, Graz, Austria

Viktorie Vlachová, Prague, Czech Republic

Invited speakers ${ }^{*}$

Alexander Sobolevsky, New York, USA

Structural and functional studies of TRP channels 
Anna Moroni, Milan, Italy

The open and closed pore conformations of the pacemaker channel HCN4

Christine Ziegler, Regensburg, Germany

Structural insights into $\mathrm{Ca}^{2+}$ and cholesterol inhibition in the TRP channel PC2

\section{Short talks ${ }^{*}$}

Adam Bartok, Budapest, Hungary

Assessment of temperature sensitivity of the TRPM2 channel

Herwig Grabmayr, Linz, Austria

A novel deletion in the CRAC protein STIM1 diminishes Stormorken syndrome pathology

Tommy Dam, Lund, Sweden

Calcium signaling in T Cells is induced by binding to nickel-chelating lipids in supported lipid bilayers

09:30-12:15

\section{MEDICAL BIOPHYSICS / IMAGING}

Chairs: Alberto Diaspro, Genoa, Italy

Jacak Jaroslaw, Linz, Austria

Invited speakers ${ }^{*}$

Dejan Žikić, Belgrad, Serbia

Biophysical method for determination of cardiovascular age by analyzing arterial blood flow waveform

Eva Rog-Zielinska, Freiburg, Germany

Nanoscopic t-tubular deformation during cardiac mechanical cycle

Leonardo Sacconi, Florence, Italy

Sub-threshold optogenetic manipulation of cardiac electrical activity

\section{Short talks ${ }^{*}$}

Jürgen Strasser, Linz, Austria

Antibodies in motion: How structural dynamics inform biological function

Alberta Trianni, Genova, Italy

Label-free quantitative phase imaging of Hutchinson-Gilford progeria syndrome (HGPS) cells

Martina Alunni Cardinali, Perugia, Italy

Chemo-mechanical investigation of human bone and cartilage in healthy and osteoarthritic conditions by Brillouin and Raman micro-spectroscopy 
09:30 - 12:15 Self-Assembled Session 1

EMERGING BREAKTHROUGH METHODS IN

MOLECULAR-SCALE BIOPHYSICS (ARBRE)

Chairs: Adriana Miele, Lyon, France

Arthur Sedivy, Vienna, Austria

Invited speakers ${ }^{*}$

Takuro Ideguchi, Tokyo, Japan

Advanced vibrational spectroscopy and microscopy for biochemical analysis

Valérie Belle, Marseille, France

Deciphering protein dynamics using nitroxide spin labels combined with EPR spectroscopy

Wouter H. Roos, Groningen, Netherlands

High Speed Atomic Force Microscopy and Fluorescent Optical Tweezers to study nano-scale dynamics in real-time

\section{Short talks*}

Markus Epe, Burlington, United States

Microfluidic Modulation Spectroscopy (MMS) - a new innovative tool for biophysical characterisation of Proteins and Peptides

Andreas Schwaighofer, Vienna, Austria

Laser-based IR spectroscopy for highly sensitive analysis of proteins

Changjiang You, Osnabrueck, Germany

Nanoscopic anatomy of dynamic multi-protein complexes at membranes resolved by graphene induced energy transfer

\section{3:15 - 14:00 PLENARY LECTURE} Sponsored by IUPAB

Francisco Bezanilla, Chicago, USA

Voltage sensors and the opening of ion channels

Chair: Peter Pohl, Linz, Austria 
14:10 - 17:00 4. MEMBRANE TRANSPORTER AND CHANNELS

Sponsored by Nanion Technologies GmbH

Chairs: Thomas Stockner, Vienna, Austria

Lubica Lacinova, Bratislava, Slovakia

Invited speakers*

Thorben Cordes, Munich, Germany

Single-molecule studies of conformational states and dynamics in the ABC importer OpuA

Bert de Groot, Göttingen, Germany

The molecular dynamics of potassium channel permeation, selectivity and gating

Crina Nimigean, New York, USA

Ball-and-chain inactivation in a calcium-gated potassium channel

\section{Short talks ${ }^{*}$}

Chloe Martens, Brussels, Belgium

Hydrogen-deuterium exchange mass spectrometry captures distinct dynamics upon substrate and inhibitor binding to a transporter

Tobias Schubeis, Villeurbanne, France Regulation of a divalent cation channel investigated by $>100 \mathrm{kHz}$ magic-angle spinning NMR

Ágota Tóth, Limoges, France

On the interplay between complex lipid bilayer membrane and multidrug resistance-associated protein 1 (MRP1) by means of molecular dynamics

\section{4:10 - 17:00 5. VIRUS BIOPHYSICS}

Chair: Oleg Batishchev, Moscow, Russia

\section{Invited speakers*}

Ismael Mingarro, Valencia, Spain

Characterizing SARS-CoV-2 membrane envelope proteins

Charlotte Uetrecht, Hamburg, Germany

Flying viruses - from biophysical to structural characterisation

David Alsteens, Louvain, Belgium

Nanomechanical Mapping of Virus Binding Sites to Animal Cells

\section{Short talks*}

Sourav Maity, Groningen, Netherlands

Recording the first steps of virus self-assembly: High Speed AFM imaging unveils assembly dynamics in real-time 
Yara Abidine, Umeå, Sweden

Role of cell-surface glycosaminoglycans and viral glycoproteins in modulating virus binding and diffusion at the cell membrane

Robert Vácha, Brno, Czech Republic

Genome release from non-enveloped RNA viruses: Capsid rupture or pore formation

$14: 10-17: 00$

6. ADVANCED OPTICAL MICROSCOPY

Chair: Gerhard Schütz, Vienna, Austria

Invited speakers ${ }^{*}$

Maria Garcia-Parajo, Barcelona, Spain

High density single molecule dynamic mapping of the cell membrane

Ralf Jungmann, Martinsried, Germany

Super-resolution microscopy with DNA molecules

Thomas Huser, Bielefeld, Germany

Live cell super-resolution optical microscopy by structured illumination microscopy with instant image reconstruction

\section{Short talks ${ }^{*}$}

Josef Lazar, Prague, Czech Republic

Using fluorescent protein directionality in microscopy imaging of membrane protein structure and function

Christian Niederauer, Amsterdam, Netherlands

Single-molecule studies of membrane protein interactions via continuous DNA-mediated fluorophore exchange

Anindita Dasgupta, Jena, Germany

Direct supercritical angle localization microscopy for nanometer 3D superresolution

14:10-17:00 INDUSTRY TALKS

Carl Zeiss GmbH

Bruker Nano Surfaces Division

Beckman Coulter GmbH Austria

Fida Biosystems 
MONDAY, JULY 26, 2021

8:30 - 9:15 PLENARY LECTURE

Raimund Dutzler, Zurich, Switzerland

The TMEM16 family of calcium-activated ion channels and lipid scramblases

Chair: Anthony J Wilkinson, York, United Kingdom

09:30-12:15 7. PROTEIN TRANSLOCATION, ASSEMBLY, AND
FOLDING

Chairs: Peter Pohl, Linz, Austria

Rainer Böckmann, Erlangen, Germany

Invited speakers ${ }^{*}$

Roland Beckmann, Munich, Germany

The AAA-ATPase Bcs1 uses an airlock-like mechanism for folded protein translocation

Sander Tans, Delft, Netherlands

Polypeptide loop translocation by single ClpB disaggregases

Alexey Amunts, Stockholm, Sweden

Protein folding in mitochondria

Short talks*

Mikhail L Kuravsky, Oxford, United Kingdom

The role of intrinsic disorder in DNA recognition and target search by a transcription factor

Mantas Liutkus, San Sebastian, Spain

Two-dimensional metal interaction-driven crystalline protein assemblies

Matthias M Schneider, Cambridge, United Kingdom

Microfluidic diffusional sizing for studying protein-protein interactions

09:30-12:15 8. BIOENERGETICS

Chairs: Karin Busch, Münster, Germany

Mario Vazdar, Zagreb, Croatia

Invited speakers ${ }^{*}$

José Antonio Enríquez Domínguez, Madrid, Spain

Sodium controls hypoxic redox signalling by the mitochondrial respiratory chain 
Edmund Kunji, Cambridge, United Kingdom

The molecular mechanism of substrate binding and transport of the mitochondrial ADP/ATP carrier

Manuela M Pereira, Oreiras, Portugal

Unveiling the membrane bound dihydroorotate:quinone oxidoreductase (DHOQO) from Staphylococcus aureus

\section{Short talks ${ }^{*}$}

Jürgen Kreiter, Vienna, Austria

The transport of fatty acid anions across the inner mitochondrial membrane by the adenine nucleotide translocase

Karim Fahmy, Dresden, Germany

Membrane protein hydration is linked to membrane lateral pressure in Copper-Transport

David Valdivieso González, Madrid, Spain

Membrane softening of microaspirated GUVs containing active F1Fo-ATP synthase

\section{9:30 - 12:15}

\section{QUANTIFICATION OF MOLECULAR FORCES}

Chair: Peter Hinterdorfer, Linz, Austria

\section{Invited speakers ${ }^{*}$}

Matthias Rief, Munich, Germany

Forces in chaperone-mediated protein unfolding and cell adhesion

David Brockwell, Leeds, UK

Length is important! Using force spectroscopy to probe protein:protein interactions

Hongbin Li, Vancouver, Canada

The mechanical design of the bacterial toxin protein RTX

\section{Short talks*}

Yoo Jin Oh, Linz, Austria

Nanomechanical mechanisms of Borrelia interactions with extracellular matrix

Zhaowei Liu, Basel, Switzerland

Optimizing mechanostable anchor points of engineered lipocalin in complex with CTLA-4

Yannic Kerkhoff, Berlin, Germany

Microfluidics-based force measurements with sub-nm resolution and sub-pN sensitivity 
09:30 - 12:15 Self-Assembled Session 2

ADVANCES AND APPLICATIONS IN CRYO-ELECTRON

MICROSCOPY (INSTRUCT-ERIC)

Chair: Ludo Renault, Leiden, Netherlands

\title{
Invited speakers*
}

Margarida Archer, Oeiras, Portugal

Membrane glycosyltransferases as promising anti-tuberculosis targets

Jiří Nováček, Brno, Czech Republic

Preparation and cryo-FIB micromachining of Saccharomyces cerevisiae for cryo-electron tomography

Marjetka Podobnik, Ljubljana, Slovenia

Development and implementation of cryo-electron microscopy in Slovenia

\section{Short talks*}

Daniel Nemecek, Brno, Czech Republic

Easy and simple way for protein structure determination using the new $100 \mathrm{kV}$ cryo-TEM microscope designed for biochemistry and biophysics laboratories

Karin Kornmueller, Graz, Austria

Structural variability and flexibility in low-density lipoprotein

Niels Fischer, Göttingen, Germany

Structural mechanism of GTPase-powered ribosome-tRNA movement

\section{3:15 - 14:00 PLENARY LECTURE}

Sponsored by Biophysical Society of America

Karolin Luger, University of Colorado, USA

Adventures in the chromatin jungle: Nucleosome remodelers, archaeal slinkies, and giant viruses

Chair: Birgit Plochberger, Linz, Austria

\author{
14:10 - 17:00 10. MECHANOBIOLOGY \\ Chair: José Luis Toca-Herrera, Vienna, Austria \\ Invited speakers ${ }^{*}$ \\ Nuria Gavara, Barcelona, Spain \\ The cytoskeleton, by the numbers
}


Andreas Janshoff, Göttingen, Germany

Viscoelastic properties of polarized cells

Malgorzata Lekka, Krakow, Poland

AFM-based differentiation between normal and cancer cells at single-cell and molecule levels

\section{Short talks ${ }^{*}$}

Hannes Witt, Amsterdam, Netherlands

The viscoelasticity of mitotic chromosomes

Johannes Rheinlaender, Tübingen, Germany

Combined SICM-TFM reveals a subcellular correlation between stiffness and traction forces in living cells

Alicja Jagiello, Irvine, United States

Dermal fibroblasts and human breast cancer cells differentially stiffen their local matrix

\section{4:10 - 17:00 11. LIGHT AS A TOOL IN BIOPHYSICS}

Chair: Thomas Klar, Linz, Austria

Invited speakers ${ }^{*}$

Alexander Gottschalk, Frankfurt, Germany

Introducing the optogenetic voltage clamp

Ivana Nikić-Spiegel, Tübingen, Germany

Minimal genetically encoded tags for fluorescent protein labelling in living neurons

Dirk Trauner, New York, USA

Controlling the fate and function of proteins with proximity

photopharmacology

\section{Short talks*}

Tony Schmidt, Graz, Austria

Light-induced gating of ion channels and action potential stimulation with photosensitive organic semiconductors

Virginia Bazzurro, Genoa, Italy

Two-Photon photoactivation of RuBi-GABA for studying the GABAA receptor's modulation by the Antisecretory Factor in cerebellar granule cells in vitro

Jürgen Pfeffermann, Linz, Austria

Gating mechanosensitive channels by photoswitchable lipids 


\section{4:10 - 17:00 12. BIOMIMETIC NANOPORES}

Chairs: Erik Reimhult, Vienna, Austria

Ivan Coluzza, Bilbao, Spain

\section{Invited speakers ${ }^{*}$}

Cees Dekker, Delft, Netherlands

Studying import and export through the nuclear pore complex using bottom up biomimetic approaches

Stefan Howorka, London, United Kingdom

Biomimetic membrane nanopores for next-generation sensing

Meni Wanunu, Boston, USA

Electrical Unfolding of Single Protein Molecules

\section{Short talks ${ }^{*}$}

Denis Knyazev, Linz, Austria

Artificial ion-selective channels formed by diblock copolymers

Sophie Marbach, New York, United States

Fractional noise in nanopores

Andreas Dahlin, Göteborg, Sweden

Solid state nanopores functionalized with polymer brushes

\section{4:10 - 17:00 Self-Assembled Session 3}

IONIC LIQUIDS BIOMOLECULES AND CELLS

Chairs: Antonio Benedetto, Rome, Italy

Hans-Joachim Galla, Münster, Germany

Invited speakers ${ }^{*}$

Samir Mitragotri, Harvard, USA

Ionic liquids for therapeutic applications

Antonio Benedetto, Rome, Italy

Mechanisms of action of ionic liquids on cell membrane mechano-elasticity, cell migration and protein amyloidogenesis

Mara Freire, Aveiro, Portugal

Ionic liquids as solvents and ligands in the purification of biopharmaceuticals

\section{Short talks ${ }^{*}$}

Ksenia S Egorova, Moscow, Russian Federation Synergistic/antagonistic cytotoxic effects in mixtures of ionic liquids with drugs 
Venus Singh Mithu, Amritsar, India

Structural insights into the interaction of amphiphilic cations in ionic liquids with lipid bilayers using solid-state NMR spectroscopy

Harekrushna Sahoo, Rourkela, India

Impact of ionic liquids on the structure, refolding, and activity of proteins 
TUESDAY, JULY 27, 2021

8:30 - 9:15 PLENARY LECTURE

Gerhard Hummer, Frankfurt am Main, Germany

Molecular simulations of SARS-CoV-2 spike and its interactions with the human host

Chair: Helmut Grubmüller, Göttingen, Germany

09:30 - 12:15 13. NEW AND NOTABLE

Chair: Anthony Watts, Oxford, United Kingdom

Invited speakers ${ }^{*}$

Jacob Piehler, Osnabrück, Germany

Resolving cytokine receptor assembly and dynamics in the plasma membrane by single molecule imaging techniques

Dorit Hanein, Paris, France

Coupling molecular activation and its functional output through multiscale imaging

Sebastien Fica, Cambridge, United Kingdom

Cryo-EM reveals molecular basis for conformational equilibrium of the spliceosome

Peter J Judge, Oxford, United Kingdom Structures of the archaerhodopsin-3 transporter reveal that disordering of internal water networks underpins receptor sensitization

09:30 - 12:15 14. MEMBRANE ACTIVE PEPTIDES

Sponsored by Nanion Technologies GmbH

Chairs: Ruth Prassl, Graz, Austria

Nuno Correia Santos, Lisbon, Portugal

\section{Invited speakers ${ }^{*}$}

Burkhard Bechinger, Strasbourg, France

The mechanisms of action of antimicrobial peptides and their synergistic interactions in membranes

Karl Lohner, Graz, Austria

Development of synthetic antimicrobial peptides killing antibiotic resistant bacteria and preventing septic complications

Efstratios Stratikos, Athens, Greece

Travel Award by MDPI Biomolecules

Molecular mechanisms of antigenic peptide generation 


\section{Short talks*}

Christian Nehls, Borstel, Germany

Mode of action of virulence factors of intracellular pathogens studied with time-resolved and high-resolution atomic force microscopy

Nessim Kichik, London, United Kingdom

Candidalysin is a pore-forming peptide toxin that destabilises the cell membrane

Selen Manioglu, Zürich, Switzerland

Investigating the effect of polymyxin class antibiotics on the outer membrane of gram-negative bacteria

09:30 - 12:15 15. METHODOLOGICAL ADVANCES IN BIOMOLECULAR SIMULATIONS

Chair: Bojan Zagrovic, Vienna, Austria

Invited speakers ${ }^{*}$

Lucie Delemotte, Stockholm, Sweden

Elucidation of G-protein-coupled receptor activation via data-driven modeling

Kresten Lindorff-Larsen, Copenhagen, Denmark

Biophysical experiments and biomolecular simulations: A perfect match?

Sereina Riniker, Zürich, Switzerland

Using molecular dynamics simulations to gain insights into biological systems

\section{Short talks*}

Roberto Covino, Frankfurt Am Main, Germany

Discovering molecular mechanisms from computer simulations with deep reinforcement learning

Monica L Fernández-Quintero, Innsbruck, Austria Ensembles in Solution as a New Paradigm in Antibody Structure Prediction and Design

Emmi Pohjolainen, Göttingen, Germany

Free energy along transition pathways from structure refinement simulations

13:15 - 14:00 PLENARY LECTURE

Marina Rodnina, Göttingen, Germany

Protein folding inside the ribosome

Chair: Jesús Pérez-Gil, Madrid, Spain 
14:10-17:00 16. MEMBRANE SIGNAL TRANSDUCTION

Chairs: Peter Jönsson, Lund, Sweden

Eva Sevcsik, Vienna, Austria

Invited speakers ${ }^{*}$

Ana-Suncana Smith, Erlangen-Nürnberg, Germany

Biomechanical of adhesion - from basic principles to mechanisms of immune cell activity

Anton van der Merwe, Oxford, United Kingdom

The critical importance of molecular size in signal transduction at the interface between immune cells and target cells

Martha E Sommer, Berlin, Germany

An ERNEST assessment of arrestin-GPCR interactions

\section{Short talks ${ }^{*}$}

Rainer A Böckmann, Erlangen, Germany

On the loss of inhibitory function due to FcgRIIB-I232T transmembrane polymorphism

Hauke Winkelmann, Osnabrück, Germany

Quantifying cytokine receptor dimerization dynamics in the plasma membrane by smFRET

Isabel D Alves, Pessac, France

Impact of lipid polyunsaturation on dopamine D2 receptor activation, signaling and internalization

14:10 - 17:00 17. CYTOSKELETON / MOTOR PROTEINS

Session is sponsored by DFG, SFB 1456 Mathematics of Experiment

Chairs: Sarah Köster, Göttingen, Germany

Stefan Wieser, Barcelona, Spain

Invited speakers*

Stefan Diez, Dresden, Germany

Torque generation by microtubule-crosslinking motors

David A Weitz, Cambridge, USA

Mechanics of interpenetrating biopolymer networks

Marileen Dogterom, Delft, Netherlands

Reconstituting cytoskeletal systems in artificial cells

Short talks*

Swathi Sudhakar, London, United Kingdom

Germanium nanospheres for ultraresolution picotensiometry of kinesin motors 
Charlotta Lorenz, Göttingen, Germany

Vimentin intermediate filaments stabilize dynamic microtubules by direct interactions

Maxim Igaev, Göttingen, Germany

Bending-torsional elasticity and energetics of the plus-end microtubule tip

14:10 - 17:00 18. MEMBRANE ARCHITECTURE AND ASYMMETRY

Session is sponsored by MDPI / Membranes

Chairs: Georg Pabst, Graz, Austria

John Seddon, London, United Kingdom

Invited speakers ${ }^{*}$

Heiko Heerklotz, Freiburg, Germany

The rise and fall of lipid asymmetry in liposomes

Syma Khalid, Southampton, United Kingdom

Towards computational microbiology of bacterial cell envelopes

Erdinc Sezgin, Stockholm, Sweden

Nanoscale biophysical properties of plasma membrane

Short talks ${ }^{*}$

Eva Sevcsik, Vienna, Austria

Probing the membrane environment of plasma membrane proteins

Marius Trollmann, Erlangen, Germany

Characterization of a lipid nanoparticle for mRNA delivery using molecular dynamics

Iztok Urbancic, Ljubljana, Slovenia

Aggregation and immobilisation of membrane proteins interplay with local lipid order in the plasma membrane of $\mathrm{T}$ cells

18:30 - 20:00 EBSA General Assembly

Plenary Hall 
WEDNESDAY, JULY 28, 2021

\section{8:30 - 9:15 PLENARY LECTURE}

\section{Carol Robinson, Oxford, United Kingdom}

Protein complexes subjected to tandem mass spectrometry reveal allosteric binding partners

Chair: László Mátyus, Debrecen, Hungary

\section{9:30 - 12:15 19. CELL AND TISSUE BIOPHYSICS}

Chair: Carl-Philipp Heisenberg, Vienna, Austria

\section{Invited speakers ${ }^{*}$}

Jean-Léon Maître, Paris, France

Mechanics of blastocyst morphogenesis

Pierre-François Lenne, Marseille, France

Cell surface mechanics beyond the bounds of adhesion and tension

Pavel Tomancak, Dresden, Germany

Comparative approach to tissue mechanics

\section{Short talks ${ }^{*}$}

Hanna-Maria Häkkinen, Barcelona, Spain

Molecular and biophysical mechanisms of protrusion plasticity in epithelial cells during phagocytic tissue clearance

Yoav G Pollack, Göttingen, Germany

Competition drivers in tissue-like confined cellular active matter

Ivan Palaia, London, United Kingdom

Durotaxis of passive nanoparticles on elastic membranes

\section{9:30-12:15 20. LIPID-PROTEIN INTERACTIONS}

Chairs: Markus Axmann, Linz, Austria

Ilpo Vattulainen, Helsinki, Finland

Invited speakers*

Sandro Keller, Graz, Austria

Lipid-bilayer nanodiscs for membrane biophysics

Nathalie Reuter, Bergen, Norway

Interfacial protein-lipid interactions from atomistic molecular simulations

Gregor Anderluh, Ljubljana, Slovenia

Interactions of Nep1-like proteins with lipid membranes 


\section{Short talks*}

Federica Scollo, Prague, Czech Republic

Calmodulin interactions with model lipid membranes: the interplay of $\mathrm{Ca}^{2+}$ and lipid composition

T. Bertie Ansell, Oxford, United Kingdom

Obtaining affinities of protein-lipid interactions from equilibrium molecular dynamics simulations

Fabio Lolicato, Heidelberg, Germany

Cholesterol promotes both head group visibility and clustering of

PI(4,5)P2 driving unconventional secretion of Fibroblast Growth Factor 2

\section{9:30 - 12:15 21. DNA ARCHITECTURE AND GENE REGULATION}

Chair: Anton Goloborodko, Vienna, Austria

\section{Invited speakers ${ }^{*}$}

Eugene Kim, Frankfurt, Germany

Recent insights into DNA loop extrusion revealed by single-molecule imaging: From Z- to SC-loop

Francisco Balaguer Pérez, Madrid, Spain

Cytidine triphosphate promotes efficient ParB-dependent DNA

condensation by facilitating one-dimensional spreading from parS

Johan Elf, Uppsala, Sweden

Live cell imaging reveals that RecA finds homologous DNA by reduced dimensionality search

\section{Short talks*}

Alexandra Teslenko, Lausanne, Switzerland Development of a single-molecule approach to observe ubiquitination dynamics in defined chromatin states

Gabriel Rosenblum, Rehovot, Israel Allostery through DNA drives phenotype switching

Marcus G Bage, Dundee, United Kingdom

Molecular details of mRNA capping enzyme recruitment and activation A computational study 
13:15 - 16:00 22. SYNTHETIC CELL

Chairs: Petra Schwille, Martinsried, Germany

Mauro Dalla Serra, Rome, Italy

Invited speakers*

Irep Gözen, Oslo, Norway

The possible role of solid interfaces in protocell formation at the origin of life

Tobias Erb, Marburg, Germany

Photosynthesis 2.0: From natural photosynthesis to artificial chloroplasts and back

Rumiana Dimova, Potsdam, Germany

To bud or not to bud: remodeling of artificial cells

Short talks ${ }^{*}$

Kevin Jahnke, Heidelberg, Germany

Actuation of DNA-based functional assemblies for synthetic cells

Sunidhi C Shetty, Potsdam, Germany

Directed enzymatic signaling networks in microfluidics-based synthetic eukaryotic cells

Fu Meifang, Munich, Germany

Non-equilibrium large-scale membrane deformation driven by a protein pattern formation system

\section{3:15 - 16:00 23. LIQUID-LIQUID PHASE SEPARATION AND INTRINSICALLY DISORDERED PROTEINS}

Chair: Andreas Horner, Linz, Austria

\section{Invited speakers*}

Edward A. Lemke, Mainz, Germany

Decoding molecular plasticity in the dark proteome

Monika Fuxreiter, Padua, Italia

Travel Award by MDPI Biomolecules

The generic nature of the condensed state of proteins

Salvador Ventura, Barcelona, Spain

Role of alternative splicing in phase separation and pathogenic aggregation

Short talks*

Vito Foderà, Copenhagen, Denmark

Conformational heterogeneity of amyloid protein condensates 
Juan Torreno-Pina, Castelldefels, Spain

Phase separation of tunable transcriptional condensates predicted by an interacting particle model

Emil G. Stender, Kgs. Lyngby, Denmark

High throughput determination of kinetic and thermodynamic parameters of biomolecular liquid-liquid phase separation [Capflex]

Chairs: Maria T Alonso, Valladolid, Spain Klaus Groschner, Graz, Austria

Invited speakers ${ }^{*}$

Jin Zhang, San Diego, USA

Illuminating the biochemical activity architecture of the cell

Roland Malli, Graz, Austria

Targeting recombinant fluorescent biosensors to image microenvironmental ion signals locally on the surface of living cells

Andrea Rentmeister, Münster, Germany

Chemo-enzymatic modifications to investigate and control mRNAs

\section{Short talks*}

Alba del Río Lorenzo, Valladolid, Spain

In vivo calibration of fluorescent genetic-encoded $\mathrm{Ca}^{2+}$ indicators within high $\mathrm{Ca}^{2+}$ content organelles

Ornella Cavalleri, Genova, Italy

Viral RNA detection through DNA-based biosensing

Til Schlotter, Zurich, Switzerland

Force-controlled interface nanopores for single-molecule and single-cell secretion sensing

16:15 - 17:30 AWARD SESSION

Chair: Jesús Pérez-Gil, Complutense University of Madrid, Spain

Yaser Hashem, Bordeaux, France

EBSA Young Investigator Award Lecture

Elina Ikonen, Helsinki, Finland

EBSA/Avanti Award Lecture

Student Awards

17:30 - 18:00 Closing Ceremony

Congress Dinner 


\section{Table of Contents}

\section{Oral presentations}

Page Session title

S39 Plenary Lectures

S41 1 - Interfacial Protons

S43 2 - Channels and $\mathrm{Ca}^{2+}$ signaling

S45 3- Medical biophysics / Imaging

S47 - Self-Assembled Session 1

Emerging breakthrough methods in molecular-scale biophysics (ARBRE)

S49 4- Membrane transporter and channels

S51 5 - Virus biophysics

S53 6-Advanced optical microscopy

S55 7 - Protein translocation, assembly, and folding

S57 8 - Bioenergetics

S59 9-Quantification of molecular forces

S61 - Self-Assembled Session 2

Advances and applications in cryo-electron microscopy (Instruct-ERIC)

S63 10 - Mechanobiology

S65 11 - Light as a tool in biophysics

S67 12 - Biomimetic nanopores

S69 - Self-Assembled Session 3

Ionic liquids biomolecules and cells

S71 13 - New and Notable

S72 14 - Membrane active peptides

S74 15 - Methodological advances in biomolecular simulations

S76 16 - Membrane signal transduction

S78 17 - Cytoskeleton / Motor proteins

S80 18 - Membrane architecture and asymmetry

S82 19 - Cell and tissue biophysics

S84 20 - Lipid-Protein interactions

S86 21 - DNA architecture and gene regulation

S88 22 - Synthetic cell

S90 23 - Liquid-liquid phase separation and intrinsically disordered proteins

S92 24 - Biosensors 


\section{Posters}

Page Session title

S94 1 - Interfacial Protons

S95 2 - Channels and $\mathrm{Ca}^{2+}$ signaling

S97 3- Medical biophysics / Imaging

S102 - Self-Assembled Session 1

Emerging breakthrough methods in molecular-scale biophysics (ARBRE)

S105 4-Membrane transporter and channels

S112 5-Virus biophysics

S115 6 - Advanced optical microscopy

S120 7 - Protein translocation, assembly, and folding

S126 8 - Bioenergetics

S129 9-Quantification of molecular forces

S131 - Self-Assembled Session 2

Advances and applications in cryo-electron microscopy (Instruct-ERIC)

S132 10 - Mechanobiology

S139 11 - Light as a tool in biophysics

S146 12 - Biomimetic nanopores

S148 - Self-Assembled Session 3

Ionic liquids biomolecules and cells

S150 13 - New and Notable

S151 14 - Membrane active peptides

S156 15 - Methodological advances in biomolecular simulations

S161 16 - Membrane signal transduction

S164 17 - Cytoskeleton / Motor proteins

S168 18 - Membrane architecture and asymmetry

S173 19 - Cell and tissue biophysics

S179 20 - Lipid-Protein interactions

S190 21 - DNA architecture and gene regulation

S193 22 - Synthetic cell

S196 23 - Liquid-liquid phase separation and intrinsically disordered proteins

S199 24 - Biosensors 
Oral Presentations

- Plenary Lectures -

0-1

From origin of life to modern medicine

Ada Yonath

Weizmann Institute of Science, Rehovot, Israel

Ribosomes possess spectacular architecture accompanied by inherent mobility, which allow their smooth performance in providing the environment needed for amino acids polymerization. Thus, peptide-bonds are being formed within a universal pocket-like semi-symmetrical region connecting all of the ribosomal features involved in nascent chain creation and elongation. The elaborate architecture of this region positions the amino-acids in appropriates stereochemistry for peptide bond formation, substrate-mediated catalysis, substrate translocation and nascent chain insertion into their exit tunnel.

This pocket-like region is still functioning within all contemporary ribosomes and its high conservation implies its existence irrespective of environmental conditions. Hence, indicating that it may represent an ancient RNA apparatus, therefore called by us the proto-ribosome.

We suggest that it evolved from an earlier molecular machine with bonding capabilities, which could perform RNA needs. When amino acids showed up, they snatched the apparatus and turned it into enabling peptide bond formation. Among the so generated random oligo-peptides, those that were found useful survived and triggered the formation of a primitive genetic code, which was co-optimized simultaneously with the evolvement of the bonding apparatus into contemporary ribosome, alongside the genetic code products: mature proteins.

Currently owing to the vital role of the ribosomes they are targeted by many antiatoms, and mutations in their genome are associated with acute human diseases such as cancer and anemia.

\section{0-3}

Voltage sensors and the opening of ion channels Francisco Bezanilla

Department of Biochemistry and Molecular Biology University of Chicago. Chicago, Illinois, USA - Centro Interdisciplinario de Neurociencia de Valparaíso, University of Valparaiso, Chile

The generation of the nerve impulse (action potential) depends on voltage-dependent sodium channels that must open before voltage-dependent potassium channels. We will review structure-function relation of the voltage sensors that give voltage dependence of the ion channels. The voltage sensors have intrinsic charges in the channel protein which move in the cell membrane electric field and generate gating currents. Experiments with voltage clamp and site-directed fluorescence describe molecular details of the voltage sensor operation indicating the paths followed by the charged arginine residues within the protein core. A detailed study of the residues in the core show that the nature of the side chains determine that $\mathrm{Na}$ channels are faster than $\mathrm{K}$ channels. The canonical coupling of the voltage sensor to the conduction pore is via the linker between transmembrane segments S3 and $\mathrm{S} 4$. We will describe that the proximity of the $\mathrm{S} 4$ segment of the voltage sensor and the S5 segment of the pore region makes another noncanonical coupling pathway. The molecular basis of this pathway will be described. (Support: NIH R01GM030376)

\section{0-2}

The molecular logic of synapse formation: From structure to function

Thomas C Südhof

HHMI \& Dept. of Molecular and Cellular Physiology, Stanford University School of Medicine, Stanford, USA

The brain processes information in millions of parallel and intersecting neural circuits. In neural circuits, neurons are connected by synapses that compute a circuit's information by transmitting and processing neuronal signals. Neural circuit computations critically depend on the number, location and properties of their constituent synapses. We hypothesize that the construction of neural circuits by formation of defined synapses is based on a molecular logic that is based on interactions between pre- and postsynaptic recognition and signaling molecules. Moreover, we hypothesize that at least a subset of autism and schizophrenia disorders are produced by specific impairments in the molecular logic of neural circuits, such that the input/output relations in affected circuits exhibit a skewed information processing capacity because of synaptic impairments. Understanding how synaptic adhesion molecules build synapses and control their properties requires insight into the identity, function, and mechanism of action of these adhesion molecules. The goal of work in my laboratory is to characterize the key adhesion molecules of central synapses at all levels of analysis, ranging from their atomic structures to they in vivo functions, in an effort to gain insight into how these molecules build diverse synapses as computational units with distinct properties. In my presentation, I will discuss synaptic adhesion molecules, such as neurexins and latrophilins and their multifarious ligands, as key drivers of the molecular logic of neural circuits.

\section{0-4}

The TMEM16 family of calcium-activated ion channels and lipid scramblases

Raimund Dutzler

University of Zurich, Switzerland

The TMEM16 proteins constitute a family of membrane proteins, which comprises lipid scramblases and $\mathrm{Cl}^{-}$channels. Both functional branches share a common architecture and are activated by intracellular $\mathrm{Ca}^{2+}$ by a similar mechanism. All family members form homodimers of subunits that are composed of ten membrane-spanning helices. The subunits are functionally independent and contain a regulatory $\mathrm{Ca}^{2+}$ binding site embedded within the transmembrane domain and a close-by site of catalysis located at the periphery of the protein, which either facilitates ion or lipid permeation. In the fungal lipid scramblase nhTMEM16, $\mathrm{Ca}^{2+}$-binding opens a hydrophilic membrane-spanning furrow, which is hidden in the $\mathrm{Ca}^{2+}$-free protein, to provide a pathway for lipid headgroups to move between both leaflets of the bilayer. In contrast, in the anion channel TMEM16A, $\mathrm{Ca}^{2+}$-binding triggers the opening of a protein-enclosed ion conduction pore located in the same region, which remains shielded from the membrane. In this case, $\mathrm{Ca}^{2+}$ serves a dual role in promoting a conformational change to release a gate that impedes conduction in the closed state and by shaping the electrostatics to enhance anion permeation. Finally, in the lipid scramblase TMEM16F, we find both functions as lipid scramblase and ion channel contained within the same protein, presumably mediated by an equilibrium of distinct conformations that are assumed in the $\mathrm{Ca}^{2+}$-bound state. 


\section{$0-5$}

Adventures in the chromatin jungle: Nucleosome remodelers, archaeal slinkies, and giant viruses Yang Liu [1], Chelsea Toner [1], Samuel Bowerman [1], Hugo Bisio [1,2], Sandra Jeudy [1,2], Nadège Philippe [1,2], Keda Zhou[1], Alison White[1], Dustin Woods [3], Jeff Wereszczynski [3], Chantal Abergel [2,1], Karolin Luger [1]

[1] University of Colorado, USA; [2] Aix-Marseille University, France; [3] Illinois Tech, USA

Invariably, all eukaryotes organize their DNA into nucleosomes, consisting of an octamer of the four core histone proteins $\mathrm{H} 2 \mathrm{~A}, \mathrm{H} 2 \mathrm{~B}, \mathrm{H} 3$, and $\mathrm{H} 4$, around which 147 base pairs of DNA are wrapped in two tight superhelical turns. Nucleosomes pack into higher order structures and require histone chaperones and ATP-dependent remodelers to make the DNA accessible. I will discuss our recent progress in understanding the structure of chromatin at the centromere, and into mechanisms by which chromatin is remodeled in an ATP dependent manner.

With the discovery of small histone-like proteins in most known Archaea, the likely origin of histones and thus the nucleosome identified. Most Archaea encode only one or two minimal histones that form polymers around which DNA coils in a dynamic quasi-continuous superhelix that opens and closes stochastically. Our exploration of non-eukaryotic chromatin continues with the discovery of genes encoding fused histone genes in giant viruses that infect amoeba. Our structural and functional analysis shows that these histones assemble into nucleosome-like structures, and that histones are essential for viral infectivity.

\section{0-7}

Protein folding inside the ribosome

Marina V Rodnina

Max Planck Institute for Biophysical Chemistry, Göttingen, Germany

Protein domains start to fold co-translationally inside the polypeptide exit tunnel of the ribosome. Co-translational folding is vectorial, confined by the space of the tunnel and is modulated by the speed of translation. Defects in protein folding cause many human diseases; thus, understanding the co-translational folding is of eminent importance. We mapped the pathways of co-translational folding for two proteins of different topology, a small five-helix domain HemK and a five-stranded beta-barrel protein CspA. In solution, both proteins fold by a concerted two-state mechanism. On the ribosome, HemK and CspA start to fold inside the exit tunnel and undergo several structural rearrangements that are not sampled in solution. HemK folds vectorially as soon as the N-terminal $\alpha$-helical segments are synthesized. As nascent chain grows, consecutive helical segments dock onto each other and continue to rearrange at the vicinity of the ribosome. CspA forms compact non-native structures inside and at the vestibule of the exit tunnel and adopts the native fold only after the release from the ribosome. These results show how the ribosome defines the folding landscape of protein domains.

\section{0-6}

Molecular simulations of SARS-CoV-2 spike and its interactions with the human host Gerhard Hummer [1,4], Sikora Mateusz [1,2], Schäfer Stefan [1], Blanc Florian [1], Von Bülow Sören [1], Gecht Michael [1], Covino Roberto [1,3], Mehdipour Ahmadreza [1] 1] Max Planck Institute of Biophysics, Frankfurt am Main, Germany; [2] Fac of Physics, Univ of Vienna, Austria; [3] Frankfurt Institute for Advanced Studies, Germany; [4] Dept of Physics, Goethe University Frankfurt, Germany

Molecular dynamics (MD) simulations of membrane proteins open up a window to the molecular processes in SARS-CoV-2 infections. We concentrate on the structure of the spike $(\mathrm{S})$ protein at the viral surface, its interactions with the host cell, and the epitopes it presents for antibody targeting. In molecular dynamics simulations of full-length $\mathrm{S}$ with a palmitoylated transmembrane domain and a fully glycosylated ectodomain, we identified three hinges in the stalk connecting the $\mathrm{S}$ head to the viral membrane. Hinge flexibility and glycosylation have been confirmed by high-resolution cry-electron tomography (Turonova, Sikora, Schürmann et al., Science 2020). We have used the detailed structural and dynamic models for a computational antibody epitope scan (Sikora et al., PLoS Comp. Biol 2021). In addition, we have studied the interactions of $S$ with the host-cell receptor ACE2 (Mehdipour, Hummer, PNAS 2021). The glycan coats of S and ACE2 emerge as important factors both in viral infection and in the host antibody response. MD simulations have also given us a molecular understanding of how SARS-CoV-2 initiates the membrane fusion process that leads to infection (Schäfer, Hummer, bioRxiv 2021). Overall, MD simulations help us to uncover some remarkable biology associated with viral infection and, as we hope, guide our fight against COVID-19.

\section{$0-8$}

Protein complexes subjected to tandem mass spectrometry reveal allosteric binding partners Carol V Robinson

University of Oxford, UK

Recent discoveries have enabled transmission into the gas phase of membrane complexes from detergent micelles in solution. By maintaining interactions between membrane and cytoplasmic subunits in the mass spectrometer, it is now possible to investigate the effects of lipids, nucleotides and drugs on intact membrane assemblies. These investigations reveal allosteric and synergistic effects of small molecule binding and expose the consequences of post-translational modifications. Whilst very insightful, the choice of detergent can sometimes perturb interactions within the membrane leading to changes in the lipid environment, loss of small molecules and disruption of protein interactions. In my lecture, I will present recent progress in our quest to eject complexes directly from membrane mimetics or native membranes and to fragment the small molecules harboured within them. 


\section{Oral Presentations}

\section{- 1. Interfacial Protons -}

\section{$0-9$}

Dynamic hydrogen-bond networks for proton binding and conformational coupling

Ana-Nicoleta Bondar $[1,2]$

[1] University of Bucharest, Faculty of Physics, Romania; [2] Forschungszentrum Jülich, Institute for Advanced Simulations, Computational Biomedicine (IAS-5/INM/9), Germany

Dynamic hydrogen bond networks that couple proton binding to changes in protein conformational dynamics are central to protein function mechanisms. Description of such networks, particularly of the response of the network to protonation change, can be challenging in the case of large biomolecules. To tackle this challenge we have recently developed graph-based algorithms that enable efficient computations of dynamic protein-water hydrogen bond networks and measures of centrality of groups contributing to the network. To ease comparison of hydrogen-bond networks in protein structures, we developed algorithms to compute conserved and difference hydrogen-bond graphs.

Analyses using our graph-based algorithms revealed that the closed conformation of SARS-CoV-2 protein S has extended hydrogen clusters that tend to have three-fold compositional symmetry; this symmetry is largely lost in the open and prefusion conformations of the protein. N501 is predicted as high-centrality group of an extended hydrogen-bond network at the interface between protein S and the ACE2.

Analyses of static protein structures and numerical simulations of G Protein Coupled Receptors suggest protein dynamics and water are required for the receptor to visit conformations with extended hydrogen bond networks potentially important for activation. In a dimeric ion channel, a dynamic hydrogen-bond path connects transiently the primary proton donors, suggesting long-distance couplings.

\section{0-11}

Time resolved structural biology - Bacteriorhodopsin paves the way to a dynamic future

Tobias Weinert

Paul Scherrer Institut, Villigen, Switzerland

The time scale of proteins in action stretches over twelve orders of magnitude in time. Tapping into this pool of dynamic information with the near-atomic resolution of crystallography holds the key to understand protein structure and function [1].

Breakage, formation and isomerization of chemical bonds are ultrafast reactions that drive the processes of life. Time resolved serial femtosecond crystallography (TR-SFX) at free electron lasers allows to observe such reactions in atomic detail. Our TR-SFX results show how bacteriorhodopsin isomerizes its retinal ligand within femtoseconds by exploiting the ultrafast motion of a bound water molecule. The protein scaffold selects one out of four isomers forming in solution and increases the quantum efficiency [2] .

Protein side chain motions reshape ligand binding sites within nanoseconds to microseconds. TR-SFX revealed how concerted side chain motions are used by bacteriorhodopsin to transport a retinal bound proton against a concentration gradient, nature's favorite mechanism to capture energy from light [3].

Large scale protein motions occur on the millisecond time scale and are important for cellular processes like membrane transport and signaling. We resolved the proton uptake step in the bacteriorhodopsin pumping cycle by time resolved serial millisecond crystallography at the synchrotron [4].
0-10

A water window on membrane biochemistry: Quantifying surface potential, free energy and binding constants

Sylvie Roke

Laboratory for fundamental BioPhotonics (LBP), École Polytechnique Fédérale de Lausanne (EPFL), Switzerland

Lipid membranes are essential for life: They are a dynamic compartmentalized environment, a localized space for proteins to perform their functions, and select what enters the cell. Although recognized as an essential building block, water is usually treated as a background for biology. However, water is a crucial mediator of chemical change and determines the structure of the membrane. The study of lipid membranes is generally pursued by following either a top-down approach, introducing labels to living cell membranes or a bottom-up approach with well-controlled but over-simplified membrane monolayer or supported membrane models. In the first approach molecular level hydration information is lost, while in the second approach the connection with real bilayer membranes is limited.

Recent developments in our laboratory offers an alternative path that could bring together both top-down and bottomup approaches. By using intermediate nano-, micro- and macroscale free-floating membrane systems in combination with novel nonlinear optical spectroscopy and imaging methods, we advance the understanding of realistic membranes on a more fundamental level. In this presentation I will provide examples of these, with a focus on understanding the molecular interfacial architecture of the aqueous interface of membranes and using the nonlinear optical water response to spatiotemporally quantify the electrostatic surface potential, free energy and binding constants.

\section{0-12}

The proton channel Hv1 is water-impermeable Danila Boytsov [1], Stefania Brescia [1], Christine Siligan [1], Boris Musset [2], Peter Pohl [1]

[1] Institute of Biophysics, Johannes Kepler University Linz, Austria; [2] Institute of Physiology, Clinic of Nurnberg Medical School at the Paracelsus Medical Private University, Germany

Most ion channels conduct water. Even extremely narrow channels may accommodate an uninterrupted chain of water molecules that connects the two aqueous solutions on both sides of the membrane. The water molecules in this chain may be highly mobile, i.e., their mobility may be close to bulk water molecules. Consequently, such channels, e.g., aquaporins and gramicidin channels, exhibit substantial unitary water permeability. Since interruptions of the water chain diminish the water flux, we used water permeability measurements as a diagnostic tool to distinguish between two proposed mechanisms of proton conduction through the proton channel $\mathrm{H}_{\mathrm{v}} 1$ : One hypothesis envisioned proton hopping along an uninterrupted water wire. The alternative hypothesis stipulated the existence of titratable amino acids in the proton pathway, interrupting the water wire halfway across the membrane. We reconstituted the purified proton channel into lipid vesicles and monitored their deflation in an osmotic gradient by registering the intensity of light scattered by them. The reconstituted channels were functional, as indicated by fast proton transport kinetics across the reconstituted vesicular membrane, and did not conduct water. Thus, our experiments support the hypothesis that channel occluding amino acids are part of the proton pathway. 


\section{Oral Presentations}

\section{- 1. Interfacial Protons -}

\section{0-13}

Measuring enzyme activity in proteoliposomes on single-enzyme level

Katharina G Hugentobler [1], Dorothea Heinrich [2], Johan Berg [3], Joachim Heberle [2], Peter Brzezinski [3], Ramona Schlesinger [2], Stephan Block [1]

[1] Department of Biology, Chemistry and Pharmacy, Freie Universität Berlin, Germany; [2] Department of Physics, Freie Universität Berlin, Germany; [3] Department of Biochemistry and Biophysics, Stockholm University, Sweden

Cuvette-assay based measurements of enzymatic processes at a lipid-water interface pose an important challenge to the experimenter: Without precise quantification of enzyme concentration, it is complex to disentangle the influence of environmental and enzymatic properties. Here we present a method to simultaneously study the influence of either one factor independent of the other. To demonstrate the power of our approach proton-coupled electron transfer reactions across a membrane are studied. We reconstitute $\mathrm{C} c \mathrm{O}$ from $R$. sphaeroides into liposomes containing a $\mathrm{pH}$ sensitive dye and probe $\mathrm{pH}$ changes inside single proteoliposomes using fluorescence microscopy. $\mathrm{C} c \mathrm{O}$ proton turnover rates are quantified at single-enzyme level for precise determination of enzyme activity. In addition, the distribution of the number of functionally reconstituted $\mathrm{C} c \mathrm{Os}$ across the proteoliposome population are recorded. Moreover, different lipid preparations are used to probe the influence of environmental factors. All lipid compositions tested so far have only minor effects on the $\mathrm{C} c \mathrm{O}$ proton turnover rate but can strongly impact the reconstitution efficiency of functionally active $\mathrm{C} c \mathrm{Os}$.

\section{0-14}

Lipid composition and lipid-protein interactions shape the free energy landscape of pore and stalk formation

Jochen S Hub

Saarland University, Germany

Topological transitions of membranes, such as pore formation or membrane fusion, play key roles many biophysical processes including exocytosis, viral infection, intracellular trafficking, proton transport, and many others. How the lipid composition, $\mathrm{pH}$, or lipid-protein interactions shape the free energy landscape of such processes is not well understood. We have developed computationally efficient methods for screening the effects of lipids and transmembrane domains (TMDs) on the energetics of the formation of membrane pores or membrane stalks at atomic or near-atomic resolution. The simulations reveal that the membrane composition may bias such topological transitions by tens or even hundreds of kilojoule per mole, suggesting that the complexity of membranes may have evolved to control the kinetics of such events. 


\section{Oral Presentations}

-2. Channels and $\mathrm{Ca}^{2+}$ signaling -

\section{0-15}

Structural and functional studies of TRP channels Alexander I Sobolevsky

Department of Biochemistry and Molecular Biophysics, Columbia University, 650 West 168th Street, 10032 New York, USA

Numerous physiological functions rely on distinguishing temperature by temperature-sensitive transient receptor potential (TRP) channels. While TRP channel function has been studied extensively, structural determination of their heat- and cold-activated states has remained a challenge. We determined cryo-EM structures of mouse TRPV3 in temperature-dependent closed, sensitized and open states. The heat-induced transformations of TRPV 3 are accompanied by changes in the secondary structure of the $\mathrm{N}$ - and $\mathrm{C}$ termini and represent a conformational wave that links these parts of the protein to a lipid occupying the vanilloid binding site. State-dependent differences in the behavior of bound lipids suggest their active role in TRP channel temperaturedependent gating. Our results provide an insight for understanding the molecular mechanism of temperature sensing.

\section{0-17}

Structural insights into $\mathrm{Ca}^{2+}$ and cholesterol inhibition in the TRP channel PC2

Georg Horn, Lifei Fu, Gregor Madej, Christine Ziegler University of Regensburg, Biophysics II-Structural Biology, Germany

The TRP channel Polycystin-2 (PC2) is a $\mathrm{Ca}^{2+}$-permeable, $\mathrm{Ca}^{2+}$-activated channel located in the $\mathrm{ER}$, the primary cilia, and the plasma membrane, respectively. PC2 is non-selectively permeable to both monovalent and divalent cations playing an important role in renal physiology. The inherited autosomal dominant polycystic kidney disease (ADPKD) is partly related to loss-of-function mutations in PC2. Although being not voltage-gated, PC2 displays sequence similarity in TM1-TM4 to the voltage-sensing domain of other TRP channels. The intracellular C-terminal domain includes a $\mathrm{Ca}^{2+}$-binding EF hand, a coiled-coil domain, and an ER retention sequence. Consequently, the fraction of $\mathrm{PC} 2$ is retained in the ER, where it is suggested to play an important role as $\mathrm{Ca}^{2+}$-leakage channel. Without the ER retention signal, $\mathrm{PC} 2$ is mis-trafficked within the kidney epithelial cells. We have solved the structure of a PC2 mutant missing the ER retention signal by cryo-EM to $3.1 \AA$. Our structure reveals an asymmetric opening of the lower gate, a conserved $\mathrm{Ca}^{2+}$-binding site in the VSD, which together with new cholesterol binding sites point towards an intriguing inhibition mechanism. Our structural data are supported by a lipid MS analysis and Scanning transmission electron microscopy (STEM) of PC2 expressing ER membranes. Based on our data we suggest that cholesterol binding is an important feature to modulate $\mathrm{PC} 2 \mathrm{Ca}^{2+}$ - channel activity in the ER.
0-16

The open and closed pore conformations of the pacemaker channel HCN4

Andrea Saponaro [1], Daniel Bauer [2], Hunter M Giese [3], Antonio Chavez-Sanjuan [1], Ermanno Uboldi [1], Martino Bolognesi [1,4], Bina Santoro [5], Anna Moroni [1]

[1] Department of Biosciences, University of Milan, Italy;

[2] Department of Biology, TU-Darmstadt, Germany;

[3] Department of Physiology and Cellular Biophysics, Columbia University, NY, USA; [4] Pediatric Research Center "Romeo ed Enrica Invernizzi", University of Milan, Italy; [5] Department of Neuroscience, Columbia University, NY, USA

HCN1-4 genes family generate the hyperpolarizationactivated cation current $\mathrm{I}_{\mathrm{f}} / \mathrm{I}_{\mathrm{h}}$, which controls automaticity in cardiac and neuronal pacemaker cells. By single particle cryogenic electron microscopy (cryo-EM) we obtained structures of the HCN4 hyperpolarization-activated cyclic nucleotide-gated channel in open and closed pore conformations, with and without cAMP bound. Systematic comparison of open and closed states in HCN4 shows that a concerted movement of the S5 and S6 transmembrane helices opens a cytosolic gate. Furthermore, the open state structures, in combination with molecular dynamics analyses, provide atomic level insights into mechanisms of $\mathrm{K}^{+}$and $\mathrm{Na}^{+}$permeation, revealing unique ion-binding dependent adaptation in selectivity filter diameter. Thus, our ability to compare open and closed state structures for HCN4 channels provides fundamental insights into mechanisms of $\mathrm{HCN}$ channel gating, cyclic nucleotide-dependent modulation, and ion permeation.

\section{0-18}

Assessment of temperature sensitivity of the TRPM2 channel

Adam Bartok [1,2], Laszlo Csanady [1,2]

[1] Department of Biochemistry Semmelweis University, Hungary; [2] HCEMM-SU Molecular Channelopathies Research Group, Hungary

TRPM2 belongs to the TRP family, and is expressed in neurons of the central nervous system, bone marrow, phagocytes, $\beta$-cells, and cardiomyocytes. It forms $\mathrm{Ca}^{2+}$-permeable nonselective cation channels that open under oxidative stress by simultaneous binding of intracellular $\mathrm{Ca}^{2+}$, ADP-ribose (ADPR), and $\mathrm{PiP}_{2}$ in the membrane. Malfunction of the channel has been linked to neurological disorders $(A D, P D$, ALS) as well as to pathological conditions that lead to apoptosis (cerebral stroke, myocardial infarction). Temperaturedependent activation of TRPM2 in warm-sensitive neurons underlies body heat control and the generation of fever. The channel may be a target to treat fever, chronic inflammations, diabetes or congenital hyperinsulinism. The biophysical background of temperature sensitivity of TRPM2 is yet unknown, and might reflect either temperature dependence of the intracellular concentrations of any of its three activating ligands, or intrinsic heat sensitivity of the TRPM2 itself. Therefore, we determined temperature dependence of TRPM2 gating parameters and apparent ligand binding affinities for $\mathrm{Ca}^{2+}, \mathrm{ADPR}$, and $\mathrm{PIP}_{2}$ in inside-out patches from HEK-293 cells expressing human TRPM2, under temperature-controlled perfusion of the cytosolic surface. Our results unequivocally clarify and describe the mechanism of temperature sensitivity of the TRPM2 channel. 


\section{Oral Presentations}

\section{-2. Channels and $\mathrm{Ca}^{2+}$ signaling -}

0-19

A novel deletion in the CRAC protein STIM1 diminishes Stormorken syndrome pathology

Herwig Grabmayr [1], Thilini H Gamage [2], Doriana Misceo [2], Marc Fahrner [1], Eirik Frengen [2], Christoph Romanin [1]

[1] Institute of Biophysics, Johannes Kepler University Linz, Austria; [2] Department of Medical Genetics, Oslo University Hospital and University of Oslo, Norway

Calcium $\left(\mathrm{Ca}^{2+}\right)$ is an integral cellular messenger involved in biological signaling processes required for gene expression or immune defense. A prominent example is the store-operated calcium entry (SOCE) pathway. It describes the entry of $\mathrm{Ca}^{2+}$ into cells in response to the depletion of the endoplasmic reticulum (ER) $\mathrm{Ca}^{2+}$ stores. The proteins underlying SOCE are called STIM1 and Orai1. STIM1 senses the concentration of $\mathrm{Ca}^{2+}$ in the ER and relays this information via direct interaction to Orai1, which constitutes the $\mathrm{Ca}^{2+}$ channel. Several disease-related mutations in STIM1 and Orai1 are known. One of them is the mutation R304W in STIM1, which causes the so-called Stormorken syndrome. A rare autosomal-dominant disease, this syndrome is characterized by permanent STIM1-Orai1 interaction. The resulting perpetual $\mathrm{Ca}^{2+}$ entry elicits pathologies that affect different body systems. Here, we provide evidence that deletion of E296 upstream of R304W diminishes pathological effects in vivo and in vitro: A STIM1 double mutant (E296del + R304W) mouse line appears healthy and behaves like the wild type. Consistently, electrophysiology and FRET experiments reveal that STIM1 E296del + R304W also corresponds to STIM1 wild type on the single cell level.

\section{0-20}

Calcium signaling in T Cells is induced by binding to nickel-chelating lipids in supported lipid bilayers Tommy Dam [1], Victoria Junghans [3,1], Jane Humphfrey [2], Manto Chouliara [1], Peter Jönsson [1]

[1] Lund University, Department of Chemistry, Sweden;

[2] University of Cambridge, Department of Chemistry, UK;

[3] Radcliffe Department of Medicine, John Radcliffe Hospital, University of Oxford, UK

Supported lipid bilayers (SLBs) are often incorporated with nickel-chelating lipids to bind polyhistidine-tagged proteins when studying $\mathrm{T}$ cell activation. However, the nickelchelating lipids themselves are capable of inducing calcium signaling which could contribute to false $\mathrm{T}$ cell signaling events. Jurkat $\mathrm{T}$ cells expressing non-signaling rCD48 molecules were deposited onto ligand-free SLBs and underwent calcium signaling. The fraction of signaling cells was depended on the concentration of nickel-chelating lipids, ranging from $24 \%$ to $60 \%$ when increasing the lipid concentration from $2 \mathrm{wt} \%$ to $10 \mathrm{wt} \%$. The effect of the nickelchelating lipids was also dominant in the presence of the rCD48-ligand rat CD2, showing no difference in signaling fraction. However, adding bovine serum albumin to block the SLB did reduce the signaling fraction to $11 \%$. Neither contact formation through CD2-binding or exclusion of CD45 from the contact was affected by the blocking. This suggests that CD45 exclusion alone is not sufficient to result in the observed signaling behaviour. It is possible that nickelchelating lipids can act as ligands to TCRs. This type of interaction should be blocked to avoid unwanted cell interactions and activation. (1)

1. Dam, T., V. Junghans, J. Humphrey, M. Chouliara, and P. Jönsson. 2021. Calcium Signaling in T Cells Is Induced by Binding to Nickel-Chelating Lipids in Supported Lipid Bilayers. Front. Physiol. 11. 
Oral Presentations

- 3. Medical biophysics / Imaging -

\section{0-21}

Biophysical method for determination of cardiovascular age by analyzing arterial blood flow waveform

Dejan Žikić

Institute of Biophysics, Faculty of Medicine, University of Belgrade, Serbia

Wave propagation of blood through cardiovascular vessels is affected by complex interactions between the left ventricular dynamics, mechanical properties of the artery wall, and biophysical properties of the blood. With aging, changes in collagen and elastin of artery wall lead to the increase of the Young's modulus of elasticity of the arterial wall. Due to that, the pulse wave reflection appears causing the increase of blood pressure. Prolonged human lifespan has led to new diseases of the cardiovascular system due to agerelated changes in blood vessels. For this reason, the focus of scientific research is on the development of simple and noninvasive biophysical methods for monitoring the aging of the cardiovascular system. Recently we have developed a new nonlinear mathematical method for analyzing long-term of arterial blood flow waveforms recorded by noninvasive optical sensors. The results of the study showed that the scaling factors $\alpha$ linearly decreases with age, and in young subjects it is greater than 1 , in the elderly less than 1 , and in middleaged subjects close to 1 . This method has been successfully applied to monitor the impact of physical activity on biophysical parameters of the cardiovascular system, which is especially important for the elderly. Also, this method has proven to be very useful in monitoring the effect of diet, as well as lifestyle changes.

\section{0-23}

Sub-threshold optogenetic manipulation of cardiac electrical activity

Sacconi Leonardo $[1,2]$

11] European Laboratory for Non-linear Spectroscopy, Sesto Fiorentino, Italy; [2] Institut of Experimental Cardiovascular Medicine, University Heart Center Freiburg, Germany

Cardiac action potential (AP) propagation across the heart is a physiological process regulated by several key dynamic factors. Experimental methods for modulating AP dynamics lack spatial and temporal specificity, and are often irreversible. In this work we propose a novel approach based on optogenetics to fine-tune AP dynamics. We explore the electrophysiological response of ChR2 expressing cardiomyocytes and isolated hearts during sub-threshold illumination. We find that light-mediated depolarizing current prolongs APs and reduces conduction velocity within user-defined heart regions in a totally reversible manner. Sub-threshold manipulation also affects the dynamic electrical restitution relation, increasing AP duration (APD) alternans. We use real-time feedback control to generate re-entrant ventricular tachycardias (VTs) to explore the role of APD alternans in spontaneous termination of rapid rhythms. We find that VT stability significantly decreases during sub-threshold illumination, suggesting a possible beneficial effect of APD alternans in self-termination of VT.

\section{0-22}

Nanoscopic t-tubular deformation during cardiac mechanical cycle

Eva Rog-Zielinska, Peter Kohl

Institute for Experimental Cardiovascular Medicine, University Heart Centre Freiburg/Bad Krozingen, Germany

Cardiac sarcolemma contains many proteins essential for excitation-contraction coupling. The distribution of these proteins is non-uniform between the surface plasma membrane and membrane invaginations known as transverse tubules (TT). TT are an intricate network of fluid-filled conduits that aid electro-mechanical synchronicity across myocytes. The narrow and tortuous TT can form domains of restricted diffusion - this fact, coupled with unequal ion fluxes across cell surface and TT, can lead to formation of ion gradients deep within TT.

Here we use electron tomography and live cell studies to describe a novel advective component to TT luminal homeostasis, wherein cyclic deformation of TT during stretch and contraction serves to mix TT luminal content and assists equilibration with extracellular fluid. We show that cellular deformation affects TT shape (TT become 'squeezed'). Furthermore, we show that the speed of diffusion is affected by the mechanical state of cardiomyocytes, and that the contractile activity accelerates apparent speed of diffusion inside TT.

In conclusion, we present an advective component to TT content exchange, a novel mechanism of cardiac autoregulation. The relevance of this mechanism in health and during pathological cardiac remodelling forms a promising target for further research.

\section{0-24}

Antibodies in motion: How structural dynamics inform biological function

Jürgen Strasser, Nikolaus Frischauf, Johannes Preiner

University of Applied Sciences Upper Austria, Austria

Immunoglobulin $\mathrm{G}(\mathrm{IgG})$ is one of the most important molecules of the mammalian immune system and is extensively used in the biological sciences. Despite decades of research on the structure and function of these proteins, it was only within the last years that we have come to realize that IgGs do not engage the complement system via sheer abundance on antigenic surfaces alone. The immunoactive complexes required for $\mathrm{C} 1 \mathrm{q}$ binding and cascade initiation rather form via specific IgG oligomerization. This novel IgG effector function may require us to rethink the way we use and design IgGs for both clinical and research applications. Here we present recent high-speed atomic force microscopy and quartz crystal microbalance results demonstrating the oligomerization process for a range of antibodies, highlighting a strong correlation between oligomer abundance, C1q binding, and downstream CDC efficacy. The inherently low Fc-Fc affinity prevents IgG oligomerization, and thus unwanted complement activation, at physiological concentrations in solution. Upon surface-epitope binding, oligomerization may proceed via two different pathways: recruitment from solution, or diffusion-driven lateral collisions. We are currently investigating the molecular determinants of increased or decreased oligomerization, and thus CDC efficacy, which include epitope-paratope and Fc-Fc affinities, the flexibilities of the hinge region and adjacent structures, as well as the fluidity of the antigenic membrane. 
Oral Presentations

- 3. Medical biophysics / Imaging -

0-25

Label-free quantitative phase imaging of Hutchinson-Gilford progeria syndrome (HGPS) cells

Alberta Trianni [1,2], Nicholas Anthony [1], Alberto Diaspro [1,2] [1] Nanoscopy, Istituto Italiano di Tecnologia, Italy; [2] DIFILAB, University of Genoa, Italy

Hutchinson-Gilford progeria syndrome (HGPS) is a genetic condition characterized by premature aging. HGPS is caused by a mutation in the encoding gene of lamin-A, a component of the nuclear lamina. Lamins provide shape and structural stability to the nucleus, and are involved in many cellular processes. Optical microscopy is one of the most used tools to investigate biological processes, and is commonly performed using fluorescent labeling. However, live-cell imaging is not always compatible with labeling due to photobleaching and phototoxicity effects. For this reason, "label-free imaging" gained significant interest for the study of biological systems. One such method is Ptychography, which is able to quantify the phase shift of light passing through a more optically dense object. It is a computational approach to phase microscopy, which provides mathematically derived information about a specimens phase-modulating characteristics. The obtained phase map contains information about cell thickness and refractive index and allows the quantification of cellular morphology. In this work, we show that by using the quantitative phase information from ptychography the refractive index distribution within cells is retrievable. This information can be used to observe structural changes in HEK 293 cells with HPGS.

\section{0-26}

Chemo-mechanical investigation of human bone and cartilage in healthy and osteoarthritic conditions by Brillouin and Raman micro-spectroscopy Martina Alunni Cardinali [1], Marco Govoni [2], Dante Dallari [2], Leonardo Vivarelli [2], Cesare Stagni [2], Martina Rocchi [2], Matilde Tschon [3], Silvia Brogini [3], Milena Fini [3], Maurizio Mattarelli [4], Silvia Caponi [5], Daniele Fioretto [4], Assunta Morresi [1]

[1] Dep. of Chemistry, Biology and Biotechnology, Univ. of Perugia, Italy; [2] Reconstructive Orthopaedic Surgery and Innovative Techniques - IRCCS Rizzoli, Italy; [3] Complex Structure of Surgical Sciences and Technologies, IRCCS Rizzoli, Italy; [4] Dep. of Physics and Geology, Univ.of Perugia, Italy; [5] IOM-CNR, University of Perugia, Italy

Bone and cartilage tissues are characterized by a stringent structure-function relation. Bone has a peculiar hierarchical architecture while the bearing-structure of the articular cartilage is designed to redistribute mechanical stresses. In both cases, impairment of any of the components already at the microscale can lead to a loss of the whole tissue mechanical performance. In Osteoarthritis the establishment over time of mechanical insults on the articular surface causes a severe inflammatory process leading to its erosion and to the subchondral bone rearrangement. Brillouin and Raman microSpectroscopy is a contact-less and not-destructive scattering technique, suitable for the imaging of biological tissues $e x$ vivo and in vivo, which allows simultaneous analysis of both the micro-mechanical and chemical properties. Here, we present its first application to the diagnosis of osteoarthritic damage occurring in the hip joint. 
Oral Presentations

- Self-Assembled Session 1 - Emerging breakthrough methods in molecular-scale biophysics (ARBRE) -

\section{0-27}

Advanced vibrational spectroscopy and microscopy for biochemical analysis

Takuro Ideguchi

The University of Tokyo, Japan

Molecular vibrational spectroscopy and microscopy enable the label-free biochemical analysis of live cells and tissues and can provide different ways to investigate biological phenomena than fluoresce-based measurements. Historically, spontaneous Raman scattering spectroscopy and microscopy have been the most used techniques as label-free measurements. Recent advancements of ultrashort pulsed lasers have introduced various methods by coherent Raman scattering, improving measurement speed. To further advance the label-free optical measurements, we have developed other advanced vibrational spectroscopy and microscopy techniques. In this talk, I will introduce recently developed methods, including the world's fastest infrared spectroscopy, simultaneous coherent Raman and infrared spectroscopy, and infraredabsorption-based photothermal microscopy. The new vibrational spectroscopy and microscopy techniques would stimulate biophysicists to come up with new applications.

\section{0-29}

High Speed Atomic Force Microscopy and Fluorescent Optical Tweezers to study nano-scale dynamics in real-time

Wouter H Roos

Moleculaire Biofysica, Zernike Instituut, Rijksuniversiteit Groningen, Netherlands

Cellular life harbours a fascinating variety of complex processes and we are still at the beginning of our understanding of these processes. Using High Speed-Atomic Force Microscopy (HS-AFM) and fluorescent Optical Tweezers we are now able to scrutinize the dynamics of these processes at the nano scale, in real time, in liquid. I will start off with briefly discussing the principles and background of these techniques, before I dive into the applications. Thereby, I will show how we are using these techniques to study the fascinating physics of sub-cellular dynamics and biomimetic assembly processes. This will be illustrated by discussing assembly and disassembly of ESCRT-III protein complexes (Science Adv, 2019, 5, eaau7198; Nature Comm, 2020, 11, 2663) and High speed AFM visualization of the dynamics of self-replicators (JACS, 2020, 142, 13709). Furthermore, dual-trap optical tweezers studies of the self-assembly of virus-like-particles (VLPs) are shown that reveal real time binding of capsid proteins to dsDNA and the formation of stable VLP structures around the genome (Nano Lett, 2019, 19, 5746; Science Adv, 2020, 6 , eaaz1639). Finally the formation dynamics of 2D capsid protein lattices will be discussed, particularly revealing how complex the kinetics of viral self-assembly can be, with multiple assembly pathways and continuously occurring assembly and disassembly events (ACS Nano, 2020, 14, 8724).
0-28

Deciphering protein dynamics using nitroxide spin labels combined with EPR spectroscopy Valérie Belle

Aix-Marseille University, France

Proteins are dynamic macromolecules that need to move to accomplish their function. This dynamic allows them to change their conformation and to adapt their structure for example for interacting with other biomolecules. Characterizing proteins in action at the molecular level requires appropriate biophysical techniques sensitive to protein motions. One of the technique well-suited to monitor protein dynamics is Site Directed Spin Labeling combined with Electron Paramagnetic Resonance spectroscopy (SDSL-EPR). The general principle consists in grafting an EPR-active label (generally a nitroxide radical) at a selected site of the macromolecule under interest followed by its observation using an appropriate EPR technique or by combining different EPR strategies. On the one hand, nitroxide labels are perfect reporters of their structural micro-environment and are thus particularly welladapted to reveal conformational changes, folding/unfolding events... On the other hand, bi labeling strategies combined with pulsed DEER technique allow the measurement of inter-label distance distributions in the range of 1.5 to $6 \mathrm{~nm}$. The aim of this lecture is to illustrate these complementary strategies through recent examples showing how SDSL-EPR can be successfully applied to characterize the structural dynamics of several biogical systems and to show the development of newly synthetized nitroxide spin labels that allow overcoming the limitations of the classical ones.

\section{$0-30$}

Microfluidic Modulation Spectroscopy (MMS) a new innovative tool for biophysical characterisation of Proteins and Peptides

Markus Epe

RedShift BioAnalytics Inc., Burlington, Massachusetts, USA

Microfluidic Modulation Spectroscopy (MMS) is a new and innovative technology in protein IR-spectroscopy, bringing together microfluidic optics with a powerful quantum cascade laser to provide greater measurement sensitivity and reproducibility than possible with established techniques for secondary structure determination, such as Circular Dichroism (CD) and Fourier Transform Infra-Red (FTIR) spectroscopy. Furthermore, due to the optics used, a much greater concentration range is accessible for structural analysis, from 0.1 to over $200 \mathrm{mg} / \mathrm{ml}$. Finally, measurements do not require any labelling and can be made directly, even in complex buffer systems that contain usually difficult species for spectroscopy, such as complex buffers, excipients, reducing agents, adjuvants and surfactants.

In our short presentation, we show how the system works and what kind of applications you can run on the system. 
Oral Presentations

- Self-Assembled Session 1 - Emerging breakthrough methods in molecular-scale biophysics (ARBRE) -

\section{0-31}

Laser-based IR spectroscopy for highly sensitive analysis of proteins

Andreas Schwaighofer, Christopher K Akhgar, Bernhard Lendl

Technische Universität Wien, Austria

Mid-IR spectroscopy is capable to provide both qualitative and quantitative information on proteins in a fast, nondestructive and label-free manner by probing the strong, fundamental vibrations of molecules. In protein analysis determination of the secondary structure $(\alpha$-helix, $\beta$-sheet, random coil, etc. ...) of a given protein is the most relevant qualitative information accessible by mid-IR spectroscopy. Protein analysis in aqueous solutions by conventional Fourier transform infrared (FTIR) spectroscopy is limited due to the strong water absorption overlapping with the informationrich protein amide I band. Consequently, only short pathlengths $(<10 \mu \mathrm{m})$ can be used for measurements as otherwise all light would be absorbed by the aqueous sample matrix. The progress made in mid-IR lasers has recently changed this situation. High spectral power densities and broad tuning ranges, as made possible by external cavity quantum cascade lasers (EC-QCL), now allow for pathlengths of $30 \mu \mathrm{m}$ and more, even for analysis of the amide I band.

We present a broadband EC-QCL based IR transmission setup covering the amide I+II bands for highly sensitive protein sensing that outperforms commercially available IR spectrometers by almost an order of magnitude. Furthermore, we report on application of laser-based IR spectroscopy on monitoring protein conformational changes after external perturbation (chemical, temperature, $\mathrm{pH}$ ).

\section{0-32}

Nanoscopic anatomy of dynamic multi-protein complexes at membranes resolved by graphene induced energy transfer

Nadia Füllbrunn[1], Zehao Li [1,2], Lara Jorde[1], Carola Meyer [1], Jörg Enderlein [3], Christian Ungermann [1], Jacob Piehler [1], Changjiang You [1]

[1] Osnabrück Univ., Germany; [2] Beijing Univ. of Chemical Technology, China; [3] Georg August Univ., Germany

For exploring the conformational organization and dynamics of protein complexes at membranes, we here introduce graphene-induced energy transfer (GIET) to probe the axial orientation of arrested biological macromolecules at lipid monolayers. Based on a calibrated distance-dependent efficiency within $25 \mathrm{~nm}$, we analyzed the conformational organization of protein complexes involved in tethering and fusion at the lysosome-like yeast vacuole. Ensemble and timeresolved single molecule GIET experiments revealed that the HOPS complex, when recruited via Ypt7 to membranes, is dynamically alternating between a "closed" and an "open" conformation. Our work highlights GIET as a unique spectroscopic ruler to reveal the axial orientation and dynamics of macromolecules at biological membranes with subnanometer resolution. The GIET approach could be combined with electron microscopy to achieve a detailed structural picture of proteins in action. By controlled surface interfacing with cells, GIET analysis to probe the axial organization of signaling complexes in the plasma membrane can be envisaged.

N.F., Z.L. equal contributions. C.U., J.P., C.Y. co-correspondents. 
Oral Presentations

-4. Membrane transporter and channels -

\section{0-33}

Single-molecule studies of conformational states and dynamics in the ABC importer OpuA

Konstantinos Tassis [1], Ruslan Vietrov [1], Matthijs de Koning [1], Marijn de Boer [1], Giorgos Gouridis [1,2], Thorben Cordes $[1,3]$

[1] University of Groningen, Netherlands; [2] IMBB Forth, Greece; [3] LMU Biocenter, Germany

The current model of active transport via $\mathrm{ABC}$ importers is mostly based on structural, biochemical and genetic data. We here establish single-molecule Förster-resonance energy transfer (smFRET) assays to monitor the conformational states and heterogeneity of the type-I ABC importer OpuA from Lactococcus lactis. Our studies include intradomain assays that elucidate conformational changes within the substrate-binding domain (SBD) OpuAC and interdomain assays between SBDs or transmembrane domains. Using the methodology, we studied ligand-binding mechanisms as well as ATP and glycine betaine dependences of conformational changes. Our study expands the scope of smFRET investigations towards a class of so far unstudied ABC importers, and paves the way for a full understanding of their transport cycle in the future.

\section{0-35}

Ball-and-chain inactivation in a calcium-gated potassium channel

Chen Fan [2], Nattakan Sukomon [2], Emelie Flood [1], Jan Rheinberger [2], Toby Allen [1], Crina Nimigean [2] [1] RMIT, Australia; [2] Weill Cornell Medical College, USA

Inactivation is the process by which ion channels terminate ion flux through pores while opening stimulus is still present. In neurons, inactivation of both $\mathrm{Na}$ and $\mathrm{K}$ channels is crucial for action potential generation and regulation of firing frequency. It has been proposed that a cytoplasmic domain of the channel complex plugs the open pore to inactivate it via a "ball-and-chain" mechanism, but no structural evidence of this had been observed. We used cryo-EM to determine the gating mechanism in $\mathrm{Ca}^{2+}$-activated $\mathrm{K}$ channels by obtaining structures of a purely $\mathrm{Ca}^{2+}$-gated and inactivating channel in a lipid environment. In the absence of $\mathrm{Ca}^{2+}$ we obtained a structure in closed state, shown by atomistic simulations to be flexible in lipid bilayers, with large rocking motions of the gating ring and bending of pore-lining helices. In $\mathrm{Ca}^{2+}$-bound conditions, we obtained several structures of multiple open-inactivated conformations. These conformations are distinguished by rocking of the gating rings with respect to the membrane, indicating symmetry breakage across the channel. In all conformations displaying open pores, the $\mathrm{N}$-terminus of one subunit sticks into the pore and plugs it, a strong interaction as shown by free energy simulations. Deletion of this N-terminus leads to non-inactivating channels and structures of open states without pore-plug, indicating that this previously unresolved $\mathrm{N}$-terminal peptide is responsible for a ball-and-chain inactivation mechanism.

\section{0-34}

The molecular dynamics of potassium channel permeation, selectivity and gating

Bert de Groot

Max Planck Institute for biophysical Chemistry, Germany

Ion channels facilitate the passive, selective permeation of ions such as sodium, potassium and chloride across biological membranes and as such are essential for cellular electrical signalling. Molecular dynamics simulations are used to study ion permeation across potassium channels at the atomic level. Together with crystallographic analyses and electrophysiological experiments these provide insight into the mechanisms of selective and efficient permeation of potassium, as well as the complex and subtle conformational changes involved in the gating of these channels.

0-36

Hydrogen-deuterium exchange mass spectrometry captures distinct dynamics upon substrate and inhibitor binding to a transporter

Chloe Martens [1,2], Ruyu Jia [2], Mrinal Shekhar [3], Shashank Pant [3], Heather Findlay [2], Emad Tajkhorshid [3], Paula Booth [2], Nicola Harris [2], Grant Pellowe [2], Politis Argyris [2]

[1] Université Libre de Bruxelles, Belgium; [2] King's College London, UK; [3] Univ of Illinois at Urbana-Champaign, USA

Proton-coupled transporters use transmembrane proton gradients to power active transport of nutrients into the cell. Highresolution structures often fail to capture the coupling between proton and ligand binding, and conformational changes associated with transport. The bacterial sugar transporter XylE is a proton-coupled symporter from the Major Facilitator Family that has been crystalized in multiple conformations, and with both substrate xylose and inhibitor glucose bound. The ability to discriminate between substrate and inhibitor is puzzling in light of the similarity of the bound structures. Here, we combine hydrogen-deuterium exchange mass spectrometry (HDX-MS) with mutagenesis and molecular dynamics simulations to dissect the molecular mechanism of this prototypical transporter. Using differential HDX-MS measurements, we show that protonation of a conserved aspartate triggers conformational transition from outward-facing to inward-facing state. This transition only occurs in the presence of substrate xylose, while the inhibitor glucose locks the transporter in the outward-facing state. MD simulations corroborate the experiments by showing that only the combination of protonation and xylose binding, and not glucose, sets up the transporter for conformational switch. We demonstrate that a specific allosteric coupling between substrate binding and protonation is a key step to initiate transport, and that such coupling displays a dynamic signature detectable by HDX-MS. 
Oral Presentations

-4. Membrane transporter and channels -

\section{0-37}

Regulation of a divalent cation channel investigated by $>100 \mathrm{kHz}$ magic-angle spinning NMR Tobias Schubeis, Marta Bonaccorsi, Andrea Bertarello, Guido Pintacuda

High field NMR center Lyon - CRMN (UMR 5082), France

In recent years, magic-angle spinning (MAS) NMR has developed as a powerful technique for membrane proteins, enabling the study of these systems in a native like lipid environment. In particular, faster MAS rates have paved the way for proton-detection in the solid state, allowing the acquisition of resolved proton resonances in fully protonated samples in sub-milligram amounts. This technical progress revolutionizes the atomic-level investigation of proteins, expanding the range of information exploitable for the determination of structures and opening new horizons for the investigation of dynamics.

Here we demonstrate that MAS rates of $100 \mathrm{kHz}$ and above, coupled to ultra-high magnetic fields, permit the site-specific measurement of observables connected to local and global dynamics in the bacterial divalent cation channel CorA reconstituted in lipid bilayers. CorA is a $5 \times 42 \mathrm{kDa}$ pentamer comprised of two transmembrane helices and a large cytoplasmic domain hosting a metal binding site (usually $\mathrm{Mg} 2+$ or $\mathrm{Co} 2+)$. Well-resolved spectra for backbone and sidechains allow resonance assignment which give information on structural elements. The measurement of residue-specific dynamic parameters provides insights on the transport mechanism of cations through the CorA channel, challenging models previously formulated on the basis of static structures from cryo-EM.

\section{0-38}

On the interplay between complex lipid bilayer membrane and multidrug resistance-associated protein 1 (MRP1) by means of molecular dynamics Ágota Tóth, Angelika Janaszkiewicz, Florent Di Meo INSERM U1248 IPPRITT, Univ. Limoges, France

Membrane crossing by xenobiotics is a key event in pharmacology, especially in pharmacokinetics (i.e., Absorption, Distribution, Metabolism, and Elimination - ADME). Particular attention should be paid to membrane transporters located in liver and kidneys since these organs are mostly responsible for drug eliminations. The present project focuses on multidrug-resistance associated proteins (ABCC/MRPs) which have been pointed out as "emerging clinical importance" by the International Transporter Consortium (ITC). Due to the absence of resolved human MRP structures, bovine MRP1 (bMRP1) is used as a prototype for ABCC family. us-Scale molecular dynamics (MD) simulations have been performed on both inward facing (IF) and outward facing (OF) bMRP1 conformations, in presence or absence of ATP and/or substrate. All are embedded in different lipid bilayer models made of POPC, POPE and/or cholesterol to help to understand the importance of lipid-protein interactions. MD simulations reveals the in situ spontaneous closing of IF conformations as suggested for nucleotide binding domain (NBD) degenerated ABC transporters. Internal structural variabilities of bMRP1 domains exhibits strong impact upon ATP and/or substrate binding. Structural parameters reveal relatively weak dependence on lipid bilayer membrane composition. However, lipid distribution analysis exhibits hot-spot for cholesterol-binding to MRP1. 


\section{Oral Presentations}

-5 . Virus biophysics -

\section{0-39}

\section{Characterizing SARS-CoV-2 membrane enve- lope proteins}

Gerard Duart, Juan Ortiz, Jose M Acosta-Cáceres, Maria J García-Murria, Manuel Sánchez del Pino, Luis Martínez-Gil, Ismael Mingarro

University of Valencia, Spain

The novel severe acute respiratory syndrome coronavirus2 (SARS-CoV-2) has caused a pandemic disease known as coronavirus disease 2019 (COVID-19). The SARS-CoV-2 genome encodes for up to 29 proteins, among them the highly glycosylated spike $(\mathrm{S})$ protein, the membrane $(\mathrm{M})$ protein and the envelope (E) protein are embedded in the lipid membrane forming the virus protein interface to the external environment. The role of each of these membrane proteins and their interactions are critical for the assembly of viral particles and infection. In the present talk, recent advances on membrane topology and homo- and hetero-oligomerizations among these membrane proteins will be presented.

\section{0-41}

Nanomechanical Mapping of Virus Binding Sites to Animal Cells

David Alsteens

Université catholique de Louvain, Belgium

During the last three decades, a series of key technological improvements turned atomic force microscopy (AFM) into a nanoscopic laboratory to directly observe and chemically characterize molecular and cellular biological systems under physiological conditions. I will present the key technological improvements that enable us to apply AFM as analytical laboratory to observe and quantify living biological systems at the nanoscale. I will report the use of advanced FD-based technology combined with chemically functionalized tips to probe the localization and interactions of chemical and biological sites on single native proteins and on living cells at high-resolution. I will present how an atomic force and confocal microscopy set-up allows the surface receptor landscape of cells to be imaged and the virus binding events within the first millisecond of contact with the cell to be mapped at high resolution $(<50 \mathrm{~nm})$. I will also highlight theoretical approaches to contour the free-energy landscape of early binding events between virus and cell surface receptors.
$0-40$

Flying viruses - from biophysical to structural characterisation

Charlotte Uetrecht

Leibniz Institute for Experimental Virology (HPI), Germany; European XFEL GmbH, Germany; Centre for Structural Systems Biology (CSSB), Germany

Viruses affect basically all organisms on earth. Some are detrimental to human development, whereas those targeting pathogenic bacteria or crop pathogens can be beneficial. An integral part of icosahedral viruses is the capsid protein shell protecting the genome. Many protein copies self-assemble into shells of defined size. Low binding affinity of individual subunits allows for efficient assembly and highly stable particles. These capsids can be studied by native and hydrogen/deuterium exchange mass spectrometry (MS) in terms of stoichiometry, dynamics, assembly pathways and stability to investigate strain specific traits of noroviruses, the main cause of viral gastroenteritis. Moreover, the highly dynamic replication machinery of coronaviruses has been a longstanding interest and newest results on various viruses including SARS-CoV-2 will be presented.

Despite the remarkable sensitivity, the structural resolution is limited in native MS. Transient states in capsid assembly are relevant to drug discovery but cannot be purified and are inaccessible for crystallography. Hard X-ray freeelectron-lasers (XFELs) in conjunction with native MS offer an opportunity to obtain high resolution structures of transient single particles.

\section{0-42}

Recording the first steps of virus self-assembly: High Speed AFM imaging unveils assembly dynamics in real-time

Sourav Maity [1], Alejandro Valbuena [2], Mauricio G Mateu [2], Wouter H Roos [1]

[1] Moleculaire Biofysica, Zernike Instituut, Rijksuniversiteit Groningen, Netherlands; [2] Centro de Biología Molecular "Severo Ochoa", Universidad Autónoma de Madrid, Spain

Self-assembly of viral proteins into highly accurate nanostructures is a fast developing research area due to its applications in various fields, e.g. synthesis of nanoparticles, nanomedicine, biology etc. Therefore, there is an ongoing quest for a thorough understanding of this highly dynamic process [Nat Rev Phys 2021, 3, 76]. While, we have a reasonable understanding of pre- and post- assembly states of viral capsid proteins, the study of self-assembly is extremely challenging, because of the size and the transient nature of the assembly intermediates. In the present study, we reveal how using highspeed atomic force microscopy [Sci Adv 2019, 5, eaau7198 \& $J A C S 2020,142,13709]$ we can uniquely visualize, in real time, individual transient intermediates and reaction pathways followed by single molecules during self-assembly of the mature human immunodeficiency virus (HIV) capsid protein [ACS Nano 2020, 14, 8724]. While the bidimensional protein lattice of HIV is randomly built, each lattice grows independently from separate nucleation events where individual molecules follow different pathways. Protein subunits are either added individually, or they first form oligomers before joining a lattice. Occasionally they are removed from the latter. Our direct real-time imaging at the single-molecule level of self-assembly of a viral protein lattice has revealed an unexpectedly complex jigsaw-like puzzle involving multiple routes that are inaccessible to bulk studies. 


\section{Oral Presentations}

\section{-5 . Virus biophysics -}

\section{0-43}

Role of cell-surface glycosaminoglycans and viral glycoproteins in modulating virus binding and diffusion at the cell membrane

Y. Abidine [1], E. Trybala [2], T. Bergström [2], M. Bally [1]

[1] Umeå Univ., Sweden; [2] Göteborg Univ., Sweden

Viral infection is a multistep process requiring the mobility of viruses in the glycocalyx and at the cell membrane to initiate entry. Many viruses, including herpes simplex (HSV), are recruited to the host cell by taking advantage of cellsurface glycosaminoglycans (GAGs) in an interaction tightly regulated by multivalent binding between viral glycoproteins and GAGs. This work aims at unraveling the mechanisms modulating the diffusive behavior of GAG-binding viruses, specifically how the virus hops from GAG to GAG to diffuse through the glycocalyx and at the cell surface towards entry points. Single-particle tracking of the initial interaction of HSV-1 reveals a heterogeneous diffusive behavior at the cell surface dependent on the nature of GAGs, where the virus switches between confined anomalous and normal free motions. We show here the competing functions of heparan sulfate (HS) and chondroitin sulfate (CS), the two main sulfated GAGs, where HS promotes virus confinement and CS enhances free diffusion. Another key element of the virusGAG interaction is the mucin-like region (MLR), a cluster of O-glycans found on the glycoprotein C of HSV-1, that affects the binding of the virus to GAGs. Here, we report that the MLR also plays a key role in promoting virus diffusion at the cell surface.Together, our findings provide new insights into the mechanisms modulating the initial interaction of GAG-binding viruses with the cell for optimal entry.

\section{0-44}

Genome release from non-enveloped RNA viruses: Capsid rupture or pore formation Lukáš Sukeník, Pavel Plevka, Robert Vácha CEITEC - Central European Institute of Technology, Masaryk University, Kamenice 753/5, 62500 Brno, Czech Republic

Viruses are composed of genome protected by a capsid, protein shell, from which the genome has to be released during the infection. The release mechanism of non-enveloped RNA viruses has long been the subject of speculation. Based on the structure of capsids which released their genomes, the pores at two-fold, three-fold, and five-fold axes were proposed to enable slow release of genome. However, capsids without pores or channels, and capsids with entire subunits missing were also observed. Consequently, the pathways of genome release from the capsid remained elusive. We developed a phenomenological model of an icosahedral virus capsid with a genome and used it to investigate genome release pathways of various viruses. In agreement with available experiment, we observed both rapid and slow categories of release and compared their success rates. Slow release occurred when interactions between capsid subunits were long-ranged and the genome was non-compact. In contrast, rapid release was preferred when either the interaction range was short or the genome was compact. These findings could be utilized in a design strategy of virus-like nanoparticles for drug delivery or new inhibitors for viruses. 
Oral Presentations

- 6. Advanced optical microscopy -

$0-45$

High density single molecule dynamic mapping of the cell membrane

N. Mateos [1], P. Sil [3], S. Talluri [3], C. Manzo [2], S. Mayor [3], M. F Garcia-Parajo [1]

[1] ICFO-Institute of Photonic Sciences, Spain; [2] Universitat de Vic-UCC, Spain; [3] NCBS-National Centre for Biological Sciences, India

Organization by compartmentalization is a general property of natural systems that efficiently facilitates and orchestrates biological events in space and time. In the last decade, compartmentalization of the plasma membrane of living cells has emerged as a dominant feature present at different spatiotemporal scales and regulating key cell functions. The advent of super-resolution microscopy and single molecule dynamic approaches has allowed the study of the cell membrane with unprecedented levels of details. While superresolution microscopy provides nanometer-scale snapshots of membrane organization, its poor temporal resolution precludes dynamic studies at the relevant temporal scales. On the other hand, single particle tracking (SPT) approaches gain in temporal resolution but they are limited to a subset of labeled molecules, hindering detailed studies over the whole molecular population. Here we combine SPT at different labeling densities. Low density conditions allow us to reconstruct the mobility of individual molecules and their transient interaction with other molecular partners, while high density labeling reports on the spatiotemporal scales of the cellular environment explored by molecules. We find that both molecular diffusion and dynamic re-modeling of the environment play key roles regulating biological function.

\section{0-47}

Live cell super-resolution optical microscopy by structured illumination microscopy with instant image reconstruction

Thomas Huser

Bielefeld University, Germany

Super-resolved structured illumination microscopy (SR-SIM) is among the most flexible, fastest and least perturbing fluorescence microscopy techniques capable of surpassing the optical diffraction limit. Current custom-built instruments are easily able to deliver two-fold resolution enhancement at video-rate frame rates, but the cost of the instruments is still relatively high and the physical size of the instruments is still prohibitively large. Here, I will present our latest efforts towards realizing a new generation of compact, cost-efficient and high-speed SR-SIM instruments. Tight integration of the structured illumination microscope capable of video-rate image acquisition with instant image reconstruction based on parallel computing of image information enables us to realize a super-resolving fluorescence microscope with the lookand-feel of regular wide-field microscopy. I will demonstrate this by discussing the overall integration of optics, electronics, and software that allowed us to achieve this, and then present its capabilities by visualizing the dynamics of intracellular transport and movement in living cells, in particular the dynamics of liver cell fenestrations. I will further demonstrate how denoising of SIM image data by deep learning allows us to extend these capabilities to data collected with very low signal-to-noise levels without compromising spatial resolution.

\section{0-46}

Super-resolution microscopy with DNA molecules Ralf Jungmann

Max Planck Institue of Biochemistry, Germany

Super-resolution fluorescence microscopy is a powerful tool for biological research. We use the transient binding of short fluorescently labeled oligonucleotides (DNA-PAINT) for easy-to-implement multiplexed super-resolution imaging that technically achieves sub-5-nm spatial resolution. To translate this resolution to cellular imaging, we introduce Slow Off-rate Modified Aptamers (SOMAmers) as efficient and quantitative labeling reagents. We demonstrate the achievable image resolution and specificity by labeling and imaging of transmembrane as well as intracellular targets in fixed and live cell-specimen.

While DNA-bases super-resolution allows high spatial resolution and spectrally unlimited multiplexing capabilities, image acquisition is slow compared to most other approaches. We recently overcame this limitation by designing optimized DNA sequences and repetitive sequence motifs. We achieve 100-fold faster imaging speeds without compromising image quality or spatial resolution. This improvement now makes DNA-PAINT applicable to high-throughput studies.

\section{0-48}

Using fluorescent protein directionality in microscopy imaging of membrane protein structure and function

Josef Lazar [1,2], Jitka Myšková [1], Alexey Bondar [2,1], Olga Rybakova [1], Jiří Brynda [3,1], Petro Khoroshyy [1]

[1] Institute of Organic Chemistry and Biochemistry CAS, Czech Republic; [2] Institute of Microbiology CAS, Czech Republic; [3] Institute of Molecular Genetics CAS, Czech Republic

Fluorescent proteins (FPs) are like antennas: the rate at which they absorb light depends on their orientation with respect to the incoming light wave, and the apparent intensity of their emission depends on their orientation with respect to the observer. FP directionality has allowed sensitive imaging of activation of G-proteins, changes in intracellular calcium concentration or cell membrane voltage by polarization-resolved fluorescence microscopy. Furthermore, FP directionality is important for interpreting results of FRET observations. However, without knowing the directions along which molecules of FPs absorb and emit light, it is not possible to interpret FRET or polarization microscopy observations in terms of protein structure. Here we present the results of our determinations of optical directionality of several FP molecules and illustrate the use of this information for gaining insights into membrane protein structure and function. 
Oral Presentations

-6. Advanced optical microscopy -

0-49

Single-molecule studies of membrane protein interactions via continuous DNA-mediated fluorophore exchange

Christian Niederauer [1], Miles Wang-Henderson [1], Johannes Stein [2], Florian Stehr [2], Julian Bauer [2], Ralf Jungmann [2], Petra Schwille [2], Kristina Ganzinger [1] [1] AMOLF, Netherlands; [2] MPI of Biochemistry, Germany

Quantifying protein-protein interactions in cell membranes is key to understand many cellular processes, including a cell's communication with its surroundings via cell surface receptors. While single-molecule tracking is a valuable tool to measure receptor-ligand interaction kinetics in cell membranes, photobleaching limits the observation times of individual molecules to only a few seconds with commonlyused fluorescent proteins or dyes. In practice, this makes it often hard to reliably distinguish real interactions from random colocalization events. Here, we show that this limitation can be overcome by a new labelling method based on DNA-PAINT, with fluorescently-labeled oligonucleotides transiently binding to a complementary DNA strand attached to the molecule of interest. Several fluorescentlylabeled oligonucleotides can be accommodated on the complementary DNA strand simultaneously, and enable tracking of individual molecules for unprecedented observation periods. We present this new method that can be readily combined with existing protein tagging methods, such as SNAP or CLIP tags. Furthermore, we show its implementation invitro and in live cells by studying the dimerization of FKBP proteins that were reconstituted on a supported lipid bilayer or expressed at the cell membrane.

\section{0-50}

Direct supercritical angle localization microscopy for nanometer 3D superresolution Anindita Dasgupta [1,2], Joran Deschamps [1], Ulf Matti [1], Uwe Hübner [2], Jan Becker [2], Sebastian Strauss [3,4], Ralf Jungmann [3,4], Rainer Heintzmann [2,5], Jonas Ries [1] [1] Cell Biology and Biophysics, EMBL, Germany; [2] Leibniz Institute of Photonic Technology, Germany; [3] Fac of Physics and Center for Nanoscience, Ludwig Maximilian Univ, Germany; [4] Max Planck Institute of Biochemistry, Germany; [5] Institute of Physical Chemistry, Friedrich-Schiller Univ, Germany

3D single molecule localization microscopy (SMLM) has emerged as a powerful method for structural cell biology, as it allows probing precise positions of proteins in cellular structures at a resolution of tens of nanometers in both 2D and 3D. Popular approaches to 3D imaging include PSF engineering to encode the position of single-emitters in its shape. However, such methods lead to worse localization precision axially than laterally. Complex localization microscopy techniques, such as iPALM, can provide isotropic resolution at the cost of a complex instrument. Supercritical angle fluorescence strongly depends on the z-position of the fluorophore and can be used for axial localization in a method called supercritical angle localization microscopy (SALM). Here, we realize the full potential of SALM by directly splitting supercritical and undercritical emission, using an ultra-high NA objective, and applying new fitting routines to extract precise intensities of single emitters, resulting in a several fold improved z-resolution compared to the state of the art. We demonstrate nanometer isotropic localization precision on DNA origami structures, and on clathrin coated vesicles and microtubules in cells, illustrating the potential of SALM for cell biology. 
Oral Presentations

- 7. Protein translocation, assembly, and folding -

\section{0-51}

The AAA-ATPase Bcs1 uses an airlock-like mechanism for folded protein translocation

Lukas Kater [1], Kikola Wagener [2], Otto Berninghausen [1], Thomas Becker [1], Walter Neupert [3], Roland Beckmann [1] [1] Gene Center and Center for Integrated Protein Science Munich, Department of Biochemistry, University of Munich, Germany; [2] Department of Biology, University of Munich, Germany; [3] Biomedical Center, University of Munich, Germany

Certain proteins require completion of folding before translocation across a membrane into another cellular compartment. Yet compromising the permeability barrier of the membrane must be minimized during translocation and underlying mechanisms have remained mostly enigmatic. Here, we present the structure of unusual AAA-ATPase of the inner mitochondrial membrane, Bcs1, which facilitates the translocation of the Rieske protein, which requires folding and incorporation of a Fe2-S2 cluster in the mitochondrial matrix before translocation across the inner mitochondrial membrane and subsequent integration into the bc1 complex. Surprisingly, this AAA-ATPase assembles into exclusively heptameric homo-oligomers, with each protomer consisting of an amphipathic transmembrane helix, a middle domain and an ATPase domain. Together, they form two aqueous vestibules, the first accessible from the mitochondrial matrix and the second positioned in the inner membrane, both separated by the seal-forming middle domain. Based on this unique architecture and observed dynamic conformations, we propose an airlock-like translocation mechanism for folded proteins employed by this exceptional ATPase.

\section{0-53}

\section{Protein folding in mitochondria}

Alexey Amunts

Stockholm University, Sweden

Mitochondrial ribosomes (mitoribosomes) are tethered to the mitochondrial inner membrane to facilitate the cotranslational membrane insertion of the synthesized proteins. We report cryo-electron microscopy structures of human mitoribosomes with nascent polypeptide, bound to the insertase oxidase assembly 1-like (OXA1L) through three distinct contact sites. OXA1L binding is correlated with a series of conformational changes in the mitoribosomal large subunit that catalyze the delivery of newly synthesized polypeptides. The mechanism relies on the folding of mL45 inside the exit tunnel, forming two specific constriction sites that would limit helix formation of the nascent chain. A gap is formed between the exit and the membrane, making the newly synthesized proteins accessible. Our data elucidate the basis by which mitoribosomes interact with the OXA1L insertase to couple protein synthesis and membrane delivery.

\section{0-52}

Polypeptide loop translocation by single ClpB disaggregases

Sander Tans

AMOLF, Netherlands

Re-dissolving protein aggregates is crucial to cells, but the molecular basis has remained untested in direct experiments. Using combined optical tweezers and single-molecule fluorescence detection, we show that the disaggregase ClpB extrudes loops of protein chains through its central pore, and hence forcibly extracts protein chains from aggregates. The data reveal notable processivity, power, step-dynamics, and switching between translocation modes. Protein disaggregation can thus be highly deterministic and energy-driven process, while polypeptide loop extrusion may be exploited by other systems including p97/cdc48.

\section{0-54}

The role of intrinsic disorder in DNA recognition and target search by a transcription factor Mikhail L Kuravsky, Sarah L Shammas

Department of Biochemistry, University of Oxford, S Parks Rd, Oxford, OX1 3QU, UK

A specific transcription factor must bind to its cognate sites after locating them within a large excess of genomic DNA. How can a single protein domain perform a rapid scan of non-target sequences, yet also form a tight complex with its target? We have used kinetic stopped-flow methods to uncover how the intrinsically disordered bZIP domain of cAMPresponsive element-binding protein (CREB) associates with DNA and searches for its target CRE sites. We report the results of the first experimental phi-value analysis for folding upon binding to DNA; after a rapid and indiscriminative association with DNA, dimeric CREB forms a disordered transition state sustained by non-native electrostatic interactions and subsequently the basic region partially folds. Being kinetically unstable, the CREB.DNA complex serves as a basis for target search via facilitated dissociation; target discrimination being achieved through a 300 -fold reduction in dissociation rate. Our results suggest that modulating the disorder (or helical) content of the bZIP domain can alter the balance between "search" and "recognition" modes, and that more disordered versions can find their targets faster. This may represent a general mechanism employed by disordered DNA binding domains to solve the search-speed/stability paradox. 


\section{Oral Presentations}

- 7. Protein translocation, assembly, and folding -

\section{0-55}

Two-dimensional metal interaction-driven crystalline protein assemblies

Mantas Liutkus [1], Aitziber L Cortajarena [1,2]

[1] CIC biomaGUNE, Spain; [2] Ikerbasque, Basque Foundation for Science, Spain

Crystalline two-dimensional planar assemblies based on consensus tetratricopeptide repeat (CTPR) proteins mediated through metal-coordinating interactions were engineered, exploiting the modular nature of the repeat proteins. CTPR proteins, made of small helical modules, have a propensity to form long rigid superhelical arrays. Metal ion coordination allows the resulting superhelices to pack with a high degree of order, which is maintained at macroscopic scales, i.e. in films or crystals. Crystals of CTPR proteins can therefore be considered as bundles of the superhelices, whereby the superhelices span the length of the crystal. As individual repeats can be modified without affecting the functions of the neighbouring modules, the superhelices were engineered to maintain metal interactions only in two dimensions. This resulted in the formation of two-dimensional protein assemblies, equivalent to a single plane out of a three-dimensional crystal. Supramolecular protein assemblies are highly desired for nanoscale applications as a means to confer order on the molecular level, and planar two-dimensional assemblies are particularly suitable for imposing order over a large planar area. The newly developed CTPR planar assemblies are likely to be particularly useful for the ordering of small functional elements, given the small scale of the individual repeats $(\sim 2 \mathrm{~nm})$ and the rigidity of the two-dimensional crystals.

\section{0-56}

Microfluidic diffusional sizing for studying protein-protein interactions

Matthias M Schneider [1], Christopher M Dobson [1], Adriano Aguzzi [2], F. Ulrich Hartl [3], Tuomas P J Knowles [1]

[1] Centre for Misfolding Diseases, Department of Chemistry, University of Cambridge, UK; [2] Institute of Neuropathology, University Hospital Zurich, Switzerland; [3] Department of Cellular Biochemistry, Max-Planck-Institute of Biochemistry, Martinsried, Germany

Standard techniques for investigating protein-protein interactions require volumes and concentrations significantly higher than those relevant under physiological conditions. Microfluidic diffusional sizing offers an alternative method suitable for investigating protein interactions and assembly, especially in the context of human diseases. In particular, recent work makes use of microfluidic techniques combined with chemical kinetic analysis to study the mechanism of the disaggregation of $\alpha$-synuclein fibrils with Hsc70 along with its co-chaperones DnaJB1 and Apg2. This analysis reveals that the chaperone can completely reverse $\alpha$-synuclein aggregation back to its soluble monomeric state by removing monomer predominantly from the fibril ends. Another recent application of microfluidic diffusional sizing allows to characterise affinity, antibody concentration and neutralisation potential in crude patient plasma samples from convalescent SARS-CoV-2 infected individuals, revealing that the affinities span more than two orders of magnitude while the concentrations are relatively constant. The presentation shows how advances in microfluidic technology enables answering emerging questions in biophysical chemistry and medicine. 
Oral Presentations

-8 . Bioenergetics -

0-57

Sodium controls hypoxic redox signalling by the mitochondrial respiratory chain

Pablo Hernansanz-Agustín [1], Antonio Martínez Ruiz [2], José Antonio Enríqez [1]

[1] Centro Nacional de Investigaciones Cardiovasculares Carlos III, Madrid, Spain; [2] Unidad de Investigación, Hospital Universitario Santa Cristina, Instituto de Investigación Sanitaria Princesa (IIS-IP), Madrid, Spain

The oxidative phosphorylation system (OXPHOS) uses oxygen for energy production. It was shown decades ago that hypoxia, the decreased availability of oxygen in tissues and cells, promote the paradoxical increase in reactive oxygen species (ROS). $\mathrm{Ca}^{2+}$ is a very well known second messenger as it directly modifies the activity of many enzymes and proteins in the cell. However, the role attributed to $\mathrm{Na}+$ has been ascribes to the mere control of membrane potential. Here, we show that in the first minutes of hypoxia mitochondrial complex I undergoes a conformational shift that leads to a mitochondrial matrix acidification and partial dissolution of the calcium phosphate precipitates. The release of soluble calcium activates the mitochondrial sodium/calcium exchanger (NCLX) which extrudes calcium in exchange of sodium. Mitochondrial sodium interacts with phospholipids in the inner leaflet of the IMM, forming a 1sodium:3phospholipid cluster which diminishes the IMM fluidity. As a consequence, CoQ transfer between CII and CIII decreases, but not between CI and CIII, and superoxide anion is produced at the levels of the Qo site of CIII. ROS production during hypoxia is needed for hypoxic pulmonary vasoconstriction (HPV) and adaptation.

\section{0-59}

Unveiling the membrane bound dihydroorotate:quinone oxidoreductase (DHOQO) from Staphylococcus aureus

Filipe M Sousa [1,2], Patricia N Refojo [1,2], Andreia Barreto [1,2], Patricia Pires [2], Micael Silva [3], Pedro Fernandes [1], Ana P Carapeto [2,4], Tiago T Robalo [2,4], Mário S Rodrigues [2,4], Mariana G Pinho [1], Eurico J Cabrita [3], Manuela M Pereira [1,2] [1] ITQB Nova - António Xavier, Oeiras, Portugal; [2] BioISI, Lisboa, Portugal; [3] Dept de Química, Univ Nova de Lisboa, Caparica, Portugal; [4] Dept de Física, Univ de Lisboa, Portugal

Staphylococcus aureus is an opportunistic pathogen and one of the most frequent causes for community acquired and nosocomial infections. In this work we unveil the dihydroorotate:quinone oxidoreductase (DHOQO) from $S$. aureus, the first example of a DHOQO from a Gram-positive organism. DQO is required for the biosynthesis of pyrimidines and thus to produce nucleic acids [1]. The FMN containing menaquinone reducing enzyme showed a Michaelis-Menten behavior towards the two substrates and was inhibited by Brequinar, Leflunomide, Lapachol, HQNO, Atovaquone and TFFA with different degrees of effectiveness. Mutation of the DHOQO coding gene led to lower growth rates, and effects in cell morphology and metabolism, most importantly in the pyrimidine biosynthesis, here systematized for $S$. aureus MW2 for the first time. This work unveils the existence of a functioning DHOQO in the respiratory chain of the pathogenic bacterium $S$. aureus, contributing to a better understanding of its energy metabolism with possible implications for the rational drug design targeting this organism. [1]F.M. Sousa, P.N. Refojo, M.M. Pereira, Biochim. Biophys. Acta - Bioenerg., 1862 (2021) 148321.
0-58

The molecular mechanism of substrate binding and transport of the mitochondrial ADP/ATP carrier

Vasiliki Mavridou, Martin S King, Sotiria Tavoulari, Jonathan J Ruprecht, Shane Palmer, Edmund R Kunji

University of Cambridge, Medical Research Council Mitochondrial Biology Unit, UK

Mitochondrial ADP/ATP carriers provide key transport steps in eukaryotic oxidative phosphorylation by importing ADP into the mitochondrial matrix and by exporting synthesized ATP to fuel cellular processes. Structures of the inhibited cytoplasmic- and matrix-open states have confirmed an alternating access mechanism, but the molecular nature of substrate binding is unresolved. Here, we investigate all solvent-exposed residues in the translocation pathway in both conformational states of the mitochondrial ADP/ATP carrier. By combining binding studies with functional assays using single alanine replacement variants, we identify a single substrate binding site in the center of the cavity. It consists of three positively charged residues, which could bind the phosphate groups, and a cluster of three aliphatic and one aromatic residue, which could bind the adenosine moiety of the nucleotides. In addition, two arginine/asparagine pairs are identified, which are unique to ADP/ATP carriers but play a role in binding. Importantly, the same residues are involved in binding of both ADP and ATP, implying that the import and export steps occur consecutively and in a reversible way. The features of the identified binding site explain the electrogenic nature of transport.

\section{$0-60$}

The transport of fatty acid anions across the inner mitochondrial membrane by the adenine nucleotide translocase

Jürgen Kreiter [1], Sanja Skulj[2], Zlatko Brkljaca [2], Kristina Zuna [1], Mario Vazdar [2,3], Elena E Pohl [1]

[1] University of Veterinary Medicine, Vienna, Austria; [2] Rudjer Boskovic Institute, Zagreb, Croatia; [3] Czech Academy of Sciences, Prague, Czech Republic

The protonophoric function of the adenine nucleotide translocase (ANT) is well described by the fatty acid (FA) cycling hypothesis, in which ANT facilitates the translocation of FA anions $\left(\mathrm{FA}^{-}\right)$across the inner mitochondrial membrane. However, the molecular transport mechanism remains elusive.

Here, we employ recombinant ANT reconstituted into planar lipid bilayers to investigate the ANT-mediated $\mathrm{FA}^{-}$transport. Using site-directed mutagenesis and molecular dynamics simulations, we show that $\mathrm{FAs}^{-}$are first caught by R59 at the protein-lipid interface on the matrix. They move across a large positively charged potential on the protein-lipid interface to R79, where the $\mathrm{FA}^{-}$penetrates into the hydrated protein cavity and protonates with the assistance of D134. The protonated FA is released and freely moves inside the membrane to the matrix and the cycle restarts. We conclude that R79 is a crucial binding site of ANT, which is further outlined by the competitive binding between $\mathrm{FA}^{-}$and the ANT-relevant substrates and inhibitors.

Since the identified binding sites are well conserved, we hypothesize a similar $\mathrm{FA}^{-}$transport mechanism for further proton transporting SLC25 members. 
Oral Presentations

- 8. Bioenergetics -

\section{0-61}

Membrane protein hydration is linked to membrane lateral pressure in Copper-Transport

Karim Fahmy, Lisa Nucke, Ahmed Sayed

Helmholtz-Zentrum Dresden-Rossendorf, Technische Universität Dresden, Germany

We have used the copper-ATPase LpCopA from Legionella pneumophila $^{1}$ as a model system to study intra-membrane protein hydration. Time-resolved fluorescence spectroscopy of the dye BADAN linked to the conserved copper-binding transmembrane CPC motif revealed substantial mobility of internal water in detergent, whereas dipol mobility was restricted in lipid nanodiscs (NDs) with remarkably distinct responses of the two cysteines. Functionally required lipid protein interactions thus shape an intra-membrane protein gradient of water mobility in the ion transporter as opposed to the more hydrated but non-functional detergent-solubilized protein $^{2}$. These dipolar relaxation studies of BADAN report water mobility only, whereas the amount of intra-membrane protein was determined by osmotic pressure-dependent static fluorescence of BADAN. We show that CopA undergoes volume changes that correspond to a pressure-induced release of 15-20 water molecules in the detergent-solubilized state. In NDs, membrane lateral pressure reduces the number of internal waters to 8-10. The data demonstrate that transient hydration / dehydration events during catalytic activity will be opposed or supported by lateral pressure, respectively, rendering the elastic membrane properties a transient reservoir for free enthalpy in the catalytic cycle.

References :1) P.Gourdon, P. et al, Nature 2011, 475, 59-64;

2) Fischermeier, E. et al., Angewandte Chemie 2017, 129, 1289-92

\section{0-62}

Membrane softening of microaspirated GUVs containing active F1Fo-ATP synthase

David Valdivieso González [1,2], Lillo M. Pilar [3], Víctor G. Almendro-Vedia [1,2], Iván López Montero [1,2]

[1] Dto. Química Física, Universidad Complutense de Madrid, Spain; [2] Instituto de Investigación Hospital Doce de Octubre, Madrid, Spain; [3] Dto. Química Física Biólogica, Instituto de Química-Física "Rocasolano" (CSIC), Madrid, Spain

Biomembranes are dynamic and complex mixtures of lipid and proteins that interact to define most of membrane functions and properties. Giant unilamellar vesicles (GUVs) are widely used to study lipid-protein interactions. GUVs are lipid bilayers of $10 \mu \mathrm{m}$ in diameter suitable for microscopy and micromanipulation. Micromanipulation allows determining the mechanical parameters of GUVs. In a typical configuration, a GUV is pulled out by a cylindrical micropipette in the aspiration mode and the bending modulus $(\kappa)$ is obtained from the tension-deformation curve, which is built upon different suction pressures. Here we have obtained the membrane stiffness of $\mathrm{F}_{1} \mathrm{~F}_{\mathrm{o}}$-ATP synthase proteo-GUVs under passive and active conditions. $\mathrm{F}_{1} \mathrm{~F}_{\mathrm{O}}$-ATP synthase is a fundamental membrane protein that synthesises the biochemical energy of the cell trough an electrochemical gradient of protons across the membrane or rotate in the opposite direction due to an excess of ATP with reverse flux of protons. The protein activity promoted a membrane softening where the bending modulus was reduced in consequence ( $\left.\kappa_{a c t} / \kappa_{\text {pass }} \approx 0.25\right)$. Here, a similar effect was measured using the microaspiration technique. 
Oral Presentations

-9. Quantification of molecular forces -

\section{0-63}

Forces in chaperone-mediated protein unfolding and cell adhesion

Matthias Rief

Technische Universität München, Germany

Many process in our cells are mechanical. Single molecule methods like optical tweezers allow studying the forces involved in those processes. In my talk, I will discuss 2 examples. In the first part, I will show how the concerted action of the hsp70 chaperone and its co-chaperones completely unfold the glucocorticoid receptor. In the second part of my talk, I will discuss how the cytoskeletal proteins talin and kindlin co-operate to strengthen their mechanical bond to the cell adhesion protein integrin.

\section{$0-65$}

The mechanical design of the bacterial toxin protein RTX

Han Wang, Hongbin Li

University of British Columbia, Canada

The efficient translocation of bacterial toxin adenylate cyclase toxin (CyaA) from the bacterial cytosol to the extracellular environment by the type 1 secretion system (T1SS) is essential for the toxin to function. To understand the molecular features that are responsible for the efficient translocation of CyaA, here we used optical tweezers to investigate the mechanical properties and conformational dynamics of the RTX-V and RTX-IV domains of CyaA at the single molecule level. Our results revealed that apo-RTX behaves like an ideal random coil. This property allows the T1SS to translocate RTX without overcoming enthalpic resistance. In contrast, the folded holo-RTX-V and RTX-IV are mechanically stable, and their folding occur in a vectorial, co-translocational fashion starting from its C-terminus. The folding of RTX-IV depends on the folding of RTX-V, and the folding of RTX-IV in turn stabilizes RTX-V. Moreover, the folding of RTX domains in the presence of $\mathrm{Ca}^{2+}$ generates a stretching force, which can further facilitate the translocation of RTX domains. Our results highlight the important role played by the $\mathrm{Ca}^{2+}$-triggered folding of RTX in the translocation of RTX and provide mechanistic insights into the mechanical design that governs the efficient translocation of RTX.

\section{$0-64$}

Length is important! Using force spectroscopy to probe protein:protein interactions

David J Brockwell

School of Molecular and Cellular Biology, University of Leeds, UK

Intrinsically disordered proteins (IDPs) or proteins with intrinsically disordered regions (IDRs) are recognised to play ever increasing roles in recognition and signalling. IDP/Rs can be challenging to study due to their shallow but rough energy landscapes and, for some, their propensity to aggregate leads to further conformational heterogeneity. Using AFM force spectroscopy to study IDP/Rs can thus be advantageous as it measures the interaction between single pairs of biomolecules tethered to two surfaces. Here we will illustrate the insight that can be gained into the conformation of IDP/Rs by force spectroscopy by focussing not on their disassociation force but on their end-to-end length at rupture.

0-66

Nanomechanical mechanisms of Borrelia interactions with extracellular matrix

Yoo Jin Oh [1], M Strnad [2,3], M Vancova [2,3], L Hain [1], J Salo [4], L Grubhoffer [2,3], J Nebesarova [2,3], J Hytoenen [4,5], P Hinterdorfer [1], R O M Rego [2,3]

[1] Johannes Kepler University Linz, Austria; [2] Biology Centre ASCR, Czech Republic; [3] University of South Bohemia, Czech Republic; [4] University of Turku, Finland; [5] Turku University Hospital, Finland

As opposed to pathogens passively circulating in the body fluids of their host, pathogenic species within the Spirochetes phylum are able to actively coordinate their movement in the host to cause systemic infections. Based on the unique morphology and high motility of spirochetes, we hypothesized that their surface adhesive molecules might be suitably adapted to aid in their dissemination strategies. Bio-AFM provides the ideal condition for nano-scale characterization of the microbial surface in their native, physiological environment and for elucidating processes occurring at the interface between microorganisms and cells. In this study, we probed the interaction forces between decorin binding protein $\mathrm{A} / \mathrm{B}$ (DbpA/B) from different genospecies and various ECM proteins. Using single-molecule force spectroscopy, we disentangled the mechanistic details of $\mathrm{DbpA} / \mathrm{B}$ and decorin/laminin interactions. Our results show that spirochetes, depite a limited number of adhesive molecules, are able to leverage a wide variety of adhesion strategies through force-tuning transient molecular binding to extracellular matrix components, which concertedly enhance spirochetal dissemination through the host. 
Oral Presentations

-9. Quantification of molecular forces -

0-67

Optimizing mechanostable anchor points of engineered lipocalin in complex with CTLA-4

Zhaowei Liu [1,2], Rodrigo A Moreira [3], Ana Dujmović [1,2], Haipei Liu [1,2], Byeongseon Yang [1,2], Adolfo B Poma [3], Michael A Nash [1,2]

[1] Department of Chemistry, University of Basel, Switzerland; [2] Department of Biosystems Science and Engineering, ETH Zurich, Switzerland; [3] Biosystems and Soft Matter Division, IPPT-PAN, Poland

Anticalin is a non-antibody protein scaffold with potential for targeted drug delivery to cytotoxic T-lymphocyte antigen 4 (CTLA-4) positive T-cells. One limiting factor is the ability of the anticalin:CTLA-4 complex to resist mechanical forces exerted by local shear stress. We combined singlemolecule AFM force spectroscopy, non-canonical amino acid incorporation and click chemistry to screen residues along the anticalin backbone and determine the most mechanostable anchor points. We found that pulling on the anticalin from residue 60 or 87 resulted in significantly higher rupture forces and a decrease in $k_{\text {off }}$ by 2-3 orders of magnitude over a force range of 50-200 pN. Five of the six internal pulling points tested were significantly more stable than N- or Cterminal anchor points, rupturing at up to $250 \mathrm{pN}$ at loading rates of $0.1-10 \mathrm{nN} \mathrm{sec}^{-1}$. Anisotropic network modelling and molecular dynamics simulations explained the mechanisms underlying the geometric dependency of mechanostability. These results suggest that optimization of anchor residue position for therapeutic and diagnostic cargo can provide large improvements in binding strength, allowing affinity maturation without genetic mutations of binding interface residues.

\section{0-68}

Microfluidics-based force measurements with sub-nm resolution and sub-pN sensitivity

Yannic Kerkhoff, Stephan Block

Department of Chemistry and Biochemistry, Emmy-Noether Group "Bionanointerfaces," Freie Universität Berlin, Arnimallee 22, 14195 Berlin, Germany

Current biophysical research is mainly concerned with interactions that are in the range of a few piconewton $(\mathrm{pN})$. Subsequently little detail is known about distinct receptorligand-interactions or stabilizing effects in protein folding and unfolding under this force limit. This is due to the fact that their interaction potential is comparable to thermal energy and therefore they are often subject to stochastic fluctuations. To overcome this problem, it is necessary to read out as many interactions as possible in parallel in order to obtain the statistics needed for an analysis.

To achieve this, we combined microfluidics with total internal reflection fluorescence (TIRF) microscopy to generate and apply shear forces on fluorescent beads linked to a glass surface by different macromolecules (e.g., polymers, proteins). Sufficient single-particle tracking of thousands of beads per field of view enabled the development a new method of highthroughput hydrodynamic single molecule force spectroscopy (SMFS). The application with polyethylene glycol linkers demonstrated that this methodology allows interactions to be characterized at sub-pN force resolution with sub-nm spatial resolution. 
Oral Presentations

- Self-Assembled Session 2 - Advances and applications in cryo-electron microscopy (Instruct-ERIC) -

0-69

Membrane glycosyltransferases as promising anti-tuberculosis targets

José Rodrigues [1], Yong Z Tan [2,3], Federico Issoglio [1], Vanessa Almeida[1], Cristiano Conceição[1], Rita Ventura [1], Clinton S Potter [2,3], Bridget Carragher [2,3], Filippo Mancia [2], Margarida Archer [1]

[1] Instituto de Tecnologia Química e Biológica, Universidade Nova de Lisboa (ITQB NOVA), Oeiras, Portugal; [2] Columbia University, New York, USA; [3] Simons Electron Microscopy Center, New York Structural Biology Center, New York, USA

Arabinofuranosyltransferase (AftA-D) and Arabinosyltransferase (EmbA-C) belong to a family of membrane-bound glycosyltransferases that build the lipidated polysaccharides of the mycobacterial cell envelope. Afts represent novel potential anti-tuberculosis drugs, while EmBs are targets of first-line drug ethambutol. We present the structures of $M y$ cobacterium abscessus AftD and M. smegmatis EmbB determined by single particle cryo-electron microscopy (cryo-EM). Remarkably, AftD is non-covalently complexed with an acyl carrier protein (ACP).

Nano- and mega- bodies have been produced for $70 \mathrm{kDa}$ AftA (Steyaert lab, VIB). Studies are on-going to assess their affinity towards AftA, so they can be further used for structural studies by cryo-EM. Furthermore, functional assays are underway using in-house chemically synthetized substrate analogues and putative inhibitors. Homology modeling and virtual screens may provide promising candidates for the development of novel and more effective drugs to treat tuberculosis.

\section{0-71}

Development and implementation of cryoelectron microscopy in Slovenia

Marjetka Podobnik

National Institute of Chemistry, Slovenia

Integrative structural biology aims at modelling the structures of biological systems based on data produced by multiple experimental and theoretical methods, to understand cellular biology as well to enable drug and vaccine discovery, or other applications in biotechnology. For atomic resolution approaches, X-ray crystallography has been a golden standard for a long time, however the immense power of cryoelectron microscopy (cryo-EM) has been increasingly contributing to the field for the past decade or so, providing detailed structural information on many complex (biological) macromolecular systems. Recent dramatic scientific achievements have come to life based on usage and constant development of high-end infrastructure for structural biology, large in terms of its physical size as well in costs. The latter might represent a huge draw back to smaller, however, ambitious scientific communities. In my talk I will describe our path to the recent successful foundation of a cryo-EM facility at the National Institute of Chemistry, the first one in Slovenia as well as in the region. I will describe how we integrate it with other methodological approaches and show some specific examples. Our cryo-EM facility has been since its opening in November 2019 continuously active in addressing Slovenian and foreign academic or industrial research questions, and importantly, also providing education to new generations of cryo-EM scientists.

\section{0-70}

Preparation and cryo-FIB micromachining of Saccharomyces cerevisiae for cryo-electron tomography

Jana Moravcová, Radka Holbová, Matyáš Pinkas, Radka Dopitová, Jiří Nováček

CEITEC, Masaryk University, Kamenice 5, 62500 Brno, Czech Republic

Cryo-electron tomography (cryo-ET) is nowadays the only technique that is capable of providing near-atomic resolution structural data on the macromolecular complexes in situ. Owing to the strong interaction of an electron with the matter, high-resolution cryo-ET studies are limited to specimens with athickness of less than $200 \mathrm{~nm}$ which restricts the applicability of cryo-ET only to the peripheral regions of a cell. A complex workflow that comprises the preparation of thin cellular cross-sections by cryo-focused ion beam micromachining (cryo-FIBM) was introduced during the last decade to enable the acquisition of the cryo-ET data from the interior of larger cells. We present a protocol for the preparation of cellular lamellae from the samples vitrified by plunge freezing utilizing Saccharomyces cerevisiae as a prototypical example of a eukaryotic cell with wide utilization in Cellular and Molecular Biology research. We describe protocols for vitrification of $S$. cerevisiae into isolated patches of few cells or continuous monolayer of the cells on the TEM grid and provide a protocol for lamella preparation by cryo-FIB for these two samples.

\section{0-72}

Easy and simple way for protein structure determination using the new $100 \mathrm{kV}$ cryo-TEM microscope designed for biochemistry and biophysics laboratories

Zuzana Hlavenkova [1], Dimple Karia [2], Milos Malinsky [1], Fanis Grollios [2], Vojtech Dolezal [1], Ondrej Shanel [1], Abhay Kotecha [2], Marketa Cervinkova [1], Daniel Nemecek [1], Lingbo $\mathrm{Yu}[2]$

[1] Thermo Fisher Scientific, Materials and Structural Analysis, Czech Republic; [2] Thermo Fisher Scientific, Materials and Structural Analysis, Netherlands

Protein structure determination by cryo-EM has become a routine method for study of large variety of proteins and advanced research in many scientific areas. Nevertheless, accessibility to cryo-EM is mostly limited to established cryoEM centers. The new $100 \mathrm{kV}$ cryo-TEM microscope, Thermo Scientific $^{T M}$ Tundra, is specifically designed and developed for regular biochemistry labs. The Tundra is adapted for standard laboratory rooms and ease-of-use is significantly improved over traditional side-entry TEMs. A novel semiautomated system for loading AutoGrids into the microscope was introduced to minimize risks of ice contamination, sample damage and/or breaking TEM vacuum. The Tundra brings a new level of automation, in which users are guided through every step of microscope operation. Critical parameters are monitored to optimize data quality and collection efficiency. Benchmark cryo-EM reconstructions demonstrate that Tundra can provide high-resolution datasets for de-novo protein structure determination or identification of secondary structure elements and interaction interfaces. 
Oral Presentations

- Self-Assembled Session 2 - Advances and applications in cryo-electron microscopy (Instruct-ERIC) -

\section{0-73}

\section{Structural variability and flexibility in low- density lipoprotein}

Karin Kornmueller [1], Aline Cisse [2,3], Anna-Laurence SchachnerNedherer [1], Arnold Hanser [4], Ambroise Desfosses [2], Heinz Amenitsch [5], Gerd Leitinger [1], Gerd Hoerl [1], Wolfgang Ring [4], Irina Gutsche [2], Eaazhisai Kandiah [6], Daouda Traore [3], Judith Peters [2,3], Ruth Prassl [1]

[1] Medical University of Graz, GSRC, OLRC, Graz, Austria; [2] University Grenoble Alpes, CNRS, CEA, LiPhy, IBS, F38000, Grenoble, France; [3] Institut Laue-Langevin (ILL), Grenoble, France; [4] University of Graz, Institute of Mathematics, Graz, Austria; [5] Graz University of Technology, Institute of Inorganic Chemistry, Graz, Austria; [6] European Synchrotron Radiation Facility (ESRF), Grenoble, France

Low-density lipoprotein (LDL) is a key player in human cholesterol transport, and thus intimately linked to the development of cardiovascular diseases. We here present a dynamical and structural description of LDL and its protein moiety apolipoprotein B-100 by combining cryo-electron microscopy (EM) and small angle X-ray scattering (SAXS). A multi-reference classification and refinement of the cryo-EM data with CryoSparc separates the LDL dataset into five distinct subsets. From each subset a 3D map with a resolution of $\sim 12 \AA$ was obtained, corresponding to a possible conformation. A comparison of the different maps shows well conserved parts, as well as highly flexible regions. SAXS is used as a cross-validation tool for the 3D-EM-reconstruction of LDL, complemented by a mathematical modeling approach. A good correlation of experimental and simulated solution scattering profiles was found.

\section{0-74}

Structural mechanism of GTPase-powered ribosome-tRNA movement

Valentyn Petrychenko[1], Bee-Zen Peng[2], Ana C de A. P. Schwarzer [1], Frank Peske[2], Marina V Rodnina [2], Niels Fischer $[1]$

[1] Department of Structural Dynamics, Max Planck Institute for Biophysical Chemistry, Germany; [2] Department of Physical Biochemistry, Max Planck Institute for Biophysical Chemistry, Germany

GTPases are regulators of cell signaling acting as molecular switches. The translational GTPase EF-G stands out, as it uses GTP hydrolysis to generate force and promote the movement of the ribosome along the mRNA. The key unresolved question is how GTP hydrolysis drives molecular movement. Here, we visualize the GTPase-powered step of ongoing translocation by time-resolved cryo-EM. EF-G in the active GDP-Pi form induces ribosomal subunit rotation and twisting of the sarcin-ricin loop of the $23 \mathrm{~S}$ rRNA. Refolding of the GTPase switch regions upon Pi release initiates a cascade of rearrangements and a large-scale rotation of EF-G that exerts force on the ribosome and ultimately drives tRNA movement. The findings demonstrate how a GTPase orchestrates spontaneous thermal fluctuations of a large RNA-protein complex into force-generating molecular movement. 
Oral Presentations

- 10. Mechanobiology -

\section{0-75}

The cytoskeleton, by the numbers

Núria Gavara

University of Barcelona, Spain

The cell's cytoskeleton is a fascinating and dynamic structure, both from a biophysical and a biomedical perspective. On the one hand, the cytoskeleton confers mechanical stability to the cell, being composed of 3 polymeric networks that each excel at a particular mechanical role: supporting tension (actin), compression (microtubulues) or strain (intermediate filaments). On the other hand, the cytoskeleton is crucial for cell function (such as migration or division), regulates cell fate, and is involved in a large number of pathologies including cancer and inflammation. Under the fluorescence microscope, the cytoskeleton is a striking structure that perfectly captures the concept of 'form follows function'. Taking advantage of this, we have developed advanced image analysis pipelines (CSK morphometrcs) that allow us to quantify in a multiparametric approach the amount and intracellular organization of cytoskeletal networks. We use this highthroughput multiplex approach, for example, to better understand the mechanical interplay between the cytoskeleton and the nucleus, and how this can affect nuclear and chromatin states. In addition, when coupled to machine learning algorithms, we use CSK morphometrics for cellular age diagnostics or to monitor cell fate in differentiating stem cells. Finally, when used simultaneously with other biophysical tools, such as Atomic Force Microscopy, we can further characterize the role of cytoskeletal organization in the mechanical properties of adherent cells.

\section{0-77}

AFM-based differentiation between normal and cancer cells at single-cell and molecule levels Malgorzata Lekka

Institute of Nuclear Physics, Polish Academy of Sciences, Krakow, Poland

Over two decades of atomic force microscopy (AFM) employment in measurements of elastic and rheological properties of living cells unravel the importance of biomechanics in various aspects of cell functioning. Gathered evidence showed that cell mechanics is not only limited to the mechanical properties of cells. It also includes cell-generated forces and all aspects of mechanotransduction. Cancer progression is linked not only with genetic and molecular changes but also involves the alterations in cell ability to deform and adhere. AFM, the nanoscopic technique, delivers a quantification of very local (in nanoscale) changes in cellular/molecular interactions. In our research, a special emphasis is laid on studies comparing AFM-derived properties of reference and cancerous cells applied to bladder cancers using all functionalities from cellular deformability measurements to quantify the interaction forces of single-molecule and single-cell levels. Obtained results reveal not only distinct deformability but also variability in the single-molecule interactions. Our findings show that cell morphological, mechanical and adhesive properties are significant in search of non-labelled biomarkers of various cancers.

\section{0-76}

Viscoelastic properties of polarized cells

Andreas Janshoff

Institute of Physical Chemistry, University of Göttingen, Germany

The mechanical properties of cells influence their shape and cellular functions, including cell adhesion, migration, growth, and differentiation. Cellular elasticity and cortex structure are found to be inherently interwoven and largely responsible for the cells' response to external mechanical stimuli.

We propose a shell-based tension-model to describe the mechanical response of cells to external probing and examined the impact of cell-size, cortex integrity, cortex attachment to the plasma membrane, cell-substrate adhesion, cell-cellcontacts and motor activity on the viscoelastic properties of cells.

In order to unequivocally relate the viscoelastic properties found for living cells to the mechanics of the cellular cortex we devised a top-down strategy to measure the viscoelasticity of isolated cortices both from the apical and basal side. We found that the mechanical properties of isolated cortices resemble those of living cells and follow the same universal scaling law, however, with a stiffening effect that can be resolved by invoking motor activity externally. Lastly, we also address the impact of compromised cell-cell-contacts on cortical tension leading to two types of cells with distinct mechanical features.

\section{0-78}

The viscoelasticity of mitotic chromosomes Hannes Witt [1], Anna E C Meijering [1], Christian F Nielsen [2], Janni Harju [1], Guus H Haasnoot [1], Charlotte Bruinsma [1], Ian D Hickson [2], Chase P Broedersz [1], Erwin J G Peterman [1], Gijs J L Wuite[1]

[1] Vrije Universiteit Amsterdam, Netherlands; [2] Københavns Universitet, Denmark

To ensure that each daughter cell inherits a full genome during mitotic cell division, the DNA forms a highly condensed structure - the metaphase chromosome. . Although the metaphase chromosome is of fundamental relevance for eukaryotic life, its structure and dynamics are not yet fully resolved.

In this talk I will present a novel method to study metaphase chromosomes and their mechanical properties by using optical tweezers to hold and manipulate single isolated chromosomes. To characterize their viscoelasticity we not only performed force-distance measurements, but also used small oscillations to assess the linear mechanical response of the chromosome. We observed a unique stress-stiffening behaviour, that we explain with a hierarchical worm like chain model based on the chromosomes heterogeneity. When measuring the complex stiffness of chromosomes over a wide range of frequencies $(0.001-100 \mathrm{~Hz})$, we observed a remarkable elasticity and robustness. By combining genetic modifications and chemical treatment we unravel the contributions of histones and different structural maintenance of chromosomes (SMC) proteins to the elasticity of mitotic chromosomes, which additionally also might provide insights into their functions. 
Oral Presentations

- 10. Mechanobiology -

\section{0-79}

Combined SICM-TFM reveals a subcellular correlation between stiffness and traction forces in living cells

Johannes Rheinlaender, Hannes Wirbel, Tilman E Schäffer Institute of Applied Physics, Eberhard Karls University Tübingen, Germany

As cell mechanics play an important role in many physiological processes as well as numerous diseases, a comprehensive understanding of the living cell and its cytoskeleton as an active mechanical system is of major importance. However, the interaction between the passive material properties of the cell and the actively generated contractile forces remains ambiguous. Here, we present combined scanning ion conductance microscopy (SICM) and traction force microscopy (TFM) as a novel method for parallel probing the "passive" material properties in terms of the mechanical stiffness and the "active" contractile forces of living cells with subcellular resolution. We identify a spatial correlation between the local stiffness and the traction force density in living cells. This behavior can be interpreted as local stress-stiffening of the cytoskeleton, recently postulated as the "missing piece in cell mechanics". Interestingly, we found this correlation in healthy breast epithelial cells but not in breast cancer cells, indicating that healthy cells exhibit "normal" stress-stiffening behavior while breast cancer cells do not. This might be a hint for a fundamental difference in mechanotransduction between normal and cancerous cells on the single-cell level and shows that combined SICM-TFM is a powerful method to study the complex interplay between cell stiffness and contractility.

\section{$0-80$}

Dermal fibroblasts and human breast cancer cells differentially stiffen their local matrix Alicja Jagiello [1], Micah Lim [1], Elliot Botvinick [1,2] [1] Department of Biomedical Engineering, University of California, Irvine, USA; [2] Beckman Laser Institute and Medical Clinic, University of California, Irvine, USA

Bulk ECM stiffness measurements are often used in research on cell mechanobiology. However, past studies by our group have shown that peri-cellular stiffness can span few orders of magnitude and diverges from the bulk properties. Using optical tweezers active microrheology (AMR) we can describe stiffness landscape around individual cells. In this study, we show how different cell lines cultured in 1.0 and $1.5 \mathrm{mg} / \mathrm{ml}$ type 1 collagen (T1C) create disparate patterns of peri-cellular stiffness. Dermal fibroblasts (DFs) increase peri-cellular stiffness, when embedded in $1.0 \mathrm{mg} / \mathrm{ml} \mathrm{T1C} \mathrm{hy-}$ drogels, but do not alter stiffness in $1.5 \mathrm{mg} / \mathrm{ml} \mathrm{T} 1 \mathrm{C}$ hydrogels. In contrast, invasive human breast cancer MDA-MB231 cells (MDAs) do not significantly change the stiffness of T1C hydrogels, as compared to cell-free controls. Results indicate that while MDAs adapt to the bulk ECM stiffness, DFs regulate local stiffness to levels they intrinsically "favor". Further, cells were also subjected to treatments that were previously shown to regulate their migration, proliferation and contractility. Following each treatment, cells established dissimilar stiffness patterns. Stiffness magnitude and extent of anisotropy varied with the cell line, T1C concentration and treatment. In summary, we demonstrate that AMR can reveal otherwise masked mechanical properties of the local ECM, which are known to affect cell behavior. 
Oral Presentations

-11. Light as a tool in biophysics -

\section{$0-81$}

Introducing the optogenetic voltage clamp Amelie Bergs [1], Jana Liewald [1], Qiang Liu [2], Nadja Zeitzschel [1], Hilal Durmaz [1], Johannes Vierock [3], Cornelia Bargmann [2], Peter Hegemann [3], Simon Wiegert [4], Alexander Gottschalk [1]

[1] Goethe University, Frankfurt, Germany; [2] The Rockefeller University, New York, USA; [3] Humboldt University, Berlin, Germany; [4] University of Hamburg, Germany

Unlike voltage-clamp electrophysiology, optogenetics allows perturbation, but not true control of neural activity. We established an all-optical optogenetic voltage clamp (OVC): Live readout of the membrane potential via a genetically encoded voltage indicator is used to generate light-based feedback, sent to optogenetic protein actuators for de- or hyperpolarization, thus clamping the cell at a distinct potential. In two types of muscle cells and three neuron classes of the nematode Caenorhabditis elegans, different configurations allowed reliable clamping of their membrane potential. In the GABAergic neuron DVB, that produces rhythmic action potentials, the OVC could dynamically clamp these. We also calibrated fluorescence signals to electrically measured membrane voltages. Live computation of acquired data occurred at $90 \mathrm{~Hz}$, where the OVC clamped fluorescence between -5 to $5 \% \Delta \mathrm{F} / \mathrm{F}_{0}$. Using a closed-loop feedback system, the OVC synergistically combines the non-invasive character of imaging methods with the control capabilities of electrophysiological methods. Outperforming standard patch-clamp electrophysiology in terms of non-invasiveness, throughput and ease of application, the OVC paves the way for true all-optical control of individual neurons in freely behaving animals.

\section{0-83}

Controlling the fate and function of proteins with proximity photopharmacology

Dirk Trauner

New York University, USA

Photopharmacology endeavors to control biological function with synthetic photoswitches that can be attached covalently or non-covalently to their targets - or nearby. I will discuss potential applications of photopharmacology in biology and medicine, in particular with respect to controlling signal transduction and targeted protein degradation. I will make a case that "Proximity Photopharmacology" is a particularly effective strategy, which combines the power of genetic engineering with the spatial and temporal precision that only light provides.

\section{0-82}

Minimal genetically encoded tags for fluorescent protein labelling in living neurons

Ivana Nikić-Spiegel

Werner Reichardt Centre for Integrative Neuroscience, University of Tübingen, Germany

Super-resolution microscopy techniques allow us to probe biological processes in previously unattainable detail. However, to fully utilize their potential, improvements in imaging technologies need to be matched with advances in the field of protein labelling. Breaking the resolution limit has brought about a demand for small labelling tags that bring the fluorophore closer to the protein of interest. This challenge can be addressed by labelling unnatural amino acids (UAAs) with click chemistry. UAAs are site-specifically incorporated into target proteins by genetic code expansion. If the UAA carries a strained alkene or alkyne moiety it can be conjugated to a tetrazine-bearing fluorophore via a strain-promoted inverse-electron-demand Diels-Alder cycloaddition (SPIEDAC), an ultrafast variant of bioorthogonal click chemistry. The minimal size of the incorporated tag and the possibility to couple the fluorophores directly to the protein of interest with single-residue precision make SPIEDAC particularly suitable for the labelling of complex proteins. In this regard, I will present our latest work on the application of SPIEDAC labelling for the studies of neuronal proteins, such as cytoskeletal elements and ion channels.

\section{$0-84$}

Light-induced gating of ion channels and action potential stimulation with photosensitive organic semiconductors

Tony Schmidt [1], Theresa Rienmüller [2], Karin Kornmüller [1], Eric Daniel Glowacki [3], Rainer Schindl [1]

[1] Gottfried Schatz Research Center - Biophysics, Medical University of Graz, Austria; [2] Institut für Health Care Engineering mit Europaprüfstelle für Medizinprodukte, TU Graz, Austria; [3] Department of Science and Technology, Linköping University, Sweden

Optical control of neurons is a powerful technique to study and manipulate brain functions. Optoelectronic photocapacitive stimulation is a minimal invasive method to generate electrical signals in attached cells by depolarizing the membrane potential. In this approach, we use nontoxic organic pigments that form a planar semiconductor on top of ITO. Here, we show single cell electrophysiological recordings on mammalian cells transfected with voltage-gated potassium channels. Attached to our optoelectronics, the channels' activation threshold shifts upon red light illumination by $\sim 30$ $\mathrm{mV}$ of membrane depolarization. Electric circuit models determined that maximum light dependent membrane depolarization occurs within one millisecond. Importantly, analogous optoelectronic experiments with cortical neurons triggered repetitive action potentials with single light pulses. Our findings demonstrate that organic optoelectronic foils shape neuronal signaling with millisecond precision. 
Oral Presentations

-11. Light as a tool in biophysics -

0-85

Two-Photon photoactivation of RuBi-GABA for studying the GABAA receptor's modulation by the Antisecretory Factor in cerebellar granule cells in vitro

Virginia Bazzurro [1], Elena Gatta [1], Elena Angeli [1], Aroldo Cupello [1], Stefan Lange [2], Mauro Robello [1], Alberto Diaspro $[1,3]$

[1] DIFILAB, Department of Physics, University of Genoa, via Dodecaneso 33, 16146 Genoa, Italy; [2] Department of Infectious Diseases, Institute of Biomedicine, University of Gothenburg, Gothenburg, Sweden; [3] Nanoscopy, CHT Erzelli, Istituto Italiano di Tecnologia, Genoa, Italy

Caged compounds are molecules biologically or functionally inert until a short pulse of light activates them. The caged effector, released by the illumination, allows the activation and control of selective biological functions and alterations. The patch-clamp technique coupled with two-photon microscopy offers an instrument for evaluating different processes as the precise and localized receptor response of a well-defined neuronal region. Here, we describe the uncaging method to study the Antisecretory Factor (AF) effect in the $\mathrm{GABA}_{\mathrm{A}}$ receptors modulation using the caged neurotransmitter RuBi-GABA, photoreleased in femtoliter volumes, in a specific neuronal area at a defined concentration. This factor inhibits intestinal hypersecretion and various inflammation conditions in vivo, even if its mechanism of action is mainly unknown. We analyzed the response of AF-16, a 16 amino acids peptide of $\mathrm{AF}$, thanks to the uncaging of caged GABA on the soma, neurites, and the growth cone for identifying the action on different $\mathrm{GABA}_{\mathrm{A}}$ receptor populations.

\section{0-86}

Gating mechanosensitive channels by photoswitchable lipids

Jürgen Pfeffermann [1], Barbara Eicher [2], Christof Hannesschläger [1], Danila Boytsov [1], Timur R Galimzyanov [3], Toma N Glasnov [2], Georg Pabst [2], Sergey A Akimov [3], Peter Pohl [1]

[1] Johannes Kepler University Linz, Linz, Austria; [2] University of Graz, Graz, Austria; [3] Russian Academy of Sciences, Moscow, Russian Federation

Membrane protein activity depends on the embedding medium's mechanical and structural properties. Reversible and spatiotemporally defined alterations of the latter are key to quantitative studies of mechanically-sensitive membrane proteins. We investigated whether photolipids (PL) may be used for gating membrane proteins. To that effect, we demonstrated their fundamental applicability by altering the open probability of the ion-conducting molecular force probe gramicidin A in planar bilayers comprising diacylglycerol PLs with azobenzene switches. We found a reciprocal relationship between changes in bilayer thickness and channel activity: upon switching the PLs to their more conical cis-state, capacitance changed by several percent whilst ensemble conductance and single-channel lifetime did so by tens of percent due to improved hydrophobic matching. GUV-aspiration and SAXS confirmed the observed thickness changes whereby the latter revealed a precipitous reduction in bilayer bending rigidity with cis-PL. Theoretical treatment of the obtained experimental results in the framework of continuum mechanics will aid in applying the method to the study of mechanically-sensitive membrane proteins. 
Oral Presentations

- 12. Biomimetic nanopores -

\section{0-87}

Studying import and export through the nuclear pore complex using bottom up biomimetic approaches

Cees Dekker

Delft University of Technology, Netherlands

Nuclear Pore Complexes (NPCs) regulate all transport between the nucleus and the cytoplasm. Intrinsically disordered FG-Nups line the NPC lumen and form a selective barrier, where transport of most proteins is inhibited whereas specific transporter proteins can freely pass. The mechanism underlying this selective transport through the NPC is still incompletely understood. In our research, we reconstitute the NPC bottom-up by attaching FG-Nups to solid-state nanopores and studying NPC structure and nuclear transport at the single-molecule level using ion current measurements, zero mode waveguides, DNA origami scaffolds, and cryo-electron microscopy. These biomimetic NPCs are found to exhibit selective behavior. Using rationally designed artificial FG-Nups that mimic natural Nups, we show that simple design rules can recapitulate the selective import behavior of native FG-Nups and demonstrate that no specific spacer sequence nor a spatial segregation of different FG-motif types are needed to create selective NPCs. Next to nuclear import, we also study the transport-receptor-mediated export of RNA through this biomimetic approach, where again we demonstrate selectivity. I will finish with presenting our efforts to build an 'artificial nucleus' where we insert DNA origami rings with an inner diameter of $35 \mathrm{~nm}$ into the membrane of a giant unilamellar vesicle, to which FG-Nups can be attached, thus mimicking the NPCs.

\section{0-89}

Electrical Unfolding of Single Protein Molecules Prabhat Tripathi [1], Abdelkrim Benabbas [1], Behzad Mehrafrooz [2], Hirohito Yamazaki[1], Aleksei Aksimentiev [2], Paul Champion [1], Meni Wanunu [1]

[1] Northeastern University, USA; [2] UI Urbana-Champaign, USA

Protein conformational dynamics have been conventionally studied as a function of variables such as concentration of chemical denaturants, temperature, and pressure. In principle, the electric field can be another perturbation parameter to study protein folding dynamics. However, how electric fields can unfold a protein has remained unexplored. We have developed here a new method to electrically unfold individual protein molecules during their translocation through an ultra-thin solid-state nanopore. This method will enable scientists to explore the energetics of unfolding, the number of metastable/intermediate states, short-lived hidden conformational sub-states, transition-path times, and isoform-specific differences in protein structural variants. This method require only localized electric fields that are easily generated with a pair of electrodes, and has no additional requirements such as unfolding enzymes, chemical tethers, or denaturants.

\section{0-88}

Biomimetic membrane nanopores for nextgeneration sensing

Stefan Howorka

University College London, UK

Biological membrane nanopores have brought about a stepchange in portable, scalable DNA sequencing and helped tackle the Covid pandemic. Nanopores are also used as research tools for label-free and fast single-molecule analysis. Yet, the barrel-like biological membrane pores of a few $\mathrm{nm}$ width cover only a tiny space in the possible size and shape spectrum and therefore miss out on many applications. In my talk, I show how rational design with DNA can drastically expand the structural and functional range of membrane pores. The design strategy forms tuneable pore shapes and lumen widths of up to tens of nanometres with the option to attach functional units for bimolecular recognition. The designer pores may be applied for portable and scalable sensing in diagnostics, environmental screening, or homeland security. The pores also illustrate how DNA nanotechnology can deliver functional biomolecular structures for synthetic biology.

\section{0-90}

Artificial ion-selective channels formed by diblock copolymers

Nora Hagleitner-Ertuğrul [1], Saira Ahmad [2], Hazrat Hussain [2], Peter Pohl [1], Denis Knyazev [1]

[1] Kepler University Linz, Institute of Biophysics, Austria; [2] Quaid-i-Azam University, Department of Chemistry, Pakistan

Proteinaceous ion channels acquire selectivity by offering restricted pore sizes and specific recognition sites. However, their industrial applicability is limited by their low stability, high production costs, and - if used for filter production by low porosity. Here we took advantage of diblock copolymers to create artificial cation-selective channels. By optimizing the lengths of hydrophilic and hydrophobic blocks, we obtained polymers with reasonable water solubility and yet sufficient probability of insertion into planar lipid bilayers. The hydrophilic blocks consisted of either polyethylene glycol or acryloyl morpholine units. 4 or 8 methyl methacrylatesilsesquioxane (MA-POSS) units formed the hydrophobic block. Single-channel electrophysiology revealed pores with a strong preference for monovalent cations. Single-channel conductance decreased in the order: $\mathrm{Cs}^{+}>\mathrm{K}^{+}>\mathrm{Na}^{+}$. The change was not proportional to the ions' aqueous diffusion coefficient, suggesting that MA-POSS' oxygens may partially act as surrogates for the water of hydration. This conclusion is in line with the observed exclusion of anions. 
Oral Presentations

- 12. Biomimetic nanopores -

0-91

Fractional noise in nanopores

Marbach Sophie

New York University, USA

Fluctuations are ubiquitous in biological and artificial nanopores. Their dramatic consequences on transport are subtle and highly intricate. Yet, biological and artificial pores have to optimize signal to noise to achieve complex tasks.

Here we show that even in the simplest nanoporous setting the osmotic pressure exhibits non-trivial fractional noise in time (i.e. grows sublinearly with time). We will rationalize this effect and investigate its consequences for nanoporous transport in biological and artificial systems.

\section{0-92}

Solid state nanopores functionalized with polymer brushes

John Andersson, Justas Svirelis, Andreas Dahlin

Chalmers University of Technology, Sweden

Our research group works with new types of solid state nanopores functionalized with polymer brushes. A hydrophilic polymer brush can form an excellent barrier towards protein translocation through the nanopores, while small molecules still can diffuse through. By molecular recognition or responsive polymers, it is possible to create macromolecular gates that can switch between opened and closed states. The polymer-functionalized pores offer new insights into supramolecular chemistry and enable us to create new filters or to trap biomolecules in a non-invasive manner at physiological conditions.

This contribution will focus on recent insights into interpolymer complex formation by multivalent hydrogen bonds. First, we show that an ordinary protein repelling hydrophilic neutral brush (e.g. polyethylene glycol) can alter its properties entirely when exposed to a polymeric acid at $\mathrm{pH}$ around 5. Hydrogen bonding with the (neutral) polyacid makes the brush compacted, rubbery and protein attracting. Besides being important to be aware of, this effect may be useful for interfaces that capture and release proteins. Second, the changes in brush morphology upon hydrogen bond complexation can be used to gate nanopores between an open and a closed state in a reversible manner. Third, this effect can be controlled by electrochemistry control, making it possible to locally open and close nanoscale gates by pressing a button. This provides new possibilities to trap biomolecules. 
Oral Presentations

- Self-Assembled Session 3 - Ionic liquids biomolecules and cells -

\section{0-93}

Ionic liquids for therapeutic applications

Samir Mitragotri

Havard University, USA

Ionic liquids, the liquid salts comprising organic anions and cations, offer exciting opportunities for several therapeutic applications. Their tunable properties offer control over their design and function. Starting with biocompatible ions, we synthesized a library of ionic liquids and explored them for various drug delivery applications. Ionic liquids provided unique advantages including overcoming the biological transport barriers of skin, buccal mucosa and the intestinal epithelium. At the same time, they also stabilized proteins and nucleic acids and enabled the delivery of biologics across these barriers. Ionic liquids also provided unique biological functions including adjuvancy towards vaccines and antimicrobial function. I will present an overview of the design features of ionic liquids and novel biomedical applications enabled by these unique materials.

\section{0-95}

Ionic liquids as solvents and ligands in the purification of biopharmaceuticals

Mara G Freire

University of Aveiro, Portugal

Specific needs of new affordable biopharmaceuticals to treat age- and prosperity-related diseases will considerably increase, reinforcing the demand for scalable and cost-effective production technologies. Biopharmaceuticals have largely improved the treatment of many diseases, and in some cases are the only approved therapies available for specific human disorders. These biologic-based products include recombinant therapeutic proteins, antibodies and nucleic-acid-based products. Despite all the advantages of biopharmaceuticals, their extremely high manufacturing cost is still the most challenging feature limiting their widespread use.

To overcome this drawback, while taking advantage of their designer solvents ability, novel downstream processes based on ionic liquids (ILs) have been investigated for the purification of antibodies and nucleic-acid-based biopharmaceuticals. Results achieved with both the use of aqueous biphasic systems formed by ILs and supported IL materials will be presented and discussed. Some integrated preservationseparation processes using ILs will be also overviewed. The key accomplishments and future challenges to the field will be highlighted.

Acknowledgements: This work was developed within the scope of the project CICECO-Aveiro Institute of Materials, UIDB/50011/2020 \& UIDP/50011/2020, financed by national funds through the Portuguese Foundation for Science and Technology/MCTES.

\section{0-94}

Mechanisms of action of ionic liquids on cell membrane mechano-elasticity, cell migration and protein amyloidogenesis

Antonio Benedetto [1,2]

[1] Department of Sciences, University Roma Tre, Italy; [2] School of Physics, University College Dublin, Ireland

Ionic liquids (ILs) are a relatively new class of organic electrolytes, liquid around room-temperature. In the last 20 years, several studies have shown a moderate-to-high level of toxicity of these compounds, breaking the dream of their completely green character. Toxicity is synonym with affinity, however, and this has stimulated a series of biochemicalphysical investigations focused on the mechanisms of action (MoAs) of ILs, key steps for their applications in nanotechnology. After presenting an overview of the MoAs of ILs [1], I will focus on two cases under study in our Lab [2-4]. First, I will discuss the absorption of ILs into biomembranes and their effect on the membrane mechano-elasticity. I will then present the implication of these effects at cell level, showing the ability of ILs to alter cell migration in a controllable manner [2]. I will conclude by presenting a set of new data on the effect of ILs on protein amyloidogenesis, clarifying the molecular mechanisms behind their observed antithetic effects [3,4]. The major approaches in use in our Lab include neutron scattering, atomic force microscopy, computer simulations and a variety of biochemical tools.

[1] Kumari et al. Biophys Rev 12, 1187, 2020

[2] Kumari et al. J Phys Chem Lett 11, 7327, 2020

3] Pillai and Benedetto, Biophys Rev 10, 847, 2018

[4] Gobbo et al. Phys Chem Chem Phys 23, 6695, 2021

\section{0-96}

Synergistic/antagonistic cytotoxic effects in mixtures of ionic liquids with drugs

Ksenia S Egorova, Alexandra V Posvyatenko, Valentine P Ananikov

N.D. Zelinsky Institute of Organic Chemistry, Russian Academy of Sciences, Russian Federation

The phenomena of microstructuring observed in the media of ionic liquids (ILs) has been attracting much attention recently. The impact of dynamic nano- and microheterogeneities on physicochemical properties of ILs has been established [1]; however, possible associations between such structures and biological activity of the corresponding ILs have been understudied so far.

A possible relation between synergistic/antagonistic cytotoxic effects detected in aqueous IL mixtures and microstructuring of these media [2] suggested additional benefits of using ILs as part of drug formulations. In our further work, we assessed such cytotoxic effects in binary mixtures of imidazolium ILs with the known drugs doxorubicin and mitoxantrone.

Of the ten mixtures tested, four revealed significant synergistic/antagonistic cytotoxic effects. Scanning electron microscopy demonstrated distinct microstructuring in these mixtures, whereas NMR spectroscopy detected strong binding between the drug and the IL in some of the mixtures. These results suggested possible advantages of employing ILs in drug formulations [3].

[1] K.S. Egorova, E.G. Gordeev, V.P. Ananikov. Chem. Rev. 2017, 117(10): 7132-7189.

[2] K.S. Egorova, A.V. Posvyatenko, A.N. Fakhrutdinov, A.S. Kashin, V.P. Ananikov. J. Mol. Liq. 2020, 297: 111751.

[3] K.S. Egorova, A.V. Posvyatenko, A.N. Fakhrutdinov, A.S. Galushko, M.M. Seitkalieva, V.P. Ananikov. J. Mol. Liq. 2021, 323: 114870 . 
Oral Presentations

- Self-Assembled Session 3 - Ionic liquids biomolecules and cells -

0-97

Structural insights into the interaction of amphiphilic cations in ionic liquids with lipid bilayers using solid-state NMR spectroscopy

Sandeep Kumar [1], Navleen Kaur [1], Markus Fischer [2], Holger A Scheidt [2], Gagandeep K Gahlay [1], Daniel Huster [2], Venus S Mithu [1,3]

[1] Guru Nanak Dev Univ, Amritsar, India; [2] Institute for Medical Physics and Biophysics, Leipzig Univ, Germany; [3] MPI for Biophysical Chemistry, Göttingen, Germany

The interaction of amphiphilic cations with lipid membranes has always drawn the attention of the scientific community. Recently, this is due to a newfound interest in understanding the bioactivity of ionic liquids. The toxicity is directly linked to the interaction of amphiphilic cations with the cell membrane. We aim to build a structure-property model correlating the cytotoxicity and membrane interaction of amphiphilic cations in ionic liquids. Here, I will discuss the work where we have compared the impact of length/number of alkyl-chains and head-group structure on the cytotoxicity and membrane permeability of amphiphilic cation-based ionic liquids. The observed differences are explained based on ${ }^{1} \mathrm{H},{ }^{31} \mathrm{P}$, and ${ }^{2} \mathrm{H}$ solid-state NMR studies which helped to determine the location of amphiphilic cations in lipid membranes and their impact on the chain order, headgroup orientation, and packing density of the lipid bilayer. Ionic liquids containing double-chained cations, or carrying a relatively polar head-group like morpholinium are relatively less cytotoxic and membrane-permeabilizing than the cations bearing a single long alkyl chain, or carrying a large-sized aromatic head-group like benzimidazolium. Our results suggest that there exists a direct correlation between ionic liquid-induced structural changes in the bilayer and their ability to permeabilize/disrupt the membrane and be cytotoxic.

\section{0-98}

Impact of ionic liquids on the structure, refolding, and activity of proteins

Harekrushna Sahoo

National Institute of Technology Rourkela, India

Different types of co-solvents (Sugars, polyhydric alcohols, etc.) were reported in the past, which serve the purpose of protein stabilization to some extent. In the recent years, ILs have emerged as a new class of solvents which can strengthen the stability of proteins. Herein, several ionic liquids such as imidazolium, ammonium, and morpholinium based are employed to understand their impact on the structure, stability, and activity of two proteins, bovine serum albumin (BSA) and lysozyme (LYZ). Our findings revealed that hydrophobicity of cationic part of ILs has a significant impact on the stabilization and refolding of proteins as well as the concentration in a direct relation. Here the most hydrophobic IL have stabilizing effect on the thermally unfolded BSA and higher concentrations have shown a positive effect. The thermal stability of BSA shows a direct correlation with the type of anion counterpart of the ionic liquid (EmimESO $4>\mathrm{BmimCl}$ ) as well as cation (EmimCl $>\mathrm{BmimCl})$. Specifically, triethyl hexyl ammonium bromide shows better thermal refolding for BSA. Furthermore, the synergistic effect of ILs and polymers (carboxymethylcellulose and polyethyleneglycol) on the activity and structure of lysozyme reveals that all the IL formulations have a protecting effect. 
Oral Presentations

- 13. New and Notable -

\section{0-99}

Resolving cytokine receptor assembly and dynamics in the plasma membrane by single molecule imaging techniques

Jacob Piehler

Department of Biology, Osnabrück University, Germany

The spatiotemporal organization of class I/II cytokine receptors in the plasma membrane and the molecular mechanisms leading to the activation of their associated Janus family tyrosine kinases (JAKs) has remained controversially debated. We have established single molecule fluorescence imaging techniques to unravel the molecular and cellular determinants of cytokine receptor activation in living cells. Binding of site-specifically fluorescence-labeled cytokines revealed very low cell surface densities of cytokine receptors and stochastic distribution within the plasma membrane. Based on efficient and selective extracellular labeling with photostable fluorescent dyes, we have established dual color single molecule assays to quantify equilibrium and dynamics of receptor dimerization in live cells. In the absence of ligand, we observed randomly distributed receptor subunits that were efficiently dimerized by the ligand. Strikingly, constitutively activating, oncogenic receptor and JAK mutations were found to induce ligand-independent dimerization. In conjunction with atomistic molecular dynamics simulations, these analyses yielded a comprehensive mechanistic model of JAK activation by ligand-induced receptor dimerization promoting regulatory interactions between the JAK pseudokinase (PK) domains. Single molecule FRET confirmed intrinsic dimerization affinity mediated by JAK PK domains and indicated a critical role of the membrane environment.

\section{0-101}

Cryo-EM reveals molecular basis for conformational equilibrium of the spliceosome

Max E Wilkinson [1], Sebastian M Fica [1,2], Wojciech P Galej [1,3], Kiyoshi Nagai [1]

[1] MRC Laboratory of Molecular Biology, UK; [2] The University of Oxford, UK; [3] EMBL Grenoble, France

The spliceosome excises introns from pre-mRNAs in two sequential transesterifications - branching and exon ligation. The ATPase Prp16 governs an equilibrium between the branching $(\mathrm{C})$ and exon-ligation $\left(\mathrm{C}^{*}\right)$ conformations of the spliceosome. Here we present the cryo-EM reconstruction of a novel C-complex intermediate $\left(\mathrm{C}_{\mathrm{i}}\right)$ from Saccharomyces cerevisiae that elucidates the molecular basis for this equilibrium. Contrary to previous models, the exon-ligation factors Prp18 and Slu7 bind to $\mathrm{C}_{\mathrm{i}}$ before ATP hydrolysis by Prp16 can destabilise the branching conformation. Biochemical assays suggest these pre-bound factors prime C complex for conversion to $\mathrm{C}^{*}$ by $\operatorname{Prp} 16$ and act as a Brownian ratchet to stabilise the higher-energy $\mathrm{C}^{*}$ conformation. Without stable binding of Slu7 and Prp18, Prp16 action causes the $\mathrm{C}_{\mathrm{i}}$ spliceosome to reverse branching and produce pre-mRNA. A newly identified $\mathrm{K}^{+}$ion bound near the catalytic $\mathrm{Mg}^{2+}$ ions in the active site modulates reversal. Our results show how the energy of ATP hydrolysis by Prp16 promotes thermodynamic control of splicing by reducing the activation barrier to transition from the very stable branching conformation to the higher-energy exon-ligation conformation. Prp16 action allows dynamic binding of exon-ligation factors to the $\mathrm{C}$ or $\mathrm{C}^{*}$ conformation, thus establishing equilibrium of the spliceosome during catalysis.

\section{$0-100$}

Coupling molecular activation and its functional output through multiscale imaging

Dorit Hanein

Scintillon Institute, USA

Professor Hanein has vast experience in developing and applying cryo-EM technology for high-resolution structural studies of biological macromolecules and cells. Prof. Hanein is leading efforts in combining light microscopy with cellular tomography to permit the placement of dynamic multimolecular protein complexes into their functional context in whole mammalian cells. Through these efforts, she made seminal contributions to our knowledge of the structure, assembly and regulation of actin cytoskeleton and its associated macromolecular assemblies, to the development of techniques and protocols for correlative light and in-situ cellular tomography, and to the evolution of quantitative cryo-EM. Prof. Hanein's primary biological interest is the nanometerscale structure of the actin cytoskeleton, the girders and cables that control the shapes and movements of cells. These structures control tissue development and maintenance, play crucial roles in metastatic cancers, and are central to many processes involved in pathogen invasion. Here, Prof. Hanein will describe recent efforts in unrevealing cell nanoarchitecture through multiscale imaging, coupling molecular activation, biosensors and their functional output.

\section{0-102}

Structures of the archaerhodopsin-3 transporter reveal that disordering of internal water networks underpins receptor sensitization

Peter J Judge [1], Juan F Bada Juarez [1], Danny Axford [2], Javier Vinals [1], Tristan O Kwan [3], Isabel Moraes [3], Anthony Watts [1]

[1] Biochemistry Department, University of Oxford, South Parks Road, Oxford, OX1 3QU, UK, UK; [2] Diamond Light Source, Harwell Science and Innovation Campus, Didcot, OX11 0DE, UK, UK; [3] National Physical Laboratory, Teddington, TW11 0LW, UK, UK

Like other photoreceptor proteins, the archaerhodopsin-3 (AR3) protein has a desensitized, inactive state which is formed in the prolonged absence of light. This dark-adapted (DA) state must be converted to the light-adapted (LA) or resting state, before the protein can generate a proton motive force. By combining high-resolution crystal structures with data from native mass spectrometry, atomic force microscopy and QM/MM calculations, we demonstrate that remodelling of internal water networks facilitates the process of photoreceptor sensitisation. The data show how the displacement of charged and hydrophilic groups within the low dielectric environment of the membrane can induce changes in ligand conformation and explain how changes in the polarity of the chromophore environment affect the relative stability of the LA and DA states. These high-resolution structures $(1.1 \AA$ and $1.3 \AA$ resolution for the LA and DA states) will facilitate the design of more efficient AR3 mutants for applications in optogenetics. 
Oral Presentations

- 14. Membrane active peptides -

\section{0-103}

The mechanisms of action of antimicrobial peptides and their synergistic interactions in membranes Burkhard Bechinger

University of Strasbourg/CNRS, Chemistry, UMR7177 \& Institut Universitaire de France, France

Biophysical and structural studies of peptide-lipid interactions, peptide topology and dynamics have changed our view how antimicrobial peptides interact with membranes. Both the peptides and the lipids are highly dynamic, mutually adapt their conformation, membrane penetration and detailed morphology on a local and a global level. Thus the peptides and lipids can form a wide variety of supramolecular assemblies such as transmembrane helical bundles or lipid-driven mesophase structures of cationic amphipathic sequences intercalated at the membrane interface. Although the membranes are soft and can adapt, at increasing peptide density transient membrane openings, rupture and ultimately lysis occur (SMART model). Interestingly mixtures of peptides such as magainin 2 and PGLa which are stored and secreted naturally as a cocktail exhibit considerably enhanced antimicrobial activities when investigated together in antimicrobial essays but also in pore forming experiments applied to biophysical model systems. Our most recent investigations reveal that these peptides do not form stable complexes but act by specific lipid-mediated interactions and through the nanoscale properties of phospholipid bilayers. Aisenbrey, C. et al., Scie Rep 10(1):11652 (2020). Bechinger, B. et al. Front Med Technol, 2, 615494 (2020). Aisenbrey, C. et al, B.Adv Exp Med Biol., 1117, 33-64 (2019). Marquette, A. \& Bechinger, B., Biomolecules 8(2). pii: E18. (2018). Harmouche, N. \& Bechinger, B., Biophys J 115:1033-1044 (2018).

\section{0-105}

Molecular mechanisms of antigenic peptide generation

Efstratios Stratikos $[1,2]$

[1] National and Kapodistrian University of Athens, Greece; [2] National Centre for Scientific Research Demokritos, Greece

The human adaptive immune system recognizes small peptides bound onto Major Histocompatibility Class I molecules (MHCI) on the surface of all somatic cells and proceeds to kill the infected cells if these peptides signify infection. These peptides are derived from intracellular proteins of pathogens and are generated intracellularly by complex proteolytic cascades. A key enzyme for generating antigenic peptides is ER aminopeptidase 1 (ERAP1). ERAP1 trims N-terminally extended antigenic peptide precursors to generate mature antigenic peptides, but can also over-trim effectively destroying them. By this function, ERAP1 shapes the repertoire of peptides available for binding onto MHCI and can indirectly regulate adaptive immune responses, therefore is an emerging target for cancer immunotherapy and the treatment of autoimmune disease. ERAP1 belongs to the M1 family of zinc aminopeptidases but has some unique molecular properties that are consistent with its biological function. Crystallographic, biophysical and enzymatic analyses during the last few years have provided clear insight on the mechanism of function and substrate specificity of ERAP1. The importance of these findings on our understanding of cellular immunogenicity and on opportunities to regulate it pharmacologically are discussed.

\section{0-104}

Development of synthetic antimicrobial peptides killing antibiotic resistant bacteria and preventing septic complications

Dagmar Zweytick [1], Enrico S Semeraro [1], Guillermo Martinez de Tejada [2], Georg Pabst [1], Karl Lohner [1]

[1] University of Graz, Austria; [2] University of Navarra, Spain

Increase of antibiotic resistant pathogens results in inadequate treatments of bacterial infections. In addition, release of pathogenicity factors, in case of Gram-negative endotoxin, can induce an excessive and uncontrolled release of inflammatory cytokines leading towards harmful septic complications. Thus, an optimized antibiotic should neutralize endotoxin in addition to killing of bacteria. A promising strategy is based on modifications of endogenous proteins containing an endotoxin-binding domain, in this study a N-terminal fragment of human lactoferrin. In a series of optimization steps, peptides were selected and modified based on the peptide structure, biophysical experiments on the interaction of peptides with membrane mimics, and biological assays in order to improve both the antimicrobial activity and the ability to bind bacterial endotoxin. Recently, we were able to link results from membrane-mimetic systems to live bacteria gaining further detailed insight into the mode of action particularly on the loss of cell envelope integrity. Further, in vivo experiments showing that the synthetic peptides do not induce resistance can be explained by the observed fast killing kinetics and non-receptor mediated action. Finally, using a neutropenic mouse model demonstrates the potential of the peptides to combat septic complications.

\section{0-106}

Mode of action of virulence factors of intracellular pathogens studied with time-resolved and high-resolution atomic force microscopy

Christian Nehls, Thomas Gutsmann

Research Center Borstel, Leibniz Lung Center, Germany

An essential part of the innate immune defenses is the phagocytosis of invading pathogenic microbes by professional phagocytes and their subsequent digestion within the phagosome. It is often forgotten that not all pathogens surrender without a fight - in particular, some species succeed in surviving inside the phagosome. These intracellular pathogens have evolved various individual strategies to counteract the killing mechanisms of the host cell, many of which have been poorly understood.

Using state-of-the-art atomic force microscopy on model membrane systems, we investigated the mode of action of host defense peptides (HDPs) and various mycobacterial pathogenicity molecules. These include the mycobacterial lipid trehalose dimycolate (TDM) and the virulenceassociated Protein A (VapA) of Rhodococcus equi. For VapA, we show the formation of protein clusters on the membrane and the dynamics within these clusters. For TDM we show the organization into domains within bacterial and model membranes. The activity of different HDPs against these membrane systems will be compared.

Our data show how the spatially and temporally resolved visualization of processes can make a decisive contribution to an understanding of the underlying molecular interactions - especially in the context of intracellular survival strategies of pathogens. 
Oral Presentations

- 14. Membrane active peptides -

0-107

Candidalysin is a pore-forming peptide toxin that destabilises the cell membrane

Nessim Kichik, Olivia Hepworth, Sejeong Lee, Jonathan Richardson, Julian Naglik

King's College London, UK

Candida albicans is a fungal human pathogen found in biofilms and a main causative agent of invasive candidiasis. We have identified candidalysin as the first cytolytic peptide toxin in a human fungal pathogen. Candidalysin is an amphipathic peptide capable of damaging epithelial cell membranes. Understanding the molecular basis of membrane destabilization and cell damage by candidalysin is necessary for the development of drugs for the treatment of candida infections.

Using a combination of biochemical, biophysical, electrophysiological and structural approaches we have studied how candidalysin interacts with the cell membrane. Our results allow us to propose a molecular model for candidalysin's lytic activity. In this model, candidalysin's the interaction with the membrane is characterised by a conformational change observed when the peptide binds to the membrane. In the subsequent step, candidalysin forms oligomers of increasing sizes. Further analysis suggests that these oligomers form pore-like structures. We will present the NMR-based structure of candidalysin in its monomeric form and will describe our hypothesis on the atomic interactions that drive oligomerisation and pore formation. We conclude that candidalysin interacts with the cell membrane to form pore-like oligomeric structures of increasing sizes that are responsible of cell damage. Our research aims to shed light on the molecular mechanism of membrane destabilisation that the fungal pathogen $C$. albicans uses to successfully invade human tissues during infection.

\section{$0-108$}

Investigating the effect of polymyxin class antibiotics on the outer membrane of gramnegative bacteria

Selen Manioglu [1], Seyed M Modaresi [2], Noah Ritzmann [1], Parthasarathi Rath [2], Sebastian Hiller [2], Daniel J Müller [1] [1] D-BSSE, ETH Zürich, Switzerland; [2] Biozentrum, University of Basel, Switzerland

The increasing and diversifying drug resistance problem in microbial infection treatment imposes a threat on global health. Therefore, it is required to develop new antibiotics by studying the action mechanism of effective ones on their bacterial counterparts. Here, we utilize outer membrane vesicles (OMVs) produced from different Escherichia coli strains as a native platform for the outer membrane of gramnegative bacteria. Using OMVs, we employ high-resolution AFM imaging and structural biology assays to investigate the effect of polymyxins as the last-resort antibiotics on lipopolysaccharide (LPS) of the bacterial outer membrane. The treatment of OMVs with different polymyxin derivatives can induce supramolecular structures that disrupt vesicles. The dimensions of the supramolecular structures get smaller when OMVs produced from E. coli strains with altered LPS lengths are incubated with polymyxins. Further, introducing an amine-mediated positive charge on the lipid A moiety of LPS abolishes the formation of supramolecular structures. Finally, different polymyxins show variance in their effect on the membrane thickness and surface area of OMVs. Our experimental assay allows us to examine the structural changes of bacterial outer membranes in response to antibiotics and assay new antibiotic designs. 
Oral Presentations

- 15. Methodological advances in biomolecular simulations -

\section{0-109}

Elucidation of G-protein-coupled receptor activation via data-driven modeling

Oliver Fleetwood, Chen Yue, Perez-Conesa Sergio, Delemotte Lucie

Department of Applied Physics of Science for Life Laboratory, KTH Royal Institute of Technology, Sweden

Ligand binding stabilizes different $\mathrm{G}$ protein-coupled receptor states via a complex allosteric process that is not completely understood. Using enhanced sampling molecular dynamics simulations, we derive free energy landscapes describing activation of the $\beta 2$ adrenergic receptor bound to ligands with different efficacy profiles. These reveal that the ligands modulate the conformational ensemble of the receptor by tuning protein microswitches. The distribution of these molecular switches is furthermore predictive of cyclic adenosine monophosphate signaling responses. Dimensionality reduction and examining the output of classifiers further enables us to propose a model of regulation of the conformational ensemble by different ligands.

\section{0-111}

Using molecular dynamics simulations to gain insights into biological systems

Sereina Riniker

ETH Zürich, Switzerland

Molecular dynamics (MD) simulations have become an important tool to provide insight into molecular processes involving biomolecules such as proteins, DNA, carbohydrates and membranes. The advances in computer power in combination with enhanced sampling techniques have enabled the study of systems that cover increasing spatial and time scales. Here, we present our findings about the conformational behaviour of molecules ranging from cyclic peptides to collagens and glycoproteins using extensive MD simulations and kinetic modelling. The effect of posttranslational modifications on the structure can be studied by reverting these modifications in silico, for example for the cytotoxic natural product polytheonamide $\mathrm{B}$ that forms an unusual beta-helix. MD simulations allowed us to elucidate the underlying structural effects for the thermal stability of synthetic collagen peptides. We used kinetic models to rationalize the passive membrane permeability of cyclic peptides, which gained much interest as drug candidates for difficult targets such as protein-protein interactions but often suffer from low bioavailability. A similar approach can be used to study how the interactions between glycans and the protein surface of glycoproteins affect oligosaccharide processing.

\section{0-110}

Biophysical experiments and biomolecular simulations: A perfect match?

Kresten Lindorff-Larsen

University of Copenhagen, Denmark

Biological macromolecules are dynamic entities and I will discuss methods and applications for how simulations and experiments can be used synergistically to study protein and RNA dynamics. Functional protein motions are often described as an exchange between a ground state structure and one or more minor states. The structural and biophysical properties of these transiently and sparsely populated states are, however, difficult to study, and an atomic-level description of those states is challenging. Using proteins with extensive NMR data available as test systems, we have shown how enhanced sampling simulations can be used to capture accurately complex conformational changes in proteins, and I will briefly discuss such examples. Despite recent progress, one may still find that a simulation does not quantitatively match experiments. Then, experiments and simulations may be combined in a very direct fashion to provide a description of the molecular motions that combines the details of atomic simulations with the accuracy afforded by experiments. The resulting conformational ensembles may provide novel insight into biomolecular systems that are not obtainable by simulations of experiments alone. I will discuss how this may be achieved, and give examples of the application of such approaches using both NMR and small-angle scattering experiments to describe both proteins and RNA, and possible future approaches to include also timescales of motion and time-resolved processes.

\section{0-112}

Discovering molecular mechanisms from computer simulations with deep reinforcement learning Roberto Covino [1], Hendrik Jung [2], A Arjun [3], Peter G Bolhuis [3], Gerhard Hummer [2,4]

[1] Frankfurt Institute for Advanced Studies, Germany; [2] Max Planck Insitute of Biophysics, Germany; [3] University of Amsterdam, Netherlands; [4] Goethe University Frankfurt, Germany

We present an artificial intelligence (AI) agent that extracts molecular mechanisms from computer simulations. The agent simulates complex molecular self-organization phenomena and learns how to predict their outcome. We integrate path theory, transition path sampling (TPS), deep learning, and symbolic AI. Using reinforcement learning, we iteratively train a neural network on the outcome of path sampling simulations. In this way, we increase the rare-event sampling efficiency while gradually revealing the underlying mechanism. The AI learns the committor function of the rare event encoded in the trained neural network. By using symbolic regression, we distil quantitative models that reveal human-understandable mechanistic insight. With this algorithm, we study the spontaneous assembly of a model transmembrane dimer using Martini simulations. In less than 20 days, the agent accumulates a total of $5 \mathrm{~ms}$ simulation time distributed over 10000 trajectories, collecting 4000 unbiased transition paths. Simplified data-driven models reveal distinct assembly mechanisms. In conclusion, our AI enables the sampling of rare events by autonomously driving many parallel simulations with minimal human intervention and aids their mechanistic interpretation. 
Oral Presentations

- 15. Methodological advances in biomolecular simulations -

\section{0-113}

Ensembles in Solution as a New Paradigm in Antibody Structure Prediction and Design

Monica L Fernández-Quintero, Patrick K Quoika, Klaus R Liedl University of Innsbruck, Austria

The ability of antibodies to specifically recognize a broad variety of antigens is determined by the antigen-binding fragment $(\mathrm{Fab})$, in particular the variable fragment $(\mathrm{Fv})$.

The diversity of an antibody is concentrated on six hypervariable loops, forming the antigen-binding site, the paratope. Five of the six complementarity determining region (CDR) loops have been assigned to canonical structures, assuming that the loops can only adopt a limited number of main-chain conformations.

For decades CDR loops have been thought to be limited to static canonical conformations determining their binding properties. Here, we escape this paradigm of static canonical structures determining binding properties and specificity of antibodies.

In contrast to this static view of the binding interface show that antibodies exist as ensembles of paratope states. These paratope states are defined by a characteristic combination of CDR loop conformations, which interconvert into each other in the micro-to-millisecond timescale by correlated loop and interdomain rearrangements. We demonstrate that crystal packing effects can distort the paratope state and result in misleading X-ray structures. We achieve a complete description of conformations, thermodynamics and kinetics of the binding paratope in solution. These findings have broad implications for antibody design and the development of biotherapeutics as they provide a new understanding of CDR loop states in antibody-antigen recognition and their dynamics.

\section{0-114}

Free energy along transition pathways from structure refinement simulations

Emmi Pohjolainen, Andrea Vaiana, Maxim Igaev, Helmut Grubmüller

Max Planck Institute for Biophysical Chemistry, Germany

Modern cryo-EM provides high-resolution structures of multiple conformational states of biomolecules, yielding insights into their conformational heterogeneity and functional mechanisms. Molecular dynamics simulations can complement such information by providing an atomistic description of the dynamics and the transitions between states in terms of the free energy landscape. Unfortunately, most complex systems cannot be fully sampled with current computational resources. In these cases, it is possible to compute the part of the free energy landscape corresponding to the transition of interest by performing biased sampling along a suitable reaction coordinate. To define a general and system-independent reaction coordinate, we turned to the real-space correlation coefficients that describe the similarity between two structures. These correlations are conventionally used in structure fitting simulations in driving the initial state to the target state, with the recorded trajectory representing a transition path between the two states. We have compared the transition paths derived from structure fitting simulations to the path derived from unbiased sampling. To this end, we investigated the transition between open and closed states of a ligand free Adenylate-kinase protein in solution. Our approach yields similar results to the 1 millisecond unbiased sampling. We are currently testing our approach for larger systems for which unbiased sampling is impossible. 
Oral Presentations

- 16. Membrane signal transduction -

\section{0-115}

Biomechanical of adhesion - from basic principles to mechanisms of immune cell activity

Ana-Suncana Smith

Institute of Theoretical Physics, Friedrich Alexander University of Erlangen-Nürnberg, Germany

Inhibitory receptors of $\mathrm{T}$ cells and natural killer (NK) cells sustain self-tolerance of the immune system and prevent collateral damage during a physiological immune response. Upon binding to their ligands expressed by target cells, these receptors initiate cascades that target signaling pathways of activating and costimulatory receptors, suppressing the activation of T cells and NK cells. It has long been hypothesized that the biological function of activating and inhibitory receptors, besides on chemical recognition, depends on their biomechanical characteristics such as length, flexibility and spatial arrangement. Especially the physical proximity of activating and inhibitory receptors has been considered essential for the integration of their signals.

In this talk, I will present quantitative measurements of cell activation as a function of the mentioned biomechanical parameters as well as mathematical models accounting for the biomechanics of the adherent cells that predict and rationalize the organisation of activating and inhibitory bonds in the adhesion zone of an NK cells. I will furthermore establish the role of bond the lengths, and flexibilities on the stability of their bonds. Finally, I will introduce discuss the effect of visco-elastic response to the formation of the bond and the present a model for the lipid-protein interactions that leads to the stabilisation of a particular lipid environment around a macro-molecular complex.

\section{0-117}

An ERNEST assessment of arrestin-GPCR interactions

Martha E Sommer [1], Martin Heck [1], Agnieszka K Sztyler [1], Ciara C Lally [1], Björn Bollmann [1], Jana Selent [2]

[1] Institute of Medical Physics and Biophysics (CC2), Charité Medical University Berlin, Germany; [2] Research Programme on Biomedical Informatics, Department of Experimental and Health Sciences, Pompeu Fabra University, Barcelona, Spain

The COST Action CA18133, the European Research Network on Signal Transduction (ERNEST), is an international multidisciplinary network of investigators focused on developing a holistic multidimensional understanding of GPCR signal transduction. This effort currently involves collecting members' diverse data into a 'blueprint' of commonly studied signalling pathways, which will enable future computational modelling of these complex pathways in space and time. Within the framework of the Action's approach, I will present my own research on the GPCR-regulatory protein arrestin. Recent years have witnessed many new highresolution structures of arrestin subtypes in various states of activation. While these structures illustrate how flexible and variable arrestin is in its interactions with different GPCRs, they do not reveal the mechanism of arrestin activation and GPCR coupling. Filling this critical gap in knowledge is ultimately necessary to understand how different functional inputs (ligand-bound receptor conformation, receptor phosphorylation pattern, membrane composition, etc.) determine the eventual cellular activities of arrestin ( $\mathrm{G}$ protein signalling attenuation, receptor internalization, scaffolding, etc.). Here, I will present my group's latest (and surprising!) findings on how arrestin recognizes and binds active, phosphorylated GPCRs.

\section{0-116}

The critical importance of molecular size in signal transduction at the interface between immune cells and target cells

Philip A van der Merwe

University of Oxford, UK

Leukocytes detect abnormal cells using immunoreceptors or non-catalytic tyrosine-phosphorylated receptors (NTRs). One NTR, the T cell receptor (TCR) plays a major role in this process by probing the surfaces of cells for the presence of 'foreign' peptides (p) presented on MHC molecules (pMHC). We have shown that the TCR uses a novel signal transduction process that depends critically on the small size $(\sim 14 \mathrm{~nm})$ of the TCR/pMHC complex, which we term the kinetic-segregation (KS) mechanism. In the KS mechanism, TCR binding to pMHC passively segregates the engaged TCR from inhibitory receptor tyrosine phosphatases with large $(>25 \mathrm{~nm})$ extracellular domains, such as CD45 and CD148, for a period long-enough for recruitment and activation of signalling proteins to the engaged TCR. More recently, we and others have provided evidence that other NTRs also use the KS mechanism. Activating and inhibitory NTRs integrate their signals when they engage ligands, a process critical for discriminating between normal and abnormal cells. We have shown that intimate-co-localization at the cell-cell interface is critical for signal integration, and that NTR/ligand complexes need to span similar intermembrane distances to colocalize. We have recently developed a generic ligand system to facilitate investigation of signal transduction by, and signal integration between, NTRs, which may be useful to other researchers.

\section{0-118}

On the loss of inhibitory function due to FcgRIIB-I232T transmembrane polymorphism Matthias Pöhnl[1], Falk Nimmerjahn [2], Rainer A Böckmann [1,3]

[1] Computational Biology, University Erlangen, Germany; [2] Genetics, University Erlangen, Germany; [3] Erlangen National High Performance Computing Center, Germany

Fc receptors play a key role in balancing the antibody mediated immune response by binding to the Fc region of $\operatorname{IgG}$ and up- or downregulating immune activity. FcgRIIB is the only receptor expressing an immunoreceptor tyrosine-based inhibitory motif and thereby the only Fc receptor with antiinflammatory properties. A single nucleotide polymorphism changing an isoleucine to a threonine in the transmembrane (TM) part of the receptor leads to a loss of function and is associated with systemic lupus erythematosus (SLE). The mechanisms underlying disease association are still under debate. Possible explanations include a reduced lateral mobility and bending of the ectodomain towards the membrane, both connected to stronger inclination of the TM region.

Here, we performed atomistic molecular dynamics simulations of the wild type FcgRIIB and the I232T mutant embedded in a plasma membrane mimic. We simulated models of the TM domain alone as well as including the ectodomain on a microsecond time scale. No significant changes in the tilt of the TM region nor of the ectodomain orientation were observed. In addition, the ectodomain orientation renders IgG-binding to monomeric FcgRIIB (wt/mut) unlikely. Together, our results hint to a receptor signaling mechanism involving additional factors affecting receptor orientation. 
Oral Presentations

- 16. Membrane signal transduction -

\section{0-119}

Quantifying cytokine receptor dimerization dynamics in the plasma membrane by smFRET

Hauke Winkelmann [1], Maximillian Hafer [1], Stephan Wilmes [1,2], Christian P Richter [1], Rainer Kurre [1], Jacob Piehler [1]

[1] Department of Biology and Center of Cellular Nanoanalytics, University of Osnabrück, Osnabrück, Germany; [2] Division of Cell Signalling and Immunology, School of Life Sciences, University of Dundee, Dundee, UK

Class I/II cytokine receptors (CR) signal via the JAK/STAT pathway, which is initiated by protein ligand binding. The spatiotemporal organization and dynamics of CR assembly in the plasma membrane (PM) is currently controversially debated. By dual-color single molecule (co-)tracking in living cells, we have identified ligand-induced dimerization as the key switch of signal activation. However, we found weak intrinsic receptor dimerization affinity leading to ligand-independent dimerization at artificially elevated CR expression levels. Strikingly, ligand-independent dimerization was substantially enhanced by oncogenic mutations in $\mathrm{CR}$ or kinase. To pinpoint interaction dynamics of $\mathrm{CR}$ dimers in the PM, we have established single molecule FRET (smFRET) imaging in living cells. For the thrombopoietin receptor, smFRET enabled unambiguous detection of transient, ligand-independent dimerization and increased lifetime for constitutively activating mutations. We increased the labeling precision by using engineered nanobodies. Combined with alternating laser excitation (ALEX) we achieved unambiguous detection of $\mathrm{CR}$ association and dissociation events in the PM with high spatiotemporal resolution.

\section{$0-120$}

Impact of lipid polyunsaturation on dopamine D2 receptor activation, signaling and internalization Rim Baccouch [1], Véronique de Smedt-Peyrusse [2], MarieLise Jobin [4], Thierry Durroux [3], David Perrais [4], Pierre Trifilieff [2], Isabel D Alves [1]

[1] CBMN UMR 5248 CNRS, U. of Bordeaux, France; [2] NutriNeuro, INRA UMR 1286, U. Bordeaux, France; [3] INSERM U1191, IGF, U. Montpellier 1 et 2, France; [4] IINS, UMR 5297 CNRS, U. of Bordeaux, France

The dopamine D2 receptor (D2R) has been implicated in the etiology of several psychiatric disorders and is a main target of most antipsychotics. Interestingly, a "whole-body" decrease in long-chain polyunsaturated fatty acids (PUFA) levels - n-3 PUFAs such as docosahexaenoic acid (DHA) in particular - has been consistently described in these psychiatric disorders. However, the mechanisms by which alteration in PUFA levels may contribute to pathogeneses and could alter the functionality and pharmacology of the D2R are unknown. Our project aims at unraveling the impact of membrane PUFAs on D2R pharmacological properties and conformation through biochemical, biophysical and modeling studies in both PUFA enriched cells and membrane model systems of controlled lipid composition. To this aim, we have investigated the impact of membrane PUFAs in the first stages of receptor activation, on the recruitment and activity of D2R signaling effectors and receptor internalization. Overall the data indicate that membrane PUFA composition impact ligand binding and receptor conformational changes. PUFA cell enrichment enhances $\beta$-arrestin recruitment and impacts receptor internalization. The results could have a significant impact in the development of adjuvant therapeutic strategies for psychiatric disorders implicating the D2R. 
Oral Presentations

- 17. Cytoskeleton / Motor proteins -

\section{0-121}

\section{Torque generation by microtubule-crosslinking} motors

Stefan Diez

TU Dresden, B CUBE - Center for Molecular Bioengineering, Germany

Within the mitotic spindle, kinesin motors cross-link and slide overlapping microtubules. Some of these motors exhibit off-axis power strokes, but their impact on motility and force generation in microtubule overlaps has not been extensively investigated. We utilize three-dimensional in vitro motility assays to explore the kinesin- 5 and kinesin- 14 driven sliding of cross- linked microtubules. We observe that free microtubules, sliding on suspended microtubules, not only rotate around their own axis but also move around the suspended microtubules with helical trajectories. Importantly, the associated torque is large enough to cause microtubule twisting and coiling. Further, our technique allows us to measure the in situ spatial extension of the motors between cross-linked microtubules. We argue that the capability of microtubulecrosslinking kinesins to cause helical motion of overlapping microtubules around each other allows for flexible filament organization, road- block circumvention and torque generation in the mitotic spindle.

\section{0-123}

Reconstituting cytoskeletal systems in artificial cells

Marileen Dogterom

Department of Bionanoscience, Faculty of Applied Sciences, TU Delft, Netherlands

In my group we are interested in understanding how dynamic and force-generating properties of the cytoskeleton contribute to the spatial organization of cells. I will highlight recent advances (and challenges) in our efforts to reconstitute minimal, functional cytoskeletal systems in artificial confinement. An example is the reconstitution of basic mitotic spindles in microfluidic droplets. These efforts fit in a long-term ambition to build, in collaboration with others, a minimal synthetic cell from scratch.

\section{0-122}

Mechanics of interpenetrating biopolymer networks

David A Weitz

Wyss Institute at Harvard University, Boston, USA

The three major structural proteins in the cell, F-actin, microtubules and intermediate filaments, each form networks of semi-flexible filaments in the cell. Here we provide evidence that these networks are better considered as interpenetrating networks, and this has important consequences on their properties and their mechanics. We combine studies of the structure and properties of these interpenetrating networks in cells with those formed from reconstitutes proteins and show how the interpenetrating character has a strong impact on their behavior.

\section{0-124}

Germanium nanospheres for ultraresolution picotensiometry of kinesin motors

Swathi Sudhakar, Mohammad Abdosamadi, Tobias Jachowski, Michael Bugiel, Anita Jannasch, Erik Schäffer Eberhard Karls University at Tuebingen, Germany

Force spectroscopy on single molecular machines generating piconewton forces is often performed using optical tweezers. Since optical forces scale with the trapped particle volume, piconewton force measurements require micron-sized probes practically limiting the spatiotemporal resolution. Here, we have overcome this limit by developing high-refractive index germanium nanospheres as ultraresolution trapping probes. Using these probes, we have dissected the molecular motion of the cytoskeletal motor kinesin-1 that transports vesicles along microtubule filaments. With a superior spatiotemporal resolution, we have resolved a controversy unifying its stepping and detachment behavior. We found that single motors took 4-nm-center-of-mass steps with alternating force dependence of their dwell times. At maximum force, motors did not detach but they slid on the microtubule with 8-nm steps on a microsecond time scale. Kinesins remained in this intermediate slip state before either truly detaching or reengaging in directed motion. Surprisingly, reengagement and, thus, rescue of directed motion occurred in about 80 percent of events. Such rescue events suggest that macroscopically observed run lengths of individual motors are concatenations and rescues need to be accounted for to understand longrange transport. Apart from ultraresolution optical trapping, germanium nanospheres are promising candidates for applications ranging from nanophotonics to energy storage. 
Oral Presentations

- 17. Cytoskeleton / Motor proteins -

\section{0-125}

Vimentin intermediate filaments stabilize dynamic microtubules by direct interactions

Charlotta Lorenz [1], Laura Schaedel [1], Anna V Schepers [1,3], Stefan Klumpp [2,3], Sarah Köster [1,3]

[1] Institute for X-Ray Physics, University of Göttingen, Friedrich-Hund-Platz 1, 37077 Göttingen, Germany; [2] Institute for the Dynamics of Complex Systems, University of Göttingen, Friedrich-Hund-Platz 1, 37077 Göttingen, Germany; [3] Max Planck School "Matter to Life", Germany

Many cellular functions such as cell shape, mechanics and intracellular transport rely on the organization and interaction of actin filaments, microtubules (MTs) and intermediate filaments (IFs), which are the main constituents of the eukaryotic cytoskeleton. Here, we study in particular the interaction between vimentin IFs and MTs in a minimal in vitro system and show that MTs are stabilized against depolymerization by the presence of vimentin IFs. To explore the nature of this interaction and in particular probe for electrostatic and hydrophobic contributions, we directly measure attractive forces occurring between individual MTs and vimentin IFs using optical tweezers in different buffer conditions. Theoretical modeling enables us to determine the corresponding energy landscape. Feeding back the physical parameters describing the interactions into a Monte Carlo simulation that mimicks dynamic MTs confirms that the additional interaction with IFs stabilizes them. We suggest that within cells, the interactions we observe might be a mechanism for cells to fine-tune cytoskeletal crosstalk and MT stability.

www.biorxiv.org/content/10.1101/2020.05.20.106179v2

\section{$0-126$}

Bending-torsional elasticity and energetics of the plus-end microtubule tip

Maxim Igaev, Helmut Grubmüller

Max Planck Institute for Biophysical Chemistry, Göttingen, Germany

Microtubules (MTs), mesoscopic cellular filaments, grow primarily by the addition of GTP-bound tubulin dimers at their dynamic flaring plus-end tips. They operate as chemical-tomechanical energy transducers with stochastic transitions to an astounding shortening motion upon hydrolyzing GTP to GDP. Mechanical stability of the MT tip - a key determinant of this behavior - as a function of nucleotide state, internal lattice strain, and stabilizing lateral interactions has remained an important issue. Here, we used atomistic simulations to study the spontaneous relaxation of complete GTPMT and GDP-MT tip models from unfavorable straight to relaxed splayed conformations and to comprehensively characterize the elasticity of MT tips. Our simulations revealed the dominance of viscoelastic dynamics of MT protofilaments driven mainly by the stored bending-torsional strain during the relaxation process, despite the nucleotide-dependent stability of lateral contacts. Combined together, our calculations, unexpectedly, implied at least a threefold higher mechanical work done by protofilaments curling outwards from relaxing GDP-MT tips compared to those of GTP-MTs. Not only does this support a 'gain of excess stiffness' mechanism for MT dynamic instability, the higher elastic power generation also explains the development of large pulling forces by disassembling GDP-MTs under external load. 
Oral Presentations

- 18. Membrane architecture and asymmetry -

0-127

The rise and fall of lipid asymmetry in liposomes Heiko Heerklotz [1,2]

[1] Institute of Pharmaceutical Sciences, University of Freiburg, Germany; [2] Leslie Dan Faculty of Pharmacy, University of Toronto, Canada; [3] Signaling Research Centers BIOSS and CIBBS, Freiburg, Germany

Finally, the transversal asymmetry of biomembranes is attracting the attention it deserves and considerable progress is being made. A prerequisite for this progress has been the increasing availability of protocols to prepare asymmetric model membranes. PS-inside liposomes were prepared using PS decarboxylase. PG-outside proteoliposomes comprising a functional, bacterial sodium-proton antiporter were produced using cyclodextrin complexes.

Once prepared, the lipid asymmety is decaying by molecular flip-flop. In suitable lipid mixtures, this may take many days so that there is plenty of time to work with such liposomes. However, membrane-active biomolecules, drugs or surfactants may challenge the kinetic stability of the asymmetry. As demonstrated for several detergents, such molecules can speed up lipid flip-flop without inducing aqueous contents leakage. That means, lipid scrambling must be considered another potential mode of action of membrane-active compounds. Furthermore, detergents may be applied to create symmetric controls in studies of asymmetry effects.

Lateral pressure asymmetry can be created by asymmetric membrane insertion of amphiphilic solutes. The resulting asymmetry stress can drive processes such as lipid flip-flop, membrane budding or transient leakage.

\section{0-129}

Nanoscale biophysical properties of plasma membrane

Erdinc Sezgin

Karolinska Institutet, Solna, Sweden

Plasma membrane is a hub for signaling events. Recent evidence shows that collective biophysical properties of plasma membrane are crucial for the function of membrane components. These properties are strictly linked to nanoscale architecture of plasma membrane. By developing and applying new methodologies with high spatiotemporal resolution, we reveal how plasma membrane structure and biophysical properties modulate cell signaling. Moreover, we investigate how cellular states and diseases such as obesity, diabetes, cancer, aging, CVDs and infection change these biophysical properties.

\section{0-128}

Towards computational microbiology of bacterial cell envelopes

Syma Khalid, Conrado Pedebos, Iain Smith

School of Chemistry, University of Southampton, UK

Gram-negative bacteria are protected by a complex, tripartite cell envelope. This consists of two membranes separated by the aqueous periplasmic space. All three regions are crowded with proteins and the periplasm also contains a range of small molecules known collectively as osmolytes. The details of molecular interactions within each region, but also across all three regions that lead to the correct functioning of the cell envelope as a whole are largely still elusive.

We are using atomistic level and more coarse-grained models and molecular dynamics simulations to model the molecular interactions within the two membrane and the periplasmic space of $E$. coli. Our results show that the crowded environments give rise to many expected but also unexpected behaviours, some of which may have mechanistic impact on e.g. movement of antibiotics through the periplasm. I will discuss the models, results and also some challenges we face in system preparation and analysis as the complexity of the simulated systems increases.

\section{0-130}

Probing the membrane environment of plasma membrane proteins

Veronika Brumovska, Gerhard J Schütz, Gergö Fülöp, Eva Sevcsik

Institute of Applied Physics, TU Wien, Austria

It is well established that lipids and proteins are not just independent components of the plasma membrane of eukaryotic cells but that their arrangement, dynamics and function are interdependent. Besides specific lipid-protein interaction, transmembrane proteins are thought to bind a shell of annular lipids, which are more or less tightly associated with the proteins. Furthermore, highly ordered nanoscopic membrane domains have been proposed to act to compartmentalize proteins and their interactions, but have thus far not been directly observed.

We use a combination of single molecule tracking and protein micropatterning to examine these interactions directly in the plasma membrane of living cells. In our experimental approach, different proteins of interest are immobilized within defined patterns in the plasma membrane, where they act as steric obstacles to the diffusion of lipid tracers and thus locally decrease their mobility. In the presence of lipid-protein interactions of any type, lipid mobility will be even further decreased within protein patterns. We use this experimental platform to examine the membrane environment of different types of membrane proteins - single- and multi-spanning transmembrane proteins, a multi-subunit protein and GPIanchored proteins. 
Oral Presentations

- 18. Membrane architecture and asymmetry -

\section{0-131}

Characterization of a lipid nanoparticle for mRNA delivery using molecular dynamics Marius Trollmann [1,2], Rainer A Böckmann [1,2] [1] Computational Biology, Department Biology, FriedrichAlexander University Erlangen-Nürnberg, Erlangen, Germany; [2] Erlangen National High Performance Computing Center (NHR@FAU), Germany

mRNA-based vaccines have recently gained attention for their promising therapeutic potential in the prevention of a severe SARS-CoV-2 infection. An important part in their mode of action are lipid nanoparticles which act as a carrier system to deliver the bioactive mRNA into the target cells. The nanoparticles protect the nucleotides against a premature degradation leading to an increased expression of the encoded protein. In addition, the nanoparticles increase the transfection rate of the vaccine through an enhanced interaction with the membrane of the target cells. Unfortunately, less is known about the molecular organization of the nanoparticles.

The present work characterizes the structural and dynamical properties of the lipid composition used in the BioNTech \& Pfizer vaccine at atomistic detail. Our simulations provide an in-depth understanding of such drug delivery systems. Analysis of microsecond self-assembly simulations suggest that the BioNTech membranes are extremely flexible and fluid connected to a reduced lateral diffusion coefficient. Results for the thermodynamical properties as well as the interaction between the lipids and mRNA will be presented.

\section{0-132}

Aggregation and immobilisation of membrane proteins interplay with local lipid order in the plasma membrane of $\mathbf{T}$ cells

Iztok Urbančič [1,2], Lisa Schiffelers[2], Edward Jenkins [2], Weijian Gong [2], Ana Mafalda Santos [2], Falk Schneider [2], Erdinc Sezgin [2,3], Christian Eggeling [2,4]

[1] Jožef Stefan Institute, Slovenia; [2] University of Oxford, UK; [3] Karolinska Institute, Sweden; [4] Friedrich Schiller University Jena, Germany

To disentangle the elusive lipid-protein interactions in $\mathrm{T}$ cell activation, we investigate how externally imposed variations in mobility of key membrane proteins (T-cell receptor (TCR), kinase Lck, and phosphatase CD45) affect the local lipid order and protein colocalisation. To this end, we use spectral imaging with polarity-sensitive membrane probes in passive model membranes and live Jurkat $\mathrm{T}$ cells. Upon aggregation and partial immobilisation, proteins (including TCR and CD45) change their preference towards more ordered lipid environments, which can in turn passively recruit Lck. Our data suggest that the cellular membrane is poised to modulate the frequency of protein encounters upon alterations of their mobility, e.g. in ligand binding, which offers new mechanistic insight into the involvement of lipidmediated interactions in membrane-hosted signalling events. 
Oral Presentations

- 19. Cell and tissue biophysics -

\section{0-133}

\section{Mechanics of blastocyst morphogenesis}

Jean-Léon Maître

Institut Curie - CNRS UMR3215/INSERM U934 - 26 rue d'Ulm, 75005 Paris, France

During pre-implantation development, the mammalian embryo forms the blastocyst. The architecture of the blastocyst is essential to the specification of the first mammalian lineages and to the implantation of the embryo. Consisting of an epithelium enveloping a fluid-filled lumen and the inner cell mass, the blastocyst is sculpted by a succession of morphogenetic events. These deformations result from the changes in the forces and mechanical properties of the tissue composing the embryo. Combining microscopy, image analysis, biophysical tools and genetics, we study the mechanical and cellular changes leading to the formation of the blastocyst. In particular, we uncovered how pulsatile contractility compacts the mouse embryo, how asymmetric division of contractile domains couples cell positioning and specification, and how hydraulic fracturing and active coarsening position the lumen of the mouse blastocyst.

\section{0-135}

Molecular and biophysical mechanisms of protrusion plasticity in epithelial cells during phagocytic tissue clearance

Hanna-Maria Häkkinen [1], Esteban Hoijman [1], Stefan Wieser [2], Verena Ruprecht [1]

[1] CRG, Spain; [2] ICFO, Spain

Cellular errors lead to spontaneous cell death in the earliest stages of development. We identified that the embryonic surface epithelium mediates efficient phagocytic clearance of apoptotic cells by forming two types of actin-based protrusions during target interactions: 1) phagocytic cups responsible for apoptotic target uptake and 2) "epithelial arms" that are able to exert mechanical pushing forces on targets. We here addressed how epithelial cells form two types of actinbased protrusions by studying the molecular and biophysical factors regulating phagocyte-target interaction dynamics. We showed that epithelial protrusion formation and phagocytic uptake is mediated by PS recognition. Inhibition of Rac1, a downstream effector of PS receptor activation regulating actin dynamics, prevents both cup and arm formation. Pharmacological interference with actin polymerization regulators revealed that Arp2/3 is essential for both cup and arm formation while Formins only for phagocytosis. Our data suggest that a lack of Formin prevents the normal function of the phagocytic cup, while a lack of Arp $2 / 3$ inhibits its entire formation. We further identify a key role of cell adhesion in regulating the epithelial protrusion formation. These findings reveal distinct functions of actin regulators and cellular adhesion in controlling epithelial protrusions plasticity and efficient phagocytic tissue clearance.

\section{0-134}

\section{Comparative approach to tissue mechanics}

Pavel Tomancak

Max Planck Institute of Molecular Cell Biology and Genetics, Dresden, Germany

The early embryo of the red flour beetle, Tribolium castaneum, initially consists of a single-layered blastoderm covering the yolk uniformly that differentiates into an embryonic rudiment as well as extraembryonic amnion and serosa. The germband anlage forms inside the egg during gastrulation when the embryonic rudiment condenses and folds along the ventral midline; this process is accompanied by large-scale flow and expansion of the extraembryonic serosa which ultimately covers the entire surface of the egg, thus engulfing the growing embryo. The mechanical properties of these tissues and the forces governing these processes in Tribolium, as well as in other species, are poorly understood. Here, we present our findings on the dynamics of myosin in the early blastoderm of Tribolium using multiview lightsheet live imaging of transiently labeled wild type embryos. We quantitatively measure the global distribution of myosin throughout the flow phase and present a physical description that couples the contractile forces generated by myosin to the mechanical properties of the blastoderm. In particular, we describe the overall tissue as a thin, actively contractile, viscous bulk medium that exhibits friction with the vitelline membrane. This description accurately captures the large-scale deformation the tissue undergoes during the initial stages of gastrulation. Our findings lay a foundation for the physical description of gastrulation in Tribolium and will allow, in combination with the well-studied Drosophila paradigm, for the first time the comparative analysis of blastoderm tissue morphogenesis.

\section{0-136}

Competition drivers in tissue-like confined cellular active matter

Yoav G Pollack [1], Philip Bittihn [1], Ramin Golestanian $[1,2]$

[1] Dept. of Living Matter Physics, Max Planck Institute for Dynamics and Self-Organization (MPIDS), Germany; [2] Rudolf Peierls Centre for Theoretical Physics, University of Oxford, UK

In growth-driven dense cellular active matter and tissues in particular, non-adversarial statistical competition is governed by an intricate interplay between growth, proliferation, death and mechanical interactions. While for expanding systems the mechanically unhindered division and death rates play a critical role, in confinement fitness is determined by emergent homeostatic properties; properties that can already be observed in homogeneous cell assemblies before competition takes place. Here we propose, based on a continuum description, that the main driver of competition is the homeostatic density of growing cells. By including the finite-time mechanical persistence of dead cells, we decouple the density of growing cells from the homeostatic pressure that was previously suggested as the competition driver, which allows comparison of their respective fitness predictions. Numerical simulations of an agent-based model show strong evidence supporting the theory. 
Oral Presentations

- 19. Cell and tissue biophysics -

0-137

Durotaxis of passive nanoparticles on elastic membranes

Ivan Palaia [1], Alexandru Paraschiv [1], Vincent Debets [2], Cornelis Storm [2], Andela Saric [1]

[1] University College London (UCL), UK; [2] Eindhoven University of Technology, Netherlands

Transporting macromolecules and nanoscopic particles to a target cellular site is crucial in many physiological processes. This directed motion must be ensured also on membranes and is generally controlled via active processes. By combining simulations and theory, we show that even fully passive nanoparticles exhibit directional motion in non-uniform mechanical environments.

We study the motion of a passive nanoparticle adhering to an elastic membrane with a bending rigidity gradient. We observe a non-trivial adhesion affinity of the particle to the membrane. We look at the combination of adhesion, bending and entropy: their interplay results in transport of the particle, that, depending on the values of the involved rigidities, can be both up or down the rigidity gradient (corresponding, respectively, to positive and negative durotaxis). This behaviour is retained when the membrane is crowded with many nanoparticles. We show that rigidity gradients can therefore accumulate passive macromolecules in areas of preferred mechanical properties and possibly sort them according to size and adhesion properties.

This novel physical mechanism might contribute to transport across membranes with heterogeneous elasticity, that are widely observed in living beings and may fulfill key biological functions.

bioRxiv: https://doi.org/10.1101/2021.04.01.438065 
Oral Presentations

- 20. Lipid-Protein interactions -

\section{0-138}

Lipid-bilayer nanodiscs for membrane biophysics Bartholomäus Danielczak [1], Eugenio Pérez Patallo [1], Julia Lenz [1], Sophie Weyrauch [1], Michael T Agbadaola [1,2], Jonathan O Babalola [2], Annette Meister [3], Carolyn Vargas [4], Sandro Keller [4]

[1] Technische Universität Kaiserslautern, Germany; [2] University of Ibadan, Nigeria; [3] Institute of Biochemistry \& Biotechnology, ZIK HALOmem, Univ. Halle, Germany; [4] Institute of Molecular Biosciences, NAWI Graz, University of Graz, Austria

Nanodiscs that harbour individual membrane proteins or membrane-protein complexes in a lipid-bilayer environment hold great promise for biophysical investigations under wellcontrolled yet native-like conditions. We have developed a series of amphiphilic polymers that directly extract membrane proteins and lipids from cellular membranes to form such nanodiscs that are compatible with a broad range of ensemble and single-molecule biophysical techniques. These bioinspired polymers possess increased hydrophobicity and reduced charge density as compared with established systems but nevertheless retain excellent solubility in aqueous solutions. The new polymers solubilise lipid vesicles of various compositions as well as native membranes much more efficiently, thereby furnishing smaller, more narrowly distributed nanodiscs that preserve a bilayer architecture and exhibit rapid lipid exchange. Nanodisc-embedded membrane proteins thus become amenable to chemical conjugation for immobilisation or fluorescence labelling and, thus, to singlemolecule spectroscopy and ligand binding assays based on microfluidic diffusional sizing.

\section{0-140}

\section{Interactions of Nep1-like proteins with lipid membranes}

Gregor Anderluh

Department of Molecular Biology and Nanobiotechnology, National Institute of Chemistry, Slovenia

NLPs (Necrosis and ethylene-inducing peptide 1 (Nep1)-like proteins) are secreted virulence factors that are produced with some of the most pressing plant pathogens such as Phytophthora and have important role in pathogenesis. NLPs are cytolysins that damage lipid membrane of plant cells. We used various biophysical approaches to provide molecular insights into the mechanism of cytolytic activity of toxic NLPs towards plant membranes. NLPs initially bind to plant sphingolipids, glycosyl inositol phospho ceramides (GIPCs) and susbsequently oligomerize into small oligomers. Membrane damage is thus a multistep process that includes plantspecific lipid recognition, shallow membrane binding, protein oligomerization and transient pore formation. Pores formed by NLPs are permeable for small molecules such as fluorescent dyes or dextran of $4 \mathrm{kDa}$, but not for larger molecules. NLPs pores may aid in pathogenesis by providing pathogens with ions and small moleculear weight nutrients.

\section{0-139}

Interfacial protein-lipid interactions from atomistic molecular simulations

Nathalie Reuter

Department of Chemistry \& Computational Biology Unit, University of Bergen, Norway

Membrane-binding interfaces of peripheral membrane proteins are restricted to a small part of their exposed surface, so the ability to engage in strong selective interactions with membrane lipids at various depths in the interface is an advantage. Driven by their hydrophobicity, aromatic amino acids preferentially partition into membrane interfaces, often below the phosphates. Yet enthalpically favorable interactions with the lipid headgroups, above the phosphate plane, are likely to further stabilize high interfacial positions.

We will focus on three types of phospholipases: a bacterial phosphatidylinositol-specific phospholipase C, three SicTox phospholipases D from recluse spider venoms, and a phospholipase A2 from cobra snake venom. Using molecular dynamics simulations, we showed that tyrosine amino acids of BtPI-PLC engage in cation- $\pi$ interactions with phosphatidylcholine lipids. Then, using free-energy perturbation we evaluated that tyrosine and tryptophane amino acids involved in cation $-\pi$ interactions with choline headgroups contributed ca. 2.5 to $3.3 \mathrm{kcal} / \mathrm{mol}$ to the protein-membrane affinity. Finally, we found that some SicTox PLDs have a conserved aromatic cage that binds a PC lipid in MD simulations. Both enzymes have a high activity against cholinecontaining substrates.

Our observations, all confirmed by experiments, strongly indicate that interfacial cation $-\pi$ interactions serve as a mechanism for specific recognition of choline-containing lipids.

\section{0-141}

Calmodulin interactions with model lipid membranes: the interplay of $\mathrm{Ca}^{2+}$ and lipid composition Federica Scollo [1], Carmelo Tempra[2], Hüseyin Evci[1], Hector Martinez-Seara [2], Pavel Jungwirth [2], Piotr Jurkiewicz [1], Martin Hof [1]

[1] J. Heyrovsky Institute of Physical Chemistry, Prague, Czech Republic; [2] Institute of Organic Chemistry and Biochemistry, Prague, Czech Republic

Interactions of ionic proteins and lipids are thought to be crucial for the structure and function of membrane receptors and ion-channels. Calmodulin $(\mathrm{CaM})$ is a multifunctional calcium-modulated protein which binds to a wide variety of target proteins, influencing their function. Although the interplay between calmodulin and calcium has been already pointed out, these processes occur in proximity of the cell membrane. Thus, it is reasonable to hypothesize that calcium spikes might favour the calmodulin-membrane interaction and the subsequent one with signaling membrane proteins. However, the mechanism has not yet been completely understood. In this work we investigate how calmodulin behaves in presence of the lipid bilayer and how the physico-chemical properties of the bilayer are affected, by using a combination of molecular dynamic simulations and Fluorescence-Correlation Spectroscopy, Forster Resonance Energy Transfer, Confocal Microscopy, General Polarization techniques. To the best of our knowledge, our results show for the first time that calmodulin interacts with model membranes depending on lipid composition and calcium concentration. 
Oral Presentations

- 20. Lipid-Protein interactions -

\section{0-142}

Obtaining affinities of protein-lipid interactions from equilibrium molecular dynamics simulations

T. Bertie Ansell, Robin A Corey, Mark S P Sansom

Department of Biochemistry, University of Oxford, UK

Equilibrium molecular dynamics (MD) simulations are routinely used to identify specific lipid binding sites on membrane proteins, providing a detailed picture of the membrane environment directly surrounding the protein. Additionally, biased MD simulations can be applied to obtain thermodynamic estimates of lipid binding free energies, supplementing sparse experimental data on differences in lipid binding affinities. Here, we develop a new method for determining lipid-protein binding affinities from equilibrium coarsegrained MD simulations using binding saturation curves. We apply this method to obtain affinities for cholesterol binding to multiple sites on a range of membrane proteins and compare our results with free energies obtained from densitybased equilibrium methods and with potential of mean force calculations, getting good agreement with respect to the ranking of affinities for different sites. Thus, our method draws conceptual links between current experimental protocols and in silico methods for determining lipid binding affinities, important for determining the relative consequence of individual sites seen in e.g. cryo-EM derived membrane protein structures surrounded by a plethora of ancillary lipid densities.

\section{$0-143$}

Cholesterol promotes both head group visibility and clustering of $\mathrm{PI}(4,5) \mathrm{P} 2$ driving unconventional secretion of Fibroblast Growth Factor 2 Fabio Lolicato [1,2], Roberto Saleppico [1], Ilpo Vattulainen [2], Walter Nickel [1]

[1] Heidelberg University Biochemistry Center, Germany; [2] University of Helsinki, Finland

Fibroblast Growth Factor 2 (FGF2) is a cellular survival factor involved in tumor-induced angiogenesis. It is one of the most prominent examples of extracellular proteins that lack signal peptides and are secreted by ER/Golgiindependent secretory pathways. Biochemical reconstitution experiments and imaging in living cells have shown that FGF2 is secreted by direct translocation across the plasma membrane. This process is initiated by $\mathrm{PI}(4,5) \mathrm{P} 2$ dependent FGF2 recruitment at the inner plasma membrane leaflet. This in turn results in the formation of membranespanning FGF2 oligomers within toroidal membrane pores. Here, using both biochemical reconstitution experiments and live-cell imaging, we demonstrate that $\mathrm{PI}(4,5) \mathrm{P} 2$-dependent FGF2 recruitment at the inner plasma membrane leaflet is positively modulated by cholesterol in both . Based on extensive molecular dynamics simulations and free energy calculations, we propose cholesterol to increase the negative charge density of the membrane surface and to induce clustering of $\mathrm{PI}(4,5) \mathrm{P} 2$ molecules stabilizing FGF2 binding through increased avidity. Our findings have general implications for phosphoinositide-dependent protein targeting membranes and explain the highly selective targeting of FGF2 towards the plasma membrane. 
Oral Presentations

-21. DNA architecture and gene regulation -

\section{0-144}

Recent insights into DNA loop extrusion revealed by single-molecule imaging: From $\mathrm{Z}$ - to SC-loop

Eugene Kim

Max-Planck Institute of Biophysics, Germany

It is becoming clear that loop extrusion mediated by structure maintenance of chromosome (SMC) proteins is the universal mechanism that organizes genome for all kingdoms of life. Recent single molecule experiments solidified this hypothesis via direct imaging of DNA loop extrusion by condensin and cohesin in vitro. In this talk, I will focus on two of our recent in vitro studies which reveal novel properties of loop extruding condensin by employing this visualization method. In the first part, we study mutual interactions of condensin. We discover that upon mutual contact condensin complexes form a novel loop structure, called Z-loop. Imaging of its formation process reveals the ability of condensin to bypass one another and to reel-in 'inter-strand' DNA, a DNA strand where the protein is not bound on. In the second part, we present how additional torsional strain on DNA, a consequent of genomic processes like transcription and replication, affects condensin-mediated loop extrusion. We find that condensin binding and DNA looping is stimulated by overwinding of DNA (positive supercoils). We further discover that condensin preferentially loads near the tips of intertwined DNA (plectonemes) and absorbs all nearby plectonemes into a supercoiled (SC) loop that is highly stable. Our findings indicate how condensin may achieve chromosomal compaction using a variety of looping structures from Z-loop to SC-loop.

\section{0-146}

Live cell imaging reveals that RecA finds homologous DNA by reduced dimensionality search

Jakub Wiktor [1], Arvid H Gynnå[1], Prune Leroy [1], Jimmy Larsson [1], Giovanna Coceano [2], Ilaria Testa [2], Johan Elf $[1]$

11] Department of Cell and Molecular Biology, Science for Life Laboratory, Uppsala University, Sweden; [2] Department of Applied Physics, Science for Life Laboratory, KTH Royal Institute of Technology, 100 44, Stockholm, Sweden

Homologous recombination (HR) is essential for the accurate repair of double-stranded DNA breaks (DSBs); it begins when the RecBCD complex resects the ends of the DSB into 3' single-stranded DNA (ssDNA) on which a RecA filament assembles. HR depends on the RecA-ssDNA filament to locate the homologous repair template on the sister chromosome. The mechanism by which the homology is located among vast amounts of heterologous DNA is not yet understood. I will describe how we directly visualize the repair of DSBs in single cells, using high-throughput microfluidics and fluorescence microscopy. We find that in E. coli, DSB repair between segregated sister loci is completed in $15 \pm 5$ (mean $\pm \mathrm{SD}$ ) minutes with minimal fitness loss. We further show that the search takes less than $9 \pm 3$ minutes and is mediated by a thin, highly dynamic RecA filament that stretches throughout the cell. We propose a model in which the architecture of the RecA filament effectively reduces search dimensionality to two dimensions.

\section{0-145}

Cytidine triphosphate promotes efficient ParBdependent DNA condensation by facilitating one-dimensional spreading from parS

Francisco Balaguer Pérez [1], Clara Aicart Ramos [1], Gemma Lm Fisher [3], Sara de Bragança [1], César L Pastrana [4], Mark S Dillingham [2], Fernando Moreno Herrero [1]

[1] Dept of Macromolecular Structures, Centro Nacional de Biotecnología, Spain; [2] DNA:Protein Interactions Unit, University of Bristol, UK; [3] Cell cycle group, MRC London Institute of Medical Sciences, UK; [4] Physics of Complex Biosystems, Technical University of Munich, Germany

Faithful segregation of bacterial chromosomes relies on the ParABS partitioning system and the SMC complex. In this work, we used single molecule techniques to investigate the role of cytidine triphosphate (CTP) binding and hydrolysis in the critical interaction between centromere-like parS DNA sequences and the ParB CTPase. Using a combined dual optical tweezers confocal microscope, we observe the specific interaction of $\mathrm{ParB}$ with parS directly. Binding around parS is enhanced 4 -fold by the presence of CTP or the nonhydrolysable analogue CTPS. However, ParB proteins are also detected at a lower density in distal non-specific regions of DNA. This requires the presence of a parS loading site and is prevented by roadblocks on DNA, consistent with one dimensional diffusion by a sliding clamp. Magnetic tweezers experiments show that the spreading activity, which has an absolute requirement for CTP binding but not hydrolysis, results in the condensation of parS-containing DNA molecules at low nanomolar protein concentrations. We propose a model in which ParB-CTP- $\mathrm{Mg}^{2+}$ complexes move along DNA following loading at parS sites and protein:protein interactions result in the localised condensation of DNA within ParB networks.

\section{0-147}

Development of a single-molecule approach to observe ubiquitination dynamics in defined chromatin states

Alexandra Teslenko, Beat Fierz

École Polytechnique Fédérale de Lausanne (EPFL), Switzerland

Dynamic regulation of transcription is orchestrated by a large cohort of enzymes, among which chromatin modifiers or 'writers' install histone post-translational modifications (PTMs) controlling the recruitment of chromatin 'readers'. A specific subset of chromatin modifiers includes Polycomb group $(\mathrm{PcG})$ proteins, which drive the inheritance of a repressed chromatin state during development and cell differentiation by preventing abnormal oncogenic transformations.

PcG members Polycomb Repressive Complex (PRC) 1 in its canonical (cPRC1) or variant (vPRC1) form function as H2A-specific E3 ligases and drive gene repression.

We hypothesize that chromatin modification, i.e. ubiquitination, by vPRC1 is controlled by its recruitment dynamics on underlying chromatin and furthermore its subunit composition. Here, we present a single-molecule approach, which allows to directly measure vPRC1 binding dynamics at defined chromatin states.

In the following, this will allow us to identify protein-protein interactions (PPIs) that are key for enzymatic activity and reveal chromatin states that are specifically targeted. This will allow us to gain a mechanistic view of 'reading' and 'writing' by vPRC1 in real time and to elucidate its contribution to gene regulation. 
Oral Presentations

-21. DNA architecture and gene regulation-

0-148

Allostery through DNA drives phenotype
switching

Gabriel Rosenblum [1,4], Nadav Elad [2,4], Haim Rozenberg [1,4], Felix Wiggers [1,4], Jakub Jungwirth [3,4], Hagen Hofmann $[1,4]$

[1] Department of Structural Biology, Israel; [2] Department of Chemical Research Support, Israel; [3] Department of Chemical and Biological Physics, Israel; [4] Weizmann Institute of Science, Herzl St. 234, 76100 Rehovot, Israel

Allostery is a pervasive principle to regulate protein function. Here, we show that DNA also transmits allosteric signals over long distances to boost the binding cooperativity of transcription factors. Phenotype switching in Bacillus subtilis requires an all-or-none promoter binding of multiple ComK proteins. Using single-molecule FRET, we find that ComKbinding at one promoter site increases affinity at a distant site. Cryo-EM structures of the complex between ComK and its promoter demonstrate that this coupling is due to mechanical forces that alter DNA curvature. Modifications of the spacer between sites tune cooperativity and show how to control allostery, which paves new ways to design the dynamic properties of genetic circuits.

\section{0-149}

Molecular details of mRNA capping enzyme recruitment and activation - A computational study

Marcus G Bage [1,2], Rajaei Almohammed [2], Victoria H Cowling [2], Andrei V Pisliakov [1,3]

[1] Computational Biology, School of Life Sciences, University of Dundee, DD1 5EH, UK; [2] Centre for Gene Regulation and Expression, School of Life Sciences, University of Dundee, DD1 5EH, UK; [3] Physics, School of Science and Engineering, University of Dundee, DD1 5EH, UK

Recruitment of the mRNA capping enzyme (CE/RNGTT) to the site of transcription is essential for the formation of the 5' mRNA cap, which in turn ensures efficient transcription, splicing, polyadenylation, nuclear export and translation of mRNA in eukaryotic cells. The CE is recruited and activated by the Serine- 5 phosphorylated carboxyl-terminal domain (CTD) of RNA polymerase II. Through the use of molecular dynamics simulations and enhanced sampling techniques, we provide a systematic and detailed characterization of the human CE-CTD interface. Our computational analyses identify novel CTD interaction sites on the human CE GTase surface and quantify their relative contributions to CTD binding. We also identify, for the first time, allosteric connections between the CE GTase active site and the CTD binding sites, allowing us to propose a mechanism for allosteric activation. Through binding and activity assays we validate the novel CTD binding sites and show that the CDS2 site is essential for CE GTase activity stimulation. 
Oral Presentations

-22. Synthetic cell -

\section{0-150}

The possible role of solid interfaces in protocell formation at the origin of life

Irep Gözen

University of Oslo, Norway

My soft matter research group investigates the autonomous transformation of lipid agglomerates into membrane compartments through a sequence of topological changes on solid interfaces. This process is initiated by contact and wetting of solid surfaces by the lipids, and proceeds via a network of interconnected lipid nanotubes to produce nearly uniform bilayer compartments. Under minimal assumptions it is conceivable that such process could have occurred on the early Earth, where the autonomous formation of simple membrane compartments is presumed to have enabled encapsulation of nucleotides and prebiotic chemistry precursors. According to the currently accepted "bulk hypothesis", such compartments could have spontaneously formed under moderate environmental conditions from lipids freely suspended in bulk aqueous medium. This model explains initial, but not subsequent events in the development process towards structured protocells. Solid surfaces can be potential supporting structures for the assembly and development of prebiotic compartments. I will report on new evidence for the involvement of surfaces in protocell nucleation, growth, and spontaneous sub-compartmentalization and encapsulation of external material. My talk will highlight the implications of the new findings for our understanding of possible origin of life, and argue that materials properties-driven autonomous processes on solid interfaces might have greater role in the development of life than currently considered.

\section{0-152}

To bud or not to bud: remodeling of artificial cells Rumiana Dimova

Max Planck Institute of Colloids and Interfaces, Science Park Golm, Potsdam, Germany

Cell membranes exhibit a large variation in curvature. It is a common perception that curvature is caused by the activity of specific protein species. Here, we will demonstrate that it can be readily generated by various other asymmetries across the membrane, which plausibly represent a governing factor for defining shapes of membrane organelles. As a workbench for artificial cells, we employ giant vesicles (see "The giant vesicle book", Dimova \& Marques, CRC Press, 2019). In this talk, we will introduce approaches employing them for the precise quantification of the membrane spontaneous curvature. Several examples for generating curvature will be considered: asymmetric distribution of ions on both sides of the membrane (Nano Lett. 18:7816, 2018), insertion/desorption of the ganglioside GM1 (PNAS 115:5756, 2018), asymmetric lipid distribution (Sci. Rep. 8:11838, 2018) and PEG adsorption (PNAS 108:4731, 2011; ACS Nano 10:463, 2016). We will also show how spontaneous curvature generation by protein adsorption at low surface density is able to modulate membrane morphology and topology to the extent of inducing vesicle fission (Nature Commun. 11:905 2020). Finally, the process membrane wetting by molecularly-crowded aqueous phases will be shown to induce vesicle budding and tubulation (Adv. Mater. Interfaces 4:1600451, 2016). The presented examples will demonstrate that even in the absence of proteins and active processes, the membrane is easily remodeled by simple physicochemical factors.

\section{0-151}

Photosynthesis 2.0: From natural photosynthesis to artificial chloroplasts and back

Tobias J Erb

Max Planck Institute for Terrestrial Microbiology, Germany

Carbon dioxide $\left(\mathrm{CO}_{2}\right)$ is a potent greenhouse gas that is a critical factor in global warming. At the same time atmospheric CO2 is a cheap and ubiquitous carbon source. Developing new ways to capture $\mathrm{CO}_{2}$ is key to create a sustainable world of tomorrow that will be able to feed an everincreasing world-population and based on a carbon-neutral economy. While Nature provides the blue-print for developing such solutions, however, natural photosynthesis alone will not be sufficient to serve these needs. In my talk I will discuss the evolution and limitation of naturally existing $\mathrm{CO}_{2}$ fixing enzymes and present strategies how to discover and engineer novel enzymes and pathways for the fixation of $\mathrm{CO}_{2}$. I will exemplify how these enzymes can be used to realize new-to-nature metabolic networks for the conversion of $\mathrm{CO}_{2}$ that outcompete those of natural photosynthesis. Finally, I will talk about the challenges of transplanting these new-tonature pathways into natural and synthetic cells to create to create new-to-nature photosynthetic systems that interface the natural and the synthetic biological worlds.

\section{0-153}

Actuation of DNA-based functional assemblies for synthetic cells

Kevin Jahnke $[1,2]$, Kerstin Göpfrich [1,2]

[1] Max Planck Institute for Medical Research, Germany; [2] Heidelberg University, Germany

Bottom-up and top-down approaches to synthetic biology each employ distinct methodologies with the common aim to harness new types of living systems. Here, we realize a strategic merger of both approaches to convert light into proton gradients for the actuation of synthetic cellular systems. We genetically engineer $E$. coli to overexpress the lightdriven inward-directed proton pump xenorhodopsin and encapsulate them as organelle mimics in artificial cell-sized compartments. Exposing the compartments to light-dark cycles, we can reversibly switch the $\mathrm{pH}$ by almost one $\mathrm{pH}$ unit and employ these $\mathrm{pH}$ gradients to trigger the attachment of DNA structures to the compartment periphery. For this purpose, a DNA triplex motif serves as a nanomechanical switch responding to the $\mathrm{pH}$-trigger of the $E$. coli. Strikingly, we find that the choice of fluorophore alters the dynamic response of the $\mathrm{pH}$-sensitive DNA nanostructure (Jahnke et al., Nucleic Acids Res. 2021, DOI: 10.1093/nar/gkab201). When DNA origami plates are modified with the $\mathrm{pH}$ sensitive triplex motif, the proton-pumping E. coli can trigger their attachment to giant unilamellar lipid vesicles (GUVs) upon illumination. A DNA cortex is formed upon DNA origami polymerization, which sculpts and deforms the GUVs. We foresee that the combination of bottom-up and top down approaches is an efficient way to engineer synthetic cells and functional DNA-based cytoskeletons. 
Oral Presentations

\section{-22. Synthetic cell -}

0-154

Directed enzymatic signaling networks in microfluidics-based synthetic eukaryotic cells Sunidhi C Shetty, Naresh Yandrapalli, Kerstin Pinkwart, Tom Robinson

Max-Planck Institute of Colloids and Interfaces, Germany

Artificial cells offer a promising tool to study specific enzymatic reaction cascades without the interference of complex networks of biomolecules present in real cells. Multivesicular vesicles (MVVs), also known as vesosomes, with multiple compartments, serve as an ideal platform to study eukaryotic cells. Here we employ microfluidic strategies to attain highthroughput monodisperse populations of multicompartment MVVs. We report a polydimethylsiloxane(PDMS)-based microfluidic device with a two-inlet channel design able to coencapsulate different populations of liposomes within giant unilamellar vesicles (GUVs) to form MVVs. A three-step enzymatic reaction cascade is constructed between the compartments to mimic the eukaryotic cell environment. Chemical communication between the compartments proceeds in a specific direction due to size-selective transport via nanopore insertion in the lipid bilayers. We thereby present a widely applicable strategy to construct artificial eukaryotic cells with optimizable intravesicular conditions that further pave the way to study the effects of compartmentalization. Moreover, such constructs serve as a platform to develop more complex multi-step nanoreactors, drug delivery systems, and synthetic cells

\section{0-155}

Non-equilibrium large-scale membrane deformation driven by a protein pattern formation system

Fu Meifang, Schwille Petra

Max-Planck-Institute of Biochemistry, Germany

The E. coli MinDE system has become a paradigmatic reaction-diffusion system in biology. The membrane-bound ATPase MinD and ATPase-activating protein MinE oscillate between the cell poles, positioning the main division protein FtsZ at midcell. In vitro reconstruction of MinDE on supported lipid bilayer (SLB) results in Min travelling wave and Min stationary patterns that that closely resemble "Turing patterns". Here, we fabricate a new membrane system, SLB supported flat vesicles, and the reconstruction of Min travelling wave and Min stationary pattern on this membrane system induce complex membrane dynamics. I will demonstrate two main results in the talk, the first is that the flat vesicles are dynamically deformed into tubules by Min traveling wave; the second is that Min stationary patterns drive the movement of flat vesicles, which was further confirmed by using giant vesicles (GUVs). 
Oral Presentations

-23. Liquid-liquid phase separation and intrinsically disordered proteins -

\section{0-156}

Decoding molecular plasticity in the dark proteome Edward A Lemke JGU \& IMB Mainz, Germany

Nuclear pore complexes (NPCs) are central to eukaryotic function. We follow a seeing is believing approach, and aim to visualize dynamics of NPCs with advanced single molecule and superresolution fluorescence tools. Our focus are the intrinsically disordered proteins (FG-Nups), which are central to the function of this machinery, but particularly hard to study by conventional approaches. We have recently developed a microfluidic device to study the phase separation and permeability barrier properties of FG-Nups in vitro (under biochemical conditions). Since site-specific labeling of proteins with small but highly photostable fluorescent dyes inside cells remains the major bottleneck for directly performing high resolution studies of NPCs in the interior of the cell, I will demonstrate an approach how to overcome this limitation. We have now developed a semi-synthetic strategy based on novel artificial amino acids that are easily and site-specifically introduced into any protein by the natural machinery of the living cell via a newly developed semisynthetic designer membraneless organelle which equips the living cell with two genetic codes. This allows rapid, specific "click" labeling and even multi-color studies of living cells and subsequent super resolution microscopy.

Nikić I. . Lemke EA. Debugging eukaryotic genetic code expansion for site-specific click-PAINT super-resolution microscopy. Angew Chem Int Ed Engl. 2016

Reinkemeier CD, Estrada Girona G, Lemke EA, Designer membraneless organelles enable codon reassignment of selected mRNAs in eukaryotes. Science 2019

\section{0-158}

Role of alternative splicing in phase separation and pathogenic aggregation

Cristina Batlle [1], J. Paul Taylor [2,3], Salvador Ventura [1] [1] Institut de Biotecnologia i Biomedicina and Departament de Bioquímica i Biologia Molecular, Universitat Autónoma de Barcelona, Spain; [2] Department of Cell and Molecular Biology, St Jude Children's Research Hospital, Memphis, TN, 38105, USA; [3] Howard Hughes Medical Institute, Chevy Chase, MD 201815, USA

Prion-like proteins form multivalent assemblies and phase separate into membraneless organelles. hnRNPDL is a RNAprocessing prion-like protein with three alternative splicing (AS) isoforms, which lack none, one or both of its two disordered domains. It has been suggested that AS might regulate the assembly properties of RNA-processing proteins by controlling the incorporation of multivalent disordered regions in the isoforms. This, in turn, would modulate their activity in the downstream splicing program. Here, we demonstrate that AS controls the phase separation of hnRNPDL, as well as the size and dynamics of its nuclear complexes, its nucleus-cytoplasm shuttling and amyloidogenicity. Mutation of the highly conserved D378 in the disordered C-terminal prion-like domain of hnRNPDL causes limb-girdle muscular dystrophy $1 \mathrm{G}$. We show that disease-linked mutations impact hnRNPDL assembly properties, accelerating aggregation and dramatically reducing the protein solubility in the muscle, suggesting a genetic loss-of-function mechanism for this muscular disorder.
0-157

Generic interactions in the condensed state of proteins

Monika Fuxreiter [1], Michele Vendruscolo [2]

[1] Department of Biomedical Sciences, University of Padova, Italy; [2] Centre for Misfolding Diseases, Department of Chemistry, University of Cambridge, UK

Dense, liquid-like state of proteins plays key roles in structural and functional organisation of the cellular matter. We propose that at high protein concentrations, such as in the cell, the droplet state should be considered as a fundamental state together with the native and amyloid states. We show that the interactions driving formation of the droplet state can be realised by a wide variety of sequence motifs, leading to a multitude of binding configurations. We have demonstrated that most protein sequence possess such regions, which are capable to form non-native, generic interactions and these can be predicted from the protein sequence. Generic interactions, however, are highly dependent on the cellular context, which makes them vulnerable to changing dynamic behaviour leading to dysfunction.

\section{0-159}

Conformational heterogeneity of amyloid protein condensates

Dirk Fennema Galparsoro [1], Giuseppe de Luca [1], Giuseppe Sancataldo [1], Valeria Vetri [1], Vito Foderà [2]

[1] Dipartimento di Fisica e Chimica, Università degli Studi di Palermo, Viale delle scienze Edificio 18, 90128 Palermo, Italy; [2] Department of Pharmacy, University of Copenhagen, Universitetsparken 2, 2100, Copenhagen, Denmark

Increasing experiments suggest that protein amyloid selfassembly may proceed via liquid-liquid phase separation. A key question is related to the protein conformational changes paralleled with the phase separation and the growth of the aggregates. We developed an approach based on advanced microscopy to monitor the secondary structure of a variety of amyloid protein microparticles with high spatial resolution. Once formed by bulk mixing in water solution and high temperature, we identify a high heterogeneity of the secondary structure along the spherical protein condensates and their structure and stability change upon further thermal incubation. Combining fluorescence lifetime microscopy and spectroscopy methods, we prove that this process, named maturation, is characterized by an increase of amyloid structure in the protein microparticles, an enhancement in surface roughness and in molecular compactness, providing a higher stability of the structure in acidic environments. Our findings suggest conformational transitions localized at the interface as a critical step in the formation and maturation of amyloid protein condensates, promoting this phenomenon as an intrinsic knob to tailor the properties of protein microparticles formed via bulk mixing in aqueous solution. 
Oral Presentations

- 23. Liquid-liquid phase separation and intrinsically disordered proteins -

$0-160$

Phase separation of tunable transcriptional condensates predicted by an interacting particle model

Juan Torreno-Pina [1,2], Gorka Munoz [1], Catalina Romero [2], Nicolas Mateos [1], Lara de Llobet Cucalon [2], Miguel Beato [2], Maciej Lewenstein [1], Maria GarciaParajo [1]

[1] ICFO-Institut de Ciencies Fotoniques, 08860 Barcelona, Spain., Spain; [2] Centre de Regulació Genomica (CRG), Dr. Aiguader 88, Barcelona, Spain., Spain

In the last decade, it has been proposed that by means of liquid-liquid phase separation (LLPS), the cell can locally modulate the condensation of transcription factors thereby modulating gene function. Yet, despite its biological significance, a quantitative understanding of transcription factor condensation is largely missing. Here, we apply singlemolecule sensitive techniques to study condensate formation of the Progesterone Receptor (PR) in the nucleus. By applying a machine learning architecture to individual PR trajectories, we show that PR mobility inside a condensate can be best described by means of fractional Brownian motion and anomalous diffusion. Moreover, by using Cartography Maps, we show that PR forms condensates that coalescence in time following Brownian Motion Coalescence. Lastly, to predict our experimental results using theory, we develop a minimal model that fully recapitulates the condensate properties obtained from our experimental data. Altogether, this work presents an unique approach to characterize LLPS in the living cell and in any soft matter based system.
0-161

High throughput determination of kinetic and thermodynamic parameters of biomolecular liquid-liquid phase separation [Capflex] Emil G P Stender [1], Rasmus K Norrild [1], Aunstrup Larsen Jacob [1], Henrik Jensen [2], Alexander K Buell [1] [1] DTU Bioengineering, Denmark; [2] FIDA Biosystems APS, Denmark

Liquid-liquid phase separation (LLPS) is the condensation of molecules into a concentrated phase and a dilute phase. LLPS is receiving increased attention from life sciences and industry. However, current methodology is labour intensive and high throughput methods that are easily approachable are lacking. Here we present a fully automated high throughput method with capillary flow experiments (Capflex) that characterizes lower critical concentration, relative droplet size distribution, droplet formation and maturation kinetics as well as the affinity between a polypeptide undergoing LLPS and binding partners that either increases or decreases the driving force for LLPS. We used Capflex to characterize the biologically relevant LLPS system human Ddx4n1 and the membraneless organelle model system $\mathrm{RP}_{3} / \mathrm{ssDNA}$. We found that PEG3000 and $\mathrm{Ca}^{2+}$ increases the driving force of Ddx4n1 for LLPS and ssDNA it even though ssDNA is partitioned into the $\mathrm{Ddx} 4 \mathrm{n} 1$ droplets. The apparent affinity between Ddx4n1 and ssDNA is $50.9 \pm 11.1 \mu \mathrm{M}$. for $\mathrm{RP}_{3}$ ssDNA is required for LLPS. The apparent affinity of $\mathrm{RP}_{3}$ for ssDNA is $5.4 \pm 0.3 \mu \mathrm{M}$ reflecting the stronger interactions in a coacervating system. We believe our Capflex method will be of great use to characterize LLPS and that it provides information unique to the method at high throughput. 
Oral Presentations

-24. Biosensors -

\section{0-162}

Illuminating the biochemical activity architecture of the cell

Jin Zhang

University of California, San Diego, USA

The complexity and specificity of many forms of signal transduction are widely suspected to require spatial microcompartmentation and dynamic modulation of the activities of signaling molecules, such as protein kinases, phosphatases and second messengers. We have developed a series of fluorescent biosensors to probe the compartmentalized signaling activities in living cells. In this talk, I will discuss a suite of newly developed fluorescent biosensors and present a study where we applied these genetically encoded fluorescent biosensors to probe the biochemical activity architecture of the cell.

\section{0-164}

Chemo-enzymatic modifications to investigate and control mRNAs

Andrea Rentmeister

Institute of Biochemistry, University of Münster, Germany

Understanding how RNA molecules orchestrate gene expression pathways and malfunction in disease requires investigations of localization, interactions, and dynamics of RNA in living cells. ${ }^{[1]}$ Labeling RNA is in most cases required to make RNA compatible with state-of-the-art methodology. Combining the specificity of RNA-modifying enzymes with non-natural substrates has emerged as a valuable strategy to modify RNA site- or sequence-specifically with functional groups suitable for subsequent bioorthogonal reactions and thus label RNA with reporter moieties such as affinity or fluorescent tags. This talk will highlight recent chemo-enzymatic methods developed in our group to make RNA accessible to analyses focusing on non-natural modifications at the 5' cap and the poly(A) tail. ${ }^{[2]}$ Furthermore, I will present our progress towards controlling functions of mRNAs with the help of enzymatically introduced photo-caging groups. ${ }^{[3]}$

[1] N. Muthmann, K. Hartstock and A. Rentmeister, WIREs RNA 2020, 11, e1561. [2] L. Anhäuser, S. Hüwel, T. Zobel and A. Rentmeister, Nucleic Acids Research 2019, 47, e42e42. [3] a) L. Anhäuser, N. Klöcker, F. Muttach, F. Mäsing, P. Špaček, A. Studer and A. Rentmeister, Angew Chem Int Ed 2020, 59, 3161-3165; b) A. Ovcharenko, F. P. Weissenboeck and A. Rentmeister, Angew Chem Int Ed 2021, 60, 4098-4103; c) D. Reichert, H. D. Mootz and A. Rentmeister, Chemical Science 2021, 12, 4383-4388.

\section{0-163}

Targeting recombinant fluorescent biosensors to image microenvironmental ion signals locally on the surface of living cells

Roland Malli

Gottfried Schatz Research Center, Molecular Biology and Biochemistry, Medical University of Graz, Neue Stiftingtalstraße 6/6, 8010 Graz, Austria, Austria

Extracellular alterations of ion concentrations influence cell functions and fate in health and disease. Monitoring ions locally at the exterior of the plasma membrane is, however, technically challenging. Targeting of genetically encoded biosensors to cell surfaces can lead to accumulations within cellular vesicles and adverse modifications of sensor domains. We recently generated recombinant fluorescent ion biosensors fused to avidin derivates, which enabled robust and specific coupling of the purified protein-based sensors to a biotinylated cell surface tag. We show that purified chimeras of such fluorescent protein-based biosensors can be immobilized specifically on cell surfaces and thereby remain functional. The approach was used to record ion efflux from neurons in response to cytotoxic glutamate. Our study suggests that the approach can be used to image microenvironmental transport and signaling processes in diverse experimental settings.

\section{0-165}

In vivo calibration of fluorescent genetic-encoded $\mathrm{Ca}^{2+}$ indicators within high $\mathrm{Ca}^{2+}$ content organelles Alba Delrio-Lorenzo, Jonathan Rojo-Ruiz, Patricia TorresVidal, Javier García-Sancho, M Teresa Alonso IBGM, CSIC, Universidad de Valladolid, Spain

$\mathrm{Ca}^{2+}$ handling is essential for life, involved in most physiological processes, including cell division, development or cell death. Synthetic $\mathrm{Ca}^{2+}$ indicators are widely considered the standard for quantitative intracellular $\mathrm{Ca}^{2+}$ measurements, but they are not appropriate to accurately measure $\mathrm{Ca}^{2+}$ concentration inside organelles. We have recently developed a novel family of fluorescent $\mathrm{Ca}^{2+}$ indicators, the GAP (GFP-Aequorin Protein) indicators. A low $\mathrm{Ca}^{2+}$-affinity member, GAP3, can be targeted to high $\mathrm{Ca}^{2+}$-content organelles, such as the endoplasmic reticulum (ER) or the Golgi. GAP3 is ratiometric, with two excitation peaks, at 405 and $470 \mathrm{~nm}$ that enables ratiometric measurements. It has a $\mathrm{K}_{\mathrm{D}}$ for $\mathrm{Ca}^{2+}$ of $489 \mu \mathrm{M}$ and a dynamic range $\left(\mathrm{R}_{\max } / \mathrm{R}_{\min }\right)$ of $3-4$. In vitro calibration is performed by completely emptying the ER with a depletion cocktail containing an $\mathrm{IP}_{3}$-producing agonist along with a SERCA inhibitor. This strategy is, however, not feasible in in vivo experiments. We found that the $R_{\min }$ value can also be obtained by heating at $50{ }^{\circ} \mathrm{C}$. The protocol is reproducible in different cells (e.g., HeLa, HEK, MEF, or cortical astrocytes) and it can be applied to other $\mathrm{Ca}^{2+}$ affinity indicators, both green (GCaMP) or red (R-CEPIA). Importantly, GAP remains functional and is sensitive to $\mathrm{Ca}^{2+}$ variations after heating. We showed that this method is valid for calibration in vivo in Drosophila where it allowed to obtain the $\mathrm{R}_{\min }$ in the thoracic muscles and other tissues, with highly reproducible values among individuals. 
Oral Presentations

-24. Biosensors -

0-166

Viral RNA detection through DNA-based biosensing

Giulia Pinto[1], Silvia Dante[2], Paolo Canepa [1], Pietro Parisse [3], Loredana Casalis [4], Maurizio Canepa [1], Ornella Cavalleri [1]

1] Physics Department, University of Genova, Genova, Italy;

[2] Materials Characterization Facility, IIT, Genova, Italy;

[3] IOM-CNR, Trieste, Italy; [4] Elettra Sincrotrone Trieste

S.C.p.A., Trieste, Italy

COVID-19 outbreak has spread rapidly, resulting in a worldwide critical health situation. RT-PCR is the standard method to diagnose SARS-CoV-2 infection, but faster and cheaper diagnostic tools are needed and continuously developing. We focus on an optical DNA-based biosensor, where DNA strands immobilized on gold are used to detect specific SARS-CoV-2 RNA sequences through hybridization. DNA self-assembled monolayers are studied by a multi-technique approach to optimize the sensing platform, by tuning ssDNA immobilization time, concentration of ssDNA and molecular spacer and solution ionic strength. The target RNA sequence is detected by measuring changes in the film thickness (by spectroscopic ellipsometry (SE) and AFM nanolithography), in the film molecular UV-Vis absorption (by SE), and coverage (by X-ray photoemission spectroscopy (XPS) and quartz crystal microbalance (QCM)). SE allows to monitor the hybridization in situ, in a non-destructive and extremely fast way. Hybridization experiments carried on by SE and $\mathrm{QCM}$ as a function of the concentration of RNA indicate, at present, a detection limit of $10 \mathrm{nmol} / \mathrm{L}$, which is expected to decrease through an ongoing upgrade of the optical setup.
0-167

Force-controlled interface nanopores for singlemolecule and single-cell secretion sensing

T. Schlotter [1], T. Kloter [1], S. Weaver [1], N. Nakatsuka [1], D. Momotenko [3], M. Aramesh [2], J. Vörös [1], T. Zambelli [1]

[1] D-ITET, ETHZ, Switzerland; [2] D-HEST, ETHZ, Switzerland; [3] Phys. Chem., Uni. Oldenburg, Germany

Striving for more sensitive and selective single-molecule sensors, nanopore (NP) devices have emerged as an intriguing technology for stochastic sensing. However, on-demand pore size adjustability and the limitation in NP engineering remain bottlenecks for practical applications, such as proteomics. These shortcomings arise from current NP sensors designs: a NP integrated into a thin membrane has limited accessibility and therefore does not allow for flexible engineering solutions such as serial NPs, molecule-specific nanoconfinements, or selective pore functionalization. To overcome those challenges, we developed the concept of a pore size adjustable AFM-controlled interface NP. Hereby a nanopore is formed between the micro-channelled AFM cantilever and a soft polymeric substrate. Different conformational states of biomolecules can translocate through the $\mathrm{NP}$ by applying forces between 0.01 to $2 \mu \mathrm{N}$. This technique was robust and stable to allow secretome monitoring in neuron cultures over several hours. To achieve functionality for single-cell proteomics, our current attempts extend the forcecontrolled NP system to surface functionalization with amino acid-specific aptamers for selective peptide detection, manufacturing of serial NPs and protein-tailored nanostructures, as well as improved data processing methodologies. 


\section{Posters}

\section{- 1. Interfacial Protons -}

\section{P-1}

\section{Hydration and dynamics of function-relevant re-} gion of HpUreI

Abhinav Abhinav, Martin Hof, Jan Sykora

J. Heyrovsky Institute of Physical Chemistry, Czech Republic

ABSTRACT: Around two-thirds of the world's population have been infected with Helicobacter pylori. This gramnegative bacterium thrives in the digestive tract, causing extreme gastritis, ulcers, gastric adenocarcinoma, and even cancer. To survive in the extremely acidic environment of stomach, H.pylori produces a proton-gated urea channel HpUreI. This periplasmic inner membrane protein selectively enables only urea molecules to enter cytosol. The urea is then hydrolyzed by urease enzyme producing carbon dioxide and ammonia which are transferred back to periplasm to neutralize the $\mathrm{pH}(\sim 5)$. The opening of HpUreI is triggered by the depressed $\mathrm{pH}$ in periplasm and majorly 6 important amino acid residues (His-123, His-131, Asp-129, Asp-140, Glu-138 and Lys-132) in periplasmic loop 2 (PL 2) play a vital role in the functioning of this urea channel protein. Our research attempts to link the proton transfer and solvation dynamics to predict the mechanism of involvement of this periplasmic loop 2 in opening and closing of HpUreI by examining the hydration and dynamics in the immediate vicinity of these important aminoacid residues. Here we labeled HpUreI protein with microenvironment sensitive dye and with the use of time-dependent fluorescence shift data we analyzed the hydration and mobility at these functionally relevant regions. Our studies will elucidate the role of these regions in the proton gating mechanisms.

\section{P-2}

The electrostatic potentials due to exchange of protons between the surface of BLM and solution Vsevolod Y Tashkin [1], Yulia V Kharitonova [1,2], Timur R Galimzyanov [1], Anna N Konstantinova [1], Valery S Sokolov [1] [1] Frumkin Institute of Physical Chemistry and Electrochemistry, Russian Academy of Sciences, Moscow, Russian Federation; [2] Mendeleev University of Chemical Technology of Russia, Moscow, Russian Federation

The rate of exchange of protons between the surface of membrane and bulk solution is controlled by a potential barrier. To evaluate it, the kinetics of relaxation of protons released on the surface of bilayer lipid membrane (BLM) from photo activate compound 2-methoxy-5-nitrophenyl sulfate (MNPS) was studied. The illumination of BLM with MNPS leads to release of MNPS anions and binding of protons on the surface detected as a change of boundary potential (BP) measured by Inner Field Compensation method. Its kinetics depended on intensity of light, concentration of MNPS, buffer and $\mathrm{pH}$ of the solution. The results were explained by model assuming that sulfate released from MNPS quickly leaves the membrane while proton remains on it during about minute. The change of $\mathrm{pH}$ on BLM surface during illumination was evaluated using styryl dyes RH421 or di-4-ANEPPS. Their neutral molecules adsorb on BLM creating a dipole BP, but leave the BLM due to protonation (pK about 4.5). The illumination of BLM with MNPS and dyes led to decrease the BP created by dyes. This allows evaluation of "surface $\mathrm{pH}$ " shift due to release of protons from 8 to the value less than 4.5. Supported by RFBR \#19-04-00694. 


\section{Posters}

-2. Channels and $\mathrm{Ca}^{2+}$ signaling -

P-3

Regulation of TRPV6 activity by lipids

Christina Humer, Carmen Höglinger, Sandra Grünzweil, Sonja Lindinger, Christoph Romanin

Institute of Biophysics, Johannes Kepler University Linz, Austria

Transient Receptor Potential Vanilloid subfamily member 6, TRPV6, is a highly Calcium selective channel, predominantly expressed in small intestine, placenta and exocrine tissues, but also correlated with tumor progression. Apart from a preferential conduction of $\mathrm{Ca}^{2+}$, TRPV6 and the related TRPV5 deviate from other TRP ion channels in terms of their constitutive activity. Despite the latter, TRPV6 function underlies modulation by several factors to ensure regulation of $\mathrm{Ca}^{2+}$ uptake and to preserve physiological levels. On the one hand, permeation is restricted by means of fast and slow $\mathrm{Ca}^{2+}$ dependent inactivation, FCDI and SCDI, respectively. On the other hand, binding of lipids, such as $\mathrm{PIP}_{2}$ and cholesterol, seems to maintain the conductive state of the channel. Taking advantage of electrophysiological recordings and site-directed mutagenesis, we thus investigated the effect of a series of TRPV6 residues that seem, based on structural data, to be involved in scaffolding a $\mathrm{PIP}_{2}$ binding site. We examined mutants that have been reported to either reveal a higher affinity for $\mathrm{PIP}_{2}$ or to be compromised in $\mathrm{PIP}_{2}$ binding. Furthermore, we analyzed a possible effect on SCDI by cholesterol. Reducing the availability of cholesterol in the plasma membrane by adding cholesterol oxidase, filipin or methyl- $\beta$-cyclodextrin showed no significant effect on SCDI. On the contrary, several TRPV6 mutants with alterations in potential $\mathrm{PIP}_{2}$ binding sites showed distinct levels of SCDI.

\section{P-5}

Characterising the cation permeation and selectivity mechanisms of the $\mathrm{Ca}^{2+}$-selective TRPV5 channel

Callum M Ives, Ulrich Zachariae

University of Dundee, UK

The selective exchange of ions across cellular membranes is a vital biological process. $\mathrm{Ca}^{2+}$-mediated signalling is implicated in a broad array of physiological processes in cells, while higher intracellular concentrations of $\mathrm{Ca}^{2+}$ are cytotoxic. Due to the significance of this cation, strict $\mathrm{Ca}^{2+}$ concentration gradients are maintained across the plasma and organelle membranes and $\mathrm{Ca}^{2+}$ signalling relies on permeation through selective ion channels that control the flux of $\mathrm{Ca}^{2+}$ ions. A key family of $\mathrm{Ca}^{2+}$ permeable membrane channels are the polymodal signal-detecting Transient Receptor Potential (TRP) ion channels. While most members of this family are cation non-selective, TRPV5 and TRPV6 are unique due to their strong $\mathrm{Ca}^{2+}$-selectivity. However, the mechanistic basis of this selectivity has remained unclear. Here we present results from all-atom molecular dynamics simulations of ion currents through the TRPV 5 channel under membrane voltage, utilising a recently developed multisite $\mathrm{Ca}^{2+}$ model to be able to model continuous ion permeation. We resolve the biophysical principles that govern selectivity and permeation in $\mathrm{Ca}^{2+}$-selective TRP channels and compare them to permeation across the non-selective TRPV channels.

\section{P-4}

RyR distribution affects properties of calcium sparks in cardiac myocytes. An in silico study Bogdan Iaparov [1], Ivan Zahradnik [1], Alexander Moskvin [2], Alexandra Zahradnikova [1]

[1] Biomedical Research Center, Slovak Academy of Sciences, Bratislava, Slovakia; [2] Ural Federal University, Ekaterinburg, Russian Federation

Calcium ions are central in cardiac excitation-contraction coupling by being the messenger linking electrical activation to mechanical contraction of myocytes. Calcium ions are released from the sarcoplasmic reticulum through ryanodine receptors (RyRs) that form numerous calcium release sites (CRSs). Recent super-resolution studies found irregular arrangement of RyRs in CRSs; however, the relation between RyR distribution and calcium release dynamics is not experimentally accessible. To unravel this relationship, we developed a mathematical model to compare calcium release events produced by virtual CRSs differing in RyR number, arrangement, surface density, and single-channel calcium current. Simulations of virtual CRSs allowed to identify three types of calcium release events (CREs) differing in amplitude and duration. By introducing a new concept of RyR vicinity as a parameter of RyR arrangement in CRSs, and a new formulation of coupling strength based on a weighted product of calcium current and vicinity, we revealed a strong correlation between characteristics of calcium release events and parameters of calcium release sites. These results allow better understanding of formation of CREs in cardiac myocytes under physiological conditions.

Supported by JRP/2019/836/RyRinHeart.

\section{P-6}

On the electrical activation of voltage sensitive ion channels

H. Richard Leuchtag

Texas Southern University, USA

Considering an alternative to circuit and device models, what electromechanism activates a voltage sensitive ion channel? Coulomb repulsions between positively charged arg and lys residues in S4 segments are inversely proportional to the dielectric permittivity $\varepsilon$ of the ion channel. Liquid crystals similar to transmembrane segments with branched segments of ile, leu and val exhibit ferroelectric phases with $\varepsilon_{s}$ in the thousands, suggesting that an excitable channel at a resting field of $14 \mathrm{MV} / \mathrm{m}$ is highly polarized, with parallel dipolar branched sidechains. The Channel Activation by Electrostatic Repulsion model proposes that repulsions within and between the four S4 segments change the channel configuration: In the excitable phase, the outer S1-S4 domains act as a tight girdle around the ion-semiconducting body of S5 and S6 segments, so that protein crowding keeps ions out. Threshold depolarization leads to a relaxed nonpolar phase with reduced $\varepsilon$, greatly increasing Coulomb forces. These expand the S4 segments and drive them apart, giving the body room to swell. Widening of core $\mathrm{H}$ bonds in $\mathrm{S} 5$ and S6 $\alpha$ helices allows permeant ions to enter, replace protons and percolate across the membrane. Thermal fluctuations account for the observed stochastic single channel currents. 


\section{Posters}

-2. Channels and $\mathrm{Ca}^{2+}$ signaling -

\section{P-7}

On the molecular origin and physiological role of phasic store-operated calcium entry in skeletal muscle

Elena Lilliu [1], Karlheinz Hilber [1], Bradley S Launikonis [2], Manfred Grabner [3], Anamika Dayal [3], Stéphane Koenig [4], Maud Frieden [4], Xaver Koenig [1]

[1] Medical University of Vienna, Vienna, Austria; [2] University of Queensland, Brisbane, Australia; [3] Medical University of Innsbruck, Innsbruck, Austria; [4] Geneva Medical Center, Geneva, Switzerland

Phasic store-operated calcium entry (pSOCE) occurs in the t-system of skeletal muscle fibres upon single action potentials, possibly in response to local $\mathrm{Ca}^{2+}$ depletions at the terminal cisternae of the sarcoplasmic reticulum (SR). It was shown for the first time by our group in rat extensor digitorum longus (EDL) fibres using high time resolution confocal $\mathrm{Ca}^{2+}$ imaging combined with electrical field stimulation. To date, both pSOCE molecular correlates and physiological role are not clear. We recently refined the experimental protocol and observed pSOCE also in mouse EDL muscle. This suggests the presence of pSOCE across mammalian species and allows us to study pSOCE in genetically-modified mouse models. Measurements performed in mice expressing a nonconducting voltage-gated $\mathrm{Ca}^{2+}$ channel $\mathrm{Ca}_{\mathrm{v}} 1.1$ as well as in mice lacking a long isoform of STIM1 (STIM1L) rule out a prominent involvement of respective molecules in pSOCE. Pharmacological interventions using the Orai1 agonist IA65 suggest respective $\mathrm{Ca}^{2+}$ channel to carry pSOCE. In conclusion, our data suggest Orai1 but not STIM1L to mediate pSOCE in skeletal muscle fibres.

\section{P-9}

Potent direct inhibition of TRPC5 by an antidepressant and analgesic drug duloxetine

Lucie Zimova [1], Alexandra Ptakova [1,2], Michal Mitro[1], Leona Loudova [1], Viktorie Vlachova [1]

[1] Institute of Physiology of the Czech Academy of Sciences, Prague, Czech Republic; [2] Faculty of Science, Charles University in Prague, Prague, Czech Republic

Transient receptor potential canonical 5 (TRPC5) is a polymodal, calcium permeable, nonselective ion channel, that is predominantly expressed in the brain, liver and kidney, but also in human sensory neurons. Its activity is potentiated upon inflammation, modulated by cold temperatures and linked to nociceptive hypersensitivity under pathological states. Clinically approved drug duloxetine is prescribed mainly as an antidepressant but it has been repurposed and used also as an analgesic. In fact, it is the only drug so far which has proved effective for the treatment of chemotherapy-induced peripheral neuropathy, which is characterized by cold hypersensitivity. Here, we ask if the cold sensitive TRPC5 receptor is modulated by duloxetine and may contribute to its analgesic effect. Using the wholecell electrophysiology in HEK293T cells in combination with calcium imaging, we show that duloxetine inhibits human TRPC5 channels in a concentration-dependent manner. Inhibitory effects are more pronounced at positive membrane potentials and completely reversible upon 5 minute washout. Our results demonstrate that physiologically relevant concentrations of duloxetine significantly attenuate TRPC5 mediated currents and indicate that TRPC5 may contribute to antinociceptive effects of this compound.

\section{P-8}

Structure-function analysis and MD-simulations of STIM mediated calcium influx

Matthias Sallinger [1], Daniel Bonhenry [2], Herwig Grabmayr [1], Christina Humer [1], Sascha Berlansky [1], Irene Frischauf [1], Marc Fahrner [1], Christoph Romanin [1], Romana Leitner [1], Rainer Schindl [3]

[1] Johannes Kepler University Linz, Austria; [2] Academy of Sciences of the Czech Republic, Nove Hrady, Czech Republic; [3] Medical University of Graz, Austria

The Stromal Interaction Molecule (STIM) is a distinct singlepass transmembrane protein located in the endoplasmic reticulum (ER) membrane. Together with the plasma membrane resident, highly selective $\mathrm{Ca}^{2+}$ channel Orai, STIM initiates $\mathrm{Ca}^{2+}$ influx, depending on the ER calcium content. Structural destabilization of the compactly folded N-terminal EF-SAM domain of STIM, triggered by the loss of an EF-hand bound $\mathrm{Ca}^{2+}$ ion and followed by di/heteromerization, is only partially resolved. Mutations within this luminal domain are linked to pathological phenotypes and are associated with various forms of cancer. In our current study we analysed several amino acid residues located within the SAM domain of STIM1, potentially important in the luminal aggregation process via inter- and intramolecular EF-SAM interactions. We therefore used molecular dynamics (MD) simulations together with live-cell imaging methods to gain detailed insight into the EF-SAM di/heteromerization and activation mechanism. Structural unfolding of the EF-SAM domain, already described for constitutively active mutants, induces store independent SOCE initiation via puncta formation, while our SAM mutants lack the ability to form inducible puncta. On the basis of our results we present essential key residues in the EF-SAM domain, crucial for the initiation of the SOCE activation cascade, as they show reduced puncta formation, lower intermolecular FRET levels and are incapable to fully activate Orai channels upon mutation. 


\section{Posters}

- 3. Medical biophysics / Imaging -

\section{P-10}

HFt-LBT with $\mathrm{Eu}^{3+}$ : a new nanovector for bioimaging applications

Luisa Affatigato [1], Giuseppe Sancataldo[1], Alessandra Bonamore [2], Alberto Boffi [2], Valeria Militello [1]

[1] University of Palermo, Italy; [2] Sapienza University of Rome, Italy

One of the major challenges in drug delivery is to release the drug in the right organ: unspecific uptake by healthy cells leads to sever cytotoxic side effects and to low therapeutic levels at disease sites. Ferritin H-homopolymers have been extensively used as nanocarriers for different applications in the targeted delivery of drugs and imaging agents, due to their unique ability to bind the transferrin receptor (CD71), highly overexpressed in most tumor cells. We are studying the interaction between an engineered nanoparticle (HFt-LBT), that is made of the mouse heavy ferritin and a lanthanide binding tag (LBT), and the Europium in order to use this vector in the diagnostic field. LBT sequence has been genetically fused at the C-terminal end of the heavy chain of mouse ferritin. The construct acts both as carrier targeted to CD71 receptors and as a FRET sensitizer by an antenna effect. Fluorescent probes based on trivalent lanthanide ions are becoming widespread due to their unique photophysical properties. Lanthanide f-orbitals are capable of efficiently radiating most of the absorbed energy, although their small absorption cross sections pose limits to their use. To improve the luminescent signals, small organic fluorophores are used in complex with the metal ion. Preliminary studies show a clear FRET effect between tryptophan in the tag and Europium and a high affinity binding of $\mathrm{Eu}(\mathrm{III})$ atoms.

\section{P-12}

Scanning probe microscopy study of biofilm degradation induced by bacteriophages and polysaccharide depolymerases

Evgeniy Dubrovin [1], Natalia Kuzmina [1], Ekaterina Varlamova [1], Vasilii Kolmogorov [2], Anna Tolstova [3], Petr Gorelkin [2], Alexander Erofeev [2], Anastasia Popova [4], Oleg Batishchev [1]

[1] A.N. Frumkin Institute of Physical Chemistry and Electrochemistry, Russian Academy of Sciences, Moscow, 119071, Russian Federation; [2] National University of Science and Technology MISIS, Moscow, Russian Federation; [3] Engelhardt Institute of Molecular Biology, Moscow, Russian Federation; [4] Moscow Institute of Physics and Technology (State University), Dolgoprudny, Russian Federation

Biofilms are surface associated communities of bacterial cells immersed in an extracellular polymeric substance (EPS), composed of polysaccharides, proteins, nucleic acids, and lipids. Since biofilm organization increases the resistance to antibiotic treatment, their disintegration is an important problem in biomedicine. Utilization of depolymerase associated bacteriophages is believed to be a promising approach for the development of biofilm degradation strategies. In this work using atomic force microscopy and scanning ion conductance microscopy we have studied the morphological changes of A. baumannii biofilms upon their treatment with the specific bacteriophages and tailspike depolymerases. Using molecular dynamics simulations we have revealed possible mechanisms of depolymerase binding with the EPS polysaccharides. The reported study was funded by RFBR, project number 20-34-70040.

\section{P-11}

Reconstruction of 3D information from limited set of projections: Microscopic biological objects and destructive $\mathrm{X}$-ray imaging

Ondrej Bernát, Jozef Uličný, Patrik Vagovič

Pavol Jozef Šafárik University, Slovakia

X-ray imaging of microscopic non-reproducible biological particles by short intense laser pulses, such as provided by Free electron lasers, is limited by radiational damage to $2 \mathrm{D}$ projections only. To achieve the full potential of diffractbefore-destroy technique, several simultaneous projections of the same system must be achieved. However, the amount of information obtained using full tomographic scan, as known in both classical and microtomography is not achievable due to experimental and technical limitations.

Due to Nyquist sampling theorem, the few projections of the system lack the complete information for full $3 \mathrm{D}$ reconstruction. However, decreasing spatial resolution and restricting the number of classes of recognizable objects, the pattern recognition techniques can be used to assign the resulting set of projections to the most likely class of objects, in an ideal case, the class of single object.

In my contribution I will discuss the suitability of the mesoscopic objects for such approach in light of current experimental capabilities.

\section{P-13}

Effects of Immunoglobulin G subclass on the classical complement activation

Nikolaus Frischauf, Jürgen Strasser, Johannes Preiner

University of Applied Sciences Upper Austria, Linz, Austria

Elimination of Immunoglobulin ( $\mathrm{IgG}$ ) opsonized cells is mediated by different branches of the innate immune system powerful natural defense mechanisms that are increasingly utilized in immunotherapy to eliminate infectious agents, regulatory immune cells, or cancer cells. We have previously shown that antigen-mediated IgG oligomerization is crucial for engaging the complement system through $\mathrm{C} 1 \mathrm{resp} . \mathrm{C} 1 \mathrm{q}$ binding. Although the tertiary structures of the four IgG subclasses are largely comparable, complement recruitment and further activation do depend strongly on subclass. The origins of these differences have not yet been fully elucidated but one key point of variation between the subclasses is the hinge and its surrounding regions, which differ in length, flexibility, and amino acid sequence. Here we used High Speed Atomic Force Microscopy (HS-AFM) to examine the structure of the four $\operatorname{IgG}$ subclasses as well as their hexamers on mica as well as on antigenic supported lipid bilayers (SLB). Moreover, we analyzed oligomer distributions of wild type IgGs and oligomerization-enhanced mutants on antigenic SLBs and demonstrate that subclass directly impacts IgG oligomerization. Finally, $\mathrm{C} 1$ and $\mathrm{C} 1 \mathrm{q}$ binding to IgG opsonized SLBs was measured using Quartz Crystal Microbalance, revealing a link between the flexibility constraint introduced by the zymogens $\mathrm{C} 1 \mathrm{r}$ and $\mathrm{C} 1 \mathrm{q}$ and the oligomer binding capability of complement proteins. 


\section{Posters}

- 3. Medical biophysics / Imaging -

\section{P-14}

Hybrid lipid-polymer nanoparticles modified by aptamers for novel cancer therapy

Zuzana Garaiová [1], Matúš Matko [1], Beata Čunderliková [2,3], Anton Mateašík [2], Tibor Hianik [1]

[1] Faculty of Mathematics, Physics and Informatics, Comenius University, Bratislava, Slovakia; [2] International Laser Centre-CVTI, Bratislava, Slovakia; [3] Institute of Medical Physics, Biophysics, Informatics and Telemedicine, Faculty of Medicine, Comenius University, Bratislava, Slovakia

The fluorescently labeled sgc8c aptamer specific to PTK7 receptors located in the membrane of Jurkat $\mathrm{T}$ lymphoblastic leukemia cells has been used. The aptamers were covalently immobilized on the nanoparticles comprising lipid (Lecithin), lipid-polyethyleneglycol (DSPE-PEG) shells having the poly(lactic-co-glycolic acid) (PLGA) copolymer core. Hydrodynamic diameter and polydispersity index (PDI) for bare nanoparticles was $105,6 \pm 12,5 \mathrm{~nm}(\mathrm{PDI}=0,22)$ and $136,8 \pm 7,4 \mathrm{~nm}(\mathrm{PDI}=0,14)$ for sgc8-conjugated nanoparticles. Corresponding values of zeta potential were - 30,3 $\pm 3,4$ $\mathrm{mV}$ and $-39,9 \pm 1,4 \mathrm{mV}$, respectively. Nanoparticle-cell interactions were studied by confocal microscopy. Internalization of PLGA-Lecithin/DSPE-PEG-sgc8c nanoparticles into the Jurkat cells was observed and their effect on the viability of cells studied by propidium iodide assay.

This work was supported by the Science Grant Agency; project no. VEGA 1/0756/20; Slovak Research and Development Agency, APVV: SK-PL-18-0080; APVV: SK-BY-RD19-0019; KEGA: 041UK-4/2020 and by NAWA International Academic Partnership Programme EUROPARTNER.

\section{P-16}

In vivo tumor treatment with Gemcitabine Ionic Pumps (GemIPs)

Verena Handl, Linda Waldherr, Silke Patz, Rainer Schindl Medical University Graz, Austria

Glioblastoma multiforme (GBM) is the most aggressive of all brain tumors, with a median survival of only 15 months after diagnosis. Treatment typically comprises maximal safe resection, followed by chemotherapy with BBB-passing alkylating agents (e.g. TMZ) and radiotherapy. Full resection is, however, rarely feasible due to tumor cell infiltration of surrounding normal tissue. Residual tumor cells are, moreover, in $50 \%$ of cases, resistant to the standard chemotherapeutic drug used to treat GBM patients. There has only been little progress in the past few decades to find new strategies for treatment. Aims: We have recently shown that Gemcitabine Ionic Pumps (GemIPs) are able to induce apoptosis in microtumors and disrupt cohesion in vitro. Now, our primary goal is to prove the efficiency of GemIPs in vivo using the chorioallantoic membrane (CAM) assay. Methods: Different human and rat GBM cell lines were seeded onto the CAM and treated with Iontronic Pumps. The grown tumors were removed and histologically and immunohistochemically analysed. To also investigate the effect of the GemIPs on more "naturally grown" tumors we used patient derived xenografts (PDX) in our assay. Results: GBM cell lines generate solid tumors with high vascularization. We showed that GemIP tumor treatment is feasible, also combined with irradiation. Conclusion: The main important goal is the optimization of GemIP treatment on CAM, for observing the chemotherapeutic tumor effects in vivo.

\section{P-15}

Machine learning approach to pattern recognition in atomic force microscopy images

Magdalena Giergiel [1], Bartlomiej Zapotoczny [2], Jerzy Konior [1], Marek Szymonski [1]

[1] Marian Smoluchowski Institute of Physics, Jagiellonian University, Poland; [2] Institute of Nuclear Physics, Polish Academy of Science, Poland

Transmembrane porous structures, called fenestrae, regulate the flow of fluids and particles through the endothelial cells. They change their diameter dynamically in the range from 50 to $300 \mathrm{~nm}$. Their fundamental role as ultrafilters allows for maintaining proper cell function and homeostasis. Quantitative description of those fragile and sensitive to external factors structures is possible using the Atomic Force Microscopy (AFM).

It is difficult to define simple rules for recognising fenestrae in AFM images. Neither algorithms based on height of the sample nor graphical approaches are efficient enough to quantitatively determine the porosity of the measured images. In recent years, convolutional neural networks have revolutionised the automated image recognition. Therefore, an attempt of using them to find fenestrae was made, with promising results. It turns out that the accuracy of this method surpasses the manual analysis. Moreover, the method is much faster, not sensitive to chosen image contrast, and fully deterministic, therefore, the results are more reliable. The presented scheme can be easily modified to different objects of interest, which promotes the use of neural networks as a universal tool for the analysis of many kinds of microscopy images.

\section{P-17}

Investigation of platelet aggregation on individual injured endothelial cells in a microfluidic using localisation microscopy

Fabian Hauser [1], Eleni Priglinger [2], Sandra Milic [1], Boris Buchroithner [1], Christoph Naderer [1], Jaroslaw Jacak [1]

[1] University of Applied Sciences Upper Austria - Campus Linz, Austria; [2] Ludwig Boltzmann Institute for Experimental and Clinical Traumatology, Austria

Microfluidics (MF) are used to study the formation of biological barriers between cells or blood clots. Yet, no studies on a nanoscopic level for quantification of these subcellular processes have been performed. Here we present a blood-vessel MF-model, which is established to investigate cellular processes at a single-molecule level. In the MF, we have grown endothelial cells (ECs) under flow conditions. Hence, we used a femto-seconds pulsed laser to introduce a slow singlecell death. Next, we introduced platelets into the flow and observed their aggregation on previously injured cell. For quantification of platelet number, we applied 3D localization microscopy (LM) for Mitochondria imaging. Antibodies targeting Mitochondria (Alexa488) and CD62p (Alexa647) have been used for 2-color LM. Mitochondria arrangement in platelets is vastly different compared to ECs, thus it is possible to estimate the number of activated platelets by counting their specific mitochondria. For quantification, we trained a deep neuronal network (3D U-NET). Additionally, we analyzed the impact of oxidized low-density lipoproteins (Atto647N), introduced into the MF flow, on the number of activated platelets per injured EC. 


\section{Posters}

- 3. Medical biophysics / Imaging -

\section{P-18}

Optimization strategies for the optoacoustic stimulation of the hearing organ

Larissa Heimann [1], Katharina Sorg [1], Christopher Carlein [2], Gabriela Moreira Lana [3,4], Patricia Stahn [1], Eduard Arzt [3,4], Achim Langenbucher [5], Bernhard Schick[1], Gentiana Wenzel[1] [1] Dept of Otolaryngology, Saarland Univ, Germany; [2] Dept of Biophysics, CIPMM, Saarland Univ, Germany; [3] Materials Science \& Engineering Dept, Saarland Univ, Germany; [4] INM, Saarbrücken, Germany; [5] Experimental Ophthalmology Dept, Saarland Univ, Germany

Through the optoacoustic effect, mechanical vibrations of the hearing organ can be induced. Pulsed laser light irradiating e.g. the tympanic membrane (TM) induces ultrasound within the structure leading to the activation of the auditory pathway. Optoacoustic stimulation is used in future hearing devices and we herein present two optimization approaches to reduce the required light energy.

In vitro, optoacoustic-induced vibrations of the explanted guinea pig's TM were measured using laser Doppler vibrometry after the stimulation of the TM with ns-laser light of 400 to $2200 \mathrm{~nm}$. The resulting spectrum demonstrated that the highest vibration amplitude of the TM was induced through stimulation with $420 \mathrm{~nm}$ pulses.

In vivo, an absorbing film attached to the TM was introduced increasing the resulting neural activity recorded through auditory brainstem responses (ABR) in mice. The optoacoustic stimulation with $1.6 \mu \mathrm{J}$ per pulse $(50 \mathrm{kHz}$ repetition rate) applied on the absorbing film, reached $60 \%$ of acoustically induced ABRs (click ABR), whereas stimulation of the bare TM achieved $15 \%$ of click ABRs. Ongoing studies will further improve the optoacoustic stimulation.

\section{P-20}

Investigating neutrophils differentiation: towards automatic screening of nuclear morphological abnormalities with Digital Holographic Microscopy

Maria Augusta do R. B. F. Lima [1,2], Dan Cojoc [2]

[1] Department of Physics,University of Trieste, Italy; [2] CNR-IOM Materials Foundry, Italy

The demand for fast and automated screening tools grows worldwide in modern medicine. Digital Holographic Microscopy (DHM) is an optical imaging technique that allows the investigation of the cell morphology without the need of previous sample preparation or labeling. Nuclear morphology is closely related to cellular functioning, and during human neutrophil differentiation, the nucleus undergoes significant changes which are further correlated with its biophysical characteristics. Morphological abnormalities in human neutrophils have been reported in severe infections and have recently being observed in patients with SARS-CoV-2 infection. Since the use of ex-vivo primary mature blood neutrophils in large scale is very limited, mature neutrophil-like granulocytes are differentiated from human myeloid leukemia cell lines. We investigated on the DHM usefulness to characterize the cells at different differentiation stages and propose an automatic segmentation method to estimate the nuclear region, which could be applied also for nuclear and cell characterization in other contexts. From this research it emerges that DHM can be used as a valid automated label-free solution, alternatively to the standard staining technology.

\section{P-19}

Singlet oxygen quenching in presence of cytochrome c

Andrej Hovan [1], Martin Berta [1], Dagmar Sedláková [2], Pavol Miskovsky [3], Gregor Bánó [1], Erik Sedlák [3]

[1] Department of Biophysics, Faculty of Science, P. J. Šafárik University, Jesenná 5, 04154 Košice, Slovakia; [2] Department of Biophysics, Institute of Experimental Physics, Slovak Academy of Sciences, Watsonova 47, 04001 Košice, Slovakia; [3] Center for Interdisciplinary Biosciences, P. J. Šafárik University, Jesenná 5, 04154 Košice, Slovakia

The quenching of the lowest electronic excited state of molecular oxygen, the so-called singlet oxygen, by proteins is usually described as an interaction of certain amino acids with singlet oxygen. The singlet oxygen phosphorescence kinetics is influenced by changing the accessibility of these amino acids to singlet oxygen. In cellular environment however, proteins with covalently bonded or encapsulated cofactors are present. These cofactors could be potential singlet oxygen quenchers, which has received little attention in the past. To investigate this possibility, we used cytochrome $c$ (cyt $c$ ) and apocytochrome $c$ (apocyt $c$ ) to demonstrate how heme prosthetic group (a cofactor) influences the rate constant of singlet oxygen quenching in the range from neutral to acidic $\mathrm{pH}$. Flavin mononucleotide (FMN) was used for photosensitized singlet oxygen production. Our work revealed that the heme group is an excellent singlet oxygen quencher when it is exposed to solvent (in acidic $\mathrm{pH}$ ). Besides that, the effect of protein structure on triplet state of FMN was investigated.

\section{P-21}

Studying the influence of NV Nano-Diamonds on living cells and their applications in ODMR based sensing techniques to biological systems Armin Mirzaki Ebrahimi

Jagiellonian University, Poland

Nanodiamonds (NDs) have been recently playing an essential role in many different biophysical studies due to their chemical inertness, nontoxicity and high biocompatibility. NDs, containing nitrogen-vacancy (NV) centers, are highly fluorescent and photostable which makes them a popular tool to study biological systems such as individual cells. They have also been investigated for use in drug delivery applications, infection diagnosis, as imaging probes, and as contrast agents e.g. in MRI. Apart from a simple fluorescence, NV centers offer additional advantages, enabled by the, so called, optically detected magnetic resonance (ODMR), where microwave field is used for $\mathrm{NV}$-spin manipulation while the spin-state is prepared and read out optically. ODMR technique allows live-cell thermometry, nano-scale magnetometry, as well as measurements of pressure and strain with high sensitivity and spatial resolution.

We present our preliminary results on studying NDs influence on MEF 3T3 cells using confocal and wide-field fluorescence microscopy techniques. We focus on the possible interactions of NDs with different cytoskeleton's components like actin and microtubules, as a function of NDs size and concentration, incubation time, and cell viability. Moreover, we aim to study the cell membrane properties using NDs trapped with optical tweezers and, in parallel, performing ODMR-based sensing experiments. 
Posters

- 3. Medical biophysics / Imaging -

P-22

Phasor analysis in circular polarization-resolved optical scanning microscopy for biological organization imaging

Ali Mohebi [1,2], Aymeric Le Gratiet [1], Fabio Callegari [1,2], Paolo Bianchini [1], Alberto Diaspro [1,2]

[1] Nanoscopy and NIC@IIT, Italian Institute of Technology (IIT), Italy; [2] Department of Physics, University of Genova, Italy, Italy

The final possible spatial resolution any way in fluorescent biological microscopy techniques suffers limitations defined by the fluorophore molecular sizes linked to the components of biomaterials, motives to apply laser light directly for imaging rather than attachment of dye molecules. Circular intensity differential scattering microscopy is a powerful label-free technique based on light circular polarization effect on chiral biomaterials. In our work, we present a distinctive way to obtain additional information at the molecular level, integrating this imaging microscopy technique with a phasor data analysis approach to provide an intuitive information out of the reciprocal spatial frequeancy space in a graphical manner to track fast changes in terms of compaction of the chiral material throughout the microscopic image pixelby-pixel. Based on discrimination of the polarimetric contrasts, the graphical representation can applied to further applications in biological fields with also high lateral spatial resolution to recognize different molecular species in the illumination volume. Using the integration of a phasor image analysis approach, shows an advantage in easy and fast molecular temporal evolution without any necessity to huge computations.

\section{P-24}

Correlation analysis of mutual interactions between haematological and coagulation parameters upon addition of amphiphilic dendrons

Šimon Sutý [1], Veronika Oravczová [1], Dzmitry Shcharbin [2], Maksim Ionov [3], Tibor Hianik [1], Maria Bryszewska [3], Iveta Waczulíková [1]

[1] Faculty of Mathematics, Physics and Informatics, Comenius University, Slovakia; [2] Institute of Biophysics and Cell Engineering of NASB, Belarus; [3] Faculty of Biology and Environmental protection, University of Lodz, Poland

Based on the fact that 1) undesirable interactions of dendrons with blood and plasma components may pose safety concerns, and 2) the mutual interactions between platelets and coagulation pathways are potent and not fully explored, in our study we have focused on the evaluation of interactions between haematological and coagulation parameters upon addition of a $1^{\text {st }}$ and a $2^{\text {nd }}$ generation amphiphilic dendrons to human blood. Evaluation of the correlation matrices showed that the pattern of correlations changed across experimental conditions in a non-linear fashion with a clear generation and concentration dependence. Abnormalities were found only for some platelet-related parameters associated with a combination of the higher concentration and higher generation. Our findings can help optimize dendronbased nanotherapeutics.

Acknowledgement: Supported by the APVV: SK-BY-RD19-0019 and by the NAWA EUROPARTNER: Strengthening and spreading international partnership activities of the Faculty of Biology and Environmental Protection for interdisciplinary research and innovation of the University of Lodz.

\section{P-23}

AI-based virtual staining and semantic segmentation of anatomopathology sections

$\underline{\text { Riccardo Scodellaro }}[1], \quad$ Davide Panzeri [1], Margaux Bouzin [1], Laura D'Alfonso [1], Maddalena Collini [1], Giuseppe Chirico [1], Donato Inverso [2], Laura Sironi [1]

[1] Department of Physics, University of Milano-Bicocca, Italy; [2] Division of Vascular Oncology and Metastasis, German Cancer Research Center Heidelberg, Germany

Nowadays pathologists base their routine clinical practice on the visual recognition, semi-quantification and integration of morphological patterns observed in Hematoxylin/Eosin (H\&E)-stained tissue sections. This approach is subjective, poorly informative, based on expensive and time-consuming staining procedures, which lead to delays in obtaining a diagnosis. Here, we propose an AI-based pipeline that introduces a technological breakthrough in the histopathology field: we developed a convolutional neural network for digital virtual staining to provide $\mathrm{H} \& \mathrm{E}$-stained images starting from the acquisition of unstained tissue sections. Moreover, AI algorithms based on color and texture contents are applied to virtually stained images of hepatocellular carcinoma to automatically highlight timorous/healthy tissues and segment relevant biological structures. The results of both virtual staining and segmentation tasks have been compared with the manual segmentation performed by histopathologists, obtaining an accuracy above 90\%. Our novel AI tools will provide a great impact, overcoming the drawbacks due to the staining procedures and the need of additional biopsies, supporting pathologists for a quantitative and faster diagnosis, overcoming the limitations due to inter-observer variability.

\section{P-25}

Nanosized lipid particle profiling with fluorescence fluctuometry

Taras Sych [1], Birgit Plochberger [2,3], Herbert Stangl[4], Erdinc Sezgin [5]

[1] Karolinska Institutet, Stockholm, Sweden; [2] Institute of Applied Physics, TU Wien, Austria; [3] Upper Austria University of Applied Sciences, Linz, Austria; [4] Institute of Medical Chemistry, Vienna, Austria; [5] MeMRC Human Immunology Unit, Univ of Oxford, UK

The high throughput methods of cell sorting based on flow cytometry, such as Fluorescence - activated cell sorting (FACS) allow performing single-cell analysis of high statistical significance. Unfortunately, the design of such methods limits their application to cell-sized objects, whereas protein assemblies, viruses or small particles in the body such as lipoproteins cannot be analyzed in that manner. In our group, we develop the approach called Fluorescence Fluoctuometry (FFM) for high throughput analysis of nanometer-sized objects, based on the fluorescence intensity fluctuations. Similar to FACS analysis in cells, our approach is based on the read out of the emission intensities of the fluorescently labeled particles of interest in two or more color channels. The fluctuations are recorded from solution of such particles that diffuse freely through the observation volume ( $250 \times 250 \times 1000 \mathrm{~nm})$. Furthermore, using the home-made python-based software, the events that correspond to the single particles diffusion through the observation volume are identified from the fluctuations and the intensity of fluorescence emission of each particle in all color channels are quantified. The results can be presented as two-dimensional plots (such as FACS dot plots). Moreover, we employ the ratiometric environmental sensitive probes in combination with multicolor imaging to characterize the lipid packing in single nanosized lipid particles such as viral envelopes, extracellular vesicles or lipoproteins. 


\section{Posters}

- 3. Medical biophysics / Imaging -

P-26

A novel targeting approach for melanoma cancer treatment based on photodynamic therapy

Eleonora Uriati [1,2], Cristiano Viappiani [1], Paolo Bianchini [2], Alberto Diaspro [2,3], Stefania Abbruzzetti [1]

[1] Dipartimento di Scienze Matematiche, Fisiche e Informatiche, Università di Parma, Italy; [2] Nanoscopy and NIC@IIT, Istituto Italiano di Tecnologia (Genova), Italy; [3] DIFILAB, Dipartimento di Fisica, Università di Genova, Italy

Together with visible light and molecular oxygen, photosensitizers (PS) are one of the three main components at the basis of photodynamic therapy (PDT). In general, a selective treatment based on the administration of the PS to cancer cells is a clinically approved therapy. When irradiated with visible light of suitable wavelength, the photo-activated molecule starts a cascade of molecular transitions leading to cytotoxic effects, which are promoted by the formation of singlet oxygen in the proximity of the targeted cells. The achievement of effective bio-compounds with photosensitizing capabilities and increased specificity towards defined tumors is at the heart of the current research in the field. The herein research project aims to create an all-in-one multifunctional bio-molecule to be used in PDT treatments for specific cancer cells. In particular, targeting, imaging and photosensitizing features are introduced into a streptavidinbiotin based complex to be used as a theranostic agent.
P-27

Antibacterial activity of phosphorus(V) porphyrins

Ekaterina A Varlamova [1], Valeriy S Sokolov [1], Yulia G Gorbunova [1,2], Oleg V Batishchev [1]

[1] A. N. Frumkin Institute of Physical Chemistry and Electrochemistry, Russian Academy of Sciences, Russian Federation; [2] N. S. Kurnakov Institute of General and Inorganic Chemistry, Russian Federation

Bacterial resistance to existing antibiotics is an urgent task at the moment. One of the possible solutions of this problem is the death of bacteria when exposed to photosensitizing porphyrins. These molecules interact with the membranes of bacterial cells and produce singlet oxygen under illumination, leading to the death of bacteria. Due to the high reactivity of singlet oxygen, its effect is limited to its immediate surroundings. We investigated various types of phosphorus(V) porphyrins with different structures as possible antibacterial photosensitizers using Escherichia coli model. We have demonstrated that these compounds have antibacterial activity and selected the best candidates for antimicrobial agents among them. Further research in this area may expand the possibilities of using photosensitization to create antibiotics or antimicrobial agents to which bacteria cannot develop resistance.

This work was supported by the Russian Science Foundation (grant No. 19-13-00410). 
Posters

- Self-Assembled Session 1 - Emerging breakthrough methods in molecular-scale biophysics (ARBRE) -

\section{P-28}

Molecular dynamics of biological macromolecules Zahra Alavi

Loyola Marymount University, Los Angeles, California, USA

Microscopic mechanisms of friction, the relation between dissipation and nonlinearity, nonequilibrium processes in nanoscale systems, are all incompletely understood, fundamental, interconnected problems in nanoscience. These topics appear with experimental immediacy when probing enzyme mechanics by nanorheology. Using the unique capability of measuring directly dissipation occurring in the driven deformation of folded enzyme molecules, this project investigates the origin of this molecular scale friction, specifically the contribution of the surface of the molecule, which includes the hydration layer. Hydration layer dynamics, explored by nanorheology, is also the starting point of a new, dynamic understanding of kosmotropic (order inducing) and chaotropic agents, a physical chemistry topic which this research develops.

Finally, the project explores the possibility of light emission from dynamically stressed molecules, with the aim of developing a new spectroscopy to characterize dissipation at the molecular scale.

Nano-rheology allows the measurement of the stress-strain relations for a folded, native enzyme with sub- $\AA$ resolution and at different frequencies. Through recent improvements, the method now allows accurate measurements of the phase of the mechanical response, as well as the amplitude, and thus gives direct access to the dissipation. This project focuses on the dissipative part of the dynamics, which is the nonlinear but reversible mechanical regime of large amplitude deformations for these molecules.

\section{P-30}

Probing local lipid dynamics at the picosecond timescale with the Bicout model

Aline Cisse $[1,2]$, Tatsuhito Matsuo [1,4], Dominique J. Bicout [2,3], Judith Peters [1,2]

[1] Univ Grenoble Alpes, CNRS, LiPhy, Grenoble, France; [2] Institut Laue-Langevin, Grenoble, France; [3] Univ Grenoble Alpes, CNRS, EPSP, TIMC Lab, VetAgro Sup, France; [4] National Institutes for Quantum and Radiological Science and Technology, Ibaraki, Japan

Lipid membranes are at the basis of cell organization. Understanding their functionality, in the light of their structure and dynamics is then crucial.

Using quasi-elastic neutron scattering (QENS), it is possible to access picosecond timescale dynamics. However, since years, only few models addressed the question of local lipid dynamics, whereas the increase of the neutron flux and data quality called for novel general models.

Based on a timescale separation approach from Wanderlingh et al (2014), the Bicout model re-introduces the main local motions presented in the 80 s by Pfeiffer et al, but writes them all in a unique framework that requires few hypotheses.

Among others are described motions of the whole lipid, like its rotation, diffusion within the membrane, or in-out-of-theplane dynamics. In addition, it makes a distinction between the head and the tail dynamics.

Here, we would like to present a quantitative and complete study of local lipid dynamics on standard DMPC membranes. We probed the effects of known features such as the main phase transition, membrane geometry, or direction of motions, and proved the application of this new model.

\section{P-29}

The MOlecular-Scale Biophysics Research Infrastructure (MOSBRI), a new EU infrastructure Claudio Canale Physics Dept., University of Genoa, Genova, Italy

The MOlecular-Scale Biophysics Research Infrastructure (MOSBRI) enables ambitious integrative multitechnological studies of bio-systems at the crucial intermediate level between atomic-resolution structural descriptions and cellular-scale observations.

Its consortium of 2 companies and 13 academic centres of excellence from 11 countries gathers a wide complementary panel of cutting-edge instrumentation and expertise, leveraging barriers that currently hinder the optimal exploitation of molecular-scale biophysics in the fields of medicine, biotechnology, biomaterials and beyond. MOSBRI provides European researchers with a Trans-National Access to the latest technological developments in advanced spectroscopies, hydrodynamics, thermodynamics, real-time kinetics and single molecule approaches.

Networking activities will multiply the impact of MOSBRI, by sharing and disseminating knowledge through training events in Europe.

Innovative research projects will be launched to explore aspects underpinning the technologies and methodologies pipelines provided by MOSBRI and push them beyond their current limits. These projects will globally aim at improving performance, notably at the level of sensitivity, throughput, parallelisation and capacity to extract information from the biological sample.

\section{P-31}

Reproducibility and accuracy of Microscale Thermophoresis in the NanoTemper Monolith a multi laboratory benchmark study

Blanca Lopez-Mendez [1], Chad A Brautigam [2], Tom Jowitt [3], Arthur Sedivy [4], Et. Al. [5]

[1] Biophysics Platform, University of Copenhagen, Denmark; [2] Dep. of Biophysics and Microbiology, UTSouthwestern, USA; [3] Biomolecular Analysis, University of Manchester, UK; [4] ProTech, Vienna Biocenter Core Facilities GmbH, Austria; [5] various,

Microscale Thermophoresis (MST), and the closely related Temperature Related Intensity Change (TRIC), are synonyms for a recently developed measurement technique in the field of biophysics to quantify biomolecular interactions, using the NanoTemper Monolith. Although this technique has been extensively used within the scientific community due to its low sample consumption, ease of use, and ubiquitous applicability, MST/TRIC has not enjoyed the unambiguous acceptance from biophysicists afforded to other biophysical techniques.

Here we present the results of a study involving 32 scientific groups within Europe and two groups from the US, carrying out experiments on 40 Monolith instruments. A protein - small molecule interaction, a newly developed protein protein interaction system and a pure dye were used as test systems. We characterized the instrument properties and evaluated instrument performance, reproducibility, the effect of different analysis tools, the influence of the experimenter during data analysis, and thus the overall reliability of this method. 
Posters

- Self-Assembled Session 1 - Emerging breakthrough methods in molecular-scale biophysics (ARBRE) -

P-32

Investigation of nanoparticle formation and active-compound delivery in cancer cells

Raquel Lopez-Rios de Castro [1,2], Martin Ulmschneider [1], Christian D Lorenz [2]

[1] Department of Chemistry, King's College London, UK; [2] Department of Physics, King's College London, UK

Nanoparticles (NPs) tend to accumulate into tumours due to preferential uptake of NPs via the leaky vasculature of tumours. As a result, chemotherapeutics delivered by NPs have significantly lower peripheral toxicity and are able to deliver more drugs to the tumour. However, despite tremendous progress in NP formulation the mechanistic details of particle formation, drug cargo release and delivery into tumour cells remain poorly understood. This is impeding the systematic and rational design and tuning of NPs.

We present multiscale molecular dynamics (MD) simulations of an NP-formulation for a new chemotherapeutic with high selectivity towards cancer cells, that we experimentally validated. We used coarse-grain MD simulations to investigate the self-assembly of the NP in a manner representative of the experimental procedure. The results of these simulations provide unprecedented molecular-level insights into the interactions that drive the self-assembly of the NP and its internal structure. The size and shape of the simulated NP agree with that found experimentally.

To investigate the mechanisms underpinning NP selectivity towards cancer cells, as well as what drives the NP membrane interaction, and the delivery of the cargo, we have used a multiscale simulation approach to investigate the NP with model membranes representative of an eukaryotic, glioma, and oligodendroglial cell membrane. These simulations provide a molecular scale understanding of the physicochemical changes of the NP, membrane and cargo during these processes.

\section{P-34}

The response of biogenic lipid vesicles to deformations: how pressure and membrane elasticity affect stiffness

A. Ridolfi, L. Caselli, J. Cardellini, C. Montis, L. Paolini, P. Bergese, D. Berti, M. Brucale, F. Valle

Center for Colloids and Interface Science, Firenze, Italy

Extracellular vesicles (EVs) are nanosized biogenic lipid vesicles, playing a crucial role in cell-to-cell communication. Understanding the mechanical properties of these membrane delimited compartments could reveal important information about their interactions with biological interfaces. We recently found that the shape adopted by EVs upon adhesion to surfaces and the adsorption of nanoparticles on their membrane are both phenomena dependent on the vesicle stiffness. The stiffness of a vesicle describes its mechanical response to a deformation and accounts for multiple cooperating factors, the most important being the internal pressure and membrane elasticity. Based on these results, we herein present two separate methods for obtaining high-throughput stiffness characterizations of EV samples. Moreover, to shed light on what affects the mechanical response of these vesicles to deformations, we developed a spring-based model that allows understanding how internal pressure and membrane elasticity contribute to the measured stiffness. The application of this model to the results from our AFM-based Force Spectroscopy study revealed that vesicles having same size, but different membrane composition are characterized by similar pressure contributions, making membrane elasticity the key determinant for the observed differences in stiffness. The presented results enrich the current understanding on vesicle mechanics and help deciphering its role in multiple biological interactions.
P-33

Biophysical characterization of the complex between the iron-responsive transcription factor Fep1 and DNA

Adriana E Miele [1,2], Laura Cervoni [1], Aline Le Roy [3], Antimo Cutone [4], Giovanni Musci [4], Christine Ebel [3], Maria Carmela Bonaccorsi di Patti [1]

[1] Sapienza Univ. of Rome, Italy; [2] Univ. of Lyon, France; [3] Univ. of Grenoble Alpes, France; [4] Univ. of Molise, Italy

Fep1 is an iron-responsive GATA-type transcriptional repressor present in numerous fungi. The DNA-binding domain of this protein is characterized by the presence of two zinc fingers of the Cys2-Cys2 type and a Cys-X5-Cys-X8-Cys$\mathrm{X} 2$-Cys motif located between the two zinc fingers, that is involved in binding of a $[2 \mathrm{Fe}-2 \mathrm{~S}]$ cluster.

In this work, biophysical characterization of the DNAbinding domain of Komagatella pastoris Fep1 and of the complex of the protein with cognate DNA has been undertaken. The results obtained by analytical ultracentrifugation (AUC) sedimentation velocity, small-angle X-ray scattering (SAXS) and differential scanning calorimetry (DSC) indicate that Fep1 is a natively unstructured protein. The same methods and fluorescence spectroscopy have been used to assess and quantitate DNA binding. Indeed, Fep1 is able to bind DNA in 1:1 and 2:1 stoichiometry; both complexes are more compact than the individual partners.

Moreover, Fep1-DNA complex formation takes place independently of the presence of a stoichiometric amount of $[2 \mathrm{Fe}-$ $2 \mathrm{~S}$ ] cluster, suggesting that the cluster may play a role in recruiting other protein(s) required for regulation of transcription in response to changes in intracellular iron levels.

\section{$\mathrm{P}-35$}

FTIR spectroscopy as a useful tool for a labelfree characterization of exosomes: towards a novel approach for an exosome-based liquid biopsy

S. Romanò [1], F. Di Giacinto [1], A. Primiano [1], J. Gervasoni [1], A. Mazzini [1], E. Krasnowska [2], M. de Spirito [1], G. Ciasca [1]

[1] Fondazione Policlinico A. Gemelli, Roma, Italy; [2] CNRIFT, Roma, Italy

Exosomes are emerging in personalized medicine due to their diagnostic potential as biomarkers of disease, with emphasis on cancer. In this scenario, the development of novel and effective methods for a label-free characterization of these molecules is highly demanded. Since Fourier Transform Infrared Spectroscopy (FTIR) allows for a direct access to the characteristic absorption bands of biomolecules, it has the concrete potential to provide a versatile platform for exosome characterization for diagnostic and classification purposes. Here, we used FTIR in the mid-IR range to investigate the composition of exosomes and discriminate their origin from different cellular phenotypes. Human intestinal Caco-2 cell lines were chosen as a model system. Cells underwent different treatments to induce a reverse epithelial-to-mesenchymal (EMT) transition, and exosomes were extracted from both cell phenotypes. Our results showed that exosomes can be classified with high accuracy, precision, specificity and sensitivity according to their origin using logistic regression and PCA-LDA. As the EMT transition represents the first event in the cancer metastatic processes, our results can positively impact on the development of a novel exosome-based liquid biopsy approach for the early detection of cancer metastasis. 
Posters

- Self-Assembled Session 1 - Emerging breakthrough methods in molecular-scale biophysics (ARBRE) -

\section{P-36}

Influence of vitamins on the binding of levothyroxine to bovine serum albumin

Nicoleta Sandu [1], Claudia Chilom [1], Melinda David [2], Monica Florescu [2]

[1] Department of Electricity, Solid Physics and Biophysics, Faculty of Physics, University of Bucharest, Romania; [2] Department of Fundamental, Prophylactic and Clinical Disciplines, Faculty of Medicine, Transilvania University of Brasov, Romania

Levothyroxine (LT4) is an essential drug used in treating hypothyroidism and other conditions. The presence of vitamins can influence the binding of LT4 to serum proteins, such as albumin. In this study, the effect of vitamin C, vitamin B12, and folic acid on the BSA-LT4 complex was studied by spectroscopic approach, SPR, and molecular docking. Fluorescence spectroscopy reveals a static quenching mechanism of the fluorescence of the BSA-LT4 complex by the vitamin $\mathrm{C}$ and folic acid and a combined mechanism for vitamin B12. A moderate interaction of vitamin $\mathrm{C}$ and folic acid with BSALT4 was found, while vitamin B12 binds much more strongly to BSA-LT4. The vitamin effect was studied by SPR spectroscopy with the confined-BSA-LT4 complex at a gold surface chip, indicating that immobilization plays an important role. The microenvironment around Trp and Tyr residues was investigated by synchronous fluorescence. The thermal stability of the BSA-LT4 complex is more pronounced in the presence of folic acid and vitamin B12. Molecular docking studies revealed that vitamin B12 binds to the same site as LT4, while vitamin $\mathrm{C}$ and folic acid bind to another protein site.
P-37

Towards detecting the atomistic structure of phospholipids in cells using NMR spectroscopy Anika Wurl, Tiago Mendes Ferreira

NMR group - Institute for Physics, Martin Luther University Halle-Wittenberg, Germany

Detecting molecular structures with atomistic details in cellular environments is difficult specially for small molecules with a high conformational freedom. Here we present a methodological approach for determining the molecular structure of phospholipids in cellular systems. Namely, we combine state-of-the-art NMR experiments and simulations accounting for RF-inhomogeneity to determine highly accurate $\mathrm{C}-\mathrm{H}$ bond order parameters from lipid membrane systems. The methodology presented enables to increase approximately tenfold the accuracy of order parameter measurements from samples with natural abundance of isotopes and is demonstrated on a number of phospholipid model systems and E. coli cells. 


\section{Posters}

-4. Membrane transporter and channels -

\section{P-38}

Synthesis and cellular effects of a mitochondriatargeted inhibitor of the two-pore potassium channel TASK-3

M. Bachmann [1], A. Rossa [1], G. Antoniazzi [1], L. Biasutto [2,1], A. Carrer [1], M. Campagnaro [1], L. Leanza [1], M. Gonczi [3], L. Csernoch [3], C. Paradisi [1], A. Mattarei [1], M. Zoratti $[2,1]$, I. Szabò $[2,1]$

[1] University of Padova, Italy; [2] CNR Institute of Neuroscience, Padova, Italy; [3] University of Debrecen, Hungary

The $\mathrm{K}^{+}$channel TASK-3 localizes to the plasmalemma and the mitochondrial inner membrane. TASK-3 is highly expressed in melanoma and has been proposed to promote tumor formation. Here we investigate whether inhibition of mitoTASK-3 has any effect on cancer cell survival and mitochondrial physiology. A novel, mitochondriotropic version of the specific TASK-3 inhibitor IN-THPP was synthesized by adding a positively charged TPP moiety. While IN-THPP is unable to induce apoptosis, mitoIN-THPP kills melanoma cells which express mitoTASK-3. Cell death is accompanied by mitochondrial membrane depolarization and fragmentation of the network, suggesting a role of the channel in the maintenance of the correct mitochondrial function. In accordance, cells treated with mitoIN-THPP become rapidly depleted of mitochondrial ATP which results in AMPK activation. The effect of mitoIN-THPP is less pronounced in melanoma cells stably knocked down for TASK-3 expression, indicating a certain degree of selectivity of the drug. In addition, mitoIN-THPP inhibits melanoma cancer cell migration. In summary, our results point to the importance of mitoTASK-3 for melanoma cell survival and migration.

\section{P-40}

Peripheral stalk subunits and channel formation of F-ATP synthase

Andrea Carrer [1], Ludovica Tommasin [1], Francesco Ciscato[1], Riccardo Filadi [1], Andrea Urbani [1], Justina Sileikyté[3], Michael Forte [3], Andrea Rasola [1], Ildikò Szabò [2], Michela Carraro [1], Paolo Bernardi [1]

[1] Department of Biomedical Sciences, University of Padova, Italy; [2] Department of Biosciences, University of Padova, Italy; [3] Vollum Institute, Oregon Health and Sciences University, USA

F-ATP synthase is one of the leading candidates as the mitochondrial permeability transition pore (PTP), yet the mechanism(s) leading to channel formation remain undefined. To shed light on the structural requirements for PTP formation we tested cells individually ablated for g, OSCP and b subunits, and $\mathrm{R}^{0}$ cells lacking subunits a and $\mathrm{A} 6 \mathrm{~L}$. We found that $\Delta \mathrm{g}$ cells (that also lost subunit e) do not show PTP channel opening in intact cells or patch-clamped mitoplasts. $\Delta \mathrm{b}$ and $\triangle \mathrm{OSCP}$ cells displayed currents insensitive to cyclosporin A but inhibited by bongkrekate, suggesting that the adenine nucleotide translocator (ANT) can also contribute to channel formation. Mitoplasts from $\mathrm{R}^{0}$ mitochondria displayed PTP currents indistinguishable from their wild-type counterparts. These findings indicate that peripheral stalk subunits are essential to turn the F-ATP synthase into the PTP and that the ANT provides mitochondria with a distinct permeability pathway.

\section{P-39}

Pharmacological characterization of peptide toxins with inhibitory activity at the norepinephrine transporter

Oliver John V Belleza [1], Carlo Martin M Ocampo [2], Simon Miguel M Lopez [2], Aaron Joseph L Villaraza [2], Harald H Sitte [1]

[1] Institute of Pharmacology, Medical University of Vienna, Austria; [2] Institute of Chemistry, University of the Philippines - Diliman, Philippines

The norepinephrine transporter (NET) is a transmembrane protein expressed in the nervous system which plays an important role in the modulation of neurotransmission, and a therapeutic target for the treatment of mood and behavioral disorders. In addition, it is implicated in the pain pathway by the chi-conotoxin class of venom-derived peptides which have been shown to block NET activity in vitro and attenuate pain-related responses in animal models. Such peptides include MrIa and its synthetic analogue Xen2174. Although extensive structure-activity relationship and lead optimization studies have been reported on MrIa, there is still an apparent gap in diversity in the number of chi-conotoxins with reported inhibitory activity on NET. In this study, we performed a screening of peptides with amino acid sequences and disulfide bond framework similar to MrIa. Their NET activity was assessed in cell-based radioligand uptake assays. These results may confirm the reported pharmacophore region of MrIa and lead towards a NET-selective peptide with higher affinity. Amino acid residues in NET involved in binding and possible allosteric interactions can also be studied by computational methods or site-directed mutagenesis.

\section{P-41}

Exploring hERG gating through MD simulations and network analysis

Flavio Costa, Carlo Guardiani, Alberto Giacomello

Sapienza University of Rome, Italy

hERG is a non-domain swapped voltage-gated potassium channel involved in the repolarization stage of the cardiac action potential and its mutations are linked to the LongQT Syndrome (LQTS). Since the clarification of its unclear gating mechanism could provide a basis for a rational drug design against the LQTS, we addressed the problem with an integrated simulation/network-theoretical approach. At first, three isoforms of the closed state with gating charge 4, 6 and 8 were built via Steered Molecular Dynamics and then Targeted Molecular Dynamics was run to drive the channel from the open to the closed state and vice versa. The contact map analysis led to formulate two hypothetical mechanisms: an inter-subunit mechanism where loop L45 of $\mathrm{n}$ subunit pushes onto the C-linker of $n+1$ subunit causing the extension of helix S6; an intra-subunit mechanism where loop L45 and helix S5 directly push onto helix S6 of the same subunit. It is noteworthy that both mechanisms underly a dynamical communication between loop L45 and helix S6. To identify the most likely communication pathway we represented our protein as a graph with edge weights based on correlation coefficients. We then used Dijkstra's algorithm to identify the minimal paths connecting helix S4 and loop L45 with helix S6 and Brandes algorithm to identify residues with the highest betweenness centrality. The approach prevalently identified intra-subunit paths either following the route $\mathrm{L} 45 \rightarrow \mathrm{S} 5 \rightarrow \mathrm{S} 6$ or $\mathrm{S} 4 \rightarrow \mathrm{S} 1 \rightarrow \mathrm{S} 5 \rightarrow \mathrm{S} 6$. 
Posters

-4. Membrane transporter and channels -

P-42

Bidirectional crosstalk during the gating of KcsA revealed by homo-FRET methodologies

Clara Díaz-García [1], Lourdes Renart [2], Marcela Giudici[2], José A Poveda[2], Aleksander Fedorov[1], José M González-Ros [2], Mario N Berberan-Santos [1], Manuel Prieto [1], Ana Coutinho [1,3]

[1] iBB, Instituto Superior Técnico, Lisboa, Portugal; [2] IDiBE, Univ. Miguel Hernández, Elche, Spain; [3] Faculdade de Ciências, Univ. Lisboa, Lisboa, Portugal

KcsA is a homotetrameric prokaryotic potassium channel. Its conduction pathway is controlled by the inner gate, activated by acidic $\mathrm{pH}$, and the selectivity filter (SF), adopting a non-conductive or conductive state. Previously, a single Trp mutant (W67 KcsA) allowed us to investigate the SF dynamics using time-resolved homo-FRET measurements. Here, the mutation G116C was introduced in W67 KcsA and subsequently conjugated to tetramethylrhodamine $(\mathrm{TMR})$ at different $\mathrm{D} / \mathrm{P}$ ratios. Steady-state fluorescence anisotropy (ssFA) of the two independent fluorescent reporters (W67 and TMR) allowed us to simultaneously monitor the conformations adopted by the inner and outer gates of the detergent-solubilized channel. $\mathrm{pH}$ titration experiments showed that the ssFA of W67 was essentially $\mathrm{pH}$ independent, while the ssFA of the conjugated TMR dye increased upon media acidification. This is due to a decrease in the homo-FRET efficiency between the TMR fluorescently labelled subunits as the inner gate opens. The activation$\mathrm{p} K_{\mathrm{a}}$ of the inner gate was $\approx 4.2\left(\mathrm{~K}^{+}\right.$-conductive $\left.\mathrm{SF}\right)$ and $\approx 5.1\left(\mathrm{Na}^{+}\right.$-non-conductive $\left.\mathrm{SF}\right)$, indicating that the channel opens more easily when the SF is not fully filled.

\section{P-44}

The molecular mechanism of substrate-triggered occlusion in the human serotonin transporter

Ralph Gradisch, Dániel Szöllősi, Harald H Sitte, Thomas Stockner

Center for Physiology and Pharmacology, Institute of Pharmacology, Medical University of Vienna, Austria

The human serotonin transporter (hSERT) shapes serotoninergic signalling in the central nervous system by fast reuptake of serotonin (5HT) from the synaptic cleft. As a secondary active transporter, SERT exploits the sodium gradient across the membrane to energize substrate translocation. As improper function is associated with diverse neuropsychiatric disorders, hSERT is a clinical relevant drug target. Since high-resolution crystal structures of SLC6 members including LeuT and hSERT became available, representing averaged static snapshots of highly flexible proteins, investigations of the structure-function relationship have been immensely accelerated. The transport cycle proposes that several conformational states are visited during substrate translocation. After initial ion binding to the outward-open conformation, $5 \mathrm{HT}$ binds to the central substrate binding site. This induces substrate occlusion by forcing the bundle domain to move towards the scaffold domain. While comprehensive studies have shown that occlusion is mainly achieved by the rearrangements of extracellular parts of transmembrane helices, the molecular mechanism remains enigmatic. In this study, unbiased all atom molecular dynamics simulation of hSERT embedded in a membrane are used. Our results i) unravelled the sequence of events occurring during substrate occlusion ii) depict the key interactions and forces that are active between 5HT and hSERT and iii) shed light on the impact of water on the process of occlusion.

\section{P-43}

A disease mutation in the selectivity filter of GIRK2 is associated with a loss of $\mathrm{K}+$ selectivity and dynamics in the $G \beta \gamma$ binding site Theres Friesacher, Harald Bernsteiner, Anna StaryWeinzinger

University of Vienna, Austria

Inwardly rectifying potassium (Kir) channels are essential for numerous physiological processes, including neuronal and cardiac excitability. Recently, the extremely rare KeppenLubinsky syndrome (KPLBS), caused by de novo heterozygous mutations in the Kir3.2 (GIRK2) channel, has been described. KPLBS leads to severe developmental and intellectual disabilities, microcephaly, tightly adherent skin and severe generalized lipodystrophy. Recent breakthroughs in x-ray crystallography and cryo-electron microscopy provide insights into the structure of inward rectifier potassium channels and enable detailed characterization of the structural effects of disease-causing mutations. We performed multi- $\mu \mathrm{s}$ molecular dynamics simulations of the WT as well as mutant GIRK2, which carries a KPLBS causing point mutation the selectivity filter. Our simulations provide insights into the structural changes evoked by the KPLBS mutation, as well as the associated molecular mechanisms leading to the loss of $\mathrm{K}+$ selectivity. Furthermore, a Functional Mode analysis (FMA) was performed in order to identify the collective motion of the protein backbone, which is maximally correlated with the mutation-induced dynamics. We determined a region comprising the binding site for a GIRK2 activator as maximally affected by the altered motions observed in the mutant channel.

\section{P-45}

Bispyridinium compounds act as open channel blockers of nicotinic acetylcholine receptors

Yves Haufe [1], Karin V Niessen [2], Thomas Seeger [2], Annette Nicke [1]

[1] Walther Straub Institute of Pharmacology and Toxicology, Faculty of Medicine, LMU Munich, Nußbaumstraße 26, 80336 Munich, Germany; [2] Bundeswehr Institute of Pharmacology and Toxicology, Neuherbergstrasse 11, $80937 \mathrm{Mu}-$ nich, Germany

Current treatments against organophosphate $(\mathrm{OP})$ poisoning do not address effects mediated by nicotinic acetylcholine receptor (nAChR). Bispyridinium (BP) compounds act on nAChRs promoting recovery of organophosphate-induced muscle block, supposedly acting as positive allosteric modulators (PAMs) or open channel blocker. To determine the potency and molecular function of 9 C3-Linker BPs we combined mutagenesis and two-electrode voltage clamp analysis of Xenopus laevis oocyte-expressed nAChRs. BP binding to a PAM binding site was excluded by binding-site mutation and co-application of PNU-12596 but revealed real-time binding kinetics of BPs. All investigated BPs blocked human $\alpha 7$ and muscle-type nAChRs in a non-competitive, voltagedependent way with $\mathrm{IC}_{50}$-values in the high $\mathrm{nM}$ range for the most potent analogue. Increased size and hydrophobicity correlated with stronger inhibition. Additional mutants localized the BP binding site to the transmembrane domains 1-3. Computer docking studies supported a binding to the outer hydrophobic part of the pore. BP compounds act as potent channel blockers at $\alpha 7 \mathrm{nAChR}$. Their potential application in OP poisoning requires further investigation. 


\section{Posters}

-4. Membrane transporter and channels -

P-46

Dimerization of cardiac sodium channel Nav1.5 Oksana Iamshanova [1], Arbresh Seljmani [1,2], Elise Ramaye [1], Sabrina Guichard [1], Maria Essers [1], Anne-Flore Hämmerli [1], Jean-Sebastien Rougier [1], Hugues Abriel [1]

[1] Institute of Biochemistry and Molecular Medicine, National Center of Competence in Research NCCR TransCure, University of Bern, Bern, Switzerland; [2] medi - Center for Medical Education, Bern, Switzerland

Not long ago, voltage-gated sodium channels were sought to function as monomers. However, this dogma was challenged by the recent studies that pointed to dimerization of neuronal $\mathrm{Na}_{\mathrm{v}} 1.1$. $\mathrm{Na}_{\mathrm{v}} 1.2, \mathrm{Na}_{\mathrm{v}} 1.7$ and cardiac $\mathrm{Na}_{\mathrm{v}} 1.5$ channels. Accordingly, functional cooperativity of the $\mathrm{Na}_{\mathrm{v}}$ dimers was emphasized for neuronal and cardiac diseases.

By using immunoblotting analysis, we have detected both dimers and monomers of $\mathrm{Na}_{\mathrm{v}} 1.5$ in HEK293 cells transiently or stably expressing human hH1 variant. Furthermore, we have shown that these $\alpha-\alpha$ dimers are present at the plasma membrane and that reducing agents are disrupting them into monomers. In our hands, $\mathrm{Na}_{\mathrm{v}} 1.5$ dimers were not affected by 14-3-3 proteins as suggested in previous reports. Surprisingly, we were able to detect only monomers of $\mathrm{Na}_{\mathrm{v}} 1.5$ in healthy mice heart tissues. It is unclear at this point, whether our research method lacks sensitivity or whether endogenous $\alpha-\alpha$ interaction of $\mathrm{Na}_{\mathrm{v}} 1.5$ is preconditioned by some pathological cardiac state.

Taken together, our results are bringing closer the understanding of the (patho)physiology of cardiac sodium channel on fundamental level.

\section{P-48}

Hippocampal excitability in early postnatal offsprings is only moderately affected by prenatal exposure to delta-opioid receptor agonist SNC80

Lubica Lacinova [1], Bohumila Jurkovicova-Tarabova [1], Daniil Grinchii [1], Eszter Bogi [2], Eliyahu Dremencov [1] [1] IMPG, Center of Biosciences SAS, Slovakia; [2] IEPT, Center of Experimental Medicine SAS, Slovakia

We investigated an effect of prenatal exposure to delta-opioid receptor (DOR) agonist SNC80 on hippocampal excitability of offsprings. SNC80 or vehicle was administered during gestation days $11-18$ at $2.5 \mathrm{mg} / \mathrm{kg} /$ day (s.c.). Hippocampal excitability was measured in acute slices prepared from P11P13 pups using whole-cell patch clamp in current clamp configuration. 17 slices from control and 16 slices from SNC80treated pups originating from 3 experimental runs were measured. Resting membrane potential was not significantly affected. Single action potentials (APs) and AP series were activated by series of 5 and $300 \mathrm{~ms}$ long depolarizing current pulses with increasing amplitudes, respectively. Significantly higher current injection was required for activation of single AP. Same tendency for increase was observed for AP series, but this increase was not significant. Voltage threshold for activation of single action potential and a series of action potentials was insignificantly increased in exposed pups. Latency for AP series was significantly increased and the number of AP in a series was significantly decreased at higher depolarizations. To conclude, suppression of hippocampal excitability after prenatal exposure to DOR agonist is only moderate.
P-47

Molecular modeling of xenobiotic membrane crossing by the organic anion transporter 1

Angelika Janaszkiewicz, Ágota Tóth, Quentin Faucher, Marving Martin, Hélène Arnion, Chantal Barin Le Guellec, Pierre Marquet, Florent Di Meo

INSERM U1248 IPPRITT, Université de Limoges, France

Drug membrane crossing is a key pharmacological event since it affects both pharmacodynamics (PD) and pharmacokinetics (PK). Such an event often involves membrane transporters Solute Carrier (SLC). Despite the importance of transporters in clinical pharmacology, knowledge about drug-drug interactions or PGx involving membrane transporters still remains fragmented, especially at the molecular level. The present study focuses on the Organic Anion Transporter 1 (OAT1) which is involved in the uptake of a broad range of endogenous substrates and xenobiotics in kidney proximal tubular cells. OAT1 consists of a twelvehelix transmembrane core which undergoes large conformational transition along drug transport. Structural features of OAT1 remain to be elucidated owing to the absence of resolved structure. By means of MD-refined protein threading techniques, a thorough overview of OAT1 outward-facing conformation will be provided in terms of structure-function relationship. $\mu$ s-Scale MD simulations revealed key residues involved in substrate binding as well as residues potentially contributing to conformational changes. The influence of the lipids on protein's structure has been studied by embedding OAT1 into different membrane bilayer compositions. We observed the special role of $\mathrm{PE}$ components in agreement with previous studies. OAT1 model will be available to scientific community to help decipher the structural impact of any clinically observed SNP or suspected substrate/inhibitor of OAT1.

P-49

Development of a comprehensive model of GABA transporter function

Erika Lazzarin, Dániel Szöllősi, Thomas Stockner

Center for Physiology and Pharmacology, Institute of Pharmacology, Medical University of Vienna, Austria

The GABA transporters (GATs) belong to the Neurotransmitter Sodium Symporter (NSS) family, a group of plasma membrane proteins regulating neurotransmitter signaling and homeostasis. GATs are responsible for the reuptake of the released GABA from the synaptic cleft into neurons and glial cells. Our research aims to build solid relationships between molecular mechanisms and systemic effects. The transport mechanism of NSS can be described by the alternating-access model, in which structural rearrangements in the transporter result in the alternated exposure of substrates to the extracellular or intracellular environment, defining the outward-open, substrate-occluded, and inward-open conformation. Homology modeling and molecular dynamics simulations of the human GAT1 in the outwardopen conformation were simulated in a physiologically relevant membrane environment, allowing for interrogating the conformational dynamics of GAT1 in the initial step of the substrate translocation process. Microsecond-long simulations of apo GAT1, compared to the substrate-bound GAT1, showed that ions and the substrate GABA induce a transition from outward-open to outward-occluded state. Analysis of these simulations revealed the key interactions and sequence of events involved in the occlusion mechanism. 
Posters

- 4. Membrane transporter and channels -

P-50

The effect of hydrogen sulfide on type 7 voltagegated potassium channels

Thomas Losgott, Isabella Salzer, Stefan Böhm

Medical University of Vienna, Austria

Hydrogen sulfide $\left(\mathrm{H}_{2} \mathrm{~S}\right)$ is a toxic gas that also functions as a messenger molecule. It influences various physiological processes like vasorelaxation or pain perception. Some of these actions are, at least in part, mediated by type 7 voltage-gated potassium (Kv7) channels. There are five subtypes of Kv7 channels (Kv7.1 - Kv7.5), which can assemble into homoor heterotetramers and are differently expressed in various tissues. However, it is unclear if these subtypes have differing sensitivities to $\mathrm{H}_{2} \mathrm{~S}$ and which amino acid residues are involved in the actions of this gaseous signaling molecule. In perforated patch-clamp recordings, hydrogen sulfide increased currents through Kv7.2 and Kv7.5 channels, while Kv7.1 and Kv7.3 channels displayed a significant current decrease in response to $\mathrm{H}_{2} \mathrm{~S}$. Although Kv7.4 currents were also elevated after application of hydrogen sulfide, this change was not statistically significant. Furthermore, $\mathrm{H}_{2} \mathrm{~S}$ shifted the activation curve of $\mathrm{Kv} 7.2$ to the left, which resulted in a leftward shift of the half-maximal activation $\left(\mathrm{V}_{50}\right)$ of around $20 \mathrm{mV} . \mathrm{V}_{50}$ values of Kv7.1, Kv7.3, Kv7.4, and Kv7.5 channels remained largely unaffected.

These results show that hydrogen sulfide indeed exerts different effects on Kv7 channels depending on the subunit composition. The role of various amino acid residues in these actions will be investigated in future experiments.

\section{P-52}

Conductance mechanism in the KcsA channel with a mutant selectivity filter

Andrei Mironenko, Bert L de Groot, Wojciech Kopec

Computational Biomolecular Dynamics Group, Max Planck

Institute for Biophysical Chemistry, Am Fassberg 11, 37077 Goettingen, Germany

$\mathrm{K}^{+}$channels play a crucial role in the life of cells by establishing the membrane potential in all cells and terminating action potentials in excitable cells. $\mathrm{K}^{+}$channels are known for combining high conductivity with high ion selectivity: to explain such efficient $\mathrm{K}^{+}$permeation, two permeation mechanisms were proposed. The 'soft knock-on' mechanism involves water $/ \mathrm{K}^{+}$co-permeation, and the conserved narrowest part of $\mathrm{K}^{+}$channels that determines the permeation mechanism - selectivity filter $(\mathrm{SF})$ - is occupied by $\mathrm{K}^{+}$ions separated by water molecules. 'Direct knock-on', conversely, is water-free and allows for direct ion-ion contacts. Both mechanisms are supported by different computational and experimental data. However, recently direct knock-on was challenged by a crystal structure of a SF mutant of the KcsA channel that locked $\mathrm{K}^{+}$and water in the SF in a soft knock-on-like state. Although the mutant was much less conductive, it showed high $\mathrm{K}^{+}$selectivity, and was proposed to capture one of the states during permeation in the wildtype KcsA. Here, we use molecular dynamics simulations to study the permeation mechanism in this SF mutant. We show that it undergoes conformational changes that are incompatible with the permeation mechanism in wild-type channels. Our results are in agreement with experimental data, thus rendering it non-contradictory with direct knock-on.

\section{P-51}

Binding mode characterization of PfFNT' inhibitors through Docking and MD simulations Alejandro Martínez-León, Jochen S Hub Universität des Saarlandes, Saarbrücken, Germany

Malaria is a key thread to public health worldwide. Recently, a Formate Nitrite transporter from Plasmodium faciparum (PfFNT) has been identified as the malaria parasite's lactate transporter and novel drug target. Few putative inhibitors for PfFNT have been identified, however their mechanism of binding and inhibition is not well understood. Here, we used molecular dynamics (MD) simulation to obtain atomistic insight into the function and inhibition of PfFNT. For the ligands, we derived new parameters based on GAFF2 using a novel in-house methodology of Stochastic Conformational Analysis at semi-empirical level with ab initio refinement, complemented with the Parameterize tool of HTMD. The new parameters reproduce the dihedral potentials at DFMP2-aug-cc-pVTZ level of theory of these ligands with a remarkable improvement relative to initial GAFF2 parameters. The ligands MMV007839 and BH267.meta were docked into the putative binding site in the PfFNT structure recently resolved by single-particle cryo-electron microscopy, now allowing MD simulations at 4 -fs time step thanks to hydrogen mass repartitioning. Initial MD simulations are in agreement with the reported experimental results.

\section{P-53}

Structural plasticity of gasdermin-A3 upon formation of membrane lytic pores

Kristyna Pluhackova [1,2], Stefania A Mari [2], Andreas Engel [2], Daniel J Müller [2]

[1] Stuttgart Center for Simulation Science- Cluster of Excellence EXC 2075, University of Stuttgart, Germany; [2] Department of Biosystems Science and Engineering, ETH Zürich, Switzerland

Gasdermins, a family of main effector proteins of pyroptosis (a lytic inflammatory type of cell death), permeabilize cell membranes. Moreover, gasdermins are associated with hereditary diseases, inflammatory disorders, and cancer. Although the structure of the transmembrane pore formed by gasdermin-A3 was resolved by cryoEM, the essential details on gasdermins' ability to form lytic pores of diverse size and shape remain unclear. Here, we identify key mechanistic steps of gasdermin-A3 pore formation and structural adaptation by combining molecular dynamics simulations with high-resolution atomic force microscopy. Gasdermin-A3 inserts into membranes and assembles arc-, slit-, and ringshaped oligomers of variable size. While the transmembrane $\beta$-hairpins of gasdermin-A3 form extensive hydrogen bond pattern outlining the pores, the hydrophilic head domains expose exceptional structural plasticity to stabilize the various oligomeric shapes and sizes. The hydrophilic inner surface of the transmembrane $\beta$-hairpins attracts water and repels hydrophobic lipid tails, resulting in membrane release and opening of lytic pores. Our findings show mechanisms of membrane insertion and lytic pore formation and provide clues on how these processes may be controlled. 


\section{Posters}

-4. Membrane transporter and channels -

\section{P-54}

Multi-target effect of $\mathrm{Ca}^{2+}$ channel blockers on electrophysiological parameters of green alga Nitellopsis obtusa

Vilmantas Pupkis, Indre Lapeikaite, Vilma Kisnieriene Institute of Biosciences, LSC, Vilnius University, Lithuania

Increase in $\left[\mathrm{Ca}^{2+}\right]_{\text {cyt }}$ is linked to the initiation of plant Action Potential (AP) generation. However, precise intracellular $\mathrm{Ca}^{2+}$ dynamics still needs to be investigated in detail. Ion channel blockers provide a useful means for exploring the functional details of $\mathrm{Ca}^{2+}$ channel operation and its importance to the electrical signaling of the whole cell.

Green alga Nitellopsis obtusa was employed to test the electrophysiological effect of ion channel blockers (tetrandrine, verapamil, NED-19) known to specifically inhibit animal calcium channels. A conventional two-electrode current/voltage clamp technique was used to explore alterations in membrane resting potential and parameters of electrically-elicited APs of $N$. obtusa.

Observed effects of all three drugs exhibited a similar pattern. Depolarization of the AP excitation threshold potential indicated interference with the activity $\mathrm{Ca}^{2+}$ of channels. All three drugs also depolarized membrane resting potential and altered AP repolarization dynamics. These effects can be explained by altered activity of $\mathrm{K}^{+}$channels and/or $\mathrm{Ca}^{2+}$. pumps. Thus, the tested drugs exhibited both specific and unspecific effects on plant model system, implying differences between animal and plant ion transport systems and urging caution about potential side-effects of conventional drugs.

The research was funded by DAINA - Polish-Lithuanian joint project No. S-LL-18-1.

\section{P-56}

Cysteine-modification of Kv7 channels as analgesic mechanism of action of paracetamol

Sutirtha Ray[1], Oliver Kudlacek[2], Stefan Boehm[1], Isabella Salzer [1]

[1] Medical University of Vienna, Department of Neurophysiology and Neuropharmacology, Austria; [2] Medical University of Vienna, Institute of Pharmacology, Austria

$\mathrm{N}$-acetyl-p-benzoquinone imine (NAPQI), the active metabolite of acetaminophen (APAP), increases currents through neuronal Kv7 channels, which in turn reduces neuronal excitability in first and second-order neurons of the pain pathway. Three adjacent cysteine residues within the S2-S3 linker of Kv7.2 channels have been found to increase currents upon oxidative modification. Hence, we investigated whether the cysteine-modifying reagent NAPQI also exerted its effects via these cysteine residues.

NAPQI shifted the V50 of activation to the left and lead to an increased maximum current through wt Kv7.2 channels. Application of NAPQI to triple cysteine-mutated Kv7.2 channels, however, decreased maximum current levels and did not influence the V50 of activation. A Kv7.2 channel completely deficient of cysteine residues showed a similar response. In addition, currents through wt Kv7.1 channels also decrease upon perfusion with NAPQI. This subtype has only a single cysteine residue in the same position. In addition to cysteine residues, NAPQI can modify tyrosine, tryptophan, and methionine residues. We therefore suspect modification of such an amino acid residue, homologous between Kv7.1 and Kv7.2 channels, to decrease currents through these channels.

\section{P-55}

Protein flexibility and an Eyring barrier control gating in the selectivity filter of a $\mathbf{K}^{+}$channel Oliver Rauh [1], Jennifer Opper [1], Maximilian Sturm [1], Nils Drexler [3], Deborah-Desirée Scheub[1], UlfPeter Hansen [2], Gerhard Thiel [1], Indra Schroeder [1,3]

[1] Plant Membrane Biophysics, Technische Universität Darmstadt, Germany; [2] Department of Structural Biology, Christian-Albrechts-Universität zu Kiel, Germany; [3] Physiology II, University Hospital Jena, Friedrich Schiller University Jena, Germany

The selectivity filter $(\mathrm{SF})$ of $\mathrm{K}^{+}$channels also functions as a gate. The importance of a residue at the cytosolic end of the pore helix for this gate has been established in numerous publications (e.g. E71 in KcsA and S42 in KcvNTs. Interestingly, this residue is poorly conserved.

We isolated the energetic contribution of single mutations at this position in concatemeric constructs of the viral model $\mathrm{K}^{+}$channel KcvNTS, which shows fast voltage-depending gating in the SF. Voltage sensitivity arises from the voltagedependent ion occupation of the $\mathrm{K}^{+}$binding sites the $\mathrm{SF}$, especially the S0 binding site. The signal from this sensor is mediated to the gate via changes in protein flexibility.

The mutation S42T stabilizes the conductive SF, while leaving the closed state unaffected. The introduction of zero to four S42T mutations lead to a steady parallel shift of the voltage dependency of the rate constant of channel closure. This indicates that ion occupation in the SF and its effect on flexibility is not affected by the mutation. Instead, the Eyring barrier mediating the effect of flexibility on the gate is increased by about $0.4 \mathrm{kcal} / \mathrm{mole}$ per mutated subunit.

\section{P-57}

Gating movements and ion permeation in $\mathrm{HCN} 4$ pacemaker channels

Andrea Saponaro [1], Daniel Bauer [2], Hunter Giese [3], Paolo Swuec [1], Alessandro Porro [1], Federica Gasparri [1], Atiyeh Sadat Sharifzadeh [1], Antonio Chaves-Sanjuan [1], Ermanno Uboldi [1], Anastasia Polyakova [1], Martino Bolognesi [1], Gerhard Thiel [2], Bina Santoro [3], Anna Moroni [1] [1] Department of Biosciences, University of Milan, Italy; [2] Department of Biology, TU-Darmstadt, Germany; [3] Department of Neuroscience, Columbia University, NY, USA,

The HCN1-4 channel family is responsible for the hyperpolarization-activated cation current $\mathrm{I}_{\mathrm{f}} / \mathrm{I}_{\mathrm{h}}$ that controls automaticity in cardiac and neuronal pacemaker cells. We present cryo-EM structures of HCN4 in the presence/absence of bound cAMP, displaying the pore domain in closed and open conformations. Analysis of our structures sheds light on how ligand-induced transitions in the channel cytosolic portion mediate the effect of cAMP on channel gating, and highlights the regulatory role of a $\mathrm{Mg}^{2+}$ coordination site formed between the C-linker and the S4-S5 linker. Comparison of open/closed pore shows that the cytosolic gate opens through concerted movements of the S5 and S6 transmembrane helices. Furthermore, in combination with molecular dynamics analyses, the open pore provide insights into the mechanisms of $\mathrm{K}^{+} / \mathrm{Na}^{+}$permeation, revealing distinctive ion-binding dependent adaptation in the selectivity filter. Our results contribute new mechanistic understanding on HCN channel gating, cyclic nucleotide-dependent modulation, and ion permeation. 
Posters

-4. Membrane transporter and channels -

P-58

MitoKv1.3 potassium channel blockade as a possible pharmacological strategy to ameliorate multiple sclerosis

V. Scattolini [1], M. Puthenparampil [2], T. Varanita [1], L. Biasutto [3], A. Mattarei [4], C. Paradisi [5], M. Zoratti [3], P. Gallo [2], I. Szabò [1]

[1] Department of Biology, University of Padua, Italy; [2] Multiple Sclerosis Centre of the Veneto Region, Neurology Clinic, Department of Neurosciences, University of Padua, Italy; [3] Department of Biomedical Sciences, Italy; [4] Department of Pharmaceutical and Pharmacological Sciences, University of Padua, Italy; [5] Department of Chemical Sciences, University of Padua, Italy

Kv1.3 potassium channel is highly expressed in activated effector memory $\mathrm{T}$ lymphocytes (TEM), which are the majority of the inflammatory infiltrate in Multiple Sclerosis (MS) brain. There is a great need for therapies targeting chronically activated autoreactive $\mathrm{T}$ cells, the major mediators in MS pathogenesis, without causing general immunosuppression or toxicity. Given the essential importance of ion channels for immune cell function, their pharmacological targeting opens up the possibility of modulation of mechanisms involved in autoimmune neuroinflammation. The plasma membrane Kv1.3 $\mathrm{K}^{+}$channel is highly expressed by activated TEMs, is crucial for their proliferation and its modulation has been shown affect MS. Instead, targeting of the mitochondrial counterpart (mitoKv1.3) may lead to apoptosis. We therefore tested the effects of a derivative of PAP-1, which is a small molecule high-affinity blocker of Kv1.3, and we observed beneficial effects in the context of MS.

\section{P-60}

Microsecond-long MD simulations of substrate occlusion by the human dopamine transporter Leticia A Silva, Thomas Stockner

Center for Physiology and Pharmacology, Institute of Pharmacology, Medical University of Vienna, Vienna, Austria

The human dopamine transporter (hDAT) belongs to the neurotransmitter:sodium symporter family and is expressed in neurons. hDAT is essential for regulating dopamine levels in the synaptic cleft through a re-uptake process. Many psychopathological diseases are linked to dopaminergic regulation, and pharmacological treatments frequently target hDAT. However, available medications suffer from limited efficacy and severe side effects. Essential for overcoming these limitations is a comprehensive understanding of the transport mechanism at the molecular level. Here we show preliminary results from $\mu$ s long simulations, carried out as five independent simulations of hDAT in the apo state as well as with the bound substrate dopamine, applying the AMBER99SB-ildn force field. hDAT is stable in all simulations and the co-transported ions ( $\mathrm{Na} 1, \mathrm{Na} 2$ and $\mathrm{Cl}$ ) remain firmly bound. In the apo simulations, the outer vestibule and the outer gate salt-bridge between residues R85 and D476 remain open. In contrast, the presence of dopamine triggers substrate occlusion, revealing the conformational changes associated with the first step of the transport cycle. From the analyses of the interactions we could identify the dominant forces involved in dopamine occlusion by hDAT.

\section{P-59}

High-resolution cryo-EM structures of human GABA(A) receptors

Andrija Sente, A. Radu Aricescu

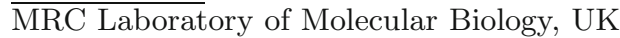

GABA(A) receptors belong to the family of pentameric ligand-gated ion channels and are the principal mediators of inhibitory neurotransmission in the brain. They are targeted by a plethora of diverse pharmacological agents with anticonvulsant, anti-anxiety, analgesic, sedative and anaesthetic properties. We recently demonstrated how a combination of new algorithms and hardware yields a $1.7 \AA$ resolution cryoEM reconstruction for a prototypical human membrane protein, the beta-3 GABA(A) receptor homopentamer bound to neurotransmitter histamine. The structure allows for a detailed understanding of small molecule coordination, visualisation of solvent molecules and alternative conformations for multiple amino acids, as well as unambiguous building of ordered acidic side chains and glycans. Moreover, the scattering potential from many hydrogen atoms can be visualised in difference maps, allowing a direct analysis of hydrogen bonding networks. This work is a step forward towards understanding the molecular principles of $\mathrm{GABA}(\mathrm{A})$ receptor assembly and gating at atomic resolution while the methods described provide a route towards routine application of cryo-EM in high-throughput screening of small molecule modulators and structure-based drug discovery.

\section{P-61}

Understanding the mechanism of CFTR ion channel bursting and of $C F$ caused by mutation $R 117 \mathrm{H}$ Márton A Simon [1,2], László Csanády [1,2]

[1] HCEMM-SU Molecular Channelopathies Research Group, Hungary; [2] Biochemistry dpt., Semmelweis Uni., Hungary

In the CFTR anion channel, an ATP Binding Cassette protein, pore gating is linked to ATP binding/hydrolysis at two cytosolic nucleotide binding domains. CFTR mutations cause cystic fibrosis (CF), a lethal incurable disease. CF mutation $\mathrm{R} 117 \mathrm{H}$ decreases anion conductance and accelerates pore closure. This position moves late during channel opening, and its mutations do not affect opening rate, suggesting that the R117 side chain stabilizes the open state. In open CFTR structures the R117 side chain forms a strong H-bond with the E1124 backbone carbonyl group, but this interaction is absent in closed CFTR structures.

We aim to investigate whether the R117-E1124 interaction stabilizes the open state. In a non-hydrolytic background (which reduces CFTR gating to reversible closedopen transitions) single-channel and macroscopic inside-out patch recordings allow quantitation of thermodynamic mutant cycles that address gating-associated changes in interaction energy between the target positions. For positions 117 and 1124, CF-causing mutation R117H and the computationally suggested E1124del were chosen, respectively. In addition to the single mutants, we also generated R117H/E1124del, to assess additivity between mutational effects. E1124del indeed accelerated closing rate similarly to R117H, and no additivity was observed in R117H/E1124del, supporting a state-dependent interaction between the target positions vital for the stabilization of the open state. 


\section{Posters}

-4. Membrane transporter and channels -

P-62

Paracetamol metabolite modifications of Kv7 channels

Jan-Luca Stampf, Jae-Won Yang, Cosmin I Ciotu, Michael J M Fischer, Stefan Boehm, Isabella Salzer Center of Physiology and Pharmacology, Medical University of Vienna, Austria

Paracetamol is often referred to as the drug used most frequently around the globe. However, its mechanism of analgesic action is unclear. In the body paracetamol is metabolized to glutathione and sulfate adducts, AM404 (N-Arachidonoylaminophenol) and NAPQI (N-Acetyl-pbenzoquinone imine), the latter inducing hepatotoxicity after paracetamol overdose. We have shown that NAPQI reduces the number of action potentials in nociceptive neurons $e x$ vivo, which is mediated by Kv7 channels. NAPQI forms adducts with nucleophilic protein residues, such as trypthophan, tyrosine and cysteine. We investigated which residues in Kv7 channels contained NAPQI adducts after exposure. Using mass spectrometry we identified NAPQI adducts almost exclusively on cysteine residues in Kv7 channel subtypes in transfected HEK293 cells. While many are located on the intracellular c-terminal region, NAPQI adducts were found on the triple cysteine sequence of Kv7.2 located on the intracellular loop between S2 and S3. Subsequent CCC150152AAA mutations showed channel dysfunction in patch clamp experiments. No modifications were found on intrinsic channel modulators such as calmodulin. In future we will analyze dorsal root ganglions of rats injected with paracetamol for NAPQI adducts on proteins. In conclusion, the study may provide new insights on analgesic mechanisms.

\section{P-64}

Investigating the mechanism of bovine complex I using solid supported membrane electrophysiology Gordon Williamson [1], Debajyoti Dutta [1,3], Olivier F Biner [2], Judy Hirst [2], Lars J C Jeuken [1]

[1] University of Leeds, UK; [2] University of Cambridge, UK; [3] Thapar Institute of Engineering \& Technology, India

Mitochondrial Complex I (NADH:ubiquinone oxidoreductase) plays a crucial role in cellular bioenergetics. As the first and largest protein in the electron transport chain, it couples oxidation of NADH to proton translocation across the mitochondrial inner membrane, thereby contributing to the proton motive force. Additionally, Complex I is a known source of radical oxygen species (ROS) and thus its malfunction has serious implications for human health and ageing. In recent years, crystallography and cryogenic electron microscopy (Cryo-EM) data have been used to form several models of Complex I mediated proton translocation. However, none of these models have been verified experimentally. Under hypoxic conditions, Complex I forms a nontransporting "deactive" state which has been reported to exhibit $\mathrm{Na}^{+} / \mathrm{H}^{+}$activity. Here we present an in vitro Solid Supported Membrane Electrophysiology (SSME) assay using Complex I from Bos taurus heart mitochondria. This assay enables investigation into potential $\mathrm{Na}^{+} / \mathrm{H}^{+}$antiport activity and enables characterisation of the mechanism of proton translocation and deuterium isotope effects in Complex I.
P-63

Alterations of TRP and Nav channels in chemotherapy-induced peripheral neuropathy: Studying their role using in vitro pre-clinical nociceptor models

Eva Villalba-Riquelme [1], Roberto de la Torre-Martínez [2], Asia Fernández-Carvajal [1], Antonio Ferrer-Montiel [1]

[1] IDiBE, Universitas Miguel Hernández, Alicante, Spain; [2] Karolinska Institutet, Stockholm, Sweden

One of the major adverse effects of cancer treatments is the development of chemotherapy-induced peripheral neuropathy (CIPN). CIPN is known to be produced by the alteration of the sensory neurons, but the mechanisms underlying the pain symptoms remain unclear. Since TRP and Nav channels play an essential role in nociception, understanding their behavior after chemotherapy could be crucial for its treatment. To address this issue, we investigated the alterations produced on these channels by two of the most common chemotherapeutics, paclitaxel and oxaliplatin. We developed an in vitro pre-clinical model that allowed us to investigate the functional and molecular changes evolving in nociceptors after direct exposure to these agents. As a result, we observed that TRPV1 and TRPM8 channels were upregulated on IB4(-) neurons after paclitaxel exposure, whereas oxaliplatin enhanced TRPV1 and TRPA1 function on IB4 $(+)$. We also found that the enhanced spontaneous activity detected correlated with alterations on different Nav channels. Altogether, our in vitro models allowed us to identify potential therapeutic targets and advance our knowledge on TRP and $\mathrm{Na}_{\mathrm{V}}$ implications on the development of paclitaxel and oxaliplatin pain symptoms.

\section{P-65}

One or two substrate/inhibitor binding site(s) in dopamine transporter?

Rong Zhu [1], Walter Sandtner [2], Thomas Stockner [2], Marion Holy [2], Oliver Kudlacek [2], Andreas Ebner [1], Hermann Gruber [1], Michael Freissmuth [2], Amy Newman [3], Harald H Sitte [2], Peter Hinterdorfer [1]

[1] Inst. Biophysics, Johannes Kepler University Linz, Austria; [2] Inst. Pharmacology, Medical University of Vienna, Austria; [3] National Institute on Drug Abuse, USA

$\mathrm{X}$-ray structures of serotonin transporter (SERT) revealed two ligand binding sites, whereas crystal structures of its close homologue, dopamine transporter (DAT) showed only one. We designed force sensors with various DAT ligands and measured their interaction forces with wild type and mutated DAT, from which two distinct populations of unbinding strengths and off-rates were detected. The population of high-force was abolished by mutation S422A, V152I, or by substitution of $\mathrm{Na}^{+}$with $\mathrm{K}^{+}$or $\mathrm{NMDG}^{+}$. In contrast, mutation G386H, acetylation of lysine residues K92 and K384, or protonation of histidine $\mathrm{H} 477$ reduced the low-force population. Our results reveal the existence of a second ligand binding site in DAT and provide information on its position and kinetics. From quantitative force measurements on DAT and SERT, we found that threshold for binding strength has to be overcome, which is between 10.1 and $13.4 \mathrm{pN}$ at force loading rate of $50 \mathrm{pN} / \mathrm{s}$, to allow for detection of an occupied binding site by X-ray structure or cryo-EM. Our finding provides a route to detect hidden binding sites in other proteins missed by X-ray structure or cryo-EM, which nevertheless are pivotal for ligand selectivity, dynamics, and function. 


\section{Posters}

- 5. Virus biophysics -

\section{P-66}

\section{Computational Microscopy of SARS-CoV-2}

Rommie E Amaro

University of California, San Diego, USA

I will discuss our lab's efforts, together with collaborators, to use computational microscopy to understand the SARSCoV-2 virus in atomic detail, with the goals to better understand molecular recognition of the virus and host cell receptors, antibody binding and design, and the search for novel therapeutics. I will focus on our studies of the spike protein, its glycan shield, its interactions with the human ACE2 receptor, our efforts to model the SARS-CoV-2 virion and escape variants.

\section{P-68}

Physico-chemical regulation of viral assembly Zaret G Denieva [1], Anna S Loshkareva [1], Liudmila A Shilova [1], Marina M Popova[1], Eleonora V Shtykova[1,2], Denis G Knyazev [1,3], Joshua Zimmerberg [1,4], Oleg V Batishchev [1]

[1] Frumkin Institute of Physical Chemistry and Electrochemistry, Russian Federation; [2] Shubnikov Institute of Crystallography, Russian Federation; [3] Johannes Kepler University Linz, Institute of Biophysics, Austria; [4] National Institute of Child Health and Human Development, USA

Nowadays, humanity has faced the emergence of many new dangerous viruses. All of them were caused by enveloped viruses, which include such pathogens as SARS-CoV-2, Influenza virus, HIV, and many others. Despite obvious differences in clinical manifestations of corresponding diseases, all the viruses share common peculiarities in their structure and lifecycle. One of the major roles in assembly and infection process of these viruses is dedicated to their capsid/matrix proteins. Combining different biophysical techniques, we demonstrated that all of these proteins can regulate the assembly of the viral scaffold and budding of progeny virions via common physico-chemical mechanisms, comprising surface activity, binding affinity, possibility to insert into lipid membrane, etc. We have shown that, despite the structural differences of matrix proteins of different viruses, their membrane activity falls into limited number of possible actions, which are common for all of enveloped viruses. Thus, our findings open a new way for search for possible antiviral drugs. This work was supported by the Russian Foundation for Basic Researches (project \# 20-54-14006).

\section{P-67}

Role of sulphation pattern in modulating the interactions between human papillomavirus and cell surface glycosaminoglycans at single particle level

Fouzia Bano [1], Laura S Martinez [2], Mario Schelhaas [2], Marta Bally $[1]$

[1] Department of Clinical Microbiology and Wallenberg Centre for Molecular Medicine, Umeå University, Umeå, Sweden; [2] Institute of Cellular Virology, ZMBE, University of Münster, Münster, Germany

Glycosaminoglycans (GAGs), negatively charged oligosaccharides, play a vital role in initially recruiting and accumulating viruses at the cell surface. However, our understanding about the interactions between non-enveloped virus and GAGs is still very limited, specifically when it comes to their dynamics. As model for our investigations, we chose the medically relevant human papillomavirus type 16 (HPV16), which is the leading cause for cervical cancer. Here, we investigate, how the interaction kinetics and resulting diffusion behaviour of a non-enveloped virus on the cell surface is regulated by specific sulfations. Using a biophysics toolbox, we study how specific sulphation patterns on highly sulphated GAGs such as heparin influence the binding kinetics and diffusion behavior of HPV16. Our single particle tracking data show that HPV16 binds efficiently to heparin in contrast to selectively $\mathrm{N}$-desulphated heparin albeit with no major difference in their diffusion behavior. Our unbinding force data shows higher binding probability and lower dissociation rate for the interaction of HPV16 and heparin further highlighting the role of $\mathrm{N}$-sulphation on the binding behavior.

\section{P-69}

Modelling of virus survival time in respiratory droplets on surfaces

N. Di Novo [1], A. Carotenuto [2], G. Mensitieri [2], M. Fraldi [2], N. Pugno [1]

[1] University of Trento, Italy; [2] University of Napoli, Italy

The microscopic droplets of saliva remain partly suspended in the air and partly settle on surfaces. Viruses, including coronaviruses, can survive hours or days in deposited drops. Experiments have shown that temperature, relative humidity and the material properties influence the persistence of the viruses contained in the drops. In particular, the survival of some viruses has a so-called "U-shaped" dependence on relative humidity $(\mathrm{RH})$, with a peak of mortality at intermediate $\mathrm{RH}$. Current hypotheses are that climatic conditions determine the physico-chemical history of a drop of saliva as they condition its evaporative process. Saliva is a complex liquid made up of water and non-volatile compounds such as salts, biomolecules and proteins. The evaporation of water affects the concentration of salts, $\mathrm{pH}$, agglomeration of compounds and crystallization of salts, all of which are non-physiological conditions, potentially harmful for a virus. However, there are still no mechanistic relations between a virus survival and the thermodynamic parameters involved. By combining the evaporation of a saline sessile droplet and a relation between virus viability and the so called cumulative dose of salts we show for the first time the mechanism leading to higher mortality at intermediate $\mathrm{RH}$, a prediction of the survival time of some SARS-CoV 2 surrogate viruses and the foundations are laid to understand the link between viability and contact angle. 


\section{Posters}

- 5. Virus biophysics -

\section{P-70}

Reovirus directly engages integrin to recruit clathrin for entry into host cells

Melanie Koehler [1], Simon J Petitjean [1], Jinsung Yang [1], Pavithra Aravamudhan [2], Xayathed Somoulay [2], Cristina Lo Giudice[1], Megane A Poncin [1], Andra C Dumitru [1], Terence S Dermody [2], David Alsteens [1]

[1] LIBST, Université catholique de Louvain, Belgium; [2] Department of Pediatrics, University of Pittsburgh, USA

As all obligate intracellular microbes, reoviruses must cross the plasma membrane to enter host cells and requires the concerted action of viral and host factors. After interaction of reovirus attachment protein $\sigma 1$ with cell-surface carbohydrates and proteinaceous receptors, additional host factors mediate virus internalization. In particular, $\beta 1$ integrin is required for endocytosis of reovirus virions following junctional adhesion molecule A binding. While integrin-binding motifs in the surface-exposed region of reovirus capsid protein $\lambda 2$ are thought to mediate integrin interaction, evidence for direct $\beta 1$ integrin-reovirus interactions and knowledge of how integrins function to mediate reovirus entry is missing. We utilize a combination of single-virus force spectroscopy, confocal and fluidic force microscopy, to discover a direct interaction between reovirus and $\beta 1$ integrins. We proved that $\lambda 2$ is the viral ligand for integrin and identified a functional role for $\beta 1$ integrin interaction in promoting clathrin recruitment to cell-bound reovirus, which may in turn facilitate endocytosis. These results offer insights into the mechanism of reovirus cell entry, providing new perspectives for the development of efficacious antiviral therapeutics, the engineering of improved viral gene delivery and oncolytic vectors.

\section{P-72}

Protein-lipid interactions in assembly of M1 protein of Influenza A virus

Marina Popova [1,2], Zaret Denieva [1], Oleg Batishchev [1,2] [1] A.N. Frumkin Institute of Physical Chemistry and Electrochemistry, Russian Academy of Sciences, Moscow, Russian Federation; [2] Moscow Institute of Physics and Technology, Dolgoprudny, Russian Federation

Influenza A virus (IAV) of the Ortomyxoviridae family is an enveloped RNA virus whose genetic material is enclosed in a lipoprotein shell. In the process of infection, the virus permeates cell through endocytosis. The acidification of the medium inside the endosome induces the fusion of the viral and endosomal membranes, followed by the destruction of the virion protein scaffold and the release of the viral genetic material into the cell cytoplasm. The M1 matrix protein of IAV is involved in all stages of the virus life cycle and highly conserved for various strains in spite of the high variability of IAV. The study of the matrix structure and disassembly depending on $\mathrm{pH}$ is an important problem for a fundamental understanding of IAV life cycle and the search for new antiviral agents.

In this work, the mechanical properties of the lipid membrane in the presence of M1 were investigated at different $\mathrm{pH}$ on the giant unilamellar vesicle (GUV) model using fluorescence microscopy. The $\mathrm{pH}$-dependent deformation of the GUV membrane by adding the M1 protein was shown, and the nature of protein-lipid interaction of various lipid compositions was established.

The work was supported by the Russian Foundation for Basic Research (grant \#20-54-14006)

\section{P-71}

\section{Lipid specificity of Viral Proteins}

Chetan S Poojari, Tobias Bommer, Jochen S Hub

Computational Biophysics Group Saarland University Theoretical Physics Campus E2 6, 66123 Saarbrücken, Germany

Viral fusion proteins drive fusion of viral and host cell membranes in a series of complex structural transition events. Although the structure of several fusion proteins has been solved, the characterization of viral protein-membrane interactions at atomistic resolution is still missing. Membrane interactions of fusion proteins are conserved and occur via fusion peptides (FPs) in class I and fusion loops (FLs) in class II/III proteins. Previously, we had characterized the glycerophospholipid binding in class II fusion protein glycoprotein $\mathrm{C}$ ( $\mathrm{gC}$ ) of Rift Valley fever virus (RVFV) [2] and the studies revealed specific binding pockets for PC lipids. Now we aim to understand if specific lipid binding site also exists in class I and III viral proteins and any preference for lipid type. Molecular dynamics (MD) simulations is an excellent technique to understand how proteins associates with lipid membrane at atomistic resolution and here we make use of MD simulations to gain structural insights into lipid contact sites and membrane insertion of FP / FL residues.

[1] M. Vallbracht et al., J Virol 92: e01203-17, (2018)

[2] P. Guardado-Calvo et al., Science 358, 663-667, (2017)

\section{P-73}

Binding Forces in Infections with SARS-CoV-2 Valentin D Reiter-Scherer $[1,2]$, Katharina G Hugentobler [2], Stefanie Wedepohl [2], Yannic Kerkhoff [2], Chuanxiong Nie[2], Paria Pouyan[2], Sumati Bhatia [2], Lutz Adam [3], Daniel Lauster [2], Christian Hackenberger [3], Kai Ludwig [2], Rainer Haag [2], Stephan Block [2], Andreas Herrmann [1]

[1] Humboldt-Universität zu Berlin, Germany; [2] Freie Universität Berlin, Germany; [3] Leibniz-Institut für Molekulare Pharmakologie, Germany

SARS-CoV-2 infects host cells by binding to the angiotensinconverting enzyme 2 (ACE2) via its spike proteins (SP). A second binding domain that interacts with the cellular receptor sialic acid has recently been identified on SP and might be a promising candidate for inhibition. For the design of potent inhibitors, the biophysical mechanisms of the interaction between SP and receptors need to be well understood. Single molecule force spectroscopy (SMFS) based on the atomic force microscope (AFM) is capable of measuring minute forces of protein-receptor bonds and has been applied by Alsteens et al. to investigate the kinetics of the bond between ACE2 and the receptor binding domain of SP. Here, we want to employ the AFM and SMFS to measure forces between the trimeric full length SP from wildtype and recent mutant virus strains and ACE2 as well as inhibitors of these interactions. To this end, supported lipid bilayers are prepared using proteoliposomes incorporating SP. AFM cantilevers will be functionalized with either ACE2 or potential inhibitors. With this setup, binding forces and bond kinetics will be determined. 
Posters

-5 . Virus biophysics -

\section{P-74}

Effect of alkaloids on calcium mediated fusion of negatively charged unilamellar vesicles

Egor V Shekunov, Svetlana S Efimova, Olga S Ostroumova Institute of Cytology of the Russian Academy of Sciences, Russian Federation

Enveloped viruses are the causative agents of numerous serious human diseases, including Influenza, Rabies, Ebola Haemorrhagic Fever, HIV, COVID-19 etc. A feature of this group of viruses is the presence of a lipid bilayer surrounding of the virion. The possibility of using this conservative element of the virion as a target for the action of new antiviral drugs is of special interest. Excellent candidates for potential antiviral compounds are secondary metabolites of plants - alkaloids. They have low cytotoxicity and high variability and availability. In this work, we investigated the ability of alkaloids to inhibit the fusion of small unilamellar vesicles (SUV) composed of negative charged phosphoglicerol. Calcein release was used to characterize the rate of leakage of the fluorescent marker from SUVs by calcium-mediated fusion. Moreover, using dynamic light scattering, we measured the size and the $\zeta$-potential of SUVs in the presence of calcium, as well at the addition of alkaloids. Using differential scanning microcalorimetry and planar lipid bilayers, the effect of compounds on the thermotropic behavior of membrane-forming lipids also were described, and changes in the membrane boundary potential respectively.

\section{P-75}

Nanomechanical crowding at the interface between RNA and soft surfaces

Horacio V. Guzman [1], Simon Poblete [2], Anže Božič [1], Matej Kanduč [1], Rudolf Podgornik [3,1]

[1] Department of Theoretical Physics, Jožef Stefan Institute, SI-1000 Ljubljana, Slovenia, Slovenia; [2] Instituto de Ciencias Físicas y Matemáticas, Universidad Austral de Chile, Valdivia 5091000, Chile, Chile; [3] School of Physical Sciences and Kavli Institute for Theoretical Sciences, University of Chinese Academy of Sciences, Beijing 100049, China, China

Nanomechanical crowding remains theoretically unexplored at the interface of RNA molecules and soft surfaces. Existing RNA molecular models tend to reach very complex ensembles on themselves to be combined to e.g. soft matter mechanics. Here, we introduce a multiscale approach which couples a tractable RNA coarse-grained model with an elastic energy component. Within this approach, we study the specific role of RNA's secondary structure patterns on the deformation of soft surfaces by characterizing representative RNA motifs. We also vary the length of the investigated molecules and propose scaling trends for longer RNAs. Under controlled molecular crowding conditions we analyze the effects of conformational entropy and the interplay between surface energy per monomer and deformation lengths. Our findings add a novel way to address the mechanisms of response of encapsulated RNA inside crowded macromolecular environments, like the ones faced during RNA delivery. 


\section{Posters}

-6. Advanced optical microscopy -

P-76

Phase-sensitive expansion microscopy

Nicholas Anthony [1], Alberta Trianni [1,2], Paolo Bianchini [1], Alberto Diaspro $[1,2]$

[1] Nanoscopy, CHT Erzelli, Istituto Italiano di Tecnologia, Italy; [2] DIFILAB, Department of Physics, University of Genoa, Italy

Label free microscopies are becoming increasingly popular as a means of imaging, and understanding, complex biological systems. Not only can they introduce image contrast, but they can also provide information unobtainable with traditional approaches. When paired with the recent and significant advances in computational approaches, label free imaging is a profoundly powerful tool to image biological systems. However, label-free approaches often suffer from poor resolution, and so the combination of label-free and superresolved methods are often used. Expansion microscopy is a super-resolved technique that physically enlarges the sample by swelling an embedded polymer network, allowing for smaller features within the sample to be resolved, as they are physically more displaced.

In this work we apply the phase-sensitive microscopy technique of ptychography to polymer-expanded biological samples (HeLa cells) to measure their phase, and hence calculate their refractive index. This novel approach, termed expansion ptychography, is shown to increase the resolution and sensitivity of the obtained images, allowing for quantification of cellular processes within HeLa cells.

\section{P-78}

Fast leaps between millisecond confinements govern Ase1 diffusion along microtubules

Łukasz Bujak [1], Kristýna Holanová [1], Verena Henrichs [2], Ivan Barvík [3], Marcus Braun [2], Zdeněk Lánský [2], Marek Piliarik [1]

[1] Institute of Photonics and Electronics CAS, Czech Republic; [2] Institute of Biotechnology CAS, Czech Republic; [3] Faculty of Mathematics and Physics, Charles University, Czech Republic

Diffusion is the most fundamental mode of protein translocation within cells. Confined diffusion of proteins along the electrostatic potential constituted by the surface of microtubules, although modeled meticulously in molecular dynamics simulations, has not been experimentally observed in real-time. Here, we used interferometric scattering microscopy to directly visualize the movement of the microtubule-associated protein Ase1 along the microtubule surface at nanometer and microsecond resolution. We resolved millisecond confinements of Ase1 and fast leaps between these positions of dwelling preferentially occurring along the microtubule protofilaments, revealing Ase1's mode of diffusive translocation along the microtubule's periodic surface. The derived interaction potential closely matches the tubulin-dimer periodicity and the distribution of the electrostatic potential on the microtubule lattice. We anticipate that mapping the interaction landscapes for different proteins on microtubules, finding plausible energetic barriers of different positioning and heights, will provide valuable insights into regulating the dynamics of essential cytoskeletal processes.
P-77

Deciphering the nanoscale structure of chromatin during neuroblastoma transformation by optical nanoscopy

Francesca Baldini [1], Isotta Cainero [1], Chantal Usai [1,2], Paolo Bianchini [1], Aldo Pagano [3,4], Laura Vergani [5], Alberto Diaspro $[1,2]$

[1] Nanoscopy, Istituto Italiano di Tecnologia, Genoa, Italy; [2] DIFILAB, University of Genoa, Italy; [3] IRCCS Ospedale Policlinico San Martino, Italy; [4] DIMES, University of Genoa, Italy; [5] DISTAV, University of Genoa, Italy

Nuclear architecture and chromatin remodeling are the main regulators of cell identity. Despite its apparent disorder, chromatin organization is a well-orchestrated mechanism involved in cellular physiology and the onset of many diseases. Neuroblastoma (NB) is the most common extracranial solid tumor that occurs in childhood and is characterized by a remarkable phenotype and clinical heterogeneity. The molecular mechanisms involved in NB are still partially unknown. Here we characterize changes of chromatin nanoscale architecture potentially correlated with NB transformation. We employ genetically engineered NB cells to overexpress the non-coding RNA NDM29, promoting differentiation in a neuronal lineage. Previous studies have shown that NDM29 can restore the expression of DNA damage repair genes. We investigate chromatin architecture in the whole nucleus, chromosome territories, and specific genes as a function of NB malignant transformation. By using STED Microscopy we unveil whether NB-associated chromatin alteration is located within specific territories or genes, paving the way towards new prognostic and therapeutic approaches.

\section{P-79}

Investigation of oncogene-induced alterations in chromatin organization by advanced optical microscopy

E. Cerutti [1,2], M. D'Amico [1], I. Cainero [2,3], G. I Dellino $[4,5]$, M. Faretta $[4]$, P. G Pelicci $[4,5]$, A. Diaspro [2,3], L. Lanzanò $[1,2]$

[1] DFA, University of Catania, Italy; [2] Nanoscopy, Istituto Italiano di Tecnologia, Genoa, Italy; [3] DIFILAB, University of Genoa, Italy; [4] Istituto Europeo di Oncologia, Milan, Italy; [5] DIPO, University of Milan, Italy

Oncogenes can induce genomic instability by interfering with fundamental processes such as DNA transcription and replication. Here we use an engineered Acute Promyelocytic Leukemia cell line, U937-PR9, which allows us to selectively activate the expression of the PML-RAR $\alpha$ oncoprotein and to analyze its effects on the organization of functional nuclear processes. First, to reach the spatial resolution required to image the nanoscale organization of chromatin in the nucleus, we perform STED-based super-resolution microscopy. As expected, the maximum achievable resolution is limited by factors such as photobleaching and/or reduction of SNR, which we overcome by application of the method of SPLIT. Second, to quantify the spatial distribution of functional sites in the nucleus, we apply Image Cross-Correlation Spectroscopy to dual color super-resolution images. ICCS provides information on the fraction of colocalized sites and on their average distances. In addition, in situ Proximity Ligation assay is performed to detect proximity at a smaller spatial scale. Our approach provides a new perspective of nuclear processes based on intact nuclei. 
Posters

-6. Advanced optical microscopy -

\section{P-80}

Diffusion and interaction dynamics of the cytosolic peroxisomal import receptor PEX5

S Galiani [1], K Reglinski [2,1], P Carravilla [2], A Barbotin [3], I Urbančič [1], E Sezgin [1], F Schneider [1], D Waithe [1], W Schliebs [4], R Erdmann [4], C Eggeling [1,2]

[1] MRC Weatherall Institute of Molecular Medicine, Oxford, UK; [2] Institute of Applied Optics and Biophysics, Jena, Germany; [3] Engineering Science, Oxford, UK; [4] Systemic Biochemistry, Bochum, Germany

Measuring diffusion dynamics in living cells is essential for understanding of molecular interactions. While many observation techniques have been used to explore such characteristics in the cellular plasma membrane, this is less developed for measurements inside the cytosol. An example of cytosolic action is the import of proteins into peroxisomes. These small organelles fulfil many metabolic functions, thereby importing all required proteins post-translationally. We combined advanced live-cell microscopy and spectroscopy techniques such as fluorescence correlation spectroscopy (FCS) and STED microscopy to characterize the diffusion and interaction dynamics of the peroxisomal import receptor PEX5, responsible for the recognition of peroxisomal matrix proteins in the cytosol. Among other features, we disclose an unexpectedly slow diffusion of PEX5, independent of many factors such as aggregation or target binding, but associated with larger cytosolic interaction partners. This sheds light on the functionality of the receptor in the cytosol. Besides these specific insights, our study highlights the potential of using complementary microscopy tools to decipher molecular interactions in the cytosol via studying their diffusion dynamics.

\section{P-82}

Simulation of molecular membrane diffusion models for an understanding of different experimental dynamic microscopy data

Agnes Koerfer [1], Francesco Reina [2], Christian Eggeling [1,2] [1] Friedrich-Schiller-Universität Jena, Germany; [2] Institut für Photonische Technologien, Germany

Diffusion of molecules in living cell membranes is influenced by several factors, such as interactions with other molecules and the characteristics of their environment. By observing the diffusion of biomolecules, we can study their interaction and hence function during different cellular processes. Here, we present a novel, versatile and open accessible program based on Python for simulating molecular membrane diffusion, specifically for three dominant models: Brownian diffusion, compartmentalized diffusion (with transient confinement in larger areas), and trapped diffusion (with transient slowdowns due to interactions). A Voronoi transformation on a uniform random distribution of seed points is performed for compartmentalized and trapping diffusion simulations to construct, respectively, the underlying network of distinct boundaries and random distributed trapping sites. We simulate signals obtained for different fluorescence microscopy measurement modes such as single-particle tracking and various Fluorescence Correlation Spectroscopy based approaches. Our ultimate goal is to develop a simulation toolbox that enables a better understanding of diffusion dynamic data obtained in living cells.

\section{P-81}

Characterization of membrane nanotubes in vitro between cultured B cells and in vivo in zebrafish embryos

Henriett Halász [1], Viktória Tárnai [2], Katalin Türmer [1], Miklós Nyitrai [1,3], János Matkó [4], Edina Szabó-Meleg [1,3] [1] Medical School, Department of Biophysics, Univ. of Pécs, Hungary; [2] Faculty of Natural Sciences, Institute of Biology, Univ. of Pécs, Hungary; [3] Szentagothai Research Centre, Univ. of Pécs, Hungary; [4] Department of Immunology, Eötvös Loránd Univ., Budapest, Hungary

Membrane nanotubes (NT) are long, actin based membrane covered cellular protrusions providing direct contact even between distant cells. These structures play crucial role in material transport processes (eg. in the transfer of cell organelles), in the progression of malignancies, in the intercellular spreading of pathogens, and in the deterioration of some neurological disorders. Molecular mechanisms mediating these transport processes are not completely understood yet. Results are available about the presence and function of NTs in vitro in the cultures of eg. immune cells, neurons, epithelial cells, although the important aspects of their growth and role in vivo need to be elucidated.

As B lymphocytes are key components of the immune response however their NTs are poorly characterized, in this study confocal and SIM microscopic techniques were used to examine the cytoskeletal components and their possible role in the transport processes via NTs of B-cells. We also aimed to reveal the in vivo existence of NTs in early-aged zebrafish embryos.

We proved that both actin and microtubule based motor proteins play role in nanotubular mediated transport processes and we found convincing evidence about the existence of NTs among the epiblast cells of 4 hour-aged zebrafish embryos.

\section{P-83}

Evaluation of red dyes performances at cryogenic temperatures

Montserrat López-Martínez, Arjun Sharma, Magdalena C Schneider, Ying-Ju Chen, Lukas Sparer, Gerhard J Schütz Institute of Applied Physics, TU Wien, Vienna, Austria

Over the last years, Single Molecule Localization Microscopy (SMLM) has established itself as an exceptional tool to address biological structures in the cellular environment. SMLM relies in the localization of the positions of individual fluorescent labels, achieving images with resolutions in the range of tens of nanometre. In a typical SMLM recording, only a small subset of the fluorophores in the sample are active at the same time. This subset of fluorophores switches or blinks stochastically, requiring to record long movies to ensure the detection of most of the labelled structures in the sample. These long acquisitions request for immobilized cells. Generally, this means chemically fixed cells, but recently the possibility to perform SMLM at cryogenic temperatures was demonstrated, opening up the door to examine cryofixated samples with SMLM.

Besides the superior preservation of biological samples, working at cryogenic temperatures comes with the added benefit of a slowing down of photobleaching of fluorophores, improving the achievable localization precision. However, it also affects the number of the fluorophore blinks. Although cryoSMLM is becoming popular in the past years, there is no clear consensus on how the cryogenic temperatures affect the blinking properties of fluorescent dyes. Here, we evaluated the emitted photons, photostability and blinking behaviour of several dyes of several SMLM dyes, with the aim of creating a library of dyes and its properties at cryogenic temperatures. 


\section{Posters}

-6. Advanced optical microscopy -

\section{P-84}

Evaluation of the effect of environmental pollution on water moss by time- and spectrally-resolved microscopy

Alzbeta Marcek Chorvatova [1,2], Martin Uherek [2], Dusan Chorvat [2]

[1] Dept of Biophysics, Fac of Natural Sciences, Univ of Ss Cyril and Methodius, Trnava, Slovakia; [2] Dept of Biophotonics, International Laser Centre, Bratislava, Slovakia

Natural sensing of changes in endogenous fluorescence is a very effective tool for monitoring responsiveness of living organisms to environmental pollution, also including the presence of microplastics and/or heavy metals. Combination of spectral and time-resolved (FLIM) microscopy was applied on intrinsic fluorophores of the sweet water bryophyte species Fontinalis antipyretica moss leafs to provide information about their modification in the presence of chosen environmental stressors. Nanoparticles (NPs) from various metals ( $\mathrm{Zn}, \mathrm{Ni}, \mathrm{Co}, \mathrm{Cu}$ ) were designed and fabricated by direct synthesis using femtosecond laser ablation in liquids. The effect of microplastics was evaluated using fluorescently labeled latex beads. We have observed differential effects of the chosen NPs on AF, as well as the capacity of the moss leafs to interact with microplastics. Gathered observations constitute the first step towards evaluation and proposal of new methodological approaches in optical microscopy for fast natural biosensing of the effects of environmental pollution directly in the water environment.

Authors acknowledge the European Union's Horizon 2020 research and innovation programme under the grant agreement no 871124 Laserlab-Europe V, as well as grant VEGA 2/0070/21.

\section{P-86}

Comprehensive fluorophore blinking analysis platform as a prerequisite for photoactivated localization microscopy

René Platzer [2], Benedikt Rossboth [1], Eva Sevcsik [1], Magdalena Schneider [1], Florian Baumgart [1], Hannes Stockinger [2], Gerhard J Schütz [1], Johannes B Huppa [2], Mario Brameshuber [1]

[1] Institute of Applied Physics, Biophysics Group, TU Wien, Austria; [2] Center for Pathophysiology, Infectiology and Immunology, Institute for Hygiene and Applied Immunology, Medical University of Vienna, Austria

Determining nanoscale protein distribution via Photoactivated Localization Microscopy (PALM) mandates precise knowledge of the applied fluorophore's blinking properties to counteract overcounting artifacts that distort the resulting biomolecular distributions.

Here, we present a readily applicable methodology to determine, optimize and quantitatively account for the blinking behavior of any PALM-compatible fluorophore. Using a custom-designed platform we revealed complex blinking of two photoswitchable fluorescence proteins (PS-CFP2 and mEOS3.2) and two photoactivatable organic fluorophores (PA Janelia Fluor 549 and Abberior CAGE 635) with blinking cycles on time scales of several seconds.

Incorporating such detailed information in our simulationbased analysis package allowed for robust evaluation of molecular clustering based on individually recorded single molecule localization maps.

\section{P-85}

A single-molecule approach to systematically study the narrow escape problem in micropatterned model membranes

Elisabeth Meiser [1], Reza Mohammadi [2], Nicolas Vogel [2], David Holcman [3], Susanne Fenz [1]

[1] Biocenter: Cell- and Developmental Biology, University of Würzburg, Germany; [2] Institute of Particle Technology, Friedrich-Alexander University Erlangen-Nürnberg, Germany; [3] IBENS - École Normale Supérieure, Paris, France

The narrow escape problem (NEP) is a common problem in biology and biophysics. It deals with Brownian particles confined to a domain with reflecting borders and one small escape window. The mean first passage time $(\tau)$, the mean time it takes a set of particles to escape, can be analytically calculated in $2 \mathrm{D}$ and $3 \mathrm{D}$ for several geometries. It depends on three parameters, which are the area of the domain, the size of the escape window, and the diffusion coefficient of the particle. We systematically tested the analytical solution of the NEP in $2 \mathrm{D}$ by variation of the escape opening $(\varepsilon)$. Experiments were complemented by matching random walk simulations. For the experimental test, we prepared micro-patterned phospholipid bilayers from a combination of colloid lithography and vesicle fusion. We then imaged fluorescently labeled lipids diffusing in circular membrane patches using single-molecule fluorescence microscopy. We present our results on membrane patterning and show how lifetime limitation of fluorescent probes can be overcome. Namely with a correction factor which rescales the measured mean first passage time by the fraction of escaped particles. We found that the dependence of the mean first passage time on the escape opening agrees well with the theoretical prediction of $\ln (1 / \varepsilon)$. We present a comparison of our experimental and simulation results with the theoretical prediction for the mean first passage time.

\section{P-87}

Ultrafast particle tracking of single lipid molecules in membranes through ISCAT and MINFLUX microscopy

Francesco Reina [1], Tobias Weihs [2], Christian A Wurm [2], B. Christoffer Lagerholm [3], Christian Eggeling [1,4]

[1] Leibniz-Institut für Photonische Technologien e.V., Germany; [2] Abberior Instruments GmbH, Germany; [3] Wolfson Imaging Centre Oxford, University of Oxford, UK; [4] Institute of Applied Optics and Biophysics, Friedrich Schiller Universität, Germany

The diffusion dynamics of lipids on cellular membranes have always proven particularly challenging to study in optical microscopy, given the highly heterogeneous nature of their environment. Given the breadth of the relevant spatiotemporal scales to detect such heterogeneities, very few optical techniques are able to probe these intervals with single molecule level of detail. Interferometric Scattering (ISCAT) microscopy, for example, can reach kilohertz sampling rates with high signal to noise ratios. We employed this technique to probe the diffusion of single lipids on cellular membranes. Using a novel analysis approach to the single particle trajectories thus collected, we were able to uncover new details pertaining to the compartmentalization of the cellular membrane at the nanoscale. The recently developed MINFLUX offers a new outlook on this problem, thanks to the simultaneous reliance on non-invasive synthetic fluorescent tags, and high signal to noise ratios obtained through minimal photon fluxes. We will also show preliminary data to demonstrate the potential of this new approach to the detection of single particle diffusion in biological samples. 
Posters

-6. Advanced optical microscopy -

\section{P-88}

Sizing oligomeric biomolecular structures with cryo-SMLM

Magdalena C Schneider [1], Roger Telschow [2], Gwenael Mercier [2], Montserrat López-Martínez [1], Otmar Scherzer [2], Gerhard J Schütz [1]

[1] Institute of Applied Physics, TU Wien, Vienna, Austria; [2] Faculty of Mathematics, University of Vienna, Vienna, Austria

Single molecule localization microscopy (SMLM) has enormous potential for resolving subcellular structures at the nanometer scale. In order to record localization microscopy data, sample fixation is inevitable to prevent molecular motion during long recording times of minutes up to hours. Crucially, preservation of the sample's ultrastructure during fixation becomes a limiting factor when using chemical fixatives. Performing SMLM at cryogenic temperatures promises to resolve this issue, thus allowing for investigation of nanoscale structures in their undistorted native state. Notably, fluorophore dipoles are fixed at low temperatures, offering the unique possibility to use the dipole orientation as additional information for analysis. Thus, localizations can be assigned to individual fluorophores with high reliability, which greatly improves the quality of particle reconstruction approaches. We developed a method for precise sizing of oligomeric structures and extensively characterized its performance based on simulations. Using the new approach for localization assignment, side lengths could be determined with a relative error of less than $1 \%$ for tetramers with a nominal side length of $5 \mathrm{~nm}$, even if the assumed localization precision for single molecules was more than $2 \mathrm{~nm}$.

\section{P-90}

Reducing the effect of saturation phenomena on the evaluation of FRET measurements

Tímea Szendi-Szatmári, Ágnes Szabó, János Szöllősi, Péter Nagy

University of Debrecen, Hungary

Although Förster resonance energy transfer (FRET) is one of the most widely used biophysical methods in biology, the effect of high excitation intensity, leading to donor and acceptor saturation, has not been addressed previously. Here, we present a formalism for the experimental determination of the FRET efficiency at high excitation intensity when saturation of both the donor and the acceptor significantly affect conventional FRET calculations. We show that the proposed methodology eliminates the dependence of the FRET efficiency on excitation intensity, which otherwise significantly distorts FRET calculations at high excitation intensities commonly used in experiments. Under most experimental conditions, the distortion effect is attributable to saturation of the donor providing a very simple way of taking this phenomenon into consideration. The work presented here adds additional rigor to FRET-based investigation of protein interactions and strengthens the device independence of such results.

\section{P-89}

Measuring the molecular dynamics at nanoscale in cell membrane with LoCCS

Komala Shivanna, Jaqulin Wallace, Samuel T Hess

Department of Physics and Astronomy, University of Maine, Orono, USA

Fluorescence photoactivation localization microscopy (FPALM) enables imaging single molecules in biological specimens with nanoscale spatial resolution. This technique is well-suited to determination of the spatial distribution of one or more species of interest at length scales as small as a few tens of nanometers. In addition, it is useful to quantify molecular kinetics at similar length scales. We therefore introduce a method of integrating FPALM with fluorescence cross-correlation spectroscopy which we name Localization cross correlation spectroscopy (LoCCS). LoCCS can also be applied to follow molecular interactions in solution and in cells, quantify colocalization, and characterize co-diffusion. This method can be implemented on a standard FPALM setup with a relatively fast camera, and has the ability to measure protein-protein interactions and diffusion dynamics in cellular membranes. We present the concept of LoCCS, experimental details, sample preparation, data acquisition and analysis. Results show the localization and diffusion of beads in vitro and molecules of the influenza viral protein hemagglutinin (HA) in living cells.

\section{P-91}

Evaluating the effect of different expansion microscopy protocols on mammalian DNAchromatin architecture

Chantal Usai [1,2], Michele Oneto [1], Isotta Cainero [1], Francesca Baldini [1], Agnieszka Pierzyńska-Mach [1], Paolo Bianchini [1], Alberto Diaspro [1,2]

[1] Nanoscopy, Istituto Italiano di Tecnologia, Italy; [2] DIFILAB, University of Genoa, Italy

Expansion microscopy (ExM) is a novel super-resolution microscopy method that enables nano-scale biophysical studies by increasing the distance among biomolecules. Such effect is achieved through the physical magnification of the specimen obtained by its embedding inside a swellable polymer network, eventually expanded in water. Several improvements to the original ExM technique were made to optimize the imaging of proteins and the isotropicity of their expansion. However, few works have been done to investigate what happens to the DNA-chromatin architecture once it's subjected to the mechanical strain associated with the expansion process. To understand whether this technique could be suitable for investigating the crowded nuclear environment, we aim to study the induced changes in the DNA conformation by the application of different expansion microscopy protocols to fixed mammalian cells and single mononucleosomes. Optical imaging will be performed using a combination of confocal, two-photon excitation and super-resolution microscopy techniques, and the performances of different fluorophores for DNA staining will also be compared. Finally, we aim to develop a reproducible protocol for the precise quantification of the expansion factor of the specimens at the microscale. 


\section{Posters}

-6. Advanced optical microscopy -

\section{P-92}

3D superresolution fluorescence microscopy on immunological synapse

Lukas Velas [1], Philipp Zelger [2], Alexander Jesacher [2], Gerhard J Schütz [1]

[1] Institute of Applied Physics, TU Wien, Vienna, Austria; [2] Institute for Biomedical Physics, Medical University of Innsbruck, Innsbruck, Austria

T-cells are responsible for recognition of antigens in our bodies. T-cell activation is triggered upon binding of T-cell receptor (TCR) to major histocompatibility complex loaded with antigenic peptide (pMHC) on the surface of antigen presenting cells (APC). According to the kinetic segregation model, T-cell topography plays a large role in the antigen recognition process. In this study we have applied a $3 \mathrm{D}$ superresolution method to study the spatial organization of the T-cell receptor within the immunological synapse with isotropic localization precision of $15 \mathrm{~nm}$. The method combines stochastic optical reconstruction microscopy (STORM) with defocused imaging that exploits effects of the supercritical angle fluorescence on the shape of the point spread function. In order to study the topography of the immunological synapse we mimicked the surface of APCs using supported lipid bilayers functionalized with $\mathrm{pMHC}$, the co-stimulatory protein B7, and the adhesion molecule ICAM. When activated, T-cells show smaller membrane fluctuations compared to the resting condition. In the resting state we could see differences between high and low concentration of ICAM on a bilayer, where the T-cell membrane topology differs already on the scale of $50 \mathrm{~nm}$.
P-93

Quantitative modeling of super-resolved structures in localisation data

$\underline{\text { Yu-Le Wu }}[1], \quad$ Philipp Hoess [1], Aline Tschanz [1], Ulf Matti [1], Markus Mund [2], Jonas Ries [1]

[1] Cell Biology and Biophysics Unit, European Molecular Biology Laboratory, Germany; [2] Department of Biochemistry, University of Geneva, Switzerland

Quantitative analysis in super-resolution microscopy is still a bottleneck, specifically for single-molecule localisation microscopy (SMLM) data, which are lists of localisation coordinates. Structures are typically analysed by simple geometric evaluation or by averaging. However, an approach to quantify spatial parameters by fitting a parametrized model (underlying geometry) directly to localisations is yet missing. To fill this gap, we developed LocMoFit (localisation model fit), an open-source framework, based on maximum likelihood estimation, for fitting an arbitrary model as a probability density function to localisation data, which are assumed to be observations drawn from the model.

With various structures (nuclear pore complex, microtubules, and the endocytic machinery, either in $2 \mathrm{D}$ or $3 \mathrm{D}$ ), we demonstrate applications of LocMoFit: how spatial parameters can be extracted, how dynamics can be reconstructed based on snapshots, how to select the most likely model among many, how protein assemblies with many colours can be reconstructed, how to perform particle fusion even without a model, and how to simulate SMLM data for proper controls and testing, along with visualization routines.

We anticipate the versatile LocMoFit can increase the quantitative information that can be extracted from SMLM data. 


\section{Posters}

- 7. Protein translocation, assembly, and folding -

\section{P-94}

Structural analysis of proteins forming the bacterial ribosome tunnel

Michaela Cerneková, Michal H Kolář

University of Chemistry and Technology, Prague, Czech Republic

Ribosomes are ribonucleoprotein particles responsible for synthesis of a nascent chain, during which is messenger ribonucleic acid translated into amino acids. They play a crucial role in all three domains of life. The nascent chain leaves the ribosome through an exit tunnel located within the large ribosomal subunit. Plentiful interactions can be found between the nascent peptide and the tunnel walls, as an illustration, with the narrowest part formed by extended loops of two ribosomal proteins named uL4 and uL22. In addition, uL4 and uL22 also have globular parts at the surface of the ribosome. The proteins can through the globular parts interact with other proteins associated with the ribosomes. It is not fully clear what roles play the two domains of the ribosomal proteins contributing to the tunnel walls and why the proteins evolved into their shapes. We address these questions by analyzing a set of experimental ribosome structures found in the Protein Data Bank. Root-mean-square fluctuation analysis reveals the flexible and the rigid sections, e.g. some of the most flexible amino acids of uL22 were at the tip of the loop intervening the tunnel. Principal component analysis of Cartesian coordinates suggests that some elements are structurally correlated. Sequence alignment complements the analyses as it offers an insight into conserved sections of the proteins and whether the critical ones are included. Observations from these analyses contribute to our understanding of ribosome function and regulation.

\section{P-96}

Effect of Hofmeister cations on $\alpha$-lactalbumin amyloid fibrillization

Andrea Antosova [1], Miroslav Gancar [1], Eva Bystrenova [2], Zuzana Bednarikova [1], Jozef Marek [1], Zuzana Gazova [1]

[1] Institute of Experimental Physics SAS, Kosice, Slovakia; [2] Consiglio Nazionale delle Ricerche - Istituto per lo Studio dei Materiali Nanostrutturati (CNR-ISMN) via P. Gobetti 101, 40129 Bologna, Italy

Amyloid fibrils have been formed in vitro from diseaseassociated as well as disease-unrelated proteins and peptides. $\alpha$-lactalbumin ( $\alpha$-LA) is a suitable model protein for amyloid aggregation study due to its ability to form a molten globular state. Using a multi-technique approach, we have compared the effect of a variety of cations from the Hofmeister series $\left(\mathrm{NH}_{4}^{+}, \mathrm{Cs}^{+}, \mathrm{K}^{+}, \mathrm{Na}^{+}, \mathrm{Mg}^{2+}, \mathrm{Ca}^{2+}\right)$ in the modality of chloride salts at two different concentrations on the amyloid formation of $\mathrm{Ca}^{2+}$-depleted $\alpha$-LA. The kinetics, the content of $\beta$-structure and amyloid fibril morphology have been studied using ThT and Trp fluorescence, FTIR spectroscopy and AFM microscopy. We found out, that the effect of cations on kinetic parameters of $\alpha$-LA amyloid formation and morphology of $\alpha$-LA fibrils is strongly correlated to salts concentration and their position in the Hofmeister series. The obtained results might contribute to a better understanding of the processes of amyloid self-assembly of globular proteins. This work was supported by the grants VEGA 2/0176/21, APVV18-0284, and the MIUR grant (PRIN 20173L7W8K).

\section{P-95}

The structure of human serum albumin upon interaction with catechin and metal ions

Karina Abrosimova, Tigran Akopian, Anton Gorokh, Sofia Paston, Svetlana Tankovskaia

Saint-Petersburg State University, Russian Federation

Human serum albumin (HSA) is the prevailing protein in the blood plasma. Its natural function as a universal lowmolecular compounds carrier as well as a promising ability to serve as a base for drug delivery systems rivet researcher's attention. Understanding the HSA structure upon its interaction with metal ions and bioactive molecules is necessary for biomedical applications. Catechin is one of the plant polyphenols displaying antioxidant activity and using in the development of new therapeutic forms and nanoparticle synthesis. This work is devoted to studying alteration in HSA structure in solutions containing mono- and divalent cations and catechin by the methods of UV absorbance, fluorescent and FTIR spectroscopy, spectrophotometrical melting, and diffusion layer potential measure.

The net charge of HSA at neutral $\mathrm{pH}$ in water solution is negative $(-15 \mathrm{e})$. In the presence of alkaline and alkaline-earth metal ions albumin's globule is more stable, than in water, and its charge tends to zero, whereas in the presence of transition metal ions strong protein aggregation is observed and assembled particles are positively charged. Catechin stabilizes tertiary and secondary structures of HSA but does not prevent its aggregation caused by transition metal ions.

A part of this work was performed at the Centre for Optical and Laser Materials Research (COLMR) in Research park of St.Petersburg State University.

\section{P-97}

The role of salt-bridge stability in the initial steps of insulin fibrillation

Z. Bednarikova $[1]$, B. N Ratha [2], R. K Kar [2], S. A Kotler [3], S. Raha [4], S. De [5], N. C Maiti [6], A. Bhunia [2], Z. Gazova [1]

[1] IEP, SAS, Kosice, Slovakia; [2] Dept. of Biophysics, Bose Institute, Kolkata, India; [3] NCATS, NIH, USA; [4] Dept. of Physics, Bose Institute, Kolkata, India; [5] School of Bioscience, IIT Kharagpur, India; [6] Struct. Biol. and Bioinformatics, CSIR - IICB, Kolkata, India

We have studied how structural and sequence similarity of insulin variants from human (HI), bovine (BI), and glargine (GI) influence their thermal stability and aggregation propensity. We investigated the structural features and kinetics of fibrillation to shed light on the role of B-chain C-ter dynamics and salt-bridge stability in the initial steps of insulin fibrillation. Kinetic analysis showed that GI fibrillation is slower than BI and HI. AFM imaging confirmed the longer lag phase of GI fibrillation. After $42 \mathrm{~h}, \mathrm{BI}$ and HI formed fibrillar species; but, only globular oligomers of GI were observed. These data point to GI's higher stability due to two additional Arg residues, Arg31B and Arg32B. NMR experiment showed atomic contacts and residue-specific interactions, particularly the salt-bridge and $\mathrm{H}$-bond formed among C-ter residues Arg31B, Lys29B and Glu4 in GI. We propose that enhanced stability of native GI by strengthening salt bridge can retard tertiary collapse, a crucial event for oligomerization. The fluctuation of the B-chain C-ter residues plays a key role in the growth phase of insulin fibrillation. 


\section{Posters}

- 7. Protein translocation, assembly, and folding -

\section{P-98}

Perturbing the unfolding of CRABP I using polyethylene glycol: An experimental and theoretical study

Krishnendu Bera [1], Suchismita Subadini [2], Jozef Hritz [1,3], Harekrushna Sahoo [2]

[1] CEITEC MU, Masaryk University, Kamenice 753/5, Brno, Czech Republic; [2] Biophysical and Protein Chemistry Lab, Department of Chemistry, NIT Rourkela, India; [3] Department of Chemistry, Masaryk University, Kamenice 5, Brno, Czech Republic

Proteins significantly affected by crowding nature of macromolecules. Thus, it is pertinent to investigate the role of crowding environment on the denaturation and renaturation kinetics of protein. Different molecular weights of Polyethylene glycol (PEG) have been considered as a molecular crowders and CRABP I (cellular retinoic acid binding protein I), as a model protein in this study. The secondary structure analysis was performed by circular dichroism (CD) spectroscopy and the unfolding kinetics using intrinsic fluorescence of CRABP I at $37^{\circ} \mathrm{C}$ to mimic the in vivo condition. Both PEG 2000 and PEG 4000 act as stabilizers by hamstring the unfolding kinetic rates. The unfolding kinetic slopes were different for both PEG, which indicating PEG favour compact conformations of protein as a function of concentration and molecular weight. The molecular dynamics (MD) studies revealed that PEG 2000 molecules assembled together during the $500 \mathrm{~ns}$ simulation, which is increasing the stability and percentage of $\beta$-sheet of the protein. The experimental findings were well supported by the theoretical results.

\section{P-100}

Monte Carlo simulations for the evaluation of oligomerization data in TOCCSL experiments Clara M Bodner [1], Dominik Kiesenhofer [2], Mario Brameshuber [1], Gerhard J Schütz [1]

[1] TU Wien, Institute of Applied Physics, Biophysics Group, Austria; [2] Institute for Quantum Optics and Quantum Information Innsbruck of the Austrian Academy of Sciences, Austria

The stoichiometry of cell-surface proteins is fundamental to cellular signaling and function. Amongst others, the singlemolecule fluorescence microscopy technique "Thinning Out Clusters while Conserving the Stoichiometry of Labeling" (TOCCSL) is used to study the oligomerization state of plasmamembrane proteins in life cells. In TOCCSL, a subregion of the plasmamembrane is stoichiometrically photobleached. At the onset of the recovery process, individual fluorescently labeled proteins can be imaged as diffraction limited singlemolecule signals.

Here, we present Monte Carlo-based computer simulations of TOCCSL experiments. Our simulations clearly show, how the selection of parameters in a TOCCSL experiment influences the results in regard to the oligomerization state. We were able to understand individual and combined error sources including incomplete photobleaching, oligomerization state-dependent mobility differences and false-positive oligomer detection due to random encounters. Strategies are suggested for optimizing TOCCSL experiments in regard to which choice of parameter-sets and data post-processing methods yield optimized results.

\section{P-99}

Modification of GPCR solubility by ribosome display

M. Berta [1], V. Dzurillová [1], M. Tomková [2], E. Sedlák [2] [1] Department of Biophysics, Faculty of Science, UPJS̆, Košice, Slovakia; [2] Center for interdisciplinary biosciences, TIP, UPJŠ, Košice, Slovakia

G-protein coupled receptors (GPCRs) are included in multitude of physiological responses to many signalling molecules and thus they belong to major drug targets. Effective drug design targeting GPCR's strongly depends on highresolution structures. The main problem in effort of crystallization of GPCRs is associated with their low solubility. In extreme case converting receptor to water-soluble form could overcame this obstacle. In the present research, we attempt to convert $\mathrm{\kappa}$-opioid receptor to a water-soluble form by redesigning the receptor surface. We substituted the hydrophobic amino acids of protein surface with suitable polar or charged residues, to produce correctly foldable and functional molecule in aqueous solution. For the reaching of desired result we have chosen an interdisciplinary approach of in vitro protein evolution by the method of ribosome display. The selected binders of the receptor library are variants of $\kappa$-opioid receptor and were purified without using a detergent. However, so far selected receptors form water-soluble oligomers. Despite the robustness of this selection method, we weren't able to evolve water-soluble functional $\kappa$-opioid receptor, which would form monomeric or dimeric functional unit. Involvement of rational design in final steps of increasing solubility might lead to preparation of water-soluble analogue of monomeric and/or dimeric structure of the receptor.

\section{P-101}

Hofmeister effect on catalytic efficiency and conformation of $3 \mathrm{C}$ protease

Eva Dušeková [2], Mária Tomková [1], Martin Berta [2], Veronika Dzurillová [2], Dagmar Sedláková [3], Erik Sedlák [1]

[1] Center for Interdisciplinary Biosciences, TIB, UPJS, Košice, Slovakia; [2] Dpt. of Biophysics, UPJS, Košice, Slovakia; [3] Institute of Experimental Physics, SAS, Košice, Slovakia

Effect of Hofmeister sulfate, chloride, bromide, and perchlorate, on catalytic properties and stability of $3 \mathrm{C}$ protease has been studied by absorbance, CD spectroscopies and differential scanning calorimetry. 3C protease specifically cleaves within a recognition sequence LEVLFQ $\downarrow$ GP. Due to its high cleavage specificity, the protease is a useful tool to cleave recombinant proteins that are expressed as a fusion protein consisting of the carrier domain and the protein of interest. The 3C-like protease is the main protease found in coronaviruses. Catalytic activity and stability of protease is dependent on salt concentration and position of anion in Hofmeister series. The catalytic efficiency of 3C protease increases more than 15-fold in the presence of $1 \mathrm{M}$ kozmotropic sulfate anions but is nearly unaffected in the presence of $1 \mathrm{M}$ chaotropic perchlorate anions when compared with the situation in low ionic buffer. Absence of any detectable conformational changes by $\mathrm{CD}$ and the positive correlation between stability and activity of chymotrypsin indicate the interdependence of the latter enzyme properties and agrees with recently developed macromolecular rate theory suggesting an important role of protein dynamics in enzyme catalysis. 
Posters

- 7. Protein translocation, assembly, and folding -

\section{P-102}

Role the conformational and colloidal stability on time-dependence solubility of human myeloma antibody $\lambda$-light chain

Veronika Džupponová [1], Gabriel Žoldák [2]

[1] Department of Biophysics, Faculty of Science, Pavol Jozef Šafárik University, Košice, Slovakia; [2] Center for Interdisciplinary Biosciences, Technology and Innovation Park, Pavol Jozef Safárik University, Košice, Slovakia

Multiple myeloma (MM) is the hematological malignancy connected with uncontrolled proliferation and accumulation of the altered plasma cells in the bone marrow. MM cells produce a light chain of IgG (LC) in huge concentrations. Under normal conditions, dimeric LCs are cleared in 3-6 hours; larger oligomers of LC, however, circulate longer time and form insoluble protein deposits in the vital organs. To understand the role of the conformational and colloidal stability on time-dependence solubility of human myeloma antibody $\lambda$-light chain, we prepared recombinant human $\lambda$-light chain protein derived from the MM patient. We found that this process contains irreversible LC unfolding, which is a unimolecular step with an activation energy of $260 \mathrm{~kJ} / \mathrm{mol}$. After the LC unfolding, the protein aggregates rapidly. The rate-limited step is the bimolecular reaction with an energy barrier of $40 \mathrm{~kJ} / \mathrm{mol}$. By combining experimental assays and kinetic modeling, we were able to extrapolate timedependent protein solubility to physiological temperatures.

\section{P-104}

Effect of mutation and a corrector molecule on the nanomechanics of CFTR nucleotide binding domain 1 (NBD1)

B Farkas [1], R Padanyi [1], H Tordai [1], B Kiss [1], H Grubmüller [2], N Soya [3], G Lukacs [3], M Kellermayer [1], T Hegedüs [1]

[1] Semmelweis University, Hungary; [2] Max Planck Institute, Germany; [3] McGill University, Canada

Mutations, in the gene encoding the CFTR chloride channel, cause cystic fibrosis $(\mathrm{CF})$, by disrupting the channel function, folding, and stability. The first nucleotide binding domain (NBD1) of CFTR, is a hot spot for mutations, including the most frequent F508 deletion ( $\Delta$ F508). CFTR folding correctors are invoked in multiple domain stabilizations, but their molecular mechanism is poorly understood. Since suppressing misfolding at the early steps of conformational maturation may manifest in improved therapies, a detailed knowledge of the channel folding pathway is required. Our goal was to characterize the wild type (WT) and $\Delta \mathrm{F} 508$ NBD1 (un)folding by using computational and experimental methods. We performed molecular dynamics simulations with constant velocity pulling of the WT and $\Delta$ F508 NBD1. To validate the in silico results, we performed atomic force microscopy experiments. The presence of NBD1 corrector molecule BIA impeded unfolding of the NBD1 $\alpha$-subdomain and its binding site was inferred from HDX-MS experiments. Our results revealed multiple unfolding pathways altered by $\Delta \mathrm{F} 508$ and weak structural elements that emerge as putative folding-sensitive points thus potential drug targets for $\mathrm{CF}$ treatment.

\section{P-103}

Development of haloalkane dehalogenases by ribosome display

Veronika Dzurillová [1], Martin Berta [1], Mária Tomková [2], Erik Sedlák [2]

[1] Department of Biophysics,Faculty of Science, P.J.Šafárik University, Jesenná 5, Košice, Slovakia; [2] Center for Interdisciplinary Biosciences, Technology and Innovation Park, P.J.Safárik University, Jesenná 5, Košice, Slovakia

Haloalkane dehalogenases (HLDs) are microbial $\alpha / \beta$ hydrolases catalyzing the hydrolytic cleavage of carbonhalogen bonds in various aliphatic halogenated hydrocarbons. Among their substrates are also prominent environmental pollutants, which HLDs help to degrade. HLDs have a significant potencial applications such as biocatalysis, bioremediation, chemical synthesis, biosensing or cell imaging, however, their widespread utilization is still hindered by many limitations. The most crucial limitations are their low stability and insufficient catalytic efficiency. To address the issue of low catalytic efficiency of selected dehalogenases, we applied the method of protein directed evolution ribosome display. Here we present successful selection of DhaA and DhaA-Halotag variants from gene library prepared by random mutagenesis upon three or four consecutive rounds of ribosome display. Based on following sequence analysis, we identified several hot-spot mutations. Surprisingly, extensive deletion of 13 aminoacids have been identified in a region important for selective recognition and kinetics of substrate binding. Biophysical and biochemical analyses of these enzyme variants suggest feasibility of using ribosome display in evolution-directed engineering of enhanced HLDs.

\section{P-105}

Controlled self-assembly of alpha-lactalbumin microparticles for pH-responsive release of protein material

Dirk Fennema Galparsoro [1], Valeria Vetri [1], Vito Foderà [2] [1] Dipartimento di Fisica e Chimica, Università degli Studi di Palermo, Italy; [2] Department of Pharmacy, University of Copenhagen, Denmark

Due to a low cytotoxicity, enhanced drug-loading and low immunogenicity, protein microparticles have been recently attracting a large interest for potential applications in drugdelivery. Such microstructures form when protein solutions are exposed to high temperature and the net charge of the protein molecules is insignificant. Temperature induces protein partial unfolding promoting a fast aggregation driven by hydrophobic interactions. Such condensation phenomena result in perfectly spherical microparticles with a similar secondary structure to the protein's native conformation.

Here we present a study on protein microparticles made of $\alpha$-lactalbumin, with a focus on how varying the initial conditions of the solution alters the properties of produced aggregates. In particular, we can control the size and structural features of the particles by changing the initial protein concentration and the time of thermal incubation. Results indicate that such changes translate into differences in the microspheres stability. It is possible to disassemble in a controlled manner these aggregates by simply changing the $\mathrm{pH}$ of the solution. The ability to release protein material at different $\mathrm{pH}$ together with the possibility of loading small molecules paves their development as drug delivery systems. 


\section{Posters}

- 7. Protein translocation, assembly, and folding -

P-106

Elongation factor $\mathrm{G}$ mutations and bacterial resistance to aminoglycosides

Sara Gabrielli, Lars V Bock, Helmut Grubmüller

Max Planck Institute for Biophysical Chemistry, Germany

Elongation Factor G (EF-G) is a GTPase that is involved in protein translation. After peptide bond formation, EF$\mathrm{G}$ binds to the ribosome, hydrolyzes GTP and accelerates tRNA translocation. It has been observed that diseasecausing bacteria containing certain mutants of EF-G display resistance towards aminoglycosides, which are a class of broad-spectrum antibiotics. Aminoglycosides induce errors in the decoding of the mRNA and, subsequently, inhibit protein synthesis. During tRNA translocation, EF-G undergoes conformational changes, e.g, a rotation of domains 4-5 relative to domains 1-3. This observation suggests that if EF-G dynamics were altered by the mutations, this effect could play a fundamental role in the resistance mechanism. Interestingly, the EF-G mutations that have been identified in different resistant bacteria are distributed over all EF-G domains in internal and in exposed regions. Specifically, the more internal locations hint that a change of the internal EF$\mathrm{G}$ dynamics, independent of the interactions with the ribosome, would contribute to the resistance mechanism. Here, we use extensive all-atom MD simulations of wild-type and mutated E. Coli EF-G in solution to investigate the effect of these mutations on the internal dynamics and energetics of EF-G. Indeed, from the simulations, we observe that for certain mutations the dynamics of EF-G domains 4-5 is restricted, providing a possible explanation for their resistance mechanism in entropic terms.

\section{P-108}

Oxidation effects in antiaggregogenic properties of Epigallocatechingallate

Daniele Gulli [1], Giuseppe Sancataldo [1], Caterina Alfano [2], Bruno Pignataro [1], Valeria Vetri [1]

[1] Department of Physics and Chemistry, University of Palermo, Italy; [2] Structural Biology and Biophysics Group, Fondazione Ri.MED, Italy

Epigallocatechin-gallate (EGCG), the most abundant flavonoid in green tea, has been extensively studied for its potential in the treatment of amyloid related disorders. This molecule was found to modulate abnormal protein selfassembly, reducing resulting cellular toxicity. EGCG is known to suppress or to slow down the aggregation processes of several proteins, thus supporting the idea that general mechanisms regulate its anti-aggregogenic effects and, interestingly, in the oxidised form it demonstrated an higher efficiency in reducing protein aggregation with respect to intact molecule.

We here investigate the effects of intact and oxidized EGCG the thermal aggregation pathway of Bovine Serum Albumin (BSA), a well-known model protein whose aggregation processes are known in details.

By means of different spectroscopic methods, we evaluate similarities and differences of the two molecules during protein aggregation. Different solution conditions are investigated, close and away from the isoelectric point of the protein, with the aim of eliciting the role of electrostatics in the observed EGCG-Protein interaction and in the supramolecular assembly which are dramatically dependent on solution conditions.

\section{P-107}

Coarse grained simulations of amyloidogenic proteins in the context of Alzheimer's disease Lianne D Gahan, Alexander I P Taylor, Buddhapriya J Chakrabarti, Rhoda J Hawkins, Rosie A Staniforth

University of Sheffield, UK

Using an in-house dynamic Monte Carlo simulation scheme in the NVT ensemble, the aim is to produce fibrillar aggregates and the formation of toxic oligomeric structures. We use a coarse grained approach whereby monomers of amyloid forming proteins are represented by spherocylinders which interact via attractive patches.

By modelling an inhibiting molecule as a sphere, the primary aim is to disrupt the formation of such aggregates to provide insight into the mechanisms of inhibition and to predict characteristics of drug treatment targets in neurodegenerative diseases.

\section{P-109}

$\mathrm{pH}$ effects on the stability and evolution of the folding landscape in caspases

Isha Joglekar, Clay Clark

University of Texas at Arlington, USA

Caspases are an ancient class of cysteinyl proteases that play an integral part in cell development and apoptosis as an evolutionarily conserved function. During apoptosis, the intracellular $\mathrm{pH}$ decreases from 7.4 to $\sim 6.8$ and cytosolic acidification affects the activation of caspase- 3 and $\mathrm{pH}-$ dependent conformational changes affect the rate of auto maturation in the dimeric procaspase-3. This protein folding landscape is sculpted through evolution by selection of stabilizing residues, while repressing the non-native ones. To understand how the energy landscapes changed over time, we resurrected an ancestral caspase (existed about 650 milllion years ago) and we examined the folding pathways of caspases from Cnidarians, specifically corals. We studied the equilibrium unfolding of caspases from O.faveolata (species susceptible to disease) and P.astreoides (species resistant to disease) as a function of $\mathrm{pH}$ (between $\mathrm{pH} 3$ and $\mathrm{pH}$ 10.5) to examine the changes in the context of stability and folding. The data shows that these monomeric proteins unfold via a two-state process at lower $\mathrm{pH}$ and a three state process at a higher $\mathrm{pH}$ with an overall free energy change of about 13 $\pm 0.5 \mathrm{kcal} / \mathrm{mol}$ for the resistant species, whereas about $19 \pm$ $0.8 \mathrm{kcal} / \mathrm{mol}$ for the susceptible species. We also observe the presence of some unstable intermediates and molten globule like structures due to hydrophobic aggregation of the partially unfolded states at a certain range of $\mathrm{pH}$. 
Posters

- 7. Protein translocation, assembly, and folding -

\section{P-110}

Long term protein stability prediction using ' $\mathrm{mi}$ cro' changes in secondary structure measured using microfluidic modulation spectroscopy

Patrick J King

RedShift BioAnalytics, USA

Microfluidic Modulation Spectroscopy (MMS) is a revolution in protein IR spectroscopy, bringing together microfluidic optics with a powerful quantum cascade laser to provide greater measurement sensitivity and reproducibility than possible with established techniques for secondary structure determination, such as Circular Dichroism (CD) and Fourier Transform Infra-Red (FTIR) spectroscopy. Furthermore, due to the optics used, a much greater concentration range is accessible for structural analysis, from 0.1 to over $200 \mathrm{mg} / \mathrm{ml}$. Finally, measurements do not require any labelling and can be made directly, even in complex buffer systems that contain usually difficult species for spectroscopy, such as complex buffers, excipients, adjuvants and surfactants.

In this poster we present a study where the sensitivity and reproducibility of MMS was used to distinguish very small differences in secondary structure between a set of samples held at elevated temperature for weeks in order to simulate long-term stability over years. Though structural differences between samples were shown to be less than $1 \%$ of their overall structure, measurements were demonstrated to be repeatable enough that this 'micro' change could be used to predict stability over long time periods. Predictions were validated by the user from longer-term studies, demonstrating how MMS can be used to greatly accelerate your stability studies where existing technologies cannot.

\section{P-112}

Natural polar phenols affect differently the structure of apoE4 forms and ameliorate apoE4 functions related to Alzheimer's disease pathogenesis Christina Mountaki, Ioannis Dafnis, Angeliki Chroni

National Center for Scientific Research "Demokritos", Athens, Greece

Apolipoprotein E4 (apoE4) is the major risk factor for Alzheimer's disease (AD). We have shown that the hereditary apoE4 mutant apoE4[L28P] and the apoE4 fragment apoE4-165 promote intraneuronal $\mathrm{A} \beta$ accumulation and oxidative stress. Polar phenols found in grapes and currants are proposed to protect against pathogenic processes underlying $\mathrm{AD}$, such as oxidative stress. The effect though of polar phenols on the functions of apoE4 forms is largely unexplored. We examined the effect of polar phenols resveratrol, quercetin, kaempferol and epigallocatechin gallate, found in currants (Vitis vinifera L.), a common Greek product, on AD-related functions of apoE4 forms. All polar phenols prevented the formation of reactive oxygen species induced by $\mathrm{A} \beta$ uptake in SK-N-SH cells treated with apoE4[L28P] or apoE4-165. Investigation of the mechanism of action of these compounds showed that resveratrol prevented cellular $\mathrm{A} \beta$ uptake via changes in cell membrane fluidity. Kaempferol prevented cellular A $\beta$ uptake by apoE4[L28P], but not by apoE4-165, due to a modulating effect on apoE4[L28P] secondary structure and stability. The action of quercetin and epigallocatechin gallate could be attributed to free radicalscavenging activity. Overall, we show for the first time that natural compounds could modify the structure of apoE4 forms and ameliorate AD-related effects of apoE4.

\section{P-111}

Dissecting the target recognition by CRISPR/Cas effector complexes with ultrafast twist measurements

Julene Madariaga-Marcos [1], Dominik J Kauert [1], Marius Rutkauskas [1], Alexander Wulfken [1], Inga Songailiene [2], Tomas Šinkūnas [2], Virginijus Šikšnys [2], Ralf Seidel [1]

[1] Universität Leipzig, Germany; [2] Vilnius University, Lithuania

CRISPR/Cas effector complexes have been recently applied in genome-editing, since they can bind practically any genomic target by tuning their intrinsic RNA component. They interrogate DNA looking for target sequences that are complementary to the RNA. Upon target recognition, base pairing between RNA and DNA forms a so-called R-loop, with a considerable tolerance for mismatches. To prevent off-targeting, quantitative modelling of the recognition process would be desirable, which requires knowledge about the energy landscapes of the R-loop formation. Determining such landscapes experimentally requires a real-time observation of the R-loop expansion/collapse at the single base-pair level. Our approach is to measure DNA unwinding during R-loop formation using ultrafast twist measurements, using DNA origami nanostructures as rotor arms, directly reporting about twist changes in DNA on a ms time scale. We resolve real-time R-loop dynamics for the Cascade effector complex and construct corresponding energy landscapes in the presence of mismatches. We achieve unique insight in the R-loop formation process, reporting mismatch penalties and resolving the 6 nucleotides periodicity for flipped out bases.

\section{P-113}

Bis-coumarin homodimers - towards understanding of anti-amyloid potential on globular and intrinsically disordered proteins

Barbora Spodniakova [1], Zuzana Bednarikova [1], Miroslav Gancar [1], Andrea Antosova [1], Slavka Hamulakova [2], Zuzana Gazova [1]

[1] Department of Biophysics, Institute of Experimental Physics, Slovak Academy of Sciences, Slovakia; [2] Department of Organic Chemistry, Institute of Chemistry, Faculty of Science, P.J. Safarik University, Slovakia

Molecular hybridization is a strategy to design molecules with improved activity compared to the mother moieties. We have studied the anti-amyloid potential 4 bis-coumarin homodimers $(\mathrm{MH})$ on globular hen egg-white lysozyme (HEWL) and the intrinsically disordered $\mathrm{A} \beta_{42}$ peptide. The compounds were prepared by connecting two coumarins by linker with different length containing 3, 4, 6 and 7 carbons. The anti-amyloid ability of homodimers was studied by spectroscopic techniques and atomic force microscopy. We have found that the activity of compounds to destroy HEWL fibrils increased with linker elongation. The best destroying activity was observed for derivative with the longest linker. However, the $\mathrm{MH}$ compounds possessed weak potential to inhibit HEWL fibrils formation. The compounds showed significant anti-amyloid ability towards $A \beta_{42}$ peptide. This was proven by significant decrease in the length and number of the fibrils without change of overall secondary structure of treated fibrils.

The present work is a step towards understanding the relationship between the structure and anti-amyloid properties. 
Posters

- 7. Protein translocation, assembly, and folding -

\section{P-114}

Organization and dynamics of MET and CD44v6 complexes at the plasma membrane

Ryshtee M Tannoo [1,2], Ludovic Richert [1], Nario Tomishige [1], Toshihide Kobayashi [1], Véronique Orian-Rousseau [2], Yves Mély [1]

[1] LBP, UMR 7021, Illkirch, France; [2] IBCS-FMS, Karlsruhe, Germany

The receptor tyrosine kinase MET and its ligand Hepatocyte Growth Factor (HGF) are key regulators of cell survival and proliferation. An uncontrolled up-regulation of MET/HGF pathway may promote tumoral progression and metastasis. We have shown that HGF binding to MET causes its dimerization by molecular biology and quantitative microscopy. The transmembrane glycoprotein CD44v6, isoform of CD44 family, acts as a co-receptor for MET activation and signaling. It also promotes the formation of a ternary complex with MET and HGF. The extracellular part of CD44v6 is involved in MET phosphorylation, while its cytoplasmic tail induces MET downstream signaling. To characterize the mechanisms of interaction between CD44v6 and MET promoted by HGF, quantitative microscopy techniques are carried out in live $\mathrm{T}$ 47D cells. By FRET-FLIM, we showed how HGF can promote the formation of CD44v6/MET complexes. In parallel, FCS allowed us to determine the diffusion and oligomerization of CD44v6 and MET on the plasma membrane before and after binding with HGF. Hence, we also track the role of membrane subdomains such as lipid rafts in this process and a possible re-localization of proteins after their activation. These results shall allow a better understanding of MET pathway.

\section{P-116}

Study of structural and conformational changes of amyloid beta peptides induced by the presence of surfactants

Michalina Wilkowska [1], Theyencheri Narayanan [2], Lauren Matthews [2], Aneta Szymańska [4], Marek Kempka [3,1], Barbara Peplińska [3], Monika Makrocka-Rydzyk [1], Maciej Kozak [1]

[1] Adam Mickiewicz University, Poland; [2] European Synchrotron Radiation Facility, France; [3] NanoBioMedical Centre UAM, Poland; [4] University of Gdańsk, Poland

Soluble oligomeric assemblies of amyloid beta peptides $(\mathrm{A} \beta)$ play the primary role in the formation of the Alzheimer's Disease (AD). The main aim of our study was to characterize the structure and conformational changes of A $\beta$ peptides which are induced by the presence of gemini surfactants. Therefore, the effect of surfactant concentration on $A \beta$ 140 and 1-42 fibrills and its shorter variants was investigated in aqueous solutions. The kinetics of aggregation process of $A \beta$ peptides in the presence of surfactant was studied with fluorescence spectroscopy and NMR methods. The structural changes were characterized using a series of SAXS measurements and time resolved SAXS. The morphology and changes in secondary structure of $A \beta$ peptides caused by the presence of surfactants were determined using FTIR, CD, AFM and TEM methods. The control of $\mathrm{A} \beta$ aggregation is very difficult to achieve, but the study on interactions of surfactants with peptides may contribute to a better understanding of this process. Acknowledgments: The study was supported by Iwanowska scholarship from NAWA-PPN/IWA/2019/1/00151 and research grant, "Grant Diamentowy"-DEC:0011/DIA2015/44.

\section{P-115}

Domain level mapping of protein-protein interaction sites in the ROCK LIMK complexes

Barbara M Végh, Attila Baksa, András Szilágyi, Péter Závodszky, István Hajdú

Institute of Enzymology, Research Centre for Natural Sciences, Budapest, Hungary

The Rho/ROCK (Rho-Associated Coiled-Coil Kinase)/LIMK (LIM domain containing kinase)/cofilin pathway is involved in several diseases including tumors, fibrosis and neurodegenerative diseases. Inhibition of ROCK kinases has potential therapeutic application, however the high number of known substrates of ROCK kinases may cause several unwanted side effects. To develop pathway specific inhibitors with fewer side effects, the specific interactions between each enzyme and its protein substrate should be clarified on structural basis. To identify the relevant binding sites between ROCK1/2 and LIMK1/2 isoenzymes, we performed comparative enzyme activity measurements using full length and truncated recombinant molecules. We found that both ROCK1 and ROCK2 phosphorylate LIMK2 faster than LIMK1 by more than an order of magnitude. To map the ROCK/LIMK protein-protein interaction surfaces, solution and surface based binding studies were conducted complemented with molecular docking. 
Posters

- 8. Bioenergetics -

\section{P-117}

The TOM receptor subunits Tom20 and Tom70 display higher mobility than the TOM complex in the outer mitochondrial membrane

Karin B Busch [1], Maniraj Bhagawati [1], Ayelen Gonzáles Montoro [2], Tasnim Arroum [1], Henning Mootz [1], Niklas Webeling [1]

[1] University of Muenster, Germany; [2] University of Osnabrueck, Germany

Mitochondria import more than $99 \%$ of their proteins via the TOM complex, the outer membrane translocase. The unfolded protein substrates are bound by the peripheral receptor proteins Tom20, and/or Tom70 before being imported through the TOM pore. The TOM pore or core complex consists of Tom40, and the small subunits Tom5, Tom6, and Tom7. Structures of the TOM complex show dimers and trimers from the core complex, in which Tom20 and Tom70 are predominantly absent suggesting that the assembly of Tom20/ Tom70 and the TOM core complex is dynamic. Using single particle tracking, we show that Tom 20 and Tom70 have higher mobility in intact mitochondria than Tom7 and Tom40. Artificially induced ligation of Tom 20 with Tom7 using ultrafast self-splicing Gp41-1-intein slows the mobility of Tom20. This effect is not observed when Tom20 is coupled to $\mathrm{hFis}$. Our data are consistent with a dynamic association of the receptor subunits Tom 20 and Tom70 with the TOM core complex. In contrast, the lower mean mobility of Tom7 can be explained if Tom7 is seen as a subunit of the dimeric or trimeric TOM core complex, which, in addition, can interact with the TIM complex in the inner mitochondrial membrane.

\section{P-119}

Unidirectional rotation of ATP synthase

Antoni Marciniak, Pawel Chodnicki, Kazi A Hossain, Joanna Slabonska, Jacek Czub

Gdansk University of Technology, Poland

F-ATP synthase is an incredibly efficient enzyme located in the inner mitochondrial membrane, responsible for synthesizing almost all of the cellular ATP. It has been historically divided into two parts: membrane-embedded Fo and soluble F1. This division is also functional; as protons flow through Fo, its centerpiece - called c-ring - rotates. C-ring rotation drives the conformational changes necessary for the ATP synthesis to occur in F1. Therefore, the c-ring converts energy accumulated in the proton gradient to the mechanical energy, which is then converted to chemical energy in form of a phosphodiester bond by the F1. The incredible efficiency implies strictly unidirectional rotation of the c-ring. Only the subunit a, which wraps around the c-ring, can be the source of the unidirectionality, as the c-ring itself is symmetric. Here, we show that evolutionarily conserved arginine is the source of unidirectionality. We show that the binding and release of protons are strictly regulated by the subunit $\mathrm{a}$ - c-ring interface. By mutating arginine, we show that charge is necessary to release protons from the c-ring. Moreover, we found that positive charge facilitates unidirectional rotation, but arginine is the only positively charged amino acid that can separate two half-channels by which protons reach the c-ring.

\section{P-118}

Model for protonophoric uncoupling: explaining discrepancies between BLM and mitochondria Andrea Ebert, Kai-Uwe Goss

Helmholtz Centre for Environmental Research GmbH - UFZ, Leipzig, Germany

Uncoupling of oxidative phosphorylation is central to bioenergetics. Understanding the processes involved is of utmost importance, not only due to toxicity, but also for various possible pharmaceutical applications, like against diabetes, obesity or cancer. Classical uncouplers such as CCCP or 2,4-dinitrophenol have been studied extensively, both in isolated mitochondria and artificial bilayer systems. Already decades ago, a good correlation between critical uncoupling effects in isolated mitochondria and electrical conductance in black lipid membranes BLM was observed. Yet some discrepancies to this correlation have been found, and nowadays the research focus has shifted to uncoupling proteins.

Here, we want to explain some of these discrepancies using a biophysical model for protonophoric uncoupling, without the need to postulate the involvement of uncoupling proteins. The model considers not only ionic permeability, but also the permeability of the neutral species, the $\mathrm{pH}$ and electrical potential across the inner mitochondrial membrane. If no experimental input parameters are available, they are calculated using quantum-chemical calculations and the COSMORS theory. With the model, we are able to explain the $\mathrm{pH}$-dependence of uncoupling induced for example by 2,4dinitrophenol. We also elucidate why pyrrolomycin D shows a higher conductance in BLM than pyrrolomycin C, but has a weaker uncoupling activity in isolated mitochondria.

\section{P-120}

Thermodynamics of the formation and decay of the P-type ferryl form of cytochrome c oxidase in the reaction of the oxidized oxidase with $\mathrm{H}_{2} \mathrm{O}_{2}$ Ludmila Mikulova [1], Ivana Pechova [2], Marek Stupak [3], Marian Fabian [1], Daniel Jancura [2]

[1] Center for Interdisciplinary Biosciences, UPJS Košice, Slovakia; [2] Biophysics dept, UPJS Košice, Slovakia; [3] Medical and Clinical Biochemistry dept, UPJS Košice, Slovakia

Cytochrome c oxidase $(\mathrm{CcO})$ participates in the energy conversion in cells of aerobic organisms. Several ferryl states of the catalytic heme $a_{3}-\mathrm{Cu}_{\mathrm{B}}$ center of $\mathrm{CcO}$ are observed during the reduction of $\mathrm{O}_{2}$ to $\mathrm{H}_{2} \mathrm{O}$. One of the $\mathbf{P}$-type ferryl forms, $\mathbf{P}_{\mathrm{M}}$, is produced by the reaction of the two-electron reduced $\mathrm{CcO}$ with $\mathrm{O}_{2}$. In this state the iron of heme $a_{3}$ is in the ferryl form and a free radical should be also present at the catalytic center. However, energetics of the $\mathbf{P}_{\mathbf{M}}$ formation has not been experimentally established, yet. Here, the generation of the $\mathbf{P}_{\mathbf{M}}$ by the reaction of the oxidized bovine $\mathrm{CcO}(\mathbf{O})$ with one molecule of $\mathrm{H}_{2} \mathrm{O}_{2}$ was investigated by ITC and UV-Vis absorption spectroscopy. Two kinetic phases, corresponding to the formation of the $\mathbf{P}_{\mathbf{M}}$ and its endogenous conversion to the $\mathbf{O}$, were resolved by both methods. The $\Delta \mathrm{H}$ of the whole process $\left(-66 \mathrm{kcal} / \mathrm{mol}\right.$ of $\left.\mathrm{H}_{2} \mathrm{O}_{2}\right)$ was larger than the heat liberated $\left(-50.8 \mathrm{kcal} / \mathrm{mol}\right.$ of $\left.\mathrm{O}_{2}\right)$ during the reduction of $\mathrm{O}_{2}$ by ferrocytochrome $c$. More importantly, $\Delta \mathrm{H}$ of $-32 \mathrm{kcal} / \mathrm{mol}$ of ferryl state, representing the first phase, far exceeds the heat production of the $\mathbf{P}_{\mathbf{M}}$ intermediate. Data indicate that during the first phase, the radical in the $\mathbf{P}_{\mathbf{M}}$ is actually quenched and a similar $\mathbf{P}$-type ferryl form $\left(\mathbf{P}_{\mathbf{R}}\right)$ is produced. Additionally, it is shown that there is only a minimal entropy contribution $\left(-0.7\right.$ cal.mol $\left.^{-1} \cdot \mathrm{K}^{-1}\right)$ to the Gibbs energy change $(\Delta \mathrm{G}$ $=-46 \mathrm{kcal} / \mathrm{mol}$ of $\mathrm{O}_{2}$ ) during the catalytic reduction of $\mathrm{O}_{2}$ by the ferrocytochrome $c$. 


\section{Posters}

-8. Bioenergetics -

\section{P-121}

Cytochrome $\mathrm{C}$ heme conformation is sensitive to membrane potential in isolated mitochondria as revealed by SERS

Evelina I Nikelshparg [1], Adil A Bayzhumanov [1], Zhanna V Bochkova [1], Vera G Grivennikova [1], Anna A Semenova [2], Evgeny A Goodilin [2], Sergey M Novikov [3], Olga Sosnovtseva [4], Georgy V Maksimov [1], Nadezda A Brazhe [1]

[1] Faculty of Biology, Moscow State University, Russian Federation; [2] Faculty of Materials Sciences, Moscow State University, Russian Federation; [3] Center for Photonics and 2D Materials, Moscow Institute of Physics and Technology, Russian Federation; [4] Department of Biomedical Sciences, Faculty of Health and Medical Sciences, University of Copenhagen, Denmark

Electron transfer on the level of cytC is believed to be the limiting stage of respiration. There is evidence that CytC heme conformation mediates efficiency of electron transfer from complex III to complex IV in electron transport chain (ETC). However, investigation of cytC heme conformation in functioning mitochondria is challenging. We developed a SERS-based approach to probe cytC heme conformation in isolated mitochondria placed on plasmonic nanostructures. We showed that the probability of planar cytC heme conformation decreases under high potential conditions (after oligomycin) and increases under low potential conditions (after FCCP or valinomycin). These findings corroborate the suggestion that planar heme conformation favors electron transfer. We believe that proposed experimental approach will advance our understanding of ETC regulation in situ.

\section{P-123}

Time-resolved electrometric study of the effect of zinc ions on cytochrome c oxidase from the P-side

Sergey A Siletsky [1], Robert B Gennis [2]

[1] Belozersky Institute of Physico-Chemical Biology, Lomonosov Moscow State University, Moscow 119991, Russian Federation; [2] Department of Biochemistry, University of Illinois at Urbana-Champaign, Urbana, Illinois 61801, USA

The effect of $\mathrm{Zn}^{2+}$ on the P-side of proteoliposomes containing membrane-incorporated Rhodobacter sphaeroides cytochrome $c$ oxidase was investigated by time-resolved electrometrics following single-electron injection into enzyme prepared in the $\mathrm{F}$ state. The wild-type enzyme was studied together with two mutants (N139D and D132N) with substitutions in the D-channel, which serves to transport both the substrate proton and the proton pumped through the membrane $(1,2)$. The data indicate that the primary influence of $\mathrm{Zn}^{2+}$ from the P-side of the membrane is to inhibit the rate of release of the pumped proton from the proton loading site (PLS) to the bulk aqueous phase on the P-side of the membrane. The results strongly suggest that there must be two pathways by which the pumped proton can exit the protein from the PLS and two separate binding sites for $\mathrm{Zn}^{2+}(3)$. This work was supported by the Russian Science Foundation (research project 19-14-00063).

1) Siletsky, S. A., et al. (2004), J. Biol. Chem., 279: 52558-52565.; 2) Siletsky, S. A., Konstantinov, A. A. (2012), Biochim. Biophys. Acta, 1817: 476-488.; 3) Siletsky, S.A., Gennis R.B. (2021), Biochemistry (Mosc.), 86(1):105-122.

\section{P-122}

Generation and transfer of charge in biological structures Yuri A Shapovalov [1], Pavel P Gladyshev [2]

[1] Al-Farabi Kazakh National Univ., Almaty, Kazakhstan; [2] Dubna State Univ., Moscow region, Russian Federation

The general principle uniting all living organisms is the energy and electrons transfer. A biological membrane (BM) containing carotenoids with semiconducting properties is considered. Charge transfer in BM is realized by the donor-acceptor mechanism through charge transfer complexes (CTC) of carotenoids with lipids.

Antioxidant properties of carotenoids are described: the radical transfers an unpaired electron to the carotene, and then, through CTC, to the active center of the enzyme-cofactor system, followed by the formation of an inactive product of the enzymatic reaction.

An important role is played by inorganic cofactors: chlorophyll $\mathrm{Mg}^{2+}$, forming CTC with xanthophylls. In the porphyrin structure, $\mathrm{Mg}^{2+}$ forms a chelate complex with astaxanthin, preserving the conjugated system of bonds and providing efficient migration of energy and electrons along BM.

The mechanism of $\mathrm{H}^{+}, \mathrm{O}_{2}$, e $\mathrm{e}^{-}$formation in the FS-II photosystem is described: atomic oxygen is formed from water by photoenzymatic reaction. Further, $\mathrm{H}_{2} \mathrm{O}_{2}$ is formed from the interaction of oxygen with water. Subsequent oxidation of $\mathrm{H}_{2} \mathrm{O}_{2}$ on the $\mathrm{Mn}_{4} \mathrm{CaO}_{5}$ forms $\mathrm{O}_{2}, \mathrm{H}^{+}$, $\mathrm{e}^{-}$. Here, $\mathrm{Mn}^{4+}$ is reduced to $\mathrm{Mn}^{2+}$, and then oxidized to $\mathrm{Mn}^{4+}$ upon transfer of the reducing equivalents of PS-I. For efficient collection of solar energy, chloroplast thylakoids are stacked into granules, which are connected by lamellas providing energy to PS I and PS II.

Studies of the two-electrode enzymatic system were carried out, and the formation of $\beta$-carotene CTC with lipids was also studied.

\section{P-124}

Engineering of chimeric carbonic anhydrases for designing isoform-selective inhibitors

Joana Smirnovienè, Alexey Smirnov, Visvaldas Kairys, Justina Kazokaitè-Adomaitienè, Aurelija Mickevičiūtè, Vilma Michailoviené, Elena Manakova, Lina Baranauskienė, Daumantas Matulis

Institute of Biotechnology, Life Sciences Center, Vilnius University, Lithuania

A family of 12 human carbonic anhydrases (CA) is drug target for cancer, glaucoma, altitude sickness, and obesity treatment. They share a high percentage of amino acid sequence homology and for this reason it is challenging to design isoform-selective inhibitors. Moreover, the production of isoforms VA, VI, and XII is complicated for large-scale drug candidate screening.

To understand the mechanism of inhibitor recognition and selectivity profiles, we have engineered chimeric carbonic anhydrases that resemble the active sites of CA VA, CA VI, or CA XII. We used the off-target isoform CA II as a core protein and replaced 5-7 amino acids in the active site to that characteristic for the CA VA, CA VI, or CA XII. The detailed thermodynamic and structural analysis revealed that chimeric CAs recognized and bound inhibitors with similar affinities and binding modes as target isoforms (CA VA, CA VI, and CA XII), but not as the off-target isoform. The compounds that bound CA VA, CA VI, or CA XII more strongly than CA II, switched their preference and bound more strongly to the engineered chimeras.

Our study validated this enzyme engineering approach to develop new inhibitors as drug candidates. 
Posters

- 8. Bioenergetics -

P-125

Mechanism of Casein Kinase 1 regulation by DDX3 Andrea Svorinic [1,2], Edoardo Fatti [1], Alexander Hirth [1], Gunter Stier [2], Irmgard Sinning [2], Christof Niehrs [1,3] [1] Division of Molecular Embryology, German Cancer Research Center (DKFZ), Germany; [2] Heidelberg University Biochemistry Center (BZH), Germany; [3] Institute of Molecular Biology (IMB), Germany

Casein Kinase 1 (CK1) family of serine/theronine kinases is involved in multiple signal transduction pathways, and is implicated in numerous human diseases and disorders. However, details of CK1 regulation remain elusive. Recently, a DEAD-box RNA helicase family member, DDX3, was identified as a novel allosteric regulator of CK1 isoform $\varepsilon$. Intriguingly, further experiments showed that other DDX family members had the potential to activate CK1ع, and that several other protein kinases were also susceptible to regulation by DDX proteins, indicating a more generic role of DDX helicases in protein kinase regulation. In order to dissect the mechanistic details of the interplay between protein kinases and DDX helicases, we performed extensive kinetic analysis in vitro, as well as in vivo experiments to establish physiological relevance of selected DDX-kinase pairs. Using the stopped-flow technique, we quantitatively characterized separate steps in the kinase cycle. At the same time, binding assays revealed that the interaction between DDX3 and CK1 is nucleotide-dependent, and that binding of RNA and CK1 to DDX3 are mutually exclusive. Current research aims to unravel the structural details of the interaction between CK1 and DDX3, using X-ray crystallography.

\section{P-127}

Mitochondrial proteins enhance the protonophoric action of 2,4-dinitrophenol

Kristina Zuna [1], Olga Jovanovic [1], Ljudmila S Khailova [2], Elena A Kotova [2], Zlatko Brkljaca [3], Sanja Skulj [3], Mario Vazdar [3,4], Yuri N Antonenko [2], Elena E Pohl [1]

[1] University of Veterinary Medicine, Vienna, Austria; [2] Belozersky Institute of Physico-Chemical Biology, Moscow, Russian Federation; [3] Rudjer Boskovic Institute, Zagreb, Croatia; [4] Czech Academy of Sciences, Prague, Czech Republic

2, 4-dinitrophenol (DNP) is a classic chemical uncoupler of mitochondria and increases the permeability of the pure lipid bilayer to protons. It is illegally used as a fat burner despite its high toxicity and the lack of antidotes. Because of the sensitivity of the DNP-mediated decrease of the mitochondrial membrane potential to carboxyatractyloside, we hypothesize that DNP modifies the proton-transporting activity of inner mitochondrial membrane proteins. We show for the first time that the addition of DNP to the bilayer membranes reconstituted with recombinant ANT1 or UCPs increases the total membrane conductance $\left(\mathrm{G}_{\mathrm{m}}\right)$ in the absence of long-chain free fatty acids, known as the only protein activators. ATP added prior to DNP decreased $\mathrm{G}_{\mathrm{m}}$, suggesting a competition mechanism for the same binding site. Using site-directed mutagenesis and molecular dynamic simulations, we found that arginine 79 of ANT1 is involved in the binding of both DNP and ATP, with a higher affinity for ATP. As proteins make up $\sim 75 \%$ of the IMM, understanding their interaction with DNP is crucial for explaining its protonophoric effect on mitochondria.

\section{P-126}

Color-tuning in Ca-binding photosynthetic bacteria

Kõu Timpmann, Margus Rätsep, Liina Kangur, Arvi Freiberg

Institute of Physics, University of Tartu, Estonia

Flexible color adaptation to available ecological niches is vital for the common thriving of photosynthetic organisms. Hence, most purple bacteria living in the shade of green plants and algae apply bacteriochlorophyll-a pigments to harvest near infra-red light around 850...875 nm. Exceptional are some $\mathrm{Ca}$ ion-containing species fit to utilize much redder quanta. Physical basis of such anomalous absorbance shift equivalent to $\sim 5.5 \mathrm{kT}$ at ambient temperature has remained unsettled for over 30 years already. Here, by applying several sophisticated spectroscopic techniques, we show that $\mathrm{Ca}$ ions bound to the structure of LH1 core light-harvesting pigment-protein complex significantly increase the couplings between the bacteriochlorophyll pigments. We thus establish the Ca-facilitated enhancement of exciton couplings as the main mechanism of the record spectral red-shift. The changes in specific interactions such as pigment-protein hydrogen bonding, although present, turned out to be secondary in this regard. Apart from solving a decades-old conundrum, these our results complement the list of physical principles applicable for efficient spectral tuning of photosensitive molecular nano-systems, native or synthetic alike. 


\section{Posters}

- 9. Quantification of molecular forces -

P-128

Global conformational change underlies the allosteric regulation in the KIX domain of CREB-binding protein

Michał Jurkowski, Cyprian Kleist, Michał Badocha, Jacek Czub

Department of Physical Chemistry, Gdańsk University of Technology, Poland

The allostery is a common process in biological systems, in which structural perturbation of one site of the macromolecule changes another, regulating its activity. Typically, binding of allosteric effector modulates the receptor affinity for a ligand. From a thermodynamic point of view, binding of allosteric effector shifts the conformational equilibrium in favor of the high-affinity state.

The KIX domain of transcription coactivator CBP (CREB Binding Protein) is a model protein for the study of allosteric regulation. This three-helix protein binds numerous transcription factors to its two distinct binding sites: c-Myb binding site and MLL binding site. Here, we examined its interactions with pKID (c-Myb binding site ligand) and MLL (allosteric regulator). Using molecular dynamics simulations, we revealed previously unknown, inactive state of the KIX domain.

First, we investigated the free energy differences of KIX active-to-inactive transition in either presence or absence of MLL. To confirm allosteric modulation of KIX by MLL, we calculated free energy profiles of pKID binding reaction to both active (MLL-bound) and inactive KIX. Finally, we characterized structural changes accompanying the shift between active and inactive conformation.

\section{P-130}

The coil-globule transition: Thermosensitive hydration of acrylamide-based polymers

Patrick K Quoika, Maren Podewitz, Yin Wang, Anna S Kamenik, Johannes R Loeffler, Klaus R Liedl

University of Innsbruck, Austria

Thermosensitive polymers undergo a counter-intuitive phase transition with a lower critical solution temperature: they form a gel above a certain temperature, whereas they exist in liquid mixture with the solvent below this temperature. This phase transition is associated with a structural collapse of the polymer chains, i.e., the Coil-Globule transition. This conformational change therefore connects the macroscopic solution properties with the dynamics of the system on a molecular level.

To understand this extraordinary phenomenon, we investigated the conformational dynamics of a selection of acrylamide-based polymers - including thermosensitive and non-thermosensitive representatives. To this end, we performed Molecular Dynamics simulations of linear polymer chains at a wide range of temperatures. Our atomistic simulations consistently reproduce the thermosensitive character of these polymers. Further, we report distinct hydration profiles for these two classes of polymers and identified the polymer-solvent interactions to be essential for the energetic balance of the transition. Moreover, we found a clear trend between the lifetime of the hydrogen bonds between the polymer-surface and water and the thermosensitive character of the polymers. Therefore, we conclude that the solvation entropy is decisive for the thermosensitivity of these substances.

\section{P-129}

The complex association behavior of the polysorbates PS20 and PS80

Hannah Knoch [1], Maximilian H Ulbrich [1], Patrick Garidel [2], Heiko Heerklotz [1]

[1] University of Freiburg, Germany; [2] Boehringer Ingelheim Pharma GmbH \& Co. KG, Germany

Polysorbates (PS) are commonly used surfactants consisting of a sorbitan core that is associated with ethylene oxide chains of variable length. One or more of these can be esterified with fatty acids. This study gives insight into the complex association behavior of PS20 and PS80, which differs tremendously from that of single-component surfactants. Isothermal titration calorimetry (ITC) provides a powerful tool to investigate the micellization behavior of surfactants. Thereto, the evaluation of serial ITC demicellization experiments with different PS concentrations is sufficient. Their experiment-dependent heats of titration are translated into a common function of the state of a sample, the micellar enthalpy $\mathrm{Qm}(\mathrm{c})$. It demonstrates the presence of initial micelles already at the lowest concentrations investigated. These micelles mainly consist of surfactant components with the lowest individual critical micelle concentration (CMC). An increase of concentration triggers the other components to enter these micelles in the sequence of increasing individual CMCs. Formation and variation of micelles proceeds up to at least $10 \mathrm{mM}$ PS. Hence, published CMC values of PS20 and PS80 may be referred to certain, major components that are incorporated into and form specific micelles. An interpretation leading to the statement, micelles are absent below and constant properties disagrees with the presented model.

\section{P-131}

AFM SMFS measurements of individual collagen molecules

Andreas Rohatschek [1], Orestis G Andriotis [1], Patrick Steinbauer [2], Stefan Baudis [2], Philipp J Thurner [1]

[1] Institute of Lightweight Design and Structural Biomechanics, TU Wien, Austria; [2] Instiute of Applied Synthetic Chemistry, TU Wien, Austria

Collagens are the most abundant and structurally the most important proteins of the human extracellular matrix. Therefore, mechanical properties of collagen molecules (tropocollagen), and the progressively larger structures they form, are crucial for tissue mechanics and function.

Here, we present an approach to experimentally characterize adhesion forces between tropocollagen molecules to substrates of choice.

Surface functionalization was used to tether individual tropocollagen molecules onto an AFM tip. In detail, we used a maleimide (MI) - polyethylene glycol (PEG) based linker system to couple individual molecules of collagen Type III onto an aminated Si AFM tip. Atomic Force Microscopy (AFM) Single Molecule Force Spectroscopy (SMFS) measurements were conducted in different media and various substrates at different retraction speeds to investigate tropocollagen-substrate interactions.

We observed a profound dependency of collagen substrate interactions on substrate type and surrounding medium.

The successful measurements enable investigating collagen adhesion to a number of substrates including other collagens, and are an important step towards future tensile tests of individual tropocollagen molecules. 
Posters

- 9. Quantification of molecular forces -

P-132

A FRET-based sensor for probing forces exerted by single $\mathrm{T}$ cell receptors on their ligands

Lukas Schrangl [1], Janett Göhring [1,2], Florian Kellner [2], René Platzer [2], Enrico Klotzsch [1], Hannes Stockinger [2], Johannes B Huppa [2], Gerhard J Schütz [1]

[1] Institute of Applied Physics, TU Wien, Austria; [2] Center for Pathophysiology, Infectiology and Immunology, Medical University of Vienna, Austria

The interaction between $\mathrm{T}$ cell receptors and antigenic peptides presented by antigen-presenting cells (APCs) constitutes the basis for the identification of harmful substances by the adaptive immune system. Prompted by indications suggesting that forces play a key role, we developed a force sensor for insertion into the immunological synapse. A spidersilk-derived peptide acting as a molecular spring serves as the sensor's core. Force-induced changes in its length are monitored via the FRET efficiency between dyes attached near the opposing termini via a combined single-molecule FRET and tracking approach. As surrogate APCs we functionalized supported lipid bilayers (SLBs). Using sensors coupled to single-chain fragments, we observed forces of 4-8 $\mathrm{pN}$ on gel-phase SLBs (i.e., immobilized sensors). On liquidphase SLBs (diffusing sensors), only weak pulling events were found. We also analyzed the force magnitude w.r.t. the duration of the $\mathrm{T}$ cell-SLB interaction under high ligand density, activating conditions as well as under low density conditions, emulating T cells probing APCs. Inspection of force time traces yielded the loading rate. Stimulating pMHCfunctionalized sensors registered reduced (activating conditions) or no (scanning conditions) forces on gel-phase SLBs.

\section{P-133}

Kinetics of hIgG antibodies and CD154 binding to CD40 viewed on the single-molecule level Hannah Seferovic [1], Patricia Sticht [1], Javier ChaparroRiggers [2], Wei Chen [2], Christian Wechselberger [3], David Bernhard [3], Peter Hinterdorfer [1]

[1] Institute for Biophysics, JKU, Linz, Austria; [2] Biotherapeutic Design \& Discovery, Pfizer Inc., San Francisco, USA; [3] Division of Pathophysiology, Medical Faculty, JKU, Linz, Austria

Cancer cells can block host immune mechanisms, leading to immune tolerance. By utilizing therapeutic antibodies, this tolerance can be disrupted by stimulating receptors through mimicking their ligands. An important receptor in immunological pathways is the transmembrane protein CD40, which is triggered by its ligand CD154, but can also be stimulated by therapeutic antibodies. Here, we investigated the binding behaviour of a set of human IgG monoclonal antibodies to CD40, using atomic force microscopy (AFM) - derived single molecule force spectroscopy (SMFS). IgG subtypes, such as IgG1, isoforms of IgG2 and IgG4 differ in the disulphide-connectivity of their hinge region, which determines the antibodies' flexibility. Using SMFS, we coupled IgG subtypes and the ligand CD154 to AFM tips and analyzed single molecular bond ruptures from bindings to CD40, stably expressed in Chinese hamster ovary (CHO) cells, to decipher interaction forces, energy landscapes and the antibodies' kinetic features. We also focused on characterizing the antibodies' monovalent and bivalent binding behavior and found that both, hIgG2B and hIgG1, can simultaneously bind with their two Fab arms to CD40 molecules. 
Posters

- Self-Assembled Session 2 - Advances and applications in cryo-electron microscopy (Instruct-ERIC) -

\section{P-134}

Effects of cryo-EM cooling on structural ensembles

Lars V Bock, Helmut Grubmüller

MPI for Biophysical Chemistry, Germany

The recent revolution in cryo-EM allows the determination of structures of macromolecular complexes at atomic resolution. Cryo-EM also provides information on the structural heterogeneity of the macromolecules. To obtain cryo-EM images, the samples are first flash-frozen. However, to what extent the structural ensemble is perturbed by the cooling is currently unknown. To quantify the effects of cooling, we estimated the temperature decay rate by solving the heat equation and started molecular dynamics simulations of the ribosome from 41 room-temperature snapshots with linearly decreasing temperature at 11 different cooling rates (simulation lengths 0.1 to $128 \mathrm{~ns}$ ). The cooling markedly decreases the structural heterogeneity. To test if this effect depends on the cooling rate, we used Bayesian statistics to test thermodynamic and kinetic models of the cooling process. The observation that a kinetic two-state model improves the prediction of the decrease in heterogeneity compared to the cooling-rate independent thermodynamic model suggests that kinetics affect the cooling of structural ensembles. Combining the estimated temperature decay rate with the kinetic model suggests that small barriers between the states $(<10 \mathrm{~kJ} / \mathrm{mol})$ are overcome during cooling and do not contribute to the structural heterogeneity of cryo-EM ensembles. The model parameters will allow one to quantify the heterogeneity of biologically relevant room-temperature ensembles from cryo-EM structures. 
Posters

- 10. Mechanobiology -

P-135

Bending compliance of lipid bilayer regulates the curvature threshold and pathway of membrane fission

P. V Bashkirov [1], P. I Kuzmin [2], P. Arrasate [3], A. V Shnyrova [3], V. A Frolov [3,4]

[1] Federal Research and Clinical Centre of PhysicalChemical Medicine, Russian Federation; [2] A.N. Frumkin Institute of Physical Chemistry and Electrochemistry, Russian Federation; [3] Biofisika Institute (CSIC, UPV/EHU) University of the Basque Country, Spain; [4] Ikerbasque, Basque Foundation for Science, Spain

Membrane bending allows to confine the membrane rearrangement sites and creates the elastic stress regarded as a trigger of local lipid rearrangements. Various pathways of the lipid rearrangements induced by the critical bending stress have been revealed. However, it remains unclear which molecular and force factors control the pathways choice and enable leakage-free membrane rearrangements. We modeled the stress action using a simple 1D model, the membrane nanotube (NT) constricted by proteins and physical forces. We used the electric field to measure the NT lumen conductance and assess changes in the material parameters of the NT membrane during the NT constriction and scission. We found that the NT membrane retained linear bending elasticity up to the moment of scission. The instability was associated with a threshold value of elastic stress and the NT monolayers' intrinsic curvature. Analysis of the NT constriction dynamic by proteins revealed that the proteins sense the lipid elastic response and generally create subcritical or transient critical stresses avoiding causing membrane leakage.

\section{P-137}

Unfolded fibrinogen alphaC-connector is a major contributor to the mechanical strength of fibrin fibers

Ali Daraei [1], Taylor C Dement [2], Heather A Belcher [2], Nathan E Hudson [2], Martin Guthold [1]

[1] Department of Physics, Wake Forest University, WinstonSalem, USA; [2] Department of Physics, East Carolina University, Greenville, USA

Blood clots perform the mechanical task of stemming the flow of blood. The main mechanical component of a blood clot is a mesh of nanoscopic fibrin fibers, which assemble from the blood protein fibrinogen. The structure and role of the two large, partially unfolded $\alpha \mathrm{C}$ regions in fibrinogen has long intrigued scientists. In human fibrinogen, the $\alpha \mathrm{C}$ region $(\alpha 221-610)$ consists of the unstructured, $60 \mathrm{~nm}$ long $\alpha \mathrm{C}$ connector ( $\alpha 221-391)$ and the folded $\alpha \mathrm{C}$-domain (392-610). The unfolded $\alpha \mathrm{C}$-connector contains ten repeat segments, each consisting of 13 amino acids, running from $\alpha 264-391$.

Using fibrinogen variant $\mathrm{A} \alpha \Delta 1-10$, in which all ten repeat units are deleted, and AFM-based nanomanipulation methods, we determined the importance of the $\alpha \mathrm{C}$-connector for uncrosslinked fibrin fiber mechanical properties.

Compared to native fibrin fibers, fibers formed from the $\mathrm{A} \alpha \Delta$ 1-10 variant showed dramatically different mechanical properties. The extensibility (fracture strain) was reduced by a factor of 1.7 (from 2.1 to 1.26 ) and the modulus (stiffness) decreased by a factor of 3 (from $3 \mathrm{MPa}$ to $1 \mathrm{MPa}$ ).

Our experiments identify the $\alpha \mathrm{C}$-connector of fibrinogen as a major contributor to fibrin fiber strength, and, thus, blood clot mechanical strength.

\section{P-136}

Spatial mapping of the collagen distribution in human and mouse tissues by force volume AFM Annalisa Calò $[1,2]$, Yevgeniy Romin [1], Ning Fan [1], Anthony Santella [1], Elvin Feng [1], Katia Manova-Todorova [1] [1] Memorial Sloan Kettering Cancer Center, USA; [2] University of Barcelona, Spain

Changes in the elastic properties of living tissues during normal development and pathological processes are often due to modifications of the collagen component of the extracellular matrix at various length scales. Force volume AFM can precisely capture the mechanical properties of biological samples with force sensitivity and spatial resolution. The integration of AFM and molecular composition data contributes to understand the interplay between tissue organization and function. The detection of heterogeneous domains at different elastic moduli in tissue sections by AFM has remained elusive so far, due to the lack of correlations with histological and biochemical assessments. Here, force volume AFM is used to identify collagen-enriched domains, present in human and mouse tissues, by their elastic modulus. Collagen identification is obtained in a robust way and affordable timescales, through an optimal design of the sample preparation method and AFM parameters for faster scan with micrometer resolution. The choice of a separate reference sample stained for collagen allows correlating elastic modulus with collagen amount and position with high statistical significance. The proposed preparation method ensures safe handling of the tissue sections, guarantees the preservation of their micromechanical characteristics over time and makes it much easier to perform correlation experiments with biomarkers.

\section{P-138}

Mechanoactivation of c-Abl kinase

Svenja de Buhr [1,2], Frauke Gräter [1,2]

[1] Heidelberg Institute for Theoretical Studies (HITS), Germany; [2] Interdisciplinary Center for Scientific Computing (IWR), Heidelberg University, Germany

Cells are frequently exposed to and need to adapt to mechanical cues from their environment. Thus, they need to be able to sense and translate physical into biochemical signals. Abl kinase is involved in cell proliferation, survival and development. Similarly to Src family kinases, the catalytic kinase domain is tightly bound by an $\mathrm{SH} 2$ and $\mathrm{SH} 3$ domain to keep it inactive. A long intrinsically-disordered region connects the kinase domain to an F-actin binding domain, localizing Abl to the cytoskeleton. Additionally, a second disordered linker called the N-cap precedes the SH3 domain and is $\mathrm{N}$ terminally myristoylated. The myristoyl moiety (Myr) binds to a hydrophobic pocket within the kinase domain, thereby aiding to stabilize the inactive conformation while at the same time providing the possibility for membrane binding. Recently, Abl has been shown to have an increased activity in stretched cells, which is independent of the known upstream kinases Src, Fyn and Yes, rendering Abl a promising candidate for a direct mechano-sensor. We performed umbrella sampling and metadynamics simulations indicating that it is favorable for Myr to bind to a lipid bilayer and that the SH3-SH2-kinase domain complex is stable after Myr unbinding. Furthermore, we used steered MD simulations to mimic forces acting on Abl through the cell membrane and cytoskeleton and found that inhibitory SH-domain interactions are released while the kinase domain remains intact. 


\section{Posters}

- 10. Mechanobiology -

\section{P-139}

Probing cell mechanics at small deformations with lateral indentation using optical tweezers Jure Derganc, Špela Zemljič Jokhadar

Institute of Biophysics, Faculty of Medicine, University of Ljubljana, Slovenia

Mechanical properties of living cells depend on a complex interplay between various cytoskeletal elements. Consequently, some cytoskeleton-disrupting drugs can make the cells softer at small deformations but stiffer at large deformation. To understand the role of different cytoskeletal elements, the cell mechanics has to be studied by complementary methods which probe the cells at the same experimental conditions but at different levels of deformation. We have therefore developed a method that is complementary to AFM and uses optical tweezers to indent the cells laterally and at small deformations, i.e. at the level of cell cortex. First, the method was applied to analyze the cortical stiffness of three keratinocyte lines (primary, WT, and mutated) and showed that the differences between these cell lines are not important only at large cellular deformations but also play a role in cortical mechanics. Next, the method was applied to analyze the effect of two actin-disrupting drugs, CK-869 and jasplakinolide, and it was found that both drugs decreased cellular cortical stiffness. These results contrasted with an AFM study, which was conducted at the same experimental conditions and showed that the cells were softened by CK-869 but stiffened by jasplakinolide. The study thus underlined the importance of a simultaneous use of complementary indentation techniques to obtain a more comprehensive picture of cell mechanics.

\section{P-141}

Fast phenotyping of monocytes mechanics under the cytokine storm

Mar Eroles [1], Richard Gerum [2], Ben Fabry [2], Felix Rico [1]

[1] Inserm, France; [2] FAU, Germany

Monocytes play a key role in the innate immune system during the inflammatory response. Serum analysis from patients infected with severe acute respiratory syndrome coronavirus 2 (SARS-CoV-2) presenting an hyperinflammatory response revealed high levels of the interleukins IL-6, IL-8, TNF-a and CD14+ CD16+ monocytes. While the biochemical response to proinflammatory proteins is well studied, little is known about the mechanical response.

The aim of this work was to determine the mechanical properties of monocytes in response to proinflammatory proteins. We used atomic force microscopy (AFM) and a viscoelastic contact model to determine the viscoelasticity of the monocytic cell line THP1 exposed to proinflammatory cytokines (IL-6, IL-8, TNF-a). We carried out similar measurements using a high throughput method: deformation cytometry (DC). Shear deformation of cells in a flux at high pressure allows us to deform our samples into ellipsoids and obtain stiffness and fluidity through an improved neural network analysis. Our results show that THP-1 cells respond mechanically, although in different degrees and directions. While IL-8 importantly softens THP-1 cells, TNF-a tends to make them stiffer. Furthermore, our AFM results are in quantitative agreement with our preliminary results using DC. Our results show that our DC approach is highly quantitative and accurate, opening the door to clinical applications.

\section{P-140}

The study of liposomes extrusion Joanna Doskocz [1,2], Paulina Dałek [1,2], Magdalena Przybyło [1,2], Marek Langner [1,2]

[1] Department of Biomedical Engineering, Wrocław University of Science and Technology, 50-377 Wrocław, pl. Grunwaldzki 13, Poland, Poland; [2] Lipid Systems sp. z o.o., 54-613, Wrocław, ul. Krzemieniecka 48C, Poland, Poland

Liposomes are lipid-based nanoparticles widely used as drug delivery systems to control drug distribution and release. Mechanical properties of liposomes are important parameters affecting their stability, size, loading capacity and ability to penetrate tissues. Nanoparticle elasticity could also affect the rate of liposomes wrapping by cells and accumulation in cancerous tissues. For these reasons, the precise determination of liposome mechanical properties is important for the successful design of the nanotechnological formulation. The new work for the quantification of liposome mechanical properties is based on the extrusion force determination. Measurements were performed using the home-made dedicated device capable to maintain a present value of volume flux of liposomes suspension and continuously monitor the employed extrusion force. The new method, supported by theoretical analysis, allows to quantitate accurately not only the bending rigidity coefficient of the lipid bilayer but also make possible to device the experimental parameter (apparent viscosity), which can be used to evaluate the extrusion process.

\section{P-142}

High-frequency cellular rheology measurements co-implemented with cell mass measurements Gotthold Fläschner [1], Cosmin I Roman [2], Nico Strohmeyer [1], David Martinez-Martin [1,3], Daniel J Müller [1] [1] Eidgenössische Technische Hochschule (ETH) Zürich, Department of Biosystems Science and Engineering, Switzerland; [2] Eidgenössische Technische Hochschule (ETH) Zürich, Department of Mechanical and Process Engineering, Switzerland; [3] The University of Sydney, School of Biomedical Engineering, NSW Sydney, Australia

Here we introduce an assay that allows rheological measurements of rounded cells in the range of $1-40 \mathrm{kHz}$, in order to elucidate the link between cellular and molecular properties, such as polymer relaxation and monomer reaction kinetics. The assay uses an actuated microcantilever to confine a single, rounded cell on a second microcantilever, which measures the cell mechanical response across a continuous high-frequency frequency range. Cell mass measurements and optical microscopy are co-implemented. The fast highfrequency measurements are applied to rheologically monitor cellular stiffening. We find that the rheology of rounded HeLa cells obeys a cytoskeleton-dependent power-law, similar to spread cells. Cell size and viscoelasticity are uncorrelated, which contrasts an assumption based on the Laplace law. Together with the presented theory of mechanical deembedding, which abolishes the condition to stay away from instrumental resonances, our assay is generally applicable to other rheological experiments. 
Posters

- 10. Mechanobiology -

\section{P-143}

Nucleotides-induced changes in the mechanical properties of living endothelial cells and astrocytes Juan Carlos Gil-Redondo[1,2], Jagoba Iturri [1], Felipe Ortega [2], Esmerilda Garcia-Delicado[2], José Luis TocaHerrera [1]

[1] Inst. for Biophysics, DNBT, BOKU Univ. for Natural Resources and Life Sciences, Austria; [2] Dept. Bioquímica y Biología Molecular, Facultad de Veterinaria, Univ. Complutense, Spain

Endothelial cells and astrocytes preferentially express metabotropic P2Y nucleotide receptors, involved in the maintenance of vascular and neural function. Among these, P2Y1 and P2Y2 receptors appear as main actors, since their stimulation induces intracellular calcium mobilization and activates signaling cascades linked to cytoskeletal reorganization. In the present work, we have analyzed by AFM the mechanical response of HUVEC cells and astrocytes upon 2MeSADP and UTP stimulation. This approach allows for simultaneous measurement of factors such as Young's modulus, maximum adhesion force and rupture event formation, which reflect the potential changes in both the stiffness and adhesiveness of the plasma membrane. The largest effect was observed in both cell types after P2Y2 receptor stimulation with UTP. In astrocytes, 2MeSADP stimulation also had a remarkable effect on AFM parameters. Additional studies performed with the selective P2Y1 and P2Y13 receptor antagonists revealed that the $2 \mathrm{MeSADP}$-induced mechanical changes were mediated by the P2Y13 receptor, although they were negatively modulated by $\mathrm{P} 2 \mathrm{Y} 1$ receptor stimulation.

\section{P-145}

Insights on the effect of cholesterol and sphingomyelin on tension and elasticity of plasmalike freestanding model membranes from natural lipids

Alessandra Griffo [1], Jean-Baptiste Fleury [1], Karin Jacobs [1], Walter Nickel [2], Ralf Seemann [1], Friederike Nolle [1], Carola Sparn [2], Hendrik Hähl [1]

[1] Saarland University, Germany; [2] Heidelberg University Biochemistry Center (BZH), Germany

The creation of model lipid membranes as free-standing platforms to study the processes occurring across the cellular membrane has been so far a field of remarkable interest. Such design in fact, allowed in vitro studies without drastically modify the physico-chemical properties and the dynamic nature of lipid bilayers. In the present study we develop unsupported membranes either for microfluidic technology than for atomic force microscopy and spectroscopy investigation and propose them as novel models for systematic studies. Our model membranes reflect the composition of the inner leaflet of plasma membrane and are enriched in sphingomyelin. Such composition in fact, revealed to be attractive to investigate the binding and insertion mechanisms of certain peripheral proteins as the Fibroblast Growth Factor 2 (FGF2), crucially involved in tumor angiogenesis processes. In addition, for a more realistic comparison with in vivo studies we chose lipids from extracts, that are more highly polydisperse. The described lipid bilayers are hence characterized mechanically and insights on the tension and the elasticity are explored.

\section{P-144}

Optomechanical detection of single bacterium mechanical modes

Eduardo Gil-Santos [1], Jose Jaime Ruz [1], Oscar Malvar [1], Ivan Favero[2], Arestide Lemaitre [3], Priscila Monteiro Kosaka [1], Sergio García-López [1], Montserrat Calleja [1], Javier Tamayo [1]

[1] Instituto de Micro y Nanotecnologia, Spain; [2] Université Paris Diderot, France; [3] Université Paris-Saclay, France

Mechanical modes of biological particles such as proteins, viruses and bacteria involve coherent structural vibrations at frequencies in the $\mathrm{THz}$ and $\mathrm{GHz}$ domains, which carry information on its structure and mechanical properties that play a pivotal role in many relevant biological processes. Despite the rapid advances of optical spectroscopy techniques, detection of low-frequency phonons of single bioparticles has remained elusive. Here we demonstrate the detection of single bacterium mechanical modes by using ultra-high frequency optomechanical resonators. We harness a particular regime in the physics of mechanical resonator sensing, which occurs when the sensor and the analyte support mechanical modes at similar frequencies, leading to their hybridization. We develop a novel theoretical framework to describe this effect and compare it with experiments, showing an excellent agreement. The model enable to retrieve the eigenfrequencies and mechanical loss of the bacterium vibration modes. We apply our method to study the effect of hydration on the vibrational properties of single bacteria. This work opens the door for a new class of vibrational spectrometry based on the use of high frequency mechanical resonators with the unique capability to obtain information on single bioentities.

\section{P-146}

Modeling and characterizing of the basement membrane mechanical prestress during epithelial folding

Karla Y Guerra [1,2], Elisabeth Fischer-Friedrich [1], Christian Dahmann $[1,2]$

[1] Cluster of Excellence Physics of Life, TU Dresden, Germany; [2] Institute of Genetics, TU Dresden, Germany

Folding is the mechanism by which epithelial sheets of cells transform into functional three-dimensional organs. In the past, folding has mainly been attributed to force generation at the apical side of an epithelium. Recent studies indicate that local decrease in mechanical prestress at the basal side of cells can drive fold formation in the larval wing imaginal disc of the fruit fly. However, how this mechanical prestress is generated and modulated remains unknown. Here, we develop a coarse-grained continuum mechanical model of epithelial folding based on a lack of balance of mechanical prestress within the tissue due to localized degradation of the basement membrane. We used atomic force microscopy to quantify the basal prestress in the explanted wing disc. We tested the influence of developmental stage and different molecular perturbations on this basal mechanical prestress. Our results show that both, the actomyosin network and the extracellular matrix play a role in building mechanical prestress. Furthermore, we monitored the turnover of collagen in the wing disc and find negligible turnover on the time scale of fold formation, suggesting an elastic behavior in the time period of folding. This research points at a novel mechanism for fold formation and highlights the importance of the extracellular matrix for epithelial folding. 


\section{Posters}

- 10. Mechanobiology -

\section{P-147}

A new approach to the mechanics of DNA

Johannes Kalliauer [1], Gerhard Kahl [2], Stefan Scheiner [1], Christian Hellmich [1]

[1] Institute for Mechanics of Solids and Structures, TU Wien, Austria; [2] Institute for Theoretical Physics, TU Wien, Austria

Traditionally, DNA sequences are considered as cylindrical straight isotropic beams ${ }^{1}$, but this lead to unrealistic ratios between torsional and bending stiffnesses ${ }^{2}$.

The deformation characteristics of long biological macromolecules, such as DNA or collagen, can be lucidly described by the terms "bending", "stretching", "torsion" and "shearing". These terms appear in a subset of continuum mechanics, called beam theory, while the standard numerical modelling procedure for macromolecules, which is molecular dynamics, does not allow for a direct introduction of these deformation characteristics ${ }^{3}$.

This deficit has motivated the theoretical development of a transition or upscaling procedure from molecular dynamics to beam theory ${ }^{4}$. It consists of two steps: (i) translation of potential energies into systems of equilibrated forces and moments; (ii) upscaling, through the principle of virtual power, the forces and moments arising from (i), to the scale where the macromolecule itself is considered as a beam structure. As a first application, the above-described novel strategy is realised for a sequence of DNA base pairs, elucidating the source of the somehow paradox stretching-torsion coupling these molecules are known for.

${ }^{1}$ Gore, J., et al. Nature 442.7104 (2006): 836-839.

${ }^{2}$ Baumann et al. Proc. Natl. Acad. Sci. 94.12 (1997): 6185-90.

${ }^{3}$ Li, C. \& Chou, T.-W. Int. J. Solids Struct. 40 (2003): 2487-99.

${ }^{4}$ Kalliauer, J., et al. J. Mech. Phys. Solids (2020): 104040.

\section{P-149}

Studying the mechanobiology of living cells during interaction with their environment

André Körnig, Tanja Neumann, Torsten Müller

Bruker Nano GmbH, JPK BioAFM, Germany

Mechanobiology encompasses the study of mechanotransduction between growing cells and their microenvironment. To this end, atomic force microscopy (AFM) remains the only technique that offers premium resolution of the analysed biological systems, while being able to simultaneously acquire information about the sample's mechanical properties at near native sample conditions and simultaneously allowing full integration of the AFM with advanced optical microscopy. Cells adapt their shape and react to the surrounding environment by a dynamic reorganization of the F-actin cytoskeleton. We will demonstrate the application of high-speed AFM in the study of membrane ruffling and cytoskeleton rearrangement in living KPG-7 fibroblasts and $\mathrm{CHO}$ cells. External mechanical stress is known to influence cell mechanics in correlation to the differences in actin cytoskeleton dynamics. A crucial aspect of investigating cellular mechanobiology is to go beyond purely elastic models, which do not reflect their complex composition. We have therefore performed rheological measurements to characterise sample response at different time scales and measure viscoelastic properties in mammalian cells over a large frequency range $(0-500 \mathrm{~Hz})$. Cell-cell and cell-substrate interactions through cytoskeletal modulation determine cell fate, shape and spreading. We will demonstrate the application of single cell force spectroscopy for quantifying the adhesion between individual cells, and cell matrix substrates.

\section{P-148}

Simultaneous cytoskeletal stiffness mapping and confocal imaging of living cells by scanning ionconductance microscopy

Vasilii S Kolmogorov [1,2], Nikita A Savin [2], Alexei P Iakovlev [2], Alexander N Vaneev [1,2], Svetlana V Lavrushkina [1], Alexander S Erofeev [1,2], Petr V Gorelkin [2], Natalia L Klyachko[1], Igor I Kireev [1], Alexander G Majouga [1,4], Christopher Edwards [3], Pavel Novak [1], Yuri E Korchev [2,3]

[1] Lomonosov Moscow State University, Moscow, Russia; [2] National University of Science and Technology "MISiS", Moscow, Russia; [3] Department of Medicine, Imperial College London, London, UK; [4] D. Mendeleev University of Chemical Technology of Russia, Moscow, Russia

In this work funded by The Russian Science Foundation, grant number № 19-19-00626, we have demonstrated possibility of simultaneous stiffness mapping and confocal imaging, allowing to study living cell topography, stiffness dynamic and live confocal visualization of actin filaments dyed with Sir-Actin for single cell analysis. Correlative imaging showed significant changes of cell topography as well as changes to the stiffness and structure of actin filaments in confocal images. We observed a decrease in the height of the periphery of the cell but not at the central part after actin disruption, which is explained by a higher concentration of actin located within the periphery of the cell. A decrease of stiffness is clearly observable during Cyto-D treatment, as expected. Disruption of actin filaments is observable at confocal image in comparison with control cell where structured filaments are seen.

\section{P-150}

A role for dihydroxyphenylalanine (DOPA) and its radical as marker for mechanical stress in collagen

Markus Kurth [1,3], Uladzimir Barayeu [2], Christopher Zapp [3], Reinhard Kappl [5], Tobias Dick [2], David M. Hudson [4], Frauke Gräter [1,3]

[1] Interdisciplinary Center for Scientific Computing (IWR), Heidelberg University, Germany; [2] German Cancer Research Center (DKFZ), Heidelberg, Germany; [3] HITS gGmbH, Heidelberg, Germany; [4] Department of Orthopaedics and Sports Medicine, University of Washington, Seattle, USA; [5] Saarland University Faculty of Medicine, Homburg, Germany

Collagen is responsible for the physical strength of tendons in which the collagen fibres are elastic to some extent, but if over-stretched can rupture. After mechanical stress, we observed the formation of phenoxy radicals on dihydroxyphenylalanine (DOPA) by electron paramagnetic resonance (EPR). In rat collagen type I, we identified DOPA by mass spectrometry (MS) as a post-translational modification of tyrosine, found close to the intermolecular crosslinking sites. By joining MS and EPR measurements, we aimed to better understand the role of DOPA as a scavenger of mechanoradicals which potentially originate from ruptures in the crosslinks. We compared tail and Achilles tendons, which both formed DOPA radicals. Achilles tendons proofed to be less flexible and more resistant to the formation of radicals. MS analysis indicated that this is because of more mature crosslinks. Finally, in case of bond ruptures, in vitro assays showed that DOPA is a more efficient radical scavenger than tyrosine. 
Posters

- 10. Mechanobiology -

P-151

Effect of lamin A mutations R527P and R249Q on cell mechanics

S. Lavrushkina [1], A. Rudenko[1], V. Kolmogorov [1,2], A. Erofeev $[1,2], \quad$ P. Gorelkin [2], G. Maksimov [1,2], I. Kireev [1]

[1] Lomonosov Moscow State University, Russian Federation; [2] National University of Science and Technology "MISiS", Russian Federation

The nuclear lamina (NL) is the main rigid component of the cell nucleus. The NL consists of a meshwork of intermediate filaments built from lamins of A- and B-types of which lamin A plays a crucial role in cell migration, proliferation, DNA repair, cell death, etc. Mutations in the lamin A gene cause many diseases, from myodystrophies, lipodystrophies, neuropathies to premature aging. The mechanisms which underlie such severe phenotypes are still unclear. The present work aimed to estimate the effect of poorly studied missense mutations of lamin A - R527P and R249Q on the morphology and mechanical properties of the cell. We evaluated the lamin A meshwork morphology in normal HT1080 cells and cells expressing mutant lamins A genes with fluorescent microscopy. We showed local disarrangement of the NL and nuclear asymmetry. Also, we found the formation of lamin A aggregates as part of the NL. Then, we measured cellular stiffness using scanning ion-conducting microscopy. We compared the elastic modulus in the central part and periphery of cells and found that the cell nucleus display higher stiffness than cytoplasm. Also, the mutant cells were found to be $\sim 1.6$ times more rigid relative to wild-type cells. The research was supported by RSF № 19-79-30062.

\section{P-153}

Trodusquemine increases the mechanical stability of lipid membranes

Davide Odino [1], Silvia Errico [2], Claudio Canale [1], Riccardo Ferrando [1], Fabrizio Chiti [2], Annalisa Relini [1]

[1] Dept. of Physics, University of Genoa, Genoa, Italy; [2] Dept. of Experimental and Clinical Biomedical Sciences, University of Florence, Florence, Italy

We have used atomic force microscopy to study the effects of trodusquemine, a potential drug against neurodegenerative disorders, on the properties of supported lipid bilayers (SLBs) exhibiting different phases: a fluid matrix and ordered domains with a larger thickness. We have found that low trodusquemine concentrations $(1-15 \mu \mathrm{M})$ maintained the phase coexistence but modulated the amount of ordered phase. Furthermore, we evaluated the membrane mechanical properties by force spectroscopy measurements (AFMFS). We found that the force required to rupture the bilayer (breakthrough force) and the bilayer Young's modulus, which represents the stiffness of the lipid layer, increase in the presence of trodusquemine. This increase in mechanical strength could contribute to an increased resistance of the membranes to the toxic action of misfolded protein oligomers. The analysis is now in progress to discriminate the Young's Modulus values of each phase and to correct these values for the effect of the rigid substrate.

\section{P-152}

\section{Adhesion of fimbriated bacteria is force-} modulated due to multivalency

Anders Lundgren [1], $\quad$ Peter D J van Oostrum [2], Erik Reimhult [1]

[1] Department of Nanobiotechnology, BOKU University of Natural Resources and Life Sciences, Austria; [2] Department of Chemistry and Molecular Biology, University of Gothenburg, Sweden

E. coli bacteria that express type-1 fimbriae (pili) migrate along surfaces when pushed by a slow flow but stick firmly upon increasing flow. Flow-modulated biological binding is often described as unique properties of lectin-glycan pair interaction. Using holographic microscopy, we quantitatively track the 3D movements of fimbriated E. coli subject to controlled flow over surfaces nanopatterned with mannose or hydrophobic binding sites. We reveal that flow-modulated surface adhesion and motion are consequences of bacteria adhering via polydisperse, elastic fimbriae, irrespective of binding affinity and specificity. The fimbria-mediated surface repulsion and the flow forces on tethered bacteria establish an equilibrium bacteria-surface separation. When the flow-rate is increased, we observe that surface-bound bacteria are pushed down due to the torque acting on the surfacetethered bacterium, allowing a larger number of shorter fimbriae to form extra tethers, determining the surface avidity and surface motion. This provides a general mechanism for adaptive bacterial surface avidity, allowing to respond super-selectively to different flow environments, binding site concentrations and affinities, essential to govern tropism and surface colonization.

\section{P-154}

Basement membranes: Not just simple barriers Raphael Reuten [1], Sina Zendehroud [2], Monica Nicolau [1], Lutz Fleischhauer [3], Denise Nikodemus [1], Tom van Agtmael [4], Eleni Maniati [5], Jae Won Kyoung [1], Wilhelm Bloch [6], Roland Netz[2], Hauke ClausenSchaumann [3], Janine Erler [1]

[1] University of Copenhagen, Denmark; [2] Freie Universität Berlin, Germany; [3] Munich University of Applied Sciences, Germany; [4] University of Glasgow, UK; [5] Barts Cancer Institute, UK; [6] German Sport University Cologne, Germany

Metastasis is a complex multistep process and the major determinant of mortality in cancer patients. During metastasis cancer cells invade through the extracellular matrix, which is divided into the basement membrane (BM) and the interstitial matrix. Interestingly, the functional role of BM architecture during metastasis has not been addressed so far. We uncovered BM stiffness as a major determinant of metastasis formation. Our exciting data reveal that the softer the BM the more resistant to metastasis formation. Remarkably, we show that stiffness is a stronger influencing factor than pore size in the regulation of cancer cell movement through the BM. Our compelling mathematical simulations and biophysical and biochemical data show that the ratio between both ECM proteins netrin- 4 and laminin gamma1 correlates with BM stiffness. These data emphasize the impact of BM composition at the primary and secondary tumor site on BM stiffness and on metastasis formation independently of cancer-mediated alterations in breast and ovarian cancer as well as melanoma patients. Finally, our data suggest that the baseline BM stiffness may pre-determine survival even before having cancer throughout lifetime. 


\section{Posters}

- 10. Mechanobiology -

\section{P-155}

Glomerular stiffness: a new driver of glomerulosclerosis

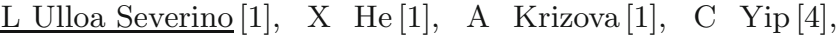
R Lennon [2], K N Campbell [3], M Krendel [5], D A Yuen [1] [1] Unity Health Toronto- St Michael's Hospital, Canada; [2] University of Manchester, UK; [3] Icahn School of Medicine at Mount Sinai, New York, USA; [4] University of Toronto, Canada; [5] SUNY Upstate Medical University, Syracuse, USA

Glomeruli are specialized collections of blood vessels in the kidney. In diabetes, they become progressively scarred, which can prevent proper blood filtration, leading to kidney dysfunction and kidney failure. Studies of glomerular scarring have focused on molecules produced in the diabetic kidney as the main cause of this scarring. However, growing evidence suggests that biomechanical forces, may also be important triggers for scarring. I have used atomic force microscopy (AFM), to study diabetic kidneys, finding that glomeruli stiffen as diabetes progresses. Several research groups, including ours, have identified the two proteins YAP and TAZ as "mechanosensors" that are activated by organ stiffening. Importantly, active YAP and TAZ promote scarring. In this project, I will study the main producers of scar in glomeruli, stiffen in response to diabetes, and whether this potential stiffening activates YAP and TAZ to cause glomerular scarring. I will used various cell and mouse models of diabetic kidney disease. Ultimately, my goal is to provide valuable new insights that will: (1) help explain why diabetes causes kidney scarring, and (2) inform the development of new treatments for diabetic kidney disease.

\section{P-157}

Morphological and mechanical dynamics of migrating platelets investigated with scanning ion conductance microscopy (SICM)

Hendrik von Eysmondt, Johannes Rheinlaender, Jan Seifert, Tilman E Schäffer

Institute of Applied Physics, Eberhard Karls University Tübingen, Germany

Platelets or thrombocytes are the central element of the hemostatic system as being the first cells adhering to the site of a vessel injury, orchestrating the blood clot formation, and thereby establishing the initial steps of sealing the wound. In addition, platelets have been identified as highly motile cells, which can actively migrate towards sites of inflammation or bacterial pathogens. However, many aspects of the underlying cellular machinery are still unknown. We therefore studied migrating platelets using scanning ion conductance microscopy (SICM), an imaging technique providing sample topography together with quantitative mechanical properties at nanoscale resolution. We found that migrating platelets exhibit a three-dimensional shape anisotropy, which is directionally correlated with the direction of migration. We recorded time-lapsed maps of the elastic modulus of migrating platelets, which revealed a characteristic subcellular distribution. Using both fluorescence microscopy and SICM, we show that this distribution is caused by a dynamic reorganization of the platelet's actin cytoskeleton and thereby give direct mechanical evidence that platelet migration is driven by active cytoskeletal reorganization.

\section{P-156}

Sensing the force from within: the nucleus as a mechanosensitive controller of adaptive dynamics Valeria Venturini [1,2], Fabio Pezzano [2], Frederic CatalàCastro [1], Monica Marro [1], Senda Jiménez-Delgado [2], Micheal Krieg [1], Stefan Wieser [1], Verena Ruprecht [2]

[1] ICFO - Institut de Ciències Fotòniques, Spain; [2] Centre for Genomic Regulation (CRG), Spain

Mechanical forces regulate single cells and tissue dynamics during development, tissue homeostasis and cancer progression. Cellular shape deformations in confined microenvironments have been shown to induce cortical myosin II activation that can transform non-motile cells into a fast-amoeboid migration mode in various cell types. How cells sense shape deformations and translate the mechanical force into myosin II activation remained unclear. Here we show that the nucleus functions as an elastic mechano-gauge that allows cells to measure shape changes. We found that inner nuclear membrane unfolding upon nucleus stretch triggers a calciumdependent mechanosensing pathway that activates cytosolic phospholipase $\mathrm{A} 2\left(\mathrm{cPLA}_{2}\right)$ and metabolite production of AA regulating myosin II activity. Highly contractile cells spontaneously acquire a stable-bleb phenotype and rapidly escape from the confined environment. Furthermore, we show that nuclear stretch combined with intracellular nucleus positioning enables cells to decode different types of shape deformations. Our data support that the nucleus establishes a functional module for cellular proprioception, enabling cells to sense and interpret shape changes and to adapt their dynamic response behavior to the $3 \mathrm{D}$ microenvironment.

\section{P-158}

Influence of hypoxia on breast cancer cell mechanics

Barbara Zbiral [1], Andreas Weber [1], Maria D Vivanco [2], José L Toca-Herrera [1]

[1] Institute for Biophysics, University of Natural Resources and Life Sciences Vienna, Austria; [2] Cancer Heterogeneity Lab, CIC bioGUNE, Spain

Compared to their healthy counterparts, breast cancer cells display an array of morphological and biochemical changes, which confer invasive properties onto the cells. Such changes are linked to changes in membrane and adhesive properties, cell-to-cell contacts, and cytoskeletal rearrangement. All these constituents strongly influence cell mechanics. These are known to change over tumour progression.

Solid tumors develop a hypoxic core associated with hallmarks of cancer progression, such as invasiveness and epithelial-to-mesenchymal transition (EMT). To gain insight into hypoxia-associated breast cancer progression from a mechanobiology perspective, the influence of hypoxia on the mechanical properties of MCF-7 and tamoxifen resistant MCF-7 cells was examined in this study.

Indentation and microrheology experiments were performed via AFM This allows the determination of mechanical properties such as the Young's Modulus, relaxation behaviour and viscoelastic parameters. CLSM, western blotting and flow cytometry experiments were done to further demonstrate changes in the cytoskeleton, adhesion proteins and gene expression. Results show that hypoxia clearly induces mechanical changes, such as cell softening, different relaxation behaviour, as well as morphological changes. 
Posters

- 10. Mechanobiology -

\section{P-159}

Viscoelastic properties of cancerous and nonmalignant bladder cells treated with microtubules targeting chemotherapeutic- MTs and actin crosstalk?

Joanna Zemła [1], Claude Verdier [2], Małgorzata Lekka [1] [1] Institute of Nuclear Physics Polish Academy of Sciences, Poland; [2] Laboratoire Interdisciplinaire de Physique, Université Grenoble Alpes, CNRS, France

Modern anticancer drugs target microtubules (MT) causing their de-/hyper-polymerization what disrupts both the cytoskeleton architecture and biochemical processes. Here, we present the study on the influence of docetaxel (DTX) on mechanical properties of bladder cancer cell lines of different grades (non-malignant bladder cancer HCV29, human urinary bladder carcinoma 5637 (grade 2), and human urinary bladder transitional cell carcinoma T24 (grade 2-3)). Microrheological measurements performed with atomic force microscope (AFM) working in force modulation mode allowed the determination of the dynamic modulus $\left(\mathrm{G}^{*}\right)$ describing both elastic (storage modulus $G^{\prime}$ ) and viscous (loss modulus G") properties of cells. The data were collected at a low-frequency range $(1-300 \mathrm{~Hz}$ ) and fitted with SGR (Soft Glassy Rheological) model. Parameters such as $\mathrm{G}_{\mathrm{N}}{ }^{0}$, elastic plateau modulus at low frequencies, and $\mathrm{f}_{\mathrm{T}}$, transition frequency in the log-log plot, were calculated. The obtained results indicate that changes in the mechanical properties of cells stem from the cytoskeleton organization. DTX exposition results in the reorganization of the MTs' network, which also induces actin mesh remodeling, and both are responsible for changes of the storage and loss moduli. 


\section{Posters}

-11. Light as a tool in biophysics -

P-160

\section{Modulating membrane shape and mechanics by} light

M. Aleksanyan [1], F. Crea [2], V. Georgiev [1], A. Grafmueller [1], J. Heberle [2], R. Dimova [1]

[1] Max Planck Institute of Colloids and Interfaces, Germany; [2] Freie Universitaet Berlin, Dept. of Physics, Germany

Over the years, light has been widely used as an effective trigger in biotechnology to interrogate biological systems and provide a conditional control over the complex cellular processes. Understanding the fundamental biophysics of lightinduced changes in biosystems is crucial for cell viability and optimization of their clinical applications in optogenetics and photopharmacology. Here, we employ giant unilamellar vesicles (GUVs) to investigate light-induced changes on the material properties of membranes doped with azobenzenephosphatidylcholine (azoPC) as a photoswitch. In particular, we examined light-induced vesicle area change, reversibility and kinetics of photoswitching and membrane bending rigidity. We show that light can be used to manipulate the shape and mechanics of these synthetic cells. Our studies demonstrated membrane area increase due to cis photoisomerization of azoPC which is consistent with results from MD simulations. At high azoPC fractions (>50 mol\%), complex shape transformations were observed. Photoswitching was reversible but showed faster kinetics from cis to trans isomerization. Trans to cis isomerization of azoPC rendered the membrane softer, which scaled with cis azoPC fraction in the membrane. Our results demonstrate membrane mechanics could be easily controlled for azoPC containing membranes through light.

\section{P-162}

Nano-lithography in microfluidics for 3D platelet aggregation analysis

Bianca Buchegger [1,2], Alexander Tanzer [2], Christian Gabriel [3], Thomas A Klar [2], Jaroslaw Jacak [1]

[1] University of Applied Sciences, Upper Austria School of Medical Engineering and Applied Social Sciences, Garnisonstraße 21, 4020, Linz, Austria; [2] Institute of Applied Physics and Linz Institute of Technology (LIT), Johannes Kepler University Linz, Altenberger Straße 69, 4040, Linz, Austria; [3] Ludwig Boltzmann Institute for Experimental and Clinical Traumatology, Donaueschingenstraße 13, 1200, Vienna, Austria

Cells cultivated on artificial 2D environments show different spread morphology, differentiation, and proliferation rates compared to cells spread on three-dimensional matrices. Hence, fabrication of two- and three-dimensional scaffolds carrying precisely shaped nanoscopic sites for external cell stimulation is an objective of paramount scientific focus. Here, we show a microfluidic (MF) device with multiphoton lithography structures carrying sub-diffractional nanoanchors written with STED-lithography, which enable von Willebrand factor (vWF) binding. The density of $\mathrm{vWF}$ is adjusted by varying the number of nanoanchors on the $3 \mathrm{D}$ structure. Our studies show that platelet activation decreases with $\mathrm{vWF}$ density on the 3D scaffolds in the MF. Our MF tool allows for studying the impact of vWF density on platelet activation.

\section{P-161}

Optogenetic high-throughput screening

Kathrin Brenker [1,2], Annik Jakob [1,2], Luis Köbele [1,2]

[1] Opto Biolabs, Freiburg im Breisgau, Germany; [2] AlbertLudwigs-Universität Freiburg, Germany

Optogenetic tools allow the remote control of cellular pathways using light. Originating from the field of neurobiology using light-gated ion channels to study neuronal behavior, more recently developed non-neuronal optogenetic tools enable the isolated, functional investigation of almost any intracellular signaling molecule within complex signaling pathways. This remote control of cellular pathways has major implications for therapeutic tools, including targeted immunotherapy.

A major obstacle for optogenetic researchers is the controlled delivery of light to the cell sample. Hence, the most popular tools for optogenetic studies are microscopy-based experiments or using self-made LED devices. A widely neglected, yet very powerful analytical tool is flow cytometry.

The flow cytometer has major advantages over a microscope, including the ability to rapidly measure thousands of cells at single cell resolution. However, it is not yet widely used in optogenetics due to a lack of suitable illumination. Opto Biolabs, a spinoff from the University of Freiburg, develops specialized illumination devices enabling the combination of optogenetics and flow cytometry. Optogenetic flow cytometry enables the easy analysis of e.g. calcium kinetics and the developed illumination device is compatible with most existing flow cytometers.

Our future work will focus on the expansion of our technology to optogenetic cell sorting. This will enable large scale single cell analysis and greatly simplify the discovery and development of novel, cell-based optogenetic tools and therapies.

\section{P-163}

The influence of small membrane proteins on bio-membrane properties - A time-resolved measurement approach

Iulia Carabadjac, Ana Natriashvili, Hans-Georg Koch, Heiko Heerklotz

Albert-Ludwigs University, Freiburg, Germany

The characterization of bio-membrane properties with and without overexpressed proteins can reveal valuable information about the role of the relevant protein in the microorganism. YohP is a model protein belonging to a largely unexplored class of small membrane proteins. Some of them are believed to be linked to stress response mechanisms in bacteria. Understanding its effects on membranes is the first step to determine the specific mechanism of its action. Timecorrelated single photon counting (TCSPC) is a powerful tool to examine fluorescence decays on the nanosecond scale. With the help of fluorescent probes showing different fluorescent behavior according to respective surroundings, i.e. in buffer compared to inner-membrane-vesicles, it is possible to estimate the membrane agility, the kinetics of the probe and the hydration level of the membrane. For timeresolved anisotropy measurements, a combination of DPH, TMA-DPH and DPH-PC probes displays a proper estimation basis for depicturing protein-dependent changes in cell membranes. Time-resolved spectra of Laurdan provide information on membrane dynamics and order. In summary, the aim of this project is to understand the impact of YohP on membranes as well as to develop a measurement protocol that is well suitable to describe changes in bio-membrane properties. 
Posters

-11. Light as a tool in biophysics -

P-164

UV-induced modifications in collagen fibers molecular structure: a fluorescence spectroscopy and microscopy study

Giuseppe de Luca [1], Giuseppe Sancataldo[2], Valeria Vetri [2] [1] Department of Biological, Chemical and Pharmaceutical Sciences and Technologies (STEBICEF), University of Palermo, Italy; [2] Department of Physics and Chemistry Emilio Segrè, University of Palermo, Italy

Collagen is one of the main components of human connective tissue (skin, bone, and tendons) and its organization and cross-linking status it is known to be at the basis of physical properties and function of these tissues.

We here present an experimental study based on spectroscopy and non-linear imaging focused at highlighting the effects of UV irradiation on collagen fibers (type I). The aim is to analyze UV-induced collagen photo degradation, which is known to be involved in premature skin aging and in the onset of pathological conditions. Bulk analysis reveals that collagen autofluorescence is strongly modified by UV-irradiation leading to the degradation of the native fluorescent species and to the rising of a small fluorescent band centered at $450 \mathrm{~nm}$ that could be related to the oxidation product of tyrosine residues.

To better analyze these modifications effect, we also took advantages of the use of ANS, a well-known fluorescent probe, that is known to bind to collagen fibers and whose fluorescence depends on peculiar properties of the environment. Bulk measurements are combined with nonlinear imaging, through the use of second harmonic generation and twophoton FLIM of ANS. Results indicate that UV-exposure modifies collagen supramolecular organization inducing critical modification in ANS binding sites reducing their overall affinity.

\section{P-166}

Changes in the fluorescence characteristics of exosomes isolated from the urine and blood of patients with bladder cancer

Daniela Džubinská, Milan Zvarík, Martina Velísková, Libuša Šikurová, Iveta Waczulíková

Department of Nuclear Physics and Biophysics, Faculty of Mathematics, Physics and Informatics, Comenius University, Mlynská dolina F1, Bratislava, Slovakia

Exosomes are nanovesicles present in body fluids, and their content carries molecular signatures or footprints resembling those of the diseased cells from which they were secreted. This molecular signature distinguishes tumor-derived exosomes from exosomes released by non-malignant cells. Exosomes thus can serve as a source of diagnostic biomarkers, many of which possess endogenous fluorescence that can be detected and further analyzed. Differences in spectral characteristics of exosomes could be potentially used as a diagnostic tool.

The presented work deals with changes in fluorescence characteristics of exosomes from the urine and blood of patients with bladder cancer (BC) and from patients with nonmalignant haematuria (HU). Exosomes were isolated using ultrafiltration and size exclusion chromatography. We measured total fluorescence of exosomes at excitation and emission wavelengths in the range of $200-800 \mathrm{~nm}$. The obtained spectra were visualized by excitation emission matrices, based on which we identified fluorescence regions of interest. Using uni- and multivariate analyses we reduced high dimensionality of the fluorescence dataset to select the fluorescence regions of exosomes providing the best classification of $\mathrm{BC}$ and $\mathrm{HU}$ patients.

\section{P-165}

Light scattering as an easy tool to measure vesicles weight concentration

Giulia Di Prima [2], Fabio Librizzi [1], Rita Carrotta [1]

[1] Institute of Biophysics, CNR, Palermo, Italy; [2] Stebicef, University of Palermo, Italy

Over the last decades, unilamellar liposomes have emerged as promising vectors for drug delivery and as effective natural membrane models, for studying biophysical and biological processes or biotechnological issues. For all applications, knowing their concentration after preparation is crucial.

A new assay to determine weight concentration is presented, based on a suitable analysis of light scattering intensity from liposome dispersions. The method is born and tested for extrusion preparations, a common method to create unilamellar liposomes, but it can be easily extended to other preparation nethods. It is precise, easy, fast, non-destructive and uses a tiny amount of sample. Furthermore, the scattering intensity can be measured indifferently at different angles, or even by using the elastic band obtained from a standard spectrofluorimeter. To validate the method, the measured concentrations of vesicles of different matrix compositions and sizes are compared to the data obtained by the standard Stewart assay.

\section{P-167}

Photoactivation of YAP for controlled growth of cell collectives

Bernhard Illes [1], Adrian Fuchs [1], Florian Gegenfurtner [1], Evelyn Ploetz [1], Stefan Zahler [1], Angelika Vollmar [1], Hanna Engelke [2,1]

[1] Ludwig-Maximilians-Universität München, Germany; [2] University of Graz, Austria

The mechanical properties of the cell environment strongly influence cell behavior. For an understanding of how external mechanical stimuli impact cell behavior it is important to investigate the underlying mechanosensing and -signaling within cells and cell collectives. A key protein regulating these processes is Yes-associated protein (YAP). It translocates into the nucleus upon mechanical activation. Once it is in the nucleus it triggers enhanced proliferation and migration. In order to study its impact on cells and cell collectives, we locally control YAP activation with light. We genetically prepend a photoactivatable nuclear localization signal to YAP to trigger its translocation into the nucleus with light. This photoactivatable YAP (optoYAP) stayed cytoplasmic in single cells until its nuclear translocation was induced with light. In the nucleus it activates downstream signaling. Transfecting optoYAP into cell spheroids allows for control of YAP-signaling within the spheroid. Illumination of the entire spheroid enhanced its general growth. The use of light enables us to locally activate YAP in a few cells of a spheroid. This leads to a directed growth selectively on the illuminated side of the spheroid resembling invasion. Thus, our optoYAP facilitates the study on how local changes in mechanosignaling may influence behavior of cells. 
Posters

-11. Light as a tool in biophysics -

\section{P-168}

Molecular dynamics of interactions of phospho$\operatorname{rus}(\mathrm{V})$ porphyrins with lipid bilayer

Maksim Kalutsky [1], Yulia Gorbunova [1,2], Oleg Batishchev [1] [1] A.N. Frumkin Institute of Physical Chemistry and Electrochemistry, Russian Academy of Sciences, Russian Federation; [2] N.S. Kurnakov Institute of General and Inorganic Chemistry, Russian Academy of Sciences, Russian Federation

The growing number of drug-resistant bacteria is a major problem in modern medicine. Antibiotic resistance significantly complicates the treatment of bacterial infections making commonly used drugs ineffectual. Antibacterial photodynamic therapy is an antibiotic-free method of bacterial inactivation. It implies the application of non-toxic photosensitizers (PS), which provide photoinduced generation of reactive oxygen species damaging structural elements of bacterial cells.

The antimicrobial activity of various PS molecules differs in solution and upon application to bacteria. Thus, it can be significantly altered by their interactions with the bilayer lipid membrane (BLM). Molecular dynamics (MD) with its ability to provide the structural information at a molecular level can give insight into the mechanisms of PS-BLM interactions. In the present work, we developed CHARMM compatible force field parameters for 6 phosphorus(V) containing porphyrins with different axial and peripheral groups and used MD to study their interactions with BLM. We obtained information about the location, orientation, and dynamics of PS molecules in the lipid membrane as well as the tendency of different PS to aggregate.

The work was supported by the Russian Science Foundation (grant \#19-13-00410)

\section{P-170}

Correlative single-molecule force-FRET measurements of DNA hairpin conformational dynamics

Aida Llauro-Portell, Ann Mukhortava, Maurice Hendriks, Philipp Rauch

LUMICKS, Netherlands

The biological function of proteins and nucleic acids is tightly coupled to their conformation dynamics. Therefore, studying how macromolecules fold and undergo conformational changes is crucial to understand the underlying mechanisms of biological processes. In particular DNA/RNA hairpins play essential roles in regulating interactions with proteins in transcription, recombination or replication. Single-molecule force spectroscopy represents an ideal tool for elucidating those biological roles because of its unique capability to isolate individual biomolecules and observe conformational transitions in real-time.

We study FRET-labelled DNA hairpin folding transitions using high-resolution optical tweezers combined with fluorescence microscopy. Unprecedented trap-to-trap distance stability enabled through an active feedback mechanism allows to measure conformational state distributions over hours without altering the energy landscape. This in turn is crucial to capture rare conformational states that might be overlooked in measurements limited by instrumental drift. Kinetic, energetic and structural properties of these states could be determined quantitatively from a correlative information source - force and fluorescence data, thereby providing new insights into folding pathways and energy landscape.

\section{P-169}

Dynamic second-harmonic imaging of lipid membrane hydration induced by copper ions

Seonwoo Lee, David Roesel, Sylvie Roke

École Polytechnique Fédérale de Lausanne (EPFL), Switzerland

Cell membranes are composed of a hydrated lipid bilayer that is molecularly complex and diverse, and the link between molecular hydration structure and membrane macroscopic properties is not well understood. This is mainly due to a lack of technology that can probe hydration on the molecular level and relate this information to micro- and macroscopic properties, such as membrane organization, electrostatics, free energy and curvature. Here, we use second harmonic (SH) microscopy to study ion-induced transient domains of ordered water at the membrane interface. Specifically, $\mathrm{Cu}^{2+}$. membrane binding is examined in terms of membrane hydration and local specific interaction using Phosphatidic acid (PA) and Phosphatidylserine (PS) lipids.

Email: seonwoo.lee@epfl.ch

\section{P-171}

Dynamic insights into Orai channel opening mechanisms using optoproteomics

Lena Maltan, Sarah Weiß, Hadil Najjar, Isabella Derler

Johannes Kepler University Linz, Institute of Biophysics, Austria

Calcium $\left(\mathrm{Ca}^{2+}\right)$ ions are essential second messengers that control a diversity of human body functions such as immune response, neuronal processes and muscle contraction. At the level of a single cell, $\mathrm{Ca}^{2+}$ signaling processes are tightly controlled by proteins that sense or transport $\mathrm{Ca}^{2+}$. An important, cellular $\mathrm{Ca}^{2+}$ entry pathway is the $\mathrm{Ca}^{2+}$ release-activated $\mathrm{Ca}^{2+}$ (CRAC) ion channel. It consists of two proteins, the stromal interaction molecule 1 (STIM1), a $\mathrm{Ca}^{2+}$ sensor localized in the ER and Orai, a cell membrane localized $\mathrm{Ca}^{2+}$ ion channel. $\mathrm{Ca}^{2+}$ store-depletion induces the activation and coupling of the two proteins, enabling $\mathrm{Ca}^{2+}$ to enter the cell via Orai. This activation cascade involves a global conformational channel of the entire channel complex, which is essential for pore opening. However, the detailed dynamics of the Orai channel are far from being understood. To visualize the dynamic structural changes of the Orai channel as it transitions from the closed to the open state, we use the emerging optoproteomics technology, which allows to site-specifically manipulate amino acids within a protein via light. We use photo-crosslinking to track key moving sites within Orai transmembrane domains that lead to Orai channel activation upon UV light irradiation. Collectively, we designed and deployed for the first time lightsensitive Orai ion channels to obtain dynamic insights into ion channel motions at the structural level. 
Posters

- 11. Light as a tool in biophysics -

P-172

Raman spectroscopy for in vivo study of carotenoids in juvenile forms of insects

Matvey I Nikelshparg [1], Daniil N Bratashov [2], Vasily V Anikin [2], Evelina I Nikelshparg [3]

[1] MAOU "Gymnasium 3" Saratov, Russian Federation; [2] Saratov State University named after Chernyshevsky, Russian Federation; [3] Lomonosov Moscow State University, Russian Federation

Raman spectroscopy (RS) allows evaluating local concentration and composition of carotenoids. $532 \mathrm{~nm}$ laser excitation provides resonance Raman conditions to study carotenoids. One of the main advantages of RS is non-invasiveness, which is especially important in studying rare animals. Such a strategy of investigation is essential for insects that undergo complete transformation and are unidentifiable in the larval stage. We applied resonance RS to probe carotenoids in living larvae and pupae of the gall wasp Aulacidea hieracii (Hymenoptera: Cynipidae), which induces gall formation on Hieracium $x$ robustum. We revealed that carotenoids distribute unevenly within insect body, and this distribution changes as insect mature. Carotenoids accumulate presumably in thoracic segments of the larvae and pupae. Though carotenoid amount differs in certain segments of insect, the carotenoid composition is the same. The present novel application of RS to study insects in vivo could advance the field of entomology and biochemistry of carotenoids.

\section{P-174}

Nanoresolved infrared spectroscopy at SISSI beamline of Elettra sincrotrone

Federica Piccirilli [1], Giovanni Birarda [1], Stefano Lupi [2,3], Lisa Vaccari [1]

[1] Elettra Sincrotrone Trieste, Italy; [2] Sapienza Università di Roma, Italy; [3] CNR Istituto Officina dei Materiali, Italy

The SISSI (Synchrotron Infrared Source for Spectroscopy and Imaging) beamline of Elettra Sincrotrone Trieste delivers light over a broad spectral range, from $\mathrm{THz}$ to visible. The laboratory represents a valuable tool in several research fields, from biophysics to solid state physics. SISSI experimental end stations allows to probe matter with diffraction limited lateral resolution of few microns. The beamline has been further upgraded since the recent installation of the nano-FTIR instrument. Exploiting infrared light of DFG and QCL lasers, as well as of SR, the instrument extends indeed the IR lateral resolution below diffraction-limit, up to $10-20 \mathrm{~nm}$. We present here selected nano-FTIR experiments performed at SISSI beamline on systems of biophysical interests. Nanoresolved experiments performed on DNA adsorbed on clay nanotubes are shown. The presented results reveal partial covering of nanotube outer surface by DNA molecules, with some DNA structural modification. A nanoscopic study on vaccine adjuvants development is also presented, showing model proteins structural modification induced by adsorption on alumina nanoparticles. NanoFTIR experiments applied to the study of biomaterials for wound dressing are also shown. The presented experiments aim to provide the audience of the conference the flavor of the new capabilities that will be achievable throguh Nano-FTIR.

\section{P-173}

Computational modeling reveals the influence of lipid membrane phases on fluorescent probes' optical properties

Silvio Osella [1], Markéta Paloncýová [2], Gustav Aniander [3], Emma Larsson [3], Maryam Sahi [3], Stefan Knippenberg [2,4] [1] Biological Systems Simulation Lab, University of Warsaw, Poland; [2] Czech Advanced Technologies and Research Institute, Palacký University, Czech Republic; [3] Department of Theoretical Chemistry and Biology, KTH, Sweden; [4] Theory Lab, UHasselt, Belgium

Organic fluorophores embedded in lipid bilayers can nowadays be described by a multiscale computational approach. We developed over the years a methodology to characterize probes in view of their different positions, orientations, conformational versatilities, and (non) linear optical as well as fluorescence properties when they are embedded in model membranes. In this presentation, computations on Laurdan, indocarbocyanine, diphenylhexatriene, and azobenzene derived probes are presented, sketching how a multiscale approach based on extended molecular dynamics and hybrid quantum mechanics-molecular mechanics frameworks can predict probes' optical properties like two-photon absorption, second harmonic generation, and fluorescence lifetime and time resolved fluorescence anisotropy. We show how computation can uncover novel functionalities of wellused probes and can guide the analysis and interpretation of experimental data. The properties can be used to gain information on the phase and condition of the surrounding biological environment.

\section{P-175}

An untapped tool for biological samples: spectroscopic ellipsometry

Giulia Pinto, Paolo Canepa, Chiara Toccafondi, Ornella Cavalleri, Maurizio Canepa

Department of Physics, University of Genova, Genova, Italy

Spectroscopic ellipsometry (SE) is a non-perturbative and extremely sensitive method for the analysis of thin films. From the measure of the changes in polarization of a light beam upon reflection from the sample surface, it provides information on film thickness and optical properties. We employed SE to investigate in situ and in real time the deposition of ultrathin films of biological molecules, like DNA and yeast cytochrome c (YCC). Through the analysis of SE difference spectra we were able to detect UV-Vis molecular absorptions at the monolayer level and correlate changes in the molecular absorptions to structural changes within the molecular layers. By in situ SE dynamic scans we could monitor in real time the self-assembly of thiolated single-stranded DNA on flat gold surfaces. By measuring the increase in the DNA absorption at $260 \mathrm{~nm}$ we could detect the hybridization with complementary DNA. An accurate optical model allowed us to optically characterize ssDNA and dsDNA films, providing a new tool for nucleic acid sequence recognition. By SE we investigated the visible absorptions of YCC monolayers deposited both on gold and $\mathrm{SiO}_{2} / \mathrm{Si}$ surfaces. The position of the Soret band as well as the intensity ratio of the Q-bands of deposited YCC were found to deviate from the YCC absorption in solution (the deviations observed on $\mathrm{SiO}_{2} / \mathrm{Si}$ being larger than those observed on gold), and suggest YCC conformational changes upon adsorption. 
Posters

-11. Light as a tool in biophysics -

\section{P-176}

Optofluidic force induction scheme for the characterization of nanoparticle ensembles

Marko Šmić [1,2], Gerhard Prossliner [2,3], Ruth Prassl [3], Christian Hill [2,3], Ulrich Hohenester [1]

[1] Institute of Physics, University of Graz, Austria; [2] Brave Analytics GmbH, Graz, Austria; [3] Institute of Biophysics, Medical University of Graz, Austria

Momentum transfer from light to matter provides the basic principle of optical tweezers. Most studies have hitherto employed this principle for trapping and manipulation of single nanoparticles. However, in a microfluidic channel one can also monitor the effect of optical forces exerted on ensembles of dielectric nanoparticles, to acquire knowledge about various nanoparticle parameters, such as size, shape or material distributions.

Here, we present an optofluidic force induction scheme (OF2i) for real-time, on-line optical characterization of nanoparticles. Our experimental setup builds on precisely controlled fluidics as well as optical elements, in combination with a focused laser beam with orbital angular momentum. By monitoring the single particle light scattering and trajectories in presence of optical and fluidic forces, we obtain detailed information about the properties of the individually tracked particles.

We analyse the trajectories using a detailed simulation approach based on Maxwell's equations and Mie's theory, in combination with laser fields and fluidic forces. We discuss the basic physical principles underlying the OF2i scheme and demonstrate its applicability. Our results prove that OF2i provides a flexible work bench for numerous applications.

\section{P-178}

Phosphorus (V) porphyrins on bilayer lipid membrane: Influence of structure on binding and photodynamic efficiency

Valery S Sokolov [1], Irene Jimenez-Munguia [1], Anna N Konstantinova [1], Kirill I Makrinsky [1], Ivan N Meshkov [1], Yuri A Ermakov[1], Oleg V Batishchev[1], Yulia G Gorbunova $[1,2]$

[1] Frumkin Institute of Physical Chemistry and Electrochemistry, Russian Academy of Sciences, Moscow, Russian Federation; [2] Kurnakov Institute of General and Inorganic Chemistry, Moscow, Russian Federation

The novel photosensitizers for photodynamic therapy - phosphorus (V) with porphyrins bearing pyridine peripheral groups and hydroxyl or ethoxy axial ligands $\left(\mathrm{PorP}^{+}\right)$- were studied by measurements of boundary potentials (BP) on bilayer lipid membranes (BLM). Adsorption of $\mathrm{PorP}^{+}$on BLM led to BP changes of positive sign detected by 3 methods: inner membrane field compensation (IFC), BLM conductance with nonactin and -potential on liposomes. Changes of BP by $\mathrm{PorP}^{+}$were observed only when it contained pyridine peripheral groups. The values of $\mathrm{BP}$ measured by IFC or with nonactin were much higher than -potentials. It was explained by immersion of $\mathrm{PorP}^{+}$charged groups into BLM. The $\mathrm{PorP}^{+}$with hydroxyl axial ligands added to one side of BLM penetrated through the membrane decreasing the difference of $\mathrm{BP}$ on it. The photodynamic efficiency of MPyP was evaluated as a rate $\mathrm{R}$ of oxidation of styryl dye di-4ANEPPS. The value of $\mathrm{R}$ did not significantly differ for all $\mathrm{PorP}^{+}$derivates. Supported by Russian Science Foundation (№ 19-13-00410) and RFBR (№ 19-04-00694).

\section{P-177}

The Brownian motion of flexible microstructures - towards viscosity measurements in microfluidic channels

Cyril Slabý [1], Jana Kubácková [2], Andrej Hovan [1], Pavol Miskovský [3], Zoltán Tomori [2], Gregor Bánó [1]

[1] Department of Biophysics, Faculty of Science, P. J. Šafárik University, Jesenná 5, 04154 Košice, Slovakia; [2] Department of Biophysics, Institute of Experimental Physics, Slovak Academy of Sciences, Watsonova 47, 04001 Košice, Slovakia; [3] Center for Interdisciplinary Biosciences, P. J. Šafárik University, Jesenná 5, 04154 Košice, Slovakia

The thermal motion of microbeads, either free or trapped by optical tweezers, is frequently used for micro-rheological measurements in microfluidic environments. In this work we investigate the possibility of an alternative method for viscosity measurements utilizing microbeads attached to flexible cantilevered nanowires. The used microstructures were prepared by two-photon polymerization direct laser writing (TPP-DLW) and were incorporated into microfluidic channels. The low stiffness of the nanowires gives rise to significant Brownian oscillation of the microbeads. The stochastic oscillations are described by an analytical model, solving the corresponding Langevin equation. The nanowires possess viscoelastic material properties, which needs to be considered. Based on the theoretical results, we propose a new method of viscosity measurements inside microfluidic channels.

\section{P-179}

Vibrational spectroscopy and nanotomography reveal the intracellular interactions of gold nanostars

Cecilia Spedalieri [1], Gergo P Szekeres [1,2], Janina Kneipp [1,2] [1] Humboldt-Universität zu Berlin, Department of Chemistry, Germany; [2] Humboldt-Universität zu Berlin, School of Analytical Sciences Adlershof (SALSA), Germany

Gold nanostars, anisotropic nanostructures with sharp tips, are promising candidates for a wide range of biotechnological and biomedical applications, as well as for optical probing, including that of nanostructure-biomolecule interactions that may affect further applications. In this work, we present results on the distribution of gold nanostars in cells using soft-X-ray tomography (SXT) together with detailed vibrational spectroscopic information on the structure and composition at the surface of the nanostars residing in cells of different cell lines. Surface-enhanced Raman scattering (SERS) signals observed after cellular uptake of gold nanostars indicate a close interaction mainly with protein components in the endolysosomal system. Moreover, modifying the morphology of the nanostructures, the interaction with lipids increases. Complementary to this, SXT was used to characterize the processing of the intracellular aggregates of the nanostars that are formed, their properties and their interaction with the biological matrix. The $3 \mathrm{D}$ nanotomography results revealed variation in localization, size morphology and density of the intracellular gold nanoaggregates. Combining SERS and SXT will improve the design of safe and efficient nanoprobes as tools for biomedical use. 
Posters

-11. Light as a tool in biophysics -

P-180

Near-infrared treatment effects on rotenone challenged U87 MG cells

Katarina Stroffekova, Simona Kolesarova, Silvia Tomkova

Faculty of Science, PJ Safarik University, Košice, Slovakia

The civilization diseases common denominator, including cardiovascular and neurodegenerative ones, is increased oxidative stress and mitochondrial dysfunction. The clinical treatments using compounds to target a signaling pathway involved in regulation of apoptosis are not effective in case of mitochondria dysfunction due to mitochondria population and various level of mitochondria damage within the population. Alternative approach is a global improvement of mitochondria population. Near-infrared (NiR) treatment has the potential to fulfill protective and regenerative functions for mitochondria and cells. In our study, we have focused on the acute NiR effects at the direct cellular level in control and rotenone, complex I inhibitor, challenged U87 MG cells with respect on cell viability, mitochondria morphology and ROS. Our results indicate that NiR effects depend on irradiation wavelength and dose. Chronic treatment (48hr) with 1 and $10 \mu \mathrm{M}$ rotenone resulted in decreased cell viability to 58 and $47 \%$, respectively, mitochondria fragmentation, and increased ROS. NiR treatment of $2.5 \mathrm{~J} / \mathrm{cm}^{2}$ with $808 \mathrm{~nm}$ laser did improved cell survival by up to $20 \%$, decreased level of ROS and mitochondrial fragmentation. Our data indicate that $808 \mathrm{~nm} \mathrm{NiR}$ treatment has a potential to improve challenged mitochondria function. Funding provided by the Slovak grants VEGA 1-0421-18; APVV-15-0485 and APVV-16-0158.

\section{P-182}

Spectral characteristics of body fluids in bladder cancer diagnostics

Martina Velísková, Milan Zvarík, Daniela Džubinská, Libuša Šikurová, Iveta Waczulíková

Department of Nuclear Physics and Biophysics, Faculty of Mathematics, Physics and Informatics, Comenius University, Bratislava, Slovakia

Measuring of spectral characteristics of body fluids provides valuable information on the processes in human body. Blood and urine contain metabolites and other molecules that reflect the current state of an organism. Separation of samples by a high-performance liquid chromatography (HPLC) with complex absorption a fluorescent detection belongs to simple methods of molecular subgroups detection.

In our research, we have focused on the analysis of spectral characteristics of blood and urine obtained from patients with bladder cancer and those with non-malignant haematuria caused by other benign conditions (e.g. urinary tract infections, trauma). Samples of urine and blood collected from both groups of patients were analysed using HPLC with different settings of absorption and fluorescence detector. We acquired 3D complex chromatograms and subsequently evaluated peaks from both blood and urine samples of each patient. The mutual correlations of identical characteristics between blood and urine samples were examined as well. Using data obtained from urine, the discriminant models (PLS-DA and OPLS-DA) were created. These models were able to distinguish patients with haematuria from bladder cancer patients with $100 \%$ sensitivity and $96 \%$ specificity. Based on our results we conclude that spectral analysis of blood plasma and urine may support future clinical diagnostics of early stages of bladder cancer.

\section{P-181}

Fluorescence excitation enhancement in nanowires for biosensing

Ivan Unksov [1], Nicklas Anttu [2,3], Damiano Verardo[1], Fredrik Höök [4,1], Christelle Prinz [1], Heiner Linke [1]

[1] NanoLund and Solid State Physics, Lund University, Sweden; [2] Department of Electronics and Nanoengineering, Aalto University, Finland; [3] Physics, Faculty of Science and Engineering, Ảbo Akademi University, Finland; [4] Department of Physics, Chalmers University of Technology, Sweden

Nanowires (NWs) are promising for optical biosensors as they have a high surface-to-volume ratio and can enhance and guide the signal from fluorophores located in proximity of a NW. For a NW sensor that outperforms conventional biosensors, the fluorescence guiding, which involves excitation enhancement and emission modification, needs to be characterized. We study excitation enhancement by GaP NWs coated with $\mathrm{Al}_{2} \mathrm{O}_{3}$ and functionalized with BSA enabling binding of Alexa Fluor 647 fluorophores. We introduce photobleaching rate analysis, independent on the number of fluorophores in a sample. Using that, we show the strongest enhancement for NW diameters around $100 \mathrm{~nm}$. We also demonstrate a drop in the enhancement with increasing thickness of $\mathrm{Al}_{2} \mathrm{O}_{3}$, indicating that the distance between a fluorescent biomarker and the NW core is crucial. We support our experimental findings with modelling. The modelling agrees excellently with the measurements, and we use it to show how the optimal NW diameter for the enhancement shifts with excitation wavelength. Our data provide guidelines for lightguiding NW biosensors.

\section{P-183}

Optomechanical coupling in a protein system with azobenzene functionalized sulfonylurea: ground versus excited state dynamics

Katarzyna Walczewska-Szewc, Łukasz Pepłowski, Wiesław Nowak

Nicolaus Copernicus University in Toruń, Poland

The concept of using light to control the action of drugs is not science-fiction anymore. Adding exogenous chromophores opens the possibility for precise light-control of protein switches. This idea is extensively exploited in optogenetics and optopharmacology. Using light allowed experimentalists to induce insulin from cells. The inward rectifying potassium channel KATP, which controls the insulin secretion in beta-cells, was opened and closed at will due to the photoisomerization of carefully designed molecules. The signal transduction goes from SUR1 to Kir6.2 complex due to a long-distance mechanical coupling between the chromophore and the pore. Here we present results of extensive (1 microsecond) molecular dynamics simulations of SUR1 protein having the chromophore in the ground and excited singlet state conformations. We delineate differences in dynamics of these systems and provide a plausible explanation of artificially created photosensitivity in this complex system. 


\section{Posters}

-11. Light as a tool in biophysics -

\section{P-184}

Allostery in Hsp90 elucidated by combining advanced fluorescence methods and MD simulations Steffen Wolf [1], Benedikt Sohmen [2], Björn Hellenkamp [3], Johann Thurn [2], Gerhard Stock [1], Thorsten Hugel [2,4]

[1] Biomolecular Dynamics, Institute of Physics, University of Freiburg, Freiburg, Germany; [2] Institute of Physical Chemistry, University of Freiburg, Freiburg, Germany; [3] Engineering and Applied Sciences, Columbia University, New York, USA; [4] Signalling Research Centers BIOSS and CIBSS, University of Freiburg, Freiburg, Germany

Combining single molecule FRET and time-resolved fluorescence correlation spectroscopy with massive multi-us molecular dynamics (MD) simulations allows to delineate allosteric changes within proteins with atomistic resolution. Using this state-of-the-art combination, we here show how ATP hydrolysis in the chaperone Hsp90 causes structural changes throughout the full protein complex. The investigated allosteric processes occur via hierarchical dynamics, starting with nanosecond- and Ångstrom-scale bond breaking and leading to millisecond- and nanometer-scale protein conformational changes. We find that hydrolysis of one ATP in the $\mathrm{N}$-domain is coupled to a conformational change of Arg380, passing structural information to an $\alpha$-helix in the M-domain and from there to the whole protein. The changes finally result in a structural asymmetry leading to the collapse of the central protein folding substrate binding site. We expect that similar hierarchical mechanisms are a general feature of allosteric information transfer in many other proteins. Details in: Wolf, S., Sohmen, B., et al. (2021) Chem. Sci., 12, 3350-3359. 
Posters

- 12. Biomimetic nanopores -

\section{P-185}

Dynamics of driven polymer transport through a nanopore

Kaikai Chen [1], Ining Jou [2], Niklas Ermann [1], Murugappan Muthukumar [2], Ulrich F Keyser [1], Nicholas A W Bell [1]

11] Cavendish Laboratory, University of Cambridge, UK; [2] Polymer Science and Engineering Department, University of Massachusetts, USA

Polymer transport across a nanoscale pore underpins many biological processes, for example, the ejection of phage DNA and the transfer of genes between bacteria. The movement of polymers into and out of confinement also underlies a wide range of sensing technologies used for single-molecule detection and sequencing. For quantitative analysis of polymer structure, including localisation of binding sites or sequences, accurate understanding of the translocation dynamics is essential. In this paper, we use synthetic nanopores and nanostructured DNA molecules to directly measure the velocity profile of driven polymer translocation through synthetic nanopores. Our results reveal a two-stage behaviour where the translocation initially slows with time before accelerating close to the end. We also find distinct local velocity correlations as the DNA polymer chain passes through the nanopore. Brownian dynamics simulations show that the two-stage behaviour is associated with tension propagation with correlations arising from the random walk starting conformation of the DNA.

\section{P-187}

Investigating the molecular interactions of local anesthetics in model cell membranes

Dilara Kilicarslan [1], Wanjae Choi [1], Hyunil Ryu [1], Ahmed Fuwad [2], Chaoge Zhou [1], Sun Min Kim [2,3], TaeJoon Jeon $[1,3]$

[1] Department of Biological Engineering, Inha University, Incheon, South Korea; [2] Department of Mechanical Engineering, Inha University, Incheon, South Korea; [3] Department of Biological Sciences and Bioengineering, Inha University, Incheon, South Korea

Local Anesthetics (LAs) are the medications that can be used to treat painful conditions during an operation or relieve the pain after surgeries. LAs block neuron's sodium channels and repress the membrane potential and neurotransmission thus overdose usage may be damaging. In this research, we used two different LAs, tetracaine, and lidocaine. LAs may have toxic effects on cell membranes, and it may affect the membrane`s fluidity, permeability, thermal stability. These parameters will influence the stability of the membrane. To investigate these effects, the fibroblast membrane is performed for local anesthetics and compared by IC50 measurements. Molecular-level membrane effect of these LAs, the physical interpolation of the membrane is understood by using the colorimetric assay of polydiacetylene (PDA) and membrane toxicity was assessed by electrophysiology of the planar lipid membrane studies. Various experiments indicated that membrane solubility is important in toxicity studies. This research will be helpful for further investigations on other local anesthetics and can be used to develop new Local Anesthetics with fewer side effects.

\section{P-186}

Molecular recognition in nanoscale confinement Nora Hagleitner-Ertuğrul [1], Yongzheng Xing [2], Denis Knyazev [1], Peter Hinterdorfer [1], Peter Pohl [1], Stefan Howorka $[1,2]$

[1] Institute of Biophysics, Johannes Kepler University Linz,Gruberstrasse 40, 4020 Linz, Austria; [2] Department of Chemistry, Institute of Structural Molecular Biology, University College London,London WC1H 0AJ, UK

Biomolecular recognition is essential in biology, biomedicine, and biotechnology and occurs in a range of environments. Solution processes are well established, but our understanding of recognition in nanoscale restricted confinements is lagging behind. Here we study how binding kinetics and selectivity are influenced in tunable, yet nanoscale confined, hollow nanopores. The DNA origami pore of $46 \mathrm{~nm}$ height and $7.5 \times 7.5 \mathrm{~nm}^{2}$ inner lumen carries precisely positioned recognition sites for DNA analytes. Fluorescence cross-correlation spectroscopy helps to determine the concentration and diffusion of co-localized fluorophore-tagged pore and short DNA oligonucleotides to derive the kinetics for binding and dissociation. Our highly tuneable system allows to probe the effect of cargo size, pore dimensions, and recognition site density on cargo dissociation and re-binding probability. The insight will help to design nanopores for optimized transport selectivity.

\section{P-188}

Negative differential resistance and thresholdswitching in biomimetic conical nanopores with fluoride ion solutions

Patricio Ramirez-Hoyos [1], Jose Joaquin Perez-Grau [1], Javier Cervera [2], Vladimir Garcia-Morales [2], Saima Nasir [3,4], Mubarak Ali [3,4], Wolfgang Ensinger [3], Salvador Mafe [2] [1] Universidad Politecnica de Valencia, Spain; [2] Universitat de Valencia, Spain; [3] Technische Universitaet Darmstadt, Germany; [4] GSI Helmholtzzentrum fuer Schwerionenforschung, Germany

Negative differential resistance (NDR) phenomena are commonplace in ion channel proteins but remain under-explored in biomimetic nanostructures. We characterize experimentally and theoretically new NDR phenomena observed when conical nanopores are immersed in KF solutions at low concentration, emphasizing the role of the pore tip in the observed phenomena. Sharp current drops in the nA range are obtained in the current-voltage curves when the applied voltages exceed thresholds of the order of $1 \mathrm{~V}$ over a wide frequency range. They can be explained as the consequence of the non-instantaneous redistribution of ions and water molecules at the pore tip following the external timedependent perturbations. The observed abrupt threshold switching can be used to amplify small electrical perturbations because a small change in voltage typically results in a large change in current. Memory and memristor-like characteristics are also discussed, showing that the biomimetic nanopores can act as biological synaptic mimics. 
Posters

- 12. Biomimetic nanopores -

P-189

Protein aggregation and chaperones: nature inspired technology

Peter van Oostrum [1], Erik Reimhult [1], Angelo Cacciuto [3], Tung Clarion [3], Alexander Mair [4], Ivan Coluzza [2] [1] Life Science University Vienna, Austria; [2] CIC biomaGUNE, San Sebastian, Spain; [3] Columbia University New York, USA; [4] University of Vienna, Austria

Protein misfolding and aggregation are critical bottlenecks in the downstream processing of protein recombinant drugs for the pharma industry. Large amounts of proteins are lost, and current methods to restore the folded structure are cumbersome and with limited yield, leading to low throughput and prohibitive costs.

Taking inspiration from natural chaperones, we propose optimally designing soft nanopores through which aggregated proteins can be extruded to refold into their functional structures. The nanopores have a cylindrical geometry and are coated with a dense brush of polymers in good solvent conditions. Protein clusters pushed by a flow against the pore and forced to translocate through the deformable polymer brush in the pore are broken up into isolated denatured chains. Due to its polymer functionalization, the pore does not get clogged by the protein clusters, as would be the case for hard nanopores. Instead, protein clusters get peeled off by the steric interaction with the brush and leave the cluster with a separation distance that allows the proteins to refold in isolation into their functional structures.

\section{P-191}

Jamming-free transport in polymer-coated channels inspired by the nuclear pore complex Tiantian Zheng [1], Anton Zilman [1,2]

[1] Department of Physics, University of Toronto, Canada; [2] Institute for Biomedical Engineering, Canada

The nuclear pore complex (NPC) is the main conduit for transporting materials into and out of the nucleus of eukaryotic cells. Artificial nanopores recapitulating the main aspects of NPC transport have been built. The central channel of the NPC is occupied by an assembly of polymer-like intrinsically disordered proteins (known as FG nups), which is a main component of the NPC selective transport mechanism. Much of the remaining free space inside the channel is taken up by many translocating cargoes and transport proteins (which interact attractively with FG nups), resulting in a highly crowded interior. How the NPC can efficiently and rapidly transport cargoes through such a crowded channel remains incompletely understood. Surprisingly, past experimental studies show no signs of jamming occurring within the NPC, and the accumulation of cargoes inside the pore appears to have no negative effects on transport efficiency or times. We construct a computational model of the NPC based on a minimal set of principles according to which the NPC operates. This model qualitatively reproduces past experimental results, and identifies a set of mechanisms that prevent jamming from occurring even at extreme levels of crowding, and give rise to a novel regime where crowding increases transport efficiency through the pore. These results suggest how crowding can be avoided in artificial nanopores.

\section{P-190}

Flow of water and proteins through soft, polymer-decorated

Mudassar Virk, Andrea Scheberl, Peter van Oostrum, Erik Reimhult

University of Natural Resources and Life Sciences, Vienna, Austria

In recent years, solid-state nanopores have left the research lab and come to the forefront as a research tool to probe biopolymers and nanoparticles. We introduce nanopores decorated with densely grafted thermoresponsive polymers (PNiPAAm) such that the flow through the nanopores leads through the polymer matrix. We demonstrate that the flow through such pores leads to a novel type of flow circuit where energy goes into deforming the polymer brush and then fed back into the flow. By thermally actuating the brush, we demonstrate that the effect only occurs for nanopores closed by the polymer brush in a good solvent. The flow-induced actuation of the polymer brushes in the nanopores also influences its interactions with proteins pushed through the pores by the flow. We will present the design of the fluidic system that allows for these measurements as well as our preliminary data on the factors that determine the response of the polymer brush. 
Posters

- Self-Assembled Session 3 - lonic liquids biomolecules and cells -

P-192

Discerning phase separation in lipid multilayers induced by ionic liquids

Ritika Gupta, Sajal K Ghosh

Shiv Nadar University, Dadri, Gautam Buddha Nagar, India

Ionic liquids (ILs) are widely used as solvents, lubricants, electrolytes, heat storage, etc. owing to their tunable physical and chemical properties. They show strong and selective interactions towards cellular membranes disrupting their structural integrity. Lipids being the major constituents of the membrane play the vital role in this interaction. Here, the X-ray diffraction has been employed to examine the modifications induced by ILs in the self-assembly of phospholipid multilayers. The ILs lead to phase separated micro domains across the bilayer plane which is evident by two distinct sets of peaks in the X-ray diffractogram. While one set of peaks representing a smectic liquid crystalline phase corresponds to the regular lipid multilayer, the other set of peaks indicates a new phase having lower inter-bilayer spacing. This newly formed bilayers are analyzed to be an interdigitated one. The bilayer interdigitation was observed for globular as well as rod-like shape cations of the ILs. This was also observed for small as well as bulky anions.

\section{P-194}

Subtoxic concentrations of ionic liquids enhance cell migration by reducing the elasticity of the cellular lipid membrane

Pallavi Kumari [1,2], Visakh Pillai [1], Brian Rodriguez [1], Maria Prencipe [3], Antonio Benedetto [1,2]

[1] School of Physics, and Conway Institute, University College Dublin, Ireland; [2] Department of Sciences, University Roma Tre, Italy; [3] School of Biomolecular and Biomedical Science, and Conway Institute, University College Dublin, Ireland

Cell migration is a universal and crucial mechanism for life. It is required in a series of physiological processes and is involved in several pathological conditions. Among the several biochemical and biophysical routes, changing cell membrane elasticity holds the promise to be a universal strategy to alter cell mobility. Due to their affinity with cell membranes, ionic liquids (ILs) may play an important role. This research focuses on the effect of subtoxic concentrations of imidazolium-ILs on mechano-elasticity of a model cellular lipid membrane and migration of the model cancer cell line MDA-MB-231. Cell membrane elasticity and cell migration studies have been carried out varying IL-concentrations and IL-cation chain length. Here we show that ILs are able to enhance cell migration by reducing the elasticity of the cellular lipid membrane, and that both the migration and the elasticity can be tuned by IL-concentration and IL-cation chain length. This bio-chemical-physical mechanism is potentially valid for all mammalian cells and can lead to new applications in bio-nanomedicine and bio-nanotechnology, e.g., in cancer cell diagnostics and stem cell differentiation.

\section{P-193}

Folding free energy landscape of Initiator Caspase 8 Mithun Nag KaraDi Giridhar, Clay Clark Allan University of Texas at Arlington, USA

Caspases are an important family of enzymes that are highly regulated in a cell to perform a variety of functions like apoptosis, inflammation, cell differentiation and necroptosis. In the Apoptosis cascade, Initiator Caspases: 8, 10 and cFLIP are monomers and they have to dimerize in order for them to be activated; Executioner caspases however exist as dimers under physiological conditions and have to be processed at specific regions for them to be activated. Post translational modifications and allosteric regulation of initiator caspases fine tune them to act on specific substrates, which carry out different functions. We have studied the equilibrium unfolding of inactive (active site mutant) monomeric initiator caspase 8 . By fitting a three state model to the caspase 8 data we have determined a free energy of unfolding from the native state to the intermediate to be $5 \pm 0.5 \mathrm{kcal} / \mathrm{mole}$ and $2 \pm 0.8 \mathrm{kcal} /$ mole from the intermediate to the unfolded state. Executioner caspases have a total free energy of $25.8 \mathrm{kcal} /$ mole and dimerization contributes to 18.8 which is enormous, whereas initiator caspases have a total free energy of $7 \mathrm{kcal} /$ mole which indicates that the cell can shift the equilibrium and maintain these enzymes under tight control with less expenditure of energy compared to controlling the dimerization of executioner caspases. It is crucial for a bioactive organism to shift conformations with minimal energy expenditure, fine tuning these precursors and direct them in the required pathway to decide the fate of the cell.

\section{P-195}

CHIMs - A class of versatile cholesterol analogs with unique properties

Anna Livia Linard Matos [1], Fabian Keller [2], Tristan Wegner [3], Carla E C del Castillo [4], David Grill [1], Sergej Kudruk[1], Anne Spang [4], Frank Glorius [3], Andreas Heuer [2], Volker Gerke [1]

[1] Institute of Medical Biochemistry, Center for Molecular Biology of Inflammation, University of Münster, Germany; [2] Physical Chemistry Institute, University of Münster, Germany; [3] Institute of Organic Chemistry, University of Münster, Germany; [4] Biozentrum, University of Basel, Switzerland

Cholesterol is an essential component of cellular membranes regulating the structural integrity and fluidity of biological bilayers and cellular processes. However, tools to investigate the role and dynamics of cholesterol in live cells are still scarce and often show limited applicability. To address this, we developed a class of imidazolium-based cholesterol analogs, CHIMs. We confirmed that CHIM membrane integration characteristics largely mimic those of cholesterol. Computational studies in simulated phospholipid bilayers and biophysical analyses of model membranes reveal that in biologically relevant systems CHIMs behave similarly to natural cholesterol. The analogs can functionally replace cholesterol in membranes, can be readily labeled by click chemistry and follow trafficking pathways of cholesterol in live cells. Thus, CHIMs represent chemically versatile cholesterol analogs that can serve as a flexible toolbox to study cholesterol behavior and function in live cells and organisms. 
Posters

- Self-Assembled Session 3 - Ionic liquids biomolecules and cells -

P-196

Imidazolium-based ionic liquids cause mammalian cell death due to modulated structures and dynamics of cellular membrane

Saheli Mitra, Sajal Kumar Ghosh

Shiv Nadar University, G. B. Nagar, Uttar Pradesh, India

Ionic liquids (ILs) are the attractions of researchers due to their vast area of potential applications. For biomedical uses, it becomes essential to understand their interactions with cellular membrane. We have studied the toxic effects of various imidazolium-based ionic liquids (ILs) with varying hydrocarbon chain lengths, on different human cell lines. Multiple biological assays have shown that the ILs with long hydrocarbon chains have stronger adverse effect especially on human liver cancer cells (Huh-7.5 cells). To understand the molecular mechanism of such an effect, the cellular membranes were mimicked with lipid bilayer and monolayer composed of liver lipids extract. The pressure-area isotherms measured from the monolayer have shown that the interaction of ILs with the lipid layer is energetically favorable. The addition of these ILs reduces the in-plane elasticity of the self-assembled molecular layer. Further, the isothermal titration calorimetry (ITC) measurements showed that the long chain IL interacts strongest with the liver lipid membranes and the interaction is found to be mainly driven by entropy. On the other hand, x-ray reflectivity (XRR) measurements showed a concentration dependent change in the monolayer structure. At low concentration of the IL, the monolayer thickness decreases, exhibiting an increase in the electron density of the layer. However, at higher concentrations, the monolayer thickness increases proving a concentration dependent effects of the IL on the arrangement of the molecules.

\section{P-198}

Effect of n-alkyl imidazolium-based ionic liquids on the kinetics of insulin fibrils formation and their morphology

V. Vanik, Z. Bednarikova, Z. Gazova, D. Fedunova

Institute of Experimental Physics, SAS, Slovakia

Amyloid fibrils (AF) are a special type of protein aggregates associated with several human diseases. AF have a potential to be used as progressive biomaterials due to their unique properties (stability, elasticity, degradation resistance). The nature of the solvent is one of the crucial conditions affecting the amyloid fibrillization and the morphology of fibrils. Ionic liquids (ILs) are a class of solvents with tunable physicochemical properties depending on ion composition. This work assesses the effect of n-alkyl $(n=2-10)$ imidazoliumbased ILs on fibrillization kinetics and morphology of insulin AF. We have found that the ILs effect depends on the concentration and alkyl chain length of a cation. ILs with short chain at lower concentration induced the fibrils formation, whereas higher concentration prolongs lag-phase and fibrillization halftime. The short fibrils $\leq 1 \mu \mathrm{m}$ were observed in ILs with 2-4 alkyl groups, while $2-5 \mu \mathrm{m}$ twisted AF were formed in ILs with $n=6$. The ILs containing cation with the longest chain (higher hydrophobicity) inhibited the fibrillization. Specific interactions between alkyl chain and insulin hydrophobic regions presumably prevent the intermolecular contacts necessary for fibrilization. Elucidation of the ILs effect on amyloid aggregation can contribute to understanding the aggregation mechanism. The formation of morphologically distinct aggregates is crucial for toxicity determination in organism, and important for biomaterials development.
P-197

Mechanism of action of ionic liquids on the amyloidogenesis of lysozyme

Visakh Pillai [1], Katarzyna Tych [2], Marina Rubini [4], Brian Rodriguez [1], Antonio Benedetto [1,3]

[1] School of Physics and Conway Institute, University College Dublin, Ireland; [2] Groningen Institute, University of Groningen, Netherlands; [3] Department of Sciences, University Roma Tre, Italy; [4] School of Chemistry, University College Dublin, Ireland

Ionic liquids(ILs) are a vast class of organic non-aqueous electrolytes, composed by an organic cation and either an organic or inorganic anion and have a marked affinity towards biomolecules. In this context, it has been shown that ILs can either prevent or favour the amyloidogenesis [1]. More specifically, high concentrations of ethyl ammonium nitrate (EAN) and tetramethylguanidinum acetate (TMG-A) have shown to enhance and inhibit, respectively, the amyloidogenesis of the model protein lysozyme [1]. However, the effect of these ILs at the lower concentration regime and the microscopic mechanism behind the interaction is still unexplored. To shed a light on this microscopic mechanism, we combine a palette of experimental techniques. The way in which these ILs affects the morphology and surface potential of amyloid fibrils was studied by tapping mode atomic force microscopy and dual harmonic kelvin probe force microscopy. This has been correlated to the unfolding force of single proteins measured using optical tweezer and to the alteration in the protein-hydration water picosecond dynamics probed by neutron scattering.

[1] Pillai and Benedetto (2018) Biophys Rev 10:847

\section{P-199}

An iontronic pump for targeted drug delivery to glioblastoma spheroids

Linda Waldherr [1], Maria Seitanidou [2], Marie Jakešová [2], Verena Handl [1], Meysam Karami Rad [2], Silke Patz [3], Daniel T Simon [2], Rainer Schindl [1]

1] Gottfried Schatz Research Center, Med Uni Graz, Austria; [2] Lab. of Organic Electronics, Linköping Univ., Norrköping, Sweden; [3] Univ. Clinic of Neurosurgery, Med Uni Graz, Austria

Glioblastoma multiforme (GBM), the most aggressively growing and most frequent brain tumor, remains hard incurable due to limited surgical resection possibilities and hindrance of promising chemotherapeutics such as Gemcitabine (Gem), which is shielded by the blood brain barrier (BBB). We here show that Gem is a promising drug for GBM treatment as it effectively kills GBM cells, is more potent than the gold standard temozolomide, but is at the same time unharmful to neurons and astrocytes.

Nevertheless, Gem has to be administered via alternative methods to the brain. We engineered Gemcitabine Ion Pumps (GemIPs), which utilize electrophoretic technology for drug delivery. GemIPs are able to administer Gem with $\mathrm{pmol}^{*} \mathrm{~min}^{-1}$ delivery precision at currents in the nanoampere range. This electrical and temporal control was further linked to applications in the cell monolayer and 3D cell culture. Most noticeable was the disintegration of targeted GBM tumor spheroids among GemIP treatment.

The here exemplified electrically-driven chemotherapy has the potential to increase the efficacy of GBM treatment and represent a highly-targeted and locally-controlled drug delivery tool that is independent from $\mathrm{BBB}$ permeability of potent chemotherapeutics. 
Posters

- 13. New and Notable -

P-200

Exchangeable polarity-sensitive dyes for real-time quantification of membrane biophysical properties using STED super-resolution microscopy

Pablo Carravilla [1], Anindita Dasgupta [1], Gaukhar Zhurgenbayeva [1], Dmytro I Danylchuk [2], Andrey S Klymchenko [2], Erdinç Sezgin [3], Christian Eggeling [1,4]

[1] Leibniz Institute of Photonic Technology and FriedrichSchiller-University Jena, Germany; [2] Université de Strasbourg, France; [3] Karolinska Institute, Sweden; [4] University of Oxford, UK

The understanding of the plasma membrane nano-scale organisation in living cells requires microscopy techniques with high temporal and spatial resolution that allow for the quantification of membrane biophysical properties. Among the most popular super-resolution techniques, stimulated emission depletion (STED) microscopy offers high temporal resolution. However, monitoring live processes using STED microscopy is limited by photobleaching. A remedy are exchangeable membrane dyes that only temporarily reside in the membrane. Here, we show that NR4A, a polaritysensitive exchangeable plasma membrane dye, permits the super-resolved quantification of membrane biophysical parameters in real time. STED-fluorescence correlation spectroscopy (STED-FCS) enables the simultaneous quantification of membrane dynamics and lipid packing, which correlate in model and live cell membranes. To showcase potential applications of polarity-sensitive exchangeable dyes, we use live 3D-STED imaging to monitor (i) the formation of giant plasma membrane vesicles, and (ii) lipid exchange during membrane fusion. 


\section{Posters}

- 14. Membrane active peptides -

P-201

Enhanced translocation of amphiphilic peptides across membranes by transmembrane proteins Ladislav Bartoš [1,2], Ivo Kabelka [1,2], Robert Vácha [1,2] [1] CEITEC - Central European Institute of Technology, Masaryk University, Czech Republic; [2] National Centre for Biomolecular Research, Faculty of Science, Masaryk University, Czech Republic

Cell membranes are phospholipid bilayers with a large amount of embedded transmembrane proteins. Some of these proteins, such as scramblases, have properties which facilitate lipid flip-flop from one membrane leaflet to another. Scramblases and similar transmembrane proteins could also affect the translocation of other amphiphilic molecules including cell-penetrating or antimicrobial peptides.

We studied the effect of transmembrane proteins on the translocation of amphiphilic peptides through the membrane. Using two very different models, we consistently demonstrate that transmembrane proteins with a hydrophilic patch enhance the translocation of amphiphilic peptides by stabilizing the peptide in the membrane. Moreover, there is an optimum amphiphilicity, because the peptide could become 'overstabilized' in the transmembrane state, in which the peptide-protein dissociation is hampered, limiting the peptide translocation.

The presence of scramblases and other proteins with similar properties could be exploited for more efficient transport into cells. The described principles could also be utilized in the design of a drug delivery system by the addition of a translocation-enhancing peptide that would integrate into the membrane.

\section{P-203}

Variation of the elasticity and Volta potential of lipid monolayers: effects of surface charge and dipole moment

Yury A Ermakov, Kirill I Makrinsky, Rodion J Molotkovsky, Timur R Galimzyanov

Frumkin Institute of Physical chemistry and Electrochemistry, RAS, Russian Federation

We have measured compression diagrams for monolayers composed of charged and neutral phospholipids in the presence of various positively charged ions and polypeptides. The shape of diagrams and electrostatic effects was analyzed by the empirical model that represents lipid molecules as an incompressible kernel with a soft shell. We show that in the region of its liquid expanded state (LE) the measured Volta potential linearly depends on the lipid density, which corresponds to the effective value of the dipole moment according to Helmholtz relation. Such linearity chmkbremains independent of the $\mathrm{pH}$ solution, while a slope of curves chmkbdecreases markedly in the presence of polypeptideschmkb. We attribute this effect to the overpowering contribution of water molecules to the Volta potential in comparison to the surface charge changes. That is why the alteration in hydration of lipid head groups leads to the major changes in the dipole moment. A small, but noticeable deviation from linearity in the LE region was detected in the presence of chlorpromazine and polyarginine because of their insertion into the monolayer. A quantitative description of diagrams was made by the previously developed model, which takes into account the inhomogeneity of the polymer adsorption layer. The study was supported by RFBR grant 19-04-00242.

\section{P-202}

Quantifying the association of antimicrobial peptides to live bacterial cells: insights in the mechanism of action, selectivity and inoculum effect Sara Bobone [1], Cassandra Troiano [1], Francesco Riccitelli [1], Filippo Savini [1], Daniela Roversi [1], Yoonkyung Park [2], Maria Rosa Loffredo[3], Bruno Casciaro [3,4], Maria Luisa Mangoni [3], Lorenzo Stella [1]

[1] Dept of Chemical Science \& Technologies, Tor Vergata, Rome, Italy; [2] Chosun University, Gwangju, South Korea; [3] Lab affiliated to Pasteur Italia-Fondazione Cenci Bolognetti, Rome, Italy; [4] Center For Life Nano Science@Sapienza, Rome, Italy

Antimicrobial peptides (AMPs) kill bacteria mainly by perturbing their cell membranes, with low toxicity towards host cells, and are promising compounds to fight drug-resistant microbes. However, their success towards clinical application is still limited, partly due to many unanswered questions on their activity and function. Our current understanding of AMP behavior has been reached by two parallel, but largely independent, approaches: microbiological studies on AMP effects on cells, and biophysical investigations on model membranes. All current models for the mechanisms of AMP membrane perturbation and cell selectivity were derived from the latter kind of studies. We recently developed a simple spectroscopic approach to quantify the interaction of AMPs with live bacterial cells. These data allowed us to compare membrane binding and peptide-induced pores in bacteria and liposomes, to determine the number of cell-bound peptide molecules needed to kill a bacterium, to predict the variation of peptide activity and toxicity with the density of cells (inoculum effect) and to examine selectivity in a mixture of target and host cells. Overall, these approaches led to a new understanding of AMPs that will be helpful for their development into effective antibiotic drugs.

\section{P-204}

Characterisation of the folding and interactions of de-novo designed transmembrane helices Ciaran A Guy, Ann M Dixon, Phillip J Stansfeld University of Warwick, UK

The design, synthesis and study of de-novo designed proteins is developing into a functional tool and keystone of bottom-up synthetic biology research, however, despite ongoing efforts, membrane proteins remain poorly represented in the field. We have explored the design of $\alpha$-helical transmembrane domain (TMD) interactions by inserting wellknown interaction motifs into TMDs of minimal sequence complexity and measuring the resulting structural impact in vitro and in silico. We found that all TMD peptides tested adopted the predicted $\alpha$-helical fold, inserted across membranes and the relative strengths of interaction facilitated by the motifs tested were largely in agreement with our previous in vivo work. However, the oligomeric states observed are much less predictable than expected. Our data shows that motifs hypothesised in the literature to stabilise strong dimeric interactions instead lead to trimeric or higher oligomeric states. We also observed considerable, to date unreported, membrane disruption activity for scaffold sequences suggesting more complex membrane assembly than originally thought. Coarse-grained molecular dynamics simulations reinforce these results and reveal sites of helix-helix interaction in our TMD peptides to be at odds with previous findings. Taken together, our results illustrate that large gaps still exist in current understanding of membrane protein sequence design and further research is required into the fundamental rules of interactions and assembly. 
P-205

Membrane interactions of virus-like mesoporous silica nanoparticles as carriers of antimicrobial peptides

Sara M Häffner [1], Elisa Parra-Ortiz [1], Martin Malmsten $[1,2]$

[1] Department of Pharmacy, University of Copenhagen, DK2100, Copenhagen, Denmark; [2] Department of Physical Chemistry 1, University of Lund, SE-22100, Lund, Sweden

We investigated here the lipid membrane interactions of silica nanoparticles with different surface topography as carriers for the antimicrobial peptide LL-37, comparing smooth versus virus-like (spiky) mesoporous nanoparticles, as well as non-porous silica. We employed a combination of neutron reflectometry, ellipsometry, dynamic light scattering and zetapotential measurements to characterize the interactions of bare and LL-37 loaded silica nanoparticles with bacteriamimicking phospholipid bilayers. We found that nanoparticle topography strongly influenced membrane binding and destabilization, and that the effect of particle spikes became further accentuated by peptide loading, proving that LL37-loaded virus-like nanoparticles displayed enhanced membrane disruption capabilities. Furthermore, neutron reflectometry indicated the creation of transmembrane defects by LL-37-loaded virus-like nanoparticles, promoting LL-37 insertion through both bilayer leaflets. The relevance of these effects for bacterial membrane rupture was proven by confocal microscopy and viability assays on Escherichia coli. Taken together, these findings point to virus-like mesoporous nanoparticles as interesting delivery systems for antimicrobial peptides.

\section{P-207}

Single-molecule detection of multiple transition states of membrane-perforating peptide toxin candidalysin

Sejeong Lee, Nessim Kichick, Olivia Hepworth, Jonathan Richardson, Julian Naglik

Centre for Host-Microbiome Interactions, Faculty of Dentistry, Oral \& Craniofacial Sciences, Guy's Campus, Kings College London, UK

Fungal infections affect over 1 billion individuals worldwide and kill 1.5 million annually. In 2016, the first peptide toxin in any human fungal pathogen was discovered in Candida albicans. This toxin was named candidalysin. Candidalysin directly causes cellular damage and immune activation during mucosal and systemic infections. However, the mechanism of cellular import remains unknown on a molecular level. Here, we reconstituted planar lipid bilayers in a multiple array device and monitored the membrane permeation properties of candidalysin using electrical recording. We observed a transition from the surface-associated state of candidalysin to its transmembrane state, leading to a transient pore formation. Multiple current levels at the transmembrane state indicate that candidalysin can create membrane pores of various sizes. Our kinetic analysis reveals that candidalysin binding to the membrane becomes faster in proportion to the strength of electrostatic interactions, which is directly affected by both the density and the distribution of Lys residues at the Cterminus of candidalysin. In summary, our work provides valuable insights into the dynamics of the peptide toxin import, which can be used to design therapeutic agents for the fight against fungal infections.

\section{P-206}

High-throughput synthesis of multi-tail lipidoids for membrane-targeting antimicrobials

James Jennings, Nermina Malanovic, Karl Lohner, Georg Pabst

University of Graz, Austria

Membrane-targeting antimicrobials offer potential solutions to the growing problem of antibiotic resistant pathogenic bacteria. In particular, antimicrobial peptides are proven to be highly selective against a range of bacteria, ${ }^{1}$ however challenges in preparing peptides at scale and their vulnerability to enzymatic degradation impede their application in all settings. Meanwhile, synthetic cationic compounds are known to have broad spectrum activity but often at the expense of cytotoxicity. Here, we present investigations into the antibacterial efficacy of synthetic "lipidoids" comprising cationic headgroups and multiple hydrophobic tails. Such compounds were recently found to mimic the salient aspects of the bacterial "lipid A" liquid crystalline assembly behaviour. $^{2}$ Furthermore, lipidoids could coordinate strongly with lipopolysaccharide aggregates and disrupt the melting transitions of lipid A aliphatic chains. Here, we report the high-throughput synthesis of a lipidoid library with diverse molecular structures by simple one-pot reactions of commercially available polyamines with hydrophobic acrylates. This library can be used to screen for chemical structures that (i) mimic the shape and aggregation behaviour of lipid A, (ii) selectively disrupt bacterial over mammalian membranes, and (iii) impart membrane-targeting antimicrobial properties.

${ }^{1}$ Malanovic et al, Biochim Biophys Acta 2020, 1862, 183275

${ }^{2}$ Jennings et al, Langmuir 2020, 36, 8240

\section{P-208}

Inside out - The role of mycobacterial ESX secretion systems in phagosome escape

C Nehls [1], F Vasquez [1], L Paulowski [1], M Grensemann [1], S Groth [1], Y-H Song [2], S Homolka [1], F Maurer [1], $\underline{\text { T Gutsmann }}[1,2]$

[1] Research Center Borstel, Germany; [2] University of Lübeck, Germany

In phagosome escape of mycobacteria the role of type VII secretion systems (T7SS) seems to be important. These systems and, in particular, the structural and functional variability of T7SS substrates belonging to the WXG100 superfamily are the focus of our research. The best studied example of Esx protein complex is the Mycobacterium tuberculosis pair ESAT-6 and CFP-10. The current model states that the secreted substrate ESAT-6 is required for membrane permeabilization. Recently, a similar protein pair, EsxT and EsxU, was identified in the ESX-4 locus of Mycobacterium abscessus. To decipher the complex interaction of Esx proteins with biological membranes we used various biophysical techniques (spectroscopy, electrophysiology, binding assays, atomic force microscopy) on natural and reconstituted lipid membrane systems. The specific role of the Esx proteins in mycobacteria was investigated with a particular focus on $\mathrm{pH}-$ , lipid-, voltage-, and protein-structure-specificities. ESAT-6 has the potential to induce membrane permeabilization by heterogeneous transient lesions at $\mathrm{pH} 7$ and in the presence of a transmembrane voltage. Comparable properties could be found for Mycobacterium abscessus EsxU and EsxT. Taken together, the results suggest that these Esx pairs may play different physiological roles in host-pathogen interaction. 


\section{Posters}

- 14. Membrane active peptides -

P-209

Amphipathic peptides modulate lateral interaction of ordered membrane domains

Konstantin V Pinigin, Timur R Galimzyanov, Sergey A Akimov

Laboratory of Bioelectrochemistry, Frumkin Institute of Physical Chemistry and Electrochemistry, Russian Academy of Sciences, Russian Federation

Ordered domains of plasma membranes enriched with sphingomyelin and cholesterol are called rafts. Lateral interaction of domains can have a significant effect on cell processes that require colocalization of several membrane proteins. The thickness of the raft bilayer exceeds the thickness of the surrounding membrane. Therefore, elastic deformations arise at the boundary in order to smooth the jump in the thickness. The characteristic length of deformations is several nanometers. As two domains approach each other, the deformations induced by their boundaries begin to overlap, thus leading to lateral interaction. We theoretically examined the energy profile of the interaction of ordered domains in the framework of theory of membrane elasticity. Bringing two parallel boundaries of ordered domains into a contact requires overcoming some energy barrier. The barrier increases several times in the presence of amphipathic peptides, which are able to partially incorporate into the membrane, causing elastic deformations. We analyzed various configurations of ordered domains and amphipathic peptides, and demonstrated that the peptides should significantly hinder the fusion of ordered domains and, thereby, contribute to the stabilization of the nanodomain ensemble.

\section{P-211}

Biophysical characterization of the novel antibiotic peptide lugdunin

Dominik Ruppelt [1], Hendrik Flegel [1], Sophia Mönnikes [1], Claudia Steinem $[1,2]$

[1] Georg-August-Universität Göttingen, Germany; [2] Max Planck Institute for Dynamics and Self Organization, Germany

Dissemination of infectious diseases caused by antibioticresistant bacterial strains represents a major global health threat. One way to overcome this issue is the development of new antibiotic entities based on natural occurring antimicrobial peptides. Recently, the cyclic peptide lugdunin was isolated from Staphylococcus lugdunensis and displayed promising antimicrobial activity against gram-positive pathogens. Previous studies showed that lugdunin transports protons across bacterial cell membranes resulting in the disruption of the membrane potential and in cell death. However, its exact proton translocation mechanism is still elusive.

Here we present an approach to provide a biophysical characterization of lugdunin activity. By monitoring intrinsic tryptophan fluorescence, we investigated the peptide-membrane interaction and show that lugdunin penetrates strongly into the hydrophobic core of membranes. Which kind of structure lugdunin adopts inside the lipid bilayer is assessed by ATR-FTIR spectropscopic mesaurements. By analyzing the amide I region, we propose a preliminary model of lugdunins structure which is based on the self-assembly to peptide nanotubes. These nanotubes could be able to act as membrane channels. To quantify the proton translocation capability, we use an approach involving microfluidic trapping of giant vesicles (GUVs) filled with a $\mathrm{pH}$-sensitive fluorescent dye. These GUVs will be used to quantify the proton transport induced by lugdunin.

\section{P-210}

Building a mimetic system for unraveling protein-protein interactions on membranes

Shahenda Ramadan [1], Paula Guerrero [1], Ruslan Nedielkov[2], Nikolai Klishin [2], Rumiana Dimova [1], Daniel V Silva [1], Heiko Möller [2]

[1] Max Planck Institute for Colloids and Interface (MPIKG), Germany; [2] University of Potsdam, Germany

Protein-protein interactions taking place at the cell's surface can control the function and communication processes between cells. Eukaryotes have proteins anchored to the cells' plasma membrane via the glycolipid anchor glycosylphosphatidylinositol (GPIs). GPI anchored proteins (GPI-APs) play a role in the protection, regulation and activation of cells. Studies of GPIs and GPI-APs are hindered by the difficulty of isolating and producing these complex molecules in pure forms. Herein, we apply a semi-synthetic method to synthesize well-defined GPI-APs by combining protein expression and chemo-selective attachment of synthetic GPI to proteins. Specifically, we will express the $19 \mathrm{KDa}$ fragment of the Plasmodium berghei merozoite surface protein 1 (MSP1-19) and ligate it to a synthetic GPI. The GPI and the GPI-anchored MSP1-19 will be inserted into cell-sized model membranes (Giant Uni-lamellar Vesicles) of different composition and to supported lipid bilayers. Using fluorescence microscopy based approaches and surface plasmon resonance, we will investigate the role of GPIs on the properties and behavior of conjugated MSP1-19 GPI complex, their partitioning between liquid-ordered and liquid-disordered lipid phases, clustering and interaction with other proteins.

\section{P-212}

Inflammation control by membrane active peptides: A novel mechanism targeting host cell membrane domains

Andra B Schromm [1], Laura Paulowski [1], Franziska Kopp [1], Christian Nehls [1], Guillermo Martinez-deTejada [2], Karl Lohner [3], Mario Brameshuber [4], Gerhard J Schütz [4], Thomas Gutsmann [1]

[1] Research Center Borstel, Borstel, Germany; [2] University of Navarra, Pamplona, Spain; [3] University of Graz, Graz, Austria; [4] TU Wien, Vienna, Austria

Antimicrobial peptides (AMPs) exert their antibacterial effects by interaction with bacterial membranes and lipids, such as lipopolysaccharide (LPS; endotoxin). Endotoxin is a potent inducer of hyper-inflammation in infectious diseases. We have performed a systematic analysis of the interaction of the cathelicidin-derived peptide LL-32 and the peptide antibiotic polymyxin B (PMB) with endotoxin to characterize the physico-chemical changes. Biological experiments revealed a so far unrecognized interaction of LL-32 and PMB also with host cell membranes. Peptide-host cell interaction conferred a strong attenuation of the inflammatory response. Model membranes and eukaryotic cell lines were analyzed by an array of methods to characterize the effect of the peptides on host cell membranes. These investigations revealed i) differential effects of the two peptides, ii) modifications of cholesterol-rich domains and iii) effects on the association of GPI-anchored proteins participating in signaling complexes. Our data support multimodal activities of the peptides to disarm endotoxin and enhance our understanding of how membrane active peptides can regulate inflammation. 
Posters

- 14. Membrane active peptides -

\section{P-213}

Bridging the activity of lactoferricin derivatives in $E$. coli and lipid-only membranes: partitioning and kinetics

Enrico F Semeraro [1,2], Lisa Marx [1,2], Johannes Mandl [1,2], Helmut Bergler [1,2], Karl Lohner [1,2], Georg Pabst [1,2]

[1] University of Graz, Institute of Molecular Biosciences, Graz, Austria; [2] BioTechMed Graz, Graz, Austria

Novel antibiotics based on membrane active antimicrobial peptides (AMPs) are promising candidates for defending the spread of diseases caused by multi-resistant pathogenic bacteria. Notwithstanding the number of works that explore the relationship between AMP activity and membrane architecture, the full mechanism that lead to cell death is currently not clear. This prompted us to investigate the modeof-action of lactoferricin derivatives on both live $E$. coli and biological lipid-membrane mimics. In particular, we explored AMP partitioning in bacteria and lipid-only vesicles revisiting susceptibility and leakage assays, respectively, and zetapotential and Trp-fluorescence spectroscopy. The structural rearrangement in vesicles and bacteria upon mixing with AMPs was probed by transmission electron microscopy and small-angle neutron and X-ray scattering. The latter permitted to access the kinetics in live cells with an unprecedented time (milliseconds) and length (nanometre to submicrometre) scales. To name but a few, results suggest that these AMPs strongly partition into the lipid membranes, quickly translocating into the cytoplasm/lumen $(<1 \mathrm{~s})$ but, at the same time, cause weak leakage and negligible lipid flip-flop.

\section{P-215}

Exploring the environment of a cyclic lipopeptide by time-resolved-fluorescence of tryptophan J. Steigenberger [1], N. Geudens [2], B. Kovács [2], V. de Roo [2], Y. Verleysen [2], J. C Martins [2], H. Heerklotz [1] [1] Institute of Pharmaceutical Sciences, University of Freiburg, Germany; [2] NMR and Structural Analysis Unit, Ghent University, Belgium

Cyclic lipopeptides (CLiP) of bacterial origin show plant beneficial and antimicrobial properties presumably by perturbing cellular membranes and appear to be a promising alternative to conventional antimicrobials. Improving our biophysical understanding of CLiP induced membrane permeabilization will aid a rational development of new drugs with high selectivity. This work aims to explore the localization and the mobility of a CLiP in the bilayer to investigate whether and how this correlates with its membrane activity. We measure time-resolved emission spectra and anisotropy of four CLiP-analogs, in each of which one specific hydrophobic amino acid is exchanged for tryptophan (Trp). Trp acts as an intrinsic fluorophore and its fluorescence properties are highly dependent on the details of its local surrounding. Monitoring continuous solvent relaxation and analyzing timescale and strength of the time-dependent shift in the emission spectra yields information on mobility and polarity of molecules in direct vicinity to the Trp. The rotational correlation time provides information on Trp mobility as well as on the viscosity of molecules in direct vicinity. Both methods combined describe a detailed picture of the CLiP's immediate environment allowing us to draw conclusions on the mode of action responsible for its membrane activity.

\section{P-214}

Pore formation mechanisms of antimicrobial peptides

Valeriy S Sokolov [1], Sergey A Akimov [1], Oleg V Batishchev [1,2], Marta V Volovik $[1,2]$

[1] A.N. Frumkin Institute of Physical Chemistry and Electrochemistry, Russian Academy of Sciences, Russian Federation; [2] Moscow Institute of Physics and Technology, Russian Federation

With the increase of resistance to conventional antibiotics development of new antimicrobial therapy becomes extremely relevant. Antimicrobial peptides (AMP) tend to be a perspective antibiotic class that kills drug-resistant strains of bacteria. To define the balance between AMP toxicity and efficiency in medical therapy it is utterly important to have a full picture of peptide activity. Using unique intramembrane electric field compensation technique and planar lipid bilayers, we clarified the adsorption mechanisms of two antimicrobial peptides, melittin and magainin-I, on the membrane surface. Using patch-clamp technique, we also studied blocking mechanisms of pore formation. According to the kinetic change of membrane conductivity the presence of peptides on both membrane surfaces inhibits pore formation. Thus, using two different techniques we succeed to clarify mechanisms of AMP melittin and magainin-I activity on the first stage of adsorption and subsequent processes of pore formation and deactivation.

The work was supported by the Ministry of Science and Higher Education of the Russian Federation (grant \# 07515-2020-782).

\section{P-216}

Testing the "sand in the gearbox" model: effects of antimicrobial peptides on the fluidity of the membranes of live $\boldsymbol{E}$. coli cells Cassandra Troiano [1], Francesco Riccitelli [1], Maria Rosa Loffredo [2], Maria Luisa Mangoni [2], Yoonkyung Park [3], Sara Bobone [1], Lorenzo Stella [1]

[1] Dept. of Chemical Science and Technologies, Univ. of Rome Tor Vergata, Italy; [2] Lab. affiliated to Pasteur ItaliaFondazione Cenci Bolognetti, Sapienza Univ. of Rome, Italy; [3] Dept. of Biotechnology, Chosun Univ., South Korea

Antimicrobial peptides (AMPs) represent a promising class of compounds to fight resistant infections. In most cases, they kill bacteria by making their membrane permeable. We have shown that bacterial killing requires complete coverage of the membrane surface by bound peptides $\left(10^{7}\right.$ bound molecules per cell) [BBA 2020, 1862:183291]. Therefore, it is possible that accumulation of the peptides on the cell surfaces causes other phenomena such as perturbation of the fluidity of the cell membranes, a mechanism that has been termed "sand in the gearbox". We have studied such effects by labeling the membranes of live E. coli cells with three fluorescent probes, DPH, Laurdan and pyrene. These probes position at different depths in the bilayer and allow monitoring bilayer viscosity (by fluorescence anisotropy), water penetration (by spectral shifts) and lateral lipid mobility (by excimer formation), respectively. These studies indicated that the cathelicidin AMP PMAP-23 causes a significant increase in membrane rigidity. This effect could contribute to the antimicrobial activity of AMPs, by affecting the function of membrane proteins, which is strongly dependent on the physico-chemical properties of the bilayer. 


\section{Posters}

- 14. Membrane active peptides -

\section{P-217}

Quantification of membrane fluidity in live epithelial cells treated by Candidalysin, a fungal peptide toxin secreted by C.albicans during invasion

Gaukhar Zhurgenbayeva [1,2], Christian Eggeling [3,4]

[1] Institute of Applied Optics and Biophysics, FriedrichSchiller-University Jena, Germany; [2] Jena School for Microbial Communication, Friedrich-Schiller-University Jena, Germany; [3] Leibniz Institute of Photonic Technology e.V., Germany; [4] MRC Human Immunology Unit, Weatherall Institute of Molecular Medicine, University of Oxford, UK

To become pathogenic and translocate across the intestinal epithelium C.abicans fungus needs to change its morphology from yeast to invasive filamentous. Fungal cytolytic peptide toxin, Candidalysin secreted during fungal invasion, is found to be crucial in direct cell membrane damage, the stimulation of a danger response signaling pathway, and the activation of epithelial immunity. Due to its amphiphilic alpha-helical structure peptide has a high binding affinity to plasma membranes. With increasing accumulation on the membrane surfaces, the alpha-helix peptide starts penetration of the lipid bilayer until the plasma membrane is damaged. Here, we used spectral imaging to assess the lipid packing changes in model membranes and plasma membranes of living epithelial cells under the treatment of candidalysin. Solvatochromic polarity-sensitive dye was used to label live oral and gut epithelial cells. Spectral imaging data allowed to calculate generalized polarization map of the labelled plasma membrane, and showed a significant change in membrane packing of Candidalysin treated epithelial cells. 
Posters

- 15. Methodological advances in biomolecular simulations -

P-218

How realistic are the conformational dynamics in contemporary lipid bilayer simulations? Hanne S Antila [1], Tiago M Ferreira [2], Samuli Ollila [3], Markus S Miettinen [1]

[1] MPI of Colloids \& Interfaces, Germany; [2] Martin-Luther Univ Halle--Wittenberg, Germany; [3] Univ of Helsinki, Finland

Molecular dynamics (MD) simulations are widely used to visualize the spatio-temporal motions of biomolecules. Yet, it is often unclear how well the conformational dynamics of the molecules in the simulations reflect the dynamics occurring in real life. Here, we extract a large set of phosphatidylcholine (PC) lipid bilayer MD simulation trajectories from an open-access database and benchmark the conformational dynamics produced by the MD models (force fields) used in those simulations against experimental nuclear magnetic resonance data. We utilize two measurables, the spin-lattice relaxation rate and the effective correlation time, to cover a wide range of dynamical processes. We find that none of the force fields perfectly captures the real-life dynamics of PC lipids. That said, CHARMM36 and Slipids force fields have more realistic dynamics than Amber Lipid14, OPLS-based MacRog, and GROMOS-based Berger force fields where a typical flaw is the too slow conformational sampling of the lipid glycerol backbone. The quality of dynamics produced by CHARMM36 and Slipids persists when cholesterol is added to the bilayer, although CHARMM36 tends to overestimate the relative weights of the $\sim 1$-ns processes in the headgroup dynamics. We stress that no new simulations were run to conduct this study, demonstrating the potential of open-access MD trajectory databanks in creating new scientific knowledge at lowered labor and CPU cost, which we hope will inspire other novel applications of these databanks.

P-220

Investigating the interaction of enzymes with functionalized surfaces : Lessons from multiscale modeling approaches

Nicolas Bourassin, Marc Baaden, Sophie Sacquin-Mora

Laboratoire de Biochimie Théorique, CNRS UPR9080, Institut de Biologie Physico-Chimique, Paris, France

Redox enzymes represent promising tools for H2-based technologies such as biofuel cells. However, many aspects of theses enzymes remain to be understood. In particular, designing efficient biofuel cells requires us to grasp details of the interaction between the enzymes and the electrode surfaces on the molecular level. Such information can be obtained using molecular modeling approaches on different scales, either with classical all-atom Molecular Dynamics simulations, or with coarse-grain calculations based on Elastic Network Models. Applications on [NiFe]-hydrogenases (which catalyze hydrogen oxidation) and copper-billirubin oxidase (which catalyzes oxygen reduction) show how simulations give us insight on factors determining enzymes orientation on the electrode surfaces, and how the adsorption on a solid surface can impact proteins structure, dynamics and mechanical properties, and therefore their catalytic activity. Keywords: Redox proteins, biofuel cells, protein mechanics, protein dynamics, Molecular Dynamics, coarse-grain simulations

\section{P-219}

Improving thermostability of biotechnological enzyme: nitrile hydratase case Julia Berdychowska, Łukasz Pepłowski Nicolaus Copernicus University, Poland

Nitrile hydratase (NHase) was discovered in $R$. rhodochrous bacteria during search for degradation of toxic nitriles (1). NHases are used in the industry to produce acrylamide from acrylonitrile and currently more than 600.000 tons of amides are obtained each year in that way. The enzyme has $\alpha$ and $\beta$ subunits. In $\alpha$ chain the active site composed of Co III (or Fe III) ion and post-translationally modified cysteines is present (2). Several natural stains of NHases are known.

Most of the NHases are active at temperatures $20^{\circ} \mathrm{C}$ to $35^{\circ} \mathrm{C}$. The catalyzed reaction is exoenergetic. During the industrial process, to avoid denaturation of enzyme, the bioreactor needs to be cooled. That generates huge costs and affect global warming. Protein engineering is an effective way of increasing protein thermostability. Our team designed mutants of PtNHase using of FireProt online server (3). They were more stable than the WT NHase (4). Next, PpNHases with linkers fusing subunits were created as well (5). It improved the stability and activity of proteins. Using molecular dynamics simulations we explain here the reasons of the increased thermostability and catalytic activity of both modified NHase variants.

(1) Yamada, H. and M. Kobayashi, Biosci Biotechnol Biochem, 1996. 60(9): p. 1391-400. (2) Prasad, S. and T.C. Bhalla, Biotechnol Adv, 2010. 28(6): p. 725-41. (3) Musil, M., et al., Nucleic Acids Res, 2017. 45(W1): p. w393-w399. (4) Cheng, Z., et al., Molecules, 2020. 25(20): p. 4806. (5) Junling, G., et al., Int J Biol Macromol, 2021.

\section{P-221}

Simulation of liquid jet explosions and shock waves induced by X-ray free-electron lasers Leonie Chatzimagas, Jochen S Hub

Saarland University, Germany

X-ray free-electron lasers (XFELs) produce X-ray pulses with very high brilliance and short pulse duration. These properties enable structural investigations of nanocrystals or single biomolecules, and they allow resolving the dynamics of biomolecules down to the femtosecond timescale. To deliver the samples rapidly into the XFEL beam, liquid jets are used. The impact of the X-ray pulse leads to vaporization and explosion of the liquid jet, while the expanding gas launches shock wave trains traveling along the jet that can affect biomolecular crystals before they have been probed. Here, we used atomistic molecular dynamics simulations to reveal the structural dynamics of shock waves after an X-ray impact. Analysis of the density in the jet revealed shock waves that form close to the explosion center and travel along the jet. A trailing shock wave formed after the first shock wave, similar to the shock wave trains in experiments. Although using purely classical models in the simulations, the resulting explosion geometry and shock wave dynamics closely resemble experimental outcomes, and they highlight the importance of the jet surface in shock wave propagation and attenuation. 


\section{Posters}

- 15. Methodological advances in biomolecular simulations -

\section{P-222}

Marked structural dynamics of the active site protein-water network in the prototypical rigid enzyme human carbonic anhydrase II

C. Das [1], C. Päslack [1], H. Singh [2], R. Linser [2], L. Schäfer [1]

[1] Theo. Chem., Ruhr Uni. Bochum, Germany; [2] Chem. \& Chem. Biol., Tech. Uni. Dortmund, Germany

Water molecules buried in protein active sites often govern their structure and dynamics. X-ray crystallography are widely used to locate such buried water molecules, whereas NMR can provide insights into the dynamical picture of coupled motions of water molecule(s) and the protein matrix. However, the interpretation of such NMR dynamics data in terms of the underlying atomic motions is often challenging. MD simulations can aid the interpretation of such NMR experiments at the atomic level. Recent NMR experiments reported pronounced microsecond timescale conformational dynamics of the active site of apo hCAII, a text-book example for a prototypical rigid enzyme. HCAII regulates $\mathrm{CO}_{2} / \mathrm{HCO}_{3}{ }^{-}$interconversion and is a potential drug target. The NMR particularly pointed to Thr198 located in an active site loop and, putatively, the highly conserved active site water network as showing marked conformational dynamics. Interestingly, this conformational dynamics was completely abrogated upon binding of a sulfonamide inhibitor. Our allatom MD simulations visualize how the active site pocket is shaped by pronounced open/close conformational-exchange dynamics of the Thr198-bearing loop and associated active site water molecules, which are strongly rigidified upon inhibitor binding.

\section{P-224}

Monosaccharide puckering influences N-glycan conformational phase space distribution Isabell L Grothaus [1], Giovanni Bussi [2], Lucio Colombi Ciacchi [1]

[1] BCCMS, University of Bremen, 28359 Bremen, Germany; [2] Scuola Internazionale Superiore di Studi Avanzati (SISSA), 34136 Trieste, Italy

Glycosylation is one of the bulkiest post-translational modification of proteins but has long been overlooked in molecular dynamics simulations, despite its omnipresence in the cell. However, the structure, function and interaction of many biochemical systems is governed by N-glycans covalently linked to asparagine residues in specific protein sequences. Due to the flexibility of their glycosidic linkages and their sugar units, N-glycans assume many different conformations, unlike the more rigid protein structure to which they are attached. A complete description of their conformational phase space requires thus the consideration of their large number of internal degrees of freedom. We show that an enhanced-sampling molecular dynamics scheme based on enhancing transitions of all relevant barriers with concurrent one-dimensional energy potentials in the framework of metadynamics can in fact capture effectively all biologically relevant global conformers of branched glycans, importantly also including the monomer puckering states. Interestingly, our approach revealed altered N-glycan conformer populations depending on the puckering state of the monosaccharides. These puckering-dependent conformer distributions, so far mostly ignored in glycoprotein simulations, might be crucial in explaining biological phenomena involving $\mathrm{N}$-glycans.

\section{P-223}

Extended structural dynamics reveals SULT1A1 accommodation to large ligands

Balint Dudas [1,2], Daniel Toth [3], David Perahia [2], Arnaud B Nicot [4], Erika Balog [3], Maria A Miteva [1]

[1] Inserm U1268 MCTR, CiTCoM UMR 8038 CNRS - University of Paris, France; [2] LBPA, Ecole Normale Supérieure Paris-Saclay, UMR 8113, CNRS, France; [3] Department of Biophysics and Radiation Biology, Semmelweis University, Hungary; [4] Université de Nantes, CRTI UMR 1064, France

Cytosolic sulfotransferases (SULTs) are phase II drug metabolizing enzymes catalyzing the sulfoconjugation from the co-factor 3' -Phosphoadenosine 5' -Phosphosulfate (PAPS) to a substrate. We employed molecular dynamics (MD) simulations and the recently developed approach of Molecular Dynamics with excited Normal Modes (MDeNM) to elucidate molecular mechanisms guiding the recognition of diverse substrates and inhibitors by SULT1A1. MDeNM allowed to explore an extended conformational space of PAPS-bound SULT1A1, which has not been achieved up to now by using classical MD. The generated ensembles combined with docking of 130 SULT1A1 ligands shed new light on substrate recognition and inhibitor binding mechanisms. Unexpectedly, our simulations demonstrated that large conformational changes of the PAPS-bound SULT1A1 can occur independently of the co-factor, sufficient to accommodate large substrates such as fulvestrant suggesting that possibly a wider range of drugs could be recognized by SULT1A1. Our study highlights the utility of including MDeNM in proteinligand interactions studies where major rearrangements are expected.

\section{P-225}

Mechanistic synergism between bacterial pyranose oxidase and peroxidase in lignin depolymerization

Enikö Hermann [1,2], Clemens K Peterbauer [2], Chris Oostenbrink [1]

[1] Institute of Molecular Modeling and Simulation, BOKU, Austria; [2] Institute of Food Technology, BOKU, Austria

Lignin is an aromatic heteropolymer that could provide a renewable source of aromatic chemicals, however, its depolymerization is challenging. Studies suggest that bacterial dyedecolorizing peroxidases (DyPs) could play a key role in bacterial lignin depolymerization. DyPs also have the potential to be utilized biotechnologically for this purpose ${ }^{1}$. Enzymes such as pyranose oxidase (POx) are proposed to act as redox partners for peroxidases in the process of lignin depolymerization. The role of POx would be dual in this synergism. At the onset of lignin depolymerization $\mathrm{POx}$ would oxidize sugars, and produce $\mathrm{H}_{2} \mathrm{O}_{2}$. This $\mathrm{H}_{2} \mathrm{O}_{2}$ could fuel the DyP to produce aromatic radicals from lignin, and in some cases Mn(III)-ions. Later on, these products can be reduced by the dehydrogenase activity of the $\mathrm{POx}^{2}$. We investigate this cycling using enzymes from Kitasatospora aureofaciens as models. KaPOx has been characterized ${ }^{1}$, and a DyP (KaDyPB) was expressed and will be characterized experimentally. We constructed homology models for KaDyPB and KaPOx and molecular dynamics simulations are used to investigate their behaviour. The most recent results will be presented.

${ }^{1}$ Colpa et al. 2014 J Ind Microbiol Biotechnol. 41:1-7.

${ }^{2}$ Herzog et al. 2019 Appl Environ Microbiol. 85(13):e00390-19 
Posters

- 15. Methodological advances in biomolecular simulations -

\section{P-226}

Computational study of SARS-CoV-2 3CLpro/RdRpC60 fullerene complexes binding properties

V. Hurmach [1], M. Platonov [2], Yu. Prylutskyy [1]

[1] Taras Shevchenko National University of Kyiv, Ukraine;

[2] Institute of Molecular Biology \& Genetics, Kyiv, Ukraine

According to WHO more than 100 million cases and 2 million deaths of SARS-CoV-2 have been confirmed. That is why the analysis of the potential binding mechanism between SARSCoV-2 proteins 3CLpro/RdRp and C60 fullerene was done. The interaction with 3CLpro has revealed that C60 fullerene tightly stuck in the catalytic binding pocket and shields catalytic dyad (Cys 145 and His 41) from any other interactions. Furthermore, C60 fullerene causes significant changes in the catalytic binding pocket. The most intriguing is that His 41 and Cys 145 are forced out from their original position. So that, the integrity of the catalytic dyad is violated. For RdRp 3 potential binding pockets were detected. The inhibition of pocket 1 could cause blocking of the RNA synthesis channel while the interaction in pockets 2 and 3 prevent the RdRp-nsp7/8 complex formation, without which RdRp can't perform its initial functions.

For a better understanding of binding peculiarities in obtained complexes, MMPBSA and MMGBSA analyses have been performed. It has been found that $\mathrm{E}_{\mathrm{vdW}}$ has the largest contribution to the binding energy in each complex. The $\mathrm{E}_{\text {elect }}, \mathrm{G}_{\text {polar }}$, and $\mathrm{G}_{\text {nonpolar impact on complex formation }}$ and stability are unfavorable. The results of the study can shed the light on 3CLpro and RdRp possible inhibition mechanisms.

\section{P-228}

Calcium binding modulates the stability of type IV Pilus

Yasaman Karami, Nadia Izadi-Pruneyre, Olivera Francetic, Michael Nilges

Institut Pasteur, Paris, France

Type IV pili (T4P) are dynamic fibers displayed on the surface of many bacteria to promote biofilm formation, host cell adherence and invasion, motility, DNA uptake, and virulence. T4P are implicated in virulence of enterohemorrhagic Escherichia. coli (EHEC), a major human pathogen. A detailed structural model of the EHEC T4P was recently determined based on cryoEM and NMR data ${ }^{1}$. NMR identified putative calcium binding residues in the pilin PpdD monomer. To investigate whether calcium plays a role in pilus assembly and function, we performed atomistic molecular dynamics simulations of EHEC pili in different conditions. The results showed that calcium occupies a binding pocket at the interface of three pilin subunits and affects the network of interactions governing pilus stability and inter-subunit communications. Complementary mutagenesis and functional assays identified a subset of residues in the calcium-binding region as critical for $\mathrm{T} 4 \mathrm{P}$ assembly. Finally, in vitro thermal stability of purified T4P in presence and absence of calcium, confirmed the role of calcium in stabilizing pili. Our investigations provided strong evidence of the existence of a pocket in which small molecules could bind to affect T4P assembly and function.

${ }^{1}$ Bardiaux et al., Structure. 2019 Jul 2;27(7):1082-1093.e5.

\section{P-227}

Conformational Sampling and Cell Permeability of Macrocycles

Anna S Kamenik, Johannes Kraml, Florian Hofer, Franz Waibl, Patrick K Quoika, Ursula Kahler, Michael Schauperl, Klaus R Liedl

University of Innsbruck, Austria

Macrocycles are highly specific drug candidates, yet they challenge standard conformer generators with their unique conformational preferences. Using accelerated MD (aMD) simulations we present a molecular dynamics-based routine that bypasses current limitations in conformational sampling and extensively profiles the free energy landscape of macrocycles in solution. We investigate the structure-permeability relationship of six macrocyclic compounds with grid inhomogeneous solvation theory (GIST) to calculate solvation free energies in explicit water and chloroform as a measure of passive cell permeability. Performing aMD simulations we capture structurally diverse ensembles in water and chloroform to profile solvent-dependent conformational preferences. Subsequent GIST calculations facilitate a quantitative measure of solvent preferences via the transfer free energy, calculated from the ensemble-averaged solvation free energies in water and chloroform. Hence, the proposed method considers how the conformational diversity of macrocycles in polar and apolar solvents translates into transfer free energies. Following this strategy, we find a striking correlation of 0.92 between experimentally determined cell permeabilities and calculated transfer free energies. For the studied model systems, we find that the transfer free energy exceeds the purely water-based solvation free energies as a reliable estimate of cell permeability and that conformational sampling is imperative for a physically meaningful model.

\section{P-229}

Ensuring the goodness-of-fit when refining molecular simulation ensembles against onedimensionally ordered data

Jürgen Köfinger [1], Gerhard Hummer [1,2]

[1] Max Planck Institute of Biophysics, Frankfurt am Main, Germany; [2] Goethe University, Frankfurt am Main, Germany

Small- and wide-angle X-ray scattering (SAXS/WAXS) data are prototypical examples of one-dimensionally ordered data against which we fit models and refine molecular simulation ensembles to extract information. Other examples are DEER/PELDOR, AUC, smFRET, titration, and enzyme kinetics data. We always have to ensure the consistency of a fit with our assumptions. For SAXS/WAXS, we usually check the assumption of uncorrelated errors visually in the residuals. The commonly used $\chi^{2}$ test is blind to order. Interpreting the residuals' signs as 1D Ising spins, we use the domain length distribution $h$ to quantify order and test for randomness (1). We introduce Shannon information distributions as a flexible tool to calculate P-values as measures of surprise. For models fitted against SAXS data (www.sasbdb.org) the $h$ test and the combined $\left(\chi^{2}, h\right)$ test substantially outperform currently available tests. As transparent measures for model quality, our superior goodness-of-fit tests enhance the predictive power of ensemble refinement methods and the reproducibility and reliability of scientific results across disciplines. An open-source Python 3 implementation is available at https://github.com/bio-phys/hplusminus.

(1) Powerful statistical tests for ordered data, Köfinger J and Hummer G, DOI: 10.26434/chemrxiv.13373351 (2020) 


\section{Posters}

- 15. Methodological advances in biomolecular simulations -

P-230

Improving CHARMM36 simulations by reintroducing the missing electronic polarizability: prosECCo

Ricky Nencini, Carmelo Tempra, Denys Biriukov, Samuli Ollila, Matti Javanainen, Hector Martinez-Seara

Institute of Organic Chemistry and Biochemistry of the Czech Academy of Sciences, Czech Republic

Molecular dynamics simulations (MD) are a powerful tool in structural biology. They allow studying molecular processes at scales often unreachable by experimental techniques. They, however, suffer because of size and time limitations related to computational power. Still, their main problem is accuracy given by the models they rely on. If a model is faulty, the results are too. MD models have improved significantly, and CHARMM36 is a state of the art force field covering most biomolecules. Unfortunately, classical nonpolarizable MD has limitations on its own. It lacks the electronic polarizability that plays a significant role in the interaction between charged moieties. Membranes, with their lipids, proteins, and sugars, do not escape from this problem. To improve this deficiency, we have created prosECCo. This lipid, protein, and sugar force field is a derivative of CHARMM36 that includes the missing electronic polarizability in a mean-field way. In practice, we do implement it by simple charge scaling of the atomic partial charges. Therefore, all available MD Softwares can readily use it without modifications and does not have any computational overhead. Our new models for show significantly improved interactions between charged moieties. Overall, we can include missing physics in our models for free and this immediately improve the structural behavior.

\section{P-232}

How do glycan \& glycosaminoglycan composition influence protein dynamics? An example of small leucine-rich proteoglycans

Rajas M Rao, Marc Guéroult, Nicolas Belloy, Jean-Marc Crowet, Stéphane Brézillon, Manuel Dauchez, Stéphanie Baud

Laboratoire de Matrice Extracellulaire et Dynamique Cellulaire, Université de Reims Champagne-Ardenne, France

The extracellular matrix (ECM) provides a critical platform for numerous activities such as development, signalling, immune response to name a few. A major component of it are the glycoproteins, which are important actors in these functions. While the importance of protein part of the glycoprotein has been well-described through various studies, the importance of the sugar component and their residues is still not well understood. In this work, we describe, at the atomic level, how changes in sugar residue composition in glycosaminoglycans impact glycoprotein dynamics and ultimately their function. We demonstrate this by characterizing the behaviour of fibromodulin, a small leucinerich proteoglycan responsible for maintenance of normal organization of ECM, using molecular dynamics simulations. We show how changes in glycan composition can influence fibromodulin dynamics, including global protein dynamics as well as glycan dynamics. We provide a structural basis from which changes in sugar residues of glycoproteins impact protein-sugar interactions, perturbation of which is a major factor in numerous diseases such as rheumatoid arthritis and macular corneal dystrophy to name a few.

\section{P-231}

Lipid nanodiscs as a tool to unlock membrane dynamics

Matthias Pöhnl [1], Rainer A Böckmann [1,2]

[1] Friedrich-Alexander University Erlangen-Nürnberg, Erlangen, Germany; [2] Erlangen National High Performance Computing Center (NHR@FAU), Germany

Cholesterol is a major constituent of membranes in all animals and able to flip between leaflets on timescales orders of magnitude faster than other lipids. But both the distribution of cholesterol between the leaflets as well as the interplay between the distribution and other membrane properties remain largely unknown. Difficulties in resolving the particular role of cholesterol for specific biomembrane characteristics are connected to the limited spatial and temporal resolution in experiments and challenges in studying asymmetric membranes and connected domain formation in simulations.

Here, we developed lipid nanodisc simulation systems for the study of asymmetric membranes. The nanodisc setup avoids membrane vesiculation as well as lipid exchange between the leaflets by lateral diffusion. All investigated membrane systems showed fluctuations increased in amplitude as compared to membranes constrained by periodic boundary conditions, albeit quantitatively preserving the membrane bending moduli. Addition of cholesterol results in fluctuations substantially amplified by accompanying cholesterol flips between the membrane leaflets, suggesting a particular function of cholesterol for localized strong bending as observed e.g. in fusion.

\section{P-233}

How does glycan-glycan interaction modify protein behaviour?

Miguel Riopedre, Hector Martinez-Seara

Institute of Organic Chemistry and Biochemistry of the CAS, Czech Republic

Glycosylation is an important feature in the regulation of biological processes, via modification of protein behaviour. It is known that the interplay between sugars and the protein surface can reshape the structural properties of the latter, and thus alter its function. However, it has yet to be discovered if the change in protein behaviour is also related to sugar-sugar interactions, and with the distributions and conformations they adopt on the protein exterior. For instance, the formation of sugar clusters could hinder some interactions, whereas the repulsion between sugar moieties could free space and ease them.

Our approach to this question consists on using classical molecular dynamics simulations of glycosylating sugars bound to a surface that mimics the protein. Then, we change the distance between the sugar moieties, and we check the number of contacts between them. To compare this with experimental data, we used a search in the Protein Data Bank, where we looked for the distances between the Nglycosylation sites of those proteins that are known to be glycosylation targets.

First results show that almost half of the glycosylation sites lie at distances that allow the studied glycans to touch each other, with a clear maximum at around $5 \mathrm{~nm}$. Also, $92 \%$ of the sites are within a distance of $15 \mathrm{~nm}$ to another site. All of this indicates that the distribution of the glycosylation spots is not random with respect to sugar-sugar interactions. 
Posters

- 15. Methodological advances in biomolecular simulations -

P-234

Molecular mechanism of ligand binding pathways and activation of the succinate receptor

Aslihan Shenol Iliyaz, Michael Lückmann, Mette Trauelsen, Thomas M Frimurer, Thue W Schwartz

Novo Nordisk Foundation Center for Basic Metabolic Research, University of Copenhagen, Copenhagen, Denmark

Aim - Here we aim to characterize the molecular mechanisms of various ligands binding and activation of the succinate receptor (GPR91). We use MD simulations, metadynamics and extensive mutagenesis to understand the protein-ligand dynamic interplay.

Methods $\& 5$ Results - Long scale MDs of X-ray structures of GPR91 binding succinate molecules demonstrate that the acidic metabolite gets to the orthogonal pocket after passing through few stable binding stages. First captured between TM6 and 7 in the extracellular vestibule, followed by an intermediate stage involving ECL2 and finally binding the orthogonal site. The free energies of the stages were investigated by well-tempered metadynamics and pointed to a bottleneck, related to the release of ECL2 through breakage of a key constraining salt-bridges. The binding site in the ECV was also proven to be an allosteric binding site. We observed binding of 2 molecules of succinate with the second one either bypassing the first or binding to the initial site after the first one had moved down to the final binding site. In both sites succinate is preferentially bound in its cis conformation. Importantly, a novel antagonist demonstrated its entry instead between TM1 and 2 to bind with its acidic group to residues also involved in succinate binding, but without affecting ECL2.

\section{P-236}

Capturing the flexibility of a protein-ligand complex: Binding free energies from different enhanced sampling techniques

Sebastian Wingbermühle [1,2], Lars V Schäfer [1]

[1] Ruhr University Bochum, Germany; [2] Science for Life Laboratory (SciLifeLab, Stockholm), Sweden

During dissociation processes, the loss of favourable interactions between the binding partners can be compensated for by an increase in configurational flexibility such that the enthalpically stabilised, fully bound state competes with an entropically stabilised, partially dissociated state that is similar in free energy. It is therefore a demanding challenge even to state-of-the-art enhanced sampling techniques to compute the corresponding free energy profile.

Here, the free energy profile for the N-terminal dissociation of an antigenic peptide from the Major Histocompatibility Complex I HLA-B*35:01 was investigated with Replica Exchange with Solute Tempering 2 (REST2), Bias Exchange Umbrella Sampling (BEUS) and Well-Tempered Metadynamics (WT-MTD). Surprisingly, the free energy profiles obtained with the three techniques were even qualitatively different, disagreeing on whether the fully bound or the partially dissociated state constitutes the global free energy minimum. A careful convergence analysis revealed that, among the three techniques tested, the most balanced sampling of the two states could be achieved with BEUS, which therefore yielded the most reliable free energy profile for the dissociation of the peptide N-terminus.

\section{P-235}

The mechanism of sodium binding to SERT - in silico investigation

Dániel Szöllősi, Thomas Stockner

Institute of Pharmacology, Medical University of Vienna, Austria

The serotonin transporter (SERT) terminates neurotransmission by transporting serotonin from the synapse into the pre-synaptic nerve terminal. Altered SERT function leads to several neurological diseases including depression, anxiety, mood disorders and attention deficit hyperactivity disorders (ADHD). Accordingly, SERT is the target for pharmacological treatment. Moreover, multiple recreational drugs also interfere with normal SERT function. Transport of serotonin is energized by the electrochemical gradient of sodium across the cell membrane. We used extensive molecular dynamics simulations to investigate the process of sodium binding to SERT, the first step in the transport cycle. Comparing data from 51 independent simulations, we find a remarkably well-defined path for sodium entry and could identify two transient binding sites, while binding kinetics that are comparable to experimental data. We find that the electric field generated by the protein attracts the sodium ions and directs them towards the respective binding sites. Importantly, structure and dynamics of the sodium binding sites indicate that sodium binding is accompanied by an induced fit mechanism that stabilizes the outward-open conformation. The loss of entropy due to conformational stabilization is compensated by the energetically favorable binding of sodium.

\section{P-237}

Molecular dynamics study of the interaction of $\mathrm{K}^{+}$counterions with DNA double helix in an external electric field

Oleksii Zdorevskyi, Sergiy Perepelytsya

Bogolyubov Institute for Theoretical Physics, Ukraine

The structure of a DNA double helix is stabilized by metal counterions condensed to a diffuse layer around the macromolecule. The dynamics of counterions in real conditions is governed by the electric fields from DNA and other biomolecules. In the present work, the molecular dynamics study was performed for the system of DNA double helix with neutralizing $\mathrm{K}^{+}$counterions and for the system of $\mathrm{KCl}$ salt solution in an external electric field of different strength (up to $32 \mathrm{mV} / \AA$ ). The analysis of ionic conductivity of these systems has shown that the counterions around the DNA double helix are slowed down compared with the $\mathrm{KCl}$ salt solution. The calculated values of ion mobility are within (0.05-0.4) $\mathrm{mS} / \mathrm{cm}$ depending on the orientation of the external electric field relative to the double helix. Under the electric field parallel to the macromolecule, $\mathrm{K}^{+}$counterions move along the grooves of the double helix staying longer in the places with a narrower minor groove. Under the electric field perpendicular to the macromolecule, the dynamics of counterions is less affected by DNA atoms, and starting with the electric field values about $30 \mathrm{mV} / \AA$ the double helix undergoes a phase transition from a double-stranded to a single-strand state. 


\section{Posters}

- 16. Membrane signal transduction -

P-238

Peptide inhibitors as tool compounds targeting GPCR signaling

Henrik Daver, Kasper Harpsøe, David E Gloriam

Department of Drug Design and Pharmacology, University of Copenhagen, Denmark

Intracellular signal transduction mainly takes place via protein-protein interactions (PPIs). The more we know about the signaling pathways, the more precise can we be in designing drugs that activate only the desired response and avoid side effects. By careful analysis of the PPIs that participate in signal transduction, and using a variety of computational methods, peptide inhibitors were designed for several targets. The aim is to generate selective PPI inhibitors to be used as tool compounds in the analysis of signaling pathways. We target proteins involved in $\mathrm{G}$ protein-coupled receptor (GPCR) signaling, such as G $\alpha, G \beta \gamma$, arrestin and adenylyl cyclase. Experimental studies of the designed peptides are underway (as of April 2021) and this poster will highlight the results.

\section{P-240}

The bradycardic agent ivabradine acts as an atypical inhibitor of voltage-gated sodium channels Benjamin Hackl [1], Janine Ebner [1], Péter Lukács [2], Krisztina Pesti [2], Árpád Mike [2], Nicholas Haechl [1], Elena Lilliu [1], Klaus Schicker [1], Anna Stary-Weinzinger [3], Karlheinz Hilber [1], Hannes Todt [1], Xaver Koenig [1]

[1] Center for Physiology and Pharmacology, Medical University of Vienna, Austria; [2] Plant Protection Institute, Centre for Agricultural Research, Hungary; [3] Department of Pharmacology and Toxicology, University of Vienna, Austria

Ivabradine (iva) is clinically administered to lower the heart rate by a proposed selective inhibition of hyperpolarizationactivated cyclic nucleotide-gated channels. Here we show that, in addition, iva inhibits voltage-gated sodium channels (VGSC) within a similar concentration range. A block of these channels likely contributes to the established and newly raised clinical indications of iva. Iva was tested on VGSC in native cardiomyocytes isolated from mouse ventricles and from the His-Purkinje system and on human $\mathrm{Na}_{\mathrm{v}} 1.5$ in a heterologous expression system. Iva inhibited VGSC in a voltage- and frequency-dependent manner, but did not alter voltage-dependence of activation and fast inactivation, nor recovery from fast inactivation. Block of cardiac VGSC by iva in native cardiomyocytes isolated from the ventricles and the His-Purkinje system was akin to block of heterologously expressed cardiac $\left(\mathrm{Na}_{\mathrm{v}} 1.5\right)$, neuronal $\left(\mathrm{Na}_{\mathrm{v}} 1.2\right)$ and skeletal muscle $\left(\mathrm{Na}_{\mathrm{v}} 1.4\right)$ isoforms. Molecular drug docking revealed an interaction of ivabradine with the classical local anaesthetic binding site. Our data support the notion that iva acts as an atypical inhibitor of VGSC.

\section{P-239}

Unravelling the role of the C-terminal DEP domain of Dishevelled protein in Wnt signaling Francesco L Falginella, Robert Vácha

CEITEC - Central European Institute of Technology, Masaryk University, Czech Republic

Wnt signaling is a group of evolutionary conserved pathways crucial for tissue development and homeostasis, and defects in its function lead to numerous diseases, including cancer. The signal transduction begins with the binding of Wnt ligand to Frizzled transmembrane receptor, which then relays the signal to Dishevelled protein (DVL). DVL acts as a branch point and selectively initiates one of various intracellular pathways by a yet unknown mechanism, presumably associated with the dynamic changes in DVL intracellular localization.

By combining classic and biased all-atom Molecular Dynamics and coarse-grained Monte Carlo simulations, we provide new insights into the interactions regulating the relocalization of DVL between the cytoplasm and the plasma membrane. In particular, we focused on the role of the membrane binding C-terminal DEP domain of DVL. Our results suggest that the binding of DEP domain to anionic phospholipid membranes is primarily driven by non-specific electrostatic attraction, further optimized to preferentially interact with phosphatidic acid. However, phosphorylation modifications altering the charge of the domain did not abolish DEPmembrane binding due to local cation-mediated interactions. We believe that the improved molecular understanding of the membrane binding function of DEP domain will help us decipher the early cytoplasmic stages of Wnt signaling.

\section{P-241}

Subcellular micropatterning for visual immunoprecipitation reveals differences in cytosolic protein complexes downstream the EGFR

Roland Hager, Ulrike Müller, Nicole Ollinger, Julian Weghuber, Peter Lanzerstorfer

University of Applied Sciences Upper Austria, Austria

Analysis of protein-protein interactions in living cells by protein micropatterning is currently limited to the spatial arrangement of transmembrane proteins ('bait', e.g. receptor) and their corresponding downstream adaptor molecules ('prey'). Here we present a robust method for visual immunoprecipitation of cytosolic protein complexes by use of an artificial transmembrane bait construct in combination with micropatterned antibody arrays on cyclic olefin polymer (COP) substrates. The method was used to characterize multiple Grb2-mediated protein interactions downstream the epidermal growth factor receptor (EGFR) on a single cell level. We proofed that EGFR downstream signaling is based on constitutively bound complexes as well as on agonistdependent protein assemblies, which possess rather transient interaction properties. Spatiotemporal analysis further revealed significant differences in stability and exchange kinetics of protein interactions. Furthermore, we could show that this approach is well suited to study the efficacy of membrane-permeable protein interaction domain inhibitors in a live cell context. Altogether, this method represents a significant enhancement of existing quantitative subcellular micropatterning approaches and might be an alternative to standard biochemical analysis in future cell biology investigations. 
Posters

- 16. Membrane signal transduction -

P-242

Role of nanoscale antigen organization on T-cell activation probed using DNA origami

Joschka Hellmeier [1], Rene Platzer [2], Gerhard J Schütz [1], Johannes B Huppa [2], Eva Sevcsik [1]

[1] Institute of Applied Physics, TU Wien, Austria; [2] Institute for Hygiene and Applied Immunology, Medical University of Vienna, Austria

$\mathrm{T}$ cells recognize the presence of even a single foreign $\mathrm{pMHC}$ on the surface of APCs although their TCRs bind with micromolar affinity. How they achieve this is still poorly understood, yet indirect evidence pointed to spatial antigen arrangement on the APC surface as a critical factor for this recognition process. Thus, we designed an APC-mimicking biointerface serving the dual purpose to generate defined exclusion zones around individual ligands to isolate them as they cluster during T-cell activation as well as permit directed pre-organization of single ligands at the nanoscale. We employed DNA platforms anchored to mobile lipid bilayers and functionalized with either high-affinity single chain antibody fragments $\left(\mathrm{scF}_{\mathrm{V}}\right)$ to serve as templates for TCR organization or low-affinity antigenic pMHCs as natural ligand. When confronting $\mathrm{T}$ cells with this biointerface we found that $\mathrm{T}$-cell activation via $\mathrm{scF}_{\mathrm{V}}$ requires close proximity of ligands within units of at least two. This held no longer true for the physiological TCR ligand, cognate pMHCs, which were well capable to stimulate $\mathrm{T}$ cells as well-isolated entities. Our data indicate that early T-cell signaling emerges from small assemblies of triggered TCRs either formed by prolonged TCR:ligand engagement of closely spaced TCRs or by repeated short-lived interactions via single pMHCs.

\section{P-244}

Insights on IgG oligomer mediated Fc receptor clustering

Andreas Karner [1], Christine Siligan [2], Johannes Preiner [1] [1] TIMed Center, University of Applied Sciences Upper Austria, Austria; [2] Institute of Biophysics, Johannes Kepler University Linz, Austria

Immunoglobulin $\mathrm{G}$ ( $\mathrm{IgG}$ ) Fc receptors (Fc $\gamma \mathrm{Rs}$ ) are expressed on most immune cells and play crucial roles in antibodymediated immune responses. Effector cell activation is triggered by Fc $\gamma \mathrm{R}$ clustering upon binding to multivalent antibody-antigen complexes. Crystal structures of these complexes suggest that IgGs therein have to adopt a distinct conformation with their $\mathrm{Fc}$ region oriented parallel to the antigenic membrane. A very similar IgG conformation was recently found in complement activating IgG oligomers that assemble on cells upon binding to surface antigens.

We hypothesize that antigen-dependent IgG oligomerization (I) facilitates Fc $\gamma R$ binding by providing a suitable IgG conformation and (II) enables Fc $\gamma \mathrm{R}$ clustering and activation by providing a multivalent target-complex for $\mathrm{Fc} \gamma \mathrm{Rs}$.

To address this, we express Fc $\gamma$ RIIIa in HEK293F cells and reconstitute the purified receptor in supported membranes. We employ high-speed atomic force microscopy to visualize, and quartz crystal microbalance to quantitatively characterize the interactions of FcyRIIIa and IgGs. Additionally, cryo-electron tomography will be employed to determine the structure of complexes and single molecule fluorescence microscopy to investigate IgG-oligomer induced Fc $\gamma$ RIIIaclustering in natural killer cells.

\section{P-243}

Protein binding across immune-cell contacts and its influence on T-cell activation

Victoria Junghans [1,2], Tommy Dam [1], Manto Chouliara [1], Ana Mafalda Santos [2], Simon J Davis [2], Peter Jönsson [1] [1] Lund University, Sweden; [2] University of Oxford, UK

Binding between ligands and receptors across contacting cells is vital for several biological processes. This includes activation of $\mathrm{T}$ cells, where T-cell receptors (TCRs) bind to antigen presented by MHC complexes (pMHC). How strong this interaction needs to be to start an immune response is, however, unclear. I will here highlight some of our recent results on the affinity between TCR and pMHC displaying different peptides, from cognate to self. In addition, I will also show how the auxiliary binding pair CD2/CD58 both can stabilize and destabilize TCR binding pMHC (1). I will also discuss how the weak interaction between the co-receptor CD4 and pMHC can influence T-cell signaling (2), and present a new method of analyzing binding affinities on a single cell level. I will finally discuss how nickel-chelating lipids, commonly used in model membranes for in vitro T-cell studies, can induce TCR-dependent cell signaling without influencing CD45 exclusion (3). Altogether demonstrating that it is not only the protein-protein interaction per see that influences binding in vivo.

1. Junghans V, et al., J. Cell Sci., 133, jcs245985 (2020).

2. Jönsson P, et al., Proc. Natl. Acad. Sci. U.S.A., 113, 5682-5687 (2016).

3. Dam T, et al., Front. Physiol., 11, 613367 (2021).

\section{P-245}

Water mediated allosteric network in $\beta 2 \mathrm{AR}$ T164I variant modulates agonist binding affinity Siddhanta V Nikte[1], Krushna Sonar [1,3], Aditi Tandale [1,4], Durba Sengupta [1], Manali Joshi [2]

[1] Physical Chemistry Div, National Chemical Lab, Pune, India; [2] Bioinformatics Centre, S. P. Univ, Pune, India; [3] Curtin Univ, Bentley, Australia; [4] University of Cambridge, UK

The $\beta 2$-adrenergic receptor $(\beta 2 \mathrm{AR})$ is an important protein that is the drug target for asthma and chronic obstructive pulmonary disorder (COPD). Clinical studies report that the population variants (single position sequence mutations) of B2AR are associated with differential response to agonist based therapy. The most important variants of $\beta_{2} \mathrm{AR}$ are Gly16Arg, Gln27Glu and Thr164Ile. The variants Gly16Arg and Gln27Glu are present at the N-terminal region that is highly flexible and consequently the least studied part of the receptor. The variant Thr164Ile is present in the transmembrane region. Interestingly none of these variations are present in the ligand binding site. We present here the structural basis for the altered ligand affinity of the $\beta 2 \mathrm{AR}$ variants using molecular dynamics simulations, docking, and solvation free energy calculations. The results show that local structural rearrangements due to the T164I variation causes perturbation in the water mediated interactions with residues nearby $164^{\text {th }}$ position which leads to long-distance effects and eventually perturbs the binding site. This results in the altered binding position and binding free energy of a series of agonists. Our study shows that the Ile164 variant leads to reduced ligand binding as compared to the wild type receptor. Our study provides a structural basis to explain the differential response towards drugs used for treatment for Asthma \& COPD in the association of $\beta 2 \mathrm{AR}$ variants. 
Posters

- 16. Membrane signal transduction -

P-246

Cooperative interactions at nanoscale patterned signaling complexes in the plasma membranes Michael Philippi [1], Isabelle Watrinet [1], Marie Kappen [1], Sergej Korneev [1], Andreas Plückthun[2], Martin Steinhart [1], Jacob Piehler [1], Changjiang You [1]

[1] University of Osnabrueck, Biology/Chemistry, Germany; [2] University of Zurich, Biochemistry, Switzerland

Spatiotemporal organization of signaling complexes at the plasma membrane plays an intricate role in regulating cell signaling. Multivalent interactions and liquid-liquid phase separation (LLPS) of proteins and lipids are emerging as key determinants of efficiency and specificity of downstream signaling. To explore these phenomena, we have established a system to capture signaling complexes in the plasma membrane of live cells based on surface functionalization by capillary nanostamping. By capturing GFP-tagged membrane receptors into high-density nanodot arrays, characteristic spot diameters of $\sim 300 \mathrm{~nm}$ were achieved. GFP-tagged Wnt coreceptor Lrp6 expressed at the surface of living cells was successfully assembled into nanodot arrays. Strikingly, the co-receptor Fzd8 as well as the cytosolic scaffold proteins Axin1 and Disheveled2 were spontaneously recruited into the nanodot array to form spatially defined signalosomes in the absence of ligand pointing toward LLPS-driven signalosome assembly. Immunofluorescence staining confirmed ligand-independent Wnt/ $\beta$-catenin signaling activated by the nanodot arrays. As a reference system, assembly of EGFR signaling platforms at nanodot arrays was explored. Ligand-independent EGFR activation was confirmed by imaging effector recruitment and enzymatic activity.

\section{P-248}

Ion-water coupling controls class A G-protein coupled receptor signal transduction pathways Neil J Thomson [1], Owen N Vickery [1,2], Callum M Ives [1], Ulrich Zachariae [1]

[1] University of Dundee, UK; [2] University of Warwick, UK

Understanding G-protein coupled receptor (GPCR) signalling, the largest family of transmembrane signalling proteins, is of great pharmaceutic interest. A structurally and evolutionarily conserved activation mechanism is believed to underpin signalling in the Class A GPCR subfamily. This mechanism involves the collapse of an ionizable binding site that binds sodium in the inactive state, and the reorganization of residue side-chains, helices, and a water-mediated hydrogen bonding network. To unravel the interaction between all of these features and the receptors' functional state, we combined all-atomistic molecular dynamics simulations with principles from information theory into a technique we term 'State Specific Information'. With this, we report a well-defined transmembrane pathway in three receptor types, bridging the extracellular ion binding site with the intracellular effector binding site by coupled conformational changes, and enabling the two most conserved ionizable residues in GPCRs to communicate their states with each other. The dynamics of internal water molecules plays a key role in signalling this information. While sodium binding locks the receptors in inactive conformations, protonation of the ion binding site promotes the receptors' transition towards active conformations, which are further stabilised after neutralizing both ionizable motifs. Our findings reveal the role of ion binding and protonation in GPCR activation and signalling.

\section{P-247}

Natural killer cell activation receptor NKp30 oligomerization depends on its $\mathrm{N}$-glycosylation Ondřej Skořepa [1], Samuel Pazicky [1,2], Barbora Kalousková [1], Jan Bláha [1,2], Celeste Abreu [1], Jan Dohnálek [3], Tereza Skálová [3], Arthur Sedivy [4], Ondřej Vaněk [1]

[1] Dept of Biochemistry, Charles Univ, Prague, Czech Republic; [2] EMBL, Hamburg Unit c/o DESY, Germany; [3] BIOCEV, Vestec, Czech Republic; [4] Protein Technologies, VBCF GmbH, Vienna, Austria

NKp30 is one of the main human natural killer cell activating receptors used in directed immunotherapy. The oligomerization of the NKp30 ligand binding domain depends on the length of the stalk region, but our structural knowledge of NKp30 oligomerization and its role in signal transduction remains limited. Moreover, ligand binding of NKp30 is affected by the presence and type of N-glycosylation. We assessed whether NKp30 oligomerization depends on its Nglycosylation. Our results show that NKp30 forms oligomers when expressed in HEK293SGnTI-cell lines with simple Nglycans. However, NKp30 was detected only as monomers after enzymatic deglycosylation. We characterized the interaction between NKp30 and its ligand, B7-H6, with respect to glycosylation and oligomerization, and we solved the crystal structure of this complex with glycosylated NKp30, revealing a new glycosylation-induced mode of NKp30 dimerization. Our study provides new insights into the structural basis of NKp30 oligomerization and explains how the stalk region and glycosylation of NKp30 affect its ligand affinity. This furthers our understanding of the molecular mechanisms involved in NK cell activation, which is crucial for the design of novel NK cell-based targeted immunotherapeutics. 
Posters

- 17. Cytoskeleton / Motor proteins -

\section{P-249}

Possible mechanism of multi-step microtubule catastrophe revealed by Monte-Carlo modeling Veronika Alexandrova, Nikita B Gudimchuk

Physics department, Lomonosov Moscow State University, Moscow, Russian Federation

The growth of the cytoskeletal filaments, such as microtubules (MTs), is occasionally interrupted by periods of rapid shrinkage, called catastrophe, followed by a resumption of growth or complete disassembly. While catastrophe is believed to be triggered by hydrolysis of guanosine triphosphate (GTP) molecules, this fact is not enough to explain a well-established phenomenon of MT lifetime-dependent increase in catastrophe frequency. The onset of MT catastrophe had been proposed to be additionally affected by slow destabilization of the MT tip structure. To delve deeper into this problem, we have developed a stochastic model of MT dynamics that accounts for both conformational and nucleotide changes in tubulin dimers. Consistent with recent structural data and in contrast to the 'allosteric' model, in which higher tubulin curvature is induced by GTP hydrolysis, in our model each tubulin dimer is curved at equilibrium, regardless of the associated nucleotide. Within this model, we show that modifications in MT tip structure can increase catastrophe probability over time, however, a coupling of GTP hydrolysis to tubulin curvature is necessary to consistently reproduce experimental data. Our findings provide an insight into fundamental properties of MT dynamics and highlight the need to revise the causality between GTP hydrolysis and tubulin conformational transitions. Supported by RSF grant 21-74-20035.

\section{P-251}

Single-molecule dissection of the force generation of the kinesin-3 motor KIF1A

Breane G Budaitis [2], Shashank Jariwala [2], Lu Rao [1], Yang Yue [2], David Sept [2], Kristen J Verhey [2], Arne Gennerich [1]

[1] Albert Einstein College of Medicine, New York, USA; [2] University of Michigan, Ann Arbor, USA

The kinesin-3 motor KIF1A functions in neurons, where its fast and superprocessive motility facilitates long-distance transport, but little is known about its force-generating properties. Using optical tweezers, we demonstrate that KIF1A stalls at an opposing load of $\sim 3 \mathrm{pN}$ but more frequently detaches at lower forces. KIF1A rapidly reattaches to the microtubule to resume motion due to its class-specific K-loop, resulting in a unique clustering of force generation events. To test the importance of neck linker docking in KIF1A force generation, we introduced mutations linked to human neurodevelopmental disorders. Molecular dynamics simulations predict that V8M and Y89D mutations impair neck linker docking. Indeed, both mutations dramatically reduce the force generation of KIF1A but not the motor's ability to rapidly reattach to the microtubule. Although both mutations relieve autoinhibition of the full-length motor, the mutant motors display decreased velocities, run lengths, and landing rates and delayed cargo transport in cells. These results advance our understanding of how mutations in KIF1A can manifest in disease.

\section{P-250}

In vitro study of the microtubule rescue mechanism

Mikhail N Anisimov, Nikita B Gudimchuk

Lomonosov Moscow State University, Russian Federation

Microtubules (MTs) are dynamic intracellular tubular filaments undergoing unique stochastic switching from growth to shortening and vice versa. These transitions, called catastrophes and rescues, respectively, are essential for chromosome segregation and other processes. Here we use in vitro reconstitution approach to examine the poorly understood mechanism of MT rescue. We find that the distribution of lengths, at which MT rescues occur, has a peak situated near the nucleating seed. This distribution is closely matched by positions of the GTP-tubulin patches incorporating into MT body from solution. Given the proximity of the rescue positions to the MT seeds, we have hypothesized that non-specific contacts with the coverslip might induce damage in MT lattice, followed with MT repair with soluble GTP-tubulin. Thus formed 'GTP-tubulin islands', might then locally stabilize MT lattice, leading to increased rescue probability. To test this idea, we have assembled MTs from the seeds overhanging from micro-fabricated pedestals, well separated from the coverslip. In this setup, MT rescue frequency was substantially reduced. Our results suggest that spontaneous rescues of isolated MTs are very rare. So the primary mechanism of MT rescue is dependent on external factors, such as regulatory proteins or MT lattice damage and repair induced by non-specific interactions with other intracellular or artificial objects. This work was supported by RSF grant \#21-74-20035.

\section{P-252}

Structural characterization of $2 \mathrm{~N} 4 \mathrm{R}$ tau protein isoform by multidimensional NMR spectroscopy

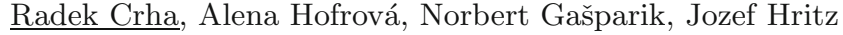
Central European Institute of Technology, Masaryk University, Kamenice 5, 62500 Brno, Czech Republic

norbert.gasparik@ceitec.muni.cz,jozef.hritz@ceitec.muni.cz

Neurodegenerative diseases are often associated with pathological conformational changes within the tau protein. Tau belongs to the group of intrinsically disordered proteins (IDP) and occurs predominantly in neuronal axons in the brain, where it regulates the formation of the microtubule system, thereby promoting proper neuron function. Under so far unknown pathological conditions, tau undergoes conformational changes, which lead to its aggregation. Aggregation causes the formation of neurofibrillary tangles (NFTs) of non-functional tau proteins. These changes prevent tau from fulfilling its biological function, which results in a gradual disruption of the proper function of the neuronal axons and later interruption of synaptic connections in the brain. In this poster, we present our interim results of structural characterization of the longest tau isoform using NMR spectroscopy. We employed 5D ${ }^{13} \mathrm{C}$-directly detected NMR experiments for the backbone assignment of tau, because of its IDP character as well as the high content of prolines in its primary structure. With this approach, we have successfully assigned $99 \%$ of the protein residues including prolines, and determined the secondary structure propensity at single residue resolution. 
Posters

- 17. Cytoskeleton / Motor proteins -

P-253

Structural and computational study of microtubule assembly, dynamics and force production Nikita B Gudimchuk [1,2], Evgeniy V Ulyanov [1], Dmitrii S Vinogradov [2], Eileen O'Toole [3], Fazoil I Ataullakhanov [1,2], J. R McIntosh [3]

[1] Physics Deparment, Lomonosov Moscow State University, Moscow, Russian Federation; [2] Center for Theoretical Problems of Physicochemical Pharmacology, Moscow, Russian Federation; [3] Department of M.C.D. Biology, University of Colorado, Boulder, USA

Microtubules (MTs) are essential tubulin polymers that stochastically alternate between assembly and disassembly. MT dynamics can generate and respond to mechanical forces, enabling vital cellular processes such as membrane remodeling, organelle positioning, cell migration and division. Recently, we and others have shown that MTs grow by addition of bent guanosine triphosphate (GTP) tubulins to the tips of curved tubulin protofilaments, challenging previous views about tubulin assembly. Building on this structural finding, here we formulate a computational model of this novel MT assembly pathway and apply electron cryotomography to test our model through characterizing the structures of MTs, grown under various conditions in vitro. Using Brownian dynamics simulations, we examine the implications of the flared MT tip morphology for dynamic instability and the ability of growing and shortening MTs to generate and respond to mechanical loads. This analysis provides new insights into MT dynamics, possible mechanisms of their regulation by drugs and proteins, and coupling of kinetochores with MT tips in mitosis.

\section{P-255}

Reversible protofilament peeling during microtubule disassembly

Kristýna Holanová, Milan Vala, Łukasz Bujak, Marek Piliarik

Institute of Photonics and Electronics of the Czech Academy of Sciences, Czech Republic

Microtubules are cytoskeletal polymers of tubulin dimers assembled into protofilaments that constitute nanotubes undergoing periods of assembly and disassembly. Static electron micrographs suggest a structural transition of straight protofilaments into curved ones occurring at the tips of disassembling microtubules. However, these structural transitions have never been observed and the process of microtubule disassembly thus remains unclear. By employing ultra-fast tracking with labels, we were capable to resolve the reversible protofilament peeling at the tip of microtubule in $3 \mathrm{D}$. The peeling proceeds the protofilament detachment from the microtubule shaft as far as hundreds of milliseconds. Furthermore, the reversible peeling was confirmed by label-free measurements. These findings bring a new perspective on the mechanism of microtubule disassembly.

\section{$\mathrm{P}-254$}

Ezrin binding as actin cortex modulator? A new quantitative approach to determine the ezrin actin binding affinity

Tim Heißenberg [1], Claudia Steinem [1,2]

[1] Institute of Organic and Biomolecular Chemistry, GeorgAugust-University Göttingen, Germany; [2] MPI for Dynamics and Self-Organization, Göttingen, Germany

Ezrin is part of the ERM protein family and works as an anchor for the actin cortex to the plasma membrane. Ezrin has a N-terminal domain called FERM domain, which contains a phosphatidylinositol- $(4,5)$ bisphosphate $\left(\mathrm{PIP}_{2}\right)$ binding motif that is needed to be recruited to the plasma membrane. The C-terminal domain of ezrin holds a filamentous actin binding domain. Therefore, when ezrin is activated within a cell, it binds to the plasma membrane via its $\mathrm{PIP}_{2}$ binding motif and connects it to the actin filaments which are part of the actin cortex.

This project will investigate the influence of ezrins with different binding affinities to actin on minimal actin cortices (MACs). Some ezrin mutations are known, that should decrease the actin binding affinity of ezrin. Those mutants were generated and their binding affinity to $\mathrm{PIP}_{2}$ and actin will be determined. For a sensitive and quantitative measurement of the ezrin actin binding affinity, a new method will be established with a reflectometric interference spectroscopy setup. After the binding affinity measurements, MACs will be created with the different ezrin mutants as linkers and analyzed. The analysis will be done with high-resolution fluorescent imaging for parameters like mesh size and node density and with video particle tracking for the viscoelastic parameters.

\section{P-256}

Post-translational modifications soften vimentin intermediate filaments

Julia Kraxner, Charlotta Lorenz, Sarah Köster

Institute for X-Ray Physics, University of Göttingen, Germany

The mechanical properties of cells greatly influence their function, such as the ability to move and they need to flexibly adapt, e.g. during cancer metastasis. These properties are determined by the cytoskeleton, a complex network consisting of 3 proteins, microtubules, actin filaments and intermediate filaments (IFs). A rather slow way to adapt cell mechanics to varying requirements is differential expression of the cytoskeleton proteins which affects the network architecture. Here, we focus on the IF vimentin and introduce post-translational modifications (PTMs), i.e. changes applied to specific amino acids in the protein. PTMs occur comparatively fast and thus provide a mechanism for mechanical modulation on short time scales. We study the impact of one PTM, phosphorylation, on IF mechanics by stretching single filaments using optical traps. Whereas full phosphorylation leads to disassembly of IFs, partial phosphorylation results in softening of IFs. By employing mutants that mimic phosphorylation as well as Monte Carlo simulations, we explain our observation through the additional charges introduced by phosphorylation. The results may provide a missing link between the role of phosphorylation of IFs in cancer metastasis and the increased motility of the metastasizing cells. 
Posters

- 17. Cytoskeleton / Motor proteins -

P-257

Evidence for Nuf2 and microtubule interaction from molecular dynamics simulations

Iuliia N Lopanskaia, Ekatherina G Kholina, Vladimir A Fedorov, Ilya B Kovalenko, Nikita B Gudimchuk

Lomonosov Moscow State University, Russian Federation

During cell division, it is necessary to distribute the duplicated genetic material between the daughter cells. The main participants of this process are tubulin microtubules (MTs) and kinetochore - a large multi-subunit complex, which enables attachment of MTs to chromosomes. NDC80 complex is a key component of the kinetochore, responsible for MT capture. It contains four proteins: Spc24, Spc25, Hec1 and Nuf2. The two latter proteins can directly bind to MTs, but the detailed understanding of their interaction has not yet been achieved. One of the puzzling aspects of it is the fact that in the cryo-EM structure of the MT and NDC80 complex, Nuf2 subunit appears not bound to the MT. However, mutations in the Nuf2 are known to weaken the affinity of the whole NDC80 complex to MTs. To shed light on this problem, we analyzed the interaction between NDC80 and MT using molecular dynamics. We have constructed a fullatomic model of a MT fragment and NDC80 complex with explicit solvent. Two possible localizations of NDC80 complex on the MT lattice (the intradimer and the interdimer positions) were examined. The results of our simulations reveal direct contacts between the Nuf2 subunit and tubulins. These interactions involve both tubulin core and the flexible C-termini. This provides an explanation for the influence of point mutations that are distant from the MT-binding interface. This study was supported by RSF grant \#21-74-20035.

\section{P-259}

Structure and mechanics of strained membranebound vimentin filaments

Sarmini Nageswaran [1], Sarah Köster [2], Claudia

Steinem $[1,3]$

[1] Institute for Biomolecular and Organic Chemistry, Germany; [2] Institute for X-ray Physics, Germany; [3] Max Planck Institute for Dynamics and Self-Organization, Germany

In eukaryotic cells, the shape and mechanical properties are determined by the cytoskeleton, which is composed of actin filaments, microtubules and intermediate filaments. Importantly, intermediate filaments are considered to be the main determinants of cell stiffness and strength. Thus, they are believed to dominate the mechanical response of cells at higher strains. Therefore, the organization of intermediate filaments at the plasma membrane and their influence on the mechanical properties of the cells under a variety of strains are of great interest.

Hence, we aim at the development of an in vitro model system to mimic the composition of the plasma membrane under strain.

Therefore, biotin-decorated lipid bilayer is spread on oxidized, elastic polydimethylsiloxane (PDMS) and biotinlabeled vimentin filaments are attached to it via neutravidin. We find that the strain speed is crucial in order to observe strain of the vimentin filaments, since the membrane-coupled system can only be stretched when stretched faster than the diffusion of the anchor lipids. Currently, we are able to stretch the membrane-bound vimentin filaments up to $30 \%$. This shows great promise in finally elucidating the question of the organization of vimentin networks as in cells.

\section{P-258}

Ciliary transport associated proteins utilize contrasting mechanisms to reach the ciliary base, assemble into trains and enter the cilia in C. elegans Aniruddha Mitra, Erwin J G Peterman

Departement of Physics and Astronomy, LaserLaB, Vrije Universiteit Amsterdam, Netherlands

Sensory neurons of $C$. elegans have signalling hubs called primary cilia, extending from dendritic endings, to sense and respond to the external environment. Cilia have a microtubulebased intraflagellar transport (IFT), which is essential for the growth and maintenance of the structure. The IFT proteins associated with cilia, have to be transported from the cell body to the cilia base, moving across the dendrites (several 10s of microns), where they assemble into IFT trains and cross the transition barrier to enter the cilia. By performing single-molecule fluorescence microscopy in the chemosensory neurons of $C$. elegans, we unravel that while motor proteins involved in IFT, kinesin-II, OSM-3 and IFT dynein, move diffusively from the cell soma to the cilia base, other proteins like CHE-11, OSM-6 (IFT train proteins) and OCR-2 (ciliary transmembrane protein) are transported in a directional manner, in small packets. Further, single-particle tracking of individual molecules at the cilia base directly illustrates that CHE-11, OSM-6 and IFT dynein pause at the base for a prolonged time, incorporating into IFT trains during the assembly process, before entering the cilia. In contrast, kinesin-II and OSM-3 are picked up by pre-packaged IFT trains, directly at the cilia base in case of kinesin-II and throughout the proximal segment of the cilia in case of OSM-3. Thus, using single-molecule live cell imaging in C. elegans, we illuminate how IFT proteins reach the cilia base, assemble into IFT trains and enter the cilia.

\section{P-260}

\section{Characterization of TTLL11 as a Tubulin Polyg-} lutamylase

Jana Nedvědová [2,1], Kseniya Ustinova [3], Cyril Bařinka [2] [1] Charles University, Czech Republic; [2] Institute of Biotechnology of the Czech Academy of Sciences, Czech Republic; [3] Centre of Genomic Regulation, Spain

Tubulin polyglutamylation is a common posttranslational modification (PTM) performed by the tubulin tyrosine ligase-like (TTLL) family of glutamylases. These PTMs endow microtubules with specialized functions as they influence microtubule stability and their interactions with microtubule associated proteins and molecular motors. Understanding the molecular mechanisms of TTLLs is thus essential to elucidate the tubulin code and its implications for microtubule functions. Our research is focused on biochemical, biophysical and structural characterization of TTLL11, one of the least studied members of TTLL glutamylases. We cloned, expressed and purified a number of TTLL11 variants and established in vitro biochemical assays to elucidate TTLL11 substrate preferences and structural determinants governing tubulin polyglutamylation. Furthermore, we used the total-internal reflection microscopy to directly visualize and quantify interactions between TTLL11 and microtubules to the single molecule level. Overall, our work will contribute to better understanding of physiological functions and functional consequences of TTLL11 activity in vitro as well as in the cellular context. 
Posters

- 17. Cytoskeleton / Motor proteins -

P-261

Pattern formation in the axon: self-assembly of actin rings

Helen Pringle, Rhoda J Hawkins

University of Sheffield, UK

Neuronal axons are crucial to the connectivity of the nervous system; however, they are vulnerable to damage through injury and neurodegenerative diseases which can disrupt the internal cell cytoskeleton, resulting in cell death or loss of function.

Recent advances in super-resolution microscopy have exposed a highly organised cytoskeletal structure within the axon, referred to as the membrane-associated periodic skeleton (MPS). This structure consists of rings of actin filaments, spaced periodically at approximately $190 \mathrm{~nm}$ intervals, and connected laterally by spectrin tetramers. The mechanism by which this structure forms, and the benefits derived from its organisation, are currently unknown.

We use in-silico models to investigate the factors driving selfassembly of the MPS structure in axons. Cytoskeletal filaments and their accessory proteins are modelled within a viscous, axon-like environment. The system is simulated using the Cytosim software, employing a combined approach of Langevin dynamics to simulate Brownian motion of filaments, along with a subset of stochastic protein-level events such as binding and polymerisation, which are evaluated at every timestep. We search parameter space and show that self-assembly of an MPS-like structure is possible under specific parameter values.

\section{P-263}

Multiscale mechanics and temporal evolution of vimentin intermediate filament networks

Anna V Schepers, Charlotta Lorenz, Peter Nietmann, Andreas Janshoff, Stefan Klumpp, Sarah Köster

University of Göttingen, Germany

The cytoskeleton consists of F-actin, microtublues and intermediate filaments (IFs), which together form a complex composite network. This composite network provides stability and flexibility for cells and enables them to adapt to a variety of external conditions. F-actin and microtubule networks have been studied extensively and a large number of different cross-linkers are known. By contrast, the interactions within reconstituted IF networks are less well understood. Microrheological measurements on vimentin networks reveal slow network dynamics, and allow us to separate the filament and network formation time scales. By amplifying electrostatic attractions or diminishing hydrophobic interactions between filaments, we are able to study the impact of the respective effect on intra- and inter-filament interactions. Combining optical trapping and fluorescence microscopy enables us to bring two single vimentin IFs in contact and directly study the interactions between them. These results, in combination with studies of the mechanical properties of single IFs, allow us to model the interactions with MonteCarlo simulations, thereby gaining a deeper understanding of cytoskeletal structures.

\section{P-262}

Jumping on the treadmill-how FtsA co-migrates with motile filaments of FtsZ and its implication for the precision of bacterial cell division Philipp Radler, Natalia Baranova, Mar López-Pelegrín, Martin Loose

Institute of Science and Technology Austria, Austria

Activation of constriction in E.coli is proposed to be regulated by FtsA, an actin-homolog widely conserved across bacteria. Previous studies suggest that FtsA activity states are determined by its oligomerization state. Switching to the active (=monomeric) state is thought to facilitate maturation of the cell division machinery and constriction of the septum. Extensive genetic studies have identified mutants of FtsA, which display reduced self-interaction and bypass otherwise essential cell division proteins. We reconstituted parts of the cell division machinery on supported lipid bilayers and compared behavior of FtsA WT and its hyperactive variant R286W. We confirmed that FtsA R286W shows decreased self-interaction and found that it exhibits significantly reduced membrane-bound lifetime. With increasing the complexity of the system, we found that the difference in self-interaction disappears in the presence of $\mathrm{FtsN}_{\text {cyto }}$ and FtsZ, while the faster exchange is preserved for FtsA R286W. We found that FtsA R286W follows the spatiotemporal dynamics of treadmilling FtsZ filaments more robustly, allowing more efficient recruitment of $\mathrm{FtsN}_{\text {cyto. }}$. We therefore propose that the active state of FtsA is characterized by a highly dynamic exchange of membrane-bound FtsA monomers. This rapid exchange allows the spatiotemporal signal of treadmilling FtsZ filaments to be tracked more precisely, enables more efficient downstream signal transduction, and ultimately initiates constriction.

\section{P-264}

Nonlinear characteristics of deep deformation of blood cell membranes

Viktoria Sergunova [1], Vladimir Inozemtsev [1], Elena Kozlova [1,2], Alexander Chernysh [1], Alexander Kozlov [2], Olga Gudkova [1], Alexander Onufrievich [3], Ekaterina Sherstyukova $[1,2]$

[1] Federal Research and Clinical Center of Intensive Care Medicine and Rehabilitology, V.A. Negovsky Research Institute of General Reanimatology, Moscow, Russia; [2] Sechenov First Moscow State Medical University (Sechenov University), Moscow, Russia; [3] Federal State Budgetary Institution "Main Military Clinical Hospital named after academician N.N. Burdenko" of the Ministry of defense of the Russian, Russia

Blood cells in the process of circulation bed undergo significant deformations that can cause deep membrane bending. In in vitro model experiments, the influence of various physicochemical factors on the mechanical characteristics of cell membranes under their deep deformation to $\mathrm{h}=1500 \mathrm{~nm}$ was studied. At the initial stage, the deformation obeyed a linear law and was described by the Hertz model $\mathrm{F}=\mathrm{ahb}$, where $\mathrm{b}=1.5$, Young's modulus $\mathrm{E}(\mathrm{h})=$ const. With increasing probe indentation $\mathrm{E}$ of RBC and neutrophil cells membranes began to nonlinearly depend on the depth of probe indentation $\mathrm{h}$, and $\mathrm{b} \neq 1.5, \mathrm{E}(\mathrm{h}) \neq$ const. The value of the membrane bending $\mathrm{hHz}$, at which nonlinear deformation occurs at $\mathrm{h}>\mathrm{hHz}, \mathrm{E}=\mathrm{F}(\mathrm{h})$, has been established. It was shown that the values of $\mathrm{E}$ and hHzin RBCs are determined, first of all, by the state of the cytoskeleton. 
Posters

- 18. Membrane architecture and asymmetry -

P-265

Structural study of plasmalogen-based liquid crystalline self assemblies involving docosapentaenoyl derivatives related to biological cubic membranes

Angelina Angelova [1], Borislav Angelov [2], Markus Drechsler [3], Thomas Bizien [4], Yulia E Gorshkova [5], Yuru Deng [6]

[1] Institut Galien Paris-Saclay UMR8612, Université ParisSaclay, CNRS, Châtenay-Malabry, France; [2] Institute of Physics, ELI-Beamlines, Academy of Sciences of the Czech Republic, Prague, Czech Republic; [3] Keylab "Electron and Optical Microscopy", Bavarian Polymer Institute, University of Bayreuth, Bayreuth, Germany; [4] Synchrotron SOLEIL, L'Orme des Merisiers, Saint-Aubin, France; [5] Frank Laboratory of Neutron Physics, Joint Institute for Nuclear Research, Dubna, Russian Federation; [6] Wenzhou Institute, University of Chinese Academy of Sciences, Wenzhou, China

Nanoassemblies based on plasmenyl-glycerophospholipids (plasmalogens) involving long polyunsaturated fatty acid (PUFA) chains have been scarcely studied although such lipids are of crucial importance for the organization and functions of the cellular membranes. We investigate the selfassembly of custom designed docosapentaenoyl (DPA) plasmenyl (ether) and ester phospholipids in aqueous environment ( $\mathrm{pH} 7$ ) by synchrotron small-angle X-ray scattering and cryogenic transmission electron microscopy. The obtained results reveal coexisting liquid crystalline cubic, hexagonal and lamellar phases depending on the lipid composition. The stability of the plasmalogens to radiation damage was determined for applications in drug delivery systems.

\section{P-267}

Effect of serotonin on membrane domain formation

Astrid F Brandner [1], Anna Bochicchio [1], Daniel Huster [2], Rainer A Böckmann [1,3]

[1] Computational Biology, Department Biology, FriedrichAlexander University Erlangen-Nürnberg, Erlangen, Germany; [2] Institute for Medical Physics and Biophysics, University of Leipzig, Leipzig, Germany; [3] Erlangen National High Performance Computing Center (NHR@FAU), Germany

Defined regions or domains in biomembranes with specific structural and dynamical properties were shown to be associated with receptor localisation and signalling. Despite their importance e.g. in immune signaling, only little is known about the composition and dynamics of membrane domains due to the methodological challenges in identifying highly dynamic domains on the nanometer scale.

Here, by means of atomistic molecular dynamic simulations, we characterized the unbiased spontaneous formation of nanodomains in a plasma membrane model containing phosphatidylcholine, palmitoyl-sphingomyelin and cholesterol at different temperatures in presence and in absence of the neurotransmitter serotonin. We show that serotonin binding to the membrane decreases the phase transition temperature. It affects domain composition and domain ordering as well as results in a decreased membrane elasticity. Our results suggest a novel mode of action of neurotransmitters in neuronal signal transmission.

\section{P-266}

Acyl-chain saturation regulates the order of phosphatidylinositol 4,5-bisphosphate nanodomains

Luís Borges-Araújo [1,3], Marco M Domingues [2], Alexander Fedorov[1], Nuno C Santos [2], Manuel N Melo [3], F Fernandes [1]

[1] iBB-Institute for Bioengineering and Biosciences, Instituto Superior Técnico, Universidade de Lisboa, Lisbon, Portugal; [2] Instituto de Medicina Molecular, Faculdade de Medicina, Universidade de Lisboa, Lisbon, Portugal; [3] Instituto de Tecnologia Química e Biológica António Xavier, Universidade Nova de Lisboa, Oeiras, Portugal

$\mathrm{PI}(4,5) \mathrm{P}_{2}$ plays a critical role in the regulation of various plasma membrane processes and signaling pathways in eukaryotes. A significant amount of cellular resources are spent on maintaining the dominant 1-stearoyl-2-arachidonyl $\mathrm{PI}(4,5) \mathrm{P}_{2}$ acyl-chain composition, while less abundant and more saturated species become more prevalent in response to stimuli, stress or aging. Here, we report the impact of acyl-chain structure on the biophysical properties of cationinduced $\mathrm{PI}(4,5) \mathrm{P}_{2}$ nanodomains. $\mathrm{PI}(4,5) \mathrm{P}_{2}$ species with increasing levels of acyl-chain saturation cluster in progressively more ordered nanodomains, culminating in the formation of gel-like nanodomains for fully saturated species. This is the first report of the impact of $\mathrm{PI}(4,5) \mathrm{P}_{2}$ acyl-chain composition on cation-dependent nanodomain ordering, and provides important clues to the motives behind the enrichment of $\mathrm{PI}(4,5) \mathrm{P}_{2}$ with polyunsaturated acyl-chains as the presence of highly ordered $\mathrm{PI}(4,5) \mathrm{P} 2$ domains could lead to dysregulation and disease.

\section{P-268}

How the fluid behavior of the ultralong lipid Cer[EOS] modifies the stratum corneum

Ferdinand Fandrei [1], Oskar Engberg [1], Albert Smith [1], Lukáš Opálka [2], Andrej Kováčik [2], Kateřina Vávrová [2], Daniel Huster [1]

[1] Institute for Medical Physics and Biophysics, University of Leipzig, Germany; [2] Skin Barrier Research Group, Charles University, Faculty of Pharmacy, Czech Republic

The stratum corneum (SC) as the outermost layer of the human skin prevents the body from exogenous substances. In the current model of the lipid phase of the SC, Cer[EOS] is considered essential for building the Long Periodicity Phase(LPP) and the LPP being regarded important for the barrier function of the SC. We investigated the structure and dynamics of Cer[EOS] as well as its impact on the organization and structure of the other lipids in a LPP model mixture. Model membranes were investigated by ${ }^{2} \mathrm{H}$ solidstate NMR spectroscopy at temperatures ranging from $25^{\circ} \mathrm{C}$ to $80^{\circ} \mathrm{C}$. The different components were specifically deuterated and measured in separate mixtures each containing one deuterated component. From this data, the molecular order of each deuterated component and the phase composition of the SC model could be determined quantitatively. The results illustrate the highly disordered behavior of the linoleic acid of the Cer[EOS] molecule, while in contrast most of the other lipids are in a highly ordered forming an orthorhombic (solid) state at physiological skin temperature. Our findings highlight the unique role of Cer[EOS] as a modulator for the barrier properties of the SC and complement our understanding of the biophysical properties of the LPP. 


\section{Posters}

- 18. Membrane architecture and asymmetry -

P-269

Interdigitation-induced interleaflet coupling in asymmetric liposomes

Moritz P K Frewein [1,2], Frederick A Heberle [4], Milka Doktorova [5], Enrico F Semeraro [1,3], Haden L Scott [4], Lionel Porcar [2], Georg Pabst [1,3]

[1] University of Graz, Institute of Molecular Biosciences, Graz, Austria; [2] Institut Laue-Langevin, Grenoble, France; [3] BioTechMed Graz, Austria; [4] The University of Tennessee, Knoxville, USA; [5] The University of Texas Health Science Center at Houston, Houston, USA

Cellular envelopes contain a large number of lipid species that are distributed asymmetrically between the two leaflets of the bilayer. One of the enduring questions of plasma membrane architecture and lipid asymmetry concerns the possibility of interleaflet coupling even in the absence of proteins, which may influence a number of physiological processes. Currently conceived lipid-mediated coupling mechanisms consider either intrinsic lipid curvature, headgroup electrostatics, cholesterol flip-flop, dynamic chain interdigitation, or thermal membrane fluctuations. We use asymmetric large unilamellar lipid vesicles (aLUVs), produced via cyclodextrin-mediated lipid exchange, to study the effect of interdigitation stress on membrane structure and dynamics, characterized by small-angle neutron and X-ray scattering as well as neutron spin-echo. We present data on vesicle systems containing mixed-chain phosphatidylcholine or sphingomyelin with high chainlength-mismatch, and the effect of the packing density in their opposing leaflet, demonstrated by saturated and mono-unsaturated phosphatidylcholine.

\section{P-271}

On the applicability of the Helfrich energy functional to highly curved lipid membranes

Timur R Galimzyanov [1], Pavel V Bashkirov [2], Sergey A Akimov [1]

[1] A.N. Frumkin Institute of Physical Chemistry and Electrochemistry, Moscow, Russian Federation; [2] Federal Research and Clinical Center of Physical-Chemical Medicine, 1a Malaya Pirogovskaya, Russian Federation

The theory of elasticity of lipid membranes is an important tool used for the theoretical description of processes of cell membrane remodelling. The classic Helfrich functional of elastic energy of lipid membrane is quadratic on the deformations and derived under the assumption of small deformations. Recently, molecular dynamics researches challenged the validity of the linear model, questioning the constancy of the bending modulus at high curvatures. Analyzing both cylindrical and parabolic deformations realized in molecular dynamic simulations, we have demonstrated the applicability of the quadratic Helfrich elastic model, if it is used separately to obtain the energy of each membrane leaflet. We confirmed theoretical results experimentally, additionally proving that the elastic energy of two lipid monolayers is additive. Furthermore, we found that membrane bending stiffness can be decreased twofold by the addition of a low molecular weight solvent to the lipid mixture. We attribute this effect to incorporation of solvent molecules in between the lipids of the inner monolayer, to relax the curvature stress. The study supported by RSCF grant No.19-74-00152.

\section{P-270}

Development of asymmetric liposomes to mimic plant plasma membrane

Aurélien Furlan, Guillaume Gilliard, Magali Deleu

Laboratory of Molecular Biophysics at Interfaces, Terra Research Center, Gembloux Agro-bio Tech, University of Liege, Belgium

Although liposomes can be criticized in terms of relevance to mimic real biological membranes, they give access to information at molecular or atomistic levels that are difficult to study in cellulo (compounds penetration/location into membrane, specific interaction with particular lipids, etc.). In biophysical studies on liposomes, the asymmetric nature of the lipid distribution is often overlooked despite its role in several biological mechanisms. However, in the last decades, several protocols have emerged to obtain asymmetric liposomes mimicking mainly human plasma membrane. In this work, two different approaches have been investigated in order to form asymmetric liposomes that mimic plant plasma membranes. The first one is based on the well-known cyclodextrin mediated lipid exchange strategy to produce large unilamellar vesicles (LUVs) with a well-defined size distribution and is developed with phosphatidylserine and phosphatidylcholine. The second approach uses the hemifusion to obtain asymmetric giants unilamellar vesicles (GUVs) containing phosphatidylcholine, glucosylceramide and sitosterol. The vesicles obtained with the two approaches have been characterised in terms of asymmetry, size uniformity and stability.

\section{P-272}

The effect of ERG6 gene deletion on membrane fluidity in Candida glabrata

Juraj Jacko [1], Marcela Morvová Jr. [1], Daniel Eliaš [2], Nora Toth-Hervay [2], Yvetta Gbelská [2], Libuša Šikurová [1] [1] Faculty of mathematics, physics and informatics, Comenius University in Bratislava, Mlynská dolina F1, 842 48, Bratislava, Slovakia; [2] Faculty of natural science, Comenius University in Bratislava, Ilkovičova 6, 842 15, Bratislava, Slovakia

The ERG6 gene deletion may cause the modification of the plasma membrane architecture, mainly the plasma membrane sterol composition, which also affect the membrane barrier function. It was reported that the ERG6 gene deletion change yeast susceptibility to azole antifungals. Sensitivity to azole antifungal drugs suggests the effectivity rate at which drug induces yeast cell damage or death. This is important because the increase resistance of yeast to antifungal drugs represents medical problem in the treatment of yeast infections. The aim of our work was to monitor the effect of ERG6 gene deletion on lipid bilayers arrangement of the cytoplasmic membrane between two groups of the Candida glabrata (wild-type and $\triangle E R G 6$ mutant). We used fluorescence polarization spectrometry, for measuring the fluorescence anisotropy of the DPH and TMA-DPH fluorescent probes. DPH probe registers the hydrophobic part and TMA-DPH probe describes the hydrophilic part of cytoplasmatic membrane. We observed a significant decrease in the fluorescence anisotropy values of the DPH probe and a decrease in the fluorescence anisotropy values of the TMA-DPH probe between the examined yeast groups of $C$. glabrata. 
Posters

- 18. Membrane architecture and asymmetry -

P-273

Membrane mimetic bolaamphiphiles and the role of the nature of lipid chains

Jelena Jeftic [1], Matthieu Berchel [2], Cristelle Mériadec [3], Loïc Lemiègre [1], Franck Artzner [3], Thierry Benvegnu [1]

[1] ENSCR UMR CNRS 6226, France; [2] Univ. de Bretagne Occidentale, France; [3] IPR Université de Rennes 1, France

Natural bolaamphiphiles, called Archaeolipids, are the constituents of membranes in Archaebacteria, extremophile organisms that can survive in harsh environments. Extreme conditions (for humans) can be found in Arctic and Antarctic, in geysers and volcanoes, in areas of high salinity or acidity, as well as in regions of high pressure. Archaeolipids can be isolated from natural resources, usually in form of mixtures of lipids. We undertook the synthesis of a series of bolaamphiphiles in our laboratory in order to investigate the role of the nature of the hydrocarbon chain on the formation of supramolecular structures mimicking membranes. The bolaamphiphile molecules synthesized have two polar heads of natural origin: one based on sugar moiety and the other based on glycine betaine. The main chain length between two polar heads has been varied for 12,22 and 32 methylene units. Another variation was to introduce a diacetylenic unit into the main chain in order to investigate its influence on the formation of supramolecular structures via the $\pi-\pi$ stacking. Using the small and wide-angle X-ray diffraction, we could elucidate the thickness of the formed membrane layer, as well as the type of the lamellar structure formed by one type of bolaamphiphiles. The determined structures are lamellar fluid, $\mathrm{L}_{\alpha}$, lamellar crystalline, $\mathrm{L}_{\mathrm{c}}$, lamellar tilted, $\mathrm{L}_{\beta}$ ' and lamellar isotropic, L. We also observed an important stability of lamellar structures upon thermal cycling up to $70{ }^{\circ} \mathrm{C}$.

\section{P-275}

Evidence for non-addititve intrinsic lipid curvature mixing of sphingolipids from X-ray experiments on inverted hexagonal phases

Michael Kaltenegger [1], Johannes Kremser [1], Moritz P K Frewein [1], Primoz Ziherl [2,3], Douwe J Bonthuis [4], Georg Pabst [1]

[1] University of Graz, Institute of Molecular Biosciences, Biophysics Division, Austria; [2] Faculty of Mathematics and Physics, University of Ljubljana, Slovenia; [3] Jožef Stefan Institute, Slovenia; [4] Graz University of Technology, Institute of Theoretical and Computational Physics, Austria

We report a X-ray data analysis method to determine the intrinsic curvatures of lipids hosted in inverted hexagonal phases of dioleoyl phosphatidylethanolamine . In particular, we combined compositional modelling with molecular shapebased arguments to account for non-linear mixing effects of guest-in-host lipids on intrinsic monolayer curvature. Applying the technique to palmitoylsphingomyelin we find positive lipid curvatures in the studied temperature range of $25^{\circ} \mathrm{C}$ to $50^{\circ} \mathrm{C}$. Ceramides, with chain lengths varying between $\mathrm{C} 2: 0$ and $\mathrm{C} 24: 0$, in turn displayed significant negative lipid curvature values. Moreover, we report non-linear and non-additive mixing for $\mathrm{C} 2: 0$ ceramide, leading to a pronounced shift of the ceramide's intrinsic curvature toward positive values with lipid concentration, which most likely due to ubiquitous $\mathrm{H}$-bond formation. Non-additive mixing was also observed for palmitoylsphingomyelin, although much less pronounced and toward negative intrinsic curvatures. The biophysics of lipid contribution to membrane curvatures thus appears to be more complex than previously considered.

\section{P-274}

Understanding vesicle budding as asymmetry stress relief induced by the asymmetric membrane insertion of lysolipids

Michael Kaiser [1], Lisa Hua [1], Ndjali Quarta [2], Anette Meister [3], Heiko Heerklotz [1,4]

[1] Institute of Pharmaceutical Sciences, University of Freiburg, Germany; [2] Institute of Pharmaceutical and Biomedical Sciences, University of Mainz, Germany; [3] Institute of Biochemistry and Biotechnology, MartinLuther-University Halle-Wittenberg, Germany; [4] Leslie Dan Faculty of Pharmacy, University of Toronto, Canada

Understanding the interactions of detergents with lipidbilayers is the basis for many physiological processes such as endo- and exocytosis. Although the three-stage-model is well known for describing solubilization of lipid bilayers by surfactants, it can only be applied to detergents with fast flip-flop-rates. If such an equilibration of surfactant does not happen or at least not in the observed time frame, asymmetry stress is induced by the excess surface area on the outer leaflet. This stress stops further insertion of lysolipids into the bilayer causing an equilibrated system of detergent micelles and liposomes over days ("staying out"). To relief the stress different mechanisms have been described - here we focus on the exovesiculation of small vesicles ("budding off").

Here we present lysolipids causing such stress on POPC liposomes. Using Asymmetric Flow-Field-Flow Fractionation (AF4) the extent of budded vesicles was quantified and correlated to osmotic pressure gradients. Further experiments were executed assessing the properties of the generated buds.

\section{P-276}

Free energy simulations of pore formation Gari Kasparyan, Chetan Poojari, Jochen S Hub Saarland University, Germany

Pore formation over lipid membranes plays a key role in processes such as membrane fusion and fission, the killing of bacterial cells with antimicrobial peptides, and others. Evidences of metastable pores were provided by experiments already four decades ago. Although pores are heavily studied with a variety of methods, the free energy landscape of the initial stages of the pore formation is still not fully understood. We use molecular dynamics simulations to study the mechanisms and energetics of pore formation. We overcome the challenge of exploring the free energy landscape using umbrella sampling along a recently developed reaction coordinate. Here, we present results from the effects (i) of electric fields on the free energies of pore formation, as applied during electroporation to allow cellular uptake of drugs or genes, and (ii) of the common small antifungal drug itraconazole and the solvent DMSO. We find that itraconazole and DMSO act in cooperation by two distinct mechanisms to strongly stabilize the open pore state. The potentials of mean force (PMFs) show that electric fields greatly stabilize open pores and lower the barrier for pore formation. 


\section{Posters}

- 18. Membrane architecture and asymmetry -

P-277

The role of charges on membrane stability upon electroporation

Fernanda S Leomil [1,2], Rafael B Lira [2,3], Marcelo Zoccoler [4], Karin A Riske[1], Rumiana Dimova [2]

[1] Biophysics Dep., UNIFESP, Brazil; [2] Max Planck I. of Colloids and Interfaces, Germany; [3] Moleculaire Biofysica, Zernike, Netherlands; [4] Biophysics Dep., USP, Brazil

Membrane stability is vital for cell survival. When subjected to strong stimuli, such as electric pulses, membrane pores open (electroporation). In model membrane systems, such as giant unilamellar vesicles (GUVs, tens of microns in size) the pores can be directly observed under the microscope. In vesicles made of neutral lipids (phosphatidylcholine, PC), pores reseal and membrane integrity is restored. However, in charged membranes, the vesicles are destabilized after electroporation: long-living submicroscopic pores are generated or the GUVs collapse completely, restructuring into membrane nanotubes. In this work, we used neutral PC GUVs containing increasing fractions of the anionic lipid phosphatidylglycerol (PG) to show that the origin of these phenomena is related to the membrane pore edge tension $(\gamma)$, which governs pore closure, and is significantly decreased in membranes containing $50 \mathrm{~mol} \%$ of PG. The pore edge tension was determined from the pore closure dynamics using a software that automatically performs the image processing steps, discloses pore dynamics and calculates the pore edge tension. We also observed that destabilization propensity is enhanced for membranes made of lipids with higher degree of unsaturation and that it can be reversed by membrane charge screening by calcium ions.

\section{P-279}

Aggregation of flexible membrane-bound proteins: thermodynamic and kinetic insights from large-scale simulations

Mohsen Sadeghi, Frank Noe

AI4Science group, Department of Mathematics and Computer Science, Freie Universität Berlin, Germany

Membrane-binding peripheral proteins are, among other tasks, responsible for shaping and remodeling biomembranes. The necessary cooperative action of a multitude of curvature-inducing proteins is facilitated by indirect membrane-mediated interactions. Despite the large body of biophysical investigation of these interactions, quantitative analysis of the dynamics of a collection of membrane-bound proteins in a consistent model that results in fully describing the membrane remodeling process is still lacking. Here, we present our coarse-grained dynamic membrane model supporting varying concentrations of flexible membrane-bound proteins. We parameterize this model based on local curvature, flexibility, and the in-plane dynamics of proteins. We present quantitative results on entropic membrane-mediated interactions between peripheral proteins and investigate the kinetics, stationary distributions, and the free energy landscape governing the formation and break-up of protein clusters. We demonstrate how the flexibility of the proteins plays a significant role in highly selective macroscopic aggregation behavior. Finally, using steered simulations, we investigate the free energy landscape of the full pathway from formation of surface clusters to large-scale membrane remodeling, and investigate the current hypotheses regarding this process in unprecedented details.

\section{P-278}

Pure protein bilayers made from fungal hydrophobins: characteristics and chances for usage Friederike Nolle [1], Alessandra Griffo [1], Kirstin Kochems [1], Michael Lienemann [2], Päivi Laaksonen [3], Ralf Seemann [1], Jean-Baptiste Fleury [1], Karin Jacobs [1], Hendrik Hähl [1] [1] Department of Experimental Physics and Center of Biophysics, Saarland University, Germany; [2] VTT Technical Research Centre of Finland Ltd., Finland; [3] HAMK Tech, Häme University of Applied Sciences, Finland

Hydrophobins are a family of surface-active proteins known for their small size and strong amphiphilicity. HFBI is a protein from this family produced by Trichoderma reese $i$ and is also associated with the formation of honeycomb-like monolayers at the water interfaces with very high lateral cohesion. When two of these monolayers are contacted with either their hydrophobic or hydrophilic side, bilayers can be formed. Compared to usual lipid bilayers, the hydrophobin bilayers feature a similar thickness but can withstand much higher lateral tension and osmotic pressure. Another fundamental property of biological membranes is their water permeability. The water permeability of HFBI membranes can be derived by contacting two water droplets of different salt concentrations covered with HFBI monolayers and observing the change of volume (DIB). Our experiments showed that HFBI bilayers exhibit a very low water permeability, which is about two or more orders of magnitude lower than for common lipid bilavers. Moreover, we already succeeded by incorporating small ion channels, such as gramicidin A or hemolysin, into our protein bilayers. Thereby, they retain their functionality as in the lipid environment and can adjust the permeability for different molecules through HFBI bilayers.

\section{P-280}

Molecular simulations of pure-protein bilayer Leonhard J Starke, Tobias Fischbach, Jochen S Hub Saarland University, Germany

Bilayer arrangements based upon the monolayer honeycomb structure are found to be unstable and therefore can not explain the experimentally observed behaviour. We can also exclude that a thin layer of oil could seal the honeycomb structure. In order to obtain a tight packing of the hydrophobins we conduct coarse grained simulations at high temperatures and lateral pressures followed by backmapping to atomistic resolution. Again, the resultant bilayers show water leakage, thus demonstrating that well defined interfacial contacts between the proteins are needed to stabilize the bilayer and a simple description as a $2 \mathrm{D}$ fluid is insufficient to explain the low permeability.

[1] Lindner,M.B. Curr. Opinion in Colloid \& Interface Science 14,356-363 (2009)

[2] Hähl et.al. Advanced Materials, 29, 1602888 (2017) 
Posters

- 18. Membrane architecture and asymmetry -

P-281

Divalent metals affect the fluidity of biomimetic model and isolated biological membranes

Kevin Sule [1], Wing-Kee Lee [2], Elmar Prenner [1]

[1] University of Calgary, Canada; [2] Witten/Herdecke University, Germany

Metals are widely used in daily anthropogenic activities and play key roles in a multitude of technical applications. The objective of this study is to investigate the interactions of both essential and non-essential metals, manganese (Mn), nickel (Ni), and cadmium (Cd), using biomimetic lipid membranes and isolated biological membranes through fluorescence spectroscopy. Previous studies have recognized lipids as targets of these divalent metals, leading to altered lipidlipid interactions. Biomimetic lipids membranes were composed of liposomes containing anionic phospholipids with a defined acyl chain composition and liposome size. In contrast, Isolated biological membranes were obtained from human proximal tubule cells (hPCT) of the kidneys, which was a suitable model organism due to its role in metal reabsorption in the body and as a site of chronic metal toxicity. Additionally, Metal speciation assessment indicated that all 3 metals formed cationic species under physiological conditions. The effects of $\mathrm{Cd}$ and $\mathrm{Ni}$ were tested on the plasma and lysosomal membranes of hPCT cells. $\mathrm{Cd}$ and $\mathrm{Ni}$ were able to rigidify the plasma membrane, whereas an increase in fluidity was observed on lysosomal membranes. On the other hand, the effects of Mn were tested on biomimetic lipid systems and negatively charged phospholipids were identified as prime targets. Overall, this work provides relevant insight into the interaction of divalent metals with model and biological membranes and their negative consequences associated with disrupting membrane properties.

\section{P-283}

Miscibility of long n-alkanes with phosphatidylcholine bilayers

Anika Wurl [1], Maria Ott [2], Alfred Blume [3], Tiago M Ferreira [1]

[1] Inst. f. Physik - NMR, ; [2] Inst. f. Biochemie und Biotechnologie, ; [3] Inst. f. Chemie - Physikalische Chemie, Martin-Luther Univ. Halle-Wittenberg, Germany

Understanding the fate of hydrophobic and amphiphilic polymers in cells is important due to their present wide range of applications and environmental concerns. Surprisingly, little is known about the interaction of long hydrophobic chains in lipid bilayers. In this work we investigate the mixing behaviour of long n-alkanes in phosphatidylcholine (PC) bilayers. Due to their simplicity, n-alkanes are a convenient starting point for trying to understand the interaction of lipid membranes with longer purely hydrophobic chains.

We combine solid-state NMR techniques with DSC, X-ray scattering and MD simulations to systematically study the molecular structure and dynamics of PC lipids and n-alkanes in mixtures of different compositions. The miscibility and morphology of the systems is strongly dependent on the degree of hydration. At reduced hydration, incorporation of n-eicosane (C20) and n-triacontane (C30) into DPPC or DMPC bilayers is possible up to alkane volume fractions of $5-10 \%$, depending on the respective chain lengths, and alkane crystallization is prevented. At higher hydration levels, n-alkane addition results in partially isotropic 31P NMR spectra, indicating the formation of smaller structures. The combination of experiments and simulations used enables to describe the systems with unprecedented atomistic detail.

\section{P-282}

Characterization of giant plasma membrane vesicles: Towards a native-like in vitro system Nikolas Teiwes [1], Phila Baumann [1], Lena Strieker [1], Tabea Oswald [1], Claudia Steinem [1,2]

[1] Institute for Organic and Biomolecular Chemistry, University of Göttingen, Germany; [2] Max Planck Institute for Dynamics and Self-Organization, Göttingen, Germany

Since decades, artificial membranes such as supported bilayers, have been applied to investigate the organization and function of individual components of biological membranes. However, if a high compositional complexity is required or the membrane components of interest are not easily reconstituted, supported membranes derived from spreading synthetic vesicles fail to mimic the natural situation. An alternative are giant plasma membrane vesicles (GPMVs) derived from the plasma membrane of living cells. Here, we show that GPMVs can be derived from HEK-293 cells by different vesiculation agents. We analyze the phase-behavior of the GPMVs. We furthermore show that these GPMVs can be spread onto solid and porous support to produce porespanning plasma membranes. 


\section{Posters}

- 19. Cell and tissue biophysics -

P-284

The influence of strain on epithelial cell monolayers

Jonathan Bodenschatz, Karim Ajmail, Andreas Janshoff Institute of Physical Chemistry, Georg-August-Universität Göttingen, Germany

The epithelium is ubiquitous and essential in mammalian biology. Epithelial cells cover internal and external body surfaces. They perform critical roles in the body. Even though these cells must withstand large strains without failure, not much is known about how static and dynamical mechanical strain influence epithelial tissues and how the strains influence the mechanical properties of the cells and cell-cell contacts. To answer this question, we have established a polydimethylsiloxane (PDMS) based device and use this novel method to stretch confluent cell monolayers. The mechanical properties of the strained cells are directly measured via indentation with an atomic force microscope (AFM). While changes in cell-cell and cell-substrate contacts, as well as cytoskeletal reorganization are investigated with immunofluorescence and confocal microscopy. This combination of cell stretcher and AFM will provide new insights into the response epithelial cells under strain.

\section{P-286}

3D bioprinting of model tissues that mimic the tumor microenvironment

Florina Bojin [1,2], Andreea Robu [3], Maria I Bejenariu [3], Valentin Ordodi [2], Emilian Olteanu [1], Ada Cean [2], Roxana Popescu [1], Monica Neagu [1], Oana Gavriliuc [1,2], Adrian Neagu [1,4], Stelian Arjoca [1], Virgil Paunescu [1,2] [1] Victor Babes University of Medicine and Pharmacy Timisoara, Romania; [2] OncoGen Institute, Romania; [3] "Politehnica" University of Timisoara, Romania; [4] University of Missouri, USA

The tumor microenvironment (TME) affects cancer progression. Thus, development of TME models is needed for fundamental research and anticancer drug screening. Here, we report the biofabrication of $3 \mathrm{D}$ printed avascular models that mimic certain features of the TME. The tumor consists of a hydrogel droplet loaded with breast cancer cells $\left(10^{6}\right.$ cells $\left./ \mathrm{mL}\right)$ which is encapsulated in the same type of hydrogel containing similar concentrations of primary cells (tumor associated fibroblasts isolated from the peritumoral environment and peripheral blood mononuclear cells). Hoechst staining of cryosectioned tissue constructs showed that cells remodelled the hydrogel and remained viable for weeks. Histological sections revealed heterotypic aggregates of malignant and peritumoral cells; moreover, the constituent cells proliferated in vitro. To better understand the experimentally observed cellular rearrangements, we built lattice models of the constructs and simulated their evolution using Metropolis Monte Carlo methods. Although unable to replicate the complexity of the TME, this approach enables the self-assembly and co-culture of several cell types of the TME.

\section{P-285}

Quantitative acoustophoresis: a contact-free assay for the mechanical characterization of bioparticles

Vadim Bogatyr, Andreas Biebricher, Gijs Wuite

Vrije Universiteit Amsterdam, Netherlands

The mechanical properties of the cell are dictated by the cytoskeleton, which consists of an interlinked network of filaments. The cytoskeleton not only determines the cell shape but also drives vital processes such as cell motility and division. Successful reconstitution of the cytoskeleton inside a liposome is thus a crucial step for creating a viable artificial cell. It would therefore be desirable to have a robust and fast technique at one's disposal to characterize the biomechanics of the cytoskeleton both in artificial and living cells. To this end, we demonstrate here a novel acoustophoresis assay as a rather simple but reliable and precise method to quantify the biomechanics of single bioparticles.

In acoustophoresis, a standing acoustic wave is generated inside a flow channel, and the velocities of bioparticles are measured as they move towards the resulting field node. These velocities depend on the acoustic contrast factor, a function of both samples' density and compressibility. Since our approach allows us to measure the density independently by different means, we are thus not only able to measure particle's compressibility but also follow its change over time.

Here, we first showcase the method capabilities using polymer microbeads in proof-of-concept experiments. We next apply our assay to successfully characterize the mechanics of artificial liposomes with cytoskeleton-mimicking structures as well as cells.

\section{P-287}

Design, conception and microfluidic flow of a vesicle prototissue

Laura Casas-Ferrer, Gladys Massiera, Laura Casanellas Laboratoire Charles Coulomb, Université de Montpellier, France

Bottom-up synthetic biology and biomimetic approaches are promising tools that allow reconstitution of biological systems with reduced degrees of complexity. The aim of this work is to build an artificial biomimetic tissue with tunable properties through the controlled assembly of Giant Unilamellar Vesicles by using the Streptavidin-Biotin pair or DNA complementary strands. By combining the ligandto-receptor ratio and the vesicle volume fraction we are able to obtain vesicle aggregates of known size and degree of internal adhesion. The latter can also be controlled by changing the type of ligand. The morphology of such systems can be changed by applying different incubation techniques: we can obtain sheet-like structures (2D) or spheroidal (3D) aggregates ). We believe that frequency of vesicle-vesicle collisions, diffusion of free ligands in solution and lateral diffusion of bound ligands are the main mechanisms that govern the kinetics of vesicle assembly.

The rheological behavior of this vesicle aggregates will be studied in microfluidic confinement, and the ability to tune its physical features will allow us to selectively probe specific mechanisms involved in their microfluidic flow. The study of this model system will help deepen the knowledge in tissue flow, which takes place in relevant biological processes like embryogenesis, metastasis, and would healing. 
Posters

- 19. Cell and tissue biophysics -

\section{P-288}

Bioelectrical coupling of single-cell states in multicellular systems

Javier Cervera [1], Jose A Manzanares [1], Michael Levin [2], Salvador Mafe [1]

[1] Department of Thermodynamics. University of Valencia, Spain; [2] Department of Biology and Allen Discovery Center. Tufts University, USA

The transmembrane potential is the electrical potential difference between the cell inside and the external environment. Although it is not a transcription factor itself, it can indirectly influence transcription via second-messengers such as calcium, serotonin, cAMP, and other signaling molecules that regulate downstream processes. Multicellular electric transmembrane potentials can act as distributed controllers encoding instructive spatial patterns in development and regeneration. Single-cell states are modulated at the multicellular level because of the coupling between neighboring cells, thus allowing memories and multicellular patterns. Our approach to bioelectricity is based on: (i) two generic voltagegated ion channels that promote the polarized and depolarized cell states; (ii) a feedback mechanism for the transcriptional and bioelectrical regulations; and (iii) voltage-gated intercellular conductances that allow a dynamic intercellular connectivity. The simulations conducted with this model provide new physical insights that complement biochemical approaches. The steady-state and oscillatory multicellular states obtained help explaining aspects of development and guide experimental procedures attempting to establish instructive bioelectrical patterns based on electric potentials and currents to regulate cell behavior and morphogenesis.

\section{P-290}

Acinetobacter Baumannii: a bacterial membrane study

Elisa Fardelli, Shadi Bashiri, Michael Di Gioacchino, Luca Persichetti, Massimiliano Lucidi, Armida Sodo, Paolo Visca, Giovanni Capellini

Roma Tre, Italy

Acinetobacter baumanni is an opportunistic nosocomial multi-drug resistant bacterium, a threat for human life, more than ever in pandemic times. Indeed, it proliferates in intense care units where it easily allies with Sars Cov 2 and the resulting co-infection is challenging to eradicate and deadly for the majority of cases. A. baumannii success relies on its extraordinary resistance towards dehydration, which enables it to easily spread through surfaces and infest life support machines. In detail, A. baumannii eludes the usual death-path followed by unicellular organisms when facing dehydration: the cell membrane transits from an healthy crystal-liquid phase to a stiffer gel phase, deteriorating the structure and ion transport ability of the membrane, leaking the inner cytoplasm and killing the organisms. This work aims to investigate the alterations of the membrane of $A$. baumannii with Raman spectroscopy and AFM by inducing the phase change with two main stresses: dehydration and the variation of temperature.

\section{P-289}

Single-cell measurements of two-dimensional binding kinetics across cell contacts

Manto Chouliara [1], Victoria Junghans [1,2], Tommy Dam [1], Peter Jönsson [1]

[1] Lund University, Sweden; [2] University of Oxford, UK

Key to understanding the first steps of an adaptive immune response is the binding kinetics between membrane proteins on the $\mathrm{T}$ cell's surface and receptors on the surface of antigen-presenting cells. We present an approach that extends the Zhu-Golan method in order to measure singlecell binding affinities as well as pave the way for weak protein interactions to be monitored in cell-cell contacts. With this method, information on the distribution of affinities within the cell population can be obtained while simultaneously reducing the experiment and data analysis time. This is achieved by replacing one of the contacting cells with a protein functionalized supported lipid bilayer (SLB) and by titrating the ligand concentration in the formed cell-SLB contacts using imidazole. As a model system, we study the interaction of fluorescently labeled rat CD2 anchored on SLBs with rat CD48 ${ }_{\text {T92A }}$ expressed on Jurkat T cells. The spread in the single-cell affinities within the cell population is low, $K_{\mathrm{d}}=4.9 \pm 0.9$ molecules $/ \mu \mathrm{m}^{2}$ (mean $\pm \mathrm{SD}$ ), and independent of the mobile receptor densities. Furthermore, singlemolecule tracking of individual ligand molecules on the SLB provides vital information on the lifetimes of the ligandreceptor complex. The lifetime of $\mathrm{rCD} 2 / \mathrm{rCD} 48 \mathrm{~T} 92 \mathrm{~A}$ is estimated to be in the order of $100 \mathrm{~ms}$ while, additionally, self and pathogenic peptide lifetimes are investigated.

\section{P-291}

Prediction of ${ }^{2} \mathrm{H}$ NMR order parameters with neural networks

Markus G Fischer, Benedikt Schwarze, Holger A Scheidt

Institute for medical physics and biophysics, University of Leipzig, Germany

${ }^{2} \mathrm{H}$ NMR order parameters are a useful tool to gain information about changes of phospholipid membrane properties upon binding of different compounds, however measurement of large amounts of samples is impractical due to time and monetary reasons.

In this paper, we established a proof-of-concept, that ${ }^{2} \mathrm{H}$ NMR order parameters of membrane mixtures of POPC in presence of up to two additional molecules can be predicted with neural networks that consider molecular properties.

We used a feedforward network, a recurrent model, as well as a sequential approach. The recurrent neural network produced the least errors compared to experimental data. Therefore, we used this architecture to establish a minimal set of molecular features with the highest impact on membrane order parameter.

Those were: the octanol-water partition coefficient $\log \mathrm{P}$, the topological polar surface area TPSA, the magnitude of the dipolar moment, the surface area, the number of electrostatic clusters, and the absolute charge difference on the electrostatic surface.

While the recurrent model performs well on a single membrane type, it will face issues when it is expanded to different membranes. A more general solution was tried with the sequential model; however, its performance was poor. An alternate model is proposed for future research. 


\section{Posters}

- 19. Cell and tissue biophysics -

\section{P-292}

Mechanical and microrheological properties of bladder cancer cells: from single cells to 3D multicellular spheroids

Kajangi Gnanachandran, Joanna Pabijan, Malgorzata Lekka Institute of Nuclear Physics - Polish Aacademy of Sciences, Krakow, Poland

Cancer progression is associated with changes in the mechanical properties of cells. Atomic Force Microscopy (AFM) is a versatile tool used to study cell elastic and rheological properties. Here, we investigate biomechanics at different complexity levels (single cells, monolayers, and 3D multicellular spheroids). Three different cell lines were used: HCV29 (non-malignant cancer), HT1376 (grade III carcinoma), T24 (grade IV transitional cell carcinoma). Three parameters were compared: Young's (compression), storage and loss (shear stress) moduli. Our results show that under compression, HCV29 is stiffer than HT1376 and T24. Thus, cancer cells are softer than non-malignant ones. HCV29 and T24 rigidity increases when cells are grown as monolayer. Similar relation is observed for cells undergoing shear stress. The elastic properties of HT1376 cells in monolayer are of the same order as for single HT1376 cells regardless of the type of applied deformation. The mechanical and rheological properties of bladder cancer cells are related to actin filament organisation as stress fibres are present in HCV29 and T24 cells, not in HT1376 ones. All three cell lines form spheroids diversely and change biomechanics at the 3D level, but still, cancer cells were softer. Both mechanical (compression) and rheological (shear stress) properties may serve as a biophysical cancer marker.

\section{P-294}

Super-resolution microscopy of GSDMD pores in polymer-supported plasma membranes

Shirin Kappelhoff, Michael Holtmannspötter, Rainer Kurre, Jacob Piehler, Katia Cosentino

Universität Osnabrück, Germany

Pyroptosis is a highly inflammatory form of regulated cell death characterized by cell lysis due to plasma membrane (PM) permeabilization by the pore forming protein Gasdermin D (GSDMD). Upon cleavage by inflammatory caspases, cytosolic activated -GSDMD translocates and oligomerizes at the PM to form pores, which allow release of cytosolic content and promote cell lysis. The supposedly small size of GSDMD pores (in the range of $20-30 \mathrm{~nm}$ ) and the strong cytosolic GSDMD background has, so far, precluded to resolve the structure of GSDMD pores in the PM of pyroptotic cells. To overcome this issue, we combined a new approach, called polymer-supported plasma membranes (PSPM), with DNAPAINT superresolution microscopy to investigate GSDMD nano-pores in their native PM environment. PSPMs are PM sheets tethered to a polymer-coated surface that were generated from removing the cell body by actin depolymerization and mechanical forces. PSPMs allow for the labeling of any intracellular membrane components and the simultaneous investigation of the PM by alternative techniques such as atomic force microscopy . This approach allowed to remove any cytosolic GSDMD fluorescence contribution while preserving membrane topography and integrity. Importantly, PSPMs and DNA-PAINT microscopy proved to be a powerful combination to resolve GSDMD structures down to few nanometers directly on the PM of pyroptotic cells.

\section{P-293}

Effect of the combined use of proton radiation and AraC on morphological changes and apoptosis in the liver of rats

Elena Ignat [1,2], Y. Severiukhin [2], M. Lalkovicova [2], I. Kolesnikova [2], Z. Bálint [1], H. Pașca [1,2]

[1] Dpt of Biomolecular Physics, "Babeș-Bolyai" Univ., Romania; [2] Dpt of Radiation Physiology, LRB, JINR, Russia

The idea of using cytostatic drugs to increase the effectiveness of radiation therapy is of great interest to radiobiologists. In our experiments, 12 out of a total of 16, male, Sprague Dawley rats received a $3 \mathrm{~Gy}$ dose of protons with $170 \mathrm{MeV}$ energy in the cranio-caudal direction. The AraC dose of $0.4 \mathrm{~g} / \mathrm{m}^{2}$ was administrated into the tail vein. The organs belonging to 4, 6 and respectively 4 animals were harvested at 4,24 and 48 hours after irradiation and fixed in paraffin. The paraffin embedded tissue samples were sectioned to $8 \mu \mathrm{m}$ with a Thermo Fisher Scientific HM 340E microtome. The slides were stained using the H\&E method and the morphological changes were studied by a LUMO Mikmed2 microscope. A qualitative analysis of the liver parenchyma of irradiated rats showed a significant dilatation of the liver sinusoids in periportal regions in the 4 hours-3Gy group. We are currently performing a statistical analysis on the morphological changes that occurred in the hepatic cells and our preliminary findings for the 4 hours groups illustrate a statistical signification between the number of binuclear hepatocytes, 4,79 $\pm 0,35$ for $3 \mathrm{~Gy}$ group and $3,41 \pm 0,42$ for $3 \mathrm{~Gy}+\mathrm{AraC}$ group, as a result of Mann-Whitney test. A quantitative, comparative analysis and a TUNEL assay are ongoing. We expect to find differences (pyknosis, karyolysis, karyorrhexis) due to the long-term effect of irradiation.

\section{P-295}

Immuno-modulating effect of temperature on early $\mathbf{T}$ cell activation

Caroline Kopittke, Joschka Hellmeier, Martin Fölser, Lukas Schrangl, Gerhard J Schütz, Mario Brameshuber Institute of Applied Physics, TU Wien, Vienna, Austria

As a part of the adaptive immune system, $\mathrm{T}$ cells rapidly screen the surface of antigen presenting cells (APCs) for signs of infection or carcinogenesis. Together with signals from co-stimulatory proteins, the binding of the $\mathrm{T}$ cell receptor (TCR) to its cognate antigen (pMHC) triggers $\mathrm{T}$ cell activation. The subsequent release of signalling molecules directs the immune response. Temperature has well-established immuno-modulating properties. While hypothermic temperatures can have anti-inflammatory effects, hyperthermic temperatures can enhance the immune response (e.g. during fever). The exact effect of temperature on $\mathrm{T}$ cells is an active field of research.

Here, we investigate the influence of temperature on $\mathrm{T}$ cell sensitivity and early $\mathrm{T}$ cell activation. We use supported lipid bilayers to mimic the APC surface and identify activated $\mathrm{T}$ cells via ratiometric calcium imaging. Determining the percentage of activating cells as a function of the antigen concentration on the bilayer results in antigen dose-response curves that allow quantifying $\mathrm{T}$ cell sensitivity under different thermal conditions. Shifts in the dose-response relationship indicate temperature induced changes in T cell sensitivity. 
Posters

- 19. Cell and tissue biophysics -

P-296

Label-free imaging of cholesterol assemblies reveals hidden nanomechanics of breast cancer cells Danahe Mohammed [1], Andra C Dumitru [1], Mauriane Maja [1], Jinsung Yang[1], Sandrine Verstraeten [1], Aranzazu del Campo[2], Marie-Paule Mingeot-Leclercq[1], Donatienne Tyteca [1], David Alsteens [1]

[1] Université catholique de Louvain, Belgium; [2] INM Leibniz-Institut für Neue Materialien gGmbH, Germany

Tumour cells present profound alterations in their composition, structural organization and functional properties. A landmark of cancer cells is an overall altered mechanical phenotype, which so far are linked to changes in their cytoskeletal regulation and organization. Evidence exists that the plasma membrane (PM) of cancer cells also shows drastic changes in its composition and organization. However, biomechanical characterization of PM remains limited mainly due to the difficulties encountered to investigate it in a quantitative and label-free manner. Here, the biomechanical properties of PM of a series of MCF10 cell lines, used as a model of breast cancer progression, are investigated. Notably, a strong correlation between the cell PM elasticity and oncogenesis is observed. The altered membrane composition under cancer progression, as emphasized by the PM-associated cholesterol levels, leads to a stiffening of the PM that is uncoupled from the elastic cytoskeletal properties. Conversely, cholesterol depletion of metastatic cells leads to a softening of their PM, restoring biomechanical properties similar to benign cells. As novel therapies based on targeting membrane lipids in cancer cells represent a promising approach in the field of anticancer drug development, this method contributes to deciphering the functional link between PM lipid content and disease.

\section{P-298}

Force-controlled tensile tests on individual collagen fibrils to study viscoelasticity of an osteogenesis imperfecta mouse model

Mathis Nalbach [1], Orestis G Andriotis [1], Georg Schitter [3], Alessandra Carriero [2], Philipp J Thurner [1]

[1] Institute of Lightweight Design and Structural Biomechanics, TU Wien, Austria; [2] Department of Biomedical Engineering of The City College of New York, USA; [3] Automation and Control Institute, TU Wien, Austria

Collagens are the major structural proteins in the extra cellular matrix (ECM) of the human body. Thus, collagen mechanics play a crucial role in cell mechanotransduction. At the cell length scale, the majority of collagens form high aspect ratio fibers - collagen fibrils $(\mathrm{CF})$. Recent studies indicate a role of ECM viscoelasticity on cell function. While quantitative properties of $\mathrm{CF}$ viscoelasticity are elusive, this knowledge is crucial to understand pathogenesis in diseases affecting collagen fibril structure, chemistry and mechanics. An example is osteogenesis imperfecta, which can be studied via the (oim/oim) mouse model. We report viscoelastic properties of individual CFs from oim/oim and wild-type (WT) mice from tension tests using a custom-built instrument. We investigate transient and steady-state deformation of individual CFs in force control with dynamic mechanical analysis and creep tests. Strikingly, we find higher storage modulus and ultimate strength in oim/oim vs. WT CFs. This is counterintuitive since oim/oim collagen forms brittle bones and molecular dynamics simulations predict it to be less stiff and strong. This may be explained by excessively increased levels of glycation cross-links in oim/oim.

\section{P-297}

Magnetic muscular cells to develop an engineering tool at tissue scale: the magnetic stretcher Irène Nagle, Claire Wilhelm, Myriam Reffay Lab. Matière et Systèmes Complexes, UMR 7057, CNRS, Univ. de Paris, France

Skeletal muscle is one of the most abundant tissue types in the human body. Creating in vitro models reproducing its multi-scale aligned structure and its function would offer a polyvalent platform for studies in tissue biology but also for the development of new drugs or gene therapies to treat muscular traumatisms or diseases. To address this challenge, the use of magnetic fields and forces, via the incorporation of biocompatible superparamagnetic nanoparticles $\left(\gamma-\mathrm{Fe}_{2} \mathrm{O}_{3}\right)$ in the cells to confer them magnetic properties, is promising. This magnetic labelling enables both the manipulation of cells at distance and the application of forces to drive their differentiation into functional muscular cells. It was applied to the immortalized mouse muscle precursor cell line $\mathrm{C} 2 \mathrm{C} 12$ to obtain a multicellular aggregate without support matrix. The aggregate deformations with a magnetic field enable to measure its macroscopic mechanical properties (surface tension, Young modulus). The expected influence of the actin cytoskeleton and cell-cell adhesions were highlighted and a major role of the intermediate filaments architecture of desmin (involved in desminopathies) was evidenced on the mechanics of this 3D tissue. A magnetic stretcher is now developed to stimulate mechanically for several days the muscle precursor cells between two mobile magnets and induce their differentiation into aligned muscular cells. This magnetic stretcher represents a new tool to study cell differentiation.

\section{P-299}

Investigation of the spatial correlation between chromatin organization and sub-nuclear pattern of oncoprotein DEK

A. Pierzyńska-Mach [1], E. Ferrando-May [2], L. Lanzanò $[1,3]$, A. Diaspro $[1,4]$

[1] Nanoscopy, Istituto Italiano di Tecnologia, Genoa, Italy; [2] Department of Biology, University of Konstanz, Germany; [3] DFA, University of Catania, Italy; [4] DIFILAB, Department of Physics, University of Genoa, Italy

The DEK protein is a ubiquitous nuclear factor that has been consistently associated with tumor progression: its expression level differs between normal and cancer cells, raising the possibility of using DEK as a tumor marker. Moreover, DEK is a non-histone architectural chromatin factor that may influence nucleosomal accessibility and therefore gene activity. The interest of this work is to investigate, using superresolution microscopy approaches, the correlation between the organization of the DEK oncoprotein and the local chromatin structure depending on the degree of cell malignancy. A model based on three well-established human breast cell lines was chosen to represent different subtypes of breast cancer. We performed dual-color confocal and STED imaging followed by the pixel intensity spatial correlation analysis, to show the differences of the nanoscale distribution of DEK in relation to the chromatin organization pattern (observed by particular eu- and heterochromatin marks IF and by RNAFISH). We expect that this study will provide novel insights into the tumor-promoting activity of DEK, its role in crucial cellular processes such as DNA replication, and thus progress our understanding of basic mechanisms of carcinogenesis. 


\section{Posters}

- 19. Cell and tissue biophysics -

P-300

Morphological, fractal, and textural features for the blood cell classification: the case of acute myeloid leukemia

Tamara Popović [1], Marko Dinčić [2], Kojadinovic Milica [1], Alexander Trbovich [2], Andjelija Ilić [3]

[1] Institute for Medical Research, University of Belgrade, Centre of Exellence in Nutrition and Metabolism, Serbia; [2] Institute of Pathological Physiology, Faculty of Medicine, University of Belgrade, Serbia; [3] Institute of Physics Belgrade, University of Belgrade, Serbia

In this work, we study different morphological, fractal, and textural descriptors for the white blood cell (WBC) classification, with an aim to indicate the most reliable parameters for the recognition of certain cell types. Structural properties of both the mature and non-mature leukocytes obtained from the (i) acute myeloid leukemia patients, or (ii) non-malignant controls, were studied in depth. An open dataset collection including the digitalized single-cell images for 200 patients, from peripheral blood smears at 100x magnification, was used in the study. Structural and textural differences among the cell types were quantified and the statistical ranges of parameters for different cell types were defined. Based on these findings, the recommendations for the blood smear WBC classification were given.

\section{P-302}

High throughput electric cell characterization Tayebeh Saghaei [1], Andreas Weber [2], Erik Reimhult [1], José Luis Toca-Herrera [2], Peter van Oostrum [1]

[1] University of Natural Resources and Life Sciences, Vienna, Department of Nanobiotechnology, Institute for Biologically inspired materials, Austria; [2] University of Natural Resources and Life Sciences, Vienna, Department of Nanobiotechnology, Institute for Biophysics, Austria

Characterization of individual cells is essential for disease diagnosis and treatment planning. The electric properties of individual cells can be characterized by studying the rotation rate of single cells in a rotating electric field. However, the throughput is extremely limited. We demonstrate a method by which many cells in a capillary spin independently of the vicinity of other rotating cells. Polarizable particles form chains in a linearly polarized AC electric field. When applying such fields to deformable colloids, e.g., oil drops or cells, we also observed a spinning motion. This rotational motion depends on the frequency of the $\mathrm{AC}$ field and varies with the design of the electrodes. In fact, we found that the rotation speed and direction of the rotation depend on the type of oil/cell, the presence of ionic surfactants, the $\mathrm{pH}$, and ionic strength. We used this method to distinguish between normal MCF7 and HeLa cells, fixed cells, and cells with depolymerized actin filaments. We also distinguish cells by their age. The rotational behavior is uniform throughout the field of view of a microscope, allowing high-throughput characterization.

\section{P-301}

AGE-related changes in indentation stiffness and surface charge of individual collagen fibrils through MGO glycation

Manuel Rufin [1], Mathis Nalbach [1], Orestis G Andriotis [1], Matthias Poik [2], Georg Schitter [2], Philipp J Thurner [1] [1] ILSB, TU Wien, Austria; [2] ACIN, TU Wien, Austria

In its fibrillar form, collagen provides mechanical strength to many load-bearing tissues such as tendons, bone and ligaments. Additionally, it offers binding sites for cell attachment and migration. Collagen fibrils are modified by several types of inter-molecular covalent cross-links, one type of which being the Advanced Glycation Endproducts (AGEs). AGEs play an important role in long-term complications in diabetes and ageing. In this study, we model physiological effects of AGEs through methylglyoxal (MGO). We performed in-situ atomic force microscopy experiments to evaluate fibril size and nano-stiffness. Amplitude-modulated kelvin force microscopy was used to indirectly assess the presence of MGO cross-linking, by measuring the surface potential before and after MGO treatment. Treatment with MGO for $4 \mathrm{~h}$ across all treated collagen fibrils led to an increased indentation modulus $+0.44(0.36) \mathrm{MPa}(\mathrm{p}=0.002)$ at almost constant fibril height, when tested in PBS, while dry fibril diameter increased by $30(9) \%(p=0.001)$. Coincidentally, collagen fibril surface potential was reduced by $21(9) \mathrm{mV}$ $(\mathrm{p}=0.0001)$. Our study allows to directly link nanomechanical and cross-linking changes due to glycation at the individual fibril level. To further evaluate cross-linking, analytical chemistry as well as nanotensile tests will be employed and correlated to the present results in the future.

\section{P-303}

Tight junction $\mathrm{ZO}$ proteins maintain tissue fluidity, ensuring efficient collective cell migration Mark Skamrahl [1], Hongtao Pang [1], Maximilian Ferle [1], Jannis Gottwald [1], Angela Rübeling [2], Riccardo Maraspini [3], Alf Honigmann [3], Tabea A Oswald [2], Andreas Janshoff [1] [1] University of Göttingen, Institute of Physical Chemistry, Göttingen, Germany; [2] University of Göttingen, Institute of Organic and Biomolecular Chemistry, Göttingen, Germany; [3] Max Planck Institute of Molecular Cell Biology and Genetics, Dresden, Germany

Tight junctions endow epithelia with their vital barrier function. However, their role in many biological processes, including collective cell migration, is unclear. Therefore, we study the tight junction proteins $\mathrm{ZO} 1$ and $\mathrm{ZO} 2$ during epithelial migration, using velocimetry, segmentation, cell tracking, and atomic force microscopy approaches. Our results imply that $\mathrm{ZO}$ proteins are required for quick and coordinated migration. Particularly, loss of ZO1 and 2 induces actomyosin remodeling away from the central cell cortex into highly contractile bundles, resulting in mechanical imbalances in the layer. A tug-of-war emerges between two cell populations with distinct morphological and mechanical properties: small, round, and extremely contractile cells pull on neighboring cells, which are stretched, flattened, and enlarged. In response, the large stretched cells proliferate more, increasing the cell density. Upon high cell densities, the small contractile cells are markedly immobile, causing a crowding-induced jamming. Our data show that $\mathrm{ZO}$ proteins maintain tissue mechanics and control proliferation, rendering them indispensable for epithelial migration. 
Posters

- 19. Cell and tissue biophysics -

P-304

Stress induces lipid droplet accumulation in brain astrocytes

Tina Smolič $[1,2]$, Petra Tavčar [1], Anemari Horvat [1,2], Urška Černe [1], Robert Zorec [1,2], Nina Vardjan [1,2]

[1] Laboratory of Neuroendocrinology-Molecular Cell Physiology, Institute of Pathophysiology, Faculty of Medicine, University of Ljubljana, Slovenia; [2] Laboratory of Cell Engineering, Celica Biomedical, Ljubljana, Slovenia

Astrocytes, neuroglial cells with homeostatic functions in the brain, are involved in the development of brain pathologies. During brain pathologies lipid droplets (LDs), the lipid storage organelles, tend to accumulate in glial cells, including astrocytes. To characterize LD biology in astrocytes, we studied the (sub)cellular localization, mobility, and the alterations in the amount of LDs in response to different stressors characteristic to brain pathologies. Astrocytes in culture and brain tissue were exposed for $24 \mathrm{~h}$ to metabolic stress (nutrient deprivation, excess of free fatty acids (FFA) or L-lactate), hypoxia $\left(1 \% \mathrm{O}_{2}\right)$, and adrenergic receptor agonists/antagonists. LDs were fluorescently labeled (Nile Red, BODIPY ${ }^{493 / 503}$ ) and evaluated by confocal and structured illumination microscopy. We found that astroglial LDs are of $\sim 450 \mathrm{~nm}$ in diameter, localized near mitochondria and ER. LDs exhibited low mobility, which was further reduced upon metabolic stress. Metabolic stress, hypoxia, and noradrenaline (stress response neuromodulator) via cAMP signaling increased LD accumulation over 2-fold. LDs may serve as an alternative energy source in astrocytes and/or may protect neural cells against the FFA lipotoxicity.

\section{P-306}

Viscoelastic properties of bacteria revisited - an AFM study

Andreas Weber [1], Benitez Rafael [2], José L. Toca-Herrera [1] [1] Institute for Biophysics, University of Natural Resources and Life Sciences, Vienna, Austria; [2] Dpto. Matemáticas para la Economía y la Empresa, Facultad de Economía, Universidad de Valencia, Spain

Gram-negative bacteria are surrounded by a tough cell wall consisting of the inner and outer membrane, separated by a viscous compartment called the periplasm. The periplasm itself contains a thin layer of peptidoglycan. Due to its structure, the bacterial cell wall plays important roles for different biological functions, such as maintenance of cell shape, conferring mechanical stability to the cell, resistance against turgor pressure and protection from hostile environments and drugs. Most of the recent AFM studies use an ideal elastic Hertzian model to describe the bacterial mechanics and determine the elastic Modulus E. Since gram-negative bacteria are viscoelastic materials, a description using viscoelastic (VE) theory is more suited to capture the nature of their mechanics. One approach was used by applying simple models such as the standard linear solid (SLS), consisting of a spring in series with a Kelvin-Voigt-element (spring in parallel to a dashpot). A thorough description of bacterial cell wall mechanics using diverse VE models is still not complete. In this work, we have used AFM using Force cycles, stress relaxation and creep experiments as well as micro-oscillations to fully describe the viscoelastic properties of gram-negative bacterial cells over different frequency and time-domains.

\section{P-305}

Microfluidic device for sorting and identification of mechanically altered cells

Renata Szydlak [1], Marcin Luty [1], Ingrid H Øvreeide [2], Vladimir Rosenov [2,3], Victorien Prot [3], Joanna Zemła [1], Bjørn T Stokke [2], Małgorzata Lekka [1]

[1] Institute of Nuclear Physics Polish Academy of Sciences, Poland; [2] Biophysics and Medical Technology, Department of Physics, NTNU The Norwegian University of Science and Technology, Norway; [3] Biomechanics, Department of Structural Engineering, NTNU The Norwegian University of Science and Technology, Norway

Cancer is one of the leading causes of death because it is often diagnosed too late. Hence, the key challenge of biomedicine and oncology is developing new diagnostic tools, which would allow the detection of the disease at an early stage, make an accurate diagnosis, and choose the best treatment. Knowing that cancer cells are more deformable than their healthy couterpartner, we present a combined approach using microfluidic and AFM methods to study cells selectively captured using lectins such as WGA, DBA, PHA-L. We present preliminary results of a study on the bladder cancer cells, i.e. HCV29 (non-malignant carcinoma of ureter), HT1376 (bladder carcinoma) and T24 (transitional cell carcinoma). Adhesion and migration activity have shown bladder cancer cells have different affinity to studied lectins showing their potential to be used in selective cell capturing. The combination of microfluidic and AFM measurements, correlates deformability and adhesive properties of cancer cells. We anticipate this could be helpful in cancer diagnosis and eventually extending to high throughput detection of cancer cells.

\section{P-307}

Noncovalent complex formation of Pseudomonas aeruginosa essential virulence factor pyocyanin with caffeine and its biological implications

Anna Woziwodzka [1], Agata Woźniak [1], Aleksandra Rapacka-Zdończyk [2], Marta Krychowiak-Maśnicka [1], Dariusz Wyrzykowski [3], Jacek Piosik [1]

[1] Intercollegiate Faculty of Biotechnology UG\&MUG, Poland; [2] Faculty of Pharmacy, Medical University of Gdansk, Poland; [3] Faculty of Chemistry, University of Gdansk, Poland

Pseudomonas aeruginosa is an opportunistic pathogen forming difficult to treat biofilms in immunocompromised or cystic fibrosis patients. Pyocyanin, a redox-active secondary metabolite produced by $P$. aeruginosa, promotes biofilm development. Here, we characterized non-covalent complex formation between pyocyanin and an important diet constituent caffeine, and evaluated caffeine impact on bacterial growth and biofilm formation. UV-Vis spectroscopy combined with statistical-thermodynamical modelling showed pyocyanin-caffeine complex formation with association constant $\boldsymbol{K}_{\boldsymbol{A} \boldsymbol{C}}$ of $30.2 \mathrm{M}^{-1}$. Enthalpy change $\boldsymbol{\Delta} \boldsymbol{H}=13.8 \mathrm{~kJ} / \mathrm{mol}$ obtained with isothermal titration calorimetry indicates that complex formation is an endogenous process. Caffeine inhibited biofilm production in a dose-dependent manner in two $P$. aeruginosa strains: ATCC 27853 and 143/p (abundant and low pyocyanin producer, respectively) with no effect on bacterial growth and survival. Our findings suggest that caffeine by binding to $P$. aeruginosa virulence factor pyocyanin can disrupt biofilm development in vitro. (Financed by National Science Centre, Poland, grant no 2016/21/D/NZ7/01524) 


\section{Posters}

-20. Lipid-Protein interactions -

P-308

Rotating ATP synthases yield lipid bilayers to behave as a driven liquid

Víctor G. Almendro-Vedia [1,2], Pablo Llombart [3], Paolo Natale [1,2], Marisela Vélez [4], María del Pilar Lillo [5], Juan L. Aragonés [3], Iván López-Montero [1,2]

[1] Departamento Química Física I, Universidad Complutense de Madrid, Spain; [2] Instituto de Investigación Sanitaria, Hospital 12 de Octubre, Spain; [3] Física Teórica de la Materia Condensada, UAM, Spain; [4] Catalysis and Oil-chemistry Institute, CSIC, Spain; [5] Instituto de Química Física Rocasolano, CSIC, Spain

The transmembrane protein ATP synthase is the most important biological motor with rotational movement. The diffusion of a set of rotating ATP synthases embedded within a lipid bilayer have been studied theoretically [Lenz $\mathrm{P}$ et al. 2003, PRL 91: 108104]. At slow spin velocities, an increase of the protein diffusion might be observed whereas beyond a critical rotational velocity, proteins might self-organize into a crystal-like structure [Oppenheimer N et al. 2019, PRL 123(14)].

We present an experimental study on the transport properties of ATP synthases based on fluorescently proteins reconstituted into supported bilayers with different lipid compositions and protein packings. We monitored the coefficient diffusion of proteins by Fluorescence Recovery After Photobleaching (FRAP) under active and passive conditions, showing differences on the diffusion coefficient. From Dissipative Particle Dynamics (DPD) simulations, we conclude that non-equilibrium density fluctuations control the lateral diffusion of rotating ATP synthases in crowded membranes.

\section{P-310}

$\alpha$-casein-membrane interactions: quantitative fluorescence analysis of the effects of protein aggregation state Sara Anselmo, Giuseppe Sancataldo, Valeria Vetri

University of Palermo, Italy

Here we present an experimental study aimed at highlighting the molecular mechanisms underlying the interaction of $\alpha$-casein with membranes. An experimental approach based on Fluorescence Microscopy methods and classical spectroscopic techniques is used to achieve a mechanistic description of the mutually disruptive interactions between $\alpha$-casein in different association states and model membranes. We exploit the capability of Raster Scan Image Correlation Spectroscopy (RICS) to monitor this spatially heterogeneous phenomenon and in particular to map the stoichiometry, the aggregation state and binding events at each pixel of fluoresce microscopy images. This allows to study in a time resolved fashion protein assembly and disassembly phenomena localising key events while monitoring morphology and structural changes of the membrane that we quantify using two photon microscopy and Laurdan generalised polarisation. The effect of monomeric proteins, protein in the micellar state and protein aggregates was analysed revealing that, while only tiny modifications occur in the presence of the first two species, the addition of protein aggregates induce profound changes in membrane conformation. Interestingly, once aggregates are added to the sample they readily reassemble in new species which are able to interact with the membrane and undergo to a new association process that occurs at the membrane inducing the formation of new lipid/protein coaggregates with a peculiar branched morphology.

\section{P-309}

Studying the structure of pulmonary surfactant protein SP-B from a recombinant version of its precursor

Alejandro Alonso, Bárbara Olmeda, Olga Cañadas, Jesús Pérez-Gil

Biochemistry and Molecular Biology Department, Complutense University of Madrid and Research Institute Hospital 12 de Octubre, Madrid, Spain

SP-B is a small protein directly involved in pulmonary surfactant homeostasis and biophysical function. The structure of SP-B remains unknown, in part due to its high hydrophobicity, its lipid interaction capabilities, and the limitation to obtain it in enough amount. Current models of the structure of SP-B and of the oligomers it apparently forms have been built by Martini simulation and need to be empirically confirmed. In this work, a recombinant variant of human SP-B precursor lacking the C-terminal propeptide (proSP$\mathrm{B}_{\Delta \mathrm{C}}$ ) has been produced in a bacterial strain engineered to synthesize proteins with complex disulphide bond patterns. This precursor, produced as a soluble protein, seems to be properly folded and tends to assemble ring-shaped oligomers, similar to those originated by $\mathrm{SP}-\mathrm{B}$, at different $\mathrm{pH}$ values. Besides, the precursor proSP-B $\mathrm{B}_{\Delta \mathrm{C}}$ shows ability to interact with different lipids or lipid mixtures, as it could be taking place during the physiological processing of the proSP-B precursor in the alveolar type 2 cells. These preliminary results suggest that proSP-B $\mathrm{B}_{\Delta \mathrm{C}}$ could be a good candidate to study the structure of the functional SP-B module.

\section{P-311}

Lipoprotein particle interaction with biomembranes

Markus Axmann [1], Florian Weber [1], Andreas Karner [1], Herbert Stangl [2], Birgit Plochberger [1]

[1] University of Applied Sciences Upper Austria, School of Medical Engineering and Applied Social Sciences, 4020 Linz, Austria; [2] Center for Pathobiochemistry and Genetics, Institute of Medical Chemistry and Pathobiochemistry, Medical University Vienna, 1090 Vienna, Austria

Excess, non-water soluble cholesterol from peripheral cells is transported to the liver by High Density Lipoprotein (HDL) particles for excretion. Thus, cholesterol has to be transferred in the periphery from the cell membrane to HDL particles and vice versa in the liver. It remains elusive whether lipoprotein particle receptors like SR-B1 directly mediate lipid flux or just keep the lipoprotein particle (LP) attached to the plasma membrane (enhancing transfer probability). We observed transfer of cholesterol from LPs to a lipid bilayer upon contact without a receptor. Initial studies suggest, that LP/membrane interactions (via specific receptor or receptor-independent) and cargo transfer are determined by two parameters: presence of specific apolipoproteins (major structural compound of LPs), and local lipid environment of the biological membrane. Hence, we plan to quantify the relative contribution of each parameter by creation of artificial LPs with known composition (i.e. different apolipoprotein combinations) and to measure their interaction with varying biomembranes using Quartz Crystal Microbalance, Fluorescence and Atomic Force Microscopy. As comparison, we measure native HDL particles from different donors. 
Posters

-20. Lipid-Protein interactions -

\section{P-312}

A neutron reflectometry study of the interaction of glycerophospholipids and the phospholipids PLA1-1 isoform of aspergillus orizae

Krishna Batchu [1], Giovanna Fragneto [1], Robert Jacobs [2], Giacomo Corucci [1]

[1] Institute Laue Langevin, Grenoble, France; [2] Chem Research Lab, Univ Oxford, UK

Phospholipases (PLAs) are lipolytic enzymes that hydrolyze phospholipid substrates at specific ester bonds. They are widespread in nature and play very diverse roles right from signal transduction and lipid mediator production to membrane phospholipid homeostasis. Phospholipases vary considerably in their structure, function, regulation, and mode of action therefore a deeper understanding of their dynamics and kinetics can be very crucial. The present study encompasses employing neutron reflectivity including other physical/chemical techniques to better understand the principles underlying the substrate specificity of phospholipases. We here, studied in detail the effect of the acyl chain length and unsaturation of phospholipids on their hydrolysis by type A1PLA (sourced from Aspergillus oryzae), that was expressed in E.Coli and purified in its pure form thus allowing us to understand the key factors that regulate its activity.

\section{P-314}

Simulating PIP2-induced gating transitions in Kir6.2 channels

Michael Bründl, Sarala Pellikan, Anna Stary-Weinzinger University of Vienna, Austria

ATP-sensitive potassium ( $\left.\mathrm{K}_{\mathrm{ATP}}\right)$ channels consist of an inwardly rectifying $\mathrm{K}^{+}$channel protein (Kir6.2) pore, to which four ATP sensitive sulfonylurea receptor (SUR) domains are attached, thereby coupling $\mathrm{K}^{+}$permeation directly to the metabolic state of the cell. Dysfunction is linked to neonatal diabetes and other diseases. $\mathrm{K}^{+}$flux through these channels is controlled by conformational changes at the helix bundle region, acting as physical barrier for $\mathrm{K}^{+}$flux. In addition, the G-loop, located at the cytoplasmic domain, and the selectivity filter might contribute to gating, as suggested by different disease-causing mutations. Gating of Kir channels is regulated by different ligands, such as $\mathrm{G}_{\beta \gamma}, \mathrm{H}^{+}, \mathrm{Na}^{+}$, adenosine nucleotides and the signaling lipid phosphatidylinositol 4,5-bisphosphate $\left(\mathrm{PIP}_{2}\right)$, which is an essential activator for all eukaryotic Kir family members. Although molecular determinants of $\mathrm{PIP}_{2}$ activation of $\mathrm{K}_{\mathrm{ATP}}$ channels have been investigated in functional studies, structural information of the binding site is still lacking due to Kir6.2 cryo-EM structures failing to resolve $\mathrm{PIP}_{2}$. Here we use Molecular Dynamics (MD) simulations to examine the dynamics of residues associated with gating in Kir6.2. By combining this structural information with functional data, we investigate the mechanism underlying Kir6.2 channel regulation by $\mathrm{PIP}_{2}$.

\section{P-313}

Revealing the mechanism behind protein-lipid interactions of curvature sensing helices in plant reticulons implicated in endoplasmic reticulum shaping

Rhiannon L Brooks, Ann M Dixon

University of Warwick, UK

Reticulons are a large family of integral membrane proteins ubiquitous across eukaryotic cells. Their primary function is to generate highly curved endoplasmic reticulum (ER) tubules via a highly conserved transmembrane domain. Recently, the presence of a C-terminal amphipathic helix (APH) has been reported for RTNLB13 from Arabidopsis thaliana that is essential for the proteins ability to constrict ER tubules but not enough by itself to influence membrane morphology. Here, biophysical techniques, including solution-state NMR, have been employed to demonstrate a dependence on curvature for membrane-binding of this APH. The results obtained suggest that the helix acts as a feedback element, only folding/binding to the ER membrane once a sufficient degree of curvature has been achieved by the transmembrane region, and that it provides additional stabilization without disrupting the bilayer. This work was then extended to other $A$. thaliana reticulons which revealed that those with the closest evolutionary relationship to RTNLB13 contain curvature sensing APHs in the same location with sequence conservation. However, a more distantly related branch of reticulons developed a 20-residue linker between the transmembrane domain and $\mathrm{APH}$, which likely enables functional flexibility.

\section{P-315}

On the way of assessing autonomous role of $\mathrm{p} 6$ in $\mathrm{HIV-1}$ spread

Laura Cantù [1], Elena Del Favero [1], Anna Moroni [1], Ulrich Schubert [2], Caterina Ricci [1]

[1] University of Milan, Italy; [2] Friedrich-Alexander Universität Erlangen-Nürnberg., Germany

During the HIV-1 replication cycle, the Gag polyprotein is known to mediate the assembly and release of progeny virions from an infected cell membrane. The Gag C-terminal p6 domain (52 amino acids) has the task to mediate incorporation of accessory proteins such as Viral protein $\mathrm{R}$ [1]. Up to $10^{10} \mathrm{HIV}-1$ particles are produced and disintegrated per day in high viraemic infected persons, leading to [2053?]5000 p6 molecules per released virion, the destiny of which is either to disintegrate in extracellularly or to infect a new host cell [2]. Although free p6 can be found in mature virions, it is hardly detectable in infected cells or in cell-free spaces, raising the question about its outcome [3]. The fate of $p 6$ is linked to its ability to interact with membranes, and has not been fully undisclosed. We investigated by QCM, Small Angle Xray Scattering and Neutron Reflectometry the structure of p6 and its interaction with biomimetic membranes. The interaction reveals to be modulated by the lipid composition, giving insight on mature p6 behaviour and shedding light on unexpected spreading mechanisms of HIV-1.

[1] Fossen, T. et al.., J. of Biol. Chem. 280.52 (2005).

[2] Briggs, J. A.G., et al. Nat. Struct. Mol. Bio. 11.7 (2004).

[3] Hahn, F. et al. Plos one 12.4 (2017).

Work partially supported by ERC Project "noMAGIC, Noninvasive Manipulation of Gating in Ion Channels, ID: 695078" 


\section{Posters}

-20. Lipid-Protein interactions -

P-316

Detergent-free membrane protein reconstitution into hybrid polymer-lipid vesicles

Rosa Catania [1], Jonathan Machin [1], Rashmi Seneviratne [1], Michael Rappolt [2], Stephen P Muench [1], Paul A Beales [1], Lars J C Jeuken [1]

[1] Astbury Centre of Structural Molecular Biology, University of Leeds, UK; [2] School of Food Science and Nutrition, University of Leeds, UK

Membrane proteins (MPs) are essential to many vital cellular processes, and their importance is also reflected in an increasing number of promising applications, from drug discovery to energy production. Amphiphilic polymers have shown promise in resolving stability limitations encountered when studying MPs in detergents or liposomes. First, amphiphilic polymers like styrene-maleic acid copolymer (SMA) are able to solubilise MPs without the need for detergents. Second, hybrid vesicles (HVs) that consist of mixtures of copolymers and lipids have shown to be robust biomimetics of liposomes, extending the lifetime of reconstituted MPs. Previously, we reconstituted the membrane protein cytochrome $b o_{3}$ from $E$. coli in HVs after purification in detergent. Here, we show for the first time that it is possible to functionally reconstitute cyt bo 3 directly from SMA lipid particles (SMALPs) into HVs without the use of any detergent. Interestingly, reconstitution directly into liposomes from SMALPs was not possible for this protein under the same experimental conditions. This suggests that the copolymer-lipid mixtures are more amenable to MP incorporation than liposomes. Finally, we show that this transfer method is not limited to cyt bo 3 and can be performed with complex MPs mixtures.

\section{P-318}

Lipid bilayer insertion and nanodisc formation of the maleic acid diisobutylene copolymer DIBMA Sebastian Daum, Jan Ebenhan, Kirsten Bacia

Martin-Luther-Universität Halle-Wittenberg, Germany

Copolymers of maleic acid with styrene (SMA) or diisobutylene (DIBMA) have been known to directly insert into and fragment phospholipid membranes, yielding polymerstabilized lipid nanoparticles called SMALPs and DIBMALPs. In contrast to the formation of lipid 'nanodiscs' with a membrane scaffolding protein (MSP), the preparation of SMALPs or DIBMALPs does not require the intermediate step of solubilizing membrane components with a detergent. Also, lipid exchange between polymer-stabilized lipid nanoparticles appears to be more efficient than between MSP-nanodiscs. Applying single molecule sensitive techniques i.e. fluorescence correlation spectroscopy (FCS), fluorescence cross correlation spectroscopy (FCCS) to labelled particles, we are able to study the diffusion properties of DIBMALPs over a wide range of lipid and polymer concentrations. Measurements of the fluorescence resonance energy transfer between different fluorescence labels provide additional information on the interaction between the polymer and the lipids. Initial results indicate that lipid bilayer insertion and nanodisc formation by DIBMA is a more complex process than previously described.

\section{P-317}

Lipid binding to the transient receptor potential canonical channel 3

Amy V Clarke [1], Thomas Stockner [1], Klaus Groschner [2] [1] Medical University of Vienna, Austria; [2] Medical University of Graz, Austria

In humans, calcium signalling is an essential mechanism for regulating an array of processes, such as muscle contraction, nerve conductance, and fertilisation. Accordingly, the molecular toolkit of calcium signalling encompasses a considerable number of proteins, including transient receptor potential (TRP) channels, which open in response to a variety of signals to allow $\mathrm{Ca}^{2+}$ influx. One such TRP channel, the transient receptor potential canonical channel 3 (TRPC3), is activated by diacylglycerol (DAG) and positively modulated by phosphotidylinositol-4,5-bisphosphate $\left(\mathrm{PIP}_{2}\right)$. However, despite the solution of three cryo-EM structures of closed state TRPC3, the $\mathrm{PIP}_{2}$ binding site has not been identified. Here we present data from molecular dynamics simulations suggesting PIP2 binds to a cluster of positively-charged residues on the pre-S1 domain of TRPC3. Through varying the simulation concentration of $\mathrm{PIP}_{2}$, we were able to identify a higher affinity binding site, comprised of residues Lys-377, Arg-380 and Lys-385. Moreover, by simulating TRPC3 embedded in a complex, asymmetric membrane comprised of five lipid types, we were able to gain further insights into the lipid fingerprint of TRPC3. Our results represent the first computational exploration of the TRPC3 lipid environment, and we anticipate that they will provide an important launching pad for further investigations into lipid regulation of TRPC3, including how $\mathrm{PIP}_{2}$ exerts its effect on the channel.

\section{P-319}

Gag polyprotein of HIV-1 interacts with lipid membranes like a surfactant

Z. G Denieva [1], P. I Kuzmin [1], T. R Galimzyanov [1], S. A Akimov [1], J. Zimmerberg [2], O. V Batishchev [1]

[1] A.N. Frumkin Institute of Physical Chemistry and Electrochemistry, Russian Academy of Sciences, Moscow, Russian Federation; [2] Eunice Kennedy Shriver National Institute of Child Health and Human Development, National Institutes of Health, Bethesda, MD, USA

Enveloped viruses represent a large family of pathogens including such a dangerous one like the human immunodeficiency virus (HIV). The genome of such viruses is wrapped in a protein scaffold and a bilayer lipid membrane. This lipid shell is taken by the virus during its budding from the plasma membrane of the infected cell. Therefore, lipid-protein interactions play one of the decisive roles in the processes of viral infection, assembly of new viral particles and their budding from the surface of the cell. Gag polyprotein is the main structural protein of HIV. In the virus life cycle, it is responsible for the assembly and release of virions from the cell. However, the question remains about the genesis of the HIV membrane curvature, which is necessary for the initiation of this process. Using a system of lipid nanotubes, which allows the creation of highly curved lipid surfaces, we investigated the mechanisms of membrane curvature generation during of the polyprotein Gag adsorption and showed that this protein tends to behave like a surfactant rather than to impose its intrinsic curvature to the membrane.

The work was supported by the Russian Foundation for Basic Research (grant \#20-54-14006). 
Posters

- 20. Lipid-Protein interactions -

P-320

In vitro reconstitution reveals phosphoinositides as cargo-release factors and activators of the ARF6 GAP ADAP1

Christian Duellberg, Albert Auer, Nikola Canigova, Katrin Loibl, Martin Loose

Institute of Science and Technology Austria, Austria

The differentiation of cells depends on a precise control of their internal organization, which is the result of a complex dynamic interplay between the cytoskeleton, molecular motors, signaling molecules, and membranes. For example, in the developing neuron, the protein ADAP1 (ADP-ribosylation factor GTPase-activating protein [ArfGAP] with dual pleckstrin homology [PH] domains 1) has been suggested to control dendrite branching by regulating the small GTPase ARF6. Together with the motor protein KIF13B, ADAP1 is also thought to mediate delivery of the second messenger phosphatidylinositol $(3,4,5)$-trisphosphate (PIP3) to the axon tip, thus contributing to PIP3 polarity. However, what defines the function of ADAP1 and how its different roles are coordinated are still not clear. Here, we studied ADAP1's functions using in vitro reconstitutions. We found that KIF13B transports ADAP1 along microtubules, but thatPIP3 as well as PI(3,4)P2 act as stop signals for this transport instead of being transported. We also demonstrate that these phosphoinositides activate ADAP1'senzymatic activity to catalyze GTP hydrolysis by ARF6. Together, our results support a model for the cellular function of ADAP1, where KIF13B transports ADAP1 until it encounters high PIP3/PI(3,4)P2 concentrations in the plasma membrane. Here, ADAP1 disassociates from the motor to inactivate ARF6, promoting dendrite branching.

\section{P-322}

Optical lipid clamp analysis and MD simulations suggest lipid binding domain2 (L2) as the primary site for TRPC3 regulation by diacylglycerols

Hazel Erkan [1], Oleksandra Tiapko [1], Amy Clarke [2], Matthias Gsell [1], Thomas Stockner [2], Klaus Groschner [1] [1] Medical University of Graz, Austria; [2] Medical University of Vienna, Austria

Coordination of membrane lipids within channel complexes maintain structural integrity and contribute to regulatory functions. TRPCs constitute a family of tetrameric cation channels, which are gated by DAGs. Recent single particle cryo-EM studies identified two key lipid interaction sites designated as L1 and L2, which can accommodate DAGs. To explore the role of L1 and L2 in TRPC3 regulation, we utilized a combination of the structure-guided mutagenesis and electrophysiological recording techniques along with information from MD simulations. The activation/deactivation kinetics of TRPC3 was analyzed by Optical Lipid Clamp to gain mechanistic insight into DAG-TRPC3 interactions. MD simulations indicated a strong propensity of DAG to accumulate within the L2 domain. A mutation in the L2 region (G652A) significantly altered activation kinetics in response to an initial OptoDArG photoisomerization. This 1st activation kinetics was best fitted by exponential functions with a power $(\mathrm{N})$ in the range of 2-4 for wild type channels. Both the time constant as well as $\mathrm{N}$ was found significantly altered in the L2 mutant. In contrast, several mutations in L1 retained wild-type-like kinetics. Our data identify L2 as a main regulatory site in TRPC3, and indicate a potential asymmetry of the gating structures.

\section{P-321}

Structural organization and dynamics of the endocytic protein FCHo2 on membranes

F El Alaoui [1], I Casuso [2], D Sanchez-Fuentes [1], C André-Arpin [1], R Rathar [1], V Baecker [1,2], A Castro [1], T Lorca[1], J Viaud [1], S Vassilopoulos [2], A CarreteroGenevrier [1], L Picas [1]

[1] CNRS, France; [2] INSERM, France

Clathrin-mediated endocytosis is a paradigm of a cellular process that is orchestrated by phosphoinositides, where the plasma membrane phosphatidylinositol-4,5-bisphosphate $\left(\mathrm{PI}(4,5) \mathrm{P}_{2}\right)$ plays an instrumental role in the formation of clathrin-coated structures. Among the early endocytic proteins that reach at the plasma membrane, the F-BAR domain only protein 1 and 2 complex (FCHo1/2) is thought to orchestrate the early stages but, the exact mechanism triggering its nucleation on membranes is not well understood. By combining bottom-up synthetic approaches on in vitro and cellular membranes, we show by sub-diffraction microscopy and High-Speed Atomic Force microscopy the molecular dynamics of FCHo2 self-assembly on membranes. Our results indicate that $\mathrm{PI}(4,5) \mathrm{P}_{2}$ domains assist $\mathrm{FCHo} 2$ docking at specific regions of the membrane, where it self-assembles into ring-like shape protein patches. We show that binding of FCHo2 on cellular membranes promotes the enrichment of $\mathrm{PI}(4,5) \mathrm{P}_{2}$ at the boundary of cargo receptors, such as the epidermal growth factor receptor (EGFR), and that this accumulation enhances the formation of clathrin spots. Thus, our results provide a mechanistic framework that could explain recruitment of early $\mathrm{PI}(4,5) \mathrm{P}_{2}$-interacting proteins at endocytic sites.

\section{P-323}

$\alpha$-synuclein binding on lipid membranes as a function of lipidic composition, $\mathrm{pH}$ and salt concentration

Marco Fornasier [1], Ricardo Gaspar [1,2], Jon Pallbo [1], Aleksandra Dabkowska [1], Kira Bartnik [1], Ulrich Weininger [3], Anders Malmendal [2], Ulf Olsson [1], Sara Linse [2], Peter Jönsson [1], Emma Sparr [1]

[1] Division of Physical Chemistry, Lund University, Lund, Sweden; [2] Department of Biochemistry and Structural Biology, Lund University, Lund, Sweden; [3] Institute of Physics, Martin-Luther-University Halle-Wittenberg, Germany

Understanding the interactions between $\alpha$-synuclein and lipid membranes can shed light on the protein's function in vivo and its role in neurodegenerative diseases. We investigated on the interaction between $\alpha$-synuclein and anionic lipid bilayers of different composition using complementary bulk and surface techniques. NMR and CD experiments identified that the N-terminus is in direct contact with the membrane in an $\alpha$-helical conformation. The binding of $\alpha$ synuclein on supported lipid bilayers was evaluated using QCM-D and TIRF fluorescence microscopy under different conditions ( $\mathrm{pH}$ and salt concentration). The QCM-D experiments showed that the binding was stronger for bilayers containing PS and at $\mathrm{pH} 5.5$ without added salt. TIRF studies highlighted that the mobility of the protein and its adsorption onto the SLB are reduced by increasing the $\mathrm{pH}$ of the buffer for each lipid composition. These investigations show the significance of lipid composition, $\mathrm{pH}$ and salt concentration as key parameters to study lipid- $\alpha$-synuclein interactions in vivo. 


\section{Posters}

- 20. Lipid-Protein interactions -

\section{P-324}

Unraveling the phospholipid identity of the gene expression compartments by single molecule localization microscopy

Peter Hoboth [1,2], Martin Sztacho [1], Ondrej Sebesta [2], Pavel Hozák [1]

[1] Institute of Molecular Genetics ASCR, Prague, Czech Republic; [2] Charles University, Prague, Czech Republic

Current models of gene expression acknowledge protein clustering and formation of transcriptional condensates as a driving force of gene expression. These models are mostly based on single-molecule localization microscopy (SMLM) which provided an unprecedented insight into the sub-nuclear organization of proteins and nucleic acids. However, the roles of nuclear lipids in the establishment of the functional nuclear architecture, apart from the nuclear envelope, has been neglected. Nevertheless, accumulating evidence suggests the involvement of nuclear lipids and particularly of phosphatidylinositol phosphates (PIPs) in gene expression. We used quantitative SMLM for the evaluation of the nuclear PIP distribution while preserving the context of nuclear architecture. We showed phosphatidylinositol 4,5-bisphosphate $(\mathrm{PI}(4,5) \mathrm{P} 2)$, phosphatidylinositol 3,4-bisphosphate $(\mathrm{PI}(3,4) \mathrm{P} 2)$ and phosphatidylinositol 4monophosphate $(\mathrm{PI}(4) \mathrm{P})$ within nuclear speckles and in the nucleoplasmic foci. Moreover, we found $\mathrm{PI}(4,5) \mathrm{P} 2$ and $\mathrm{PI}(3,4) \mathrm{P} 2$ in the close proximity with the subset of RNA polymerase II (RNAPII) foci either in the nucleoplasm or nuclear speckles. We continue to uncover the PIP fingerprints in the subsequent stages of RNAPII transcription. Our efforts aim at elucidating the roles of nuclear PIPs in the compartmentalization of gene expression.

\section{P-326}

The STIM1 SOAR $\alpha 2$ domain controls multiple steps in the CRAC channel activation cascade Carmen Höglinger [1], Herwig Grabmayr [1], Lena Maltan [1], Ferdinand Horvath [2], Heinrich Krobath [2], Martin Muik [1], Adela Tiffner [1], Thomas Renger [2], Christoph Romanin [1], Marc Fahrner [1], Isabella Derler [1]

[1] Institute of Biophysics, JKU, Austria; [2] Institute of Theoretical Physics, JKU, Austria

The calcium release-activated calcium channel is composed of STIM1, a $\mathrm{Ca}^{2+}$ sensor in the endoplasmic reticulum (ER), and the $\mathrm{Ca}^{2+}$ ion channel Orail. $\mathrm{Ca}^{2+}$ store depletion triggers a conformational transition within the STIM1 protein, leading to STIM1 homomerization as well as coupling to and gating of Orai1. When activated, the STIM1 C-terminus takes on an extended conformation which exposes the socalled CAD/SOAR region for coupling to Orai1. In this study, we discovered the multiple roles of a small alpha helical segment (STIM1 $\alpha 2$ ) within CAD/SOAR. It contributes to the stabilization of the STIM1 resting state and controls STIM1 C-terminal activation, STIM1 homomerization as well as STIM1-Orai1 coupling. Using molecular dynamics simulations, we found that a loss-of-function mutation F394D in the $\alpha 2$ region impairs STIM1 activation due to electrostatic interactions between F394D and positively charged head groups of ER membrane phospholipids. Experimentally, we observed that homomerization was impeded only in STIM1 F394D fragments that were directly attached to the ER membrane. Taken together, our findings provide evidence that STIM1 activity is fine-tuned by interactions between CAD/SOAR and the ER membrane.

\section{P-325}

The killer's armour: Physical membrane properties protect cytotoxic lymphocytes from accidental death

Adrian Hodel [1,2], Jesse Rudd-Schmidt [2], Ilia Voskoboinik [2], Bart Hoogenboom [1]

[1] University College London, UK; [2] Peter MacCallum Cancer Centre, Melbourne, Australia

Perforin is a key protein of the vertebrate immune system. Secreted by killer lymphocytes as soluble monomers into an immunological synapse, perforin can self-assemble into oligomeric pores of 10-20 $\mathrm{nm}$ inner diameter in the membranes of virus-infected and cancerous cells. These large pores facilitate the entry of pro-apoptotic granzymes, thereby rapidly killing the target cell. Unlike the target cells, however, cytotoxic lymphocytes remain unscathed in this process, although they are similarly exposed to perforin in the immunological synapse. Using nanoscale imaging by atomic force microscopy, combined with cell-based assays, we have established the pathways of perforin pore assembly and resolved the mystery of how the lymphocytes are protected against the perforin they secrete. We have found that such protection is provided by physical membrane properties: high lipid order repels perforin and, in addition, exposed negatively charged lipids (phosphatidylserine) sequester and inactivate perforin. The resulting perforinresistance of killer lymphocytes explains their ability to kill target cells in rapid succession and to survive these encounters. Furthermore, these mechanisms imply an unsuspected role for plasma membrane organization in protecting cells from immune attack.

\section{P-327}

Alpha-synuclein affects differently the internal and external leaflet of the the lipid membranes Samira Jadavi [1,3], Silvia Dante [2], Alberto Diaspro [1,3], Claudio Canale [3]

[1] Nanoscopy, CHT Erzelli, ITT, Genova, Italy; [2] Materials Characterization Facility, ITT, Genova, Italy; [3] DIFILAB, Department of Physics, University of Genoa, Genova, Italy

It is known that $\alpha$-synuclein ( $\alpha$-syn), a peptide involved in Parkinson's disease, is present in both the inner (cytosol) and extracellular space. Both the endogenous and exogenous components seem to have a role in the neurodegenerative process typical of the disorder. It is also demonstrated that the physiological membrane has an asymmetric structure, i.e., the lipid compositions of the inner and outer leaflet are different. As a consequence of this asymmetry, also the organization of the lipid phase in the two leaflets are different. $\alpha$-syn interacts with supported lipid bilayers (SLBs) with two different compositions, mimicking the inner and the outer leaflet of the cell membrane respectively, to reveal the possible destabilization induced by the pathological peptides. It is known that the presence of the rigid substrate, that support the membrane, reduces the diffusion of the single lipid molecules within the membrane and induce a mechanical stabilization of the membrane, that increase the resistance of the bilayer toward the action of an external agent. We proposed a new approach, making the peptides interact with lipid vescicles in solution, creating a planar bilayer after this interaction. In this way, $\alpha$-syn interacts with a tridimensional membrane, that is better resembling the properties of the cell membrane. Before and after the interaction with $\alpha$-syn the membrane were characterized by atomic force microscopy, quartz micor-balance, and fluorescence techniques. 
Posters

-20. Lipid-Protein interactions -

P-328

The signaling lipid PIP2 promotes specific dimerization of the membrane region of the EphA2 receptor

Stefanski M Katherine, Russell M Charles, Westerfield M Justin, Lamichhane Rajan, Barrera N Francisco

University of Tennessee, USA

The EphA2 receptor can induce anti-oncogenic signaling after ligand binding. However, EphA2 ligand-independent activation is pro-oncogenic. The transmembrane (TM) domain of EphA2 can adopt two alternate conformations in the two activation states. However, it is poorly understood how the different TM helical crossing angles that characterize the two conformations impact the activity and regulation of EphA2. We devised a method that uses hydrophobic matching to stabilize two conformations of a peptide comprising the EphA2 TM domain and a portion of the intracellular juxtamembrane (JM) segment. The two conformations show different TM crossing angles that agree with the ligand-dependent and ligand-independent states of EphA2. We developed a singlemolecule technique that uses SMALP nanodiscs to measure dimerization. We observed that the signaling lipid PIP2 (phosphatidylinositol 4,5-bisphosphate) promotes dimerization, but only in the conformation with a small crossing angle (ligand-independent state). In this conformation the two TM are almost parallel, and the positively charged JM segments are expected to be close to each other and cause electrostatic repulsion. Our results indicate a conformational coupling between the TM and JM regions of EphA2, and suggest that PIP2 regulates EphA2 activation in cells specifically for the ligand-independent conformation of the receptor.

\section{P-330}

Influence of Charged Residues on the Membrane Insertion of Gasdermin A3

Viktoria H Korn, Kristyna Pluhackova

SC SimTech EXC 2075, University of Stuttgart, Germany

Gasdermins are a family of proteins that facilitate pyroptosis, a defense mechanism against infections, by forming pores in membrane bilayers. However, the mechanism by which gasdermins form pores and permeate membranes remains unclear. Molecular dynamics (MD) simulations have become a valuable tool to study such biological processes and unravel their biochemical mechanisms. Earlier MD studies show that charged amino acids change their protonation state while moving through the membrane, leading to smaller free energy barriers. While aspartic and glutamic acid protonate close to the membrane surface with a local free energy barrier of $\approx 2-3 \mathrm{kcal} / \mathrm{mol}$, lysine has to enter deep into the bilayer to deprotonate. As the free energy barrier at this depth amounts to $\approx 6-9 \mathrm{kcal} / \mathrm{mol}$, such a spontaneous deep penetration is a rare event. Here, we investigated the membrane insertion of gasdermin A3 using MD and the impact of protonation state of the Glu94, Lys97 and Lys100 on the free energy barriers of the insertion process. Our results demonstrate that the protein context has substantial impact on the free energy barriers of membrane permeation of charged amino acids. These include the pair formation between Glu94 and Lys100 as well as stabilizing effects initiated by the protein's surroundings. Understanding pore formation in membrane bilayers could not only help us understand cell death more profoundly but open the gate to novel methods of transporting therapeutics into cells.

\section{P-329}

Gramicidin interactions mediated by lipid membrane deformations lead to changes in channel lifetime and monomer-dimer equilibrium

Oleg V Kondrashov [1], Tatyana I Rokitskaya [2], Elena A Kotova [2], Yuri N Antonenko[2], Sergey A Aki$\operatorname{mov}[1]$

[1] Frumkin Institute of Physical Chemistry and Electrochemistry, Russian Academy of Sciences, Russian Federation; [2] Belozersky Institute of Physico-Chemical Biology, Lomonosov Moscow State University, Russian Federation

Gramicidin A (gA) is a well-known pentadecapeptide that forms ion channels - conductive dimers - through transbilayer dimerization of gA monomers. It has been previously demonstrated that the lifetime of tandem gA channels, i.e. couples of gA dimers, constructed from pairs of linked gA monomers in each lipid monolayer, is about 1000 times greater than the lifetime of a single gA dimer. Besides, for gA analogs with $\mathrm{N}$-terminal valine replaced by glycine or tyrosine, the time of relaxation to equilibrium increased, but not decreased, upon elevation of the total membrane conductance, thus contradicting the classical kinetic theory. In the framework of the elastic theory, we calculated lipid membrane deformations near gA molecules. If $\mathrm{gA}$ monomers/dimers are close enough, the deformations produced by them overlap, thereby leading to membranemediated interactions between gA molecules. According to our present results, the lifetime of tandem gA channels and dimerization constant depend on gA concentration. We also demonstrated that these effects may be modulated by incorporation of other peptides into the membrane.

\section{P-331}

Human autophagy-related protein Atg3 contains a membrane-sensing amphipathic helix

Gianmarco Lazzeri, Roberto Covino

Frankfurt Institute for Advanced Studies, Germany

Autophagy is an essential cellular maintenance program involving a concerted choreography between proteins and membranes. In humans, one of the key actors is the protein Atg3. Its N-terminus recognizes and associates to specific lipid membranes. Several in vivo and in vitro studies revealed that binding depends on the curvature and composition of the target membrane. Here, we report 15 us-long atomistic molecular dynamics (MD) simulations revealing that the Atg3's N-terminus contains an amphipathic helix $(\mathrm{AH})$.

The simulations show that the AH stably associates on membranes, where it induces a specific footprint, affecting both the membrane's geometry and lipids' distribution. Additional simulations of a mutated AH show significant alterations in the footprint. This specificity suggests that Atg3's $\mathrm{AH}$ is a fine-tuned sensor that recognizes both membrane curvature and lipid composition. 


\section{Posters}

-20. Lipid-Protein interactions -

\section{P-332}

Protein based targeted delivery systems for antimicrobial PDT

Andrea Mussini [1], Eleonora Uriati [1,2], Pietro Delcanale [1], Paolo Bianchini [2,3], Alberto Diaspro [3,4], Stefania Abbruzzetti [1], Cristiano Viappiani [1], Cormac Hally [5], Montserrat Agut [5], Santi Nonell [5]

[1] Dipartimento di Scienze Matematiche, Fisiche e Informatiche, Università di Parma, Italy; [2] Nanoscopy, Istituto Italiano di Tecnologia, Genoa, Italy; [3] Nikon Imaging Center, Istituto Italiano di Tecnologia, Genoa, Italy; [4] Department of Physics, University of Genoa, Genoa, Italy; [5] Institut Químic de Sarrià, Universitat Ramon Llull, Spain

Water soluble proteins are natural candidates as biocompatible delivery systems for photosensitizers. We report the development of a supramolecular structure endowed with photosensitizing properties and targeting capability for antimicrobial photodynamic inactivation. Our synthetic strategy uses the tetrameric bacterial protein streptavidin, labelled with eosin, as the main building block. Biotinylated immunoglobulin $\mathrm{G}$ ( $\mathrm{IgG}$ ) from human serum, known to associate with $S$. aureus protein A, was bound to the complex streptavidin-eosin. The supramolecular complex was used to demonstrate photoinactivation of $S$. aureus suspensions. FCS and STED nanoscopy experiments confirmed binding of the complex to $S$. aureus. Efficient photoinactivation is observed for $S$. aureus suspensions treated with IgG-streptavidin-eosin at concentrations higher than $0.5 \mathrm{M}$ and exposed to green light. The proposed strategy offers a flexible platform for targeting a variety of molecular species.

\section{P-334}

Cholesterol-dependent substrate binding by gamma-secretase is governed by hydrophobic mismatch

Lukasz P Nierzwicki, Michal M Olewniczak, Pawel Chodnicki, Jacek M Czub

Gdansk University of Technology, Poland

Gamma-secretase is a membrane bound aspartyl protease which is composed of four protein units: presenilin, nicastrin, APH-1 and PEN-2. The enzyme complex mediates regulated intramembrane proteolysis of more than ninety different proteins, including Notch and APP, which lead to development of cancer or Alzheimer's disease when processed improperly. The key groups that are responsible for processing the substrates, aspartic acids 257 and 385 are located in the presenilin unit. Yet despite the extensive research, the mechanism by which gamma-secretase recognizes and cuts other proteins is still unclear. Although it is known that increase of cholesterol content enhances the activity of gamma-secretase, its role in aforementioned processes is also poorly understood. In this work we use all-atom MD simulations to investigate the role of cholesterol in the initial binding of APP by gamma-secretase. Our results suggest that in cholesterol-rich membranes, both the substrate and the enzyme region in the vicinity to the active site induce a local membrane thinning. These results were then compared to the non-raft membrane environment: taken together, our results show that membrane thickness is a key feature in the binding process and it is the negative hydrophobic mismatch between the membrane and substrate that governs this process.

\section{P-333}

Phase behavior of ternary systems - what can we learn about the in vivo state of mixed micelles? Leonie C Naßwetter, Heiko Heerklotz

Institute of Pharmaceutical Sciences, University of Freiburg, Germany

Mixed micelles (MM) composed of a bile salt (BS) and lecithin can serve as formulation vehicles for poorly soluble drugs. Despite various advantages, little is known about their in vivo state after intravenous injection. Dilution and interactions with blood components give room for transformation processes of the vehicle with different possible outcomes. To establish a better mechanistic understanding of these systems, the phase behavior of BS, lecithin (the formulation components) and bovine serum albumin (BSA) as model protein was investigated using Isothermal Titration Calorimetry. By titrating GC to lecithin vesicles and BSA, the phase boundaries at which micelles transform to vesicles have been detected at various BSA concentrations. This allowed the establishment of a ternary phase diagram using a simple plane equation for the saturation and solubilization phase boundaries. The diagram shows which concentration compositions of BS, lecithin and BSA lead to the existence of MM, liposomes or the coexistence of both. At theoretical concentrations, reached through the dilution of a typical MM formulation (120 mM GC, $100 \mathrm{mM}$ lecithin) to the plasma volume $(\sim 3 \mathrm{l})$, the saturation phase boundary is distinctly crossed. Accordingly, the possibility of persisting MM in the blood system can be excluded. Although not a perfect in vivo model, it is a first step towards a possible estimation of the actual state of such formulations after application.

\section{P-335}

Study of the interaction of dendrons with liposomes and lipid monolayers

Veronika Oravczová, Šimon Šutý, Veronika Šubjaková, Zuzana Garaiová, Iveta Waczulíková, Tibor Hianik

Faculty of Mathematics, Physics and Informatics, Comenius University, Slovakia

Dendrons are nanosized dendritic structures with good potential in medical applications such as cancer therapy. Their hyperbranched structure offers plenty of terminal groups, which are suitable for further functionalization in targeted drug delivery. We studied interactions between dendrons and model lipid membranes such as liposomes and monolayers composed from DMPC/DMPG/Cholesterol (Lipid:Cholesterol $=70: 30$ weight \%). We used a first (jq108) and a second (jq111) generation of dendrons in various concentrations ranging from $0.1 \mu \mathrm{M}$ to $15.0 \mu \mathrm{M}$ at temperatures $25^{\circ} \mathrm{C}$ and $37^{\circ} \mathrm{C}$. We found that dendrons interacted with liposomes and changed their size and Zeta potential depending on the concentration applied. The liposome size varied from $250 \mathrm{~nm}$ to $1 \mu \mathrm{m}$ following addition of $6-12.5 \mu \mathrm{M}$ (jq108) or 3-12.5 $\mathrm{\mu M}$ (jq111), which suggests formation of aggregates at higher dendron concentrations. Simultaneously we observed an increase of Zeta potential from approximately -40 $\mathrm{mV}$ up to almost $-7 \mathrm{mV}$ with increasing concertation of dendrons. We also measured the change in the surface tension of lipid monolayers using Langmuir-Blodgett trough after injection of jq108 into the water subphase in the concentration range $1.0 \mu \mathrm{M}-10.0 \mu \mathrm{M}$. The initial surface tension of the lipid monolayer simulated surface tension typical for cancer or healthy cells $(20 \mathrm{mN} / \mathrm{m}$ and $30 \mathrm{mN} / \mathrm{m}$ respectively). Addition of dendrons resulted in a significant increase of surface tension under all experimental conditions even at low dendron concentrations. 
Posters

- 20. Lipid-Protein interactions -

\section{P-336}

Lipid membrane as an interaction interface of the two major listerial virulence factors

Nejc Petrišič, Maksimiljan Adamek, Andreja Kežar, Gregor

Anderluh, Marjetka Podobnik

National Institute of Chemistry, Ljubljana, Slovenia

Broad-range phospholipase C (PC-PLC) is a zinc metalloenzyme and a major virulence factor of the bacterial pathogen Listeria monocytogenes. PC-PLC, together with the cholesterol-dependent pore-forming toxin listeriolysin $\mathrm{O}$ (LLO) and other bacterial and host proteins, disintegrates the lipid membrane of the phagosome and releases the pathogen into its replicative niche, the cytosol. PC-PLC cleaves different phospholipids and sphingomyelin. We recombinantly produced the active PC-PLC, determined its previously unknown crystal structure, and constructed mutants exhibiting varying degrees of phospholipase activity. We investigated the interaction between PC-PLC and LLO via the lipid membrane by pre-incubating POPC/cholesterol lipid vesicles with PC-PLC. Enzyme activity increased LLO binding to liposomes and LLO-induced vesicle leakage, whereas PC-PLC alone did not cause any permeabilization. Preincubation with less active PC-PLC mutants resulted in reduced LLO binding and vesicle leakage. In addition, PCPLC preincubation also increased the rate of LLO-induced hemolysis. We hypothesize that PC-PLC may increase membrane cholesterol availability by cleaving polar head groups of lipids, thereby exposing membrane cholesterol. Our results suggest an important interplay of listerial virulence factors in a key pathophysiological step of L. monocytogenes phagosomal escape.

\section{P-338}

Tilt-curvature coupling and curvature gradient contributions to elastic energy of lipid membranes Konstantin V Pinigin, Petr I Kuzmin, Sergey A Akimov, Timur R Galimzyanov

A.N. Frumkin Institute of Physical Chemistry and Electrochemistry, Russian Academy of Sciences, Moscow, Russian Federation

The energy of lipid bilayer deformations controls the principal features of numerous cell processes. Although various phenomenological geometrical theories of membrane elasticity were developed to address these issues, they lack a microscopic physical rationale behind the deformation modes taken into account in energy functionals, especially concerning such a small-scale deformation mode as tilt, i.e. the deviation of lipid molecules from the local membrane normal. Here, we further elaborate a classical microscopic continuum model developed by Hamm and Kozlov [Eur. Phys. J. E $3,323(2000)]$ and later revised by Terzi and Deserno [J. Chem. Phys. 147, 084702 (2017)]. We show that the transverse shear mode gives rise not only to the tilt squared as was previously considered but also to the following quadratic terms: the square of gradients of curvature, of stretching, and their coupling with tilt. We analyze the influence of these energy contributions on the membrane-mediated interaction of amphipathic peptides. The curvature gradient solves the problems of membrane instability and divergence of fluctuation spectra of the previously derived energy functional. We also show that the Gaussian curvature causes membrane instability and should be either neglected or included together with the Gaussian curvature squared.

\section{P-337}

Hydrolytic activity of OmpLA in symmetric and asymmetric lipid membranes

Paulina Piller, Nadine Angerer, Enrico F Semeraro, Gerald N Rechberger, Sandro Keller, Georg Pabst

Institute of Molecular Biosciences, University of Graz, Austria

The outer membrane phospholipase A (OmpLA) is an integral membrane enzyme located in the outer membrane of Gram-negative bacteria, which catalyzes the hydrolysis of phospholipids. We reconstituted OmpLA in symmetric liposomes composed of palmitoyl oleoyl phosphatidylcholine (POPC) and in asymmetric large unilamellar vesicles (aLUVs), with an inner leaflet containing POPC and an outer leaflet enriched in palmitoyl oleoyl phosphatidylethanolamine (POPE). First, we focused on the effect of lipid asymmetry on protein activity using a wellestablished colorimetric assay. Results indicated a significantly reduced activity of OmpLA in aLUVs. However, the assay uses 2-hexadecanoylthio-1-ethylphosphorylcholine (HEPC), which may, due to its detergent-like nature, lead to increased lipid flip/flop affecting this observation. We therefore developed a second assay based on high performance thin layer chromatography (HPTLC) sensitive to unsaturated hydrocarbons. Focusing on the formation of lyso-PC and -PE, we report first insights into the differential activity of the protein in symmetric and asymmetric lipid membranes as a function of OmpLA concentration.

\section{P-339}

Molecular dynamics and lipid interactions of the complete $\mathrm{T}$-cell receptor in a plasma membrane model

Dheeraj Prakaash [1,2], Graham P Cook [3], Oreste Acuto [4], Antreas C Kalli [1,2]

[1] Leeds Institute of Cardiovascular and Metabolic Medicine, University of Leeds, UK; [2] Astbury Center for Structural Molecular Biology, University of Leeds, UK; [3] Leeds Institute of Medical Research, University of Leeds, UK; [4] Sir William Dunn School of Pathology, University of Oxford, UK

The T-cell receptor (TCR) is a membrane protein embedded in the T-cell plasma membrane which recognizes foreign antigens presented by Major Histocompatibility Complexes (MHC). The TCR is an octameric complex consisting of the TCR $\alpha \beta$, CD $3 \varepsilon \gamma$, CD $3 \varepsilon \delta$ and $\zeta \zeta$ dimers, where the TCR $\alpha \beta$ directly interacts with MHCs, and the CD3 and $\zeta \zeta$ dimers allosterically propagate signals to the intracellular region. The structure of the TCR intracellular region remains unknown and challenging to resolve using structural techniques due to its dynamic nature. This limits our understanding of the T-cell signalling mechanism. Here, we employed in silico molecular modelling to obtain the complete structure of the TCR and performed molecular dynamics simulations to study its dynamics when embedded in a complex membrane mimicking the TCR activation membrane domain. Our simulations provide novel insights into the dynamics of the complete TCR at near-atomic resolution and reveal preferential interactions and clustering of phosphatidylinositol-4,5biphosphate lipids around the TCR which could be key in recruiting signalling proteins to the activation site. 


\section{Posters}

-20. Lipid-Protein interactions -

P-340

Binding of Histidine tagged molecules to vesicles with DGS-NTA (Ni) anchor lipid

Shreya Pramanik [1], Jan Steinkühler [1], Rumiana Dimova [1], Joachim Spatz [2], Reinhard Lipowsky [1]

[1] Max Planck Institute of Colloids and Interfaces, Potsdam, Germany; [2] Max Planck Institute for Medical Research, Heidelberg, Germany

Giant unilamellar vesicles (GUV) are model systems to study simple cell membranes. Peripheral proteins on the cell membranes are important for signaling and membrane trafficking. Binding of peripheral proteins to the GUV membrane can be achieved by direct interaction with specific lipids. Histidine tag molecules attach to 1,2-dioleoyl-sn-glycero3 -[(N-(5-amino-1-carboxypentyl)iminodiacetic acid)succinyl] (nickel salt) DGS-NTA (Ni) lipids forming an octahedral coordinate complex. DGS-NTA (Ni) lipids have been used in many studies for biofunctionalisation of GUVs but what is missing is a comparison of the different protocols of GUV formation and their efficiency of binding the histidine tagged molecules. In this study we have explored different protocols for the preparation of GUVs as well as different solution conditions which affect the binding of two histidine tagged molecules, green fluorescent protein (GFP) and Fluorescein isothiocyanate (FITC), to the NTA lipids. This study is important to explore the effect of proteins bound to lipid membrane at low densities, i.e. in a dilute, non-crowding regime.

\section{P-342}

Lipid interaction with the nucleoid occlusion protein SlmA modulated by DNA and FtsZ

Miguel Ángel Robles-Ramos [1], William Margolin [2], Marta Sobrinos-Sanguino [1], Carlos Alfonso [1], Germán Rivas [1], Begoña Monterroso [1], Silvia Zorrilla [1]

[1] Centro de Investigaciones Biológicas Margarita Salas, Consejo Superior de Investigaciones Científicas (CSIC), Spain; [2] Department of Microbiology and Molecular Genetics, McGovern Medical School, University of Texas, USA

Successful division in E. coli relies on precise spatiotemporal positioning of the divisome at midcell by multiple regulatory systems, one of them being nucleoid occlusion mediated by the effector protein SlmA. This key mechanism inhibits the formation of the divisome by blocking assembly of the essential protein FtsZ in the vicinity of SlmA bound to its chromosomal DNA binding sites (SBSs), preventing constriction over unsegregated nucleoids during cytokinesis and hence protecting the chromosome from aberrant scission. Following an orthogonal approach involving reconstruction in minimal membrane systems, we have found that SlmA binds to lipids matching the composition of the $E$. coli inner membrane. Thus, the interaction of SlmA with lipids and its modulation by its binding partners SBS and FtsZ was studied through encapsulation in cell-like containers generated by microfluidics, lipid-coated microbead assays, fluorescence anisotropybased competition experiments and biolayer-interferometry. The emergence of the lipid membrane as a new binding partner of SlmA, with SBS and FtsZ tuning that interaction, should shed new light on our understanding of the function of this regulatory factor.

\section{P-341}

Role of seipin in lipid droplet biogenesis

Xavier Prasanna, Veijo Salo, Shiqian Li, Katharina Ven, Helena Vihinen, Eija Jokitalo, Ilpo Vattulainen, Elina Ikonen University of Helsinki, Finland

Lipid droplets (LDs) serve as reservoirs of neutral lipids for metabolic energy and membrane components. However, excess storage of neutral lipids underlies metabolic diseases, such as obesity. Synthesis of LDs occurs in the endoplasmic reticulum (ER). LDs have a core of neutral lipids surrounded by a phospholipid monolayer. One of the key proteins involved in $L D$ biogenesis is seipin which plays an essential role, especially in its formation and maturation. Cryo-EM structure of seipin oligomer has been elucidated recently and helps suggest possible functional role. The underlying mechanism, however, is largely unknown.

Using experiments and molecular dynamics simulations, we have shown that seipin segregates triacylglycerol (TAG) within its ring-like oligomeric structure. The membrane associated helices in the luminal domain serves as a nucleation site for LD aggregation. We identified S166 to have a high affinity towards TAG and mutating this residue largely reduces the ability of seipin to sequester TAG within its lumen. Mutant seipin induces ER membrane deformation and shows reduced association with promethin. Our findings provide valuable insight into the moleular mechanism of seipin in initiation of LD formation.

\section{P-343}

Structural characterization of a pre-fibrillar $\alpha$ Synuclein aggregation intermediate

Vrinda Sant, Leif Antonschmidt, Kumar T Movellan, Riza Dervisoglu, Stefan Becker, Loren Andreas, Christian Griesinger

Max Planck Institute for Biophysical Chemistry, Göttingen, Germany

$\alpha$-Synuclein $(\alpha S)$ aggregation is a key event implicated in neurodegeneration. We isolated and characterized a prefibrillar $\alpha \mathrm{S}$ intermediate during aggregation in the presence of negatively charged phospholipids. We show prolonged stability of the intermediate when free monomer is depleted making three-dimensional solid-state NMR measurements possible. De novo backbone assignments were obtained using proton detected NMR spectroscopy. Proton-proton z-mixing experiments reveal the lipid and water exposure profile along the $\alpha \mathrm{S}$ sequence, specific for the pre-fibrillar aggregation intermediate. The secondary structure of the pre-fibrillar intermediate is predominantly $\beta$-sheet and it has a Thioflavin$\mathrm{T}$ reactivity $\sim 20$ times less than for the fibril. These results are a step towards understanding the atomic scale characteristics of a misfolded nucleus responsible for rapid aggregation of amyloid fibrils. In the presentation, more details of the structure will be revealed. 
Posters

- 20. Lipid-Protein interactions -

P-344

Regulation of actin dynamics by phosphoinositides

Yosuke Senju [1], Maria Kalimeri [2], Ilpo Vattulainen [2], Pekka Lappalainen [2]

[1] Okayama University, Japan; [2] University of Helsinki, Finland

The actin cytoskeleton provides forces for vital cellular processes involving membrane dynamics. Membrane phosphoinositides regulate many actin-binding proteins including cofilin, profilin, mDia2, N-WASP, ezrin and moesin, but the underlying mechanisms have remained elusive. By applying a combination of biochemical assays, photobleaching/activation approaches, and atomistic molecular dynamics simulations, we revealed that these proteins interact with membranes through multivalent electrostatic interactions, without specific binding pockets or penetrations into the lipid bilayers. However, their membrane-binding kinetics differ drastically. Cofilin and profilin exhibit transient, lowaffinity interactions with phosphoinositide-containing membranes, whereas $\mathrm{F}$-actin assembly factors $\mathrm{mDia} 2$ and $\mathrm{N}$ WASP reside on phosphoinositide-containing membranes for longer periods to perform their functions. Ezrin and moesin, which link actin cytoskeleton to the plasma membrane, bind membranes with very high affinities and slow dissociation dynamics, and do not require high stimulusresponsive phosphoinositide density for membrane binding. Thus, membrane-interaction mechanisms of actin-binding proteins evolved to precisely fulfill their specific functions in cytoskeletal dynamics.

\section{P-346}

Molecular insight into the mechanism of nuclear PIP2 regulation of RNA Polymerase II transcription

Martin Sztacho [1], Can Balaban [1], Barbora Šalovská [1], Jakub Červenka [2], Pavel Hozák [1]

[1] Institute of Molecular Genetics of the Czech Academy of Sciences, Czech Republic; [2] Institute of Animal Physiology and Genetics of the Czech Academy of Sciences, Czech Republic

Specific nuclear sub-compartments that are regions of fundamental processes such as gene expression or DNA repair, contain phosphoinositides (PIPs). PIPs potentially represent signals for the localization of specific proteins into different nuclear functional domains. We performed limited proteolysis followed by label-free quantitative mass spectrometry and identified nuclear protein effectors of phosphatidylinositol 4,5-bisphosphate (PIP2). We identified 515 proteins with PIP2-binding capacity. Gene ontology analysis revealed that these proteins are involved in regulation of $\mathrm{Pol}$ II, mRNA splicing, transport and cell cycle. They localize to non-membrane bound organelles and are connected to actin nucleoskeleton. We provided the evidence for presence of MPRIP, an F-actin-binding protein in the cell nucleus. The MPRIP protein binds to PIP2 and localizes to the nuclear speckles and nuclear lipid islets which are known to be involved in transcription. We identified MPRIP as a component of Pol2/Nuclear Myosin 1 complex and showed that MPRIP forms phase-separated condensates which are able to bind nuclear F-actin fibers.

\section{P-345}

The structure of Gb3 receptors influences their partitioning in membranes: effect on bacterial Shiga Toxin B insertion in lipid monolayers Larissa Socrier [1,2], Celine Bail [2], Elena Ackermann [2], Ann-Kathrin Beresowski [2], Daniel Werz [3], Ahadi Somayeh [3], Claudia Steinem [1,2]

[1] Max Planck Institute for Dynamics and SelfOrganization, Germany; [2] Georg-August-Universität, Institute of Organic and Biomolecular Chemistry, Germany; [3] Technische Universität Braunschweig, Institute of Organic Chemistry, Germany

Infections with Shiga Toxin (STxB) producing bacteria constitute a worldwide public health issue as they cause hundreds of thousands of deaths each year. Therefore, it is of great importance to investigate the interactions between STxB and globotriaosylceramide $\left(\mathrm{Gb}_{3}\right)$ receptors as well as the molecular mechanisms leading to the internalization of bacteria into cells. The phase behavior of $\mathrm{STxB}$ and $\mathrm{Gb}_{3}$ lipids has widely been studied in our group over the last few years. After binding, STxB was evidenced to be present in the liquid-ordered phase of the membrane but the localization of $\mathrm{Gb}_{3}$ lipids prior to binding remained unclear.

In the present work, we investigated $\mathrm{Gb}_{3}$ distribution in phase-separated monolayers prior to Shiga Toxin binding as well as its impact on the insertion of the protein in membranes. Interestingly, $\mathrm{Gb}_{3}$ partitioning did not influence Shiga Toxin penetration in monolayers, suggesting proteinprotein lateral interactions prevail regarding cluster formation leading to the internalization of the toxin into cells.

Correspondance : larissa.socrier@ds.mpg.de

\section{P-347}

Membrane composition affects the binding of pore-forming $\gamma$-hemolysin monomers

Thomas Tarenzi [1,2], Raffaello Potestio [1,2]

[1] University of Trento, Trento, Italy; [2] INFN-TIFPA, Trento, Italy

Pore-forming toxins (PFTs) are one of the largest classes of bacterial virulence factors. Once secreted from the bacterium in the extracellular space, they undergo a functional rearrangement that leads inactive, soluble, and monomeric proteins to assemble as a multimeric pore in the membrane of the target cell [1]. PFTs are currently exploited in different fields of biomedicine and nanotechnology; in particular, targeted cytolysis of malignant cells represents a promising field of application of PFT-based immunotoxins for cancer treatment [2]. Here, through a large set of molecular dynamics simulations at different levels of resolution, we investigate the effect of membrane composition on the ability of LukF, a monomer of the bicomponent PFT gamma-hemolysin and the one that initiates membrane anchoring, to steadily bind the lipid bilayer. In addition, a comparison between the monomer in solution and in the bound form is performed in terms of conformations, key interactions, and intra-protein network analysis. Results from the simulations are compared to experimental data of gamma-hemolysin pore formation on model membranes. These insights in the mechanism of transition between soluble and membrane bound-forms and the role played by the lipids can be instrumental in the design of PFT-analogs with enhanced efficacy.

[1] Dal Peraro et al. (2016) Nat. Rev. Microbiol., 14(2).

[2] Allahyari et al. (2017) Tumor Biol., 39(2). 
Posters

-20. Lipid-Protein interactions -

P-348

Evidence for PIP2 interaction with TRPC3 as a requirement for inward rectification Oleksandra Tiapko [1], Amy Clarke [2], Hazel Erkan [1], Thomas Stockner [2], Klaus Groschner [1]

[1] Medical University of Graz, Austria; [2] Medical University of Vienna, Austria

TRPC3 is a distinct member of TRPC channel family with significant constitutive activity at the cells resting state. Physiological activation of TRPC3 involves direct interaction of DAG produced by $\mathrm{PIP}_{2}$ hydrolysis. $\mathrm{PIP}_{2}$ by itself is considered as a positive regulatory lipid for TRPC3, but direct binding has not been shown. Therefore, we performed MD simulations of TRPC3-lipid interactions and identified a potential $\mathrm{PIP}_{2}$ binding region, which is tightly linked to the cytoplasmic TRP helix and features a cluster of positively charged residues. Neutralization of four charged residues (R374, K377, R380 and K385) by alanine preserved plasma membrane targeting of the proteins. Electrophysiological characterization revealed impaired physiological activation of R374, R380 and K385 mutant channels. However, solely K385A displayed significantly reduced basal currents and reduction of inward current when challenged with the synthetic agonist GSK1702934A. $\mathrm{PIP}_{2}$ depletion by a membrane-targeted $\mathrm{PIP}_{2}$ scavenger reduced inward currents through TRPC3wt and generated a rectification pattern similar to the K385A phenotype. Importantly, the $\mathrm{PIP}_{2}$ scavenger lacked any effects on K385A channels. We suggest that K385A introduces a conformational state in TRPC3 similar to that of reduced $\mathrm{PIP}_{2}$ lipidation. We propose that $\mathrm{PIP}_{2}$ governs the function of TRPC3 by affecting the conformation of the channel's inner vestibule to alter inward current-to-voltage rectification.

\section{P-350}

Membrane order change due to lipoprotein particles

Florian Weber [1,3], Markus Axmann [1], Taras Sych [3], Herbert Stangl [2], Erdinc Sezgin [3], Birgit Plochberger [1]

[1] University of Applied Sciences Upper Austria, School of Medical Engineering and Applied Social Sciences, 4020 Linz, Austria; [2] Center for Pathobiochemistry and Genetics, Institute of Medical Chemistry and Pathobiochemistry, Medical University Vienna, 1090 Vienna, Austria; [3] Karolinska Institutet, Department of Women's and Children's Health, 17177 Stockholm, Sweden

Lipoprotein particles (LPs) transport cholesterol within the body. LPs interact with membranes and transfer their cargo even in the absence of receptors. However, it remains unclear to what extent receptor-independent cargo transfer, as well as the influence of target membrane properties, contribute to the overall LP transport yield. Here we present two aspects, first, how LP cargo transfer alters the properties of the target membrane, and second, which membrane properties enable the interaction in the first place. We use fluorescence correlation spectroscopy, spectral imaging, and spectral fluorescence automated cell sorting to determine the transfer efficiency as well as the membrane order change due to LP interaction. We use bead-supported lipid bilayers with variable composition and thus biophysical properties as mimics of the target cell membrane. Thus, we can show that, on the one hand, the lipid properties of the head groups as well as the fatty acid chains of the target membrane have an influence on the interaction with LP, and that, on the other hand, these changes the composition of the target membrane.

\section{P-349}

Mapping amino acids at protein-membrane interfaces to update the current membrane binding model

Thibault Tubiana [1,2], Nathalie Reuter [1,2]

[1] Department of Chemistry, University of Bergen, Norway; [2] Computational Biology Unit, University of Bergen, Norway

Peripheral Membrane Proteins (PMP) are essential components of multiple biological processes like lipid metabolism, membrane remodelling and other complex cellular pathways. They bind transiently to the surface of biological membranes through short helices or loops, and their Interfacial Binding Site (IBS) has been described as displaying basic and hydrophobic amino acids. However, recent studies indicate that this model is too simplistic to account for the fine-tuned lipid specificity achieved by many PMPs.

Terms like "hydrophobic spikes" or "protruding loops" have been used to describe PMP IBS. Recently we proposed a hydrophobic protrusion model for IBSs. This model is based on the calculation of the protein convex hull and its vertices and captures a structural pattern common to PMP families. Yet it does not have the resolution to capture fine differences between families, and these differences may be decisive for their membrane specificity. In order to characterize the IBS at a finer resolution, we collected a curated dataset of structures from the CATH database. We developed a statistical framework and could map the composition of the IBS in several PMP superfamilies. We will present and discuss our results and assess how we can relate them to certain domains function. 
Posters

-21 . DNA architecture and gene regulation -

\section{P-351}

Simulation studies of DNA denaturation dynamics Amal J Al Qanobi, Issam A Al Lawatia

Sultan Qaboos University, Oman

We use Langevin dynamics simulations to study the dynamics of DNA denaturation. We use a coarse-grained single nucleotide model for dsDNA which is implemented in LAMMPS (Large scale Atomic/Molecular Massively Parallel Simulator). The model considers the characteristic helical structure. We study the melting dynamics of a free DNA of length $300 \mathrm{pb}$ in the melting temperature $70 \mathrm{C}$ as well as one that is fixed from one side. We find the melting is easier and faster when the DNA is free to move rather than when it is restricted from one end of the DNA. In addition, we investigate the size distribution of bubbles formed during the melting process. We find a maximum bubble size of about $50 \mathrm{bp}$ on average for a free DNA ( $1 / 6^{\text {th }}$ of the DNA length) and about $100 \mathrm{pb}$ for a DNA fixed from one side $\left(1 / 3^{\text {rd }}\right.$ of the DNA length), with bubbles of size $1 \mathrm{bp}$ most prevalent. The number of bubbles appearing in a DNA fixed from one side is noticeably larger than that appearing in a free DNA. It is entropically advantageous to create more bubbles when the DNA is fixed from one side.

\section{P-353}

Nanoscale investigation of Nucleoplasmic Reticulum and chromatin organization

Isotta Cainero [1], Francesca Baldini [1], Chantal Usai [1,2], Paolo Bianchini [1], Alberto Diaspro [1,2]

[1] Nanoscopy, Istituto Italiano di Tecnologia, Italy; [2] DIFILAB, University of Genoa, Italy

Chromatin organization and maintenance is fundamental to ensure proper genome expression. Therefore, changes in its architecture can lead to different conditions including cancer. In this context, the Nucleoplasmic Reticulum (NR) has an important role not only in chromatin organization by anchoring chromatin itself, but also in chromatin regulation by being involved in many fundamental cellular processes such as DNA replication, DNA transcription and in the DNA Damage Repair. Despite NR recent discovery its role remains unclear. The NR is an extension of the endoplasmic reticulum inside the cell nucleus. It consists in a complex network of Nuclear Envelope invaginations, including nuclear membranes and Lamins proteins which interact with chromatin. Our aim is to investigate the relation between NR and chromatin distribution in interphase cell nuclei. In particular, we perform staining of Lamins and histone's modification markers for euchromatin and both constitutive and facultative heterochromatin. We visualize and analyze the data collected with Super-Resolution imaging techniques, such as SIM and STED, to investigate the nanoscale spatial arrangement of NR and chromatin structure in live and fixed cells The final aim of this work will be characterizing the functional role of NR and nuclear Lamins in relation to the organization and regulation of fundamental cell processes.

\section{P-352}

A continuous complete RNA translocation cycle by the DEAH-box helicase Prp43 in atomic detail Robert A Becker, Jochen S Hub

University of Saarland, Germany

Understanding conformational cycles of complex macromolecular machines in atomic detail remains a central goal of molecular biophysics. Here, we focus on helicases that are crucial for every living organism to carry out functions such as DNA/RNA transcription, translation, DNA/RNA repair, recombination and splicing. The largest group among helicases is the Superfamily 2 (SF2), which includes the DEADand DEAH-box helicases as key players in the splicing pathway. Despite the wide interest in understanding the detailed mechanism of ssRNA translocation during splicing, the exact movements are still unknown. Using molecular dynamics simulations and enhanced sampling techniques, we observed a complete RNA translocation cycle of the DEAH-box helicase Prp43 in atomic detail. The simulations reveal the collective behaviour of the three main domains RecA1, RecA2 and CTD, like the detachment and the formation of the interface of the RecA domains or the rotation of the CTD. Additionally, the simulations give detailed insight in the essential and atomistic processes during the large domain motions, e.g. a movement cascade induced by an arginine finger in the ATP binding side, the conformational change of a serine loop to a helical state, the cleavage and formation of various hydrogen-bonds, including the so called Hook-loop and Hook-turn, the behaviour of the ssRNA during the process and more.

\section{P-354}

Soft DNA Curtains: high-throughput SM biophysics method to investigate protein-DNA interaction Aurimas Kopūstas [2,1], Tomas Rakickas [1], Justè Paksaitè [2], Ernesta Pocevičiūtè [1,2], Tautvydas Karvelis [2], Mindaugas Zaremba [2], Elena Manakova [2], Marijonas Tutkus [2,1]

[1] FTMC, Lithuania; [2] Vilniaus University, Institute of biotechnology, Lithuania

During last 20 years, single-molecule (SM) fluorescence microscopy methods have evolved and widely spread allowing answering many biological problems. SM fluorescence methods enables monitoring various dynamic interactions, conformational fluctuations, and movements of biomolecules. Next-generation in vitro SM biophysics approaches are characterized by a much greater complexity. One well-working method that belongs to the next-generation approaches is called DNA curtains.

Here I will present a strategy that we recently started to employ in our laboratory to create a nanoscale platform for protein-DNA interaction studies at the SM level - Soft DNA Curtains. This platform relies on stretching of long DNA molecule along the surface using the buffer flow. Biotin and digoxigenin (dig) labeled DNA molecules are immobilized on the nanoscale lines, composed of Streptavidin or Traptavidin protein, formed on the surface of silanized and PEGylated glass coverslip. The second DNA end is treated using biotinylated anti-dig antibodies. These immobilized DNA molecules can be fluorescently labeled. I will present our newest results of fluorescently labeled CRISPR-Cas proteinDNA interaction studies. 


\section{Posters}

-21 . DNA architecture and gene regulation -

P-355

Soft DNA Curtains platform for single-molecule level DNA-protein interaction studies

Aurimas Kopūstas [1,2], Tomas Rakickas [1], Justè Paksaitė [2], Ernesta Pocevičiūtè [1,2], Tautvydas Karvelis [2], Mindaugas Zaremba[2], Elena Manakova [2], Marijonas Tutkus $[1,2]$

[1] Center for Physical Sciences and Technology, Vilnius, Lithuania; [2] Vilnius University, Life Sciences Center, Institute of Biotechnology, Vilnius, Lithuania

The DNA Curtains platform was developed for facile DNAprotein interaction studies at the single-molecule (SM) level using total internal reflection fluorescence (TIRF) microscopy almost ten years ago. This platform allowed aligning of DNA fragments, which were anchored on a supported lipid bilayer, along the nano-fabricated chromium barriers using a buffer flow. This fabrication process is technically challenging.

Recently we presented an alternative strategy for fabrication of more stable and straightforward DNA Curtains "Soft" DNA Curtains - using DNA tethering onto a nanopatterned protein template via high-affinity molecular recognition. This strategy is based on lift-off micro-contact printing $(\mu \mathrm{CP})$ of streptavidin lines on silanized and PEGylated glass coverslips. We demonstrated the feasibility of our platform for tethering biotinylated DNAs: a single-tethered $5 \mathrm{~kb}$ DNAs and single- or double-tethered $48.5 \mathrm{~kb}$ DNAs. Here we will present the strategy for "soft" DNA Curtains fabrication and our latest results on the platform optimization. Also, we will present our new results on SM level DNA-protein interaction studies using our "soft" DNA Curtains.

\section{P-357}

A single-molecule multiplexed approach to study Cas9 nucleosomes invasion

Kristina Makasheva, Louise Bryan, Beat Fierz

Ecole Polytechnique Fédérale de Lausanne, Switzerland

In eukaryotic cells the Cas9 nuclease must target chromatinized DNA. The position of PAM sites within nucleosomes, the presence of histone modifications as well as sgRNA specificity are important factors for Cas9 recognition and binding, but the mechanisms governing Cas9/dCas9 chromatin invasion remain unclear. Single-molecule measurements provide detailed mechanistic insight into intricate molecular processes, and are thus suitable to reveal how Cas9 dynamically engages nucleosomes. However, single-molecule experiments with complex chromatin samples are difficult to perform and the reproducible and quantitative determination of parameters can be challenging.

We have developed a method to multiplex dynamic singlemolecule observations, where the interactions of dCas9 to many different types of nucleosomes are observed simultaneously in one single-molecule experiment. Parallel experiments are subsequently spatially decoded, via the detection of specific binding of fluorescent DNA probes. Using the established multiplexing system, we reveal that the time required for stable dCas9 binding is greatly increased for target sequences located further within nucleosomes. Moreover, nucleosomes suppress non-specific binding by shortening the residence time of dCas9 for sgRNAs containing mismatches. Together, these results provide mechanistic information on the role of chromatin in directing Cas9 activity in genome targeting and editing applications.

\section{P-356}

Single-molecule monitoring of nascent RNA dynamics within native transcriptional complexes Daniel A Lafontaine [1], Juan C Penedo [2], Adrien Chauvier [1], Patrick St-Pierre[1], Jean-Francois Nadon[1], Cibran Perez-Gonzalez [2], Sebastian H Eschbach [1], AnneMaire Lamontagne [1]

[1] Department of Biology, Université de Sherbrooke, Canada; [2] Centre of Biophotonics, School of Physics and Astronomy, University of St. Andrews, UK

Single-molecule Förster Resonance Energy Transfer (smFRET) has emerged as a unique tool to investigate the structure and dynamics of post-transcriptional RNA sequences. However, in a cotranscriptional context, its use has been limited to non-native methods that intrinsically lack key RNA polymerase-dependent features that are crucial for gene regulation. Here, we present a novel approach based on the site-specific incorporation of fluorescent nucleotides within nascent transcripts that enables the real-time study of RNA folding within actively transcribing bacterial complexes. We show its potential by monitoring the cotranscriptional folding of a riboswitch within Escherichia coli complexes. Using this approach, we unveil a crucial role of the RNA polymerase controlling riboswitch function by modulating RNA structure and demonstrate an inverse relationship between elongation speed and metabolite-sensing efficiency that results in a progressively narrow time-window for RNA folding and gene regulation. Our approach provides a cotranscriptionally native platform to investigate the folding and regulatory mechanisms of other important bacterial and eukaryotic systems.

\section{P-358}

Distribution analysis of alpha satellites in chimpanzee, Neanderthal and human genome using Global Repeat Map and ALPHAsub algorithm - novel database

Ines Vlahović [2,1], Matko Glunčić[1], Hrvoje Jerković [2], Leo Mršić [2], Vladimir Paar [3,1]

[1] Department of Physics, Faculty of Science, University of Zagreb, Croatia; 2] Algebra University College, Croatia; [3] Croatian Academy of Sciences and Arts, Croatia

During recent years, more and more DNA sequences are available to research community with emergence of new sequencing technologies that are capable of closing gaps in DNA sequence assemblies. Most of those gaps were in the centromeric region of chromosomes - region that is replete with alpha satellites organized in form of tandem repeats or in higher order structures (HORs). In human chromosomes, those alpha satellites are $171 \mathrm{bp}$ long and are responsible, as in other species, for microtubule attachment to kinetochores and chromosome segregation. Also, they are associated with cancer outcomes, aneuploidy, ageing and evolutionary development. Using our novel computational Global Repeat Map (GRM) and ALPHAsub algorithm, we created database (www.genom.hazu.hr) for tandem repeats that currently have $\sim 3000$ records of alpha satellite tandem arrays, their position in chromosome, sequence length and divergence for chimpanzee, Neanderthal and human genomes. Using this data we conduct their distribution analysis that is foundation for our further research of alpha satellites between closely related species and identification of HOR structures and their organization, function and possible regulatory roles as well as usage of variation in HOR structures for diagnostic purposes. 
Posters

-21. DNA architecture and gene regulation -

\section{P-359}

Torsional diversity in nucleosome core particle structures influences the folding of closed DNA minichromosomes

Robert T Young, Stefjord Todolli, Wilma K Olson

Rutgers University, USA

Organization of DNA into compact chromosomes hinges on forming nucleosome core particles (NCPs), the fundamental packing unit of chromatin where $\sim 147$-bp of DNA wraps around a histone protein core. Nucleosomal organization is highly dynamic as binding factors such as RNA polymerase interact with DNA, resulting in local distortions that must be compensated by neighboring DNA. Recent analysis of high-resolution NCPs from the Protein Data Bank has revealed previously unrecognized differences in the end-to-end displacement and the overall twist of nucleosomal DNA. Here we show how the uptake of twist on different NCPs affects the configuration of closed circular DNA constructs bearing a lone nucleosome. Minichromosomes of lengths 341bp and 359-bp were constructed by connecting the ends of NCP structures with Bezier curves and then optimizing the elastic energy of these protein-free regions. Optimization results show that the variation in twist found in a collection of $\sim 150 \mathrm{NCP}$ structures introduces changes in loop topology and overall minichromosome shape. Simulations of nucleosomal breathing, obtained by shortening the length of the rigid NCP regions, lead to increases in loop opening, with greater resistance to structural change found in minichromosomes bearing more over-twisted NCPs. These studies lay the foundation for exploring the influence of nucleotide sequence on the elastic response of the protein-free region and the contribution of the nucleosome to the genomic organization as a whole.

P-361

Super-resolution imaging reveals spatiotemporal propagation of human replication foci mediated by CTCF-organized chromatin structures

Ziqing Winston Zhao [1], Qian Peter $\mathrm{Su}[2,3]$, Xiaoliang Sunney Xie [3], Yujie Sun [3]

[1] National University of Singapore, Singapore; [2] Uni. of Technology Sydney, Australia; [3] Peking University, China

DNA replication is initiated at numerous replication origins clustered into thousands of replication domains (RDs) across the genome. However, it remains unclear whether replication origins within each RD are activated stochastically or preferentially near certain chromatin features. To understand how replication is regulated in single human cells at the sub-RD level, we quantitatively characterized the spatio-temporal organization, morphology, and in situ epigenetic signatures of individual replication foci $(\mathrm{RFi})$ across S-phase at superresolution using STORM. We revealed a hierarchical radial pattern of $\mathrm{RFi}$ propagation dynamics that reverses directionality from early to late S-phase, and is diminished upon CTCF knockdown. Together with simulation and bioinformatic analyses, our findings point to a "CTCF-organized REplication Propagation" (CoREP) model, which suggests a non-random selection mechanism for replication activation mediated by CTCF-organized chromatin structures. These findings shed critical insights into the involvement of local epigenetic environment in coordinating replication across the genome, and have wide-ranging implications for our conceptualization of the role of multi-scale chromatin architecture in regulating nuclear dynamics in space and time.

\section{P-360}

Radioprotective Damage suppressor protein (Dsup) in model organisms: from transcriptome and physiology to molecular structure

Mikhail Zarubin, Anna Rzyanina, Elena Kravchenko

Joint Institute for Nuclear Research, Russian Federation

Ionizing radiation is one of the most harmful exposome factors, thus recently discovered tardigrade Dsup protein is highly valuable for the development of new genetically engineering way of innate radioprotection. Dsup protein (42.8 $\mathrm{kDa}$ ) has high positive charge, that provides electrostatic interaction between Dsup protein and DNA/nucleosomes, contributing to the protection of chromatin from ROS generated by radiation. In this work we obtained $D$. melanogaster lines and cell culture HEK 293 expressing Dsup with increased radioresistance to $\gamma$-rays (500-1000 Gy for D. m.) and protons (0.5-5 Gy for HEK 293). We hypothesized that addition of Dsup protein in nucleus, besides radioprotective advantages, may impede gene regulation, interrupt normal processes of translation and reparation. In that view, we performed transcriptome analysis of Dsup-expressing flies and observed alterations in expression of genes related to transcription, chromatin silencing, chromosome organization, mitotic spindle elongation, that indicates some disorder in DNA machinery or chromatin compaction. In addition, we registered changes at physiological level in Dsup-expressing lines (lifespan, climbing test). Also, we conducted transcriptome analysis of irradiated Dsup-expressing and control organisms to evaluate possible differences in response to irradiation. To determine Dsup structure and parameters of Dsup-DNA complex SANS and SAXS techniques will be applied. 


\section{Posters}

- 22. Synthetic cell -

\section{P-362}

Mechanisms for the controlled division of giant unilamellar vesicles

Yannik Dreher [1,2], Kevin Jahnke [1,2], Kerstin Göpfrich [1,2] [1] Biophysical Engineering Group, Max Planck Institute for Medical Research, Jahnstraße 29, 69120 Heidelberg, Germany; [2] Department of Physics and Astronomy, Heidelberg University, 69120 Heidelberg, Germany

Cell division is one of the hallmarks of life. Success in the bottom-up assembly of synthetic cells will, no doubt, depend on strategies for the division of protocellular compartments. Here, we demonstrate two mechanisms for the controlled division of giant unilamellar lipid vesicles (GUVs).

The first mechanism relies on the line tension of phaseseparated GUVs and a surface-to-volume ratio increase via osmosis. We derive a conceptual model based on the vesicle geometry which makes four quantitative predictions that we verify experimentally.

The second mechanism is based on osmotic deflation and an increase of the membrane spontaneous curvature. To controllably increase the spontaneous curvature, we use the photosensitizer chlorine e6 which self-assembles into lipid bilayers and leads to lipid peroxidation upon illumination. Remarkably, we achieve full division of single-phased DNAcontaining GUVs.

Overall, our work provides broadly applicable mechanisms for the division of synthetic cell compartments and adds to the strategic toolbox of bottom-up synthetic biology.

\section{P-364}

Self-programmed enzyme phase separation and multiphase coacervate droplet organization Hedi Karoui, Nicolas Martin

Centre de Recherche Paul Pascal, CNRS, Pessac, France

Compartmentalization is a hallmark of living cells allowing them to perform complex tasks by dynamically coordinating matter and energy fluxes in space and time. Recent years have witnessed growing interest in the bottom-up assembly of synthetic micro-compartments via complex coacervation to reproduce the cellular organization.

Researchers sought to increase spatial complexity via formation of multiphase droplets under thermodynamic equilibrium. On the other hand, active processes such as enzymatic reactions are explored to increase their temporal dynamics. Pioneering studies focused on using antagonistic enzymes as endogenous catalytic controllers to trigger the formation and dissolution of these droplets. Yet, attempts to combine both active regulation and spatial organization into hierarchical droplets have not yet been reported.

Here, a model for membraneless organelles based on enzyme complex coacervation controls its own phase separation: Glucose Oxidase forms droplets with DEAE-Dextran on a narrow $\mathrm{pH}$ range so that enzyme-driven monotonic $\mathrm{pH}$ changes regulate the emergence, growth, decay, and dissolution of the droplets depending on the substrate concentration. Significantly, time-programmed coacervate emergence and dissolution can be achieved in a single enzyme system. We further exploit this self-driven enzyme phase separation to produce multiphase droplets via dynamic polyion self-sorting in the presence of a secondary coacervate phase.

\section{P-363}

Effect of detergents on morphology, size distribution and concentration of copolymer-based polymersomes

Radosław Górecki [1,2], Fabio Antenucci [3], Karolis Norinkevicius [4,1], Line Elmstrøm Christiansen [1], Scott T Myers [1], Krzysztof Trzaskuś [1], Claus Hélix-Nielsen [2] [1] Aquaporin A/S, Denmark; [2] Technical Univ of Denmark, Dept of Environmental Engineering, Denmark; [3] Univ of Copenhagen, Dept of Veterinary and Animal Sciences, Denmark; [4] Technical Univ of Denmark, Dept of Chemical and Biochemical Engineering, Denmark

Polymersomes made of amphiphilic diblock copolymers are generally regarded as having higher physical and chemical stability than liposomes composed of phospholipids. This enhanced stability arises from the higher molecular weight of polymer constituents. Despite their increased stability, polymer bilayers are solubilized by detergents in a similar manner to lipid bilayers. In this work, we evaluated the stability of poly(ethylene glycol)-block-poly( $\varepsilon$-caprolactone) (PEG-PCL)-based polymersomes exposed to three different detergents: N-octyl- $\beta$-Dglucopyranoside (OG), lauryldimethylamine N-oxide (LDAO), and Triton X-100 (TX100). Changes in morphology, particle size distribution, and concentrations of the polymersomes were evaluated during the titration of the detergents into the polymersome solutions. Furthermore, we discussed the effect of detergent features on the solubilization of the polymeric bilayer and compared it to the results reported in the literature for liposomes and polymersomes. This information can be used for tuning the properties of PEG-PCL polymersomes for use in applications such as drug delivery or protein reconstitution studies. (Langmuir 2021, 37, 6, 2079-2090).

\section{P-365}

Protocell formation is enhanced by innate solid surfaces

Elif S Köksal, Inga Põldsalu, Henrik Friis, Irep Gözen University of Oslo, Norway

Protocells, or primitive prebiotic vesicular compartments, are thought to have encapsulated and protected genetic materials important for the emergence of life on the early Earth. Given the ubiquity of mineral-water interfaces on the earth's surface today as well as at the time of the origin of life, interactions with chemically diverse, potentially catalytic mineral surfaces may have aided the autonomous formation of protocells. Following a recent report that clay mineral, montmorillonite, catalyzes the polymerization of RNA from activated ribonucleotides, and accelerates the self-assembly of amphiphilic compartments, we report on the autonomous formation and growth of vesicular compartments on various types of naturally occurring solid surfaces. We observe that one of the mechanisms of formation is nanotubemediated, which we had previously characterized and reported on laboratory-made substrates. Our observations support the suggested catalytic role of interfaces in the emergence of primitive vesicular compartments under prebiotic conditions, and suggest that earliest compartments may have formed due to the interactions of amphiphiles with minerals in an aqueous environment. 
Posters

-22. Synthetic cell -

P-366

TRPC chemo-optogenetics enables precise and specific control over mast cell function

Denis Krivić, Bernadett Bacsa, Annarita Graziani, Oleksandra Tiapko, Klaus Groschner

Medical University of Graz, Division of Biophysics, Austria

Canonical transient receptor potential (TRPC) channels are considered as elements of the immune cell $\mathrm{Ca}^{2+}$ handling machinery, but their role within the immune system appears highly specific and limited to particular cell phenotypes. Utilizing a recently established TRPC3/6/7 selective, photochromic benzimidazole agonist OptoBI-1, we tested the concept of conferring light sensitivity to immune cells by introducing OptoBI-1-sensitive TRPC isoforms. RBL-2H3 mast cells were found to express TRPC 3 and TRPC7 mRNA but lacked appreciable $\mathrm{Ca}^{2+} / \mathrm{NFAT}$ signalling in response to OptoBI-1 photocycling. Genetic modification of the cells to overexpress single recombinant TRPC isoforms revealed that exclusively TRPC6 expression generated OptoBI-1 sensitivity suitable for opto-chemical control of NFAT1 activity. Expression of TRPC6 or TRPC7 enabled light-mediated generation of temporally defined $\mathrm{Ca}^{2+}$ signalling patterns. Nonetheless, only cells overexpressing TRPC6 retained essentially low basal levels of NFAT activity and displayed rapid and efficient NFAT nuclear translocation upon OptoBI1 photocycling. Importantly, OptoBI-1-induced mast cell activation lacked a significant impact on degranulation indicating that the TRPC6-OptoBI-1 strategy enables precise and highly specific control over $\mathrm{Ca}^{2+}$ transcription coupling in these immune cells by light.

\section{P-368}

Surface-assisted formation of model protocells from fatty acid and phospholipid mixtures Inga Põldsalu [1], Elif S Köksal [1], Irep Gözen [1,2]

[1] Centre for Molecular Medicine Norway, Faculty of Medicine, University of Oslo, 0318 Oslo, Norway; [2] Department of Chemistry, Faculty of Mathematics and Natural Sciences, University of Oslo, 0315 Oslo, Norway

Solid substrates have been shown to enhance the formation of prebiotic containers by supporting a spontaneous, multi-step transformation of surface-adhered phospholipid reservoirs to giant unilamellar primitive compartments connected via lipid nanotube networks. It has been previously shown that membranes from mixtures of fatty acids and phospholipids enable the coexistence of increased permeability and stability, expected to be advantageous to early protocells. Here we show the solid surface-assisted formation of primitive compartments, i.e. model protocells, from lipid membranes with gradually increasing fatty acid fractions. Upon contact with a high energy surface, reservoirs of mixed amphiphiles act in a similar way to pure phospholipid mixtures, and spontaneously transform into a network of multiple compartments. We demonstrate that the generated compartments can take up and encapsulate components present in the ambient solution, e.g. fluorescein and RNA. The preliminary findings indicate that the surface-assisted protocell formation from mixed amphiphiles may have been a plausible scenario for the early stages of the emergence of primitive cells.

\section{P-367}

Reconstitution of contractile actomyosin rings in vesicles

Thomas Litschel [1], Charlotte F Kelley [1], Danielle Holz [2], Naoko Mizuno [1], Dimitrios Vavylonis [2], Petra Schwille [1] 1] Max Planck Institute of Biochemistry, Germany; [2] Lehigh University, USA

One of the grand challenges of bottom-up synthetic biology is the development of minimal machineries for cell division. The mechanical transformation of large-scale compartments, such as Giant Unilamellar Vesicles (GUVs), requires the geometry-specific coordination of active elements, several orders of magnitude larger than the molecular scale. Of all cytoskeletal structures, large-scale actomyosin rings appear to be the most promising cellular elements to accomplish this task. Here, we have adopted advanced encapsulation methods to study bundled actin filaments in GUVs and compare our results with theoretical modeling. By changing few key parameters, actin polymerization can be differentiated to resemble various types of networks in living cells. Importantly, we find membrane binding to be crucial for the robust condensation into a single actin ring in spherical vesicles, as predicted by theoretical considerations. Upon force generation by ATP-driven myosin motors, these ring-like actin structures contract and locally constrict the vesicle, forming furrow-like deformations. On the other hand, cortex-like actin networks are shown to induce and stabilize deformations from spherical shapes.

\section{P-369}

Mimicking the minimal fusion machinery: Reconstitution of SNARE proteins into dropletstabilized giant unilamellar vesicles

Merve Sari [1], Julius von Wirén [1], Anne-Sophie Rösler [1], Claudia Steinem $[1,2]$

[1] Institute of Organic and Biomolecular Chemistry, GeorgAugust-University Göttingen, Germany; [2] Max Planck Institute for Dynamics and Self-Organization, Göttingen, Germany

Fusion of neurotransmitter containing synaptic vesicles with the planar presynaptic membrane is a fundamental process in signal transduction within neurons. The driving force for this process are soluble $N$-ethylmaleimide-sensitive-factor attachment protein receptors (SNAREs) enabling the merging of the lipid bilayers and the release of the neurotransmitter into the presynaptic cleft.

Here, we mimic the planar target membrane using porespanning membranes (PSMs) which contain target SNAREs. To produce PSMs, giant unilamellar vesicles (GUVs) are spread on porous substrates. Conventional methods of GUV production limit protein insertion due to their low tolerance under physiological conditions which often results in low reconstitution efficiency. Therefore, we use droplet-based microfluidics for high-throughput GUV production. The aim of this work is to reconstitute the neuronal fusion machinery into droplet-stabilized GUVs which are then released as freestanding GUVs and spread to PSMs. The higher reconstitution efficiency of the protein containing PSMs will be characterized in lipid and protein mobility using confocal laser scanning microscopy. A high time resolution method (spinning disk confocal microscopy) will be used to investigate the influence of the reconstitution efficiency on fusion events. 


\section{Posters}

-22. Synthetic cell -

\section{P-370}

Subcompartmentalization and pseudo-division of model protocells

Karolina Spustova [1], Elif S Köksal [1], Alar Ainla [2], Irep Gözen [1]

[1] Centre for Molecular Medicine Norway, University of Oslo, Norway; [2] International Iberian Nanotechnology Laboratory, 4715-330 Braga, Portugal

Membrane enclosed intracellular compartments have previously been exclusively associated with the eukaryotes, represented by the highly compartmentalized last eukaryotic common ancestor. Recent evidence showing the presence of membranous compartments with specific functions in archaea and bacteria makes it conceivable that the last universal common ancestor and its hypothetical precursor, the protocell, could have exhibited compartmentalization. To our knowledge, there are no experimental studies yet that have tested this hypothesis. We report on an autonomous subcompartmentalization mechanism which results in the formation of several tens of membranous subunits inside surfaceadhered protocells. The protocells, as well as the subcompartments, can encapsulate solutes from the external environment and exchange them via transient pores in the lipid membrane. In the event of the disintegration of the protocell, the subcompartments transform into individual daughter cells providing an alternative pathway for protocell division. The process is solely determined by the fundamental materials properties and interfacial events, and do not require biological machinery or externally supplied chemical energy. In the light of our findings, we propose that similar events could have taken place under early Earth conditions.

\section{P-371}

Liposome-assisted delivery of biomembraneimpermeable cargo to primitive cells

Lin Xue [1], Anna B Stephenson [2], Irep Gözen [1]

[1] Centre for Molecular Medicine Norway, Faculty of Medicine, University of Oslo, Norway; [2] Harvard John A. Paulson School of Engineering and Applied Sciences, Harvard University, USA

We report on a platform for targeted delivery, and incorporation of membrane-impermeable compounds into celllike unilamellar lipid bilayer microcompartments. We perform the delivery by means of an open-space microfluidic device, which perfuses individual surface-adhered compartments (primitive model cells) with cargo-loaded small unilamellar liposomes, composed of cationic, i.e. fusogenic, lipids. We show specifically that water-soluble dyes, fluorescently labeled genetic polymers, sugars and proteins can be successfully internalized. By labeling the membrane of the small vesicles, as well as the membrane of the targeted model cells with fluorophores of different emission wavelengths, we monitor the mixing of fluorophores, hence fusion and internalization progress, simultaneously. This delivery method can potentially be extended to other types of cargo compounds, such as globular proteins, actin filaments, or microtubules. We intend to apply the delivery technique to networks of surface-adhered model protocells, interconnected via lipid nanotubes, in order to initiate chemical reactions, and cell-free gene expression, and expect to gain deeper understanding of possible chemical processes within primitive cell populations at the origin of life. 
Posters

-23. Liquid-liquid phase separation and intrinsically disordered proteins -

P-372

Theoretical study of chromatin organization at the mesoscale

Omar Adame-Arana, Gaurav Bajpai, Samuel A. Safran

Weizmann Institute of Science, Israel

Recent experiments have shown that chromatin organizes peripherally with chromatin-depleted regions in the middle of the nucleus in intact live cells, thus challenging the conventional picture in which chromatin fills the entire nucleus. Additionally, polymer simulations offered an explanation for this mechanism, suggesting that it stems from an interplay between chromatin self-attraction and its interactions with the nuclear lamina via its lamin associated domains(LADs). Motivated by these findings, we present an analytical study of the problem of chromatin organization using two complementary approaches. On the one hand, we predict chromatin concentration profiles by means of a minimal meanfield polymer model, showing that an appropriate choice of boundary conditions resembling the bonding interaction between LADs and the nuclear lamina is necessary, in order to reproduce experimental and numerical observations. On the other hand, we describe the polymer chain as a liquid droplet and study the transitions between different types of chromatin organization at the mesoscale as a function of the polymer droplet's surface tension, the chromatin fraction made of LADs, and their interaction strength with the nuclear lamina. We then compare our analytical results with Brownian dynamics simulations of chromatin.

\section{P-374}

Recognition hierarchy of adaptor proteins to clathrin Lucas A Defelipe [1,2], Knut Kölbel [3], Charlotte Uetrech [3], Isabel Bento [1], Maria M Garcia Alai [1,2]

[1] European Molecular Biology Laboratory, Hamburg Unit, Germany; [2] Centre for Systems structural biology (CSSB), Hamburg, Germany; [3] Heinrich-Pette-Institut, LeibnizInstitut für Experimentelle Virologie, Germany

Clathrin-mediated endocytosis (CME) is a mechanism present in eukaryotes (conserved from Yeast to Human) as the main source for nutrient uptake and membrane receptor recycling. Its heavy chain (CHC) assembles into the wellknown triskelion decorated by light chains (CLC) to compose the clathrin cage. However, Clathrin does not bind to the membrane but determines the progression of endocytosis, granting its vesicle size and regularity. Adaptor proteins forming the mid-coat bind to the membrane and are the main contribution to its elongation and shaping during invagination. The interaction of adaptor proteins with CHC is mediated by intrinsically disordered regions (IDRs) containing short linear interaction motifs (SLiMs). The structural determinants of the binding in $S$. cerevisiae $(S c)$ are unknown at this point. In the present work, we present the structure of Sc CHC N-terminal Domain bound with different adaptor proteins peptides (YAP1801, Ent-1, Ent-5, AP1, SWA2, and Sla1) in the three canonical boxes (Clathrin, Arrestin and Wbox). We also determined the Kd using NanoDSF isothermal analysis of these peptides and found that each box has its characteristic $\mathrm{Kd}$ that are close in value but distinguishable from each other. These results suggest an allovalent behavior of the Clathrin-AP complex function.

\section{P-373}

Amphiphilic gold nanoparticles perturb lipid phase separation in biomimetic cell membranes Ester Canepa

Department of Physics, University of Genoa, Italy

Cell membrane functionality strongly depends on the aggregation state of membrane lipids. Special attention should be devoted to the selective lipid segregation originating the liquid ordered phase of lipid rafts, i.e. self-assembled and specialized nanodomains enriched in cholesterol, saturated lipids, and glycosylated species. Since lipid rafts perform regulatory functions for numerous cellular processes, studying the interaction between nanoparticles (NPs) and the lateral phase separation of cell membranes becomes critical when evaluating NP-based theranostic applications. Here, we consider phase-separated biomimetic cell membranes and amphiphilic Au NPs of $\sim 3 \mathrm{~nm}$. These NPs possess high biomedical potential as they can passively enter cells for diagnostic and therapeutic purposes. For enhanced biomimicry, our membranes are composed of 1,2-dioleoylglycero-3-phosphocholine, sphingomyelin, cholesterol, and ganglioside GM1. This composition is representative of neuronal plasma membranes and leads spontaneously to ordered/disordered phase separation. Through atomic force microscopy, quartz crystal microbalance, and molecular dynamics simulations we reveal that Au NPs can suppress ordered domains in a concentration-dependent manner and form stable and diffuse bilayer-embedded lattices. Notably, by developing a general thermodynamic model, we show that the enthalpy of lipid mixing is the driving force behind the NP-induced destabilization of membrane phase separation.

\section{P-375}

Physical theory of biological noise buffering by multi-component phase separation

Dan Deviri, Samuel Safran

Weizmann Institute of Science, Israel

Maintaining homeostasis is a fundamental characteristic of living systems. In cells, this is contributed to by assembly of membraneless organelles that form by the physical process of liquid-liquid phase separation (LLPS). By analogy with LLPS in binary solutions, cellular LLPS was hypothesized to contribute to homeostasis by facilitating "concentration buffering", which renders the local protein concentration within the organelle robust to global variations in the average, cellular concentration (e.g. due to expression noise). Interestingly, concentration buffering was experimentally measured in vivo, in an organelle with a single solute, while it was observed not to be obeyed in one with several solutes. We formulate theoretically and solve analytically a physical model of LLPS in a ternary solution of two solutes, $\varphi$ and $\psi$, with both $\varphi-\varphi$ and $\varphi-\psi$ interactions. Our physical theory predicts how the coexisting concentrations in LLPS are related to expression noise and thus generalizes the concept of concentration buffering to multi-component systems. This reconciles the seemingly contradictory experimental observations. Furthermore, we predict that incremental changes of the $\varphi-\varphi$ and $\varphi-\psi$ interactions, such as those that are caused by mutations in the genes encoding the proteins, may increase the efficiency of concentration buffering of a given system. Thus, we hypothesize that evolution may optimize concentration buffering, and suggest experimental approaches to test this in different systems. 


\section{Posters}

\section{-23. Liquid-liquid phase separation and intrinsically disordered proteins -}

\section{P-376}

The role of entropy in biological liquid-liquid phase separation

Oliver T Dyer, Daniel Hebenstreit, Robin C Ball

University of Warwick, UK

Liquid-liquid phase separation is a mechanism by which cell nuclei organise their constituents without using membranes. The droplets produced vary in role and composition, but share an over-abundance of proteins containing intrinsically disordered regions (IDRs), and the leading hypothesis is that these IDRs provide a large number of weak attractive interactions between the proteins, leading to a non-rigid aggregation (i.e. a liquid droplet).

In this presentation we consider whether entropy could drive this phase separation without needing attractive interactions, akin to how it drives the separation of polymers and colloids in 'depletion flocculation'. In analogy to this well-studied system, we model biomolecules as either flexible polymer chains or low-entropy hard spheres. The IDRcontaining proteins are then be modelled as 'tadpoles' with a flexible polymer tail grafted to a hard sphere head.

Through Monte Carlo simulations we find that IDRs do indeed encourage entropic phase separation and that the tadpole molecules behave as surfactants, being found preferentially at the interface between the polymer- and colloid-rich phases. By comparing biological parameters to our theoretical phase diagram, we predict whether entropy is sufficient to induce droplet formation.

\section{P-378}

Diffusion of intrinsically disordered coral acidrich proteins

Barbara Klepka, Agnieszka Michaś, Anna Niedźwiecka

Laboratory of Biological Physics, Institute of Physics, Polish Academy of Sciences, Warsaw, Poland

Coral acid-rich proteins (CARP) of skeletal organic matrix (SOM) are responsible for biomineralisation. It is anticipated that their interactions with calcium cations could determine the crystal form of the skeleton, $i$. $e$. that the proteins control early stages of crystallisation by driving the amorphous calcium carbonite to calcite or aragonite crystal nuclei. Little is known about such proteins at the molecular level. Only four CARP SOM have been cloned and partially characterised. Here we show expression, purification and hydrodynamic characterisation of two new CARPs SOM, an N-terminal part of secreted acidic protein $1 \mathrm{~A}$ and aspartic and glutamic acid rich protein, discovered recently in Acropora millepora, a model organism to study environmental changes in the oceans due to global climate-warming. At issue is how to analyse diffusion of such biopolymers to observe changes in the Stokes radius upon interactions with calcium ions. The classical polymer formalism is based on the number of units in the polymer, which is applicable for denatured proteins in the random coil form and proteins with well-defined 3D structures. However, intrinsically disordered proteins (IDPs) have more intricate characteristics, hence a sequence-dependent model for IDPs was also proposed. The goal of our study is to compare diffusion properties of the CARPs in relation to standard proteins by means of fluorescence correlation spectroscopy.

\section{P-377}

Phase separation of synapsin, alpha-synuclein and synaptic vesicles

Christian Hoffmann[1], Roberto Sansevrino[1], Giuseppe Morabito [1], Chinyere Logan [1], Marcelo Ganzella [2], Dragomir Milovanovic [1]

[1] German Center for Neurodegenerative Diseases, Germany; [2] Max Planck Institute for Biophysical Chemistry, Germany

Nerve terminals contain hundreds of synaptic vesicles (SVs) that form tight clusters. Recent compelling evidence indicates that SV clusters at the nerve terminal are assembled as liquid condensates in which one component of the phase are SVs and the other synapsin 1, a highly abundant synaptic protein. Another major family of disordered proteins at the presynapse includes synucleins, most notably alphasynuclein. To determine the effect of alpha-synuclein on the synapsin phase, we employ the reconstitution approach using natively purified SVs from rat brains and the heterologous cell system to generate synapsin condensates. Our data indicate that synapsin condensates recruit alpha-synuclein, and while enriched into these synapsin condensates, alphasynuclein still maintains its high mobility. The presence of SVs enhances the rate of synapsin/alpha-synuclein condensation. At physiological salt and protein concentrations, alpha-synuclein alone is not able to cluster isolated SVs. Excess of alpha-synuclein attenuates the kinetics of synapsin/SV condensate formation, indicating that the molar ratio between synapsin and alpha-synuclein is essential in assembling the functional condensates of SVs. Understanding the molecular mechanism of alpha-synuclein interactions at the nerve terminals is crucial for clarifying the pathogenesis of synucleinopathies, where alpha-synuclein, synaptic proteins and lipid organelles all accumulate as insoluble intracellular inclusions.

\section{P-379}

Lysolipids regulate the size of lipid rafts depending on cholesterol content

Vladimir D Krasnobaev [1,2], Timur R Galimzyanov [1], Oleg V Batishchev [1]

[1] A.N. Frumkin Institute of Physical Chemistry and Electrochemistry, Russian Academy of Sciences, Russian Federation; [2] Moscow Institute of Physics and Technology, Dolgoprudny, Russian Federation

Lipid rafts play a crucial role in signal transduction, apoptosis and other cell processes. Thus, they impact the development of different diseases, e.g., Alzheimer's and Parkinson disease, some types of cancer, etc. The exact mechanisms and ways to influence raft processes are still unknown. Although studying rafts in vivo is rather complicated, liquidordered lipid domains are observed in model systems. Misfunction of phospholipase $\mathrm{A}_{2}$ is involved in development of above-mentioned diseases and it produces lysophospholipids, which influences membrane composition. We examined how lysophospholipids regulate the formation and size of rafts in model membranes. We demonstrated that even small concentrations of lysolipids in a membrane significantly impact domain size in cholesterol dependent manner. This data helped us to build a more effective theoretical model of the domain formation, which supported the hypothesis that molecules with complex molecular geometry influence the line tension of the raft boundary, its physical properties and thus membrane processes.

The work was supported by the Russian Foundation for Basic Research (project \#18-54-74001). 
Posters

-23. Liquid-liquid phase separation and intrinsically disordered proteins -

P-380

Pyroglumate-modified $A \beta(3-42)$ monomers bind with higher affinity than $A \beta 42$ monomers to A $\beta 42$ fibrils

Soumav Nath [1,4], Alexander K Buell [2], Bogdan Barz [1,4], Lothar Gremer [1,4], Sara S Linse [3], Dieter Willbold [1,4]

[1] Heinrich-Heine-Universität Düsseldorf, Germany; [2] Technical University of Denmark, Denmark; [3] Lund University, Sweden; [4] Forschungszentrum Jülich, Germany

Extracellular deposits of amyloid fibrils in human brain originate Alzheimer's Disease (AD). Intrinsically disordered proteins like $A \beta 42$ and pyroglutamate-modified $A \beta(p E-A \beta)$ 342 are abundant variants in senile plaques of AD brains. $\mathrm{pE}-\mathrm{A} \beta(3-42)$ shows accelerated fibrillation and oligomerization rate than $\mathrm{A} \beta 42$. The aggregation of $\mathrm{pE}-\mathrm{A} \beta(3-42)$ is diminished in presence of $\mathrm{A} \beta 42$ fibril seeds, but aggregation of $A \beta 42$ is accelerated when $p E-A \beta(3-42)$ seeds are present. This could be related to different rates of primary and secondary nucleation during the aggregation process. Here, we conducted Surface Plasmon Resonance experiments to calculate binding affinities of $\mathrm{A} \beta 42$ and $\mathrm{pE}-\mathrm{A} \beta(3-42)$ monomers for $A \beta 42$ fibril surface and we found increased binding affinity of $\mathrm{pE}-\mathrm{A} \beta(3-42)$ monomers compared to $\mathrm{A} \beta 42$ monomers. To identify the key molecular interactions responsible for the different binding behavior, we performed enhanced sampling molecular dynamics simulations of single monomers or in the presence of fibrils. Simulation of $\mathrm{A} \beta 42$ and pE-A $\beta(3-$ 42) monomers alone indicate significant differences of the monomeric structure. Also, the binding to the fibrils reveal different types of attachment on the fibril for two peptides.

\section{P-382}

A precise and general FRET-based method for monitoring structural transitions in protein selforganization

Qi Wan [1,2], Sara N Mouton[3], Liesbeth M Veenhoff [3], Arnold J Boersma [1]

[1] DWI-Leibniz Institute for Interactive Materials, Germany; [2] Department of Biochemistry, Groningen Biomolecular Sciences and Biotechnology Institute, University of Groningen, Netherlands; [3] European Research Institute for the Biology of Ageing, University of Groningen, University Medical Center Groningen, Netherlands

Proteins assemble into a tremendous variety of dynamic and functional structures. Sensitive measurements directly in cells with a high spatiotemporal resolution are needed to distinguish these different assemblies. Here, we demonstrate precise and continuous monitoring of cytoplasmic protein self-assemblies and their structural transitions. Intermolecular FRET with both the donor and acceptor protein at the same target protein provides high sensitivity while retaining the advantage of straightforward ratiometric imaging. We measure different assembly structures, transient intermediate states' kinetics, and assembly formation resolved in space and time. We thus observe the dynamic structural transition between unstructured condensates and fibrils and confirm an array of previous observations on aggregations in yeast and HEK293. Combined with FACS it allows assembly measurement in a high-throughput manner crucial for screening efforts. Implementation in other native or nonnative proteins could provide insight into many studies involving protein condensation or aggregation.

\section{P-381}

Self-assembly of recombinant spider silk protein eADF4(C16) triggered by $\mathrm{pH}$

Veronika Talafová [1], Gabriel Žoldák [3], Martin Humenik [2], Thomas Scheibel [2], Erik Sedlák [3]

[1] Department of Biophysics,Faculty of Science,P.J.Šafárik University,Jesenná 5,041 54 Košice, Slovakia; [2] Department of Biomaterials,Faculty of Engineering Science,University of Bayreuth,Universitätsstraße 30,95440 Bayreuth, Germany; [3] Center for Interdisciplinary Biosciences,P.J.Šafárik University,Jesenná 5,041 54 Košice, Slovakia

Spider silk formation is a complex process involving a $\mathrm{pH}$ drop, ion exchange and sheer forces, which induce transformation of the underlined protein from an intrinsically unfolded soluble state into beta-sheet-rich fibres. This work focused on changes in self-assembly of protein eADF4(C16) in a broad range of $\mathrm{pH}$ environments ( $\mathrm{pH} 3$ to 10) in presence or absence of kosmotropic phosphate ions. The formation of aggregate states was followed using evolution of turbidity over time, whereas changes in the protein secondary structures were analyzed using CD and FT-IR spectroscopy. Presence of hydrophobic surface on aggregates or cross-beta structures in the fibrils was studied using fluorescent probes ANS and ThT. The resulting assemblies were imaged by TEM. The results indicate that the presence of phosphate ions induces fibril formation independent of $\mathrm{pH}$ environment. Interestingly, low $\mathrm{pH}$ values ( $\mathrm{pH} 3$ to 5 ) trigger fibril self-assembly even in absence of the phosphate ions, but at the same time the formation of amorphous aggregates is observed as well. In contrast, at higher $\mathrm{pH}$ values ( $\mathrm{pH} 6$ to 10), the protein retains unfolded structures and remains soluble.

\section{P-383}

Cryo-electron microscopy imaging of Alzheimer's amyloid-beta 42 oligomer displayed on a functionally and structurally relevant scaffold

Jinming Wu, Thorsten Blum, Daniel Farrell, Frank Dimaio, Jan Pieter Abrahams, Jinghui Luo

Paul Scherrer Institute, Switzerland

Amyloid- $\beta$ peptide $(A \beta)$ oligomers are pathogenic species of amyloid aggregates in Alzheimer's disease. Like certain protein toxins, $A \beta$ oligomers permeabilize cellular membranes, presumably through a pore formation mechanism. Due to their structural and stoichiometric heterogeneity, the structure of these pores remains to be characterized. We studied a functional $\mathrm{A} \beta 42$-pore equivalent, created by fusing $\mathrm{A} \beta 42$ to the oligomerizing, soluble domain of the $\alpha$-hemolysin $(\alpha \mathrm{HL})$ toxin. Our data reveal A $\beta 42-\alpha H L$ oligomers to share major structural, functional and biological properties with wildtype A $\beta 42$-pores. Single-particle cryo-EM analysis of A $\beta 42-$ $\alpha$ HL oligomers (with an overall resolution of $3.3 \AA$ ) reveals the A $\beta 42$-pore region to be intrinsically flexible. We anticipate that the A $\beta 42-\alpha \mathrm{HL}$ oligomers will allow studying many of the features of the wild type amyloid oligomers that cannot be studied otherwise, and may represent a highly specific antigen for the development of immuno-base diagnostics and therapies.

Reference: Wu, J., Blum, T..B., Farrell, D..P., DiMaio, F., Abrahams, J..P. and Luo, J. (2021), Angew. Chem. Int. Ed.. Accepted Author Manuscript. DOI: 10.1002/anie.202104497 


\section{Posters}

-24. Biosensors -

\section{P-384}

\section{Lifetime of conical nanopores for biosensing applications}

Mohammed Alawami, Filip Bošković, Jinbo Zhu, Kaikai Chen, Ulrich F Keyser

Cavendish Laboratory, University of Cambridge, JJ Thompson Avenue, Cambridge CB3 0HE, United Kingdom, UK

Nanopore sensing is a robust emerging technology that has many biosensing applications. The applications range from nucleic acid sequencing using biological pores to biomolecules detection using solid-state pores. The ability to easily fabricate solid-state nanopores that can withstand harsh conditions makes them an attractive choice for biosensing applications. Still, biomolecule interactions with nanopores reduces their reusability increasing their usage cost. In this study, we propose a simple storing method for repeated use of conical nanopores up to a few months. We investigated the current signal to noise ratio (SNR) for quartz conical nanopores with a diameter of $10-15 \mathrm{~nm}$ over months to determine their lifetime for single-molecule biosensing. Almost 100 nanopores were fabricated using laser-assisted pipette pulling. Multiple nanopores were assembled into individual PDMS chips that was used to performed almost 100 nanopore measurements over 19 weeks. We developed a protocol that allows for safe storage and repeated use of nanopore chips for up to 19 weeks. The single-molecule biosensing capability over repeated use cycles was demonstrated by quantitative analysis of structured DNA carriers designed for detection of short nucleic acids.

\section{P-386}

Simulations of DNA-carbon nanotube interactions for the design of field-effect transistors biosensors

Sebastien Cote [1,2], Normand Mousseau [1]

[1] Universite de Montreal, Departement of physics, Canada;

[2] Cegep de Saint-Jerome, Departement of physics, Canada

Bioanalytical sensors based on field-effect transistors (bioFETs) are emerging as promising tools to measure the kinetics of biopolymers such as proteins and DNA strands. This class of biosensors is based on an ultra-miniaturized electronic circuit whose conductance is very sensitive to the variations of the electrostatic potential in its environment caused by conformational changes in the biopolymer. Here, we investigate the working of a specific bioFET made of a single carbon nanotube to which is covalently grafted a single DNA strand of the G-quadruplex motif. More specifically, we use advanced sampling techniques based on molecular dynamics simulations to unveil the interactions and kinetics between the biopolymer and the carbon nanotube. We observed that, while the structural stability of the Gquadruplex motif is not significantly altered by the carbon nanotube, some interactions could modify its folding kinetics. We also investigated the origin of the device's sensitivity by characterizing the electrostatic potential around the nanotube as a function of the biopolymer's conformational ensemble. Our conclusions from computational simulations complement the experimental measurements obtained by our collaborators who characterized the same setup. Together, they support the development of this promising biosensor for monitoring the kinetics of biopolymers.

\section{P-385}

Gold nanoparticles decorated with customized self-assembled monolayers: A versatile platform toward the development of nanobiosensors Ahmed Alsadig [1,2], Hendrik Vondracek [2,3], Paolo Pengo [3], Lucia Pasquato [3], Sara Fortuna [3], Paola Posocco [4], Pietro Parisse [2], Loredana Casalis [2]

[1] PhD School in Nanotechnology, Univ of Trieste, Italy; [2] NanoInnovation Lab, Elettra-Sincrotrone Trieste, Italy; [3] Chemical \& Pharmaceutical Sciences dept, Trieste Univ., Italy; [4] Engineering \& Architecture dept, Trieste Univ., Italy

The self-assembly of ligands at the surface of nanoparticles allows the construction of platforms with advanced merits. In our lab, we exploit different nanoparticle bio-coating routes for diverse needs. To start with, DNA-modified gold nanoparticles (DNA-AuNPs) offer excellent versatility combining DNA addressability with an easy coating of AuNPs. We developed an approach based on the DNA-directed immobilization of protein-DNA conjugates onto a mixed self-assembled monolayer (SAM) composed of thiolated ssDNA alkanethiols with the possibility to adjust DNA density. The platform's biomolecular recognition ability was demonstrated utilizing the avidin-biotin complex. This general straregy represents a promising tool for anchoring functional biomolecules. Alternatively, we exploited mixed alkanethiol SAMs with nitrilotriacetic acid (NTA)/ oligo ethylene glycol termination to anchor recombinant His-tagged antigens quantifying antibodies used in immunotherapy. In our design, antibody recognition resulted in a plasmonic coupling of the particles that was readily detected by optical spectroscopy. Such an optical readout system can be used in point-of-care devices to aid personalized therapies or clinical analysis settings. Other click chemistry protocols based on alkyne SAMs are currently being implemented to overcome the challenges faced with other systems.

\section{P-387}

Photoacoustic properties of a photochromic red/green cyanobacteriochrome from Synechocystis PCC6803

Francesco Garzella [1,2], Aba Losi [2], Wolfgang Gärtner [3], Stefania Abbruzzetti [2], Paolo Bianchini [1], Cristiano Viappiani [2], Alberto Diaspro [1,4]

[1] Nanoscopy and NIC@IIT, Istituto Italiano di Tecnologia, Italy; [2] Department of Mathematical, Physical and Computer Sciences, University of Parma, Italy; [3] Institute for Analytical Chemistry, University of Leipzig, Germany; [4] Department of Physics, University of Genova, Italy

The photoacoustic (PA) effect is a physical phenomenon based on the emission of sound waves following light absorption in material samples. The photon absorption and subsequent non-radiative depletion of the chromophores rapidly raise the temperature within the sample. Thus, the pressure increases and induces a thermoelastic expansion that leads to the emission of a PA pressure wave. Such a combined use of light and acoustic waves enables high-resolution, deeply penetrating imaging in biological tissues. The difference among the absorption coefficients of tissue components or suitable transgene labels in the sample gives the base of contrast in PA imaging. Still, the few available probes showing high PA efficiency reduces the applications to living systems at the cellular and subcellular levels. Similarly to fluorescence microscopy, where the development of reversibly switchable fluorescent proteins has strongly improved contrast-to-noise ratio and spatial resolution, an analogous approach in PA microscopy is conceivable. In this work, we will present a spectroscopic characterization of the third GAF domain of the protein slr1393, a red/green cyanobacteriochrome showing interesting properties in terms of photoacoustic emission and switching quantum yield. 
Posters

-24. Biosensors -

P-388

Diamond-based temperature sensing for mechanobiology studies

Tomasz Kołodziej [1], Mariusz Mrózek [1], Saravanan Sengottuvel [1], Maciej Głowacki [2], Mateusz Ficek [2], Wojciech Gawlik [1], Zenon Rajfur [1], Adam Wojciechowski [1]

[1] Faculty of Physics, Astronomy and Applied Computer Science, Jagiellonian University in Kraków, Poland; [2] Department of Metrology and Optoelectronics, Faculty of Electronics, Telecommunications and Informatics, Gdansk Univerity of Technology, Poland

Local temperature changes can influence the behaviour of cells in living organisms, hence, the cell biology may benefit from precise temperature measurements. We employed Optically Detected Magnetic Resonance (ODMR) method, which relies on subtle changes in fluorescence intensity of NitrogenVacancy colour centres in diamonds, when interacting with a microwave field with a frequency around $2.87 \mathrm{GHz}$.

In cell biology experiments we need to provide physiological conditions. However, mechanical properties of environment are mostly neglected. Since soft tissues are $10^{4}-10^{7}$ times softer than standard microscopy dishes, seeding cells on soft substrates can influence results. To mimic tissues' elasticity, we applied polyacrylamide hydrogels, where by changing the ratio between the monomer and cross-linker we can modify their elasticity from $0.1 \mathrm{kPa}$ to up to hundreds of kilopascals. In our study, NV-microdiamonds were placed in the top layer of elastic substrate to measure the temperature just beneath the cell. We did not introduce diamonds directly into cells, preventing diamond-cell interaction, which allowed us to link temperature changes to cellular mechanobiological processes.

\section{P-390}

Hexokinase 1 prevents mitochondrial division during energy stress

Johannes Pilic, Benjamin Gottschalk, Wolfgang Graier, Roland Malli

Gottfried Schatz Research Center for Cell Signaling, Metabolism and Aging, Medical University Graz, Austria

Mitochondrial morphology, ranging from fragmented units to elongated networks, is tightly regulated. Elongated mitochondria are spared from autophagic degradation, whereas fragmentation of mitochondria promotes cell death. It is well known that amino acid starvation induces mitochondrial elongation, which is mediated by phosphorylation of the pro-fission dynamin-related protein 1 (DRP1). Using fluorescent fusion constructs and time-lapse imaging, we found that the mitochondrial bound enzyme hexokinase 1 (HK1) forms ring structures around constricted mitochondria during glucose starvation, thereby preventing DRP1 recruitment and mitochondrial fission. HK1-ring-formation is completely reversible within minutes by glucose readdition. Using fluorescent protein-based biosensors in digitonin permeabilized cells, we show that HK1 aggregation is induced by depletion of intracellular ATP and reversed by glucose-6-phosphate. Manipulations of the HK1 structure revealed that the catalytic inactive N-terminal half is crucial for ATP depletioninduced HK1 aggregation. We conclude that HK1 senses energy stress and aggregates around constricted mitochondria to prevent mitochondrial fission, thereby most likely reducing cell death pathways. Given the high expression levels of HK1 in neurons, this novel function of HK1 might have multiple implications in health and diseases of the brain.

\section{P-389}

Investigating mechanotransduction in living cells by optical manipulation and FRET-based molecular force microscopy

Laura Perego [1], Marios Sergides [2], Giulio Bianchi [1], Marco Capitanio [1]

[1] Department of Physics and Astronomy, University of Florence, Italy; [2] Department of Physics, University of Cyprus, Cyprus

Mechanotransduction, that is the conversion of mechanical forces into biomolecular signals, is at the basis of many processes which are crucial for cell's development. However, knowledge of the mechanisms at its basis is still scarce. Optical tweezers have been successfully used to measure forces mainly on the external cell membrane. Recently, direct measurement of forces on proteins inside cells has become possible by using genetically encoded tension sensors. This technique uses force-sensitive peptides inserted between couples of fluorophores which undergo efficient FRET.

To investigate how mechanical stimuli propagate from the external environment into the cell, we implemented a setup which allows the combined use of force sensors and optical tweezers. Dielectric microspheres are trapped and used to apply and measure forces on cells, while FRET microscopy monitors the force acting on distinct proteins inside the cell. The extracellular environment is also critical to define cell mechanics. We investigated this effect by growing cells on substrates with different stiffness. Force measurements on actin and actinin by FRET-based tension sensors showed that forces on the cytoskeleton grow with the substrate stiffness.

\section{P-391}

Exploring the magnetic properties of natural silk polymers

Varun Ranade, Kamal P Singh

Indian Institute of Science Education and Research (IISER) Mohali, India

Silks are fibrous proteins, which are spun by a variety of species including silkworms and spiders. Research has revealed their potential use in tissue engineering and medical applications. Being a natural fibre, silk has been demonstrated to successfully being used in designing and developing artificial muscles, tissue as well as bones, which are advantageous over present available treatments without any inflammatory effects. Understanding fundamental physical properties of silk polymers like magnetism can be beneficial for future medical applications.

Magnetic properties of natural silk fibres in their pristine state have largely remained unexplored. We experimentally try to explore the magnetic properties of silkworm cocoon membrane and spider dragline silk in their pristine states without any magnetic doping using SQUID magnetometer. Elemental analysis techniques like EDX and XPS were used to check the presence of any ferromagnetic elements. Electron paramagnetic resonance (EPR) was also used to check the presence of free radicals in the silks. A sensitive torsion technique was also employed to check the behaviour of spider dragline silk under magnetic fields.

Magnetically active silk polymers can have a huge impact in biomedical fields as they can be spatially and temporally controlled, and can additionally be operated externally to the system, providing a non-invasive approach to remote control. 


\section{Posters}

-24. Biosensors -

P-392

Real-time in vitro determination of copper ions with a nanocapillary-based sensor

Roman Timoshenko [1], Alexander Vaneev [1,2], Nikita Savin [1], Nelly Chmelyuk [1], Olga Krasnovskaya [2,1], Alexander Savchenko[1], Natalia Klyachko[2], Alexander Majouga [1], Peter Gorelkin [1], Alexander Erofeev [2,1], Yuri Korchev $[3,1]$

[1] National University of Science and Technology "MISiS", Russian Federation; [2] Lomonosov Moscow State University, Russian Federation; [3] Imperial College London, UK

The problem of quantitative control of copper has been described in many cases. This is due to the research for new approaches to the treatment of neurodegenerative diseases associated with copper dysregulation, as well as the assessment of the penetration ability of promising copper-containing compounds, both anticancer and antimicrobial.

We present a development of a low-invasive electrochemical nanocapillary-based sensor for a wide range of applications, supported by the Russian Science Foundation (No. 19-7410059). For this goal, we use heat-resistant quartz glass nanocapillaries, suitable for the subsequent pyrolytic deposition of carbon inside the capillary. Further functionalization consists of etching a carbon surface on a tip and electrochemical deposition of gold into the resulting nanocavities.

Nanoscale tip of the sensor combined with a micromanipulator on which it is fixed allows you to real-time explore solutions or micro-object (single cells). We measured the copper concentration inside MCF-7 cells under the effect of copper, as well as the depth distribution of copper in the MCF-7 spheroid (3D tumor model).

\section{P-394}

Intracellular ROS detection by electrochemical nanocapillary-based nanosensor

Alexander Vaneev [1,2], Roman Timoshenko [1], Vasilii Kolmogorov [1,2], Elena Lopatukhina [2], Nelly Chmelyuk [1], Olga Krasnovskaya [2], Emil Yamansarov [2], Alexander Majouga [3], Pavel Novak [4], Natalia Klyachko[2], Alexander Erofeev [1,2], Peter Gorelkin [1], Yuri Korchev [1,4]

[1] NUST MISIS, Russian Federation; [2] Lomonosov Moscow State University, Russian Federation; [3] D. Mendeleev University of Chemical Technology of Russia, Russian Federation; [4] Imperial College London, UK

The study of intracellular ROS and RNS levels may represent one possibility to research the effects of anticancer drugs. Effect of many chemotherapeutic drugs is due to the induction of oxidative stress. Copper complexes have promising possibilities in the treatment of cancer by the ROS/RNS formation. In order to understand mechanism of action of new developed drugs, a sensor system is required capable of controlling ROS in life systems in the real-time. Our data obtained by electrochemical nanoprobe with platinum tips showed a ROS increase using copper compounds. Development of nanoprobe was supported by the Russian Science Foundation grant (No 19-79-30062). We studied the effect of drugs based on copper complexes on the cell line MCF-7 and demonstrated that copper complexes in various degrees of oxidation, depending on the ligands, can have different effects on tumor cells. ROS concentration in spheroids depends on the penetration depth and structure of copper complexes.

\section{P-393}

A novel $\mathrm{Ca}^{2+}$ biosensor for acidic organelles Patricia Torres-Vidal, Alba Delrio-Lorenzo, Jonathan RojoRuiz, Javier García-Sancho, M Teresa Alonso

Instituto de Biología y Genética Molecular (IBGM), Universidad de Valladolid y Consejo Superior de Investigaciones Científicas (CSIC). Valladolid, Spain

$\mathrm{Ca}^{2+}$ is an universal second messenger that is compartimentalized in citoplasmic organelles. Acidic organelles are emerging as important $\mathrm{Ca}^{2+}$ stores capable of accumulating and releasing $\mathrm{Ca}^{2+}$ upon cell activation. Therefore, much effort has been devoted to develope genetically encoded $\mathrm{Ca}^{2+}$ indicators as tools for measuring lumenal $\mathrm{Ca}^{2+}$ in acidic compartmets. However, this task is extremely challenging because most of the flurorescent $\mathrm{Ca}^{2+}$ indicators are $\mathrm{pH}-$ sensitive and this produces a reduction in the fluorescent signal. By contrast, bioluminiscence-based $\mathrm{Ca}^{2+}$ sensors are potentially advantageous since they are relatively resistant to $\mathrm{pH}$ variations. Aequorin is a $\mathrm{Ca}^{2+}$ binding protein that emits blue light when reconstituted with its cofactor coelenterazine. Targeted aequorins are excellent $\mathrm{Ca}^{2+}$ indicators for monitoring subcellular $\mathrm{Ca}^{2+}$ dynamics. Moreover, a wide range of $\mathrm{Ca}^{2+}$ affinities can be achieved by aequorin reconstitution with different celenterazines. Here, we will present results obtained with a novel family of $\mathrm{Ca}^{2+}$ aequorin-based fusion proteins targeted to endolysosome. We will provide evidence for mechanisms for $\mathrm{Ca}^{2+}$ uptake and release upon direct activation. This novel biosensor will provide new insights into the role of endolysosomes within cellular $\mathrm{Ca}^{2+}$ homeostasis upon different physio-pathological conditions.

\section{P-395}

Distinguishing algal cell species by quartz crystal microbalance

Ema Vlašić [1], Nives Novosel [2], Adrianna Zalewska [3], Anna Sobiepanek[3], Tomasz Kobiela [3], Nadica Ivošević Denardis [2], Tomislav Vuletić [4]

[1] Faculty of Science, University of Zagreb, Croatia; [2] Ruđer Bošković Institute, Zagreb, Croatia; [3] Faculty of Chemistry, Warsaw University of Technology, Warszawa, Poland; [4] Institute of Physics, Zagreb, Croatia

The study aimed to examine the effect of the physicomorphological properties of algal cells on binding efficiency to a surface modified sensor of a quartz crystal microbalance with dissipation monitoring (QCM-D). Two unicellular algae were tested; Cylindrotheca closterium encased with an organosilicate cell wall and Dunaliella tertiolecta as wall-less species. Cells were grown at 3 selected temperatures to examine their temperature tolerance. We found no correlation of the concentration of algae in the solution with the number of algae bound to the sensor. However, the correlation of this number to the resonant frequency shift $[\Delta f]$ of the sensor was confirmed. The viscoelastic properties (rigidity) of algae were represented by the dissipation factor shift of the sensor normalized by $\Delta f-$ i.e. adhered mass of algae. We found that the QCM-D method may distinguish the viscoelastic properties of the studied species, in accordance with their reported mechanical properties. However, the values vary widely and the results for the two species overlap, which could be related to cell age and physiological activity. Our results could contribute to a better understanding of interactions between algae and substrate and the development of QCM-D devices towards biological research methods. 
Posters

-24. Biosensors -

\section{P-396}

Fluidic force microscopy for monitoring and quantifying the nucleo-cytoskeletal coupling

Elaheh Zare-Eelanjegh [1], Ines Lüchtefeld [1], Mi Li [2], Edin Sarajlic [3], Tomaso Zambelli [1]

[1] Laboratory for Biosensors and Bioelectronics, Institute for Biomedical Engineering, ETH Zurich, Switzerland; 2] State Key Laboratory of Robotics, Shenyang Institute of Automation, Chinese Academy of Sciences, China; [3] MESA+ Institute for Nanotechnology, University of Twente, Netherlands

Understanding the interactions between biochemical and mechanical cues in cellular behaviors is a key for revealing numerous biological functions and development of major diseases. This necessitates creating tools enabling in situ monitoring and quantifying such small forces in intact cells. Mechanosensory complexes at the cellular level are such a demanding situation in which the dynamic interconnectivity of the cytoskeleton, nuclear envelope, and nucleoskeleton is yet to be understood. Fluidic force microscopy (FluidFM), a force-controlled micropipette configured on an atomic force microscope (AFM) enables to manipulate living cells while delivering non-permeable sensory biomolecules into their cytosol (or nucleus) for parallel screening of molecular responses. This will be achieved owing to the versatility of FluidFM tips and gentleness of cantilever-membrane contact. Thereby, I will elaborate FluidFM combined with sophisticated time-resolved optical readouts such as fluorescencelifetime imaging microscopy (FLIM) and fluorescence resonance energy transfer (FRET)-based systems to address the dynamic mechanosensory of essential components of the linker of nucleoskeleton and cytoskeleton (LINC) complex. 


\section{Author index}

\author{
Abbruzzetti Stefania P-26, \\ P-332, P-387 \\ Abdosamadi Mohammad O-124 \\ Abergel Chantal O-5 \\ Abhinav Abhinav P-1 \\ Abidine Y. O-43 \\ Abrahams Jan Pieter P-383 \\ Abreu Celeste P-247 \\ Abriel Hugues P-46 \\ Abrosimova Karina P-95 \\ Ackermann Elena P-345 \\ Acosta-Cáceres Jose M O-39 \\ Acuto Oreste P-339 \\ Adam Lutz P-73 \\ Adame-Arana Omar P-372 \\ Adamek Maksimiljan P-336 \\ Affatigato Luisa P-10 \\ Agbadaola Michael T O-138 \\ Agut Montserrat P-332 \\ Aguzzi Adriano O-56 \\ Ahmad Saira O-90 \\ Ahmadreza Mehdipour O-6 \\ Aicart Ramos Clara O-145 \\ Ainla Alar P-370 \\ Ajmail Karim P-284 \\ Akhgar Christopher K O-31 \\ Akimov Sergey A O-86, P-209, \\ P-214, P-271, P-319, \\ P-329, P-338 \\ Akopian Tigran P-95 \\ Aksimentiev Aleksei O-89 \\ $\mathrm{Al}$ Lawatia Issam A P-351 \\ Al Qanobi Amal J P-351 \\ Al. Et. P-31 \\ Alavi Zahra P-28 \\ Alawami Mohammed P-384 \\ Aleksanyan M. P-160
}

Alexandrova Veronika P-249

Alfano Caterina P-108

Alfonso Carlos P-342

Ali Mubarak P-188

Allan Clay Clark P-193

Allen Toby O-35

Almeida Vanessa O-69

Almendro-Vedia Víctor G. O-62, P-308

Almohammed Rajaei O-149

Alonso Alejandro P-309

Alonso M Teresa O-165, P-393

Alsadig Ahmed P-385

Alsteens David O-41, P-70, P-296

Alunni Cardinali Martina O-26

Alves Isabel D O-120

Amaro Rommie E P-66

Amenitsch Heinz O-73

Amunts Alexey O-53

Ananikov Valentine P O-96

Anderluh Gregor O-140, P-336

Andersson John O-92

André-Arpin C P-321

Andreas Loren P-343

Andriotis Orestis G P-131, P-298, P-301

Angeli Elena O-85

Angelov Borislav P-265

Angelova Angelina P-265

Angerer Nadine P-337

Aniander Gustav P-173

Anikin Vasily V P-172

Anisimov Mikhail N P-250

Ansell T. Bertie O-142

Anselmo Sara P-310

Antenucci Fabio P-363
Anthony Nicholas O-25, P-76

Antila Hanne S P-218

Antonenko Yuri N P-127, P-329

Antoniazzi G. P-38

Antonio Enríqez José O-57

Antonschmidt Leif P-343

Antosova Andrea P-96, P-113

Anttu Nicklas P-181

Aragonés Juan L. P-308

Aramesh M. O-167

Aravamudhan Pavithra P-70

Archer Margarida O-69

Argyris Politis O-36

Aricescu A. Radu P-59

Arjoca Stelian P-286

Arjun A O-112

Arnion Hélène P-47

Arrasate P. P-135

Arroum Tasnim P-117

Artzner Franck P-273

Arzt Eduard P-18

Ataullakhanov Fazoil I P-253

Auer Albert P-320

Axford Danny O-102

Axmann Markus P-311, P-350

Baaden Marc P-220

Babalola Jonathan O O-138

Baccouch Rim O-120

Bachmann M. P-38

Bacia Kirsten P-318

Bacsa Bernadett P-366

Bada Juarez Juan F O-102

Badocha Michał P-128

Baecker V P-321

Bage Marcus G O-149

Bail Celine P-345 
Bajpai Gaurav P-372

Baksa Attila P-115

Balaban Can P-346

Balaguer Pérez Francisco O-145

Baldini Francesca P-77, P-91, P-353

Bálint Z. P-293

Ball Robin C P-376

Bally Marta O-43, P-67

Balog Erika P-223

Bano Fouzia P-67

Bánó Gregor P-19, P-177

Baranauskienè Lina P-124

Baranova Natalia P-262

Barayeu Uladzimir P-150

Barbotin A P-80

Bargmann Cornelia O-81

Barin Le Guellec Chantal P-47

Bařinka Cyril P-260

Barreto Andreia O-59

Bartnik Kira P-323

Bartok Adam O-18

Bartoš Ladislav P-201

Barvík Ivan P-78

Barz Bogdan P-380

Bashiri Shadi P-290

Bashkirov Pavel V P-135, P-271

Batchu Krishna P-312

Batishchev Oleg V P-12, P-27, P-68, P-72, P-168, P-178, P-214, P-319, P-379

Batlle Cristina O-158

Baud Stéphanie P-232

Baudis Stefan P-131

Bauer Daniel O-16, P-57

Bauer Julian O-49

Baumann Phila P-282

Baumgart Florian P-86

Bayzhumanov Adil A P-121

Bazzurro Virginia O-85

Beales Paul A P-316

Beato Miguel O-160

Bechinger Burkhard O-103

Becker Jan O-50

Becker Robert A P-352

Becker Stefan P-343

Becker Thomas O-51
Beckmann Roland O-51

Bednarikova Z. P-97, P-198

Bednarikova Zuzana P-96, P-113

Bejenariu Maria I P-286

Belcher Heather A P-137

Bell Nicholas A W P-185

Belle Valérie O-28

Belleza Oliver John V P-39

Belloy Nicolas P-232

Benabbas Abdelkrim O-89

Benedetto Antonio O-94, P-194, P-197

Bento Isabel P-374

Benvegnu Thierry P-273

Bera Krishnendu P-98

Berberan-Santos Mario N P-42

Berchel Matthieu P-273

Berdychowska Julia P-219

Beresowski Ann-Kathrin P-345

Berg Johan O-13

Bergese P. P-34

Bergler Helmut P-213

Bergs Amelie O-81

Bergström T. O-43

Berlansky Sascha P-8

Bernardi Paolo P-40

Bernát Ondrej P-11

Bernhard David P-133

Berninghausen Otto O-51

Bernsteiner Harald P-43

Berta Martin P-19, P-99, P-101, P-103

Bertarello Andrea O-37

Berti D. P-34

Bezanilla Francisco O-3

Bhagawati Maniraj P-117

Bhatia Sumati P-73

Bhunia A. P-97

Bianchi Giulio P-389

Bianchini Paolo P-22, P-26, P-76, P-77, P-91, P-332, P-353, P-387

Biasutto L. P-38, P-58

Bicout Dominique J. P-30

Biebricher Andreas P-285

Biner Olivier F P-64

Birarda Giovanni P-174
Biriukov Denys P-230

Bisio Hugo O-5

Bittihn Philip O-136

Bizien Thomas P-265

Bláha Jan P-247

Bloch Wilhelm P-154

Block Stephan O-13, O-68, P-73

Blum Thorsten P-383

Blume Alfred P-283

Bobone Sara P-202, P-216

Bochicchio Anna P-267

Bochkova Zhanna V P-121

Bock Lars V P-106, P-134

Böckmann Rainer A O-118, O-131, P-231, P-267

Bodenschatz Jonathan P-284

Bodner Clara M P-100

Boehm Stefan P-56, P-62

Boersma Arnold J P-382

Boffi Alberto P-10

Bogatyr Vadim P-285

Bogi Eszter P-48

Böhm Stefan P-50

Bojin Florina P-286

Bolhuis Peter G O-112

Bollmann Björn O-117

Bolognesi Martino O-16, P-57

Bommer Tobias P-71

Bonaccorsi di Patti Maria Carmela $\mathrm{P}-33$

Bonaccorsi Marta O-37

Bonamore Alessandra P-10

Bondar Alexey O-48

Bondar Ana-Nicoleta O-9

Bonhenry Daniel P-8

Bonthuis Douwe J P-275

Booth Paula O-36

Borges-Araújo Luís P-266

Bošković Filip P-384

Botvinick Elliot O-80

Bourassin Nicolas P-220

Bouzin Margaux P-23

Bowerman Samuel O-5

Boytsov Danila O-12, O-86

Božič Anže P-75

Brameshuber Mario P-86, P-100, P-212, P-295

Brandner Astrid F P-267 
Bratashov Daniil N P-172

Braun Marcus P-78

Brautigam Chad A P-31

Brazhe Nadezda A P-121

Brenker Kathrin P-161

Brescia Stefania O-12

Brézillon Stéphane P-232

Brkljaca Zlatko O-60, P-127

Brockwell David J O-64

Broedersz Chase P O-78

Brogini Silvia O-26

Brooks Rhiannon L P-313

Brucale M. P-34

Bruinsma Charlotte O-78

Brumovska Veronika O-130

Bründl Michael P-314

Bryan Louise P-357

Brynda Jiří O-48

Bryszewska Maria P-24

Brzezinski Peter O-13

Buchegger Bianca P-162

Buchroithner Boris P-17

Budaitis Breane G P-251

Buell Alexander K O-161, P-380

Bugiel Michael O-124

Bujak Łukasz P-78, P-255

Busch Karin B P-117

Bussi Giovanni P-224

Bystrenova Eva P-96

C Shetty Sunidhi O-154

Cabrita Eurico J O-59

Cacciuto Angelo P-189

Cainero Isotta P-77, P-79, P-91, P-353

Callegari Fabio P-22

Calleja Montserrat P-144

Calò Annalisa P-136

Campagnaro M. P-38

Campbell K N P-155

Cañadas Olga P-309

Canale Claudio P-29, P-153, P-327

Canepa Ester P-373

Canepa Maurizio O-166, P-175

Canepa Paolo O-166, P-175

Canigova Nikola P-320
Cantù Laura P-315

Capellini Giovanni P-290

Capitanio Marco P-389

Caponi Silvia O-26

Carabadjac Iulia P-163

Carapeto Ana P O-59

Cardellini J. P-34

Carlein Christopher P-18

Carotenuto A. P-69

Carragher Bridget O-69

Carraro Michela P-40

Carravilla Pablo P-80, P-200

Carrer Andrea P-38, P-40

Carretero-Genevrier A P-321

Carriero Alessandra P-298

Carrotta Rita P-165

Casalis Loredana O-166, P-385

Casanellas Laura P-287

Casas-Ferrer Laura P-287

Casciaro Bruno P-202

Caselli L. P-34

Castro A P-321

Casuso I P-321

Català-Castro Frederic P-156

Catania Rosa P-316

Cavalleri Ornella O-166, P-175

Cean Ada P-286

Černe Urška P-304

Černeková Michaela P-94

Cerutti E. P-79

Červenka Jakub P-346

Cervera Javier P-188, P-288

Cervinkova Marketa O-72

Cervoni Laura P-33

Chakrabarti Buddhapriya J

$$
\mathrm{P}-107
$$

Champion Paul O-89

Chaparro-Riggers Javier P-133

Charles Russell M P-328

Chatzimagas Leonie P-221

Chauvier Adrien P-356

Chaves-Sanjuan Antonio P-57

Chavez-Sanjuan Antonio O-16

Chen Kaikai P-185, P-384

Chen Wei P-133

Chen Ying-Ju P-83

Chernysh Alexander P-264

Chilom Claudia P-36
Chirico Giuseppe P-23

Chiti Fabrizio P-153

Chmelyuk Nelly P-392, P-394

Chodnicki Pawel P-119, P-334

Choi Wanjae P-187

Chorvat Dusan P-84

Chouliara Manto O-20, P-243, P-289

Chroni Angeliki P-112

Ciasca G. P-35

Ciotu Cosmin I P-62

Ciscato Francesco P-40

Cisse Aline O-73, P-30

Clarion Tung P-189

Clark Clay P-109

Clarke Amy P-317, P-322, $\mathrm{P}-348$

Clausen-Schaumann Hauke P-154

Coceano Giovanna O-146

Cojoc Dan P-20

Collini Maddalena P-23

Colombi Ciacchi Lucio P-224

Coluzza Ivan P-189

Conceição Cristiano O-69

Cook Graham P P-339

Cordes Thorben O-33

Corey Robin A O-142

Cortajarena Aitziber L O-55

Corucci Giacomo P-312

Cosentino Katia P-294

Costa Flavio P-41

Cote Sebastien P-386

Coutinho Ana P-42

Covino Roberto O-112, P-331

Cowling Victoria H O-149

Crea F. P-160

Crha Radek P-252

Crowet Jean-Marc P-232

Csanády László O-18, P-61

Csernoch L. P-38

Čunderliková Beata P-14

Cupello Aroldo O-85

Cutone Antimo P-33

Czub Jacek P-119, P-128, P-334

Dabkowska Aleksandra P-323

Dafnis Ioannis P-112 
Dahlin Andreas O-92

Dahmann Christian P-146

Dałek Paulina P-140

D'Alfonso Laura P-23

Dallari Dante O-26

Dam Tommy O-20, P-243, P-289

D'Amico M. P-79

Danielczak Bartholomäus O-138

Dante Silvia O-166, P-327

Danylchuk Dmytro I P-200

Daraei Ali P-137

Das C. P-222

Dasgupta Anindita O-50, P-200

Dauchez Manuel P-232

Daum Sebastian P-318

Daver Henrik P-238

David Melinda P-36

Davis Simon J P-243

Dayal Anamika P-7

de A. P. Schwarzer Ana C O-74

de Boer Marijn O-33

de Bragança Sara O-145

de Buhr Svenja P-138

de Groot Bert O-34, P-52

de Koning Matthijs O-33

de la Torre-Martínez Roberto

$$
\mathrm{P}-63
$$

de Llobet Cucalon Lara O-160

de Luca Giuseppe O-159, P-164

de Roo V. P-215

De S. P-97

de Smedt-Peyrusse Véronique O-120

de Spirito M. P-35

Debets Vincent O-137

Defelipe Lucas A P-374

Dekker Cees O-87

del Campo Aranzazu P-296

del Castillo Carla E C P-195

Del Favero Elena P-315

Delcanale Pietro P-332

Deleu Magali P-270

Dellino G. I P-79

Delrio-Lorenzo Alba O-165, P-393

Dement Taylor C P-137
Deng Yuru P-265

Denieva Zaret G P-68, P-72, P-319

Derganc Jure P-139

Derler Isabella P-171, P-326

Dermody Terence S P-70

Dervisoglu Riza P-343

Deschamps Joran O-50

Desfosses Ambroise O-73

Deviri Dan P-375

Di Giacinto F. P-35

Di Gioacchino Michael P-290

Di Meo Florent O-38, P-47

Di Novo N. P-69

Di Prima Giulia P-165

Diaspro Alberto O-25, O-85, P-22, P-26, P-76, P-77, P-79, P-91, P-299, P-327, P-332, P-353, P-387

Díaz-García Clara P-42

Dick Tobias P-150

Diez Stefan O-121

Dillingham Mark S O-145

Dimaio Frank P-383

Dimova Rumiana O-152, P-160, P-210, P-277, P-340

Dinčić Marko P-300

Dixon Ann M P-204, P-313

Dobson Christopher M O-56

Dogterom Marileen O-123

Dohnálek Jan P-247

Doktorova Milka P-269

Dolezal Vojtech O-72

Domingues Marco M P-266

Dopitová Radka O-70

Doskocz Joanna P-140

Drechsler Markus P-265

Dreher Yannik P-362

Dremencov Eliyahu P-48

Drexler Nils P-55

Duart Gerard O-39

Dubrovin Evgeniy $\quad \mathrm{P}-12$

Dudas Balint P-223

Duellberg Christian P-320

Dujmović Ana O-67

Dumitru Andra C P-70, P-296

Durmaz Hilal O-81
Durroux Thierry O-120

Dušeková Eva P-101

Dutta Debajyoti P-64

Dutzler Raimund O-4

Dyer Oliver T P-376

Džubinská Daniela P-166, P-182

Džupponová Veronika P-102

Dzurillová Veronika P-99, P-101, P-103

Ebel Christine P-33

Ebenhan Jan P-318

Ebert Andrea P-118

Ebner Andreas P-65

Ebner Janine P-240

Edwards Christopher P-148

Efimova Svetlana S P-74

Eggeling Christian O-132, P-80, P-82, P-87, P-200, $\mathrm{P}-217$

Egorova Ksenia S O-96

Eicher Barbara O-86

El Alaoui F P-321

Elad Nadav O-148

Elf Johan O-146

Eliaš Daniel P-272

Elmstrøm Christiansen Line P-363

Enderlein Jörg O-32

Engberg Oskar P-268

Engel Andreas P-53

Engelke Hanna P-167

Ensinger Wolfgang P-188

Epe Markus O-30

Erb Tobias J O-151

Erdmann R P-80

Erkan Hazel P-322, P-348

Erler Janine P-154

Ermakov Yuri A P-178

Ermakov Yury A P-203

Ermann Niklas P-185

Erofeev Christian S P-12, P-148, P-151, P-392, P-394

Eroles Mar P-141

Errico Silvia P-153

Eschbach Sebastian H P-356 
Essers Maria P-46

Evci Hüseyin O-141

Fabian Marian P-120

Fabry Ben P-141

Fahmy Karim O-61

Fahrner Marc O-19, P-8, P-326

Falginella Francesco L P-239

Fan Chen O-35

Fan Ning P-136

Fandrei Ferdinand P-268

Fardelli Elisa P-290

Faretta M. P-79

Farkas B P-104

Farrell Daniel P-383

Fatti Edoardo P-125

Faucher Quentin P-47

Favero Ivan P-144

Fedorov Aleksander P-42

Fedorov Alexander P-266

Fedorov Vladimir A P-257

Fedunova D. P-198

Feng Elvin $\quad$ P-136

Fennema Galparsoro Dirk O-159, P-105

Fenz Susanne P-85

Ferle Maximilian P-303

Fernandes F P-266

Fernandes Pedro O-59

Fernández-Carvajal Asia P-63

Fernández-Quintero Monica L O-113

Ferrando Riccardo P-153

Ferrando-May E. P-299

Ferreira Tiago M P-218, P-283

Ferrer-Montiel Antonio P-63

Fica Sebastian M O-101

Ficek Mateusz P-388

Fierz Beat O-147, P-357

Filadi Riccardo P-40

Findlay Heather O-36

Fini Milena O-26

Fioretto Daniele O-26

Fischbach Tobias P-280

Fischer Markus O-97

Fischer Markus G P-291

Fischer Michael J M P-62

Fischer Niels O-74
Fischer-Friedrich Elisabeth

$$
\text { P-146 }
$$

Fisher Gemma Lm O-145

Fläschner Gotthold P-142

Fleetwood Oliver O-109

Flegel Hendrik P-211

Fleischhauer Lutz P-154

Fleury Jean-Baptiste P-145, P-278

Flood Emelie O-35

Florescu Monica P-36

Florian Blanc O-6

Foderà Vito O-159, P-105

Fölser Martin P-295

Fornasier Marco P-323

Forte Michael P-40

Fortuna Sara P-385

Fragneto Giovanna P-312

Fraldi M. P-69

Francetic Olivera P-228

Francisco Barrera N P-328

Freiberg Arvi P-126

Freire Mara G O-95

Freissmuth Michael P-65

Frengen Eirik O-19

Frewein Moritz P K P-269, $\mathrm{P}-275$

Frieden Maud P-7

Friesacher Theres P-43

Friis Henrik P-365

Frimurer Thomas M P-234

Frischauf Irene P-8

Frischauf Nikolaus O-24, P-13

Frolov V. A P-135

Fu Lifei O-17

Fuchs Adrian P-167

Füllbrunn Nadia O-32

Fülöp Gergö O-130

Furlan Aurélien P-270

Fuwad Ahmed P-187

Fuxreiter Monika O-157

Gabriel Christian P-162

Gabrielli Sara P-106

Gahan Lianne D P-107

Gahlay Gagandeep K O-97

Galej Wojciech P O-101

Galiani S P-80
Galimzyanov Timur R O-86, P-2, P-203, P-209, P-271, P-319, P-338, P-379

Gallo P. P-58

Gamage Thilini H O-19

Gancar Miroslav P-96, P-113

Ganzella Marcelo P-377

Ganzinger Kristina O-49

Garaiová Zuzana P-14, P-335

Garcia Alai Maria M P-374

Garcia-Delicado Esmerilda P-143

García-López Sergio P-144

Garcia-Morales Vladimir P-188

García-Murria Maria J O-39

Garcia-Parajo M. F O-45

Garcia-Parajo Maria O-160

García-Sancho Javier O-165, P-393

Garidel Patrick P-129

Gärtner Wolfgang P-387

Garzella Francesco P-387

Gaspar Ricardo P-323

Gašparik Norbert P-252

Gasparri Federica P-57

Gatta Elena O-85

Gavara Núria O-75

Gavriliuc Oana P-286

Gawlik Wojciech P-388

Gazova Zuzana P-96, P-97, P-113, P-198

Gbelská Yvetta P-272

Gegenfurtner Florian P-167

Gennerich Arne P-251

Gennis Robert B P-123

Georgiev V. P-160

Gerke Volker P-195

Gerum Richard P-141

Gervasoni J. P-35

Geudens N. P-215

Ghosh Sajal K P-192

Ghosh Sajal Kumar P-196

Giacomello Alberto P-41

Giergiel Magdalena P-15

Giese Hunter O-16, P-57

Gil-Redondo Juan Carlos P-143

Gil-Santos Eduardo P-144 
Gilliard Guillaume P-270

Giudici Marcela P-42

Gladyshev Pavel P P-122

Glasnov Toma N O-86

Gloriam David E P-238

Glorius Frank P-195

Glowacki Eric Daniel O-84

Głowacki Maciej P-388

Glunčić Matko P-358

Gnanachandran Kajangi P-292

Göhring Janett P-132

Golestanian Ramin O-136

Gonczi M. P-38

Gong Weijian O-132

Gonzáles Montoro Ayelen P-117

González-Ros José M P-42

Goodilin Evgeny A P-121

Göpfrich Kerstin O-153, P-362

Gorbunova Yulia P-27, P-168, P-178

Górecki Radosław P-363

Gorelkin Petr P-12, P-148, P-151, P-392, P-394

Gorokh Anton P-95

Gorshkova Yulia E P-265

Goss Kai-Uwe P-118

Gottschalk Alexander O-81

Gottschalk Benjamin P-390

Gottwald Jannis P-303

Gouridis Giorgos O-33

Govoni Marco O-26

Gözen Irep O-150, P-365, P-368, P-370, P-371

Grabmayr Herwig O-19, P-8, P-326

Grabner Manfred P-7

Gradisch Ralph P-44

Grafmueller A. P-160

Graier Wolfgang P-390

Gräter Frauke P-138, P-150

Graziani Annarita P-366

Gremer Lothar P-380

Grensemann M P-208

Griesinger Christian P-343

Griffo Alessandra P-145, P-278

Grill David P-195

Grinchii Daniil P-48

Grivennikova Vera G P-121
Grollios Fanis O-72

Groschner Klaus P-317, P-322, P-348, P-366

Groth S P-208

Grothaus Isabell L P-224

Gruber Hermann P-65

Grubhoffer L O-66

Grubmüller Helmut O-114, O-126, P-104, P-106, $\mathrm{P}-134$

Grünzweil Sandra P-3

Gsell Matthias P-322

Guardiani Carlo P-41

Gudimchuk Nikita B P-249, P-250, P-253, P-257

Gudkova Olga P-264

Guéroult Marc P-232

Guerra Karla Y P-146

Guerrero Paula P-210

Guichard Sabrina P-46

Gulli Daniele P-108

Gupta Ritika P-192

Guthold Martin P-137

Gutsche Irina O-73

Gutsmann Thomas O-106, P-208, P-212

Guy Ciaran A P-204

Gynnå Arvid H O-146

Haag Rainer P-73

Haasnoot Guus H O-78

Hackenberger Christian P-73

Hackl Benjamin P-240

Haechl Nicholas P-240

Hafer Maximillian O-119

Häffner Sara M P-205

Hager Roland P-241

Hagleitner-Ertuğrul Nora O-90, P-186

Hähl Hendrik P-145, P-278

Hain L O-66

Hajdú István P-115

Häkkinen Hanna-Maria O-135

Halász Henriett P-81

Hally Cormac P-332

Hämmerli Anne-Flore P-46

Hamulakova Slavka P-113

Handl Verena P-16, P-199
Hanein Dorit O-100

Hannesschläger Christof $\quad \mathrm{O}-86$

Hansen Ulf-Peter P-55

Hanser Arnold O-73

Harju Janni O-78

Harpsøe Kasper P-238

Harris Nicola O-36

Hartl F. Ulrich O-56

Haufe Yves P-45

Hauser Fabian P-17

Hawkins Rhoda J P-107, P-261

He X P-155

Hebenstreit Daniel P-376

Heberle Frederick A P-269

Heberle Joachim O-13, P-160

Heck Martin O-117

Heerklotz Heiko O-127, P-129, P-163, P-215, P-274, $\mathrm{P}-333$

Hegedüs T P-104

Hegemann Peter O-81

Heimann Larissa P-18

Heinrich Dorothea O-13

Heintzmann Rainer O-50

Heißenberg Tim P-254

Hélix-Nielsen Claus P-363

Hellenkamp Björn P-184

Hellmeier Joschka P-242, P-295

Hellmich Christian P-147

Hendriks Maurice P-170

Henrichs Verena P-78

Hepworth Olivia O-107, P-207

Hermann Enikö P-225

Hernansanz-Agustín Pablo O-57

Herrmann Andreas P-73

Hess Samuel T P-89

Heuer Andreas P-195

Hianik Tibor P-14, P-24, P-335

Hickson Ian D O-78

Hilber Karlheinz P-7, P-240

Hill Christian P-176

Hiller Sebastian O-108

Hinterdorfer Peter O-66, P-65, P-133, P-186

Hirst Judy P-64

Hirth Alexander P-125

Hlavenkova Zuzana O-72 
Hoboth Peter P-324

Hodel Adrian P-325

Hoerl Gerd O-73

Hoess Philipp P-93

Hof Martin O-141, P-1

Hofer Florian P-227

Hoffmann Christian P-377

Hofmann Hagen O-148

Hofrová Alena P-252

Höglinger Carmen P-3, P-326

Hohenester Ulrich P-176

Hoijman Esteban O-135

Holanová Kristýna P-78, P-255

Holbová Radka O-70

Holcman David P-85

Holtmannspötter Michael P-294

Holy Marion P-65

Holz Danielle P-367

Homolka S P-208

Honigmann Alf P-303

Hoogenboom Bart P-325

Höök Fredrik P-181

Horn Georg O-17

Horvat Anemari P-304

Horvath Ferdinand P-326

Hossain Kazi A P-119

Hovan Andrej P-19, P-177

Howorka Stefan O-88, P-186

Hozák Pavel P-324, P-346

Hritz Jozef P-98, P-252

Hua Lisa P-274

Hub Jochen S O-14, P-51, P-71, P-221, P-276, P-280, $\mathrm{P}-352$

Hübner Uwe O-50

Hudson David M. P-150

Hudson Nathan E P-137

Hugel Thorsten P-184

Hugentobler Katharina G O-13, P-73

Humenik Martin P-381

Humer Christina P-3, P-8

Hummer Gerhard O-6, O-112, P-229

Humphfrey Jane O-20

Huppa Johannes B P-86, P-132, P-242

Hurmach V. P-226
Huser Thomas O-47

Hussain Hazrat O-90

Huster Daniel O-97, P-267, $\mathrm{P}-268$

Hytoenen J O-66

Iakovlev Aleksei P P-148

Iamshanova Oksana P-46

Iaparov Bogdan P-4

Ideguchi Takuro O-27

Igaev Maxim O-114, O-126

Ignat Elena P-293

Ikonen Elina P-341

Ilić Andjelija P-300

Illes Bernhard P-167

Inozemtsev Vladimir P-264

Inverso Donato P-23

Ionov Maksim P-24

Issoglio Federico O-69

Iturri Jagoba P-143

Ives Callum M P-5, P-248

Ivošević Denardis Nadica P-395

Izadi-Pruneyre Nadia P-228

Jacak Jaroslaw P-17, P-162

Jachowski Tobias O-124

Jacko Juraj P-272

Jacob Aunstrup Larsen O-161

Jacobs Karin P-145, P-278

Jacobs Robert P-312

Jadavi Samira P-327

Jagiello Alicja O-80

Jahnke Kevin O-153, P-362

Jakešová Marie P-199

Jakob Annik P-161

Janaszkiewicz Angelika O-38, P-47

Jancura Daniel P-120

Jannasch Anita O-124

Janshoff Andreas O-76, P-263, P-284, P-303

Jariwala Shashank P-251

Javanainen Matti P-230

Jeftic Jelena P-273

Jenkins Edward O-132

Jennings James P-206

Jensen Henrik O-161

Jeon Tae-Joon P-187
Jerković Hrvoje P-358

Jesacher Alexander P-92

Jeudy Sandra O-5

Jeuken Lars J C P-64, P-316

Jia Ruyu O-36

Jiménez-Delgado Senda P-156

Jimenez-Munguia Irene P-178

Jobin Marie-Lise O-120

Joglekar Isha P-109

Jokitalo Eija P-341

Jönsson Peter O-20, P-243, P-289, P-323

Jorde Lara O-32

Joshi Manali P-245

Jou Ining P-185

Jovanovic Olga P-127

Jowitt Tom P-31

Judge Peter J O-102

Jung Hendrik O-112

Junghans Victoria O-20, P-243, P-289

Jungmann Ralf O-46, O-49, O-50

Jungwirth Jakub O-148

Jungwirth Pavel O-141

Jurkiewicz Piotr O-141

Jurkovicova-Tarabova Bohumila P-48

Jurkowski Michał P-128

Justin Westerfield M P-328

Kabelka Ivo P-201

Kahl Gerhard P-147

Kahler Ursula P-227

Kairys Visvaldas P-124

Kaiser Michael P-274

Kalimeri Maria P-344

Kalli Antreas C P-339

Kalliauer Johannes P-147

Kalousková Barbora P-247

Kaltenegger Michael P-275

Kalutsky Maksim P-168

Kamenik Anna S P-130, P-227

Kandiah Eaazhisai O-73

Kanduč Matej P-75

Kangur Liina P-126

Kappelhoff Shirin P-294

Kappen Marie P-246 
Kappl Reinhard P-150

Kar R. K P-97

KaraDi Giridhar Mithun Nag P-193

Karami Rad Meysam P-199

Karami Yasaman P-228

Karia Dimple O-72

Karner Andreas P-244, P-311

Karoui Hedi P-364

Karvelis Tautvydas P-354, P-355

Kasparyan Gari P-276

Kater Lukas O-51

Katherine Stefanski M P-328

Kauert Dominik J P-111

Kaur Navleen O-97

Kazokaitè-Adomaitienè Justina $\mathrm{P}-124$

Keller Fabian P-195

Keller Sandro O-138, P-337

Kellermayer M P-104

Kelley Charlotte F P-367

Kellner Florian P-132

Kempka Marek P-116

Kerkhoff Yannic O-68, P-73

Keyser Ulrich F P-185, P-384

Kežar Andreja P-336

Khailova Ljudmila S P-127

Khalid Syma O-128

Kharitonova Yulia V P-2

Kholina Ekatherina G P-257

Khoroshyy Petro O-48

Kichick Nessim P-207

Kichik Nessim O-107

Kiesenhofer Dominik P-100

Kilicarslan Dilara P-187

Kim Eugene O-144

Kim Sun Min P-187

King Martin S O-58

King Patrick J P-110

Kireev I. P-151

Kireev Igor I P-148

Kisnieriene Vilma P-54

Kiss B P-104

Klar Thomas A P-162

Kleist Cyprian P-128

Klepka Barbara P-378

Klishin Nikolai P-210
Kloter T. O-167

Klotzsch Enrico P-132

Klumpp Stefan O-125, P-263

Klyachko Natalýa L P-148, P-392, P-394

Klymchenko Andrey S P-200

Kneipp Janina P-179

Knippenberg Stefan P-173

Knoch Hannah P-129

Knowles Tuomas P J O-56

Knyazev Denis O-90, P-68, P-186

Kobayashi Toshihide P-114

Köbele Luis P-161

Kobiela Tomasz P-395

Koch Hans-Georg P-163

Kochems Kirstin P-278

Koehler Melanie P-70

Koenig Stéphane P-7

Koenig Xaver P-7, P-240

Koerfer Agnes $\mathrm{P}-82$

Köfinger Jürgen P-229

Kohl Peter O-22

Köksal Elif S P-365, P-368, P-370

Kolář Michal H P-94

Kölbel Knut P-374

Kolesarova Simona P-180

Kolesnikova I. P-293

Kolmogorov Vasilii S P-12, P-148, P-151, P-394

Kołodziej Tomasz P-388

Kondrashov Oleg V P-329

Konior Jerzy P-15

Konstantinova Anna N P-2, P-178

Kopūstas Aurimas P-354, P-355

Kopec Wojciech P-52

Kopittke Caroline P-295

Kopp Franziska P-212

Korchev Yuri P-392, P-394

Korchev Yuri E P-148

Korn Viktoria H P-330

Korneev Sergej P-246

Körnig André P-149

Kornmüller Karin $\quad$ O-73, O-84

Kosaka Priscila Monteiro P-144
Köster Sarah O-125, P-256, P-259, P-263

Kotecha Abhay O-72

Kotler S. A P-97

Kotova Elena A P-127, P-329

Kováčik Andrej P-268

Kovács B. P-215

Kovalenko Ilya B P-257

Kozak Maciej P-116

Kozlov Alexander P-264

Kozlova Elena P-264

Kraml Johannes P-227

Krasnobaev Vladimir D P-379

Krasnovskaya Olga P-392, $\mathrm{P}-394$

Krasnowska E. P-35

Kravchenko Elena P-360

Kraxner Julia P-256

Kreiter Jürgen O-60

Kremser Johannes P-275

Krendel M P-155

Krieg Micheal P-156

Krivić Denis P-366

Krizova A P-155

Krobath Heinrich P-326

Krychowiak-Maśnicka Marta P-307

Kubácková Jana P-177

Kudlacek Oliver P-56, P-65

Kudruk Sergej P-195

Kumar Sandeep O-97

Kumari Pallavi P-194

Kunji Edmund R O-58

Kuravsky Mikhail L O-54

Kurre Rainer O-119, P-294

Kurth Markus P-150

Kuzmin Petr I P-135, P-319, P-338

Kuzmina Natalia P-12

Kwan Tristan O O-102

Kyoung Jae Won P-154

Laaksonen Päivi P-278

Lacinova Lubica P-48

Lafontaine Daniel A P-356

Lagerholm B. Christoffer P-87

Lalkovicova M. P-293

Lally Ciara C O-117 
Lamontagne Anne-Maire P-356

Lange Stefan O-85

Langenbucher Achim P-18

Langner Marek P-140

Lánský Zdeněk P-78

Lanzanò L. P-79, P-299

Lanzerstorfer Peter P-241

Lapeikaite Indre P-54

Lappalainen Pekka P-344

Larsson Emma P-173

Larsson Jimmy O-146

Launikonis Bradley S P-7

Lauster Daniel P-73

Lavrushkina Svetlana V P-148, $\mathrm{P}-151$

Lazar Josef O-48

Lazzarin Erika P-49

Lazzeri Gianmarco P-331

Le Gratiet Aymeric P-22

Le Roy Aline P-33

Leanza L. P-38

Lee Sejeong O-107, P-207

Lee Seonwoo P-169

Lee Wing-Kee P-281

Leitinger Gerd O-73

Leitner Romana P-8

Lekka Małgorzata O-77, P-159, P-292, P-305

Lemaitre Arestide $\quad$ P-144

Lemiègre Loïc P-273

Lemke Edward A O-156

Lendl Bernhard O-31

Lennon R P-155

Lenz Julia O-138

Leomil Fernanda S P-277

Leonardo Sacconi O-23

Leroy Prune O-146

Leuchtag H. Richard P-6

Levin Michael P-288

Lewenstein Maciej O-160

Li Hongbin O-65

Li Mi P-396

Li Shiqian P-341

Li Zehao O-32

Librizzi Fabio P-165

Liedl Klaus R O-113, P-130, P-227

Lienemann Michael P-278
Liewald Jana O-81

Lilliu Elena P-7, P-240

Lillo María Del Pilar P-308

Lim Micah O-80

Lima Maria Augusta D R B F P-20

Linard Matos Anna Livia P-195

Lindinger Sonja P-3

Lindorff-Larsen Kresten O-110

Linke Heiner P-181

Linse Sara P-323

Linse Sara S P-380

Linser R. P-222

Lipowsky Reinhard P-340

Lira Rafael B P-277

Litschel Thomas P-367

Liu Haipei O-67

Liu Qiang O-81

Liu Yang O-5

Liu Zhaowei O-67

Liutkus Mantas O-55

Llauro-Portell Aida P-170

Llombart Pablo P-308

Lo Giudice Cristina P-70

Loeffler Johannes R P-130

Loffredo Maria Rosa P-202, P-216

Logan Chinyere P-377

Lohner Karl O-104, P-206, P-212, P-213

Loibl Katrin P-320

Lolicato Fabio O-143

Loose Martin P-262, P-320

Lopanskaia Iuliia N P-257

Lopatukhina Elena P-394

López Montero Iván O-62

Lopez Simon Miguel M P-39

López-Martínez Montserrat P-83, P-88

Lopez-Mendez Blanca P-31

López-Montero Iván P-308

López-Pelegrín Mar P-262

Lopez-Rios de Castro Raquel $\mathrm{P}-32$

Lorca T P-321

Lorenz Charlotta O-125, P-256, $\mathrm{P}-263$

Lorenz Christian D P-32
Losgott Thomas P-50

Loshkareva Anna S P-68

Losi Aba P-387

Loudova Leona P-9

Lüchtefeld Ines P-396

Lucidi Massimiliano P-290

Lucie Delemotte O-109

Lückmann Michael P-234

Ludwig Kai P-73

Luger Karolin O-5

Lukacs G P-104

Lukács Péter P-240

Lundgren Anders P-152

Luo Jinghui P-383

Lupi Stefano P-174

Luty Marcin P-305

M. Pilar Lillo O-62

Machin Jonathan P-316

Madariaga-Marcos Julene P-111

Madej Gregor O-17

Mafe Salvador P-188, P-288

Mair Alexander P-189

Maiti N. C P-97

Maity Sourav O-42

Maja Mauriane P-296

Majouga Alexander P-148, P-392, P-394

Makasheva Kristina P-357

Makrinsky Kirill I P-178, P-203

Makrocka-Rydzyk Monika P-116

Maksimov Georgy P-121, P-151

Malanovic Nermina P-206

Malinsky Milos O-72

Malli Roland O-163, P-390

Malmendal Anders P-323

Malmsten Martin P-205

Maltan Lena P-171, P-326

Malvar Oscar P-144

Manakova Elena P-124, P-354, P-355

Mancia Filippo O-69

Mandl Johannes P-213

Mangoni Maria Luisa P-202, P-216

Maniati Eleni P-154 
Manioglu Selen O-108

Manova-Todorova Katia P-136

Manzanares Jose A P-288

Manzo C. O-45

Maraspini Riccardo P-303

Marcek Chorvatova Alzbeta $\mathrm{P}-84$

Marciniak Antoni P-119

Marek Jozef P-96

Margolin William P-342

Mari Stefania A P-53

Marquet Pierre P-47

Marro Monica P-156

Martens Chloe O-36

Martin Marving P-47

Martin Nicolas P-364

Martinez de Tejada Guillermo O-104

Martinez Laura S P-67

Martínez Ruiz Antonio O-57

Martinez-de-Tejada Guillermo P-212

Martínez-Gil Luis O-39

Martínez-León Alejandro P-51

Martinez-Martin David P-142

Martinez-Seara Hector O-141, P-230, P-233

Martins J. C P-215

Marx Lisa P-213

Massiera Gladys P-287

Mateašík Anton P-14

Mateos Nicolas O-45, O-160

Mateu Mauricio G O-42

Mateusz Sikora O-6

Matkó János P-81

Matko Matúš P-14

Matsuo Tatsuhito P-30

Mattarei A. P-38, P-58

Mattarelli Maurizio O-26

Matthews Lauren P-116

Matti Ulf O-50, P-93

Matulis Daumantas P-124

Maurer F P-208

Mavridou Vasiliki O-58

Mayor S. O-45

Mazzini A. P-35

Maître Jean-Léon O-133

McIntosh J. R P-253
Mehrafrooz Behzad O-89

Meifang Fu O-155

Meijering Anna E C O-78

Meiser Elisabeth P-85

Meister Anette P-274

Meister Annette O-138

Melo Manuel N P-266

Mély Yves P-114

Mendes Ferreira Tiago P-37

Mensitieri G. P-69

Mercier Gwenael P-88

Mériadec Cristelle P-273

Meshkov Ivan N P-178

Meyer Carola O-32

Michael Gecht O-6

Michailovienė Vilma P-124

Michaś Agnieszka P-378

Mickevičiūtè Aurelija P-124

Miele Adriana E P-33

Miettinen Markus S P-218

Mike Árpád P-240

Mikulova Ludmila P-120

Milic Sandra P-17

Milica Kojadinovic P-300

Militello Valeria P-10

Milovanovic Dragomir P-377

Mingarro Ismael O-39

Mingeot-Leclercq Marie-Paule P-296

Mironenko Andrei P-52

Mirzaki Ebrahimi Armin P-21

Misceo Doriana O-19

Miskovský Pavol P-19, P-177

Miteva Maria A P-223

Mithu Venus S O-97

Mitra Aniruddha P-258

Mitra Saheli P-196

Mitragotri Samir O-93

Mitro Michal P-9

Mizuno Naoko P-367

Modaresi Seyed M O-108

Mohammadi Reza P-85

Mohammed Danahe P-296

Mohebi Ali P-22

Möller Heiko P-210

Molotkovsky Rodion J P-203

Momotenko D. O-167

Mönnikes Sophia P-211
Monterroso Begoña P-342

Montis C. P-34

Mootz Henning P-117

Morabito Giuseppe P-377

Moraes Isabel O-102

Moravcová Jana O-70

Moreira Lana Gabriela P-18

Moreira Rodrigo A O-67

Moreno Herrero Fernando O-145

Moroni Anna O-16, P-57, P-315

Morresi Assunta O-26

Morvová Jr. Marcela P-272

Moskvin Alexander P-4

Mountaki Christina P-112

Mousseau Normand P-386

Mouton Sara N P-382

Movellan Kumar T P-343

Mrózek Mariusz P-388

Mršić Leo P-358

Muench Stephen P P-316

Muik Martin P-326

Mukhortava Ann P-170

Müller Daniel J O-108, P-53, $\mathrm{P}-142$

Müller Torsten P-149

Müller Ulrike P-241

Mund Markus P-93

Munoz Gorka O-160

Musci Giovanni P-33

Musset Boris O-12

Mussini Andrea P-332

Muthukumar Murugappan P-185

Myers Scott T P-363

Myšková Jitka O-48

Naderer Christoph P-17

Nadon Jean-Francois P-356

Nagai Kiyoshi O-101

Nageswaran Sarmini P-259

Nagle Irène P-297

Naglik Julian O-107, P-207

Nagy Péter P-90

Najjar Hadil P-171

Nakatsuka N. O-167

Nalbach Mathis P-298, P-301

Narayanan Theyencheri P-116 
Nash Michael A O-67

Nasir Saima P-188

Naßwetter Leonie C P-333

Natale Paolo P-308

Nath Soumav P-380

Natriashvili Ana P-163

Neagu Adrian P-286

Neagu Monica P-286

Nebesarova J O-66

Nedielkov Ruslan P-210

Nedvědová Jana P-260

Nehls Christian O-106, P-208, P-212

Nemecek Daniel O-72

Nencini Ricky P-230

Netz Roland P-154

Neumann Tanja P-149

Neupert Walter O-51

Newman Amy P-65

Nicke Annette P-45

Nickel Walter O-143, P-145

Nicolau Monica P-154

Nicot Arnaud B P-223

Nie Chuanxiong P-73

Niedźwiecka Anna P-378

Niederauer Christian O-49

Niehrs Christof P-125

Nielsen Christian F O-78

Nierzwicki Lukasz P P-334

Niessen Karin V P-45

Nietmann Peter P-263

Nikelshparg Evelina I P-121, P-172

Nikelshparg Matvey I P-172

Nikić-Spiegel Ivana O-82

Nikodemus Denise P-154

Nikte Siddhanta V P-245

Nilges Michael P-228

Nimigean Crina O-35

Nimmerjahn Falk O-118

Noe Frank P-279

Nolle Friederike P-145, P-278

Nonell Santi P-332

Norinkevicius Karolis P-363

Norrild Rasmus K O-161

Nováček Jiří O-70

Novak Pavel P-148, P-394

Novikov Sergey M P-121
Novosel Nives P-395

Nowak Wiesław P-183

Nucke Lisa O-61

Nyitrai Miklós P-81

Ocampo Carlo Martin M P-39

Odino Davide P-153

Oh Yoo Jin O-66

Olewniczak Michal M P-334

Ollila Samuli P-218, P-230

Ollinger Nicole P-241

Olmeda Bárbara P-309

Olson Wilma K P-359

Olsson Ulf P-323

Olteanu Emilian P-286

Oneto Michele P-91

Onufrievich Alexander P-264

Oostenbrink Chris P-225

Opálka Lukáš P-268

Opper Jennifer P-55

Oravczová Veronika P-24, P-335

Ordodi Valentin P-286

Orian-Rousseau Véronique $\mathrm{P}-114$

Ortega Felipe P-143

Ortiz Juan O-39

Osella Silvio P-173

Ostroumova Olga S P-74

Oswald Tabea A P-282, P-303

O'Toole Eileen P-253

Ott Maria P-283

Øvreeide Ingrid H $\quad$ P-305

Pașca H. P-293

Paar Vladimir P-358

Pabijan Joanna P-292

Pabst Georg O-86, O-104, P-206, P-213, P-269, P-275, P-337

Padanyi R P-104

Pagano Aldo P-77

Paksaitè Justė P-354, P-355

Palaia Ivan O-137

Pallbo Jon P-323

Palmer Shane O-58

Paloncýová Markéta P-173

Pang Hongtao P-303
Pant Shashank O-36

Panzeri Davide P-23

Paolini L. P-34

Paradisi C. P-38, P-58

Paraschiv Alexandru O-137

Parisse Pietro O-166, P-385

Park Yoonkyung P-202, P-216

Parra-Ortiz Elisa P-205

Päslack C. P-222

Pasquato Lucia P-385

Paston Sofia P-95

Pastrana César L O-145

Patz Silke P-16, P-199

Paulowski Laura P-208, P-212

Paunescu Virgil P-286

Pazicky Samuel P-247

Pechova Ivana P-120

Pedebos Conrado O-128

Pelicci P. G P-79

Pellikan Sarala P-314

Pellowe Grant O-36

Penedo Juan C P-356

Peng Bee-Zen O-74

Pengo Paolo P-385

Peplińska Barbara P-116

Pepłowski Łukasz P-183, P-219

Perahia David P-223

Perego Laura P-389

Pereira Manuela M O-59

Perepelytsya Sergiy P-237

Pérez Patallo Eugenio O-138

Pérez-Gil Jesús P-309

Perez-Gonzalez Cibran P-356

Perez-Grau Jose Joaquin P-188

Perrais David O-120

Persichetti Luca P-290

Peske Frank O-74

Pesti Krisztina P-240

Peterbauer Clemens K P-225

Peterman Erwin J G O-78, P-258

Peters Judith O-73, P-30

Petitjean Simon J P-70

Petra Schwille O-155

Petrišič Nejc P-336

Petrychenko Valentyn O-74

Pezzano Fabio P-156

Pfeffermann Jürgen O-86 
Philippe Nadège O-5

Philippi Michael P-246

Picas L P-321

Piccirilli Federica P-174

Piehler Jacob O-32, O-99, O-119, P-246, P-294

Pierzyńska-Mach Agnieszka P-91, P-299

Pignataro Bruno P-108

Piliarik Marek P-78, P-255

Pilic Johannes P-390

Pillai Visakh P-194, P-197

Piller Paulina P-337

Pinho Mariana G O-59

Pinigin Konstantin V P-209, P-338

Pinkas Matyáš O-70

Pinkwart Kerstin O-154

Pintacuda Guido O-37

Pinto Giulia O-166, P-175

Piosik Jacek P-307

Pires Patricia O-59

Pisliakov Andrei V O-149

Platonov M. P-226

Platzer Rene P-242

Platzer René P-86, P-132

Plevka Pavel O-44

Plochberger Birgit P-25, P-311, P-350

Ploetz Evelyn P-167

Plückthun Andreas P-246

Pluhackova Kristyna P-53, P-330

Poblete Simon P-75

Pocevičiūtè Ernesta P-354, $\mathrm{P}-355$

Podewitz Maren P-130

Podgornik Rudolf P-75

Podobnik Marjetka O-71, P-336

Pohjolainen Emmi O-114

Pohl Elena E O-60, P-127

Pohl Peter O-12, O-86, O-90, P-186

Pöhnl Matthias O-118, P-231

Poik Matthias P-301

Pollack Yoav G O-136

Polyakova Anastasia P-57

Poma Adolfo B O-67
Poncin Megane A P-70

Poojari Chetan P-276

Poojari Chetan S P-71

Popescu Roxana P-286

Popova Anastasia P-12

Popova Marina P-68, P-72

Popović Tamara P-300

Porcar Lionel P-269

Porro Alessandro P-57

Posocco Paola P-385

Posvyatenko Alexandra V O-96

Potestio Raffaello P-347

Potter Clinton S O-69

Pouyan Paria P-73

Poveda José A P-42

Prakaash Dheeraj P-339

Pramanik Shreya P-340

Prasanna Xavier P-341

Prassl Ruth O-73, P-176

Preiner Johannes O-24, P-13, $\mathrm{P}-244$

Prencipe Maria P-194

Prenner Elmar P-281

Prieto Manuel P-42

Priglinger Eleni $\quad$ P-17

Primiano A. P-35

Pringle Helen P-261

Prinz Christelle P-181

Prossliner Gerhard P-176

Prot Victorien P-305

Prylutskyy Yu. P-226

Przybyło Magdalena P-140

Ptakova Alexandra P-9

Pugno N. P-69

Pupkis Vilmantas P-54

Puthenparampil M. P-58

Põldsalu Inga P-365, P-368

Quarta Ndjali P-274

Quoika Patrick K O-113, P-130, P-227

Radler Philipp P-262

Rafael Benitez P-306

Raha S. P-97

Rajan Lamichhane P-328

Rajfur Zenon P-388

Rakickas Tomas P-354, P-355
Ramadan Shahenda P-210

Ramaye Elise P-46

Ramirez-Hoyos Patricio P-188

Ranade Varun P-391

Rao Lu P-251

Rao Rajas M P-232

Rapacka-Zdończyk Aleksandra P-307

Rappolt Michael P-316

Rasola Andrea P-40

Rath Parthasarathi O-108

Ratha B. N P-97

Rathar R P-321

Rätsep Margus P-126

Rauch Philipp P-170

Rauh Oliver P-55

Ray Sutirtha P-56

Rechberger Gerald N P-337

Reffay Myriam P-297

Refojo Patricia N O-59

Reglinski K P-80

Rego R O M O-66

Reimhult Erik P-152, P-189, P-190, P-302

Reina Francesco P-82, P-87

Reiter-Scherer Valentin D P-73

Relini Annalisa P-153

Renart Lourdes P-42

Renger Thomas P-326

Rentmeister Andrea O-164

Reuten Raphael P-154

Reuter Nathalie O-139, P-349

Rheinberger Jan O-35

Rheinlaender Johannes O-79, P-157

Ricci Caterina P-315

Riccitelli Francesco P-202,

$$
\text { P-216 }
$$

Richardson Jonathan O-107,

$$
\text { P-207 }
$$

Richert Ludovic P-114

Richter Christian P O-119

Rico Felix P-141

Ridolfi A. P-34

Rief Matthias O-63

Rienmüller Theresa O-84

Ries Jonas O-50, P-93

Ring Wolfgang O-73 
Riniker Sereina O-111

Riopedre Miguel P-233

Riske Karin A P-277

Ritzmann Noah O-108

Rivas Germán P-342

Robalo Tiago T O-59

Robello Mauro O-85

Roberto Covino O-6

Robinson Carol V O-8

Robinson Tom O-154

Robles-Ramos Miguel Ángel $\mathrm{P}-342$

Robu Andreea P-286

Rocchi Martina O-26

Rodnina Marina V O-7, O-74

Rodrigues José O-69

Rodrigues Mário S O-59

Rodriguez Brian P-194, P-197

Roesel David P-169

Rog-Zielinska Eva O-22

Rohatschek Andreas P-131

Rojo-Ruiz Jonathan O-165, P-393

Roke Sylvie O-10, P-169

Rokitskaya Tatyana I P-329

Roman Cosmin I P-142

Romanin Christoph O-19, P-3, P-8, P-326

Romanò S. P-35

Romero Catalina O-160

Romin Yevgeniy P-136

Roos Wouter H O-29, O-42

Rosenblum Gabriel O-148

Rosenov Vladimir P-305

Rösler Anne-Sophie P-369

Rossa A. P-38

Rossboth Benedikt P-86

Rougier Jean-Sebastien P-46

Roversi Daniela P-202

Rozenberg Haim O-148

Rübeling Angela P-303

Rubini Marina P-197

Rudd-Schmidt Jesse P-325

Rudenko A. P-151

Rufin Manuel P-301

Ruppelt Dominik P-211

Ruprecht Jonathan J O-58

Ruprecht Verena O-135, P-156
Rutkauskas Marius P-111

Ruz Jose Jaime P-144

Rybakova Olga O-48

Ryu Hyunil P-187

Rzyanina Anna P-360

Sacquin-Mora Sophie P-220

Sadat Sharifzadeh Atiyeh P-57

Sadeghi Mohsen P-279

Safran Samuel P-372, P-375

Saghaei Tayebeh P-302

Sahi Maryam P-173

Sahoo Harekrushna O-98, P-98

Saleppico Roberto O-143

Sallinger Matthias P-8

Salo J O-66

Salo Veijo P-341

Šalovská Barbora P-346

Salzer Isabella P-50, P-56, P-62

Sancataldo Giuseppe O-159, P-10, P-108, P-164, $\mathrm{P}-310$

Sánchez del Pino Manuel O-39

Sanchez-Fuentes D P-321

Sandtner Walter P-65

Sandu Nicoleta P-36

Sansevrino Roberto P-377

Sansom Mark S P O-142

Sant Vrinda P-343

Santella Anthony P-136

Santoro Bina O-16, P-57

Santos Ana Mafalda O-132, $\mathrm{P}-243$

Santos Nuno C P-266

Saponaro Andrea O-16, P-57

Sarajlic Edin P-396

Sari Merve P-369

Saric Andela O-137

Savchenko Alexander P-392

Savin Nikita P-148, P-392

Savini Filippo P-202

Sayed Ahmed O-61

Scattolini V. P-58

Schachner-Nedherer Anna-

Laurence

O-73

Schaedel Laura O-125

Schäfer Lars P-222, P-236
Schäffer Erik O-124

Schäffer Tilman E O-79, P-157

Schauperl Michael P-227

Scheberl Andrea P-190

Scheibel Thomas P-381

Scheidt Holger A O-97, P-291

Scheiner Stefan P-147

Schelhaas Mario P-67

Schepers Anna V O-125, P-263

Scherzer Otmar P-88

Scheub Deborah-Desirée P-55

Schick Bernhard P-18

Schicker Klaus P-240

Schiffelers Lisa O-132

Schindl Rainer O-84, P-8, P-16, P-199

Schitter Georg P-298, P-301

Schlesinger Ramona O-13

Schliebs W P-80

Schlotter T. O-167

Schmidt Tony O-84

Schneider Falk O-132, P-80

Schneider Magdalena P-83, P-86, P-88

Schneider Matthias M O-56

Schrangl Lukas P-132, P-295

Schroeder Indra P-55

Schromm Andra B P-212

Schubeis Tobias O-37

Schubert Ulrich P-315

Schütz Gerhard J O-130, P-83, P-86, P-88, P-92, P-100, P-132, P-212, $\mathrm{P}-242, \mathrm{P}-295$

Schwaighofer Andreas O-31

Schwartz Thue W P-234

Schwarze Benedikt P-291

Schwille Petra O-49, P-367

Scodellaro Riccardo P-23

Scollo Federica O-141

Scott Haden L P-269

Sebesta Ondrej P-324

Sedivy Arthur P-31, P-247

Sedlák Erik P-19, P-99, P-101, P-103, P-381

Sedláková Dagmar P-19, P-101

Seeger Thomas P-45

Seemann Ralf P-145, P-278 
Seferovic Hannah P-133

Seidel Ralf P-111

Seifert Jan P-157

Seitanidou Maria P-199

Selent Jana O-117

Seljmani Arbresh P-46

Semenova Anna A P-121

Semeraro Enrico F P-213, P-269, P-337

Semeraro Enrico S O-104

Seneviratne Rashmi P-316

Sengottuvel Saravanan P-388

Sengupta Durba P-245

Senju Yosuke P-344

Sente Andrija P-59

Sept David P-251

Sergides Marios P-389

Sergio Perez-Conesa O-109

Sergunova Viktoria P-264

Sevcsik Eva O-130, P-86, P-242

Severiukhin Y. P-293

Sezgin Erdinç O-129, O-132, P-25, P-80, P-200, P-350

Shammas Sarah L O-54

Shanel Ondrej O-72

Shapovalov Yuri A P-122

Sharma Arjun P-83

Shcharbin Dzmitry P-24

Shekhar Mrinal O-36

Shekunov Egor V P-74

Shenol Iliyaz Aslihan P-234

Sherstyukova Ekaterina P-264

Shilova Liudmila A P-68

Shivanna Komala P-89

Shnyrova A. V P-135

Shtykova Eleonora V P-68

Šikšnys Virginijus P-111

Šikurová Libuša P-166, P-182, $\mathrm{P}-272$

Sil P. O-45

Sileikyté Justina P-40

Siletsky Sergey A P-123

Siligan Christine O-12, P-244

Silva Daniel V P-210

Silva Leticia A P-60

Silva Micael O-59

Šimić Marko P-176
Simon Daniel T P-199

Simon Márton A P-61

Singh H. P-222

Singh Kamal P P-391

Šinkūnas Tomas P-111

Sinning Irmgard P-125

Sironi Laura P-23

Sitte Harald H P-39, P-44, P-65

Skálová Tereza P-247

Skamrahl Mark P-303

Skořepa Ondřej P-247

Skulj Sanja O-60, P-127

Slabonska Joanna P-119

Slabý Cyril P-177

Smirnov Alexey P-124

Smirnovienè Joana P-124

Smith Albert P-268

Smith Ana-Suncana O-115

Smith Iain O-128

Smolič Tina P-304

Sobiepanek Anna P-395

Sobolevsky Alexander I O-15

Sobrinos-Sanguino Marta P-342

Socrier Larissa P-345

Sodo Armida P-290

Sohmen Benedikt P-184

Sokolov Valeriy S P-27, P-214

Sokolov Valery S P-2, P-178

Somayeh Ahadi P-345

Sommer Martha E O-117

Somoulay Xayathed P-70

Sonar Krushna P-245

Song Y-H P-208

Songailiene Inga P-111

Sophie Marbach O-91

Sören Von Bülow O-6

Sorg Katharina P-18

Sosnovtseva Olga P-121

Sousa Filipe M O-59

Soya N P-104

Spang Anne P-195

Sparer Lukas P-83

Sparn Carola P-145

Sparr Emma P-323

Spatz Joachim P-340

Spedalieri Cecilia P-179

Spodniakova Barbora P-113

Spustova Karolina P-370
St-Pierre Patrick P-356

Stagni Cesare O-26

Stahn Patricia P-18

Stampf Jan-Luca P-62

Stangl Herbert P-25, P-311, P-350

Staniforth Rosie A P-107

Stansfeld Phillip J P-204

Starke Leonhard J P-280

Stary-Weinzinger Anna P-43, P-240, P-314

Stefan Schäfer O-6

Stehr Florian O-49

Steigenberger J. P-215

Stein Johannes O-49

Steinbauer Patrick P-131

Steinem Claudia P-211, P-254, P-259, P-282, P-345, P-369

Steinhart Martin P-246

Steinkühler Jan P-340

Stella Lorenzo P-202, P-216

Stender Emil G P O-161

Stephenson Anna B P-371

Sticht Patricia P-133

Stier Gunter P-125

Stock Gerhard P-184

Stockinger Hannes P-86, P-132

Stockner Thomas P-44, P-49, P-60, P-65, P-235, P-317, P-322, P-348

Stokke Bjørn T P-305

Storm Cornelis O-137

Strasser Jürgen O-24, P-13

Stratikos Efstratios O-105

Strauss Sebastian O-50

Strieker Lena P-282

Strnad M O-66

Stroffekova Katarina P-180

Strohmeyer Nico P-142

Stupak Marek P-120

Sturm Maximilian P-55

Su Qian Peter P-361

Subadini Suchismita P-98

Šubjaková Veronika P-335

Sudhakar Swathi O-124

Südhof Thomas C O-2

Sukeník Lukáš O-44 
Sukomon Nattakan O-35

Sule Kevin P-281

Sun Yujie P-361

Šutý Šimon P-24, P-335

Svirelis Justas O-92

Svorinic Andrea P-125

Swuec Paolo P-57

Sych Taras P-25, P-350

Sykora Jan P-1

Szabó Ágnes P-90

Szabó-Meleg Edina P-81

Szabò I. P-38, P-58

Szabò Ildikò P-40

Szekeres Gergo P P-179

Szendi-Szatmári Tímea P-90

Szilágyi András P-115

Szöllősi Dániel P-44, P-49, P-235

Szöllősi János P-90

Sztacho Martin P-324, P-346

Sztyler Agnieszka K O-117

Szydlak Renata P-305

Szymańska Aneta P-116

Szymonski Marek P-15

Tajkhorshid Emad O-36

Talafová Veronika P-381

Talluri S. O-45

Tamayo Javier P-144

Tan Yong Z O-69

Tandale Aditi P-245

Tankovskaia Svetlana P-95

Tannoo Ryshtee M P-114

Tans Sander O-52

Tanzer Alexander P-162

Tarenzi Thomas P-347

Tárnai Viktória P-81

Tashkin Vsevolod Y P-2

Tassis Konstantinos O-33

Tavčar Petra P-304

Tavoulari Sotiria O-58

Taylor Alexander I P P-107

Taylor J. Paul O-158

Teiwes Nikolas P-282

Telschow Roger P-88

Tempra Carmelo O-141, P-230

Teslenko Alexandra O-147

Testa Ilaria O-146
Thiel Gerhard P-55, P-57

Thomson Neil J P-248

Thurn Johann P-184

Thurner Philipp J P-131, P-298, P-301

Tiapko Oleksandra P-322, P-348, P-366

Tiffner Adela P-326

Timoshenko Roman P-392, P-394

Timpmann Kõu P-126

Toca-Herrera José Luis P-143, P-158, P-302, P-306

Toccafondi Chiara P-175

Todolli Stefjord P-359

Todt Hannes P-240

Tolstova Anna P-12

Tomancak Pavel O-134

Tomishige Nario P-114

Tomková M. P-99

Tomková Mária P-101, P-103

Tomkova Silvia P-180

Tommasin Ludovica P-40

Tomori Zoltán P-177

Toner Chelsea O-5

Tordai H P-104

Torreno-Pina Juan O-160

Torres-Vidal Patricia O-165, P-393

Tóth Ágota O-38, P-47

Toth Daniel P-223

Toth-Hervay Nora P-272

Traore Daouda O-73

Trauelsen Mette P-234

Trauner Dirk O-83

Trbovich Alexander P-300

Trianni Alberta O-25, P-76

Trifilieff Pierre O-120

Tripathi Prabhat O-89

Troiano Cassandra P-202, P-216

Trollmann Marius $\quad$ O-131

Trybala E. O-43

Trzaskuś Krzysztof P-363

Tschanz Aline P-93

Tschon Matilde O-26

Tubiana Thibault P-349

Türmer Katalin P-81
Tutkus Marijonas P-354, P-355

Tych Katarzyna P-197

Tyteca Donatienne P-296

Uboldi Ermanno O-16, P-57

Uetrech Charlotte P-374

Uetrecht Charlotte O-40

Uherek Martin P-84

Ulbrich Maximilian H P-129

Uličný Jozef P-11

Ulloa Severino L P-155

Ulmschneider Martin P-32

Ulyanov Evgeniy V P-253

Ungermann Christian O-32

Unksov Ivan P-181

Urbančič I P-80

Urbančič Iztok O-132

Urbani Andrea P-40

Uriati Eleonora P-26, P-332

Usai Chantal P-77, P-91, P-353

Ustinova Kseniya P-260

V. Guzman Horacio P-75

Vaccari Lisa P-174

Vácha Robert O-44, P-201, P-239

Vagovič Patrik P-11

Vaiana Andrea O-114

Vala Milan P-255

Valbuena Alejandro O-42

Valdivieso González David O-62

Valle F. P-34

van Agtmael Tom P-154

van der Merwe Philip A O-116

van Oostrum Peter P-189, P-190, P-302

van Oostrum Peter D J P-152

Vancova M O-66

Vaneev Alexander P-392, P-394

Vaneev Alexander N P-148

Vaněk Ondřej P-247

Vanik V. P-198

Varanita T. P-58

Vardjan Nina P-304

Vargas Carolyn O-138

Varlamova Ekaterina P-12, $\mathrm{P}-27$

Vasquez F P-208 
Vassilopoulos S P-321

Vattulainen Ilpo O-143, P-341, P-344

Vávrová Kateřina P-268

Vavylonis Dimitrios $\mathrm{P}-367$

Vazdar Mario O-60, P-127

Veenhoff Liesbeth M P-382

Végh Barbara M P-115

Velas Lukas P-92

Vélez Marisela P-308

Velísková Martina P-166, P-182

Ven Katharina P-341

Vendruscolo Michele O-157

Ventura Rita O-69

Ventura Salvador O-158

Venturini Valeria P-156

Verardo Damiano P-181

Verdier Claude P-159

Vergani Laura P-77

Verhey Kristen J P-251

Verleysen Y. P-215

Verstraeten Sandrine P-296

Vetri Valeria O-159, P-105, P-108, P-164, P-310

Viappiani Cristiano P-26, $\mathrm{P}-332, \mathrm{P}-387$

Viaud J P-321

Vickery Owen N P-248

Vierock Johannes O-81

Vietrov Ruslan O-33

Vihinen Helena P-341

Villalba-Riquelme Eva P-63

Villaraza Aaron Joseph L P-39

Vinals Javier O-102

Vinogradov Dmitrii S P-253

Virk Mudassar P-190

Visca Paolo P-290

Vivanco Maria D P-158

Vivarelli Leonardo O-26

Vlachova Viktorie P-9

Vlahović Ines P-358

Vlašić Ema P-395

Vogel Nicolas P-85

Vollmar Angelika P-167

Volovik Marta V P-214

von Eysmondt Hendrik P-157

von Wirén Julius P-369

Vondracek Hendrik P-385
Vörös J. O-167

Voskoboinik Ilia P-325

Vuletić Tomislav P-395

Waczulíková Iveta P-24, P-166, P-182, P-335

Wagener Kikola O-51

Waibl Franz P-227

Waithe D P-80

Walczewska-Szewc Katarzyna $\mathrm{P}-183$

Waldherr Linda P-16, P-199

Wallace Jaqulin P-89

Wan Qi P-382

Wang Han O-65

Wang Yin P-130

Wang-Henderson Miles O-49

Wanunu Meni O-89

Watrinet Isabelle P-246

Watts Anthony O-102

Weaver S. O-167

Webeling Niklas P-117

Weber Andreas P-158, P-302, P-306

Weber Florian P-311, P-350

Wechselberger Christian P-133

Wedepohl Stefanie P-73

Weghuber Julian P-241

Wegner Tristan P-195

Weihs Tobias P-87

Weinert Tobias O-11

Weininger Ulrich P-323

Weiß Sarah P-171

Weitz David A O-122

Wenzel Gentiana P-18

Wereszczynski Jeff O-5

Werz Daniel P-345

Weyrauch Sophie O-138

White Alison O-5

Wiegert Simon O-81

Wieser Stefan O-135, P-156

Wiggers Felix O-148

Wiktor Jakub O-146

Wilhelm Claire P-297

Wilkinson Max E O-101

Wilkowska Michalina P-116

Willbold Dieter P-380

Williamson Gordon P-64
Wilmes Stephan O-119

Wingbermühle Sebastian P-236

Winkelmann Hauke O-119

Wirbel Hannes O-79

Witt Hannes O-78

Woźniak Agata P-307

Wojciechowski Adam P-388

Wolf Steffen P-184

Woods Dustin O-5

Woziwodzka Anna P-307

Wu Jinming P-383

Wu Yu-Le P-93

Wuite Gijs O-78, P-285

Wulfken Alexander P-111

Wurl Anika P-37, P-283

Wurm Christian A P-87

Wyrzykowski Dariusz P-307

Xie Xiaoliang Sunney P-361

Xing Yongzheng P-186

Xue Lin P-371

Yamansarov Emil P-394

Yamazaki Hirohito O-89

Yandrapalli Naresh O-154

Yang Byeongseon O-67

Yang Jae-Won P-62

Yang Jinsung P-70, P-296

Yip C P-155

Yonath Ada O-1

You Changjiang O-32, P-246

Young Robert T P-359

Yu Lingbo O-72

Yue Chen O-109

Yue Yang P-251

Yuen D A P-155

Zachariae Ulrich P-5, P-248

Zahler Stefan P-167

Zahradnik Ivan P-4

Zahradnikova Alexandra P-4

Zalewska Adrianna P-395

Zambelli Tomaso O-167, P-396

Zapotoczny Bartlomiej P-15

Zapp Christopher P-150

Zare-Eelanjegh Elaheh P-396

Zaremba Mindaugas P-354, P-355

Zarubin Mikhail P-360 
Závodszky Péter P-115

Zbiral Barbara P-158

Zdorevskyi Oleksii P-237

Zeitzschel Nadja O-81

Zelger Philipp P-92

Zemła Joanna P-159, P-305

Zemljič Jokhadar Špela P-139

Zendehroud Sina P-154

Zhang Jin O-162

Zhao Ziqing Winston P-361

Zheng Tiantian P-191
Zhou Chaoge P-187

Zhou Keda O-5

Zhu Jinbo P-384

Zhu Rong P-65

Zhurgenbayeva Gaukhar P-200,

$$
\mathrm{P}-217
$$

Ziegler Christine O-17

Ziherl Primoz P-275

Žikić Dejan O-21

Zilman Anton P-191

Zimmerberg Joshua P-68, P-319
Zimova Lucie P-9

Zoccoler Marcelo P-277

Žoldák Gabriel P-102, P-381

Zoratti M. P-38, P-58

Zorec Robert P-304

Zorrilla Silvia P-342

Zuna Kristina O-60, P-127

Zvarík Milan P-166, P-182

Zweytick Dagmar O-104 


\section{List of participants \\ Participants list as of May 28, 2021 subject to changes}

Abhinav Abhinav, Czech Republic

Abidine Yara, Sweden

Abrosimova Karina, Russian Federation

Adame-Arana Omar, Israel

Affatigato Luisa, Italy

Akimov Sergey, Russian Federation

Al Qanobi Amal, Oman

Alawami Mohammed, United Kingdom

Alberto E Silva Ana Sofia, Austria

Aleksanyan Mina, Germany

Alexandrova Veronika, Russian Federation

Almendro-Vedia Victor G., Spain

Alonso Alejandro, Spain

Alonso M. Teresa, Spain

Alsteens David, Belgium

Alunni Cardinali Martina, Italy

Alves Isabel, France

Alves da Silva Leticia, Austria

Amaro Rommie, USA

Amunts Alexey, Sweden

Anderluh Gregor, Slovenia

Angelov Borislav, Czech Republic

Anisimov Mikhail, Russian Federation

Ansell T. Bertie, United Kingdom

Anselmo Sara, Italy

Anthony Nicholas, Italy

Anthony Raj Xavier Prasanna, Finland

Antila Hanne, Germany

Antosova Andrea, Slovakia

Araújo Luís, Portugal

Archer Margarida, Portugal

Arjoca Stelian, Romania

Auer Albert, Austria

Aurélien Barbotin, France

Axmann Markus, Austria

Baaske Martin, Netherlands

Bachmann Magdalena, Italy

Bage Marcus, United Kingdom

Balaguer Pérez Francisco, Spain

Balboni Beatrice, Italy

Baldini Francesca, Italy

Bano Gregor, Slovakia

Bano Fouzia, Sweden
Barboiu Mihail, France

Barrera Francisco, USA

Bartók Ádám, Hungary

Bartoš Ladislav, Czech Republic

Bashkirov Pavel, Russian Federation

Batishchev Oleg, Russian Federation

Bazzurro Virginia, Italy

Bechinger Burkhard, France

Becker Robert Adrian, Germany

Beckmann Roland, Germany

Bednarikova Zuzana, Slovakia

Belle Valérie, France

Belleza Oliver John, Austria

Benedetto Antonio, Italy

Bera Krishnendu, Czech Republic

Berdychowska Julia, Poland

Bernát Ondrej, Slovakia

Berta Martin, Slovakia

Bezanilla Francisco, USA

Bobone Sara, Italy

Bock Lars V, Germany

Böckmann Rainer, Germany

Bodenschatz Jonathan, Germany

Bodner Clara, Austria

Bogatyr Vadim, Netherlands

Bondar Ana-Nicoleta, Romania

Bouckaert Julie, France

Brameshuber Mario, Austria

Brandner Astrid, Germany

Brenker Kathrin, Germany

Brescia Stefania, Austria

Brockwell David, United Kingdom

Brooks Rhiannon, United Kingdom

Bründl Michael, Austria

Busch Karin, Germany

C Shetty Sunidhi, Germany

Cainero Isotta, Italy

Calò Annalisa, Spain

Canale Claudio, Italy

Canepa Ester, Italy

Carabadjac Iulia, Germany

Carravilla Pablo, Germany

Carrer Andrea, Italy 
Carrotta Rita, Italy

Casas Ferrer Laura, France

Catania Rosa, United Kingdom

Cavalleri Ornella, Italy

Černeková Michaela, Czech Republic

Cerutti Elena, Italy

Cervera Javier, Spain

Chatzimagas Leonie, Germany

Chen Kaikai, United Kingdom

Chouliara Manto, Sweden

Chroni Angeliki, Greece

Cisse Aline, France

Clarke Amy, Austria

Coluzza Ivan, Spain

Cordes Thorben, Germany

Cosentino Katia, Germany

Costa Flavio, Italy

Cote Sebastien, Canada

Covino Roberto, Germany

Crha Radek, Czech Republic

Dahlin Andreas, Sweden

Dalla Serra Mauro, Italy

Dam Tommy, Sweden

Das Chandan Kumar, Germany

Dasgupta Anindita, Germany

Daum Sebastian, Germany

Daver Henrik, Sweden

de Buhr Svenja, Germany

de Groot Bert, Germany

de Luca Giuseppe, Italy

Defelipe Lucas, Germany

Dekker Cees, Netherlands

del Río Lorenzo Alba, Spain

Delemotte Lucie, Sweden

Denieva Zaret, Russian Federation

Derganc Jure, Slovenia

Derler Isabella, Austria

Deviri Dan, Israel

Di Novo Nicolò Giuseppe, Italy

Díaz-García Clara, Portugal

Diez Stefan, Germany

Dimova Rumiana, Germany

do Rêgo Barros Fernandes Lima Maria Augusta, Italy

Dogterom Marileen, Netherlands

Dreher Yannik, Germany

Dubrovin Evgeny, Russian Federation

Dudás Bálint, France

Dunne Orla, Austria
Dutzler Raimund, Switzerland

Dyer Oliver, United Kingdom

Džubinská Daniela, Slovakia

Džupponová Veronika, Slovakia

Dzurillová Veronika, Slovakia

Ebert Andrea, Germany

Edina Szabó-Meleg, Hungary

Egorova Ksenia, Russian Federation

Elf Johan, Sweden

Engelke Hanna, Austria

England Patrick, France

Enkavi Giray, Finland

Epe Markus, USA

Erb Tobias, Germany

Enríquez Domínguez José Antonio, Spain

Erkan Hazel, Austria

Ermakov Yury, Russian Federation

Eroles Mar, France

Ezsias Bence, Austria

Fahmy Karim, Germany

Falginella Francesco Luca, Czech Republic

Fandrei Ferdinand, Germany

Fardelli Elisa, Italy

Fennema Galparsoro Dirk, Italy

Fernandez Varela Paloma, France

Fernández-Quintero Monica, Austria

Fica Sebastian, United Kingdom

Fischer Markus, Germany

Fischer Niels, Germany

Fläschner Gotthold, Switzerland

Foderà Vito, Denmark

Fornasier Marco, Sweden

Freiberg Arvi, Estonia

Freire Mara G, Portugal

Frewein Moritz, Austria

Friesacher Theres, Austria

Frischauf Nikolaus, Austria

Fu Meifang, Germany

Furlan Aurélien, Belgium

Fuxreiter Monika, Italy

Gabrielli Sara, Germany

Gahan Lianne, United Kingdom

Galimzyanov Timur, Russian Federation

Garaiova Zuzana, Slovakia

Garcia-Parajo Maria, Spain

Garzella Francesco, Italy

Gavara Núria, Spain

Gennerich Arne, USA 
Giacomo Corucci, France

Giergiel Magdalena, Poland

Gil-Santos Eduardo, Spain

Gnanachandran Kajangi, Poland

Górecki Radosław, Denmark

Gössweiner-Mohr Nikolaus, Austria

Gottschalk Alexander, Germany

Gözen Irep, Norway

Grabmayr Herwig, Austria

Gradisch Ralph, Austria

Griffo Alessandra, Germany

Groschner Klaus, Austria

Grothaus Isabell Louise, Germany

Grubmüller Helmut, Germany

Gudimchuk Nikita, Russian Federation

Guerra Yanín, Germany

Gulli Daniele, Italy

Gupta Purvi, Belgium

Gupta Ritika, India

Guthold Martin, USA

Gutsmann Thomas, Germany

Guy Ciaran, United Kingdom

Hackl Benjamin, Austria

Hagleitner-Ertugrul Nora, Austria

Häkkinen Hanna Maria, Spain

Handl Verena, Austria

Hanein Dorit, USA

Haufe Yves, Germany

Heerklotz Heiko, Germany

Hegedus Tamas, Hungary

Heimann Larissa, Germany

Heißenberg Carl-Philipp, Austria

Heißenberg Tim, Germany

Hellmeier Joschka, Austria

Hermann Enikö, Austria

Hoboth Peter, Czech Republic

Höglinger Carmen, Austria

Holanova Kristyna, Czech Republic

Hoogenboom Bart, United Kingdom

Horner Andreas, Austria

Horvath Ferdinand, Austria

Hovan Andrej, Slovakia

Howorka Stefan, United Kingdom

Hozak Pavel, Czech Republic

Hua Lisa, Germany

Hub Jochen, Germany

Hugentobler Katharina Gloria, Germany

Humer Christina, Austria
Hummer Gerhard, Germany

Hurmach Vasyl, Ukraine

Huser Thomas, Germany

Iamshanova Oksana, Switzerland

Iaparov Bogdan, Slovakia

Ideguchi Takuro, Japan

Igaev Maxim, Germany

Ignat Elena-Mădălina, Russian Federation

Iturri Jagoba, Austria

Ives Callum, United Kingdom

Jacak Jaroslaw, Austria

Jadavi Samira, Italy

Jagiello Alicja, USA

Jahnke Kevin, Germany

Janaszkiewicz Angelika, France

Jancura Daniel, Slovakia

Janshoff Andreas, Germany

Jaritz Fritz Simon, Austria

Jeftic Jelena, France

Jennings James, Austria

Joglekar Isha, USA

Jönsson Peter, Sweden

Judge Peter, United Kingdom

Jungmann Ralf, Germany

Juraj Jacko, Slovakia

Jurkowski Michał, Poland

Kalliauer Johannes, Austria

Kaltenegger Michael, Austria

Kalutskiy Maksim, Russian Federation

Kamenik Anna, Switzerland

Kappelhoff Shirin, Germany

KaraDi Giridhar Mithun Nag, USA

Karami Yasaman, France

Karner Andreas, Austria

Karoui Hedi, France

Kasparyan Gari, Germany

Keller Sandro, Austria

Kerkhoff Yannic, Germany

Khalid Syma, United Kingdom

Kichik Nesim, United Kingdom

Kim Eugene, Germany

King Patrick, United Kingdom

Klar Thomas A., Austria

Klepka Barbara, Poland

Knippenberg Stefan, Belgium

Knoch Hannah, Germany

Knyazev Denis, Austria

Koehler Melanie, Belgium 
Koerfer Agnes, Germany

Köfinger Jürgen, Germany

Köksal Elif Senem, Norway

Kolmogorov Vasilii, Russian Federation

Kolodziej Tomasz, Poland

Kondrashov Oleg, Russian Federation

Kopittke Caroline, Austria

Kopūstas Aurimas, Lithuania

Korn Viktoria, Germany

Kornmueller Karin, Austria

Köster Sarah, Germany

Krasnobaev Vladimir, Russian Federation

Kraxner Julia, Germany

Kreiter Jürgen, Austria

Krivic Denis, Austria

Kumari Pallavi, India

Kunji Edmund R, United Kingdom

Kuravsky Mikhail, United Kingdom

Kurth Markus, Germany

Lacinova Lubica, Slovakia

Lanzerstorfer Peter, Austria

Lavrushkina Svetlana, Russian Federation

Lazar Josef, Czech Republic

Lazzarin Erika, Austria

Lazzeri Gianmarco, Germany

Lee Seonwoo, Switzerland

Lee Sejeong, United Kingdom

Lekka Malgorzata, Poland

Lemke Edward, Germany

Lenne Pierre-Francois, France

Leomil Fernanda, Germany

Leuchtag H. Richard, USA

Li Hongbin, Canada

Lilliu Elena, Austria

Linard Matos Anna Lívia, Germany

Lindorff-Larsen Kresten, Denmark

Litschel Thomas, Germany

Liu Zhaowei, Switzerland

Liutkus Mantas, Spain

Llauro Portell Aida, Netherlands

Lohner Karl, Austria

Lolicato Fabio, Germany

Lopanskaia Iuliia, Russian Federation

Lopez-Martinez Montserrat, Austria

Lopez-Rios de Castro Raquel, Spain

Lorenz Charlotta, Germany

Losgott Thomas, Austria

Luger Karolin, USA
Luo Jinghui, Switzerland

Luty Marcin, Poland

Madariaga-Marcos Julene, Germany

Maître Jean-Léon, France

Maity Sourav, Netherlands

Makasheva Kristina, Switzerland

Malli Roland, Austria

Manioglu Selen, Switzerland

Marbach Sophie, USA

Marcek Chorvatova Alzbeta, Slovakia

Marciniak Antoni, Poland

Martens Chloe, Belgium

Martínez-León Alejandro, Germany

Martinez-Seara Hector, Czech Republic

Matagne André, Belgium

Maznichenko Anna, Austria

Meiser Elisabeth, Germany

Mendes Ferreira Tiago, Germany

Miele Adriana Erica, France

Milhiet Pierre-Emmanuel, France

Milovanovic Dragomir, Germany

Mingarro Ismael, Spain

Mironenko Andrei, Germany

Mirzaki Ebrahimi Armin, Poland

Mithu Venus Singh, India

Mitra Aniruddha, Netherlands

Mitra Saheli, India

Mohammed Ahmed Alsadig Ahmed, Italy

Mohammed Danahe, Belgium

Mohebi Ali, Italy

Moroni Anna, Italy

Mueller Torsten, Germany

Munene John, Austria

Mussini Andrea, Italy

Nageswaran Sarmini, Germany

Nagle Irène, France

Nagy Peter, Hungary

Nalbach Mathis, Austria

Naßwetter Leonie, Germany

Nath Soumav, Germany

Nedvedova Jana, Czech Republic

Nehls Christian, Germany

Nemecek Daniel, Czech Republic

Niederauer Christian, Netherlands

Nigro Michela, Italy

Nikelshparg Matvey, Russian Federation

Nikelshparg Evelina, Russian Federation

Nikić-Spiegel Ivana, Germany 
Nikte Siddhanta, India

Nimigean Crina, USA

Nolle Friederike, Germany

Nováček Jiří, Czech Republic

Odino Davide, Italy

Oh Yoo Jin, Austria

Olewniczak Michal, Poland

Olmeda Barbara, Spain

Oravczová Veronika, Slovakia

Pabst Georg, Austria

Palaia Ivan, United Kingdom

Parra-Ortiz Elisa, Denmark

Penedo-Esteiro Juan, United Kingdom

Perego Laura, Italy

Pereira Manuela, Portugal

Perez-Gil Jesus, Spain

Petrišič Nejc, Slovenia

Pfannenstill Veronika, United Kingdom

Pfeffermann Jürgen, Austria

Philippi Michael, Germany

Picas Laura, France

Piccirilli Federica, Italy

Piehler Jacob, Germany

Pierzynska-Mach Agnieszka, Italy

Piliarik Marek, Czech Republic

Pilic Johannes, Austria

Piller Paulina, Austria

Pinigin Konstantin, Russian Federation

Pinto Giulia, Italy

Plochberger Birgit, Austria

Pluhackova Kristyna, Germany

Podobnik Marjetka, Slovenia

Pohjolainen Emmi, Germany

Pohl Elena, Austria

Pohl Peter, Austria

Pöhnl Matthias, Germany

Põldsalu Inga, Norway

Pollack Yoav, Germany

Poojari Chetan, Germany

Popova Marina, Russian Federation

Popovic Tamara, Serbia

Prakaash Dheeraj, United Kingdom

Pramanik Shreya, Germany

Prassl Ruth, Austria

Pringle Helen, United Kingdom

Pupkis Vilmantas, Lithuania

Quoika Patrick, Austria

Radler Philipp, Austria
Ramadan Shahenda, Germany

Ramirez-Hoyos Patricio, Spain

Ranade Varun, India

Rao Rajas, France

Rauch Philipp, Netherlands

Raynal Bertrand, France

Reglinski Katharina, Germany

Reimhult Erik, Austria

Reina Francesco, Germany

Reiter-Scherer Valentin, Germany

Renault Ludovic, Netherlands

Rentmeister Andrea, Germany

Reuten Raphael, Denmark

Reuter Nathalie, Norway

Rheinlaender Johannes, Germany

Ricci Caterina, Italy

Ridolfi Andrea, Italy

Rief Matthias, Germany

Rinaldi Francesco, Italy

Riniker Sereina, Switzerland

Riopedre Fernandez Miguel, Czech Republic

Robinson Carol, United Kingdom

Robles-Ramos Miguel Ángel, Spain

Rodnina Marina, Germany

Rog-Zielinska Eva, Germany

Rohatschek Andreas, Austria

Roke Sylvie, Switzerland

Romanò Sabrina, Italy

Roos Wouter H., Netherlands

Rosenblum Gabriel, Israel

Rufin Manuel, Austria

Ruppelt Dominik, Germany

Ruprecht Verena, Spain

Sacconi Leonardo, Italy

Sacquin-Mora Sophie, France

Sadeghi Mohsen, Germany

Saghaei Tayebeh, Austria

Sahoo Harekrushna, India

Sallinger Matthias, Austria

Salzer Isabella, Austria

Sandu Nicoleta, Romania

Sant Vrinda, Germany

Santos Nuno C., Portugal

Saponaro Andrea, Italy

Sari Merve, Germany

Scattolini Valentina, Italy

Schepers Anna, Germany

Schindl Rainer, Austria 
Schlotter Til, Switzerland

Schmidt Tony, Austria

Schneider Matthias, United Kingdom

Schneider Magdalena, Austria

Schrangl Lukas, Austria

Schroeder Indra, Germany

Schromm Andra, Germany

Schubeis Tobias, France

Schütz Gerhard, Austria

Schwaighofer Andreas, Austria

Scodellaro Riccardo, Italy

Scollo Federica, Czech Republic

Seddon John M., United Kingdom

Sedivy Arthur, Austria

Seferovic Hannah, Austria

Semeraro Enrico, Austria

Senju Yosuke, Japan

Sente Andrija, United Kingdom

Sergunova Viktoria, Russian Federation

Sevcsik Eva, Austria

Sezgin Erdinc, Sweden

Shapovalov Yuri, Kazakhstan

Shekunov Egor, Russian Federation

Sheno Iliyaz Aslihan, Denmark

Shivanna Komala, USA

Siletsky Sergey, Russian Federation

Siligan Christine, Austria

Simic Marko, Austria

Simon Márton, Hungary

Sitte Harald, Austria

Skamrahl Mark, Germany

Skorepa Ondrej, Czech Republic

Slabý Cyril, Slovakia

Smirnovienè Joana, Lithuania

Smith Ana-Suncana, Germany

Smolič Tina, Slovenia

Sobolevsky Alexander, USA

Socrier Larissa, Germany

Sokolov Valery, Russian Federation

Sommer Martha, Germany

Spedalieri Cecilia, Germany

Spodniakova Barbora, Slovakia

Spustova Karolina, Norway

Stampf Jan-Luca, Austria

Starke Leonhard, Germany

Steigenberger Jessica, Germany

Stender Emil G. P., Denmark

Stockner Thomas, Austria
Strancar Janez, Slovenia

Strasser Jürgen, Austria

Stratikos Efstratios, Greece

Stroffekova Katarina, Slovakia

Sudhakar Swathi, United Kingdom

Südhof Thomas, USA

Sule Kevin, Canada

Svorinic Andrea, Germany

Sych Taras, Sweden

Szöllösi Daniel, Austria

Sztacho Martin, Czech Republic

Szydlak Renata, Poland

Talafova Veronika, Slovakia

Tannoo Ryshtee Mary, France

Tans Sander, Netherlands

Tarenzi Thomas, Italy

Tashkin Vsevolod, Russian Federation

Teiwes Nikolas, Germany

Teslenko Alexandra, Switzerland

Thomson Neil, United Kingdom

Tiapko Oleksandra, Austria

Timoshenko Roman, Russian Federation

Toca-Herrera Jose Luis, Austria

Tomancak Pavel, Germany

Tomkova Maria, Slovakia

Torreno-Pina Juan Andres, Spain

Torres Vidal Patricia, Spain

Tóth Ágota, France

Trauner Dirk, USA

Trianni Alberta, Italy

Troiano Cassandra, Italy

Trollmann Marius, Germany

Tubiana Thibault, Norway

Tutkus Marijonas, Lithuania

Uetrecht Charlotte, Germany

Ulloa Severino Luisa, Canada

Unksov Ivan, Sweden

Urbancic Iztok, Slovenia

Uriati Eleonora, Italy

V Guzman Horacio, Slovenia

V S Pillai Visakh, Ireland

Vácha Robert, Czech Republic

Valdivieso González David, Spain

van der Merwe Anton, United Kingdom

Vaneev Alexander, Russian Federation

Vanik Vladimir, Slovakia

Varlamova Ekaterina, Russian Federation

Vattulainen Ilpo, Finland 
Velas Lukas, Austria

Velísková Martina, Slovakia

Ventura Salvador, Spain

Venturini Valeria, Spain

Villalba-Riquelme Eva María, Spain

Vlahovič Ines, Croatia

Volovik Marta, Russian Federation

von Eysmondt Hendrik, Germany

Vuletic Tomislav, Croatia

Wachlmayr Johann, Austria

Walczewska-Szewc Katarzyna, Poland

Waldherr Linda, Austria

Wan Qi, Germany

Wanunu Meni, USA

Weber Andreas, Austria

Weber Florian, Sweden

Weinert Tobias, Switzerland

Weitz David, USA

Wilkinson Anthony J., United Kingdom

Wilkowska Michalina, Poland

Williamson Gordon, United Kingdom

Wingbermühle Sebastian, Germany

Winkelmann Hauke, Germany
Witt Hannes, Netherlands

Wolf Steffen, Germany

Woziwodzka Anna, Poland

Wu Yu-Le, Germany

Wurl Anika, Germany

Xue Lin, Norway

Yonath Ada, Israel

Young Robert, USA

Zare Eelanjegh Elaheh, Switzerland

Zarubin Mikhail, Russian Federation

Závodszky Péter, Hungary

Zbiral Barbara, Austria

Zdorevskyi Oleksii, Ukraine

Zemła Joanna, Poland

Zhang Jin, USA

Zhao Ziqing Winston, Singapore

Zheng Tiantian, Canada

Zhu Rong, Austria

Zhurgenbayeva Gaukhar, Germany

Ziegler Christine, Germany

Zikic Dejan, Serbia

Zimova Lucie, Czech Republic

Zuna Kristina, Austria 